\section{ENVIRONMENTAL RESTORATION PROGRAM}

\section{Level III Baseline Risk Evaluation for Building 3506 at Oak Ridge National Laboratory, Oak Ridge, Tennessee}

\author{
K. M. Golden \\ S. K. Robers \\ F. M. Cretella
}

\section{DISCLAIMER}

This report was prepared as an account of work sponsored by an agency of the United States Government. Neither the United States Government nor any agency thereof, nor any of their employees, makes any warranty, express or implied, or assumes any legal liability or responsibility for the accuracy, completeness, or usefulness of any information, apparatus, product, or process disclosed, or represents that its use would not infringe privately owned rights. Reference herein to any specific commercial product, process, or service by trade name, trademark, manufacturer, or otherwise does not necessarily constitute or imply its endorsement, recommendation, or favoring by the United States Government or any agency thereof. The views and opinions of authors expressed herein do not necessarily state or reflect those of the United States Government or any agency thereof.

MARTIN MARIETTA ENERGY SYSTEMS, INC. 
This report has been reproduced directly from the best available copy.

Available to DOE and DOE contractors from the Office of Scientific and Technical Information, P.O. Box 62, Oak Ridge, TN 37831; prices available from 615-576-8401, FTS 626-8401.

Available to the public from the National Technical Information Service, U.S. Department of Commerce, 5285 Port Royal Rd., Springfield, VA 22161. 


\section{DISCLAIMER}

Portions of this document may be illegible in electronic image products. Images are produced from the best available original document. 


\title{
Level III Baseline Risk Evaluation for Building 3506 at Oak Ridge National Laboratory, Oak Ridge, Tennessee
}

\author{
K.M. Golden \\ S.K. Robers \\ F.M. Cretella
}

Date Issued-December 1994

Prepared by

Health Sciences Research Division

Oak Ridge National Laboratory

Oak Ridge, Tennessee

\section{Prepared for}

U.S. Department of Energy

Office of Environmental Restoration and Waste Management under budget and reporting code EW 20

OAK RIDGE NATIONAL LABORATORY

Oak Ridge, Tennessee 37831-6285

managed by

MARTIN MARIETTA ENERGY SYSTEMS, INC.

for the

U.S. DEPARTMENT OF ENERGY

under contract DE-AC05-84OR21400 
THIS PAGE INTENTIONALLY LEFT BLANK 


\section{CONTENTS}

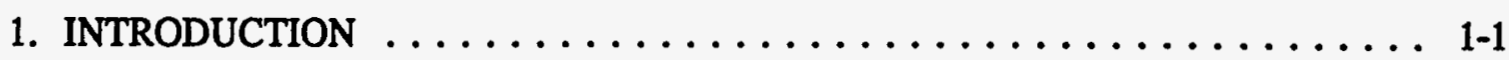

1.1 PURPOSE AND SCOPE OF THE BRE $\ldots \ldots \ldots \ldots \ldots \ldots \ldots \ldots \ldots$

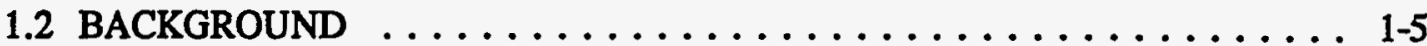

1.3 ORGANIZATION OF THE REPORT $\ldots \ldots \ldots \ldots \ldots \ldots \ldots \ldots \ldots$

2. DATA COLLECTION AND EVALUATION $\ldots \ldots \ldots \ldots \ldots \ldots \ldots \ldots$ 2-1

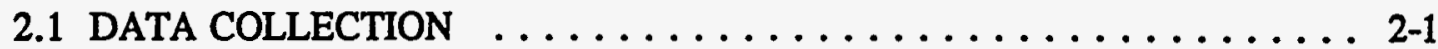

2.1.1 Characterization Data at Building $3506 \ldots \ldots \ldots \ldots \ldots \ldots \ldots \ldots$ 2-1

2.1.2 Potential Pathways and Exposure Points ............ 2-2

2.1.3 Preliminary Contaminants of Potential Concern ........... 2-9

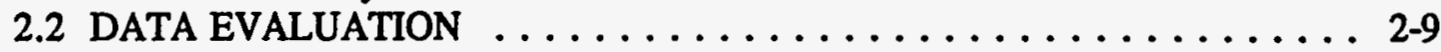

2.2.1 Data Quality Requirements . . . . . . . . . . . . 2-9

2.2.2 Evaluation of Existing Characterization Data .......... 2-10

2.3 SELECTION OF CONTAMINANTS OF POTENTIAL CONCERN . . . . . 2-11

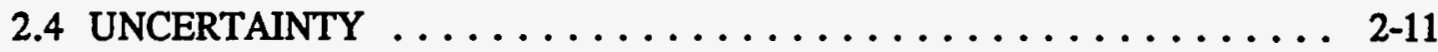

3. EXPOSURE ASSESSMENT $\ldots \ldots \ldots \ldots \ldots \ldots \ldots \ldots \ldots \ldots$ 3-1

3.1 CHARACTERIZATION OF THE EXPOSURE SETTING $\ldots \ldots \ldots \ldots \ldots 3-1$

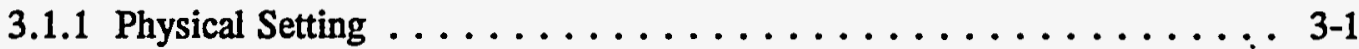

3.1.2 Potentially Exposed Populations $\ldots \ldots \ldots \ldots \ldots \ldots \ldots$ 3-12

3.2 IDENTIFICATION OF POTENTIAL EXPOSURE PATHWAYS $\ldots \ldots \ldots$ 3-20

3.2.1 Potential Sources and Receiving Media $\ldots \ldots \ldots \ldots \ldots \ldots$ 3-22

3.2.2 Potential Exposure Points and Exposure Routes $\ldots \ldots \ldots \ldots \ldots$ 3-23

3.2.3 Summary of Potential Exposure Pathways . . . . . . . . . 3-23

3.3 QUANTIFICATION OF EXPOSURE $\ldots \ldots \ldots \ldots \ldots \ldots \ldots \ldots .3 .25$

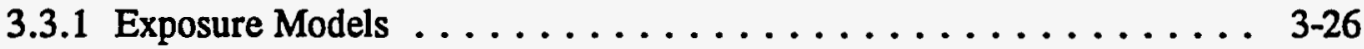

3.3.2 Exposure Parameters . . . . . . . . . . . . . . . . 3 3-29

3.3.3 Exposure Concentrations . . . . . . . . . . . . . 3-31

3.4 RESULTS OF THE EXPOSURE ASSESSMENT $\ldots \ldots \ldots \ldots \ldots \ldots \ldots .34$

3.5 UNCERTAINTY $\ldots \ldots \ldots \ldots \ldots \ldots \ldots \ldots \ldots \ldots \ldots \ldots \ldots \ldots \ldots \ldots$

4. TOXICITY ASSESSMENT $\ldots \ldots \ldots \ldots \ldots \ldots \ldots \ldots \ldots \ldots \ldots$ 4-1

4.1 TOXICITY PROFILES $\ldots \ldots \ldots \ldots \ldots \ldots \ldots \ldots \ldots \ldots \ldots \ldots \ldots \ldots$

4.1.1 Contaminants Evaluated Quantitatively ............ 4-1

4.1.2 Contaminants Evaluated Qualitatively $\ldots \ldots \ldots \ldots \ldots \ldots \ldots$ 4-23

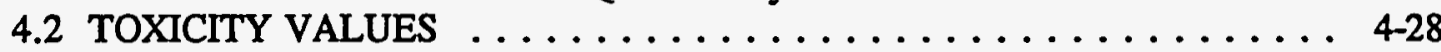

4.2.1 Toxicity Information for Noncarcinogenic Effects . . . . . . . 4-28

4.2.2 Toxicity Information for Carcinogenic Effects $\ldots \ldots \ldots \ldots \ldots$ 4-30

4.2.3 Chemicals Without EPA Toxicity Values $\ldots \ldots \ldots \ldots \ldots \ldots$ 4 4-30

5. RISK CHARACTERIZATION $\ldots \ldots \ldots \ldots \ldots \ldots \ldots \ldots \ldots \ldots \ldots$ 5-1

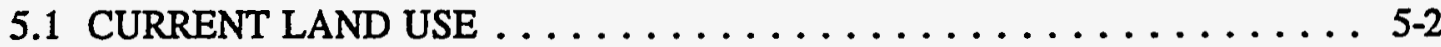

5.1 .1 Excess Cancer Risk ................... 5-3

5.1 .2 Noncarcinogenic Effects . . . . . . . . . . . . . . . . 5-9 


\section{CONTENTS (continued)}

5.2 FUTURE LAND USE $\ldots \ldots \ldots \ldots \ldots \ldots \ldots \ldots \ldots \ldots \ldots \ldots, 5-12$

5.2.1 Excess Cancer Risks ................... 5-12

5.2.2 Noncarcinogenic Effects . . . . . . . . . . . . . . . . 5 5-19

5.3 SUMMARY OF RISK CHARACTERIZATION $\ldots \ldots \ldots \ldots \ldots \ldots \ldots$ 5-19

5.4 EVALUATION OF UNCERTAINTY $\ldots \ldots \ldots \ldots \ldots \ldots \ldots \ldots .5-22$

5.4.1 Uncertainties and Assumptions for Chemical Risk Evaluation . . . . 5 5-22

5.4.2 Uncertainties in Radiological Risk Evaluation . . . . . . . 5 5-26

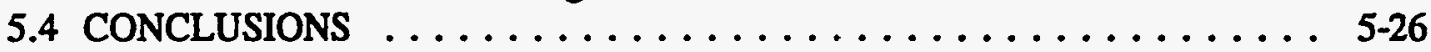

6. REFERENCES $\ldots \ldots \ldots \ldots \ldots \ldots \ldots \ldots \ldots \ldots \ldots \ldots \ldots \ldots \ldots \ldots \ldots$

APPENDIX A $\ldots \ldots \ldots \ldots \ldots \ldots \ldots \ldots \ldots \ldots \ldots \ldots \ldots \ldots \ldots \ldots \ldots \ldots \ldots \ldots$ APPENDIX B $\ldots \ldots \ldots \ldots \ldots \ldots \ldots \ldots \ldots \ldots \ldots \ldots \ldots \ldots \ldots \ldots \ldots \ldots \ldots \ldots \ldots \ldots$ APPENDIX C $\ldots \ldots \ldots \ldots \ldots \ldots \ldots \ldots \ldots \ldots \ldots \ldots \ldots \ldots \ldots \ldots \ldots \ldots \ldots \ldots \ldots, 1$

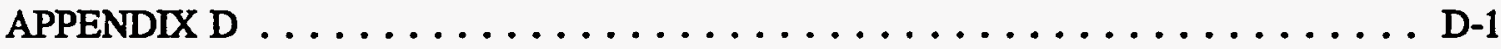

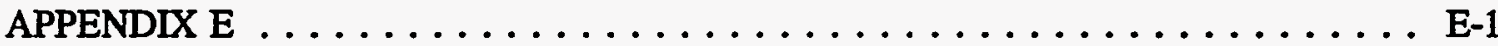




\section{FIGURES}

1.1 DOE Oak Ridge Reservation and Vicinity $\ldots \ldots \ldots \ldots \ldots \ldots \ldots \ldots$ 1-2

1.2 Overview of the BRE Process $\ldots \ldots \ldots \ldots \ldots \ldots \ldots \ldots \ldots \ldots \ldots$

1.3 Operable Units in WAG 1 at ORNL $\ldots \ldots \ldots \ldots \ldots \ldots \ldots \ldots \ldots$

1.4 Facilities in the GAAT OU $\ldots \ldots \ldots \ldots \ldots \ldots \ldots \ldots \ldots \ldots \ldots$

2.1 Building 3506 sampling locations $\ldots \ldots \ldots \ldots \ldots \ldots \ldots \ldots \ldots \ldots$

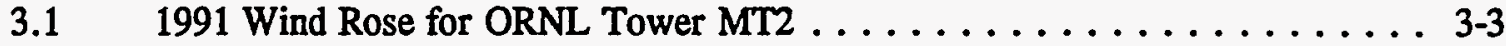

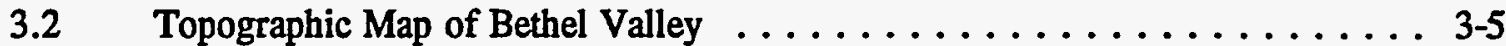

3.3 Stratigraphic Section in Bear Creek Valley, Chestnut Ridge, Bethel Valley, Haw Ridge,

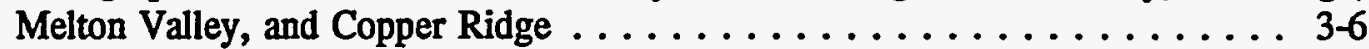

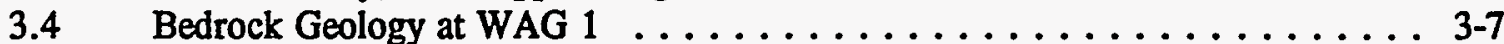

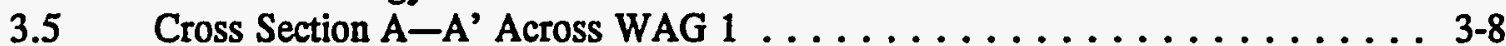

3.6 Schematic Vertical Relationship of Flow in Aquitards of the ORR . . . . . 3-10

3.7 Water Table in the Vicinity of the GAAT OU Under High Flow Conditions, April 1991 . . . . . . . . . . . . . . . . . . . . . . . 3 3-11

3.8 DOE Oak Ridge Reservation and Geographic Region . . . . . . . . 3-13

3.9 DOE Oak Ridge Reservation and Vicinity . . . . . . . . . . . 3-14

3.10 Projected 1991 Population Densities within 10 miles $(16 \mathrm{~km})$ of the Center of the Oak Ridge Reservation ....................... 3-16

3.11 Projected 1991 Population Densities within 50 miles $(80 \mathrm{~km})$ of the Center of the Oak Ridge Reservation ..................... 3-17

3.12 Conceptual Site Model for Building $3506 \ldots \ldots \ldots \ldots \ldots \ldots$. . . . . . . 


\section{TABLES}

2.1 TLD string summary data in cell $\ldots \ldots \ldots \ldots \ldots \ldots \ldots \ldots . \ldots \ldots$

2.2 Summary results of location-specific measurements in the east gallery . . . . 2-4

2.3 Summary results of location-specific measurements in the north/south cell area . 2-5

2.4 Sampling summary for Building $3506 \ldots \ldots \ldots \ldots \ldots \ldots \ldots \ldots \ldots \ldots .7$

2.5 Summary results for radiological analysis for core and sediment samples . . . . 2-8

2.6 Contaminants evaluated quantitatively $\ldots \ldots \ldots \ldots \ldots \ldots \ldots \ldots, 2-12$

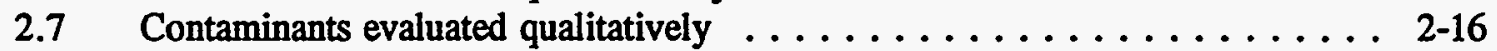

3.1 Exposure scenarios and potential receptors for the Building $3506 \ldots \ldots \ldots 3-18$

3.2 Selection of potentially complete current exposure pathways $\ldots \ldots \ldots \ldots$ 3-24

3.3 Selection of potential future exposure pathways $\ldots \ldots \ldots \ldots \ldots \ldots \ldots$ 3-27

3.4 Exposure variables for Building 3506 exposure assessment . . . . . . . . 3-30

3.5 Data evaluated for each exposure pathway $\ldots \ldots \ldots \ldots \ldots \ldots \ldots \ldots, 3-32$

4.1 Toxicity values: potential carcinogenic effects contaminants evaluated quantitatively $\ldots \ldots \ldots \ldots \ldots \ldots \ldots \ldots \ldots \ldots \ldots$ 4-31

4.2 Toxicity values: potential carcinogenic effects contaminants evaluated qualitatively $\ldots \ldots \ldots \ldots \ldots \ldots \ldots \ldots \ldots . \ldots \ldots$ 4 434

4.3 Toxicity values: potential noncarcinogenic effects for Building 3506 COPCs - 4-35

5.1 Summary risk characterization results-cancer risk estimates-maintenance worker5-4

5.2 Summary risk characterization results-cancer risk estimates-S\&M worker . . 5-6

5.3 Summary risk characterization results-noncancer hazard index estimates-maintenance worker . . . . . . . . . . . . . . . . . . . 5-10

5.4 Summary risk characterization results-noncancer hazard index estimates-S\&M worker $\ldots \ldots \ldots \ldots \ldots \ldots \ldots \ldots \ldots \ldots \ldots \ldots \ldots \ldots \ldots \ldots \ldots$ 5-13

5.5 Summary risk characterization results-cancer risk estimates-adult trespasser 5-15

5.6 Summary risk characterization results-noncancer hazard index estimates-adult trespasser . . . . . . . $5-20$

5.7 Risks that increase the chance of death by one part in one million (1E-06) $\ldots$ 5-22

5.8 Site-specific uncertainty factors $\ldots \ldots \ldots \ldots \ldots \ldots \ldots \ldots \ldots \ldots \ldots \ldots$ 


\section{ACRONYMS AND ABBREVIATIONS}

\begin{tabular}{|c|c|}
\hline AEC & Atomic Energy Commission \\
\hline ATSDR & Agency for Toxic Substances and Disease Registry \\
\hline BEIAS & Biomedical and Environmental Information Analysis Section \\
\hline BRE & Baseline Risk Evaluation \\
\hline BZA & Breathing Zone Apparatus \\
\hline CDI & Chronic Daily Intake \\
\hline CERCLA & Comprehensive Environmental Response, Compensation, and Liability Act \\
\hline CEW & Clinton Engineer Works \\
\hline CLP & Contract Laboratory Procedure \\
\hline CNS & Central Nervous System \\
\hline CSM & Conceptual Site Model \\
\hline D\&D & Decomtamination and Decommissioning \\
\hline DNA & Deoxyribonucleic Acid \\
\hline DOE & United States Department of Energy \\
\hline DQR & Data Quality Requirement \\
\hline EPA & United States Environmental Protection Agency \\
\hline ER & Environmental Restoration \\
\hline FFA & Federal Facility Agreement \\
\hline FS & Feasibility Study \\
\hline GAAT & Gunite and Associated Tanks \\
\hline GI & Gastro-Intestinal \\
\hline HEAST & Health Effects Assessment Summary Tables \\
\hline HEW & Hanford Engineer Works \\
\hline $\mathrm{HI}$ & Hazard Index \\
\hline $\mathrm{HQ}$ & Hazard Quotient \\
\hline ILCR & Incremental Lifetime Cancer Risk \\
\hline IRIS & Integrated Risk Information System \\
\hline LET & Linear Energy Transfer \\
\hline LLLW & Liquid Low Level Waste \\
\hline LOAEL & Lowest Observable Adverse Effects Level \\
\hline MF & Modifying Factor \\
\hline MMES & Martin Marietta Energy Systems, Inc. \\
\hline ND & Not Detected \\
\hline NGVD & National Geodetic Vertical Datum \\
\hline NOAEL & No Observable Adverse Effects Level \\
\hline NRC & Nuclear Regulatory Commission \\
\hline ORNL & Oak Ridge National Laboratory \\
\hline ORR & Oak Ridge Reservation \\
\hline OU & Operable Unit \\
\hline PAH & Polynuclear Aromatic Hydrocarbon \\
\hline PCB & Polychlorinated Biphenyl \\
\hline QC & Quality Control \\
\hline RAC & Risk Assessment Council \\
\hline
\end{tabular}




\section{ACRONYMS AND ABBREVIATIONS}

$\begin{array}{ll}\text { RAS } & \text { Risk Analysis Section } \\ \text { RI } & \text { Remedial Investigation } \\ \text { ROD } & \text { Record of Decision } \\ \text { S\&M } & \text { Surveillance and Maintenance } \\ \text { SCSR } & \text { Soil Characterization and Summary Report } \\ \text { SF } & \text { Slope Factor } \\ \text { SRE } & \text { Screening Risk Evaluation } \\ \text { STF } & \text { South Tank Farm } \\ \text { SWMU } & \text { Solid Waste Management Unit } \\ \text { TDEC } & \text { Tennessee Department of Environment and Conservation } \\ \text { TLD } & \text { Thermoluminescent Dosimeter } \\ \text { TVA } & \text { Tennessee Valley Authority } \\ \text { UF } & \text { Uncertainty Factor } \\ \text { UV } & \text { Ultraviolet } \\ \text { WAG } & \text { Waste Area Grouping } \\ \text { Y-12 } & \text { Oak Ridge Y-12 Plant }\end{array}$




\section{EXECUTIVE SUMMARY}

This report presents the results of the Level 3 Baseline Risk Evaluation (BRE) performed on Building 3506 located at the Oak Ridge National Laboratory (ORNL). This BRE is intended to provide an analysis of the potential for adverse health effects (current or future) posed by contaminants at the facility. The decision was made to conduct a Level 3 (least rigorous) BRE because only residual contamination exists in the building. Future plans for the facility (demolition) also preclude a rigorous analysis.

Building 3506, also known as the waste evaporator facility, was constructed during 1949. The facility consists of a cell and a gallery (east gallery). In 1954, evaporator operations ceased, and the building housed an experimental radioactive waste incinerator and ancillary equipment. The facility has been out of service since 1976, and most of the equipment and piping has been removed. Currently, no worker activity occurs within Building 3506 except for routine surveillance and maintenance ( $S \& M$ ) activities, which include routine surveys (approximately once a year) and vegetation maintenance around the building. Releases of residual contamination are not occurring from this facility.

Site characterization activities for Building 3506 were conducted in fall of 1993 . Concrete core samples were taken from the floors and walls of both the cell and the east gallery. These cores were analyzed for radionuclides and organic and inorganic chemicals. Smear samples and direct radiation measurements were also collected. Sediment exists on the floor of the cell and was also analyzed.

To adequately characterize the risks posed by the facility, receptors for both current and potential future land uses were evaluated. For the current land use conditions, two receptors were evaluated. The first receptor is a hypothetical maintenance worker who spends 250 days ( 8 hours/day) for 25 years working in the facility. The remaining receptor evaluated is a hypothetical S\&M worker who spends 2 days ( 8 hours/day) per year for 25 years working within the facility. This particular receptor best exemplifies the current worker scenario for the facility. The two current exposure scenarios and parameters of exposure (e.g., inhalation and ingestion rates) have been developed to provide a conservative (i.e. health protective) estimate of potential exposure.

This risk evaluation is intended to provide exposure and risk estimates for a "no-action" (i.e., no remedial action) future land use scenario. A hypothetical no-action scenario makes the assumption that Building 3506 is abandoned by $\mathrm{DOE}$ at the start of the risk evaluation. In this scenario, the United States Department of Energy (DOE) makes no attempt to prevent contaminant releases from the facility or restrict access to the facility. Because Building 3506 is on the Oak Ridge Reservation (ORR) and DOE does not intend to release the ORR property for development, the area around the building is expected to remain industrial even if the building is abandoned. Therefore, it is assumed that residential development will not occur at this site. However, it is further assumed that an adult trespasser could enter the building and take up residence (e.g., a squatter). An adult trespasser is evaluated as a future receptor for this risk evaluation. It is assumed that this adult lives within the facility for 30 years ( 24 hours/day, 350 days/year). While it is unlikely that an adult trespasser receptor will ever occur at this 
facility, this receptor was evaluated to provide an upperbound estimate of the potential human health risks.

If the building is abandoned by DOE (i.e., all building maintenance is discontinued), it will eventually decay and collapse. The building structure currently acts as a containment vessel for the contaminants inside. As the building decays, these contaminants will be released to the surrounding environment via wind erosion and dust transport, washout with rain, windborne vapors, and tracking of contaminants by humans and small animals potentially visiting the site. Such contaminant releases could potentially result in exposure of off-site (i.e., outside of DOE property) residential receptors. Such off-site exposures have not been included in the quantitative risk evaluation because the quantity of the radioactive and chemical contaminants is insufficient to result in significant off-site exposures. Likewise, environmental receptors are also not included in the quantitative risk evaluation. If releases of contaminants do occur from the facility, factors such as quantity and the mobility of the contaminants preclude further evaluation of risk to potential ecological receptors.

Human health risks are evaluated using human exposure results and toxicity information. Risks are estimated differently for carcinogens and noncarcinogens (systemic toxicants). For carcinogenic contaminants, risk is expressed in terms of the probability of contracting cancer over a lifetime, over and above the normal background risk. This is the Incremental Lifetime Cancer Risk (ILCR) and is estimated as the product of the estimated exposure to a carcinogen and the contaminant-specific, route-specific slope factor:

where:

$$
\mathrm{ILCR}=\mathrm{SF} \times \mathrm{CDI}
$$

$$
\begin{aligned}
& \mathrm{ILCR}=\text { Incremental lifetime cancer risk (unitless probability) } \\
& \mathrm{SF}=\text { Slope factor (mg/kg-day) })^{-1} \\
& \mathrm{CDI}=\text { Chronic daily intake (mg/kg-day) }
\end{aligned}
$$

In weighing exposures to potentially carcinogenic compounds, a reasonable level of risk must be identified. EPA specifies a risk range of $10^{-6}$ to $10^{-4}$ associated with the consideration and selection of remedial alternatives for Superfund sites (EPA, 1990). The lower end of this range is generally applied to residential risks while occupational risks may be considered acceptable at the higher end of this range. In the United States, the incidence of cancer occurrence is 3E-01 or 0.30 (American Cancer Society, 1990). The ILCR (also referred to as excess cancer risk) is defined as the estimated increased risk above background that occurs over an assumed average lifespan of 70 years as the result of exposure to a specific, known carcinogen. Therefore, an incremental lifetime cancer risk of one in one million (1E-06) may be interpreted as an increase in the baseline population cancer incidence from 300,000 per million population to 300,001 per million population. Current radiation protection standards for workers include a limit of 5 rem for the annual effective dose equivalent. This dose equates to a 25-year lifetime risk corresponding to $2 \mathrm{E}-02$. Similar protection standards for members of the public include a limit of 0.1 rem per year. This dose equates to a 70 -year lifetime risk of $4 \mathrm{E}-03$. 
Noncarcinogenic effects of contaminants are quantified as hazard quotients (HQ). An HQ is the ratio of the estimated daily intake to the allowable daily intake or RfD:

where:

$$
\mathrm{HQ}=\mathrm{CDI} / \mathrm{RfD}
$$

$\mathrm{HQ}=$ Hazard quotient (unitless)

$\mathrm{CDI}=$ Chronic daily intake (mg/kg-day)

$\mathrm{RfD}=$ Reference dose (mg/kg-day)

Because the HQ does not define exposure-response relationships, its numerical value should not be construed as a direct estimate of risk. It is a numerical indication of whether or not acceptable exposure levels are exceeded. As this quotient approaches unity, concern for the potential hazard of the contaminant increases. The HQs can be summed for each contaminant and pathway to give a hazard index (HI).

The risks and HQs calculated using the described methodology are presented in the following text. Risks and HQs were calculated for each receptor for exposure to contaminants in the cell, sediment, east gallery, and whole building (excluding sediment).

\section{Cell}

\section{Carcinogenic Risks}

Risks for the cell are presented in Table 1.

Table 1. Excess cancer risks due to external exposure from contaminants in the cell

\begin{tabular}{lc}
\hline \multicolumn{1}{c}{ Receptor } & Excess Carcinogenic Risk \\
\hline Maintenance worker & $1 \mathrm{E}-02$ \\
S\&M worker & $1 \mathrm{E}-04$ \\
Adult trespasser & $6 \mathrm{E}-02$ \\
D\&D worker & $3 \mathrm{E}-04$ \\
\hline
\end{tabular}

Risks caused by external exposure to radionuclides dominated the risks estimated for Building 3506. These risks are calculated based on the assumption that the receptors spend all of their time in the cell. The hypothetical maintenance worker excess cancer risk (calculated from core data) for external exposure to radionuclides in the cell (excluding sediment) was $1 \mathrm{E}-02$, whereas the $\mathrm{S} \& \mathrm{M}$ worker excess cancer risk (external exposure; core data) was 1E-04. The hypothetical future trespasser excess cancer risk (external exposure; core data) was 6 E-02. As mentioned previously, these risks are estimated for a worker that spends 25 years (250 days/year for the maintenance worker and 2 days/year for the S\&M worker) in the facility and a trespasser who lives in the building for 30 years. As a comparison, the excess cancer risk (external exposure to radionuclides) associated with a 
D\&D worker who would be exposed for 8 months ( 8 hours/day) is $3 \mathrm{E}-04$. It is important to note that the dose (and risk) to the D\&D worker would decrease as contaminants were removed from the building.

The external exposure risks associated with the hypothetical maintenance worker and the hypothetical aduit trespasser exceed EPA's specified risk range $\left(10^{-6}\right.$ to $\left.10^{-4}\right)$. As mentioned previously, the 25-year risk corresponding to the protection standard of 5 rem per year is 2E-02. The ORNL administrative control level during routine conditions is $1.5 \mathrm{rem} /$ year. This yearly dose equates to a 25-year lifetime risk of 6.0E-03. Using the ORNL administrative control level, only the hypothetical maintenance worker and the hypothetical adult trespasser would exceed this limit but only by a slight margin (i.e., one order of magnitude).

The radionuclide that contributed the greatest risk via the external exposure pathway is cesium-137. This trend is also evident for the other areas within the building (east gallery and whole building). Other characterization data [direct measurements and thermoluminescent dosimeter (TLD) strings] were used to calculate risks due to external exposure to radionuclides. Risks calculated using these data were comparable to that of the core data, further validating the method used to calculate risk. Risks from all other pathways (ingestion, inhalation, dermal absorption) for both chemical and radiological contaminants were all either within or below EPA's target risk range.

\section{Noncarcinogenic Effects}

No HQ exceeded unity for the individual contaminants detected in the floors and walls for the hypothetical maintenance worker, although the total HI exceeded unity. The contaminants that contributed to the HI were cadmium, mercury, and vanadium. The HQ for cadmium exceeded unity for the hypothetical adult trespasser. Other contaminants (mercury and vanadium) had HQs, although not exceeding unity, which contributed to the hazard index. The other receptors did not have HQs or HIs exceeding unity.

\section{Sediment}

\section{Carcinogenic Risks}

The greatest source of risk to receptors was associated with external exposure to radionuclides from the sediment located in the cell. Table 2 presents the risks associated with the sediment in the cell.

Table 2. Excess cancer risks due to external exposure from contaminants in the sediment

\begin{tabular}{lc}
\hline \multicolumn{1}{c}{ Receptor } & Excess Carcinogenic Risk \\
\hline Maintenance worker & $3 \mathrm{E}-01$ \\
S\&M worker & $2 \mathrm{E}-03$ \\
Adult trespasser & $8 \mathrm{E}-01$ \\
D\&D worker & $7 \mathrm{E}-03$ \\
\hline
\end{tabular}


The excess cancer risk value for the hypothetical maintenance worker was $3 E-01$. The excess cancer risks from external exposure to radionuclides for the hypothetical S\&M worker and the hypothetical adult trespasser were $2 \mathrm{E}-03$ and $8 \mathrm{E}-01$, respectively. For a comparison, a hypothetical D\&D worker would have an excess cancer risk of 7E-03. These carcinogenic risk values are all above EPA's target risk range. The hypothetical maintenance worker and the hypothetical adult trespasser also exceed the risk for the ORNL administrative control limit (6E-03) and the risk for the protection standard of $5 \mathrm{rem} / \mathrm{year}(2 \mathrm{E}-02)$. Again, the radionuclide cesium-137 contributed the greatest carcinogenic risk via external exposure to receptors. Other characterization data (direct measurements and TLD strings) were used to calculate risks due to external exposure to radionuclides. Risks calculated using these data were comparable to that of the core data, further validating the method used to calculate risk.

Unlike the excess cancer risks associated with the cell structure (i.e., walls and floors), carcinogenic risks determined for one chemical contained in the sediment exceeded EPA's target risk range. The ingestion pathway and the dermal pathway for the adult trespasser had overall risk values of $2 \mathrm{E}-03$ and $1 \mathrm{E}-03$, respectively. The contaminant responsible for these risk values is Aroclor-1254. Risks from all other pathways and receptors were either within or below EPA's risk range.

\section{Noncarcinogenic Effects}

For noncarcinogenic effects, the only chemical that had HQs exceeding unity was mercury (via the dermal pathway), indicating that safe exposure levels are exceeded. The hypothetical maintenance worker, the hypothetical adult trespasser, and the D\&D worker were the receptors that had HQs exceeding unity. No other chemical had an HQ that exceeded unity.

\section{East Gallery}

\section{Carcinogenic Risks}

Excess carcinogenic risks to receptors in the east gallery were much lower compared to the cell. These risks are calculated based on the assumption that the receptors spend all of their time in the east gallery. Only one receptor had a risk value that exceeded EPA's target risk range; the excess cancer risk for the adult trespasser (core data) was $1 \mathrm{E}-03$. As before, the radionuclide that contributed to this risk value is cesium-137. All other contaminants (chemical and radionuclides) and pathways had risk values within or below EPA's risk range.

\section{Noncarcinogenic Effects}

No HQ exceeded unity for contaminants in the east gallery, indicating that safe exposure levels have not been exceeded. 


\section{Whole Building}

\section{Carcinogenic Risks}

Excess cancer risks due to external exposure to radionuclides were the greatest risks estimated for the whole building. These risks are calculated based on the assumption that the receptors spend their time in the cell and the east gallery. Whole building risk values do not include the risks associated with the sediment in the cell. Table 3 presents the risks associated with the whole building.

Table 3. Excess cancer risks due to external exposure from contaminants in the whole building

\begin{tabular}{l|c}
\hline \multicolumn{1}{c}{ Receptor } & Excess Carcinogenic Risk \\
\hline Maintenance worker & $1 \mathrm{E}-02$ \\
S\&M worker & $1 \mathrm{E}-04$ \\
Adult trespasser & $6 \mathrm{E}-02$ \\
D\&D worker & $3 \mathrm{E}-04$ \\
\hline
\end{tabular}

Excess cancer risks due to external exposure exceeded EPA's target risk range for the hypothetical maintenance worker and the hypothetical adult trespasser. These risk values also exceeded the risk calculated for ORNL's administrative control limit (6E-03). The other two receptors were within EPA's target risk range. Again, the radionuclide cesium-137 contributed the greatest carcinogenic risk via the external exposure to receptors. Risks from all other pathways (ingestion, inhalation, dermal absorption) for both chemical and radiological contaminants were all either within or below EPA's target risk range.

\section{Noncarcinogenic Effects}

No HQ exceeded unity for the individual contaminants detected in the floors and walls for the hypothetical maintenance worker, although the total HI did exceed unity. The contaminants that contributed to the HI were cadmium, mercury, and vanadium. The HQ for cadmium exceeded unity for the hypothetical adult tresplasser. Other contaminants (mercury and vanadium) had HQs, although not exceeding unity, which contributed to the HI. The other receptors did not have HQs or HIs exceeding unity.

\section{Risk Perspective}

To place the results presented in this risk assessment in perspective, one should consider the probability of an individual's developing cancer from unavoidable exposure to naturally occurring background radiation. The lifetime risk of cancer incidence from background radiation in the general population is approximately 1E-02 (MMES, 1993). This value is 100 times greater than the upperbound $\left(10^{-4}\right)$ of EPA's target risk range. In addition, Table 4 lists activities that increase the chance of cancer fatality by $1 \mathrm{E}-06$. It should be noted that these values are for fatal cancers, not the incidence of cancer. 
Table 4. Risks that increase the chance of death by one part in one million (1E-06)

\begin{tabular}{ll}
\hline \multicolumn{1}{c}{ Activity } & \multicolumn{1}{c}{ Cause of death } \\
\hline Smoking 1.4 cigarettes & Cancer, heart disease \\
Flying 6000 miles by jet & Cancer caused by cosmic radiation \\
Living 2 months in average stone or & Cancer from natural radioactivity \\
brick building & \\
Eating 40 tablespoons of peanut butter & Liver cancer caused by aflatoxin B \\
\hline
\end{tabular}

Taken from Wilson, 1979

The results of the BRE for Building 3506 indicate that long-term exposures to contaminated media inside the building pose a potential health risk. While it is unlikely that adult trespasser exposures will ever occur at the facility, these exposures were evaluated to provide an upperbound estimate of the potential human health risks. Current exposures to contaminated media at Building 3506 indicate that worker protection is necessary to ensure worker safety. Future health risks from exposures to contaminants are estimated to be above levels of concern as set by EPA. In summary, external exposure is the major concern for this building, and sediment is the major contributing factor in terms of the potential for adverse human health effects. 
THIS PAGE INTENTIONALLY LEFT BLANK

xviii 


\section{INTRODUCTION}

As part of its ongoing Environmental Restoration Program, the U.S. Department of Energy (DOE) has reduced operations at various federal facilities, including the Oak Ridge Reservation (ORR) in Oak Ridge, Tennessee, as missions have been determined obsolete or have been relocated. Many of the buildings at these facilities may pose a threat to human health and the environment as a result of radioactive and/or chemical contaminants remaining at the site. Therefore, these buildings have been placed in the DOE's Decontamination and Decommissioning (D\&D) Program for further evaluation and remediation. Elimination of potential environmental or public health risk, the significant reduction of the risk of adverse publicity, and the decrease in the risk of potential personnel contamination caused by these buildings are significant goals to be achieved by the D\&D Program (Martin Marietta Energy Systems, 1992a).

The following information was taken from Remedial Investigation Report Feasibility Study For The Gunite and Associated Tanks Operable Unit At Waste Area Grouping 1 (Radian, 1993). DOE's ORR is in eastern Tennessee, approximately 25 miles $(40 \mathrm{~km})$ west of Knoxville (Fig. 1.1). The ORR was originally referred to as the Clinton Engineer Works (CEW), a name derived from the nearby small town of Clinton, Tennessee. The site was selected on September 19, 1942, as the location of several facilities that were part of the Manhattan Engineer District. The name "Oak Ridge" came into general use in the summer of 1943, when it was chosen for the new community's permanent housing area.

The X-10 Site, which later became the Oak Ridge National Laboratory (ORNL), is one of three facilities constructed on the ORR in 1943. The other two facilities, the K-25 Site and the Y-12 Plant, primarily focused on the production of enriched uranium, while X-10 served as a temporary pilot model for plutonium production facilities being built at the Hanford Engineer Works (HEW) in eastern Washington State. The X-10 facility originally had a planned life of only one year, but given the need for nuclear research, ORNL has operated continuously since that time.

With its wartime mission completed, the CEW was transferred in 1947 from the Manhattan Engineer District to the Atomic Energy Commission (AEC) (now known as DOE), a civilian agency created to provide oversight of the nation's nuclear industry. The CEW was renamed the ORR in 1947 after the establishment of the AEC.

The scope and direction of ORNL programs have seen many changes over the years, including the continuation and expansion of nuclear reactor fuel reprocessing research, large scale production of radioisotopes, and operation of a variety of reactors. Many of the activities of the past 50 years generated radioactive and mixed (containing both radioactive and hazardous components) waste materials. Management of these radioactive wastes has been of primary importance since the beginning of operations and has evolved over time.

Building 3506 (Waste Evaporator Facility) at ORNL, is managed by Martin Marietta Energy Systems, Inc. (Energy Systems) and has been placed in the ORNL D\&D Program within the Energy Systems Environmental Restoration (ER) Program. Actions may need to be taken with Building 3506 to avoid the potential spread of contamination and human and environmental exposures as the building and equipment deteriorate. The Risk Analysis Section (RAS) of Oak 


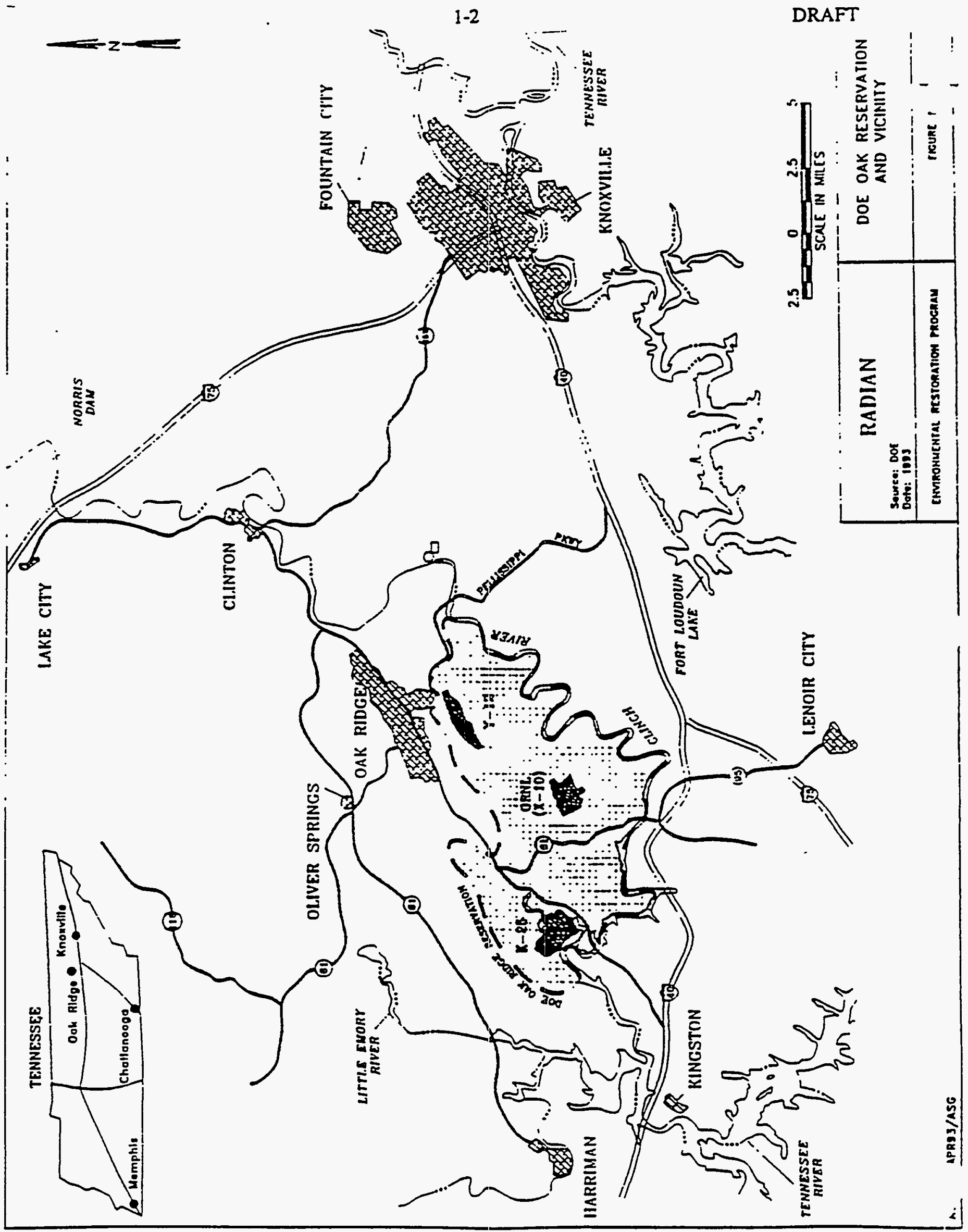

GAT OU R.

Fig. 1.1. DOE Oak Ridge Reservation and Vicinity 
Ridge National Laboratory has been tasked with the preparation of a Baseline Risk Evaluation (BRE) for Building 3506 to assess the potential risks to human health and the environment posed by current and potential future conditions at this facility. This BRE was prepared following the methodology being developed for the D\&D Program by RAS.

The methodology includes a tiered or graded approach to conducting the BRE. The purpose of the tiered approach is to save time and effort at facilities that do not warrant a rigorous baseline risk evaluation. The level of detail of the evaluation is measured by the amount and resolution of the data used and the sophistication of the analysis employed. The methodology includes guidance for conducting BREs at three levels of effort:

- Level 1 requires a full characterization of the facility and provides a complete quantitative "CERCLA-like" BRE. Two exposure scenarios are evaluated: abandonment and continued S\&M. A preliminary end-point analysis is also provided.

- Level 2 includes an extensive characterization of the facility but may be less rigorous than Level 1. The abandonment scenario is evaluated, and the continued S\&M scenario is optional. The preliminary end-point analysis is optional for this evaluation. The primary differences between the Level 2 and Level 1 evaluations are the amount of characterization data available and the level of fate and transport modeling applied.

- Level 3 uses minimal characterization data similar to that used for the screening risk evaluation (SRE). This assessment relies heavily on historic knowledge of the facility. The quantitative evaluation has a greater uncertainty than levels 1 and 2 and therefore uses more "worst-case" assumptions. Only one exposure scenario (abandonment) is evaluated.

All three levels are designed to conform to EPA $(1988,1989 a, 1989 b, 1990,1991)$ guidance for conducting BREs under CERCLA.

\subsection{PURPOSE AND SCOPE OF THE BRE}

The purpose of this report is to present the BRE for Building 3506. The decision to conduct a Level 3 BRE was based on the fact that only residual surface contamination exists in the building; therefore, a rigorous analysis is not warranted. Future plans for the facility (demolition) also preclude a rigorous analysis. This BRE uses many "worst case" assumptions for potential exposures. The purpose of this risk evaluation is to provide interested stakeholders (managers, regulators, and the general public) with a preliminary estimation of any potential human health and environmental threats posed by contaminants at Building 3506 . Characterization activities have been performed in an effort to plan the D\&D of the facility.

The BRE is a four-stage process that provides an analysis of the potential for adverse health effects (current or future) caused by hazardous substances at and/or being released from a site in the absence of any actions to control or mitigate exposures and releases (EPA, 1989). Figure 1.2 provides an overview of the risk evaluation process for $D \& D$ program facilities. Data collection/evaluation is the first stage of the process. During this stage, analytical and other site specific data are used to characterize the nature and extent of contamination. The second stage, 


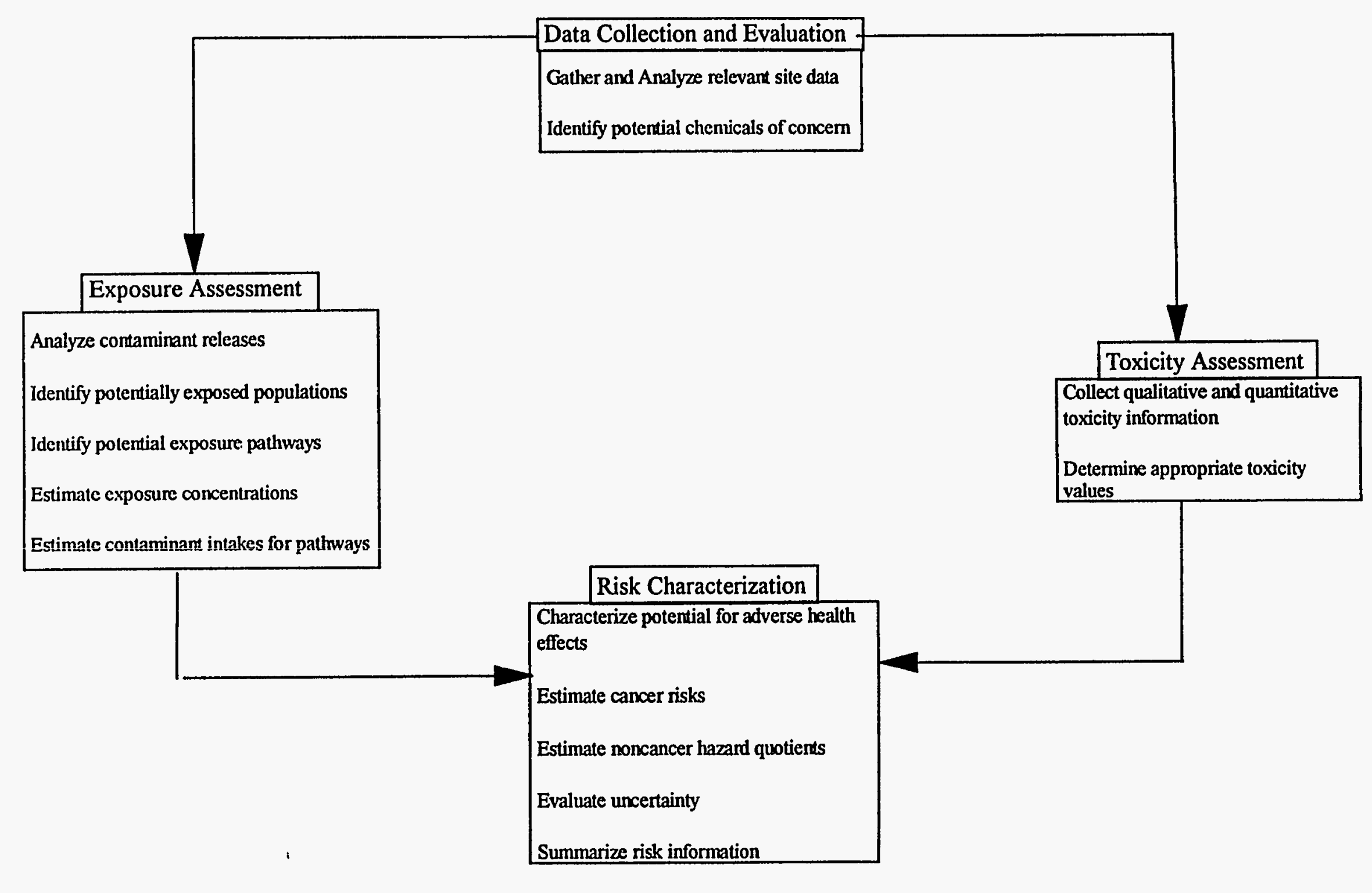

Fig. 1.2. Overview of the BRE Process 
exposure assessment, involves the estimation of exposures based on contaminant concentrations estimated from the analytical data, fate and transport models, and information collected on the demography and behavior of receptor populations. The third stage, toxicity assessment, involves the collection of information concerning the potential toxic characteristics of each contaminant. The results of the toxicity assessment are combined with the exposure estimates to quantitatively or qualitatively evaluate potential risks to human health and the environment posed by each contaminant of potential concern at the facility in the fourth stage, risk characterization (Martin Marietta Energy Systems, 1992b).

\subsection{BACKGROUND}

Building 3506 is located at ORNL within the Gunite and Associated Tanks (GAAT) Operable Unit (OU) of Waste Area Grouping 1 (WAG 1). The following background information provides the reader with information concerning the GAAT OU for orientation purposes only. This information was taken from the Remedial Investigation Report Feasibility Study For The Gunite and Associated Tanks Operable Unit At Waste Area Grouping 1 (Radian, 1993).

Oak Ridge National Laboratory is a large, multifunctional, research and development facility for the federal government, and it will continue to support applied research and engineering development programs in fission, fusion, conservation, fossil and other energy technologies, as well as perform research in the physical and life sciences. Past research and development and waste management activities have resulted in a significant number of sites or areas that have been contaminated with low-level radioactive andlor hazardous chemical wastes that will potentially require remediation. Remediation of contamination will be conducted concurrently with other ongoing operational and maintenance activities, and as a result, remediation will be technically and logistically complicated.

The approach for remediating the large number of ORNL sites is to first organize sites into Waste Area Groupings (WAGs). A WAG is a "group of solid waste management units (SWMUs) or other areas of contamination that are geographically contiguous or are located within defined hydrologic units" (DOE 1992a). Twenty WAGs were identified originally.

The GAAT OU is within ORNL WAG 1, which includes most of the original ORNL research and development facilities and associated waste management units. WAG 1 covers approximately 150 acres (60.7 ha) (Fig. 1.3). A total of 167 identified contaminated sites or areas have been classified as requiring no further action. Fourteen of the sites or areas are listed for remediation under the DOE surplus facilities program and are the responsibility of ORNL's D\&D program. The remaining 126 sites or areas include inactive underground waste storage tanks, waste burial grounds, surface impoundments, liquid low-level waste (LLLW) lines and leak sites, active underground waste storage tanks, and mercury spill areas.

The concept of the OU was developed in accordance with the Federal Facility Agreement (FFA) to address complex remediation management issues that result from the large number of sites within a WAG. According to the FFA, an OU is a discrete remedial action site designation that contributes to the final remediation of a facility or site. Each OU should be a discrete, identifiable action with a controllable scope. Where possible, physical media and waste units in 


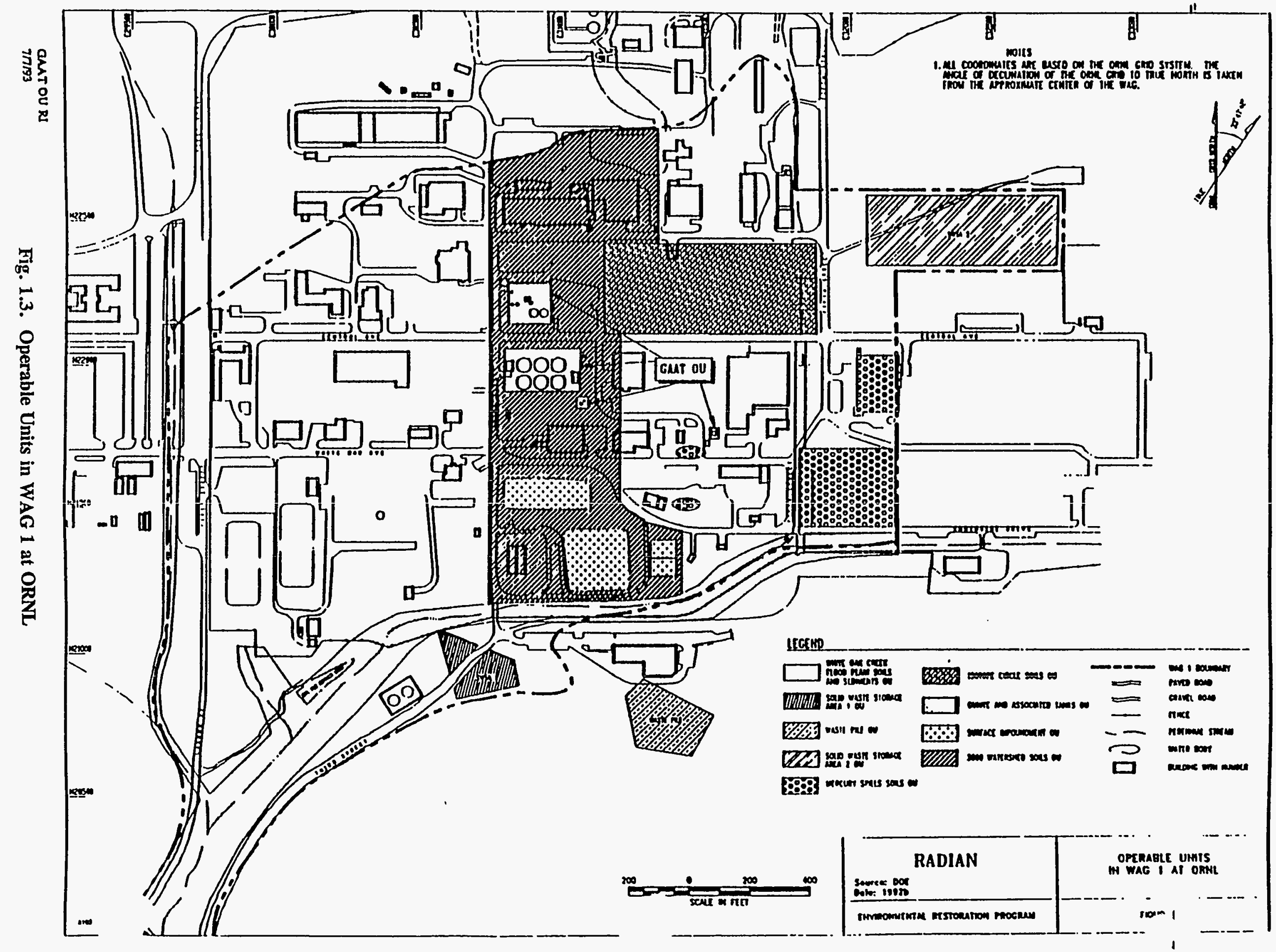


each OU should be geographically distinct from those in other OUs to minimize the potential for recontamination. However, because of the complexity of WAG 1, some overlap of OUs is inevitable and potentially desirable to ensure final remediation of WAG 1 . The flexible definition of an OU can be used to focus remediation of specific site issues. However, an OU is not defined with firmly established elements or boundaries. The boundaries of the OU can be optimized on the basis of new information or alternatives evaluation.

The WAG 1 Remedial Investigation Plan indicated that WAG 1 RI data would be collected in phases. The results of the WAG 1 Phase I RI are documented in three reports: the OU Strategy Document, the Preliminary Risk Assessment Report, and the Site Characterization Summary Report (SCSR). On the basis of information collected and documented in the SCSR, 10 OUs have been defined for WAG 1, as described in the OU Strategy Document and shown in Fig. 1.4.

The GAAT OU includes all 12 of the Gunite tanks in WAG 1 and 4 stainless steel tanks in the north tank farm which are being remediated at the same time because of their presence within the tank farm and location within the vicinity of the Gunite Tanks. These tanks have been removed from service but contain about $95 \%$ of the documented inventory of radionuclides in inactive waste management units in WAG 1.

Building 3506 is located within the GAAT OU west of the south tank farm on Third Street south of Central Avenue. The facility consists of an operation gallery and a cell area and was built in 1949 to house the first liquid waste evaporator at ORNL. The facility was operational as a liquid waste evaporator until 1954, when it underwent extensive decontamination and afterwards housed an experimental radioactive waste incinerator. In 1959, equipment for rare earth separation was installed in the building. The building was partially decommissioned some time prior to 1980 , and a filter housing and a few pipes are the only remaining equipment. In the early 1980s, the east gallery was used as a dressing room for workers in the tank farm area. No operational records that could provide information on building contamination were found.

\subsection{ORGANIZATION OF THE REPORT}

This BRE is presented in five sections. An overview of the BRE methodology and background information on Building 3506 has been presented in Sect. 1. Section 2 describes the collection and evaluation of facility characterization data. The exposure assessment is presented in Sect. 3. Section 4 presents the toxicity information for the toxicity assessment. Finally, Sect. 5 presents results of the risk characterization. 


\section{$1-8$ \\ DRAFT}

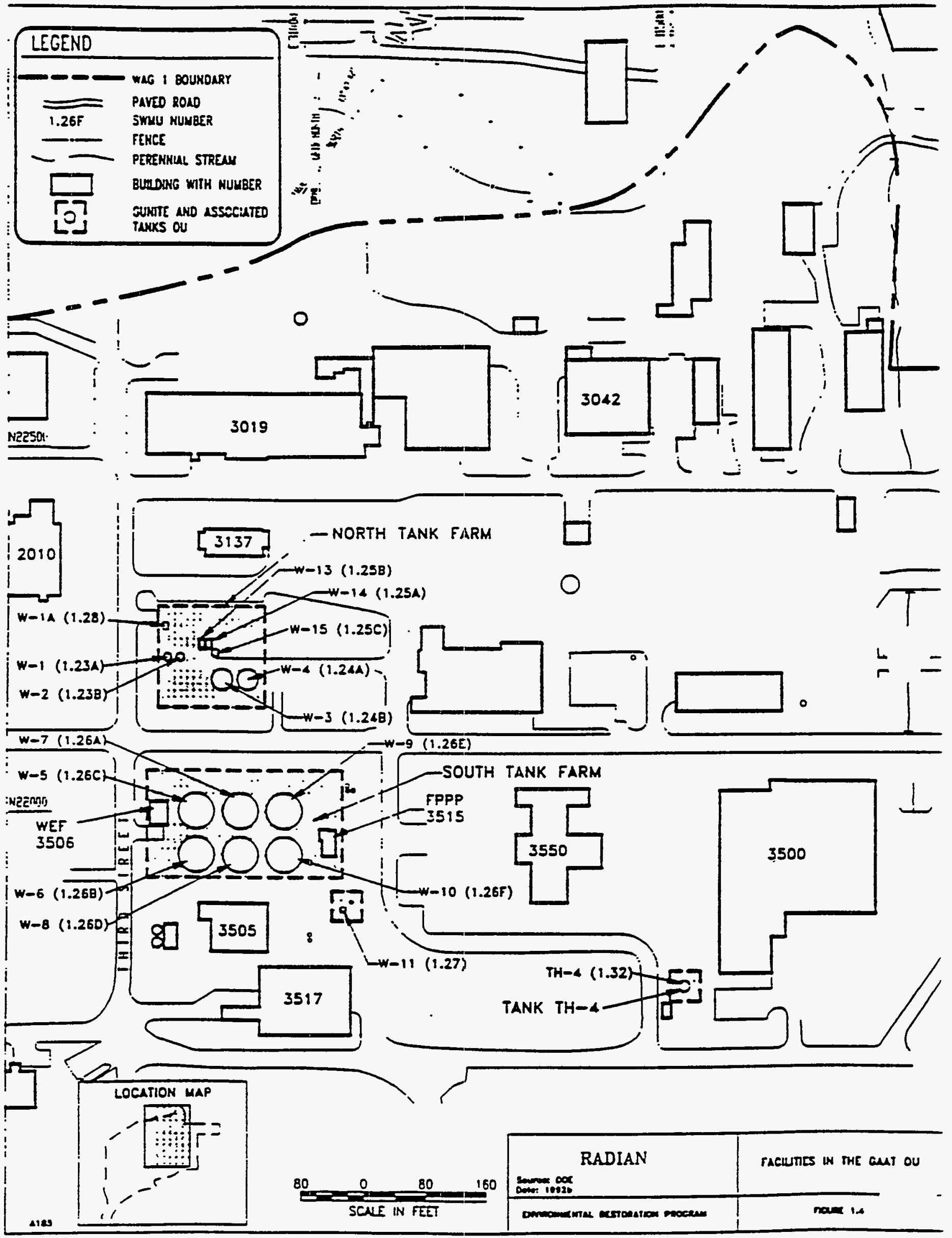




\section{DATA COLLECTION AND EVALUATION}

The objective of data collection and evaluation is the acquisition of reliable chemical, radionuclide, facility structure, and receptor population data for use in determining the potential for exposures to contaminants of potential concern that are present at or migrating from the facilities scheduled for D\&D. The purpose of the data evaluation is to assess the available data at the facility and identify any additional data that may be required to adequately characterize the potential source(s) of environmental contamination associated with the facility. This evaluation includes the collection of available data, an evaluation of data quality, and an estimation of the ability of the sampling and analytical methods used to meet the needs of a quantitative risk evaluation. A list of the chemicals of potential concern (constituents likely to be present and contribute to the potential risks) at the facility is then prepared.

The data evaluation generally is accomplished in two stages. First, an evaluation of existing data is conducted. In this initial evaluation, available site information is reviewed to 1) determine basic site characteristics, 2) initially identify potential exposure pathways and exposure points, and 3) help determine additional data needs (EPA, 1989). After this initial data evaluation, additional sampling and analyses may be performed to fill data gaps identified in the initial evaluation, and a final data analysis is conducted. The results of the final data analysis are presented in this BRE and provide quantitative and qualitative estimates of the identities, concentrations, volumes, and forms of chemical and/or radiological contaminants of concern at the facility.

This data evaluation section is organized into the following four subsections: Data Collection (Sect. 2.1), Data Evaluation (Sect. 2.2), Selection of Contaminants of Potential Concern (Sect. 2.3), and Uncertainty (Sect. 2.4.)

\subsection{DATA COLLECTION}

The primary objective of the data collection at D\&D facilities such as Building 3506 is to provide the information necessary for estimating potential contaminant sources/inventories and the magnitude of potential future releases from the facility to the surrounding environment. To ensure that the data collected at a facility meet these objectives, the risk assessor must have some knowledge of past practices at the facility, potential contaminants present, and the potential current and future pathways for exposure at the facility. This will enable the risk assessor to target data collection activities to those contaminants and areas which represent a potential risk at the facility and determine when adequate data have been collected to fulfill the needs of the risk evaluation.

\subsubsection{Characterization Data at Building 3506}

The characterization data for Building 3506 was collected during fall of 1993. These efforts were conducted to plan the D\&D of this building. The characterization activities were conducted following the Site Characterization Plan for Decontamination and Decommissioning of Buildings 3506 and 3515 at Oak Ridge National Laboratory, Oak Ridge, Tennessee (Bechtel, 1993). Please refer to this site characterization plan for further information on sampling and analysis. The data 
collected fall into two categories: 1) health and safety monitoring and 2) sampling data. Data in these categories are briefly discussed in the following paragraphs.

\section{Health and Safety Monitoring Data}

The health and safety data consist of air filter samples from personnel monitoring devices [breathing zone apparatus (BZAs)] and radiological surveys. The radiological surveys include: thermoluminescent dosimeter (TLD), smear samples, and exposure rate surveys. Data from the radiological surveys are found in Appendix A. A summary of the data is presented in Tables 2.1-2.3.

\section{Sampling Data}

The media sampled from Building 3506 consisted of concrete cores taken from the floors and walls of the building (Fig. 2.1), concrete chips (taken from the west wall of the east gallery), soils from below the gallery and cell floors, and sediment taken from the floor of the cell. Soil samples were collected in July 1991 near Building 3506 as part of the Phase I of the WAG 1 Remedial Investigation. Paint chips were taken from the facility for lead analysis. In addition, the putty surrounding the windows and the sheet rock and felt in the ceiling were sampled and analyzed for asbestos. Media samples were taken to the laboratory for chemical and radiological analysis. Table 2.4 summarizes the samples collected and the analyses performed. Summary data for the radiological analysis can be found in Table 2.5. Complete data from these analyses can be found in Appendix A.

\subsubsection{Potential Pathways and Exposure Points}

The majority of contamination at Building 3506 is dispersed throughout the walls, floors, and sediment. Therefore, the building is considered to serve as a primary containment system for the contaminants. Currently, there is no information which suggests that release of contaminants to the environment is occurring.

Current exposures are limited to direct contact with contaminants, breathing of dust and vapors, and external exposure to radiation by workers. However, health and safety measures at Building 3506 mitigate exposures to workers or visitors by requiring the use of protective clothing and prohibiting eating or smoking in the facility. Access to the facility is also restricted. The primary source of potential current and future releases is from the building structure. Secondary sources of potential future releases are from the soils around and below the building.

In the future, releases resulting from deterioration of the building, natural disasters, or D\&D operations are possible. In the event of these releases, exposures to contaminated environmental media including soil and surface water could occur, in addition to exposures to air-borne vapor or particulate phase contaminants. 


\section{2-3}

Table 2.1 TLD string summary data in cell $^{2}$

\begin{tabular}{cccccc}
\hline \multirow{2}{*}{$\begin{array}{c}\text { String } \\
\text { number }\end{array}$} & $\begin{array}{c}\text { Number of } \\
\text { Samples }\end{array}$ & H, Rate & H $_{\mathbf{d}}$ Rate & H, Rate & $\mathbf{H}_{\mathrm{d}}$ Rate \\
\cline { 3 - 6 } & 10 & $0.5-3.1$ & $0.4-1.2$ & 1.3 & 0.8 \\
\hline 1 & 10 & $1.6-5.2$ & $1.4-2.8$ & 2.9 & 2.2 \\
3 & 10 & $8.9-98.3$ & $4.8-24.2$ & 50.8 & 12.6 \\
4 & 10 & $3.1-5.8$ & $2.5-5.7$ & 4.5 & 4.2 \\
\hline
\end{tabular}

${ }^{2} \mathrm{H}_{\mathrm{a}}$ - Shallow dose; $\mathrm{H}_{d}$ - Deep dose 
Table 2.2 Summary results of location-specific measurements in the east gallery"

\begin{tabular}{|c|c|c|c|c|c|}
\hline & Soutb Wall & Weat Wall & North Walf & Ent Wall & Floor \\
\hline \multicolumn{6}{|c|}{$\begin{array}{l}\text { Direct Alpha Coumting } \\
\text { (dpm/100 cm²) }\end{array}$} \\
\hline Range & $21-37$ & $6-7,090$ & 49 & $3-68$ & $9-44$ \\
\hline Average & 29 & 743 & - & 40 & 18 \\
\hline \multicolumn{6}{|c|}{$\begin{array}{l}\text { Direct Betw/Gammer } \\
\text { Counting at } 10 \mathrm{~cm}\end{array}$} \\
\hline \multicolumn{6}{|c|}{ Open Hindow (mrad/h) } \\
\hline Renge & $\begin{array}{c}6.1 \mathrm{E}-02-2.6 \mathrm{E}- \\
02\end{array}$ & $\begin{array}{c}\text { 2.7E-02-3.6E- } \\
01\end{array}$ & $6.2 \mathrm{E}-02$ & $2.6 \mathrm{E}-02-1.4 \mathrm{E}-01$ & 2.1E-02-9.7E-02 \\
\hline Average & 4.3E-02 & 1.1E-01 & - & 6.1E-02 & $5.2 \mathrm{E}-02$ \\
\hline \multicolumn{6}{|c|}{ Clased Window (mR/h) } \\
\hline Renge & $\begin{array}{c}5.3 \mathrm{E}-02-2.1 \mathrm{E}- \\
02\end{array}$ & $\begin{array}{c}2.1 \mathrm{E}-02-4.6 \mathrm{E}- \\
01\end{array}$ & $5.2 \mathrm{E}-02$ & $2.0 \mathrm{E}-02-1.2 \mathrm{E}-01$ & $1.2 E-02-1.2 E-01$ \\
\hline Average & 3.7E-02 & 8.7E-02 & - & 5.3E-02 & $4.5 \mathrm{E}-02$ \\
\hline \multicolumn{6}{|c|}{ Smear Counting } \\
\hline \multicolumn{6}{|c|}{ Alpha $\left.(\mathrm{dpm} / 100 \mathrm{~cm})^{2}\right)$} \\
\hline Range & $24-46$ & $2-33$ & 2 & $1-6$ & $1-22$ \\
\hline Average & 35 & 10 & - & 3 & 6 \\
\hline \multicolumn{6}{|c|}{$\begin{array}{l}\text { Beta/Gamma (dpa/ } 100 \\
\left.\mathrm{~cm} \mathrm{~m}^{2}\right)\end{array}$} \\
\hline Renge & $12-13$ & $10-1,700$ & 5 & $4-1,170$ & $4-10,301$ \\
\hline Avernge & 12.5 & 381 & - & 283 & 1,550 \\
\hline \multicolumn{6}{|c|}{$\begin{array}{l}\text { CSL Cesinm-137 } \\
\text { Smears } \\
\left(\mu \mathrm{Ci} / 100 \mathrm{~cm}^{2}\right)\end{array}$} \\
\hline Range & $\begin{array}{l}\text { 7.72E } \\
06-8.49 \mathrm{E}-06\end{array}$ & $\begin{array}{c}8.88 \mathrm{E}- \\
06-3.64 \mathrm{E}-04\end{array}$ & - & 1.33E-05-5.03E-04 & 4.94E-05-1.38E-03 \\
\hline Average & 8.11E-06 & 9.1E-05 & -- & $1.52 \mathrm{E}-04$ & 3.18E-04 \\
\hline
\end{tabular}

- Taken from Bechtel, 1993

- Onty one location was relected and messured on this wall.

- Background has not been subtractod from these messurements.

- All smears were counted at the CSL, and backgrourod bas been subtracted. 


\section{$2-5$}

Table 2.3. Summary results of location-specific measurements in the north/south cell area

\begin{tabular}{|c|c|c|c|c|c|c|c|}
\hline & \multicolumn{4}{|c|}{ North Cell Area } & \multicolumn{3}{|c|}{ South Cell Area } \\
\hline & Weat Wall & North Wall & Eart Wall & $\underset{\text { System }}{\text { HEPA }}$ & East Wall & South Wall & Weat Wall \\
\hline \multicolumn{8}{|l|}{$\begin{array}{l}\text { Direct Alpha } \\
\text { Cournting } \\
\left.100 / \mathrm{cm}^{2}\right)\end{array}$} \\
\hline Renge & $44-306$ & $46-67$ & $16-206$ & 58 & $74-769$ & $93-130$ & $77-136$ \\
\hline Avernge & 104 & 54 & 80 & - & 260 & 110 & 101 \\
\hline \multicolumn{8}{|l|}{$\begin{array}{l}\text { Direct } \\
\text { Beta/Gamma } \\
\text { Counting at } 10 \\
\mathrm{~cm}\end{array}$} \\
\hline \multicolumn{8}{|l|}{$\begin{array}{l}\text { Open Window } \\
(\mathrm{mrnd} / \mathrm{h})\end{array}$} \\
\hline Range & $\underset{01-3.2 \mathrm{E}-00}{7.5 \mathrm{E}}$ & $\underset{01-8.4 \mathrm{E}-01}{5.8 \mathrm{E}-}$ & $\stackrel{6.8 \mathrm{E}}{01-1.1 \mathrm{E}+01}$ & $2.1 \mathrm{E}-00$ & $00-6.5 \mathrm{E}+01$ & $00-7.0 \mathrm{E}-00$ & $00-4.4 \mathrm{E}-00$ \\
\hline Average & $1.6 \mathrm{E}-00$ & $7.0 \mathrm{E}-01$ & $3.8 \mathrm{E}-00$ & - & $1.9 \mathrm{E}+01$ & $5.4 \mathrm{E}-00$ & $3.0 \mathrm{E}-00$ \\
\hline \multicolumn{8}{|l|}{$\begin{array}{l}\text { Cosed Window } \\
(\mathrm{mR} / \mathrm{h})\end{array}$} \\
\hline Range & $\begin{array}{c}6.4 \mathrm{E}- \\
01-1.9 \mathrm{E}-00\end{array}$ & $\underset{01-6.7 \mathrm{E}-01}{5.2 \mathrm{E}-}$ & $\underset{01-8.3 \mathrm{E}-00}{2.9 \mathrm{E}-}$ & $\underset{00}{1.75 E}$ & $\stackrel{2.4 \mathrm{E}-}{00-3.8 \mathrm{E}+01}$ & $00-5.9 \mathrm{E}-00$ & $00-2.5 \mathrm{E}-00$ \\
\hline Average & $1.1 \mathrm{E}-00$ & $5.9 \mathrm{E}-01$ & 2.TE-00 & - & $1.2 \mathrm{E}+01$ & $4.0 \mathrm{E}-00$ & $1.9 \mathrm{E}-00$ \\
\hline \multirow{2}{*}{\multicolumn{8}{|c|}{$\begin{array}{l}\text { Smear Countinge } \\
\text { Alpha (dpm/100 } \\
\text { cmon }\end{array}$}} \\
\hline & & & & & & & \\
\hline Range & $1-32$ & $2-4$ & $1-9$ & 5 & $2-7$ & $1-4$ & $2-26$ \\
\hline Average & 10 & 3.3 & 4.2 & - & 4.4 & 1.8 & 9 \\
\hline \multicolumn{8}{|l|}{$\begin{array}{l}\text { Beta/Gamma } \\
\left(\mathrm{dpm} / 100 \mathrm{~cm}{ }^{2}\right)\end{array}$} \\
\hline Range & $63-5,480$ & $198-821$ & $27-48,600$ & 524 & $573-3,640$ & $63-748$ & $67-2,640$ \\
\hline Average & 563 & 511 & 9,420 & - & 1,920 & 462 & 859 \\
\hline \multicolumn{8}{|c|}{$\begin{array}{l}\text { CSL Cesium-137 } \\
\text { Smears }(\mu \mathrm{Ci} / 100 \\
\left.\mathrm{cm}^{2}\right)\end{array}$} \\
\hline Range & $\begin{array}{c}2.68 \mathrm{E} \\
05-5.21 \mathrm{E} \\
03\end{array}$ & $\begin{array}{l}8.80 \mathrm{E}- \\
05-2.3 \mathrm{E}-04\end{array}$ & $\underset{05-1.29 \mathrm{E}-02}{7.35 \mathrm{E}}$ & $\underset{04}{2.14 \mathrm{E}-}$ & $\frac{2.44 \mathrm{E}-}{04-1.45 \mathrm{E}-03}$ & $\underset{04}{05-3.22 \mathrm{E}}$ & $\begin{array}{c}1.35 \mathrm{E} \\
04-4.46 \mathrm{E}- \\
04\end{array}$ \\
\hline Average & $1.4 \mathrm{E}-03$ & $2.22 \mathrm{E}-04$ & 2.95E-03 & -- & $8.07 \mathrm{E}-4$ & $1.94 \mathrm{E}-04$ & $1.64 \mathrm{E}-04$ \\
\hline
\end{tabular}

Taken from Bechtel, 1993

- Only one location wes eclected and measured.

- Beckground has not been aubtracted from these measurements.

4 All smears were counted in the CSL, and background hes been aubtracted. 


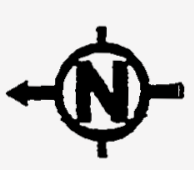

$32.0^{\circ}$

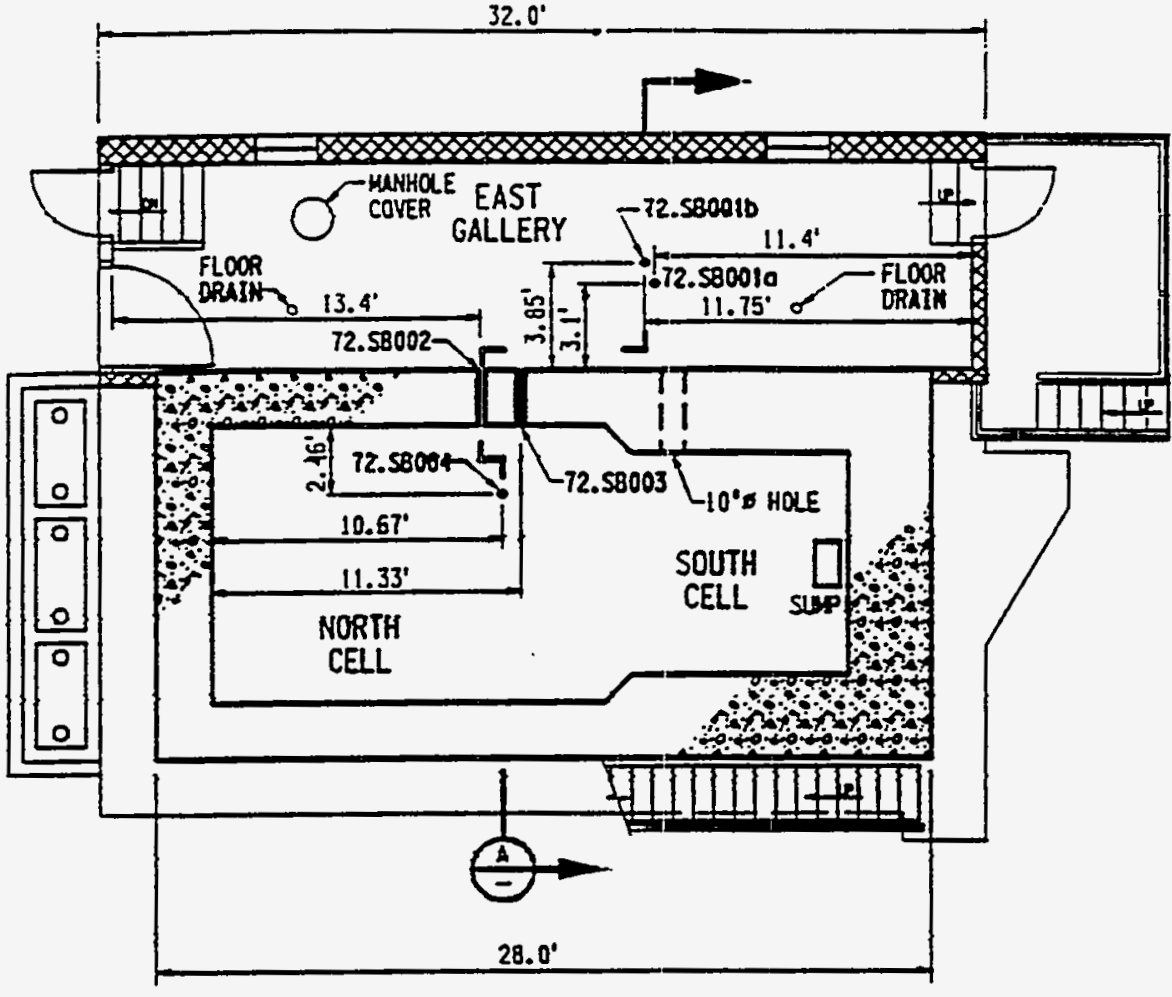

BUILDING 3506 PLAN

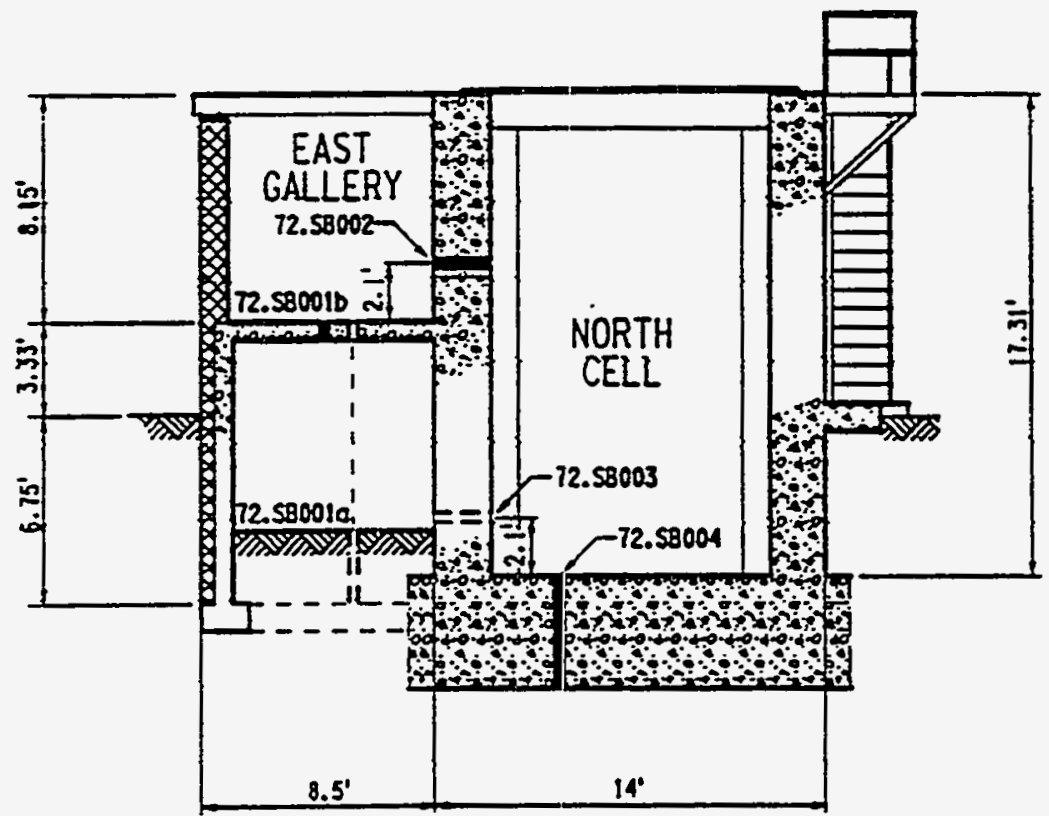

BLDG SECTION A

NOTE : For 72.58001 , locotion (a) is the soil sompling locotion and locotion (b) is the concrete core sampling location. 1 core ras atilled ot locotion (a) but it foll into tho crow spoce and wos not rotriared.

Fig. 2.1. Building 3506 sampling locations 
Table 2.4. Sampling summary for Building 3506

\begin{tabular}{|c|c|c|c|c|c|c|c|c|c|}
\hline \multirow[b]{2}{*}{$\begin{array}{c}\text { Location } \\
\text { No: }\end{array}$} & \multirow[b]{2}{*}{$\begin{array}{l}\text { Sample } \\
\text { No. }\end{array}$} & \multirow[b]{2}{*}{$\begin{array}{c}\text { Sample } \\
\text { Type }\end{array}$} & \multirow[b]{2}{*}{ Sample Description } & \multicolumn{5}{|c|}{ Chemical Anslyzis } & \multirow[b]{2}{*}{$\begin{array}{c}\text { Radiological } \\
\text { Analyais }\end{array}$} \\
\hline & & & & $\begin{array}{l}\text { TCL } \\
\text { VOCs }\end{array}$ & $\begin{array}{l}\text { TCL } \\
\text { BNAEs }\end{array}$ & $\begin{array}{l}\text { Peat/ } \\
\text { PCBs }\end{array}$ & TAL & TAL & \\
\hline \multirow[t]{4}{*}{ 72.SBCO1 } & 03936 & $\begin{array}{l}\text { QCl } \\
\text { equipme } \\
\text { nt } \\
\text { rinieate } \\
\text { (unfiltere } \\
\text { d) }\end{array}$ & $\begin{array}{l}\text { Prior to uning the core drill in Building } 3506 \text {, } \\
\text { but after using the drill in sampling team } \\
\text { practice seasions, orgenic-free water was } \\
\text { pumped through the core drill system (i.e., } \\
\text { from the poly-bottle oprayer, through } \\
\text { plantic/rubber boses, into the drill bead, and } \\
\text { through the core barrel). }\end{array}$ & & $\mathbf{x}$ & $\mathbf{x}$ & $x$ & $\mathbf{x}$ & $\mathbf{x}$ \\
\hline & 03943 & $\begin{array}{l}\text { Coocrete } \\
\text { core }\end{array}$ & $\begin{array}{l}\text { 8.5-in. core (aggregate with rebar) tuken from } \\
\text { the gallery floor. }\end{array}$ & & $\mathbf{x}$ & $x$ & $\mathbf{x}$ & $\mathbf{x}$ & $\mathbf{x}$ \\
\hline & 03933 & Soil & $\begin{array}{l}\text { Hend-eugered composite soil sample taken } \\
\text { below gallery concrete floor and crewlspace. } \\
\text { Depth for the sample was } 8.25 \text { to } 10.1 \text { ft from } \\
\text { top of floor. (Exception: the VOC contriner } \\
\text { was taken from } 8.5 \text { ft and the peaticide/PCBs } \\
\text { continer was taken from } 9.9 \mathrm{ft} \text { ) Refusal at } \\
10.1 \mathrm{ft} \text { may have been due to concrete } \\
\text { surface. Percent solids is } 78.9 \text {. }\end{array}$ & $\mathrm{x}$ & $x$ & $x$ & $\mathbf{x}$ & $\mathbf{x}$ & $\mathbf{x}$ \\
\hline & 03960 & $\begin{array}{l}\text { Trip } \\
\text { blanks } \\
\text { water }\end{array}$ & Submitted with ample 03933 & $x$ & & & & & \\
\hline \multirow[t]{2}{*}{ 72.sBO02 } & 03944 & $\begin{array}{l}\text { Concrete } \\
\text { core }\end{array}$ & $\begin{array}{l}\text { Horizootally drilled core from weet wall of } \\
\text { gallery into cell: core length is } 1.98 \mathrm{ft} \text {. }\end{array}$ & & $x$ & $\mathbf{x}$ & $\mathbf{x}$ & $\mathbf{x}$ & $\mathbf{x}$ \\
\hline & 04237 & $\begin{array}{l}\text { Concrete } \\
\text { chips }\end{array}$ & $\begin{array}{l}\text { Chip collected with hammer and chisel from } \\
\text { west wall of gallery around core bole. }\end{array}$ & & $\mathbf{x}$ & $\mathbf{x}$ & & & \\
\hline $72 . \mathrm{SB} 003$ & 04044 & $\begin{array}{l}\text { Concrete } \\
\text { core }\end{array}$ & $\begin{array}{l}\text { Horizoatally drilled core from east wall of cell } \\
\text { into crawlypace beacath gallery floor; core } \\
\text { whs drilled above the "bathtub ring," which is } \\
\text { approximntely } 17 \text { in. above the cell floor. } \\
\text { Core length is } 2 \mathrm{ft} \text {. }\end{array}$ & & $\mathbf{x}$ & $x$ & $x$ & $\mathbf{x}$ & $x$ \\
\hline \multirow[t]{4}{*}{$72 . S B 004$} & \multirow[t]{3}{*}{04045} & $\begin{array}{l}\text { Concrete } \\
\text { core }\end{array}$ & $\begin{array}{l}\text { Componite core from cell floor using core } \\
\text { intervals of } 0 \text { to } 6.5 \text { in., } 10.5 \text { to } 19.5 \text { in., and } \\
19.5 \text { to } 24 \text { in. }\end{array}$ & & $\mathrm{x}$ & $x$ & $\mathbf{x}$ & $\mathbf{x}$ & $\mathrm{x}$ \\
\hline & & $\begin{array}{l}\text { Concrete } \\
\text { core }\end{array}$ & $\begin{array}{l}\text { Relatively "bot" core interval from } 6.5 \text { to } \\
10.5 \text { in. }\end{array}$ & & $x$ & $x$ & $\mathbf{x}$ & $\mathbf{x}$ & $x$ \\
\hline & & $\begin{array}{l}\text { Concrete } \\
\text { core }\end{array}$ & $\begin{array}{l}\text { Core interval of } 24 \text { to } 47 \text { in. archived; no } \\
\text { andyes performed. }\end{array}$ & & & & & & \\
\hline & 03935 & Soil & $\begin{array}{l}\text { Auger refiral on bedrock limestone after } \\
\text { drilling } 0.2 \mathrm{ft} \text { (depth from } 4 \text { to } 4.2 \mathrm{ft} \text { below } \\
\text { floor grade). No cbemical analyses performed } \\
\text { because of insufficient sample volume. }\end{array}$ & & & & & & $\mathbf{X}$ \\
\hline \multirow[t]{2}{*}{$72 . S D 001$} & 03934 & $\begin{array}{l}\text { Grab } \\
\text { samplet } \\
\text { sediment }\end{array}$ & $\begin{array}{l}\text { Sample for VOC containers grabbed from } \\
\text { northeast comer of cell; sample for other } \\
\text { contriners is composite mix of detritus } \\
\text { covering catire stainless-steel-lined cell floor. } \\
\text { Percent solids is } 43.5 \text {. }\end{array}$ & $x$ & $\mathbf{x}$ & $x$ & $x$ & $\mathbf{x}$ & $\mathbf{x}$ \\
\hline & 04047 & $\begin{array}{l}\text { Trip } \\
\text { blankf } \\
\text { water }\end{array}$ & Submitted with sample 03934. & $x$ & & & & & \\
\hline
\end{tabular}

- Locations are shown in Fig. 2.1.

TAL-Turox Co pond L L

VoC-VT=A

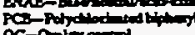


Table 2.5. Summary results for radiological analysis for core and sediment samples

\begin{tabular}{|c|c|c|c|}
\hline \multirow{2}{*}{ Radionuclide } & \multicolumn{3}{|c|}{ Location } \\
\hline & $\begin{array}{c}\text { East Gallery } \\
(\mathrm{pCi} / \mathrm{g})\end{array}$ & $\underset{(\mathrm{pCi} / \mathrm{g})}{\mathrm{Cell}}$ & $\begin{array}{l}\text { Sediment (Cell) } \\
(\mathrm{pCi} / \mathrm{g})\end{array}$ \\
\hline $\mathrm{Pu}^{299240}$ & 0.4 & 4.0 & 35.4 \\
\hline $\mathrm{Pu}^{238}$ & 0.4 & 0.6 & 1.2 \\
\hline $\mathrm{Ra}^{225}$ & 0.4 & 0.4 & ND \\
\hline $\mathrm{Th}^{203}$ & 0.6 & 0.8 & 0.5 \\
\hline $\mathrm{Th}^{230}$ & 1.2 & 1.4 & 0.04 \\
\hline $\operatorname{Th}^{232}$ & 0.4 & 0.9 & 0.6 \\
\hline$U^{233 / 234}$ & 0.9 & 1.3 & 3.4 \\
\hline$U^{235}$ & 0.1 & 0.1 & ND \\
\hline$U^{238}$ & 0.7 & 1.7 & 2.2 \\
\hline $\mathrm{Am}^{2 A 1}$ & 0.5 & ND & ND \\
\hline $\mathrm{Cs}^{137}$ & 22.8 & 1375.0 & 30800 \\
\hline $\mathrm{K}^{40}$ & 5.4 & 5.28 & 9.4 \\
\hline $\mathrm{Sr}^{90}$ & 8.1 & 11.6 & 97.5 \\
\hline $\mathrm{Ra}^{208}$ & 0.4 & ND & ND \\
\hline $\mathrm{CO}^{60}$ & $\mathrm{ND}^{\mathrm{b}}$ & 0.1 & 3.4 \\
\hline
\end{tabular}

a Values are averages of floors and walls

${ }^{b} \mathrm{ND}=$ Not Dectected 


\subsubsection{Preliminary Contaminants of Potential Concern}

A brief description of Building 3506 is given in Sect. 1.1. From the processes known to have occurred at Building 3506, the principal contaminants of potential concern associated with the building are radionuclides, particularly cesium-137; other contaminants of potential concern include asbestos, organic chemicals (including PCBs), lead in paint, and other heavy metals. Radionuclide contamination exists to varying degrees throughout the floors and walls of the building as well as in the sediment found in the cell area. The sediment also contains chemical constituents including the PCB Aroclor-1254. Samples taken of the window putty tested positive for asbestos (chrysotile at 1 to $5 \%$ ), but samples taken of the sheet rock and roofing felt paper were negative.

An additional sample collection effort took place on July 14, 1994. In this second sampling effort, two samples of the roofing felt paper were collected, one of which tested positive for asbestos (chrysotile 1 to $5 \%$ ). In addition, analysis of the two paint chips revealed concentrations of lead of 890 and $8000 \mathrm{mg} / \mathrm{kg}$.

\subsection{DATA EVALUATION}

The purpose of the data evaluation is to determine whether the data are of sufficient quality and quantity to meet the needs of the BRE. This is an iterative process. First, data quality requirements (DQR) are established by determining the site-specific objectives of the BRE and the amount of uncertainty in the data which will allow these objectives to be met. The available data are then compared to these $\mathrm{DQRs}$ to determine which data are useable and whether there is sufficient data to conduct the BRE. If adequate data are not available, the risk assessor must either 1) change the objectives of the BRE so that they can be met by the available data and/or 2) collect additional data. If additional data are collected, the comparison to DQRs is repeated with these new data. For Building 3506 , adequate data were available to satisfy the DQRs of the Level 3 BRE.

The following sections provide a description of the DQRs (Sect 2.2.1), and the evaluation of the characterization data (Sect 2.2.2) for this BRE.

\subsubsection{Data Quality Requirements}

Data Quality Requirements (DQRs) are qualitative and/or quantitative statements that specify the quality of the data required to support decisions during risk evaluation activities. The development and use of DQRs is an on-going process from initial project scoping, through the BRE and Alternatives Risk Evaluation. The development and use of DQRs are described briefly in the following paragraph.

Information obtained from historical and operational investigations is used as the foundation for the scoping of the BRE (i.e., definition of the problem and objectives of the BRE) and development of initial DQRs. The DQRs for this Level $3 \mathrm{BRE}$ are the satisfaction of three criteria: 1) determination of basic site characteristics, 2) initial identification of potential exposure pathways and exposure points, and 3) determination of future data needs. The existing 
characterization data have been compared to the DQRs established for this Level 3 BRE. The results of this evaluation are discussed in Sect. 2.2.2.

\subsubsection{Evaluation of Existing Characterization Data}

The quality and useability of the data are discussed in the following paragraphs. The documentation for both the chemical and radiological laboratory analyses met RI/FS QC level III requirements. The QC level III mandates that QC data, including raw data, be reported in the CLP data package. The data were validated and met the RI/FS QC level II requirements.

\section{Health and Safety Monitoring Data}

The data from the BZAs were unavailable for use in this BRE; only the conclusions of the study were available. Results from the air filter samples were reported to be below the derived air concentration limits reported in Federal Guidance Report No. 11 (ORNL, 1988). Based on the lack of available data, inhalation of contaminants was modeled using the data from building surfaces, structures, and the sediment. Direct measurements for alpha radiation (in the cell and east gallery) were not used in this BRE. Only the direct measurement data for beta/gamma and gamma radiation were used in calculating risk from external exposure. Since alpha radiation is known to travel only extremely short distances, the data vere not useful in estimating risk from external exposure.

Directional gamma measurements taken from the cell were excluded from analysis because information from the gamma measurements (direct measurements) as well as the TLD strings was sufficient for use in this BRE.

\section{Sampling Data}

The concrete cores taken from the walls and floors of the facility were slit-scanned in the field. The slit-scans were performed to develop a contamination profile which assisted in core sample separation for laboratory analysis; therefore, the data from the scans were not used in this BRE.

The core samples taken from the floor in the cell were separated into two separate containers: container 3 held the section from the 6.5- to 10.5-in. depth, and container 9 held sections from the 0 - to 6-in. and 10.5- to 24-in. depths. Data from container 9 were used in the risk calculations for radiological contaminants for the three receptors evaluated in this BRE because it was assumed that the contaminants were in the top 6 in. of the concrete and that there was little shielding due to concrete (a factor of 0.2 was assumed). The sample (taken from the cell) which contained the composite core ( 0 to 24.0 in.) was used in the risk calculations for the organic contaminants, rather than the hot interval (6.5 to 10.5 in.) because it was assumed that the composite core contains a representative concentration of contaminants.

Data reported for the QC/equipment rinseate were not used in the data set for this BRE. The rinsing procedure was performed to avoid cross contamination of the cores. Soil samples collected below the east gallery floor and crawlspace as well as under the cell were also not 
included in the BRE data set. In addition, data obtained from WAG 1 soil borings were also not evaluated. As stated previously, existing soil contamination is not within the scope of this BRE.

The applicability and satisfaction of the three criteria by the characterization data set is as follows:

- Determination of basic site characteristics: The characterization data available for Building $\mathbf{3 5 0 6}$ are adequate for determining basic site characteristics. From these data, it is evident that Building 3506 is contaminated to varying degrees with radionuclides, organic chemicals, metals, and possibly asbestos. The contamination is associated with the structural media of the entire building and the sediment found in the cell.

- Initial identification of potential exposure pathways and exposure points: The historical data provide adequate information to identify the following potential exposures within the building: 1) inhalation of airborne contaminants (particulates and volatiles ); 2) incidental ingestion of contaminants through hand-to-mouth transfer (eating, smoking, etc.); 3) dermal absorption of chemicals; and 4) external exposure to radiation.

- Identification of additional data needs: The valve pits and the pit on the west side of the building were not characterized; therefore, the risks associated with these areas could not be estimated.

\subsection{SELECTION OF CONTAMINANTS OF POTENTIAL CONCERN}

Section 2.2.1 presents the preliminary identification of contaminants of potential concern. The contaminants of potential concern evaluated quantitatively in this BRE are presented in Table 2.6. The contaminants of potential concern evaluated qualitatively are presented in Table 2.7. The contaminants listed in Table 2.7 could not be evaluated quantitatively because toxicity information is not available for these contaminants. Appendix $\mathrm{C}$ contains summary statistics for all data used in this BRE.

\subsection{UNCERTAINTY}

The characterization data used in this BRE were not intentionally collected for use in a risk assessment; therefore, several assumptions and approximations had to be made. The site characterization plan (Bechtel, 1993) states that, "During characterization of Buildings 3506/3515, site data relevant to formal exposure assessments, toxicity assessments, and risk estimation need not be collected." Therefore, the data collected for the characterization of Building $\mathbf{3 5 0 6}$ may not fulfill the needs of a risk assessment.

The direct measurements of radionuclide contamination did not subtract background, although the smear samples analyzed at the laboratory subtracted background levels of radiation.

Only one sample was taken from the floors and walls of the cell and east gallery; this increases the uncertainty when extrapolating the data to the entire room. 
$2-12$

Table 2.6. Contaminants evaluated quantitatively

\begin{tabular}{|c|c|c|c|c|c|}
\hline Semple Type & Location & Organic Chemicals & Inorgeric Chemicals & Radionuclides & \\
\hline \multirow[t]{32}{*}{ Core Data } & Cell & Aroclor-1254 & Anconic & ${ }^{25} \mathrm{C}$ & \\
\hline & & Aroclor-1260 & Barivm & ${ }^{20} \mathrm{Ra}$ & \\
\hline & & & Beryllium & $\mathrm{Th}$ & \\
\hline & & & Cadminem & ${ }^{200} \mathrm{Th}$ & \\
\hline & & & Chromium VI & $200 \mathrm{Th}$ & \\
\hline & & & Manganese & 2sspsu & \\
\hline & & & Mercury & ${ }^{204} \mathrm{Th}$ & \\
\hline & & & Nickel & 24YP & \\
\hline & & & Vanadium & ${ }^{25} \mathrm{Pu}$ & \\
\hline & & & Zine & 2verp & \\
\hline & & & & $20000 \mathrm{Pu}$ & \\
\hline & & & & $\omega_{\mathrm{K}}$ & \\
\hline & & & & ${ }^{\infty} \mathrm{Co}$ & \\
\hline & & & & $\infty$ & \\
\hline & & & & Tritium & \\
\hline & Emat Gallery & Aroclor-1254 & Arnenic & ${ }^{15} \mathrm{C}$ & \\
\hline & & Aroclor -1260 & Barium & ${ }^{2 x_{\mathrm{R}}}$ & \\
\hline & & Bis(2-ethythexyl)phthatale & Beryllium & ma & \\
\hline & & Dimethyl phthalate & Codminum & $20 \mathrm{Th}$ & \\
\hline & & & Chrominum VI & $20 \mathrm{Th}$ & \\
\hline & & & Menganese & $25 \mathrm{Th}$ & . \\
\hline & & & Nickel & $2002 \times \mathrm{U}$ & \\
\hline & & & Vamadium & 2000 & \\
\hline & & & Zinc & ${ }^{2 A t} \mathrm{Am}$ & \\
\hline & & & & $\omega_{K}$ & \\
\hline & & & & ${ }^{\infty} \mathrm{Sr}$ & \\
\hline & & & & Tritium & \\
\hline & Whole Building & Arockor-1254 & Arrenic & ${ }^{25} \mathrm{Cs}$ & \\
\hline & & Aroclor-1260 & Barium & ${ }^{22 x} \mathrm{Ra}$ & \\
\hline & & Bis(2-ethy thexyl)phthalate & Beryllium & ${ }^{2 m a}$ & \\
\hline & & Dimethyl phthalate & Crdminum & $200 \mathrm{Th}$ & \\
\hline & & & Chromium VI & $20 \mathrm{Th}$ & \\
\hline
\end{tabular}


2-13

Table 2.6. (continued)

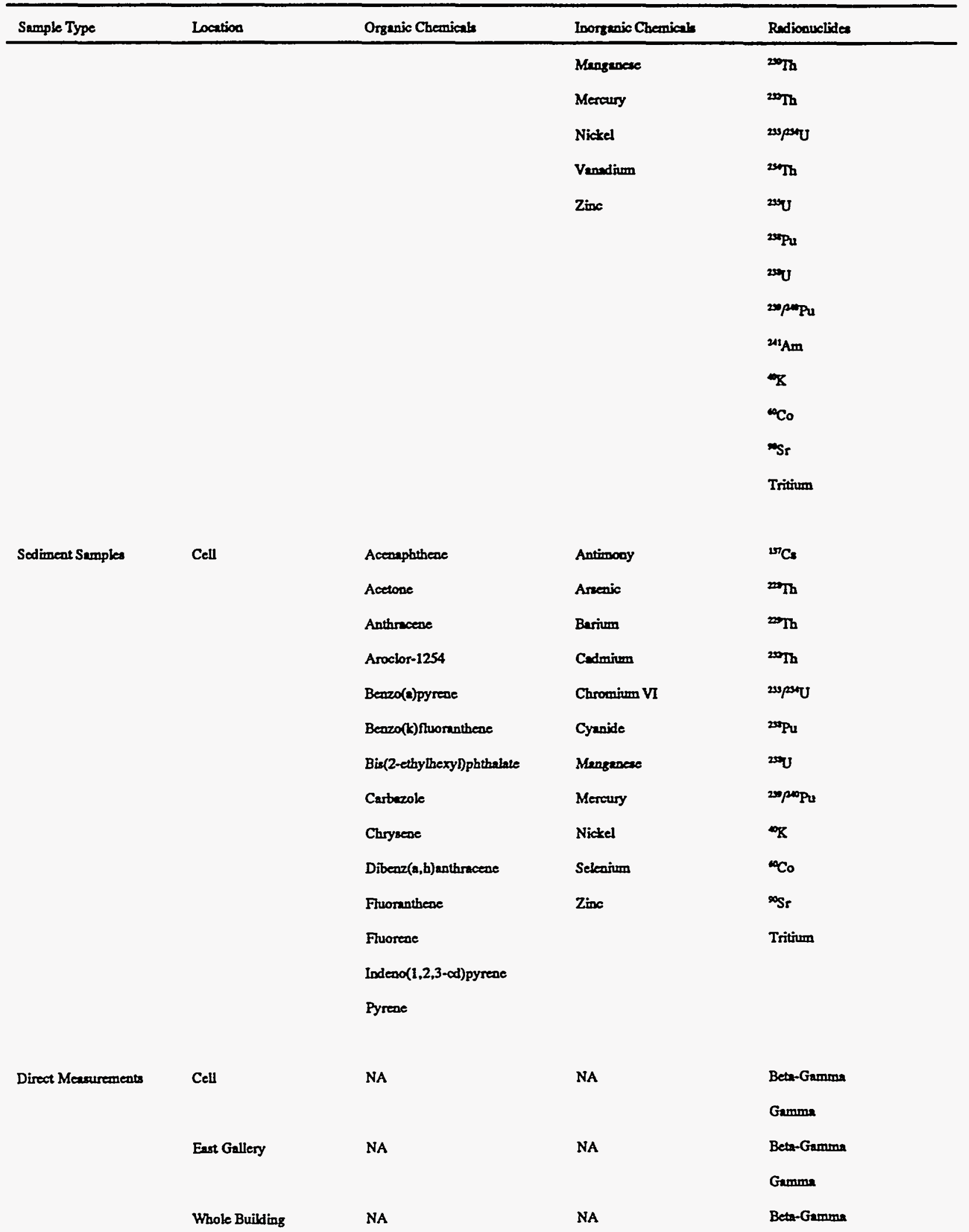


$2-14$

Table 2.6. (continued)

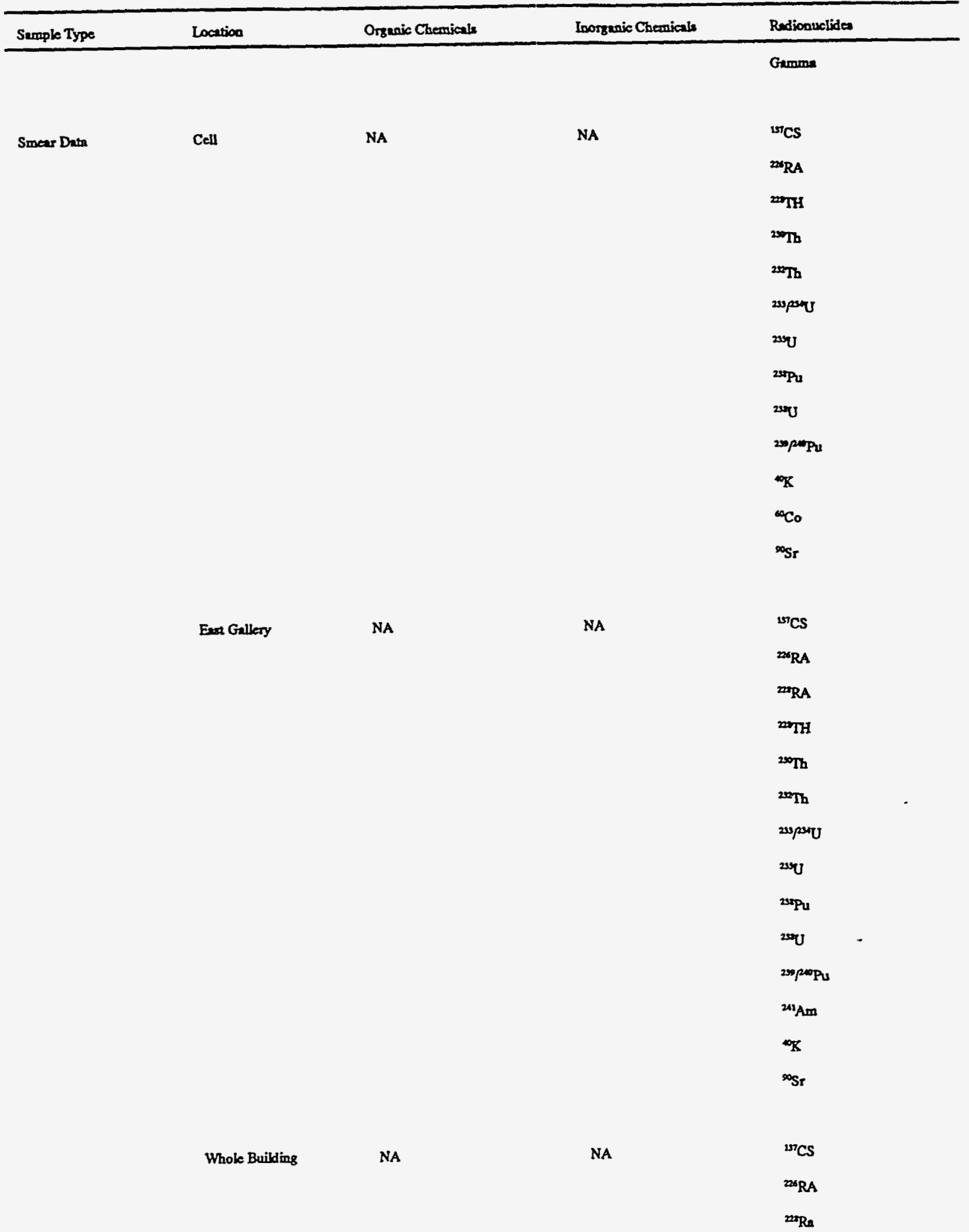


2-15

Table 2.6. (continued)

\begin{tabular}{|c|c|c|c|c|}
\hline Sumple Type & Location & Organic Chemicals & Inorgenic Chemials & Redionuclides \\
\hline ' & & & & 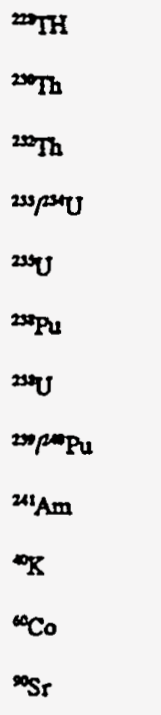 \\
\hline TID Mearurementu & Cell & NA & NA & $\begin{array}{l}\text { Beta } \\
\text { Grmma }\end{array}$ \\
\hline
\end{tabular}


Table 2.7. Contaminants evaluated qualitatively

\begin{tabular}{|c|c|c|}
\hline DATA & LOCATION & ANALYTE \\
\hline \multirow[t]{4}{*}{ Core } & Cell & Aluminum \\
\hline & & Asbestos \\
\hline & & Cobalt \\
\hline & & Lead \\
\hline \multirow[t]{7}{*}{ Core } & East Gallery & Aluminum \\
\hline & & Asbestos \\
\hline & & Cobalt \\
\hline & & Lead \\
\hline & & 2-Methylnaphthalene \\
\hline & & Naphthalene \\
\hline & & Phenanthrene \\
\hline \multirow[t]{7}{*}{ Sediment } & Cell & Aluminum \\
\hline & & Lead \\
\hline & & 2-Methylnaphthalene \\
\hline & & $\operatorname{Benzo}(g, h, i)$ perylene \\
\hline & & Dibenzofuran \\
\hline & & Naphthalene \\
\hline & & Phenanthrene \\
\hline \multirow[t]{7}{*}{ Core } & Whole Building & Aluminum \\
\hline & & Asbestos \\
\hline & & Cobalt \\
\hline & & Lead \\
\hline & & 2-Methylnaphthalene \\
\hline & & Naphthalene \\
\hline & & Phenanthrene \\
\hline
\end{tabular}




\section{EXPOSURE ASSESSMENT}

The objective of this exposure assessment is to estimate the type and magnitude of potential exposures to the chemicals and radionuclides of potential concern that are present at or migrating from Building 3506. The exposure assessment is performed assuming both current and potential future uses of the facility and involves three main steps:

- Characterizing the exposure setting (Sect. 3.1).

- Identifying potential migration routes and exposure pathways (Sect. 3.2).

- Quantitatively and qualitatively estimating chemical- and radionuclide-specific exposures (Sect. 3.3).

The results of the exposure assessment are presented in Sect. 3.4, and the uncertainties associated with this assessment are discussed in Sect. 3.5.

\subsection{CHARACTERIZATION OF THE EXPOSURE SETTING}

The first step in the exposure assessment is characterizing the facility. This establishes the setting in which potential exposures could occur. The characterization of the exposure setting includes a description of the facility (Sect. 3.1.1.1); the meteorology (Sect. 3.1.1.2); site hydrology (Sect. 3.1.1.3); demographics and land use (Sect. 3.1.2.1); and ecology (Sect. 3.1.2.2).

\subsubsection{Physical Setting}

\subsubsection{Facility Description}

The following information on the facility was taken from Site Characterization Report for Building 3506 at Oak Ridge National Laboratory, Oak Ridge Tennessee (Bechtel, 1994).

Building 3506 is southeast of the intersection of Central Avenue and Third Street, in the vicinity of South Tank Farm (STF). Approximate ORNL coordinates are N22000, E30800. The facility was constructed during 1949 to house a liquid waste evaporator. In 1954, evaporator operations ceased, and the building housed an experimental radioactive waste incinerator and ancillary equipment. The facility has been out of service since 1976, and most of the equipment and piping have been removed.

The facility is a split level building with approximate plan dimensions of $22.5 \mathrm{ft}$ wide, $32 \mathrm{ft}$ long, and $17 \mathrm{ft}$ high. The building is divided into three areas: cell area (evaporator pit) on the west side, operating gallery (east gallery) on the east side, and crawl space under the gallery floor. The finished floor elevations of the cell area and the operating gallery are $800 \mathrm{ft}$ and $809 \mathrm{ft}$, respectively, above sea level. The facility was built using a combination of concrete masonry and cast-in-place, reinforced concrete construction. Checkered steel plates with 
stiffeners were used as roofing panels over the cell area. Thin-gage stainless steel sheeting mounted on plywood over wooden joists covers the gallery roof area.

The north, south, and east walls below the operating gallery area are reinforced concrete walls that extend approximately $6 \mathrm{ft}$ below grade and butt against the east wall of the cell area (connection details to the cell wall are not known). A masonry block veneer runs up the east wall from the footing to the operating floor elevation. The floor in the operating gallery is reinforced cement concrete, except for a small area (app. $40 \mathrm{sq}$. ft.) of brick in front of the north entrance door. Double-width-inch concrete masonry unit walls along the north, east, and south side extend approximately $10 \mathrm{ft}$ above the operating floor level and match the top elevation of the cell area.

The superstructure of the gallery area includes two windows on the east wall and wooden personnel doors on the north and south walls. The doors are installed with a short wood framing system, and doors and framing appear to be in good condition. The north wall of the gallery area also has a door at floor level to facilitate loading and unloading operations.

The cell area is of reinforced cement concrete construction, and the floor is approximately $6 \mathrm{ft}$ below grade. The cell is divided into two parts approximately $10 \mathrm{ft}$ wide (east-west) $\times 15 \mathrm{ft}$ long (north-south) on the north side and $8 \mathrm{ft}$ wide (east-west) $\times 8 \mathrm{ft}$ long (north-south) on the south side. The east and west walls are $2 \mathrm{ft}$ thick in the northern part and $3 \mathrm{ft}$ thick in the southern part. The north wall is $3 \mathrm{ft}$ thick, and the south wall is $2 \mathrm{ft}$ thick. The existing floor is approximately $4 \mathrm{ft}$ thick.

\subsubsection{Meteorology}

The following information has been summarized from the Remedial Investigation Report Feasibility Study For The Gunite and Associated Tanks Operable Unit At Waste Area Grouping 1 (Radian, 1993).

The mean annual temperature for the Oak Ridge area is $58^{\circ} \mathrm{F}\left(14.4^{\circ} \mathrm{C}\right)$. The coldest month is usually January, with average temperatures of approximately $38^{\circ} \mathrm{F}\left(3.3^{\circ} \mathrm{C}\right)$ but occasionally dropping as low as $0^{\circ} \mathrm{F}\left(-17^{\circ} \mathrm{C}\right)$. July is typically the hottest month with average temperatures of $77^{\circ} \mathrm{F}\left(25^{\circ} \mathrm{C}\right)$ but with temperatures occasionally peaking at over $100^{\circ} \mathrm{F}\left(37.8^{\circ} \mathrm{C}\right)$. Average daily temperatures vary $53.6^{\circ} \mathrm{F}\left(12^{\circ} \mathrm{C}\right)$ over the course of the year. The Oak Ridge area receives an average $53.75 \mathrm{in} .(136.5 \mathrm{~cm})$ of precipitation annually. Periods of five days or more without precipitation occur on average only four to five times per year. Maximum monthly precipitation generally occurs from December through March and is associated with winter storms that are commonly of low intensity but long duration. Snowfall averages $10.4 \mathrm{in} .(26.4 \mathrm{~cm})$ per year as a contribution to the total precipitation. A second precipitation maximum occurs in July when short, heavy rainfall associated with thunderstorms is common. The higher intensity of the summer thunderstorms compared to the winter storms results in a higher percentage of runoff and less infiltration.

Winds in the Oak Ridge area are controlled to a large degree by the valley and ridge topography. Figure 3.1 shows a wind rose for the ORR at ORNL with the percentage of wind frequency by wind speed and compass orientation. Prevailing winds tend to be either up-valley (northeasterly) or down-valley (southwesterly). Daytime winds generally blow up-valley, while 
ORNL-DWG 92M-7776

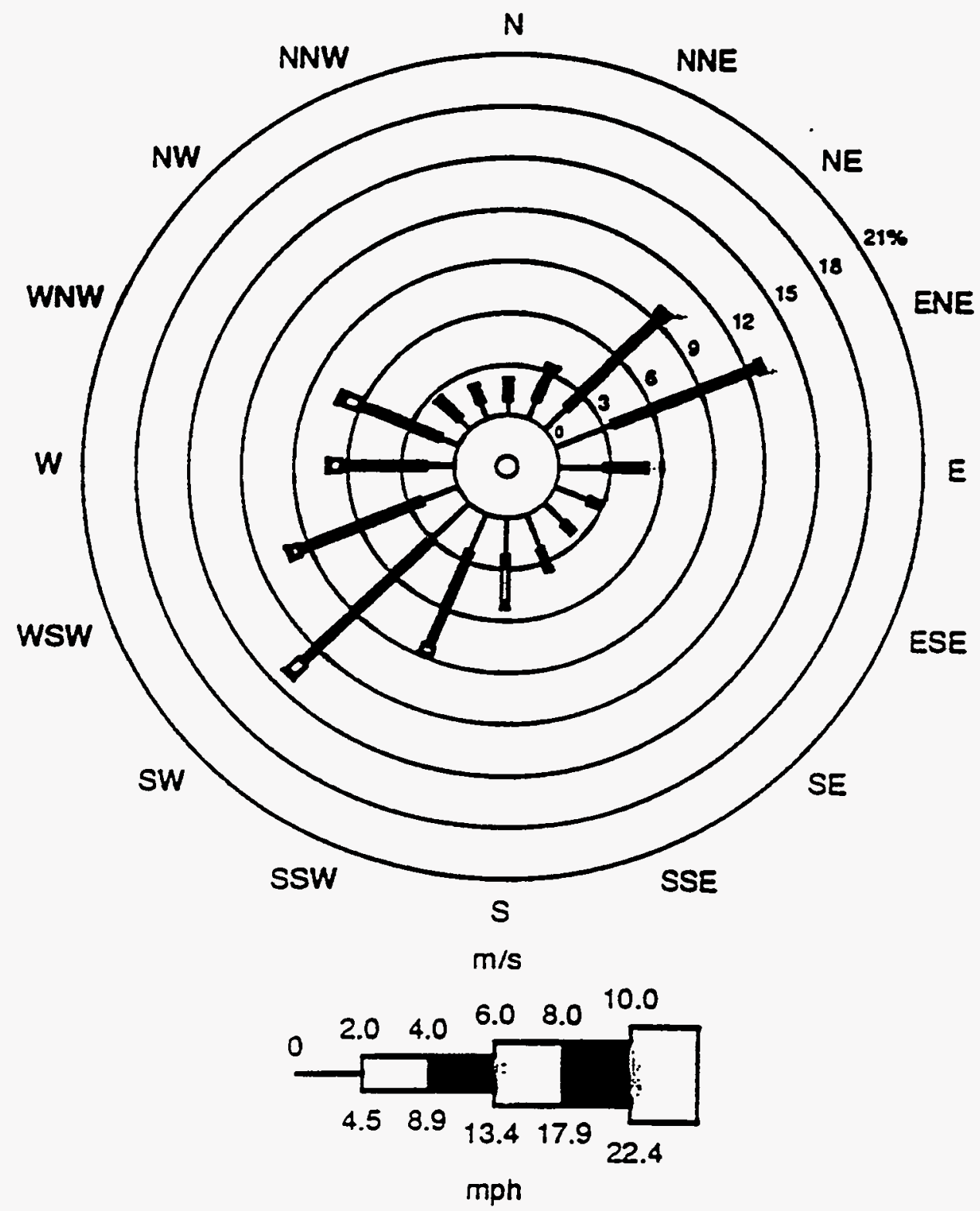

Fig. 3.1. 1991 Wind Rose for ORNL Tower MT2 (100-m level), with $80.4 \%$ of possible data. 
nighttime winds usually blow down-valley. Wind speeds are less than $7.4 \mathrm{mph}(11.9 \mathrm{~km} / \mathrm{hour})$ $75 \%$ of the time. Tornadoes and high-velocity winds are rare, as are wind speeds exceeding $18.5 \mathrm{mph}(30 \mathrm{~km} / \mathrm{hour})$.

\subsubsection{Site-Specific Topography, Geology, and Soils}

The original ORNL facilities are in Bethel Valley, between Chestnut Ridge to the northwest and Haw Ridge to the southeast. The valley floor occurs at an elevation of approximately $900 \mathrm{ft}$ (275 m) above the National Geodetic Vertical Datum of 1929 (NGVD) (Fig. 3.2). The valley floor slopes gently southward across the ORNL site with an average slope of $4 \%$. Chestnut Ridge rises above $1200 \mathrm{ft}$ (365 m) NGVD, while Haw Ridge rises above $1040 \mathrm{ft}$ (317 m) NGVD.

All of the GAAT OU (including Building 3506) is underlain by rocks of the Ordovician-aged Chickamauga Group in the upper part of the stratigraphic section (Fig. 3.3). These rocks strike northeast-southwest and dip toward the southeast. The Chickamauga Group consists of interbedded limestones and siltstones.

The most detailed, as well as the most recent, interpretation of the geology at ORNL is provided by the WAG 1 Site Characterization Summary Report. The bedrock geology at ORNL shown in Fig. 3.4. Figure 3.5 shows a geologic cross section in the vicinity of the GAAT OU facilities (Building 3506). Building 3506 is underlain by Stockdale's Unit E.

The Benbolt Formation is a relatively heterogeneous formation that is 360 to $377 \mathrm{ft}(110$ to $115 \mathrm{~m}$ ) thick consisting of thick interbeds of fossiliferous nodular limestone; unfossiliferous, amorphous micrite within a dark-gray siltstone matrix; dark-gray siltstone; and unfossiliferous calcarenite.

The Bowen Formation is a distinctive maroon-colored unit used as a reliable marker for both field and subsurface correlations. The Bowen Formation is 16 to $32 \mathrm{ft}$ (5 to $10 \mathrm{~m}$ ) thick and consists of maroon calcareous and shaley siltstone and thin beds of light-grey to olive-grey limestone and argillaceous limestone.

A relatively thin overburden has developed over the bedrock in Bethel Valley. Investigation during the site characterization of WAG 1 indicated that this overburden is typically less than $30 \mathrm{ft}(9 \mathrm{~m})$ thick. In the vicinity of the North Tank Farm and South Tank Farm, the thickness of the overburden is between 10 and $20 \mathrm{ft}(3.1$ and $6.1 \mathrm{~m})$. The overburden materials may contain three components: soil materials (residual soil, alluvium, and colluvium); saprolite (weathered bedrock containing relic structures); and fill material (recompacted natural soil, imported material, and waste). As a result of the construction activities for Building 3506, fill is probably the primary material encountered, while natural soil structure or saprolite has probably been eliminated.

\subsubsection{Site Hydrogeology}

The Knox aquifer is the only true aquifer present on ORR. Groundwater flow in the Knox aquifer is dominated by solution conduits developed from widening by dissolution of the original 


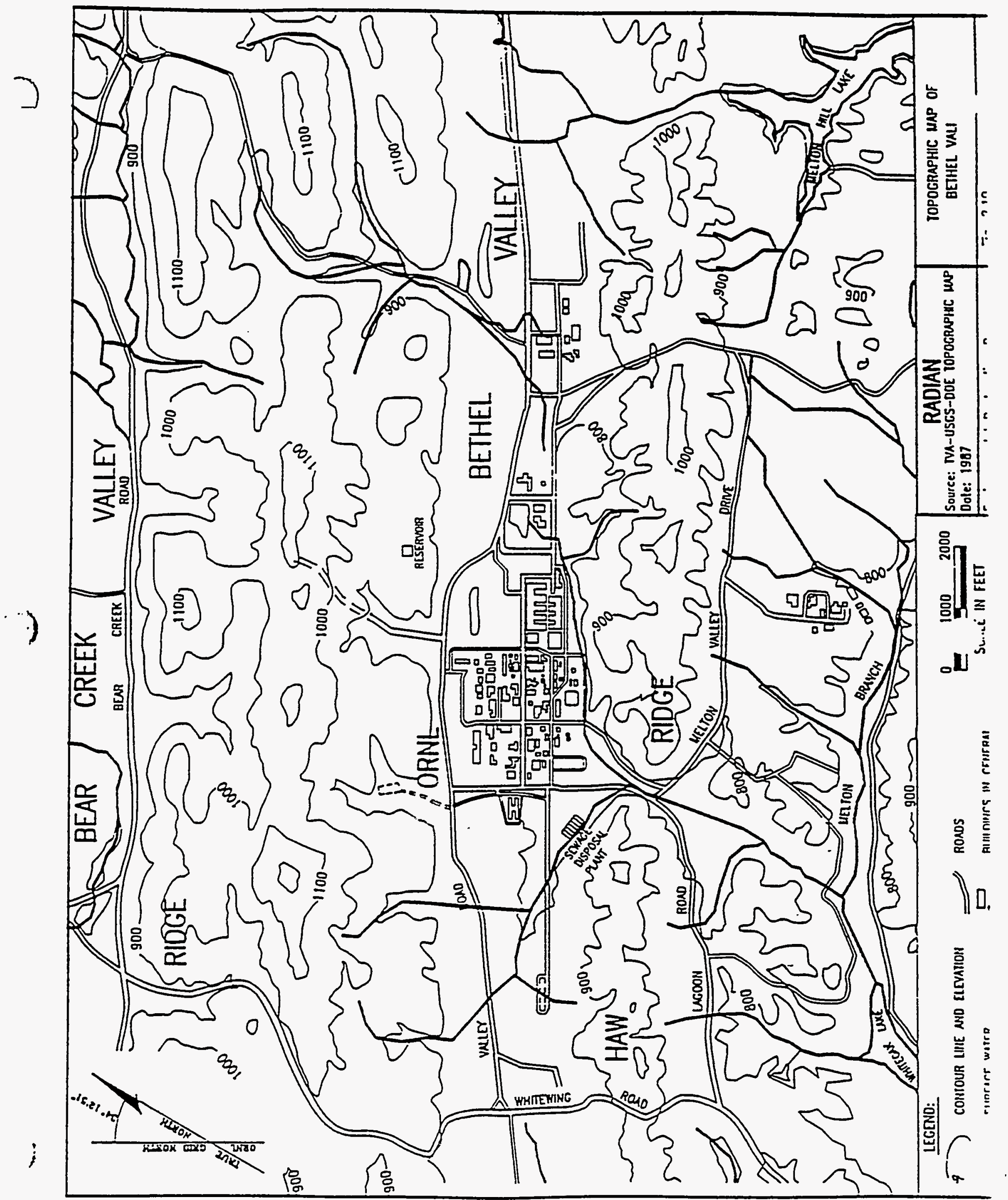




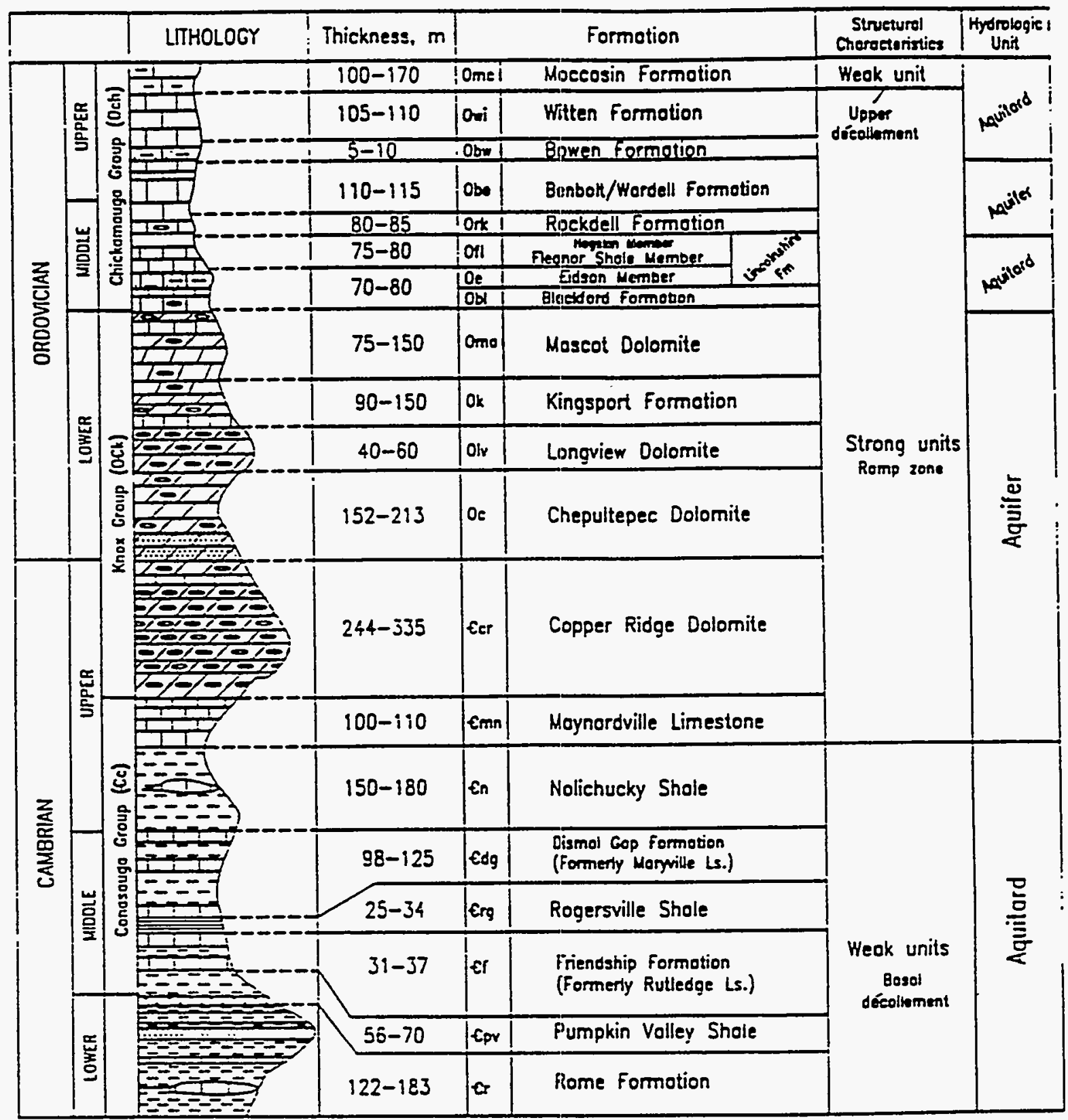

\begin{tabular}{|c|c|}
\hline $\begin{array}{l}\text { RADIAN } \\
\text { Souren: Hotcher et ol. } \\
\text { Dote: } 1992\end{array}$ & 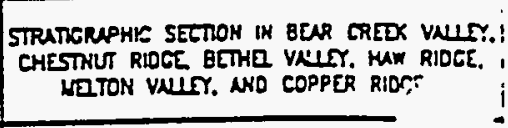 \\
\hline Environmontal Rarlorotuon Progrem & Fig. 2.9 \\
\hline
\end{tabular}




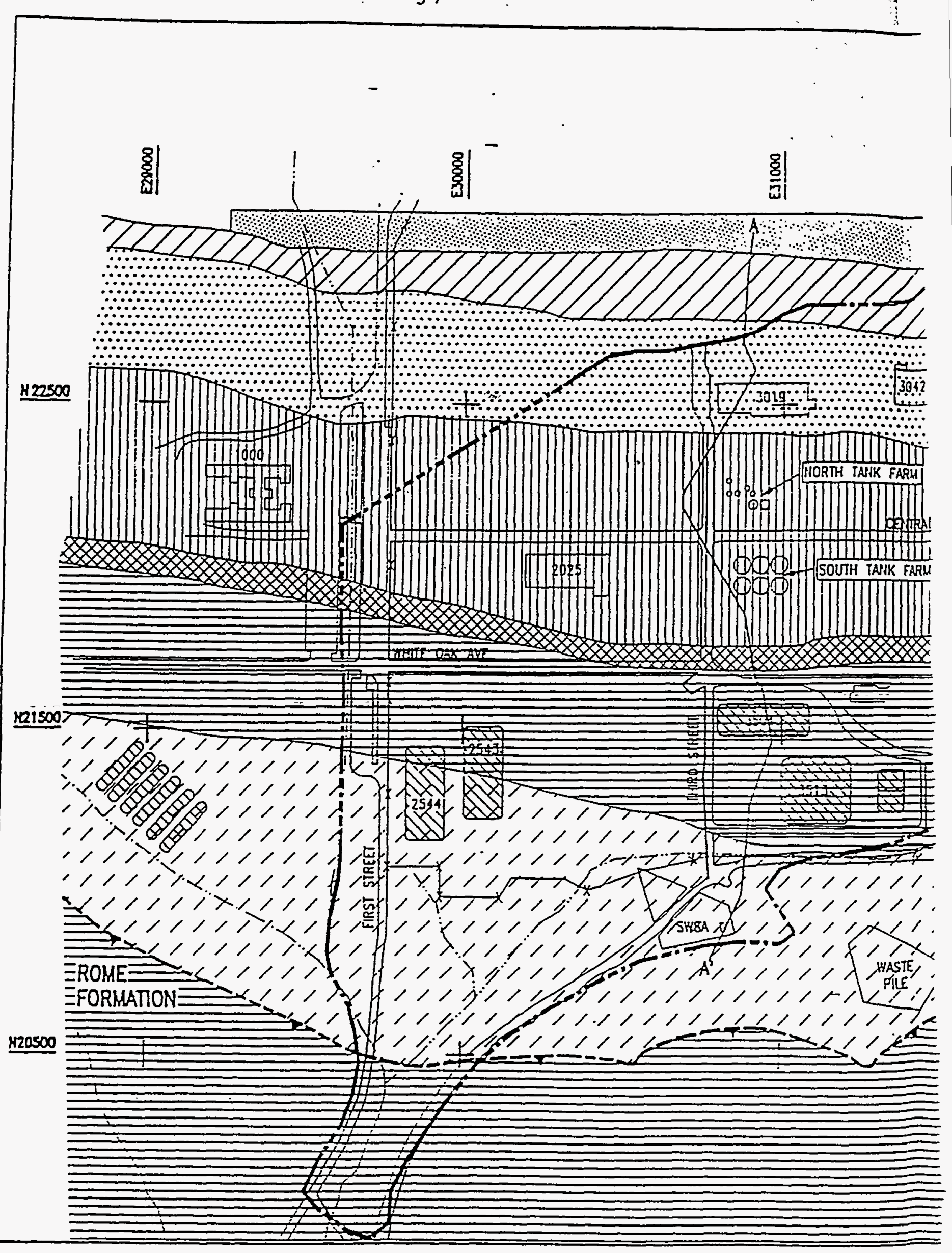

Fig. 3.4. Bedrock Geology at WAG 1 


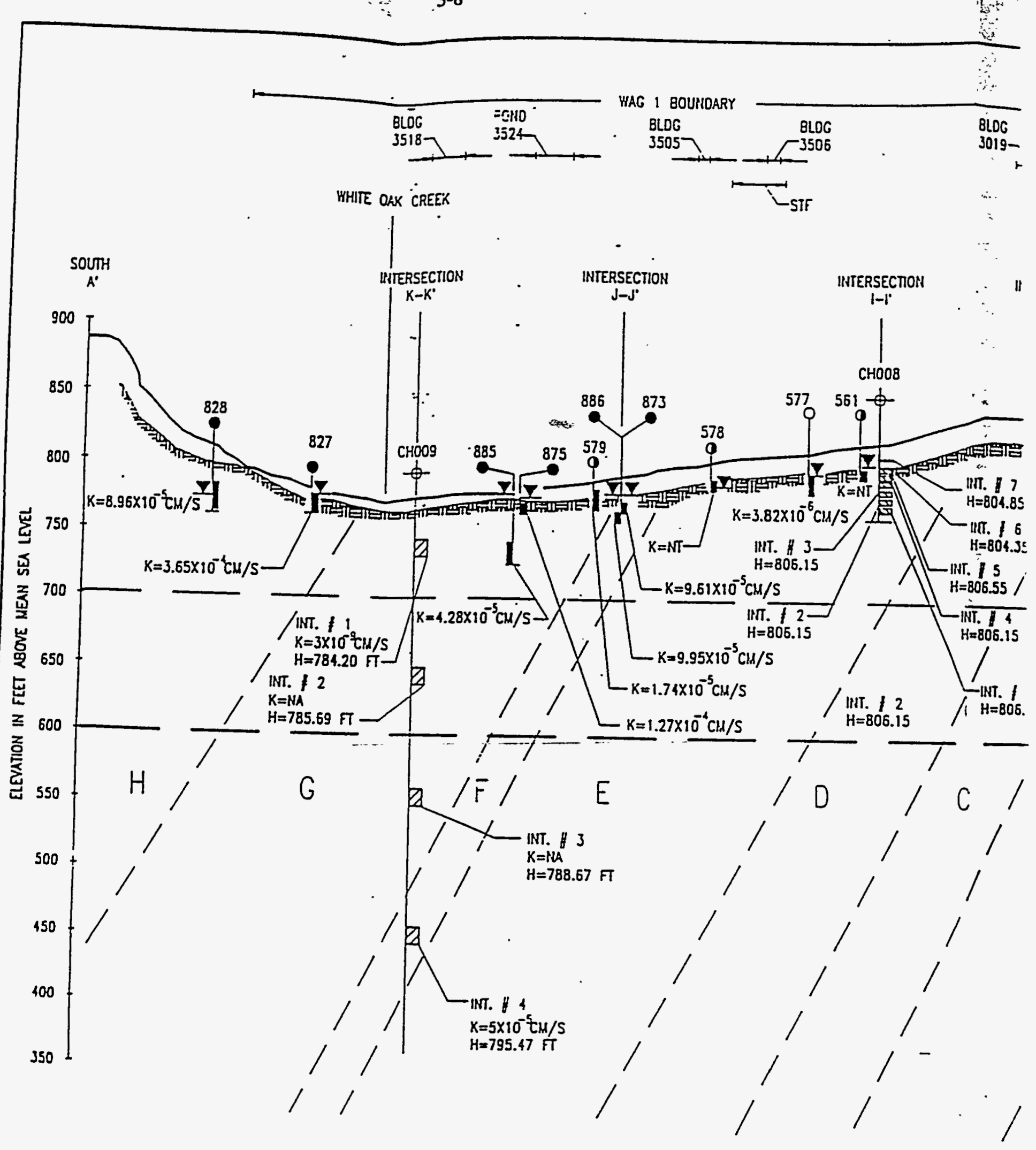

Fig. 3.5. Cross Section A-A' Across WAG 1 
fracture network in the bedrock. The solution conduits store and transmit relatively large volumes of water.

All other rock types on ORR, including the Chickamauga Group in Bethel Valley that underlies ORNL, are classified as aquitards. (Figure 3.6 shows the conceptual model for aquitards.) Six different flow zones are defined in terms of increasing depth: 1) stormflow zone, 2) vadose zone, 3) water table interval, 4) intermediate interval, 5) deep interval, and 6) aquiclude. In general, the water table interval, intermediate interval, deep interval, and aquiclude are included within the groundwater zone and are encountered at various levels in the bedrock; stormflow and vadose zones are encountered in the overburden material.

Depending on lithology; the depths generally below 100 to $300 \mathrm{ft}$ (30 to $100 \mathrm{~m}$ ) exhibit no open fractures, and no significant quantity of water is transmitted. This zone is defined as the aquiclude. Above the aquiclude is a transition between the aquiclude and the water table interval that includes both the deep and intermediate intervals. In the deep interval, a few discrete fractures will transmit water. However, active fractures are few in number and shorter in length. Wells completed in the deep zone typically yield less than 0.03 gallons $/ \mathrm{min}(0.1 \mathrm{~L} / \mathrm{min})$ with less than $1 \%$ of the total groundwater flow occurring in this zone. In the intermediate interval, additional open fractures exist, but the fractures are poorly connected. It has been estimated that less than $2 \%$ of the total groundwater flow occurs in this zone.

The water table interval coincides with a narrow zone of weathered bedrock. This zone is approximately 0.3 to $1.5 \mathrm{ft}(1$ to $5 \mathrm{~m}$ ) thick and contains many open, closely-spaced, interconnected fractures. While the bedrock in the water table interval has been weathered, it has not been reduced to the clay and silt encountered in the overburden. The water table generally corresponds with the top of the weathered bedrock. It has been estimated that approximately $8 \%$ of the total groundwater flow occurs in the water table interval.

Almost all of WAG 1 is a local discharge area for groundwater flow, and most of the groundwater flow occurs at shallow depth toward the south with discharge primarily to White Oak Creek (Fig. 3.7). Consistent with the concept of a discharge area, vertical hydraulic gradients are generally upward with the water table present in the overburden over portions of WAG 1.

The issue of the effect of fill material on groundwater conditions in the vicinity of Building 3506 remains unresolved. Various types of fill material have been used in the utility and pipe trenches underlying much of WAG 1 and in the transfer lines for the tanks. In addition, several feet of bedrock and all of the overburden appear to have been excavated for the construction of the South Tank Farm. Historical photographs show one large excavation for all six Gunite tanks. To control potential leaks from the tanks, a system of dry wells was installed. The concrete pads on which the actual tanks were constructed have larger diameters than the tanks (as if the tanks were cups sitting on the saucer-like pads). The pads include a curb and gutter system that drains by means of a 6 -in. (15-cm)-diameter terra cotta drain tile system to six individual dry wells, all of which connect to a common drain for pumping to the equalization basin. Design drawings indicate that a 3-ft $(0.9-\mathrm{m})$-thick layer of 2-in. $(5.1-\mathrm{cm})$ crushed limestone was placed around the exterior wall from the base to the top of the tank [about $12 \mathrm{ft}(3.7 \mathrm{~m})$ ]. If a tank leaked, the 

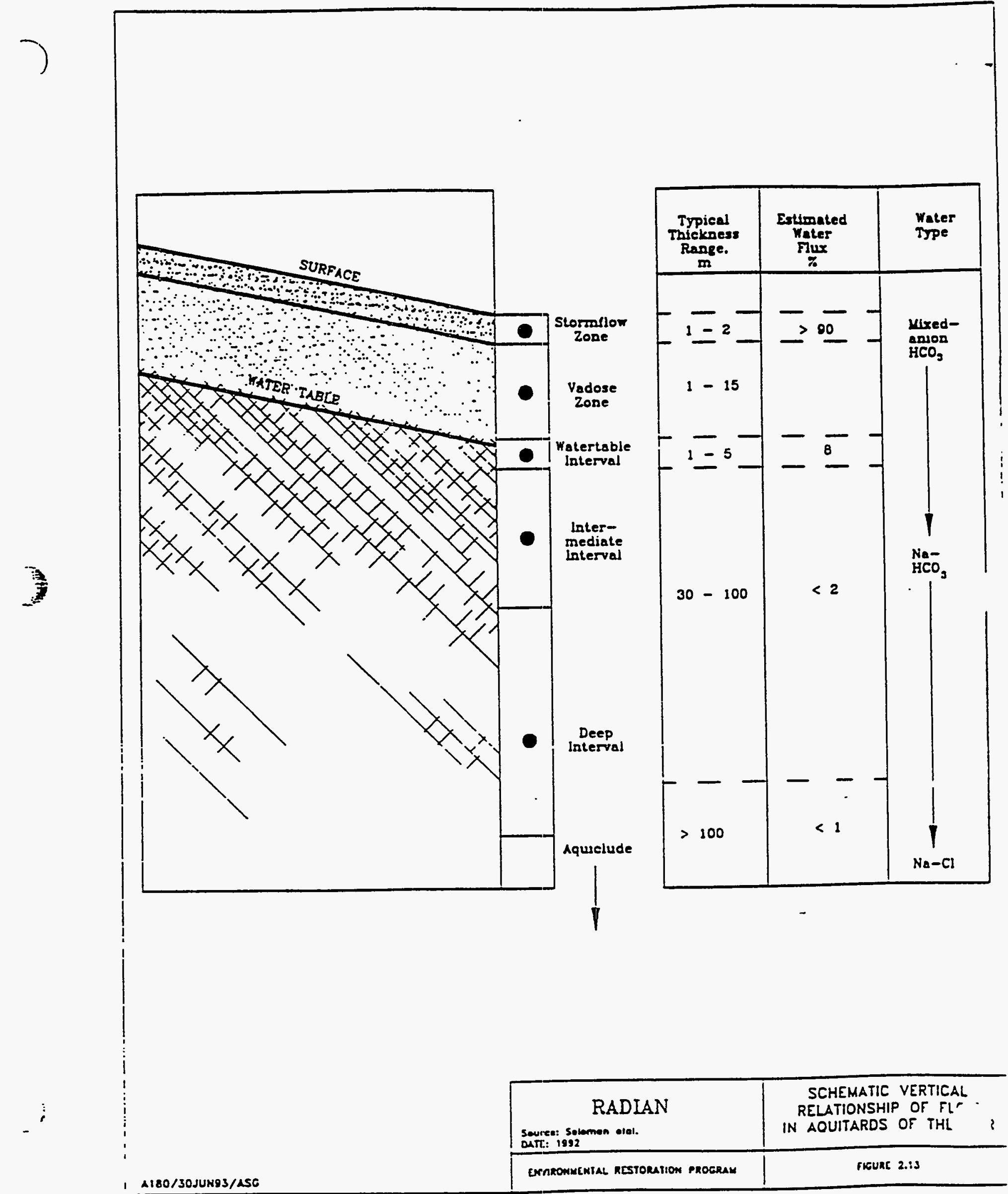
I66I ILIdV 's

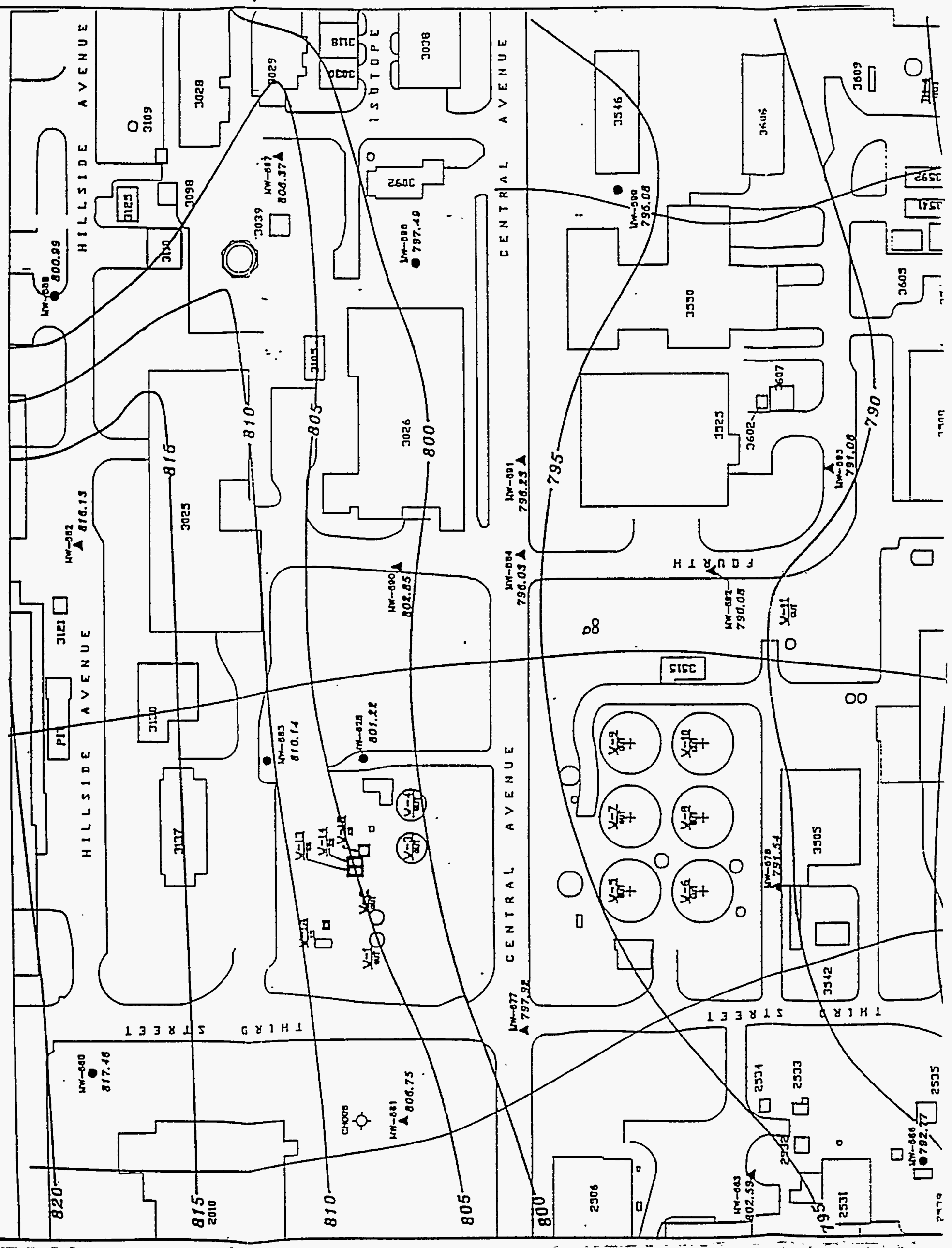


liquid would flow down through the crushed rock, collect in the curb and gutter system, and be captured in the dry wells where routine monitoring would detect the leak.

The use of fill material influences the occurrence and movement of groundwater as well as the movement of contamination at ORR. Use of granular backfill material or inadequate compaction of the native clay material in pipeline and utility trenches, as well as around some of the tanks, have resulted in higher permeability zones. Groundwater or waste liquids can move along the preferential pathways created by the more permeable material. In the early 1960 s, the dry well system at the South Tank Farm was reportedly draining an average of 26,500 gallons $(100,300 \mathrm{~L})$ per day. The source of the volume of water is believed to have been groundwater that was drawn into the dry well systems located below the local water table.

\subsubsection{Potentially Exposed Populations}

Populations potentially exposed to contaminants in Building 3506 and/or released to the environment from Building 3506 include both human. and ecological receptors. The potential human receptors can be divided into the following groups:

- employees of 1) Martin Marietta Energy Systems and its subcontractors at the ORNL, 2) DOE, 3) Tennessee Department of Environment and Conservation (TDEC), and 4) EPA employees and other individuals who visit ORNL;

- residents of Oak Ridge and surrounding communities;

- individuals who use the recreational facilities on or near the ORR; and

- individuals who trespass onto the site seeking permanent or temporary shelter under future scenarios.

Potential ecological receptors are terrestrial and aquatic organisms who spend all or part of their lives on or near the ORR. Discussions of regional demographics, current and future land use, and ecology are presented in the following sections.

\subsubsection{Demography and Land Use}

ORR covers approximately 35,300 acres (14,300 ha) of federally-owned land in eastern Tennessee (Fig. 3.8). ORNL is in the approximate center of the ORR (Fig. 3.9). The Tennessee Valley Authority's (TVA's) Melton Hill reservoir on the Clinch River and Watts Bar Reservoir on the Tennessee River form the eastern, southern, and western boundaries of the ORR. The residential area of the city of Oak Ridge forms the northern boundary.

Oak Ridge, with a population of 27,000 , is the closest population center. [All population levels are based on 1990 U.S. census data (U.S. Department of Commerce 1991).] Although located predominantly to the west and south of the population center, the ORR is located inside the city limits of Oak Ridge. In addition to the city of Oak Ridge, the nearest population centers to the ORR include Oliver Springs (pop. 3400), 6.8 miles $(11 \mathrm{~km})$ to the northwest; Clinton (pop. 9,000), 10 miles $(16 \mathrm{~km})$ to the northeast; Lenoir City (pop. 6,100), 6.8 miles $(11 \mathrm{~km})$ to 


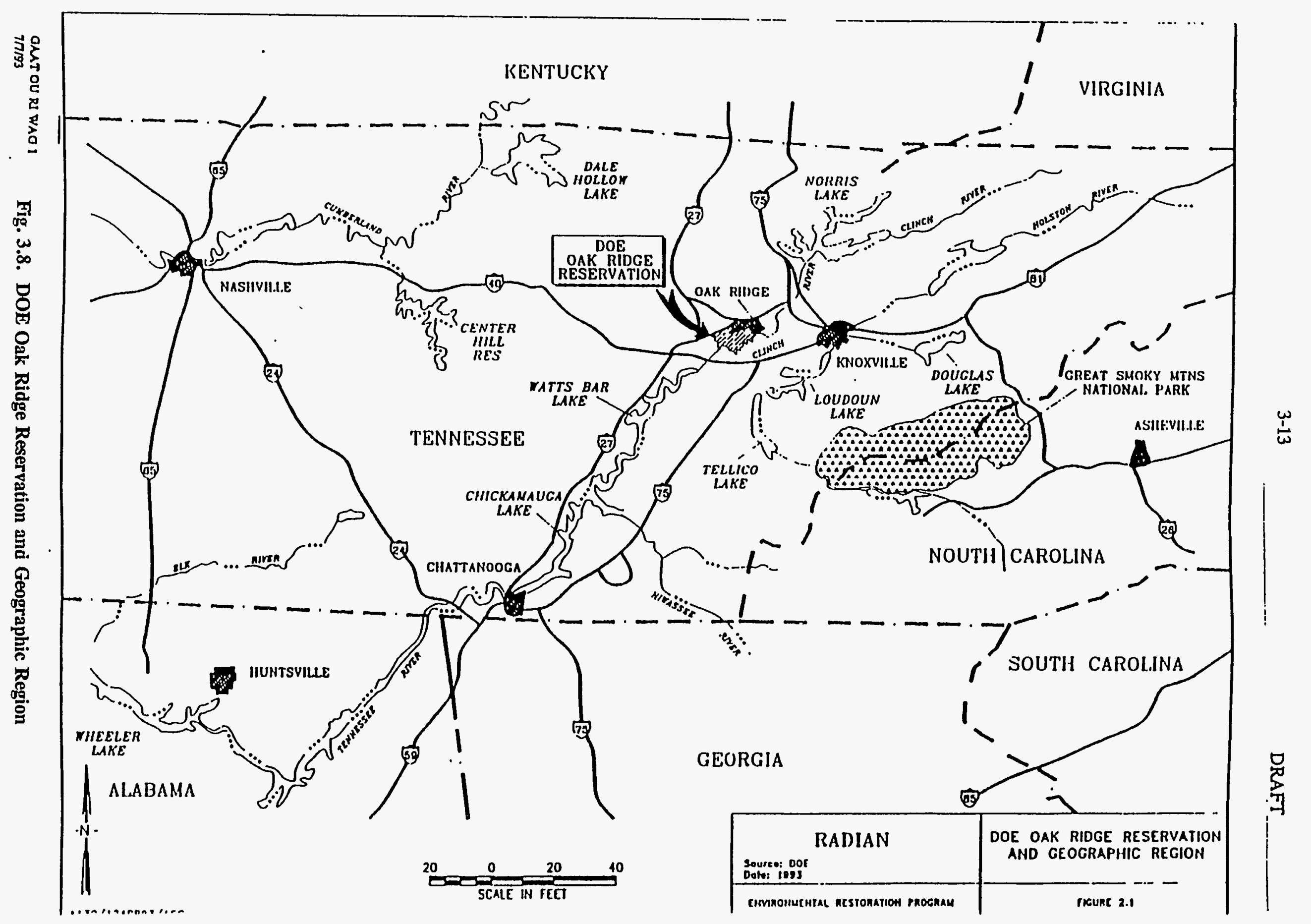




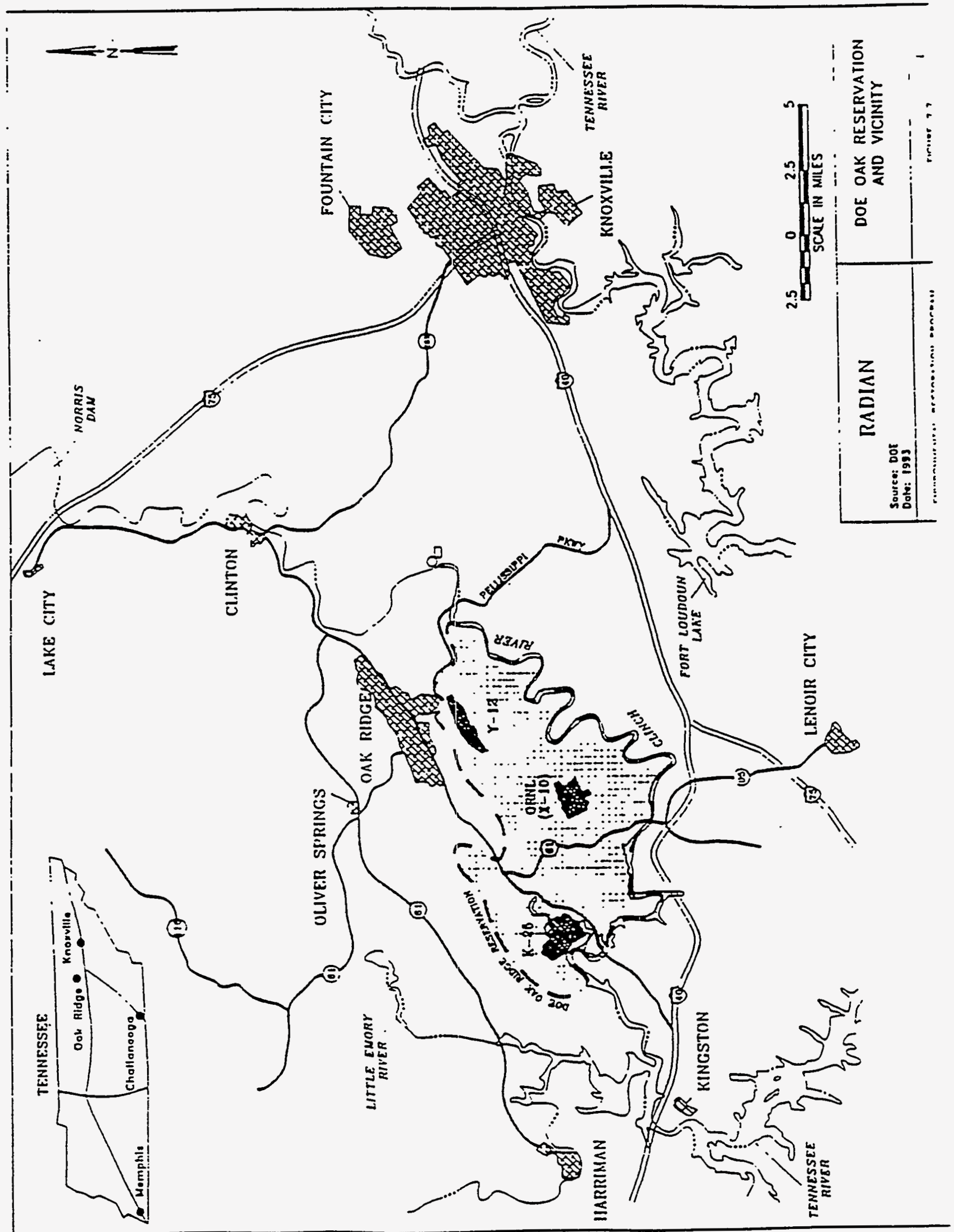


the southeast; Kingston (pop. 4600), 6.8 miles $(11 \mathrm{~km})$ to the southwest; and Harriman (pop. 7100), 8 miles $(13 \mathrm{~km}$ ) to the west. The population of Anderson County, which includes Oak Ridge, Oliver Springs, and Clinton, is 68,250. Knoxville, Tennessee, is the closest major metropolitan area. Knoxville is approximately 25 miles $(40 \mathrm{~km})$ east of the ORR and has a population of approximately 165,000 . Knox County, which includes Knoxville, has a population of 335,749 .

Figure 3.10 shows the population density within 10 miles $(16 \mathrm{~km})$ of the center of the ORR. Except for the city of Oak Ridge, land within 5 miles $(8 \mathrm{~km})$ of ORR is predominantly rural and is used primarily for residences and small farms. Fewer than 13,000 people live within 5 miles $(8 \mathrm{~km})$ of the center of the ORR, which includes a portion of the city of Oak Ridge. Figure 3.11 shows the population density within 50 miles $(80 \mathrm{~km})$ of the ORR.

As of February 1993, ORR employs approximately 16,000 regular employees, with 5782 employees working at ORNL. Most employees at ORNL work at facilities that are located near Building 3506.

\section{Current Land Use}

Worker activities in the vicinity of Building 3506 are those common to industrial complexes. These include pedestrian and motor vehicle traffic, utility repair, and grounds maintenance. Currently, there is no worker activity within Building 3506 except for routine surveillance and maintenance (S\&M) activities. Activities for this facility include routine surveys (approximately once a year) and vegetation maintenance around the building. The cell portion of the facility is rarely accessed. All activities are performed under applicable regulations and monitored by the Industrial Hygiene and Health Physics departments as necessary. Protective clothing (coveralls and booties) are required when entering the cell.

For this Level 3 BRE two hypothetical current exposure scenarios have been developed to provide a conservative (i.e., health protective) estimate of potential exposure. The first hypothetical current receptor is a maintenance worker who spends 8 hours per day, 250 days per year, for 25 years working in the building. This receptor provides a worst-case or upper-bound estimate of potential exposure for a current receptor at the facility. A second more realistic receptor is represented by a S\&M worker who spends two 8-hour days per year in the facility for 25 years. This second receptor provides a more realistic, but still health-protective (by virtue of the conservative exposure parameters), estimate of potential exposure at the facility. It is also assumed that protective measures are not taken by either worker. Current exposure scenarios and receptors evaluated in this BRE are summarized in Table 3.1 .

\section{Future Land Use}

Building 3506 is located in the main plant area of ORNL. This area is highly developed, and land use is expected to remain industrial for the foreseeable future. The population of Oak Ridge is currently stable. Growth is hindered due to a lack of developable land. If DOE releases portions of the ORR for to the public, expansion of Oak Ridge would be expected to follow. 

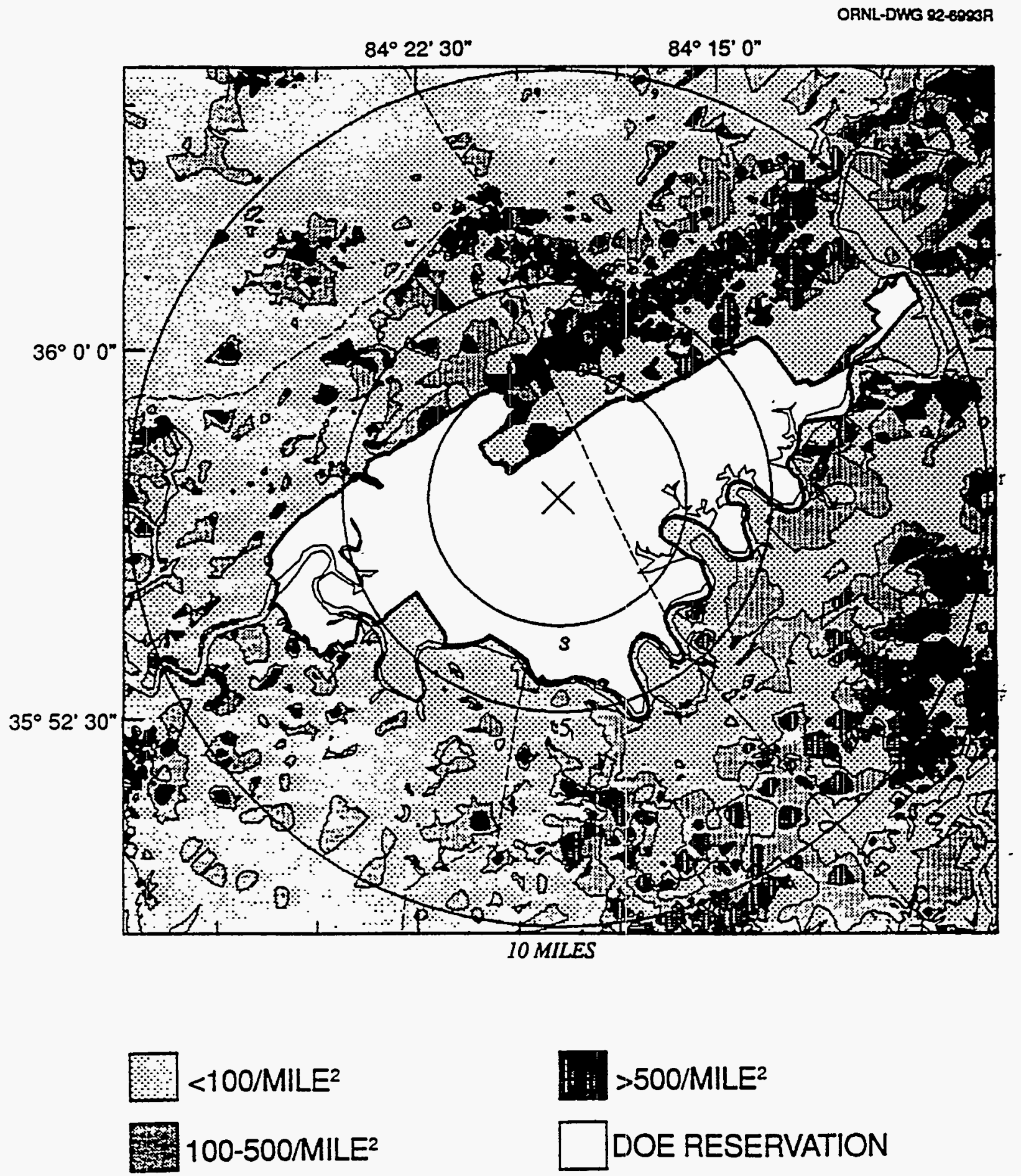

Fig. 3.10. Projected 1991 Population Densities Within $16 \mathrm{~km}(10$ miles) of the center of the Oak Ridge Reservation, based on 1990 Census Data (U.S. Departrnent of Commerce, 1991). 
ORNL-DWG 92-6904R

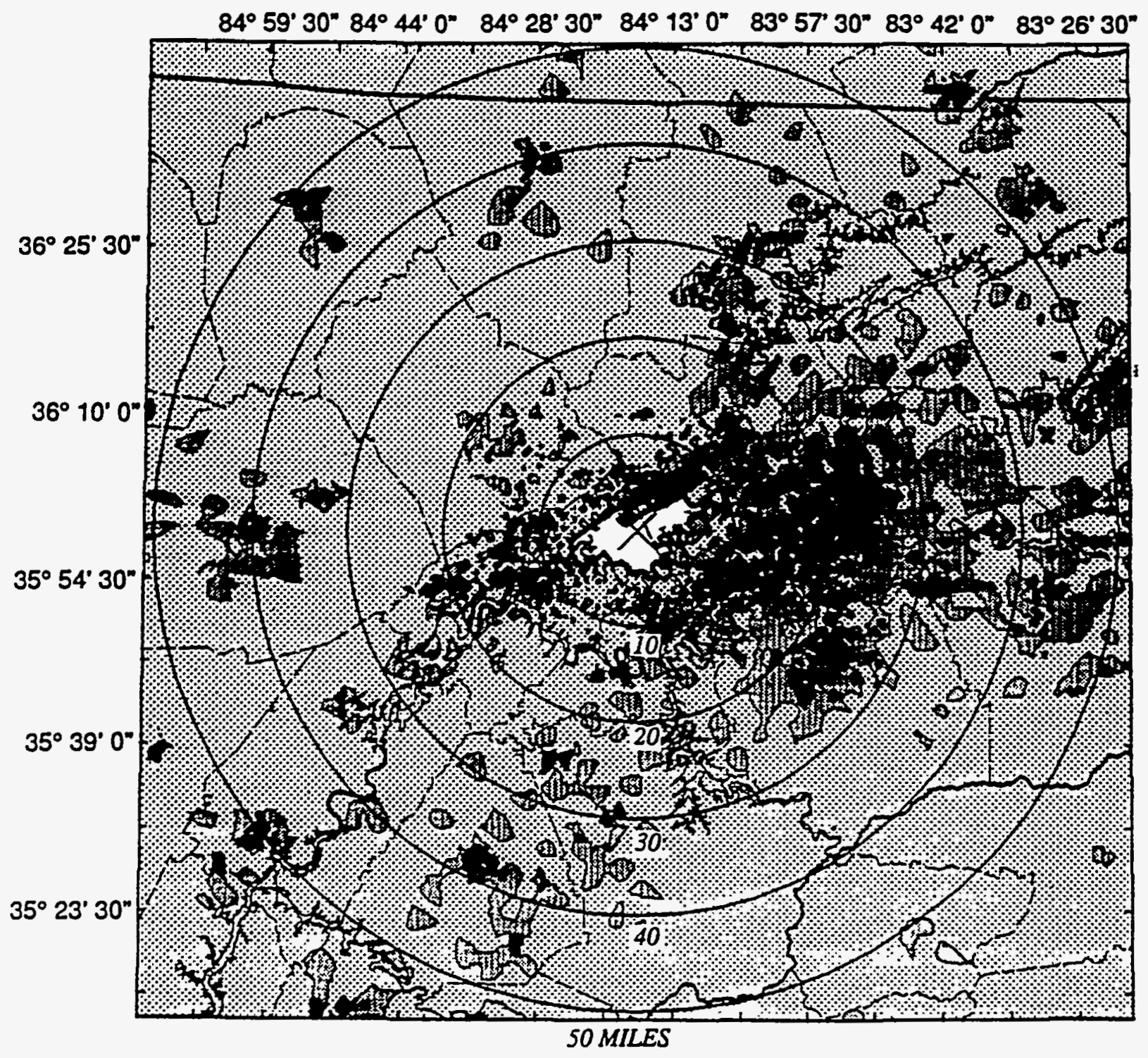

\% $<100 /$ MILE 2 100-500/MILE2
Fi $>500 /$ MILE $^{2}$ DOE RESERVATION

Fig. 3.11. Projected 1991 Population Densities Within $80 \mathrm{~km}(50$ miles) of the center of the Oak Ridge Reservation, based on 1990 Census Data (U.S. Department of Commerce, 1991). 
Table 3.1. Exposure scenarios and potential receptors for Building $\mathbf{3 5 0 6}$

\begin{tabular}{|c|c|c|c|}
\hline Scenario & Action & Final Property Dispersement & Receptors \\
\hline \multicolumn{4}{|l|}{ Current Land Use } \\
\hline $\begin{array}{l}\text { "Worst-case" Worker } \\
\text { Exposure }\end{array}$ & $\begin{array}{l}\text { Maintenance and other work } \\
\text { requiring daily access by workers }\end{array}$ & $\mathrm{NA}$ & Worker $=$ routine, daily access \\
\hline Normal S\&M & $\begin{array}{l}\text { Normal S\&M activities require } \\
\text { worker access twice per year }\end{array}$ & NA & $\begin{array}{l}\text { Worker = limited access to } \\
\text { facility }\end{array}$ \\
\hline \multicolumn{4}{|l|}{ Future Land Use } \\
\hline Abandonment & $\begin{array}{l}\text { Complete immediate abandonment } \\
\text { of the building. } \\
\text { Loss of institutional controls on the } \\
\text { building. }\end{array}$ & $\begin{array}{l}\text { None-DOE releases property } \\
\text { without converting the land to } \\
\text { any predetermined use (i.e., "as } \\
\text { is" conditions). }\end{array}$ & $\begin{array}{l}\text { Trespasser - an adult trespasser } \\
\text { may take up residence in the } \\
\text { vacant building. }\end{array}$ \\
\hline
\end{tabular}


This BRE is intended to provide exposure and risk estimates for a "no-action" (i.e., no remedial action) future land use scenario. A hypothetical no-action scenario makes the assumption that Building 3506 is abandoned by DOE at the start of the BRE. In this scenario, DOE makes no attempt to prevent contaminant releases from the facility or restrict access to the facility. Because Building 3506 is on the ORR and DOE does not intend to release the ORR property for development, the area around the building is expected to remain industrial even if the building was abandoned. Therefore, it is assumed that residential development will not occur at this site. However, it is assumed that an adult trespasser can enter the building and take up residence (e.g., a squatter). This is a conservative exposure scenario designed to fulfill the intent of future no-action. It is unlikely that a trespasser could enter the building even if access to the building is not restricted because the building is on DOE property with controlled access. Actual future exposures are likely to be limited to S\&M workers (as described for current land use) or the occasional trespassing worker if S\&M activities are discontinued.

If the building is abandoned by DOE (i.e., all building maintenance is discontinued), it will eventually decay and collapse. The building structure currently acts as a containment vessel for the contaminants inside. As the building decays, these contaminants will be released to the surrounding environment via wind erosion and dust transport, washout with rain, wind born vapors, and tracking of contaminants by humans and small animals potentially visiting the site. Such contaminant releases could potentially result in exposure of off-site (i.e., outside of DOE property) residential receptors. Such off-site exposures have not been included in the quantitative risk evaluation because the quantity of radioactive and chemical contaminants is insufficient to result in significant off-site exposures. The potential exposure scenario and receptor associated with the future land-use scenario evaluated in this BRE are summarized in Table 3.1.

\subsubsection{Potential Ecological Receptors}

\section{Potentially Affected Habitats}

Building 3506 is located inside the main industrial area of ORNL and has little natural habitat. The vegetation surrounding Building 3506 consists primarily of grass lawns and landscaping plants. The following information taken from the Preliminary Risk Assessment Report for-Waste Area Grouping 1 at Oak Ridge National Laboratory, Oak Ridge, Tennessee (Bechtel, 1992) also pertains to Building 3506, which as mentioned previously, is located within WAG 1.

The aquatic habitat at WAG 1 consists of White Oak Creek, First Creek, Fifth Creek, and part of the Northwest Tributary. These small streams are within the White Oak Creek watershed and receive discharges directly from ORNL facilities. Nonpoint sources such as solid waste storage areas or process ponds may also contribute contaminants to these streams. Surface impoundments constricted for use as waste settling basins provide additional aquatic habitat within WAG 1 (Bechtel, 1992).

\section{Potentially Exposed Populations}

Thirty-seven species of mammals, 151 species of birds, and 61 species of reptiles and amphibians have been found on the ORR. The area, however, provides little natural terrestrial habitat, and the most common species are those generally associated with ore urban and 
developed areas. In the lawn areas, mammalian species are likely to be those typical of old field areas such as house mice (Mus musculus), cotton rats (Sigmodon hispidus), white-footed mice (Peromyscus leucopus), short-tail shrews (Blarina brevicauda), eastern harvest mice (Reithrodontomys humilis), and groundhogs (Marmota monax). Shrubs and low-growing herbaceous plants provide food and nesting areas for birds such as sparrows, rufous-sided towhees (Pipilo erythrophthalmus), blue grosbeaks (Guiraca caerulae), brown thrashers (Toxostoma rutum), cardinals (Cardinalis cadinalis), mockingbirds (Mimus polyglottos), and robins (Turdus migratorius). Waterfowl, such as Canada geese (Branta canadensis), frequent the area, as do frogs, toads, and reptiles (Bechtel, 1992).

The most common fish species in the White Oak Creek watershed are two native minnows: blacknose dace (Rhinichtys atratulus) and creek chubs (Semotilus atromaculatus). Fathead minnows (Pimephales promelas), and introduced species, are also common, as are sunfish species [e.g., redbreast (Lepomis auritus) and bluegill (L. macrochirus)]. Benthic macroinvertebrate communities include primarily taxa that are pollution-tolerant, mainly chironomids.

The Ecological Evaluation in the Preliminary Assessment Report (Bechtel, 1992), which relied on data from the Biological Monitoring and Abatement Program and data collected during the Remedial Investigation, concluded that there is a present or potential risk of detrimental effects to the environment in the absence of remediation at WAG 1. As mentioned previously, there are currently no known releases of contaminants from Building 3506. Contamination released as a result of catastrophic events or through building decay may reach ecological receptors, but the effect, if any, caused by the contaminants released from Building 3506 would be hard to differentiate from the level of contamination (and their effects) that already exists within WAG 1. A quantitative ecological risk evaluation would not be useful for decision-making because contminant releases from the building would not be distinguishable from other contamination in the area.

\subsection{DENTIFICATION OF POTENTIAL EXPOSURE, PATHWAYS}

The purpose of this section is to identify the potential pathways for human exposure to contaminants found in Building 3506 at ORNL. An exposure pathway is defined as the course a chemical or physical agent takes from its source to a potentially exposed receptor. For exposures to occur, complete exposure pathways must exist. A complete exposure pathway consists of the following four elements:

- A source and mechanism for release of a contaminant

- A retention or transport medium

- A point of potential human contact with the contaminated medium

- An exposure route at the exposure point 
1)
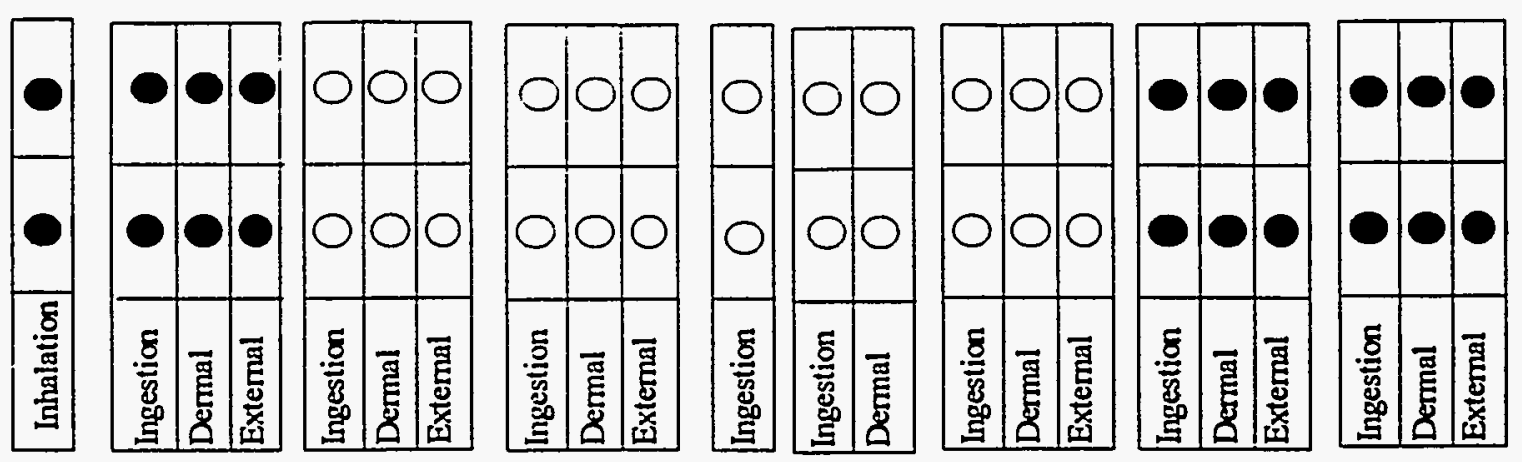

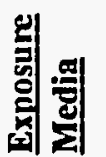

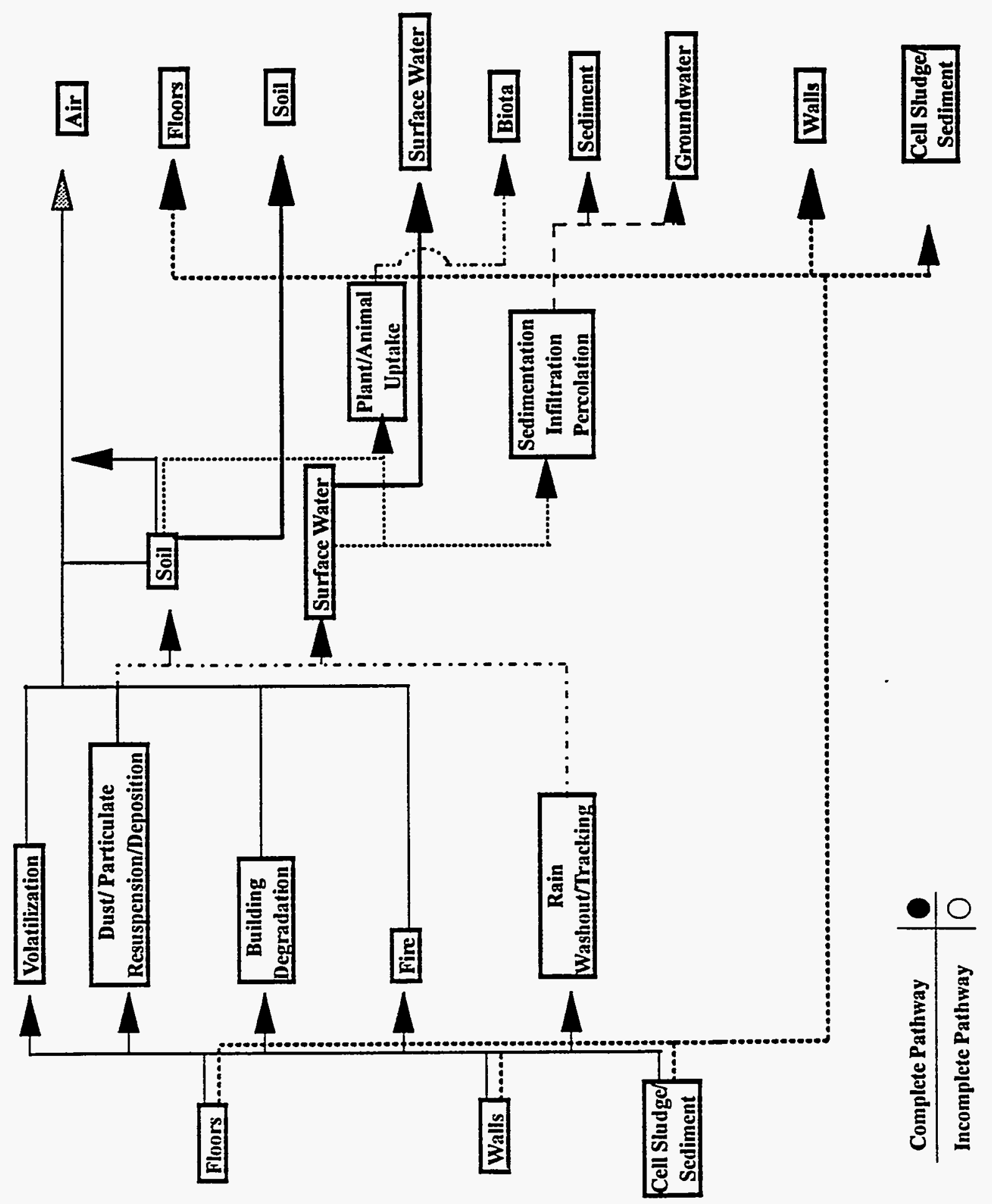



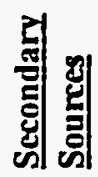

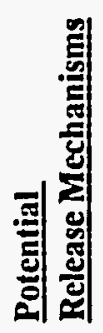

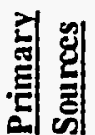


If any one of these elements is missing, the pathway is not complete and there is no exposure. A Conceptual Site Model (CSM) is a graphic representation of the potential routes of contaminant migration from a source to a receptor (i.e., exposure pathway). The conceptual site model for Building 3506 is depicted in Figure 3.12 and is discussed in the following subsections.

\subsubsection{Potential Sources and Receiving Media}

The primary sources of contaminants at Building 3506 are the floors and walls of the building and the sediment in the cell. These sources are also exposure media for receptors within Building 3506. Exposure media are those contaminated media where actual or potential contact by receptors may occur. Potential release mechanisms can release contaminants from the walls, floors, and sediment to secondary sources and exposure media. Secondary sources include soil, water, and air. As depicted in Fig. 3.12, releases from the secondary sources can result in contamination of the exposure media.

Potential release mechanisms within the building include dust/particulate resuspension/deposition of contaminated concrete dust from the floors and walls and from sediment. Volatilization of chemicals may also occur from the sediment. Potential release mechanisms for contaminants to leave the building and enter the surrounding environment include particulate resuspension/deposition associated with building degradation or fire, tracking of contaminated dust and/or sediment, and rain washout. The magnitude of the release is controlled by the initiating event, integrity of the containment unit, and form of the contaminant. Once contaminants are in the environment, they become available for continued transport through the environment and uptake into the food chain. Releases of large volumes of the contaminants associated with the walls and floors are highly unlikely because of their association with these structural media. However, contaminants found in the sediment could potentially be released via rain washout (if roof integrity is lost) or by tracking.

Specific sources and receiving media evaluated for the current and future exposure scenarios in this BRE are presented in Subsects. 3.2.1.1 and 3.2.1.2.

\subsubsection{Current sources and receiving media}

Current contaminant release from the floors, walls, and sediment can occur by generation of dust resulting from pedestrian traffic within the building. This release of contaminants may be minimal due to the low numbers of individuals who access Building 3506 . Receiving media for dust within the building are the air and exposed surfaces. Releases of volatile chemicals to the air may also occur. Transport of dust borne contaminants outside the building is expected to be kept at a minimum because shoe scuffs are required to be worn in the cell portion of the facility.

\subsubsection{Future sources and receiving media}

Further release of contaminants within the building will occur as described for the current scenario. Releases are expected to increase as the building structure deteriorates over time. Future sources of contaminant released to the environment outside the building include all media inside Building 3506 (building structure and sediment). As noted previously (Sect. 3.1.2.1), the 
quantity of contaminants in Building 3506 is not large enough to result in significant off-site release.

\subsubsection{Potential Exposure Points and Exposure Routes}

An exposure point is the location and medium at which a receptor contacts a contaminant (e.g., concrete dust from the floor). Exposure routes are the potential uptake mechanisms by which exposure occurs once a receptor comes in contact with the contaminated media (i.e., ingestion, inhalation, etc.).

The walls and floors of Building 3506 (both the east gallery and the cell) serve as locations with which a receptor may come in contact with contaminants. Contaminants in the walls and floors of Building 3506 reside in the concrete and concrete dust (particulates). Another medium with which a receptor may contact contaminants is the sediment located in the cell. Exposure routes for these media include incidental ingestion of dust and sediment, inhalation of air borne contaminants (including dust and volatile chemicals), dermal contact with chemicals, and external exposure from radionuclides.

\subsubsection{Summary of Potential Exposure Pathways}

Exposure pathways combine the contaminant source, receiving medium, exposure point, and exposure route. Exposure pathways selected for evaluation in this BRE for the potential current and future exposure scenarios are described below. Once all potential pathways have been identified, the potentially significant ones are selected for more detailed evaluation in the BRE. Potential exposure pathways may be eliminated from further consideration in the BRE if they are not complete, if their contribution to total exposure is negligible compared to other pathways, or due to the definition and scope of the BRE.

\subsubsection{Potential Current Exposures}

Exposures at Building 3506 are limited to direct contact with contaminants, inhalation of dust, incidental ingestion, and external exposure to radiation by workers. Health and safety measures at ORNL mitigate exposures to workers by requiring the use of protective clothing and prohibiting eating or smoking in the facility. Potential exposure are described in the following paragraphs for each medium of concern. The current exposure pathways evaluated in this BRE are listed in Table 3.2 .

Air

Inhalation of contaminated dusts and volatile chemicals is a pathway of concern at this site. Maintenance and S\&M workers at Building 3506 entering the building are potentially exposed via this route. 


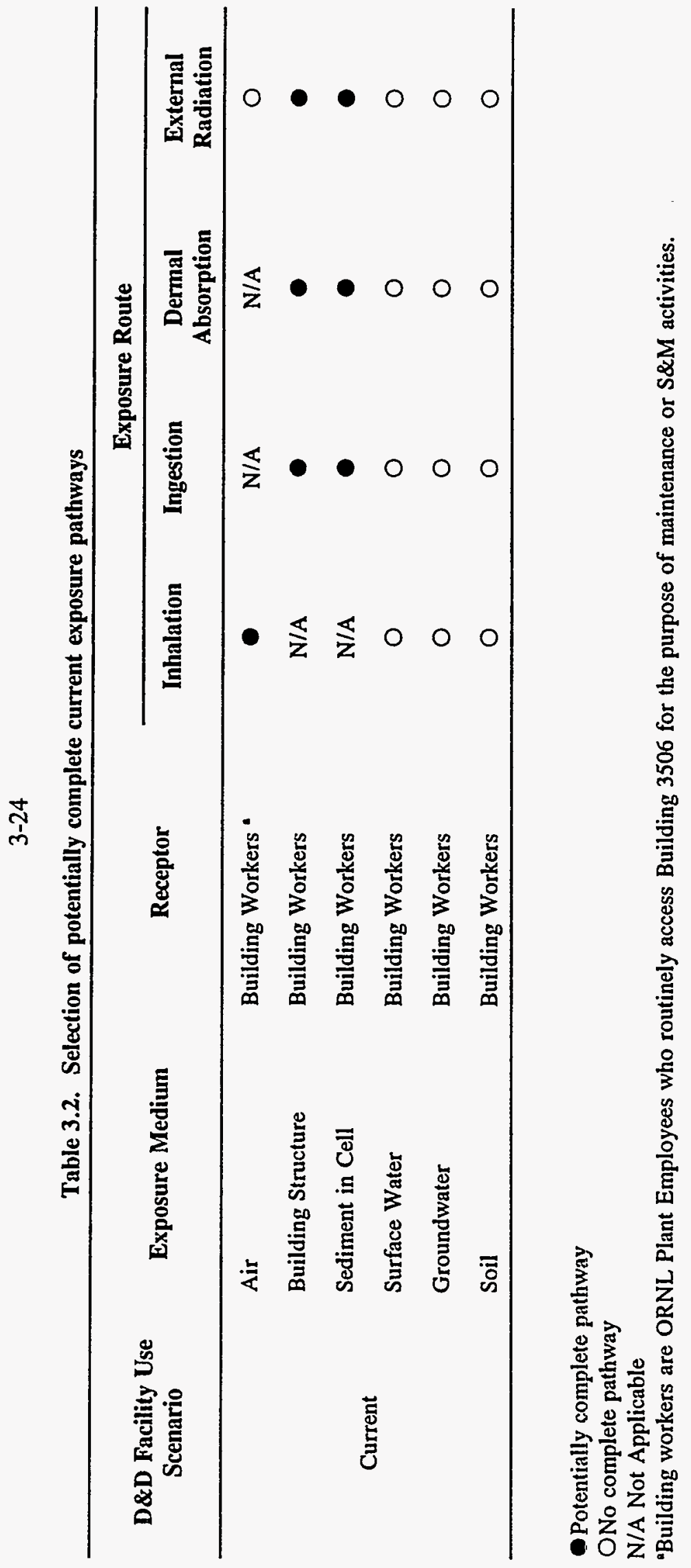




\section{Building Structure (Floors and Walls)}

Dermal absorption (chemicals), incidental ingestion, and external exposure (radionuclides) to contaminants on the inside surfaces of the building structure are potential exposure pathways of concern for the hypothetical current receptor at Building 3506.

\section{Sediment in Cell}

Dermal absorption (chemicals only), incidental ingestion, and external exposure (radionuclides) to contaminants present in the sediment are also potential exposure pathways of concern for the hypothetical current receptor at Building 3506.

\subsubsection{Potential Future Exposures}

Future exposures to contaminants within Building 3506 may include direct contact with contaminated media (ingestion or dermal absorption), inhalation of volatiles or particulate phase contaminants, and external exposure to radionuclides. Future exposures could also result from releases of contaminants to the environment. Potential exposures are discussed in the following paragraphs for each medium of concern. The future exposure pathways evaluated in this BRE are listed in Table 3.3.

Air

Inhalation of contaminated dust and volatiles is a pathway of concern at this site as previously described for current exposures. Trespassers at Building 3506 would be potentially exposed via this route.

\section{Building Structure (Floors and Walls)}

Dermal absorption(chemicals), incidental ingestion, and external exposure (radionuclides) to contaminants present on the inside surfaces of the building structure are potential exposure pathways of concern for trespassers at Building 3506.

\section{Sediment}

Dermal absorption (chemicals), incidental ingestion, and external exposure (radionuclides) to contaminants present in the sediment in the cell are also potential exposure pathways of concern for trespassers at Building 3506.

\subsection{QUANTIFICATION OF EXPOSURE}

This section describes the quantitative estimation of exposures for each of the exposure pathways described above. The process involves:

- identifying applicable human exposure models and input parameters, 
- determining the concentration of each contaminant of potential concern in the exposure media at the point of human exposure, and

- estimating human intakes.

The potential exposure pathways at Building 3506 are identified in Tables 3.2 and 3.3.

\subsubsection{Exposure Models}

Exposures were estimated for ingestion, inhalation, dermal absorption, and external radiation exposure using the following equations (EPA 1989a):

Ingestion of dust and sediment

$C D I=\frac{C \times I R \times C F \times F I \times E F \times E D}{B W \times A T}$

Chemicals

$C D I=C \times I R \times E D \times E F \times F I$ Radionuclides

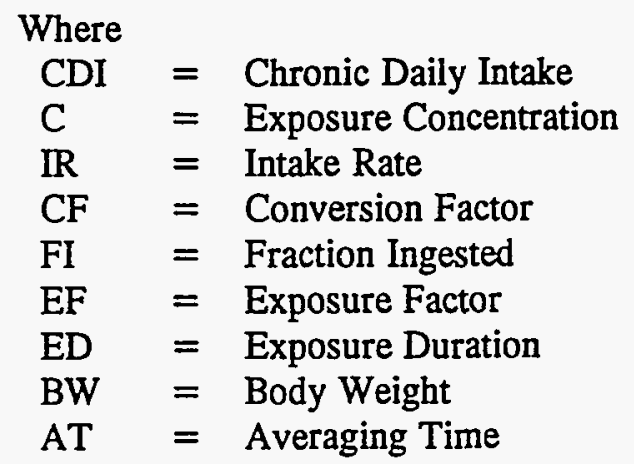

Inhalation of dust

$C D I=\frac{C \times I R \times E F \times E D \times \frac{1}{P E F}}{B W \times A T}$ Chemicals 
Table 3.3. Selection of potential future exposure pathways

\begin{tabular}{|c|c|c|c|c|c|c|}
\hline \multirow{2}{*}{$\begin{array}{l}\text { D\&D Facility Use } \\
\text { Scenario }\end{array}$} & \multirow[b]{2}{*}{ Exposure Medium } & \multirow[b]{2}{*}{ Receptor } & \multicolumn{4}{|c|}{ Exposure Route } \\
\hline & & & Inhalation & Ingestion & $\begin{array}{c}\text { Dermal } \\
\text { Absorption }\end{array}$ & $\begin{array}{l}\text { External } \\
\text { Radiation }\end{array}$ \\
\hline \multirow{6}{*}{ Future Abandonment } & Air & Trespasser & 0 & $\mathbf{N} / \mathbf{A}$ & $\mathbf{N} / \mathbf{A}$ & 0 \\
\hline & $\begin{array}{l}\text { Building Structure (Floors } \\
\text { and Walls) }\end{array}$ & Trespasser & N/A & 0 & 0 & - \\
\hline & Sediment in cell & Trespasser & 0 & - & 0 & 0 \\
\hline & Surface Water & Trespasser & 0 & O & 0 & N/A \\
\hline & Groundwater & Trespasser & 0 & 0 & 0 & $N / A$ \\
\hline & Soil & Trespasser & 0 & 0 & 0 & 0 \\
\hline
\end{tabular}

- Potentially complete pathway

ONo complete pathway

N/A Not Applicable

-The Trespasser is an adult individual who takes up residence in the building after the loss of institutional controls. 
$C D I=C \times I R \times E F \times E D \times \frac{1}{P E F} \quad$ Radionuclides

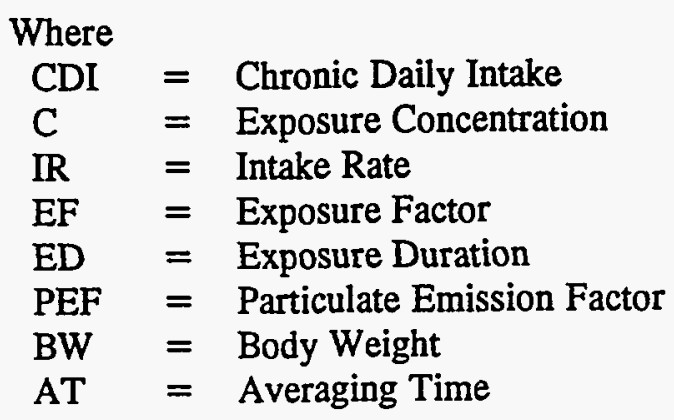

External Exposure to Radionuclides

$C D I=C \times E D \times\left(1-S_{e}\right) \times T_{e} \quad$ Radionuclides $(\mathrm{pCi} / \mathrm{g})$

Radionuclides (mrem/hr)

$C D I=C \times D R \times E F \times E D$

Where

$\mathrm{CDI}=$ Chronic Daily Intake

$\mathrm{C}=$ Exposure Concentration

$\left(1-S_{e}\right)=$ Gamma shielding factor

$\mathrm{T}_{\mathrm{e}}=$ Gamma exposure time factor

$\mathrm{DR}=$ Dose Equivalent Rate

$\mathrm{EF} \quad=$ Exposure Frequency

$\mathrm{ED}=$ Exposure Duration

Dermal contact with dust and sediment

$$
C D I=\frac{C \times C F \times S A \times A F \times A B S \times E F \times E D}{B W \times A T}
$$

Where

$\mathrm{CDI}=$ Chronic Daily Intake

$\mathrm{C}=$ Exposure Concentration

$\mathrm{CF}=$ Conversion Factor

SA $=$ Skin Surface Area Available for Contact 


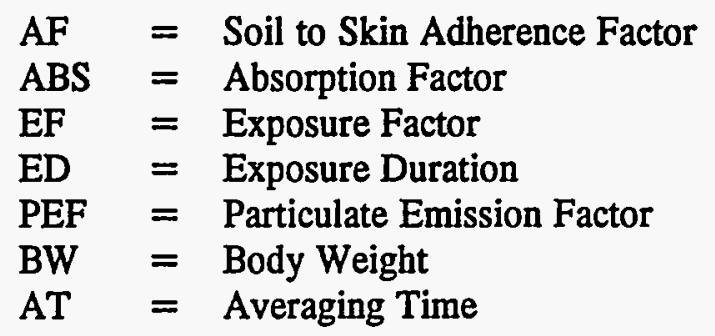

\subsubsection{Exposure Parameters}

Two current and one future exposure scenarios were evaluated. The current exposure scenarios are a worst-case worker scenario and a more likely S\&M worker exposure. The future exposure scenario is a residential scenario for an adult trespasser/squatter in the building. The exposure parameters used to quantify exposure for each exposure pathway evaluated for these three scenarios are described and summarized in Table 3.4. These exposure parameters are based on EPA guidance (EPA, 1989a, 1989b). All receptors were assumed to be represented by an average adult weighing $70 \mathrm{~kg}$ with a 70 -year lifespan.

\section{Current Worst-Case Worker}

Workers are assumed to ingest $50 \mathrm{mg}$ of dust or sediment per day at the facility. For inhalation exposure, workers are assumed to have an inhalation rate of $20 \mathrm{~m}^{3} /$ workday (Table 3.4). A PEF of $4.28 \times 10^{9} \mathrm{~m}^{3} / \mathrm{kg}$ was assumed for dust and sediment being disturbed by worker activities. For dermal contact an exposed surface area of $3120 \mathrm{~cm}^{2}$ representing the average adult's head, hands, and forearms was used. This was combined with a soil-to-skin adherence factor of $1.0 \mathrm{mg} / \mathrm{cm}^{2}$ and absorption factors of 0.001 for metals and 0.01 for organic chemicals. The receptor is assumed to work at the facility 250 days/year for 25 years.

\section{Current S\&M Worker}

This receptor is assumed to work at the facility 2 days/year for 25 years. As previously described for the worst-case scenario, workers were assumed to ingest $50 \mathrm{mg}$ of dust or sediment per day at the facility (Table 3.4). For inhalation exposure, workers were assumed to have an inhalation rate of $20 \mathrm{~m}^{3} /$ workday. A PEF of $4.28 \times 10^{9} \mathrm{~m}^{3} / \mathrm{kg}$ was assumed for dust and sediment being disturbed by worker activities. For dermal contact, an exposed surface area of $3120 \mathrm{~cm}^{2}$ representing the average adult's head, hands, and forearms was used. This was combined with a soil-to-skin adherence factor of $1.0 \mathrm{mg} / \mathrm{cm}^{2}$ and absorption factors of 0.001 for metals and 0.01 for organic chemicals.

\section{Future Trespasser}

The receptor for this scenario is an adult who has taken up residence in the abandoned building at some time in the future. This receptor is assumed to ingest $100 \mathrm{mg}$ of dust or sediment per day at the facility (Table 3.4). For inhalation exposure, workers are assumed to have an inhalation rate of $20 \mathrm{~m}^{3} / \mathrm{day}$. A PEF of $4.28 \times 10^{9} \mathrm{~m}^{3} / \mathrm{kg}$ was assumed for dust and sediment being disturbed by worker activities. For dermal contact an exposed surface area of $5300 \mathrm{~cm}^{2}$ representing the average adult's head, hands, forearms, and lower legs was used. This was 
Table 3.4. Exposure variables for Building 3506 exposure assessment

\begin{tabular}{|c|c|c|c|}
\hline & Maintenance Worker & S\&M Worker & Adult Trespasser \\
\hline C & Contaminant Specific & Contaminant Specific & Contaminant Specific \\
\hline IR (inhalation) & $20 \mathrm{~m}^{3} /$ day & $20 \mathrm{~m}^{3} /$ day & $20 \mathrm{~m}^{3} /$ day \\
\hline IR (ingestion) & $50 \mathrm{mg} /$ day & $50 \mathrm{mg} / \mathrm{day}$ & $100 \mathrm{mg} /$ day \\
\hline $\mathrm{CF}$ & $1 \times 10^{-6} \mathrm{~kg} / \mathrm{mg}$ & $1 \times 10^{-6} \mathrm{~kg} / \mathrm{mg}$ & $1 \times 10^{-6} \mathrm{~kg} / \mathrm{mg}$ \\
\hline FI & 1 Unitless & 1 Unitless & 1 Unitless \\
\hline ED & 25 years & 25 years & 30 years \\
\hline BW & $70 \mathrm{~kg}$ & $70 \mathrm{~kg}$ & $70 \mathrm{~kg}$ \\
\hline AT (Noncarcinogens) & $\mathrm{ED} \times 365 \mathrm{day} / \mathrm{yr}$ & ED $\times 365$ day/yr & ED $\times 365$ day/yr \\
\hline AT (Carcinogens) & 70 yrs $x 365$ day/yr & $70 y r s \times 365$ day/yr & $70 \mathrm{yrs} \times 365 \mathrm{day} / \mathrm{yr}$ \\
\hline PEF & $4.28 \times 10^{9} \mathrm{~m}^{3} / \mathrm{kg}$ & $4.28 \times 10^{9} \mathrm{~m}^{3} / \mathrm{kg}$ & $4.28 \times 10^{9} \mathrm{~m}^{3} / \mathrm{kg}$ \\
\hline ABS (Metals) & 0.001 (unitless) & 0.001 (unitless) & 0.001 (unitless) \\
\hline ABS (Organics) & 0.01 (unitless) & 0.01 (unitless) & 0.01 (unitless) \\
\hline $\mathrm{EF}$ & $250 \mathrm{day} / \mathrm{yr}$ & $2 \mathrm{day} / \mathrm{yr}$ & $350 \mathrm{day} / \mathrm{yr}$ \\
\hline SA & $3120 \mathrm{~cm}^{2} /$ day & $3120 \mathrm{~cm}^{2} /$ day & $5300 \mathrm{~cm}^{2} /$ day \\
\hline $\mathrm{AF}$ & $1.0 \mathrm{mg} / \mathrm{cm}^{2}$ & $1.0 \mathrm{mg} / \mathrm{cm}^{2}$ & $1.0 \mathrm{mg} / \mathrm{cm}^{2}$ \\
\hline DR & mrem/day (8 hours) & mrem/day ( 8 hours) & mrem/day (24 hours) \\
\hline $\mathrm{T}_{\mathrm{e}}$ & 0.33 & 0.33 & 1.0 \\
\hline $\mathrm{S}_{c}$ & 0.2 & 0.2 & 0.2 \\
\hline
\end{tabular}


combined with a soil-to-skin adherence factor of $1.0 \mathrm{mg} / \mathrm{cm}^{2}$ and absorption factors of 0.001 for metals and 0.01 for organic chemicals. The receptor is assumed to be in the facility 350 days/year for 30 years.

\subsubsection{Exposure Concentrations}

The exposure concentrations for this BRE are derived for the ingestion, inhalation, dermal contact, and external exposure pathways using many different types of data (e.g., pCi/g concentrations for the smear samples versus mrem doses for direct measurements). The type of measurements used for evaluation of each exposure pathway are listed in Table 3.5. Exposure concentrations were calculated separately for the cell, east gallery, sediment as well as for the whole building (see Table 3.5).

The exposure concentration is the average of the concentration contacted over the exposure period (EPA, 1989a). Although this concentration does not reflect the maximum concentration that could be contacted at any one time, it is regarded as a reasonable estimate of the concentration likely to be contacted over time.

Because of the uncertainty associated with estimating the true average exposure concentration, the upper confidence limit (i.e., the $95 \%$ upper confidence limit) on the arithmetic mean of each contaminant's concentrations was used as the concentration exposure. Where the upper confidence limit value was greater than the maximum detected value, the maximum detected value was used as the exposure concentration. For samples with no detected concentration, the detection limit was used to estimate the average and upper confidence limit concentrations.

The following paragraphs discuss how exposure concentrations were calculated for each type of data.

\section{Core Samples}

Core samples were taken from the walls and floors of the cell and the east gallery. An exposure concentration (upper $95 \%$ confidence limit or maximum detect) for each specific contaminant was calculated for the cell and the gallery. If contaminants were not.detected in the floor but were detected in the walls, the value from the walls was used to represent the entire room and vice versa. This method is a conservative approach. The wall and floor values from both the cell and the east gallery were used to calculated contaminant concentrations for the whole building. This was done by averaging all values for the floors, walls and sediment. Again, if contaminants were not detected in one of the rooms (e.g., cobalt-60 was not detected in the east gallery), then the values from the other room were used to represent the concentration of that contaminant for the whole building (i.e., it is assumed that the concentration is throughout the entire building), which again is a conservative approach.

\section{Smear Samples}

Measurements of transferrable radioactive contamination (smear samples) were converted from $\mathrm{dpm} / 100 \mathrm{~cm}^{2}$ to $\mathrm{pCi} / \mathrm{kg}$ using the conversion equations in Appendix B. The smear data were reported as alpha or beta radiation rather than as concentrations of specific radionuclides. To 


\section{3-32}

Table 3.5. Data evaluated for each exposure pathway

\begin{tabular}{|c|c|c|}
\hline Data Type & Location & Exposure Pathway \\
\hline \multirow[t]{12}{*}{ Core Samples } & Cell & Ingestion \\
\hline & & Dermal Absorption \\
\hline & & Inhalation \\
\hline & & External Radiation \\
\hline & East Gallery & Ingestion \\
\hline & & Dermal Absorption \\
\hline & & Intralation \\
\hline & & External Radiation \\
\hline & Whole Building & Ingestion \\
\hline & & Dermal Absorption \\
\hline & & Inhalation \\
\hline & & External Radiation \\
\hline \multirow[t]{4}{*}{ Sediment } & Cell & Ingestion \\
\hline & & Dermal Absorption \\
\hline & & Inhalation \\
\hline & & External Radiation \\
\hline \multirow[t]{9}{*}{ Smear } & Cell & Ingestion \\
\hline & & Inhalation \\
\hline & & External Radiation \\
\hline & East Gallery & Ingestion \\
\hline & & Inhalation \\
\hline & & Extemal Radiation \\
\hline & Whole Building & Ingestion \\
\hline & & Inhalation \\
\hline & & External Radiation \\
\hline \multirow{3}{*}{$\begin{array}{l}\text { Direct } \\
\text { Measurements }\end{array}$} & Cell & Externsl Radiation \\
\hline & East Gallery & External Radiation \\
\hline & Whole Building & External Radiation \\
\hline TLD Strings & Cell & Extermal Radiation \\
\hline
\end{tabular}


evaluate the smear sample data by radionuclide, it was assumed that the transferable contamination had the same distribution of radionuclides as that detected in the core samples from the walls and floors. This was accomplished by adding the concentration of the radionuclides from the core samples for the cell, east gallery, and whole building, respectively, then determining the percent contribution of each radionuclide. The total smear concentration for each room was multiplied by the percent contribution of each radionuclide to determine the contribution of each radionuclide for the smear samples. An example of this calculation is illustrated in the following exhibit using data from the cell.

Core Data

$\underline{\text { Smear Data (scaled) }}$

Radionuclide Concentration(pCi/g)

\begin{tabular}{lllll} 
& Alpha & $\%$ Contribution & $(\mathrm{pCi} / \mathrm{g})$ & Total alpha $\mathrm{pCi} / \mathrm{g}=1.48 \mathrm{E}-01$ \\
${ }^{2391240} \mathrm{Pu}$ & 4 & 35.49 & $5.25 \mathrm{E}-02$ & \\
${ }^{238} \mathrm{Pu}$ & 0.64 & 5.68 & $8.40 \mathrm{E}-03$ \\
${ }^{206} \mathrm{RA}$ & 0.401 & 3.56 & $5.27 \mathrm{E}-03$ \\
${ }^{228} \mathrm{TH}$ & 0.84 & 7.45 & $1.10 \mathrm{E}-02$ \\
${ }^{230} \mathrm{Th}$ & 1.4 & 12.42 & $1.84 \mathrm{E}-02$ \\
Core Data & & & Smear Data (scaled) \\
\hline
\end{tabular}

$\underline{\text { Radionuclide }}$ Concentration(pCi/g)

$\begin{array}{llll}{ }^{232} \mathrm{Th} & 0.87 & 7.72 & 1.14 \mathrm{E}-02 \\ { }^{233 / 234} \mathrm{U} & 1.3 & 11.53 & 1.71 \mathrm{E}-02 \\ { }^{235} \mathrm{U} & 0.12 & 1.06 & 1.58 \mathrm{E}-03 \\ { }^{238} \mathrm{U} & \frac{1.7}{11.271} & \frac{15.08}{100} & \underline{2.23 \mathrm{E}-02} \\ \text { Total } & & 1.48 \mathrm{E}-01\end{array}$

\begin{tabular}{|c|c|c|c|c|}
\hline & & & & Total beta $\mathrm{pCi} / \mathrm{g}=1.45 \mathrm{E}+02$ \\
\hline & Beta & $\%$ & $(\mathrm{pCi} / \mathrm{g})$ & \\
\hline${ }^{137} \mathrm{Cs}$ & 1375 & 98.78 & $1.43 E+02$ & - \\
\hline${ }^{40} \mathrm{~K}$ & 5.28 & 0.38 & $5.49 \mathrm{E}-01$ & \\
\hline${ }^{x 0} \mathrm{Sr}$ & 11.6 & 0.83 & $1.21 E+00$ & \\
\hline${ }^{60} \mathrm{Co}$ & $\underline{0.13}$ & $\underline{0.01}$ & $1.35 \mathrm{E}-02$ & \\
\hline Total & 1392.01 & 100 & $1.45 E+02$ & \\
\hline
\end{tabular}

\section{Direct Radiation Measurements}

The direct measurement and TLD string exposure concentrations were derived based on a lifetime exposure in mrems. Direct measurements were reported for the cell and the east gallery for both beta/gamma and beta. 


\subsection{RESULTS OF THE EXPOSURE ASSESSMENT}

The exposure assessment for Building 3506 was conducted to evaluate potential exposures to the contaminants of potential concern identified for the building. The assessment resulted in the identification of potential receptor populations, the pathways of exposure to contaminants within Building 3506, and the concentrations at which receptor populations may be exposed. The results of the quantitative exposure assessment are shown in Appendix D. Using the equations presented in Sect. 3.3.1, Chronic Daily Intakes (CDIs) were calculated for each receptor (current maintenance worker, current S\&M worker, future trespasser) and contaminant present. These CDI values will be used along with toxicity values (Sect. 4) to derive Excess Cancer Risk estimates for the carcinogenic contaminants and Hazard Quotients for noncarcinogenic contaminants in Sect. 5 of this BRE.

\subsection{UNCERTAINTY}

The objective of this exposure assessment is to estimate the type and magnitude of potential . exposures to the chemicals and radionuclides of potential concern that are present at Building 3506. Three major types of uncertainties should be considered when reviewing the results of the exposure assessment: uncertainties associated with predicting future land use, uncertainties associated with estimating contaminant concentrations at receptor locations, and uncertainties associated with assumptions used in the exposure models. Uncertainties in the Exposure Assessment are discussed in the following paragraphs.

It is rarely possible to know what will occur at a site in the future. Assumptions must, therefore, be made regarding future land-use. The conservative assumption that a receptor could trespass into the area and take up residence at the site is designed to provide an upper-bound estimate of exposure and risk. Uncertainty in the estimated exposure concentrations result from normal analytical error and the fact that a few samples must be used to represent the concentration in a larger area. The true distribution of radionuclides was not evaluated in the smear samples; therefore, it was assumed that the same distribution of radionuclides detected in the floor and wall cores was the same for the smears. Smear samples were analyzed for cesium-137, strontium- 90 , cobalt- 60 , and radium-226. These radionuclide-specific data confirm that most of the radioactivity detected in the smears is cesium-137 as estimated from the core sample data.

Smear samples were not collected from the floor of the cell because of standing water resulting from a leak in the roof. The data from the walls were used to represent the entire cell. This assumption could underestimate the concentration of transferrable radionuclide contamination in the cell.

Based on the lack of air monitoring data, a particulate emission factor was used to model the release of contaminants to the air. Using an emission factor increases the uncertainty in the inhalation exposure.

Exposure parameters (e.g., inhalation rate, exposure frequency, exposure duration) used to model hypothetical receptors are a combination of reasonable and upper bound (generally upper 
95\% confidence limit) values. The use of upper bound values will tend to overestimate exposure. This provides a conservative health protective approach for the risk assessment. 
THIS PAGE INTENTIONALLY LEFT BLANK 


\section{TOXICITY ASSESSMENT}

The following contaminant-specific information provides a discussion on health effects relating to the contaminants of potential concern for Building 3506. Data from human and laboratory animal research and data from occupational studies are used to characterize potential health effects resulting from exposure to the contaminants of potential concern.

The carcinogenic and noncarcinogenic effects listed here may not necessarily be indicative of risks associated with exposure conditions at the site. This information does, however, give an indication of the kinds of potential health effects associated with these contaminants. For the purpose of this BRE, some contaminants were evaluated on a quantitative basis, while others were evaluated on a qualitative basis.

\subsection{TOXICITY PROFILES}

\subsubsection{Contaminants Evaluated Quantitatively}

\subsubsection{Inorganics}

\section{Antimony}

Antimony ( $\mathrm{Sb})$ is a naturally occurring metal that is used in various manufacturing processes. It exists in valence states of 3 and 5 ( ATSDR, 1990b). Antimony is a common urban air pollutant. Exposure to antimony may be via inhalation, oral and dermal routes (ATSDR, 1990b).

Antimony is sparingly absorbed following ingestion or inhalation (ATSDR, 1990b). Both gastrointestinal and pulmonary absorption are a function of compound solubility. Antimony is transported in the blood, its distribution varying among species and dependent on its valence state. Antimony is not metabolized but may bind to macromolecules and react covalently with sulfhydryl and phosphate groups (ATSDR, 1990b). Excretion of antimony is primarily via the urine and feces, and is also dependent upon valence state (ATSDR, 1990b).

Acute oral exposure of humans and animals to high doses of antimony or antimony-containing compounds (antimonials) may cause gastrointestinal disorders (vomiting, diarrhea), respiratory difficulties, and death at extremely high doses ( ATSDR, 1990b). Subchronic and chronic oral exposure may affect hematologic parameters (ATSDR, 1990b). Long-term exposure to high doses of antimony or antimonials has been shown to adversely affect longevity in animals. Limited data suggest that prenatal and postnatal exposure of rats to antimony interferes with vasomotor responses.

Acute inhalation exposure of humans may cause gastrointestinal disorders (probably due to ingestion of airborne antimony) (ATSDR, 1990b). Exposure of animals to high concentrations of antimony and antimonials (especially stibine gas) may result in pulmonary edema and death. Long-term occupational exposure of humans has resulted in electrocardiac disorders, respiratory disorders, and possibly increased mortality(. Antimony levels for these occupational exposure evaluations ranged from 2.2 to $11.98 \mathrm{mg} \mathrm{Sb} / \mathrm{m}^{3}$. Based on limited data, occupational exposure 
of women to metallic antimony and several antimonials has reportedly caused alterations in the menstrual cycle and an increased incidence of spontaneous abortions. Reproductive dysfunction has been demonstrated in rats exposed to antimony trioxide (BEIAS Toxicity Profiles).

The primary target organ for acute oral exposure to antimony appears to be the gastrointestinal tract (irritation, diarrhea, vomiting) and targets for long-term exposure are the blood (hematological disorders) and liver (mild hepatotoxicity) (ATSDR, 1990b). Inhalation exposure to antimony affects the respiratory tract (pneumoconiosis, restrictive airway disorders), with secondary targets being the cardiovascular system (altered blood pressure and electrocardiograms) and kidneys (histological changes). Only limited evidence exists for reproductive disorders due to antimony exposure.

\section{Arsenic}

The toxicity of inorganic arsenic (As) depends on its valence state $(-3,+3$, or +5$)$, and also on the physical and chemical properties of the compound in which it occurs. Trivalent $\left(\mathrm{As}^{+3}\right)$ compounds are generally more toxic than pentavalent $\left(\mathrm{As}^{+5}\right)$ compounds, and the more water soluble compounds are usually more toxic and more likely to have systemic effects than the less soluble compounds, which are more likely to cause chronic pulmonary effects if inhaled. One of the most toxic inorganic arsenic compounds is arsine gas $\left(\mathrm{AsH}_{3}\right)$. It should be noted that laboratory animals are generally less sensitive than humans to the toxic effects of inorganic arsenic. In addition, in rodents the critical effects appear to be immunosuppression and hepatorenal dysfunction, whereas in humans the skin, vascular system, and peripheral nervous system are the primary target organs (BEIAS Toxicity Profiles).

Water soluble inorganic arsenic compounds are absorbed through the G.I. tract (>90\%) and lungs; distributed primarily to the liver, kidney, lung, spleen, aorta, and skin; and excreted mainly in the urine at rates as high as $80 \%$ in $61 \mathrm{hr}$ following oral dosing. Pentavalent arsenic is reduced to the trivalent form and then methylated in the liver to less toxic methylarsinic acids (ATSDR, 1989a).

Symptoms of acute inorganic arsenic poisoning in humans are nausea, anorexia, vomiting, epigastric and abdominal pain, and diarrhea. Dermatitis (exfoliative erythroderma), muscle cramps, cardiac abnormalities, hepatotoxicity, bone marrow suppression and hematologic abnormalities (anemia), vascular lesions, and peripheral neuropathy (motor dysfunction, paresthesia) have also been reported ( ATSDR, 1989a). Oral doses as low as 20-60 $\mu \mathrm{g} / \mathrm{kg} / \mathrm{day}$ have been reported to cause toxic effects in some individuals (ATSDR, 1989a). Severe exposures can result in acute encephalopathy, congestive heart failure, stupor, convulsions, paralysis, coma, and death. The acute lethal dose to humans has been estimated to be about $0.6 \mathrm{mg} / \mathrm{kg} / \mathrm{day}$ (ATSDR, 1989a). General symptoms of chronic arsenic poisoning in humans are weakness, general debility and lassitude, loss of appetite and energy, loss of hair, hoarseness of voice, loss of weight, and mental disorders. Primary target organs are the skin (hyperpigmentation and hyperkeratosis), nervous system (peripheral neuropathy). Anemia, leukopenia, hepatomegaly, and portal hypertension have also been reported. In addition, possible reproductive effects include a high male to female birth ratio (BEIAS Toxicity Profiles). 
In animals, acute oral exposures can cause gastrointestinal and neurological effects. Oral $\mathrm{LD}_{50}$ values range from about 10 to $300 \mathrm{mg} / \mathrm{kg}$ (ATSDR, 1989a). Low subchronic doses can result in immunosuppression, and hepato-renal effects . Chronic exposures have also resulted in mild hyperkeratosis and bile duct enlargement with hyperplasia, focal necrosis, and fibrosis . Reduction in litter size, high male/female birth ratios, and fetotoxicity without significant fetal abnormalities occur following oral exposures; however, parenteral dosing has resulted in exencephaly, encephaloceles, skeletal defects, and urogenital system abnormalities (BEIAS Toxicity Profiles).

Acute inhalation exposures to inorganic arsenic can damage mucous membranes, cause rhinitis, pharyngitis and laryngitis, and result in nasal septum perforation. Chronic inhalation exposures, as occurring in the workplace, can lead to rhino-pharyno-laryngitis; tracheobronchitis; dermatitis, hyperpigmentation, and hyperkeratosis; leukopenia; peripheral nerve dysfunction as indicated by abnormal nerve conduction velocities; and peripheral vascular disorders as indicated by Raynaud's syndrome and increased vasospastic reactivity in fingers exposed to low temperatures. Higher rates of cardiovascular disease have also been reported in some arsenic-exposed workers. Possible reproductive effects include a high frequency of spontaneous abortions and reduced birth weights. Arsine gas $\left(\mathrm{AsH}_{3}\right)$, at concentrations as low as 3-10 ppm for several hours, can cause toxic effects. Hemolysis, hemoglobinuria, jaundice, hemolytic anemia, and necrosis of the renal tubules have been reported in exposed workers.

Animal studies have shown that inorganic arsenic, by intratracheal instillation, can cause pulmonary inflammation and hyperplasia, lung lesions, and immunosuppression. Long-term inhalation exposures have resulted in altered conditioned reflexes and CNS damage. Reductions in fetal weight and in the number of live fetuses and increases in fetal abnormalities due to retarded osteogenesis have been observed following inhalation exposures.

\section{Barium}

Barium is a divalent alkaline-earth metal found only in combination with other elements in nature. The most important of these combinations are the peroxide, chloride, sulfate, carbonate, nitrate, and chlorate. The pure metal oxidizes readily and reacts with water emitting hydrogen; it is chemically similar to calcium. The most likely source of barium in the atmosphere is from industrial emissions. Since it is usually present as a particulate form, it can be removed from the atmosphere by wet precipitation and deposition. Due to the element's tendency to form salts with limited solubility in soil and water, it is expected to have a residence time of hundreds of years and is not expected to be very mobile. Acidic conditions, however, will increase the solubility of some barium compounds facilitating their movement from the soil to the groundwater. Trace amounts of barium were found in more than $99 \%$ of the surface waters and finished drinking water samples across the United States.

The soluble salts of barium, an alkaline earth metal, are toxic in mammalian systems. They are absorbed rapidly from the gastrointestinal tract and are deposited in the muscles, lungs, and bone. Barium is excreted primarily in the feces.

At low doses, barium acts as a muscle stimulant and at higher doses affects the nervous system eventually leading to paralysis. Acute and subchronic oral doses of barium cause vomiting and 
diarrhea, followed by decreased heart rate and elevated blood pressure. Higher doses result in cardiac irregularities, weakness, tremors, anxiety, and dyspnea. A drop in serum potassium may account for some of the symptoms. Death can occur from cardiac and respiratory failure. Acute doses around 0.8 grams can be fatal to humans (BEIAS Toxicity Profiles).

\section{Beryllium}

Beryllium is present in the earth's crust, in emissions from coal combustion, in surface water and soil, and in house dust, food, drinking water, and cigarette smoke. However, the highest risk for exposure occurs among workers employed in beryllium manufacturing, fabricating, or reclamation industries (ATSDR, 1988a). Workers encounter dusts and fumes of many different beryllium compounds; the current occupational standard for worker exposure to beryllium is $2 \mu \mathrm{g} / \mathrm{m}^{3}$ during an 8-hour workshift.

Inhaled beryllium is absorbed slowly and localizes mainly in the lungs, bone, liver, and kidneys. Ingested beryllium undergoes limited absorption and localizes in liver, kidneys, lungs, stomach, spleen, and large and small intestines. Significant absorption of beryllium or its compounds through intact skin is unlikely because of its chemical properties. Beryllium per se is not biotransformed, but soluble salts may be converted to less soluble compounds in the lung. Most orally administered beryllium passes through the gastrointestinal tract unabsorbed and is excreted in the feces, whereas inhaled water-soluble beryllium salts are excreted mainly by the kidneys.

In contrast, the toxicity of inhaled beryllium is well-documented. Humans inhaling "massive" doses of beryllium compounds (such as the water soluble sulfate, fluoride, chloride, and oxide) may develop acute berylliosis. ATSDR (1988a) estimated that, based on existing data, the disease could develop at levels ranging from approximately 2-1000 $\mu \mathrm{g} \mathrm{Be} / \mathrm{m}^{3}$. This disease usually develops shortly after exposure and is characterized by rhinitis, pharyngitis, and/or tracheobronchitis, and may progress to severe pulmonary symptoms. The severity of acute beryllium toxicity correlates with exposure levels, and the disease is now rarely observed in the United States because of improved industrial hygiene.

Humans inhaling beryllium may also develop chronic berylliosis which, in contrast to acute berylliosis, is highly variable in onset, is more likely to be fatal, and can develop a few months to $\geq 20$ years after exposure. Chronic beryllium disease is a systemic disease that primarily affects the lungs and is characterized by the development of non-caseating granulomas. The disease most likely results from a hypersensitivity response to beryllium as evidenced by positive patch tests and positive lymphocyte transformation tests in exposed individuals. Granulomas may also appear in the skin, liver, spleen, lymph nodes, myocardium, skeletal muscles, kidney, bone, and salivary glands (BEIAS Toxicity Profiles).

\section{Cadmium}

Cadmium is a naturally occurring metal that is used in various chemical forms in metallurgical and other industrial processes, and in the production of pigments. Environmental exposure can occur via the diet and drinking water (ATSDR, 1989b). 
Cadmium is absorbed more efficiently by the lungs ( 30 to $60 \%$ ) than by the gastrointestinal tract, the latter being a saturable process. Cadmium is transported in the blood and widely distributed in the body but accumulates primarily in the liver and kidneys. Cadmium burden (especially in the kidneys and liver) tends to increase in a linear fashion up to about 50 or 60 years of age after which the body burden remains somewhat constant. Metabolic transformations of cadmium are limited to its binding to protein and nonprotein sulfhydryl groups, and various macromolecules, such as metallothionein, which is especially important in the kidneys and liver (ATSDR, 1989). Cadmium is excreted primarily in the urine.

Acute oral exposure to 20-30 $\mathrm{g}$ have caused fatalities in humans. Exposure to lower amounts may cause gastrointestinal irritation, vomiting, abdominal pain, and diarrhea (ATSDR, 1989b). An asymptomatic period of one-half to one hour may precede the onset of clinical signs. Oral $\mathrm{LD}_{s 0}$ values in animals range from 63 to $1125 \mathrm{mg} / \mathrm{kg}$, depending on the cadmium compound . Longer term exposure to cadmium primarily affects the kidneys, resulting in tubular proteinosis although other conditions such as "itai-itai" disease may involve the skeletal system. Cadmium involvement in hypertension is not fully understood (BEIAS Toxicity Profiles).

Inhalation exposure to cadmium and cadmium compounds may result in effects including headache, chest pains, muscular weakness, pulmonary edema, and death . The 1-minute and 10-minute lethal concentration of cadmium for humans has been estimated to be about 2500 and $250 \mathrm{mg} / \mathrm{m}^{3}$, respectively (BEIAS Toxicity Profiles). An 8-hour TWA (time-weighted-average) exposure level of $5 \mathrm{mg} / \mathrm{m}^{3}$ has been estimated for lethal effects of inhalation exposure to cadmium, and exposure to $1 \mathrm{mg} / \mathrm{m}^{3}$ is considered to be immediately dangerous to human health. Renal toxicity (tubular proteinosis) may also result from inhalation exposure to cadmium.

The target organ for cadmium toxicity via oral exposure is the kidney. For inhalation exposure, both the lungs and kidneys are target organs for cadmium-induced toxicity (ATSDR, 1989b; BEIAS Toxicity Profiles).

\section{Chromium III, VI}

Elemental chromium $(\mathrm{Cr})$ does not occur in nature, but is present in ores, primarily chromite $\left(\mathrm{FeOCr}_{2} \mathrm{O}_{3}\right)$. Only two of the several oxidation states of chromium, $\mathrm{Cr}$ (III) and $\mathrm{Cr}(\mathrm{VI})$, are reviewed in this report based on their predominance and stability in the ambient environment and their toxicity in humans and animals.

Chromium plays a role in glucose and cholesterol metabolism and is thus an essential element to man and animals. Non-occupational exposure to the metal occurs via the ingestion of chromium-containing food and water, whereas occupational exposure occurs via inhalation. Workers in the chromate industry have been exposed to estimated chromium levels of 10 $50 \mu \mathrm{g} / \mathrm{m}^{3}$ for $\mathrm{Cr}$ (III) and $5-1000 \mu \mathrm{g} / \mathrm{m}^{3}$ for $\mathrm{Cr}(\mathrm{VI})$; however, improvements in the newer chromeplating plants have reduced the $\mathrm{Cr}(\mathrm{VI})$ concentrations 10 - to 40 -fold.

Chromium(III) is poorly absorbed, regardless of the route of exposure, whereas chromium(VI) is more readily absorbed. Humans and animals localize chromium in the lung, liver, kidney, spleen, adrenals, plasma, bone marrow, and red blood cells (RBC). There is no evidence that chromium is biotransformed, but $\mathrm{Cr}(\mathrm{VI})$ does undergo enzymatic reduction, resulting in the 
formation of reactive intermediates and $\mathrm{Cr}(\mathrm{III})$. The main routes for the excretion of chromium are via the kidneys/urine and the bile/feces (BEIAS Toxicity Profiles).

Animal studies show that $\mathrm{Cr}(\mathrm{VI})$ is generally more toxic than $\mathrm{Cr}(\mathrm{III})$, but neither oxidation state is very toxic by the oral route. In long-term studies, rats were not adversely affected by $-1.9 \mathrm{~g} / \mathrm{kg} /$ day of chromic oxide [Cr(III)] (diet), $2.4 \mathrm{mg} / \mathrm{kg} /$ day of $\mathrm{Cr}$ (III) as chromic chloride (drinking water), or $2.4 \mathrm{mg} / \mathrm{kg} /$ day of $\mathrm{Cr}(\mathrm{VI})$ as potassiurn dichromate (drinking water).

The respiratory and dermal toxicity of chromium are well-documented. Workers exposed to chromium have developed nasal irritation (at $<0.01 \mathrm{mg} / \mathrm{m}^{3}$, acute exposure), nasal ulcers, perforation of the nasal septum (at $\sim 2 \mu \mathrm{g} / \mathrm{m}^{3}$, subchronic or chronic exposure) (ATSDR, 1989c) and hypersensitivity reactions and "chrome holes" of the skin. Among the general population, contact dermatitis has been associated with the use of bleaches and detergents (BEIAS Toxicity Profiles).

Compounds of both $\mathrm{Cr}(\mathrm{VI})$ and $\mathrm{Cr}(\mathrm{III})$ have induced developmental effects in experimental animals that include neural tube defects, malformations, and fetal deaths.

\section{Cyanide}

Cyanide most commonly occurs as hydrogen cyanide and its salts-sodium and potassium cyanide. Cyanides are both man-made and naturally occurring substances. They are found in several plant species as cyanogenic glycosides and are produced by certain bacteria, fungi, and algae. In very small amounts, cyanide is a necessary requirement in the human diet. Cyanides are released to the environment from industrial sources and car emissions (ATSDR, 1989d).

Cyanides are readily absorbed by the inhalation, oral, and dermal routes of exposure. The central nervous system (CNS) is the primary target organ for cyanide toxicity. Neurotoxicity has been observed in humans and animals following ingestion and inhalation of cyanides. Cardiac and respiratory effects, possibly CNS-mediated, have also been reported. Short-term exposure to high concentrations produces almost immediate collapse, respiratory arrest, and death. Symptoms resulting from occupational exposure to lower concentrations include breathing difficulties, nervousness, vertigo, headache, nausea, vomiting, precordial pain, and electrocardiogram (EKG) abnormalities. Thyroid toxicity has been observed in humans and animals following oral and inhalation exposure to cyanides. In animal studies, cyanides have produced fetotoxicity and teratogenic effects, including exencephaly, encephalocele, and rib abnormalities (BEIAS Toxicity Profiles).

\section{Manganese}

Manganese is an essential trace element in humans, which can elicit a variety of serious toxic responses upon prolonged exposure to elevated concentrations either orally or by inhalation. The central nervous system is the primary target. Initial symptoms are headache, insomnia, disorientation, anxiety, lethargy and memory loss. These symptoms progress with continued exposure and eventually include motor disturbances, tremors and difficulty in walking, similar to symptoms seen in Parkinsonism. These motor difficulties are most often irreversible. Based on human epidemiological studies, $0.8 \mathrm{mg} / \mathrm{kg} / \mathrm{day}$ for drinking water exposure and $0.34 \mathrm{mg} / \mathrm{m}^{3}$ 
in air for inhalation exposure have been estimated as lowest-observed-adverse-effect levels (LOAELs) for central nervous system effects.

Effects on reproduction (decreased fertility, impotence) have been observed in humans with inhalation exposure and in animals with oral exposure at the same or similar doses that initiate the central nervous system effects. An increased incidence of coughs, colds, dyspnea during exercise, bronchitis and altered lung ventilatory parameters have also been seen in humans and animals with inhalation exposure. A possible effect on the immune system may account for some of the respiratory symptoms.

\section{Mercury}

Mercury is a naturally occurring element existing in multiple forms and in various oxidation states. It is used in a wide variety of products and processes. In the environment, mercury may undergo transformations among its various forms and among its oxidation states. Exposure to mercury may occur in both occupational and environmental settings, the latter primarily involving dietary exposure (ATSDR, 1989e).

Absorption, distribution, metabolism, and excretion of mercury is dependent upon its form and oxidation state (ATSDR, 1989e). Organic mercurials are more readily absorbed than are inorganic forms. An oxidation-reduction cycle is involved in the metabolism of mercury and mercury compounds by both animals and humans (ATSDR, 1989e). The urine and feces are primary excretory routes. The elimination half-life is 35 to 90 days for elemental mercury and mercury vapor, and about $\mathbf{4 0}$ days for inorganic salts.

Ingestion of mercury metal is usually without effect (BEIAS Toxicity Profiles). Ingestion of inorganic salts may cause severe gastrointestinal irritation, renal failure and death with acute lethal doses in humans ranging from $1-4 \mathrm{~g}$ (ATSDR, 1989e). Mercuric (divalent) salts are usually more toxic than are mercurous (monovalent) salts. Mercury is also known to induce hypersensitivity reactions such as contact dermatitis and acrodynia (pink disease). Inhalation of mercury vapor may cause irritation of the respiratory tract, renal disorders, CNS effects characterized by neurobehavioral changes, peripheral nervous system toxicity, renal toxicity (immunologic glomerular disease), and death (ATSDR, 1989e, BEIAS Toxicity Profiles).

Toxicity resulting from subchronic and chronic exposure to mercury and mercury compounds usually involves the kidneys and/or nervous system, the specific target and effect being dependent on the form of mercury (ATSDR, 1989e). Organic mercury, especially methyl mercury, rapidly enters the central nervous system (CNS) resulting in behavioral and neuromotor disorders (ATSDR, 1989e). The developing CNS is especially sensitive to this effect, as documented by the epidemiologic studies in Japan and Iraq where ingestion of methyl mercury-contaminated food resulted in severe toxicity and death in adults and severe CNS effects in infants. Blood mercury levels of $<10 \mu \mathrm{g} / \mathrm{dL}$ and $300 \mu \mathrm{g} / \mathrm{dL}$ corresponded to mild effects and death, respectively. Teratogenic effects due to organic or inorganic mercury exposure do not appear to be well-documented for humans or animals, although some evidence exists for mercury-induced menstrual cycle disturbances and spontaneous abortions (BEIAS Toxicity Profiles). 
Methyl mercury is formed by biotic and abiotic methylation of mercury. Methyl mercury has been used as a fungicide, disinfectant, and in industrial processes. Methyl mercury is highly toxic and is readily absorbed by the body following ingestion or inhalation . Methyl mercury may be metabolized to inorganic mercury by the liver and kidneys, with further transformation occurring to form the divalent cation (ATSDR, 1989e). Methyl mercury is excreted as inorganic mercury, primarily in the feces (BEIAS Toxicity Profiles).

The target organ for methyl mercury toxicity is the central nervous system (CNS), especially the brain, and may occur at doses as low as $3 \mu \mathrm{g} / \mathrm{kg}$ in humans. Methyl mercury is neurotoxic to several species of experimental animal and to humans. The $\mathrm{LD}_{50}$ values for various rodent species range from 21 to $57.6 \mathrm{mg} / \mathrm{kg}$. Manifestation of toxic effects (neurobehavioral alterations and degenerative changes in the central and peripheral nervous system) is probably a function of accumulation of critical levels of mercury. Histopathologic correlates have been identified in the brains of humans and animals prenatally exposed to methyl mercury (BEIAS Toxicity Profiles).

Exposure to methyl mercury in the diet (fish and contaminated grain) has caused epidemic poisonings in Iraq and Japan, characterized by severe developmental effects (impaired motor and cognitive functions) in infants of exposed mothers. The primary target organ for oral exposure to methyl mercury is the brain; the effects on this organ accounting for the developmental toxicity of the chemical.

\section{Nickel}

Nickel is a naturally occurring element that may exist in various mineral forms. Nickel is used in a wide variety of applications including metallurgical processes and electrical components, such as batteries (ATSDR, 1988b). There is some evidence that nickel may be an essential trace element for mammals.

The absorption of nickel is dependent on its physicochemical form, with water soluble forms being more readily absorbed. The metabolism of nickel involves conversion to various chemical forms and binding to various ligands (ATSDR, 1988b). Nickel is excreted in the urine and feces with relative amounts for each route being dependent on the route of exposure and chemical form. Most nickel enters the body via food and water consumption, although inhalation exposure in occupational settings is a primary route for nickel-induced toxicity (BEIAS Toxicity Profiles).

In large doses ( $>0.5 \mathrm{~g}$ ) some forms of nickel may be acutely toxic to humans when taken orally (BEIAS Toxicity Profiles). Oral $L^{2} D_{s 0}$ values for rats range from $67 \mathrm{mg} \mathrm{Ni} / \mathrm{kg}$ (nickel sulfate hexahydrate) to $>9000 \mathrm{mg} \mathrm{Ni} / \mathrm{kg}$ (nickel powder) (ATSDR, 1988b). Toxic effects of oral exposure to nickel usually involve the kidneys with some evidence from animal studies showing a possible developmental/reproductive toxicity effect (ATSDR, 1988).

Inhalation exposure to some nickel compounds will cause toxic effects in the respiratory tract and immune system (ATSDR, 1988b). Inhalation $\mathrm{LC}_{50}$ values for animals range from $0.97 \mathrm{mg}$ $\mathrm{Ni} / \mathrm{m}^{3}$ for rats (6-hour exposure) to $15 \mathrm{mg} \mathrm{Ni} / \mathrm{m}^{3}$ for guinea pigs (time not specified). Acute inhalation exposure of humans to nickel may produce headache, nausea, respiratory disorders and death. Asthmatic conditions have also been documented for inhalation exposure to nickel. 
Soluble nickel compounds tend to be more toxic than insoluble compounds. Nickel carbonyl is known to be extremely toxic to humans upon acute inhalation exposure.

Sensitivity reactions to nickel are well documented and usually involve contact dermatitis reactions resulting from contact with nickel-containing items such as cooking utensils, jeweiry, coins, etc. (ATSDR, 1988b).

The primary target organs for nickel-induced systemic toxicity are the lungs and upper respiratory tract for inhalation exposure and the kidneys for oral exposure (ATSDR, 1988b). Other target organs include the cardiovascular system, immune system, and the blood (BEIAS Toxicity Profiles).

\section{Selenium}

Selenium is an essential trace element important in many biochemical and physiological processes including the biosynthesis of coenzyme $Q$ (a component of mitochondrial electron transport systems), regulation of ion fluxes across membranes, maintenance of the integrity of keratins, stimulation of antibody synthesis, and activation of glutathione peroxidase (an enzyme involved in preventing oxidative damage to cells). Recommended human dietary allowances (average daily intake) for selenium are as follows: infants up to 1 year, $10-15 \mu \mathrm{g}$; children 1-10 years, 20-30 $\mu \mathrm{g}$; adult males 11-51+ years, 40-70 $\mu \mathrm{g}$; adult females $11-51+$ years, $45-55 \mu \mathrm{g}$; pregnant or lactating women, 65-75 $\mu \mathrm{g}$. There appears to be a relatively narrow range between levels of selenium intake resulting in deficiency and those causing toxicity.

Selenium occurs in several valence states: -2 (hydrogen selenide, sodium selenide, dimethyl selenium, trimethyl selenium, and selenoamino acids such as selenomethionine; 0 (elemental selenium); +4 (selenium dioxide, selenious acid, and sodium selenite); and +6 (selenic acid and sodium selenate). Toxicity of selenium varies with valence state and water solubility of the compound in which it occurs. The latter can affect gastrointestinal absorption rates.

Gastrointestinal absorption in animals and humans for various selenium compounds ranges from about $44 \%$ to $95 \%$ of the ingested dose (BEIAS Toxicity Profiles). Respiratory tract absorption rates of $97 \%$ and $94 \%$ for aerosols of selenious acid have been reported for dogs and rats, respectively. Selenium is found in all tissues of the body; highest concentrations occur in the kidney, liver, spleen, and pancreas. Excretion is primarily via the urine (0-15 $\mu \mathrm{g} / \mathrm{L})$; however, excretory products can also be found in the feces, sweat, and in expired air.

In humans, acute oral exposures can result in excessive salivation, garlic odor to the breath, shallow breathing, diarrhea, pulmonary edema, and death. Other reported signs and symptoms of acute selenosis include tachycardia, nausea, vomiting, abdominal pain, abnormal liver function, muscle aches and pains, irritability, chills, and tremors. Acute toxic effects observed in animals include pulmonary congestion, hemorrhages and edema, convulsions, altered blood chemistry (increased hemoglobin and hematocrit); liver congestion; and congestion and hemorrhage of the kidneys (BEIAS Toxicity Profiles).

General signs and symptoms of chronic selenosis in humans include loss of hair and nails, acropachia (clubbing of the fingers), skin lesions (redness, swelling, blistering, and ulcerations), 
tooth decay (mottling, erosion and pitting), and nervous system abnormalities attributed to polyneuritis (peripheral anesthesia, acroparaethesia, pain in the extremities, hyperreflexia of the tendon, numbness, convulsions, paralysis, motor disturbances, and hemiplegia). In domesticated animals, subchronic and chronic oral exposures can result in loss of hair, malformed hooves, rough hair coat, and nervous system abnormalities (impaired vision and paralysis). Damage to the liver and kidneys and impaired immune responses have been reported to occur in rodents following subchronic and/or chronic oral exposures (Ganther and Baumann, 1962; Beems and van Beek, 1985; NCI, 1980; Tinsley et al., 1967; Harr et al., 1967; Schroeder, 1967).

Selenium is teratogenic in birds and possibly also in domesticated animals (pigs, sheep, and cattle), but evidence of teratogenicity in humans and laboratory animals is lacking (ASTDR, $1989 \mathrm{~g}$ ). However, adverse reproductive and developmental effects (decreased rates of conception, increased rates of fetal resorption, and reduced fetal body weights) have been reported for domesticated and laboratory animals (BEIAS Toxicity Profiles).

In humans, inhalation of selenium or selenium compounds primarily affects the respiratory system. Dusts of elemental selenium and selenium dioxide can cause irritation of the skin and mucous membranes of the nose and throat, coughing, nosebleed, loss of sense of smell, dyspnea, bronchial spasms, bronchitis, and chemical pneumonia. Other signs and symptoms following acute inhalation exposures include lacrimation, irritation and redness of the eyes, gastrointestinal distress (nausea and vomiting), depressed blood pressure, elevated pulse rate, headaches, dizziness, and malaise (ATSDR, 1989g). In animals, acute inhalation exposures also result in severe respiratory effects including edema, hemorrhage, and interstitial pneumonitis as well as in splenic damage (congestion, fissuring red pulp, and increased polymorphonuclear leukocytes) and liver congestion and mild central atrophy (BEIAS Toxicity Profiles).

\section{Vanadium}

Vanadium is a metallic element that occurs in six oxidation states and numerous inorganic compounds. Some of the more important compounds are vanadium pentoxide $\left(\mathrm{V}_{2} \mathrm{O}_{5}\right)$, sodium metavanadate $\left(\mathrm{NaVO}_{3}\right)$, sodium orthovanadate $\left(\mathrm{Na}_{3} \mathrm{VO}_{4}\right)$, vanadyl sulfate $\left(\mathrm{VOSO}_{4}\right)$, and ammonium vanadate $\left(\mathrm{NH}_{4} \mathrm{VO}_{3}\right)$. Vanadium is used primarily as an alloying agent in steels and non-ferrous metals (ATSDR, 1990h). Vanadium compounds are also used as catalysts and in chemical, ceramic or specialty applications.

Vanadium compounds are poorly absorbed through the gastrointestinal system $(0.5-2 \%$ of dietary amount), but slightly more readily absorbed through the lungs (20-25\%). Absorbed vanadium is widely distributed in the body, but short-term localization occurs primarily in bone, kidneys, and liver. In the body, vanadium can undergo changes in oxidation state (interconversion of vanadyl $(+4)$ and vanadate $(+5)$ forms) and it can also bind with blood protein (transferrin) (Harris et al., 1984). Vanadium is excreted primarily in the feces following oral exposures and primarily in the urine following inhalation exposures (ATSDR, 1990h; BEIAS Toxicity Profiles).

The toxicity of vanadium depends on its physico-chemical state; particularly on its valence state and solubility. Based on acute toxicity, pentavalent $\mathrm{NH}_{4} \mathrm{VO}_{3}$ has been reported to be more than twice as toxic as trivalent $\mathrm{VCl}_{3}$ and more than 6 times as toxic as divalent $\mathrm{VI}_{2}$. Pentavalent $\mathrm{V}_{2} \mathrm{O}_{5}$ 
has been reported to be more than 5 times as toxic as trivalent $\mathrm{V}_{2} \mathrm{O}_{3}$. In animals, acutely toxic oral doses cause vasoconstriction, diffuse desquamative enteritis, congestion and fatty degeneration of the liver, congestion and focal hemorrhages in the lungs and adrenal cortex). Minimal effects seen after subchronic oral exposures to animals include diarrhea, altered renal function, and decreases in erythrocyte counts, hemogloblin, and hematocrit. In humans, intestinal cramps and diarrhea may occur following subchronic oral exposures. These studies indicate that for subchronic and chronic oral exposures the primary targets are the digestive system, kidneys, and blood (BELAS Toxicity Profiles).

Inhalation exposures to vanadium and vanadium compounds result primarily in adverse effects to the respiratory system ATSDR, 1990h). In laboratory studies, minimal effects (throat irritation and coughing) occurred after an 8-hr exposure to $0.1 \mathrm{mg} \mathrm{V} / \mathrm{m}^{3}$. In studies on workers occupationally exposed to vanadium, the most common reported symptoms were: irritation of the respiratory tract, conjunctivitis, dermatitis, cough, bronchospasm, pulmonary congestion, and bronchitis (BEIAS Toxicity Profiles).

\section{Zinc}

Zinc is used primarily in galvanized metals and metal alloys, but zinc compounds also have wide commercial applications as chemical intermediates, catalysts, pigments, vulcanization activators and accelerators in the rubber industry, UV stabilizers, and supplements in animal feeds and fertilizers. They are also used in rayon manufacture, smoke bombs, soldering fluxes, mordants for printing and dyeing, wood preservatives, mildew inhibitors, deodorants, antiseptics, and astringents (ATSDR, 1989i). In addition, zinc phosphide is used as a rodenticide.

Zinc is an essential element with recommended daily allowances ranging from $5 \mathrm{mg}$ for infants to $15 \mathrm{mg}$ for adult males (NRC, 1989).

Gastrointestinal absorption of zinc is variable (20-80\%) and depends on the chemical compound as well as on zinc levels in the body and dietary concentrations of other nutrients. In individuals with normal zinc levels in the body, gastrointestinal absorption is 20-30\% (ATSDR, 1989i). Information on pulmonary absorption is limited and complicated by the potential for gastrointestinal absorption due to mucociliary clearance from the respiratory tract and subsequent swallowing. Zinc is present in all tissues with the highest concentrations in the prostate, kidney, liver, heart, and pancreas. Zinc is a vital component of many metalloenzymes such as carbonic anhydrase, which regulates $\mathrm{CO}_{2}$ exchange (BEIAS Toxicity Profiles). Homeostatic mechanisms involving metallothionein in the mucosal cells of the gastrointestinal tract regulate zinc absorption and excretion (ATSDR, 1989i).

In humans, acutely toxic oral doses of zinc cause nausea, vomiting, diarrhea, and abdominal cramps and in some cases gastric bleeding (ATSDR, 1989i). Ingestion of zinc chloride can cause burning in the mouth and throat, vomiting, pharyngitis, esophagitis, hypocalcemia, and elevated amylase activity indicative of pancreatitis. Zinc phosphide, which releases phosphine gas under acidic conditions in the stomach, can cause vomiting, anorexia, abdominal pain, lethargy, hypotension, cardiac arrhythmias, circulatory collapse, pulmonary edema, seizures, renal damage, leukopenia, and coma and death in days to weeks. The estimated fatal dose is $40 \mathrm{mg} / \mathrm{kg}$. 
Animals dosed orally with zinc compounds develop pancreatitis, gastrointestinal and hepatic lesions, and diffuse nephrosis (BEIAS Toxicity Profiles).

Gastrointestinal upset has also been reported in individuals taking daily dietary zinc supplements for up to 6 weeks. There is also limited evidence that the human immune system may be impaired by subchronic exposures. In animals, gastrointestinal and hepatic lesions, pancreatic lesions, anemia, and diffuse nephrosis have been observed following subchronic oral exposures (BEIAS Toxicity Profiles).

Chronic oral exposures to zinc have resulted in hypochromic microcytic anemia associated with hypoceruloplasminemia, hypocupremia, and neutropenia in some individuals . Anemia and pancreatitis were the major adverse effects observed in chronic animal studies. Teratogenic effects have not been seen in animals exposed to zinc; however, high oral doses can affect reproduction and fetal growth.

Under occupational exposure conditions, inhalation of zinc compounds (mainly zinc oxide fumes) can result in a condition identified as "metal fume fever", which is characterized by nasal passage irritation, cough, rales, headache, altered taste, fever, weakness, hyperpnea, sweating, pains in the legs and chest, leukocytosis, reduced lung volume, and decreased diffusing capacity of carbon monoxide (ATSDR, 1989i). Inhalation of zinc chloride can result in nose and throat irritation, dyspnea, cough, chest pain, headache, fever, nausea and vomiting, and respiratory disorders such as pneumonitis and pulmonary fibrosis (ATSDR, 1989i). Pulmonary inflammation and changes in lung function have also been observed in inhalation studies on animals (BEIAS Toxicity Profiles).

Although "metal fume fever" occurs in occupationally exposed workers, it is primarily an acute and reversible effect that is unlikely to occur under chronic exposure conditions when zinc air concentrations are less than $8-12 \mathrm{mg} / \mathrm{m}^{3}$ (ATSDR, 1989i). Gastrointestinal distress, as well as enzyme changes indicative of liver dysfunction, have also been reported in workers occupationally exposed to zinc ; however, it is unclear as to what extent these effects might have been caused by pulmonary clearance, and subsequent gastrointestinal absorption. Consequently, there are no clearly defined toxic effects that can be identified as resulting specifically from pulmonary absorption following chronic low level inhalation exposures. Animal data for chronic inhalation exposures are not available.

\subsubsection{Organics}

\section{Acenaphthene}

Acenaphthene, also known as 1,2-dihydroacenaphthylene or 1,8-ethylenenaphthalene, is a tricyclic aromatic hydrocarbon that occurs in coal tar. It is used as a dye intermediate, in the manufacture of some plastics, and as an insecticide and fungicide. Acenaphthene has been detected in cigarette smoke, automobile exhausts, and urban air; in effluents from petrochemical, pesticide, and wood preservative industries and in soils, groundwater, and surface waters at hazardous waste sites (ATSDR, 1990e). 
No absorption data are available for acenaphthene; however, by analogy to structurally-related polycyclic aromatic hydrocarbons (PAHs), it would be expected to be absorbed from the gastrointestinal tract and lungs. The anhydride of naphthalic acid was identified as a urinary metabolite in rats treated orally with acenaphthene (BEIAS Toxicity Profiles).

Although a large body of literature exists on the toxicity and carcinogenicity of PAHs, primarily benzo[ $a]$ pyrene, toxicity data for acenaphthene are limited. Acenaphthene is irritating to the skin and mucous membranes of humans and animals. Acute toxicity data for animals include oral $\mathrm{LD}_{50} \mathrm{~s}$ of $10 \mathrm{~g} / \mathrm{kg}$ for rats and $2.1 \mathrm{~g} / \mathrm{kg}$ for mice and an intraperitoneal $\mathrm{LD}_{50}$ of $600 \mathrm{mg} / \mathrm{kg}$ for rats. Oral exposure of rats to daily $2-\mathrm{g}$ doses of acenaphthene for 32 days produced peripheral blood changes, mild liver and kidney damage, and pulmonary effects. Subchronic oral exposure to acenaphthene at doses of $\geq 350 \mathrm{mg} / \mathrm{kg}$ for 90 days produced increased liver weights, hepatocellular hypertrophy, and increased cholesterol levels in mice. Reproductive effects included decreased ovary weights at doses of $\geq 350 \mathrm{mg} / \mathrm{kg}$ and decreased ovarian and uterine activity as weil as smaller and fewer corpora lutea at $700 \mathrm{mg} / \mathrm{kg} /$ day. Adverse effects on the blood, lungs, and glandular tissues were reported in rats exposed daily to $12 \mathrm{mg} / \mathrm{m}^{3}$ of acenaphthene for 5 months (BEIAS Toxicity Profiles).

\section{Acetone}

Acetone (also 2-propanone or dimethyl ketone) is an industrial solvent synthesized from isopropyl alcohol. It is a highly volatile, flammable liquid with a sweetish taste. Prolonged or repeated exposure may cause erythema and dryness. Inhalation may produce headaches, fatigue, excitement, bronchial irritation, and, in large amounts, narcosis. The $\mathrm{LD}_{s 0}$ in rats is $10.7 \mathrm{mg} / \mathrm{kg}$ orally.

\section{Anthracene}

Anthracene, also referred to as paranaphthalene or green oil, is a polycyclic aromatic hydrocarbon ( $\mathrm{PAH})$ derived from coal tar and is primarily used as an intermediate in the production of dyes. It has also been used in the production of smoke screens. Anthracene is ubiquitous in the environment as a product of incomplete combustion of fossil fuels. Although a large body of literature exists on the toxicity and carcinogenicity of a number of PAHs, toxicity data for anthracene are limited.

Evidence indicates that anthracene is absorbed following oral and dermal exposure. Targets for anthracene toxicity are the skin, hematopoietic system, lymphoid system, and gastrointestinal tract. Adverse dermatologic effects have been observed in humans and animals in conjunction with acute and subchronic exposure to anthracene. In humans, anthracene may cause acute dermatitis with symptoms of burning, itching, and edema. Prolonged dermal exposure produces pigmentation, cornification of skin surface layers, and telangiectasis. Anthracene is photosensitizing, potentiating skin damage elicited by exposure to ultraviolet (UV) radiation (BEIAS Toxicity Profiles).

Hematologic toxicity was observed in patients receiving intraperitoneal injections of anthracenecontaining chemotherapeutic agents and in rats exposed to anthracene by oral gavage and by inhalation (BEIAS Toxicity Profiles). Mice receiving subcutaneous injections of anthracene 


\section{4-14}

exhibited adverse lymphoid effects. Long-term use of anthracene-containing laxatives produced melanosis of the colon and rectum. Human exposure to anthracene has also been associated with headache, nausea, loss of appetite, inflammation of the gastrointestinal tract, slow reactions, and weakness.

\section{Benzo(a)pyrene}

Benzo(a)pyrene, formerly known as 1,2-benzpyrene, is a polycyclic aromatic hydrocarbon (PAH) with a molecular formula of $\mathrm{C}_{20} \mathrm{H}_{12}$. It is found associated with coal tar and petroleum residues. There is no commercial production or known use of this compound. Benzo[a]pyrene is found in fossil fuels and occurs ubiquitously in products of incomplete combustion and in soils, groundwater, and surface waters at hazardous waste sites (ATSDR, 1990e; BEIAS Toxicity Profiles).

\section{Benzo(k)fluoranthene}

Benzo[ $k]$ fluoranthene, a crystalline solid with a chemical formula of $\mathrm{C}_{20} \mathrm{H}_{12}$ and a molecular weight of 252.32, is a polycyclic aromatic hydrocarbon (PAH) with one five-membered and four six-membered rings. There is no commercial production or known use of this compound. Benzo[k]fluoranthene is found in fossil fuels and occurs ubiquitously in products of incomplete combustion and in soils, groundwater, and surface waters at hazardous waste sites (ATSDR, 1990e; BEIAS Toxicity Profiles).

\section{Bis(2-ethylhexyl)phthalate}

Bis(2-ethylhexyl)phthalate is a colorless, oily liquid that is extensively used as a plasticizer in a wide variety of industrial, domestic and medical products. It is an environmental contaminant and has been detected in groundwater, surface water, drinking water, air, soil, plants, fish and animals. It is rapidly absorbed from the gastrointestinal tract primarily as mono(2-ethylhexyl)phthalate. The diester can be absorbed through the skin and from the lungs. It is rapidly metabolized in the blood and tissues to the monoester, which can be excreted as a glucuronide conjugate or further hydrolyzed to phthalic acid and excreted (BEIAS Toxicity Profiles).

Animal studies have indicated that the primary target organs are the liver and kidneys; however, higher doses are reported to result in testicular effects and decreased hemoglobin and packed cell volume. The primary intracellular effects of bis(2-ethylhexyl)phthalate in the liver and kidneys are an increase in the smooth endoplasmic reticulum and a proliferation in the number and size of peroxisomes. An epidemiological study reported no toxic effects from occupational exposure to air concentrations of bis(2-ethylhexyl)phthalate up to $0.16 \mathrm{mg} / \mathrm{m}^{3}$. Other studies on occupational exposures to mixtures of phthalate esters containing bis(2-ethylhexyl)phthalate have reported polyneuritis and sensory-motor polyneuropathy with decreased thrombocytes, leukocytes and hemoglobin in some exposed workers. Developmental toxicity studies with rats and mice have shown that bis(2-ethylhexyl)phthalate is fetotoxic and teratogenic when given orally during gestation. Oral exposure has also been shown to result in decreased sperm count in rats (BEIAS Toxicity Profiles). 
Bis(2-ethylhexyl)phthalate is known to induce the proliferation of peroxisomes, which has been associated with carcinogenesis. Dose-dependent, statistically-significant increases in the incidences of hepatocellular carcinomas and combined carcinomas and adenomas were seen in mice and rats exposed to bis(2-ethylhexyl)phthalate in their diet for 103 weeks. An increased incidence of neoplastic nodules and hepatocellular carcinomas was also reported in rats (BELAS Toxicity Profiles).

\section{Carbazole}

Carbozole, also known as dibenzopyrrole, is a three ringed aromatic organic compound with a molecular formula of $\mathrm{C}_{12} \mathrm{H}_{9} \mathrm{~N}$. It is found associated with coal hydrogenation products and is used as an intermediate in dye manufacture. It is also used as in photographic plates that are sensitive to ultraviolet light. The LD50 for rats is $>5 \mathrm{~g} / \mathrm{kg}$ orally.

\section{Chrysene}

Chrysene, a polycyclic aromatic hydrocarbon, is an ubiquitous environmental contaminant formed primarily by the incomplete combustion of organic compounds. Although present in coal and oil, chrysene found in the environment is the result of anthropogenic activities such as coal combustion and gasification; gasoline, diesel and aircraft exhausts; emissions from coke ovens, wood burning stoves, and waste incineration (ATSDR, 1990e). Chrysene is not produced or used commercially; its use is limited strictly to research applications.

Little information on the absorption, distribution, metabolism and excretion of chrysene in humans is available. Animal studies have shown that approximately $75 \%$ of the administered chrysene may be absorbed by oral, dermal, or inhalation routes. Following its absorption, chrysene is preferentially distributed to highly lipophilic regions of the body, most notably adipose and mammary tissue. Phase I metabolism of chrysene, whether in the lung, skin, or liver, is mediated by the mixed function oxidases. The metabolism results in the formation of 1,2-, 3,4-, and 5,6-dihydrodiols as well as the formation of 1-, 3-, and 4-phenol metabolites. Additional Phase I metabolism of chrysene 1,2-dihydrodiol forms chrysene 1,2-dihydrodiol-3,4epoxide and 9-hydroxychrysene 1,2-diol-3,4-oxide, metabolites that have mutagenic and alkylating activity. Phase II metabolism of chrysene results in the formation of glucuronide and sulfate ester conjugates; however, glutathione conjugates of diol- and triol-epoxides are also formed. Hepatobiliary secretion with elimination in the feces is the predominant route of excretion (BEIAS Toxicity Profiles).

\section{$\operatorname{Dibenz}(\mathrm{a}, \mathrm{h})$ anthracene}

Dibenz(a,h)anthracene, also known as 1,2:5,6-dibenzanthracene, is a five-ringed aromatic compound with a molecular formula of $\mathrm{C}_{22} \mathrm{H}_{14}$. It is associated with coal and petroleum residues and is classified as a PAH.

\section{Dimethylphthalate}

Dimethylphthalate, also known as DMP, is an aromatic compound with a molecular formula of $\mathrm{C}_{10} \mathrm{H}_{10} \mathrm{O}_{4}$. It is used as a solvent and plasticizer for cellulose acetate and cellulose acetate-butyrate 
based compositions. It is also used as an insect repellent for biting insects. It does cause irritation to mucous membranes and does cause CNS depression when ingested.

\section{Fluoranthene}

Fluoranthene is a polycyclic aromatic hydrocarbon (PAH) that can be derived from coal tar. Occurring ubiquitously in products of incomplete combustion of fossil fuels, fluoranthene has been identified in ambient air, surface, drinking, and waste water, and in char-broiled foods. Currently, there is no commercial production or use of this compound (BEIAS Toxicity Profiles).

Fluoranthene can be absorbed through the skin following dermal exposure and, by analogy to structurally-related PAHs, would be expected to be absorbed from the gastrointestinal tract and lungs. An in vitro study identified 2-methylfluoranthene and 3-methylfiuoranthene and their dihydrodiols as metabolites of fluoranthene (BEIAS Toxicity Profiles).

Although a large body of literature exists on the toxicity and carcinogenicity of PAHs, primarily benzo[a]pyrene, toxicity data for phenanthrene are very limited. No human data were available that addressed the toxicity of fluoranthene. Acute toxicity data for animals include an oral $\mathrm{LD}_{\text {so }}$ of $2000 \mathrm{mg} / \mathrm{kg}$ for rats; a dermal $\mathrm{LD}_{50}$ of $3180 \mathrm{mg} / \mathrm{kg}$ for rabbits; and an intravenous $\mathrm{LD}_{50}$ of $100 \mathrm{mg} / \mathrm{kg}$ for mice (BEIAS Toxicity Profiles). Subchronic oral exposure to fluoranthene at doses of $\geq 250 \mathrm{mg} / \mathrm{kg}$ produced nephropathy, increased liver weights, and increased liver enzyme levels in rats. A single intraperitoneal injection of fluoranthene to pregnant rats caused an increased rate of embryo resorptions. Fluoranthene was photosensitizing, enhancing erythema elicited by ultraviolet radiation in guinea pig skin and was irritating to the eyes of rabbits .

\section{Fluorene}

Fluorene, also know as o-biphenylenemethane, is a polycyclic aromatic hydrocarbon that is found associated with coal tars and petroleum residues. Occurring ubiquitously in products of incomplete combustion of fossil fuels, fluoranthene has been identified in ambient air, surface, drinking, and waste water, and in char-broiled foods. Currently, there is no commercial production or use of this compound (BEIAS Toxicity Profiles).

\section{Indeno(1,2,3-cd)pyrene}

Indeno[1,2,3-cd]pyrene, a crystalline solid with a chemical formula of $\mathrm{C}_{22} \mathrm{H}_{12}$ and a molecular weight of 276.3 , is a polycyclic aromatic hydrocarbon (PAH). There is no commercial production or known use of this compound. Indeno[1,2,3-cd]pyrene is found in fossil fuels and occurs ubiquitously in products of incomplete combustion and has been identified in soils, groundwater, and surface waters at hazardous waste sites (ATSDR, 1990).

No absorption data were available for indeno[1,2,3-cd]pyrene; however, by analogy to structurally-related PAHs, primarily benzo[a]pyrene, it would be expected to be absorbed from the gastrointestinal tract, lungs, and skin. In vivo metabolites identified in mouse skin include the trans-1,2-dihydrodiol and 8- and 9-hydroxy forms of indeno[1,2,3-cd]pyrene. Similar metabolites were formed in vitro in rat liver microsomes (BEIAS Toxicity Profiles). 


\section{Polychlorinated Biphenyls (Aroclor 1254 and Aroclor 1260)}

Aroclor is a trade name for a mixture of chemicals in the class of polychlorinated biphenyls or PCBs. There are several hundred chemicals in the class of PCBs. Mixtures of PCBs were formulated to have differing industrial uses ranging from heat transfer agents, lubricants, insulating agents and to reduce flammability of other parts. Aroclor 1254 and 1260 are two of those mixtures. Although PCBs are no longer manufactured, they are prevalent in the environment and are readily taken up in the food chain, especially in fish, and are stored in the fat of humans and animals. PCBs are transferred to infants via breast milk. Because of the widespread distribution of PCBs in the environment, most humans have been exposed to PCBs. PCBs can enter the human body through the skin, be inhaled as contaminated dust particles or vapors, or be ingested in contaminated foods. Occupational studies of workers exposed to PCBs and specifically to Aroclor 1254 and 1260 vapors have shown symptoms of acne or skin rashes, respiratory tract and eye irritation, and altered levels of liver enzymes as measured in the blood. PCBs are considered to be potential human carcinogens because some mixtures of PCBs have produced liver and stomach cancer in animals. Studies of PCB workers have not shown any specific type of cancer because the studies have generally been small and the results are not easily statistically verifiable. Studies of humans indicate a possible link between birth weight, head circumference, gestational age and neonatal behavior between PCB ingestion or maternal body burden (ATSDR 1991, BEIAS Toxicity Profiles).

\subsubsection{Radionuclides}

Radionuclides (or radioisotopes) are unstable isotopes of a chemical element that decay or disintegrate spontaneously, emitting radiation, to achieve a more stable state. The charged particles are termed "alpha and beta radiation" and the neutral particles are termed "gamma radiation or photons." Interaction of charged particles and photons with matter will produce ionization events, which may cause living cell tissue damage. Because the deposition of energy by ionizing radiation is a random process, sufficient energy may be deposited (in a critical volume) within a cell and result in cell modification or death (ICRP 1991). In addition, ionizing radiation has sufficient energy that interactions with matter will produce an ejected electron and a positively charged ion (known as free radicals) that are highly reactive and may combine with other elements, or compounds within a cell, to produce toxins or otherwise disrupt the overall chemical balance of the cell. These free radicals can also react with deoxyribonucleic acid (DNA), causing genetic damage, cancer induction, or even cell death.

A radionuclide is characterized by its half-life, which is the time required for half of the number of atoms in a sample to decay, and by the type and energies of the radiations emitted. Radiation emissions fall into two major categories: particulate (electrons, alpha particles, beta particles or positrons) or electromagnetic radiation (gamma and x-rays) (ATSDR 1989). Some radionuclides also emit protons or neutrons, but are not of concern with the radionuclides in this evaluation. All radionuclides are classified by EPA as Group A carcinogens based on their property of emitting ionizing radiation and on the extensive weight of evidence provided by epidemiological studies of humans with cancers induced by high doses of radiation.

Alpha particles are nuclei of ${ }^{4} \mathrm{He}$ atoms and consist of two protons and two neutrons. Alpha particles are emitted mostly in the decay of radioisotopes of elements with high atomic number 
(e.g., uranium). The alpha particles emitted by a particular radionuclide occur at discrete energies characteristic of that radioisotope. Alpha particles have the ability to react (and/or ionize) with other molecules, but they have very little penetrating power and lack the ability to pass through a piece of paper or human skin. Therefore, external exposure is not a concern with respect to alpha particles. However, alpha-emitting radionuclides are of concern when there is a potential for inhalation or ingestion of the radionuclide. Alpha particles are directly ionizing and deposit their energy in dense concentrations [termed high linear energy transfer (high LET)], resulting in short paths of highly localized ionization reactions. The probability of cell damage increases as a result of the increase in ionization events occurring in smaller areas; this may also be the reason for increased cancer incidence caused by inhalation of radon gas. In addition, the cancer incidence in smokers may be directly attributed to the naturally occurring alpha emitter, polonium-210, common in tobacco products.

Beta emissions usually refer to the continuous spectrum of negative electrons emitted in beta decay, but some radionuclides also decay by positron emission resulting in a continuous spectrum of positively charges electrons. Beta or positron radiation, like alpha radiation, is directly ionizing but, unlike alpha activity, deposit their energy along a longer track length (low-LET), resulting in more space between ionization events. Beta-ernitting radionuclides can cause injury to the skin and superficial body tissue but are most destructive when inhaled or ingested. Many beta emitters are similar chemically to naturally occurring essential nutrients and will therefore tend to accumulate in specific tissues. For example, strontium- 90 is chemically similar to calcium and, as a result, accumulates in the bones, where it causes continuous exposure. The health effects of alpha and beta particle emissions depend upon the target organ. Those seeking the bones would cause a prolonged exposure to the bone marrow and affect blood cell formation, possibly resulting in leukemia, other blood disorders, or bone cancers. Those seeking the liver would result in liver diseases or cancer, while those seeking the thyroid would cause thyroid and metabolic disorders. In addition, beta radiation may lead to damage of genetic material (DNA), causing hereditary defects (BEIAS Toxicity Profiles).

Transformation of the atomic nucleus can result in gamma emissions or $\mathrm{x}$-rays. The resulting energy released have differing origins; gamma emissions are released from the atomic nucleus, whereas $\mathrm{x}$-rays result from changes in the orbiting electron structure. Both external exposure (exposure to gamma radiation emitted by radionuclides outside the body) and internal exposure (exposure to gamma radiation emitted by radionuclides taken into the body) are of concern for gamma-emitting radionuclides. Gamma rays have high penetrating ability in living tissue and are capable of reaching all internal body organs. Without such sufficient shielding such as, lead, concrete, or steel, gamma radiation can penetrate the body from the outside and does not require ingestion or inhalation to penetrate sensitive organs. Gamma rays are characterized as low-LET radiation, as is beta radiation; however, the behavior of beta radiation differs from that of gamma radiation in that beta particles deposit most of their energy in the medium through which they pass, while gamma rays often escape the medium because of higher energies, thereby creating difficulties in determining actual internal exposure. For this reason, direct whole-body measurements are necessary to detect gamma radiation, while urine/fecal analyses are usually effective in detecting beta radiation (BEIAS Toxicity Profiles).

People continuously receive background radiation from naturally occurring radioactive decay processes that occur on the earth's surface including: radiation naturally occurring inside their 
bodies, from the atmosphere as fallout from nuclear testing or explosions, and from space or cosmic sources. cesium-137 (from nuclear fallout) decays to barium-137m, the highest contributor to fallout-induced gamma radiation (NCRP 1977). Beta radiation from the soil is a less penetrating form of radiation but has many contributing sources, most commonly: potassium-40, cesium-137, lead-214, and bismuth-214 (NCRP 1977). Alpha radiation is also emitted by radionuclides in the soil, but is not measurable more than a few centimeters from the ground surface. The majority of alpha emissions are attributable to radon-222 and radon-220 and their decay products (NCRP 1977).

The general health effects of radiation can be divided into stochastic and nonstochastic effects. Stochastic effects are those which the probability of occurrence, but not the severity, is assumed to be a function of dose (without threshold). There is no clear causal relationship between exposure and observed effect. Nonstochastic effects are those which the severity varies with dose, but a threshold for occupance of the effects is assumed. The risks from exposure to radionuclides calculated in this evaluation are those associated with stochastic effects.

Radiation can damage cells in different ways. It can cause damage to DNA within the cell, and the cell either may not be able to recover from this type of damage or may survive but function abnormally. If an abnormally functioning cell divides and reproduces, a tumor or mutation in the tissue may develop. The rapidly dividing cells that line the intestines and stomach and the blood cells in bone marrow are extremely sensitive to this damage. Organ damage results from the damage caused to the individual cells. This type of damage has been reported with doses of 10 to 500 rads (0.1 to $\mathbf{5 . 0}$ gray, in SI units). Acute radiation sickness is seen only after doses of $>50$ rads ( 0.5 gray) which is a dose rate usually achieved only in a nuclear accident (ATSDR 1989h).

When the radiation-damaged cells are reproductive cells, genetic damage can occur in the offspring of the person exposed. The developing fetus is especially sensitive to radiation. The type of malformation that may occur is related to the stage of fetal development and the cells that are differentiating at the time of exposure. Radiation damage to children exposed in the womb is related to the dose the pregnant mother receives. Mental retardation is a possible effect of fetal radiation exposure (ATSDR $1989 \mathrm{~h}$ ).

The most widely studied population that has had known exposure to radiation is the atomic bomb survivors of Hiroshima and Nagasaki, Japan. Data indicate an increase in the rate of leukemia and cancers in this population. However, the rate at which cancer incidence is significantly affected by low radiation exposures, such as results of exposure to natural background and industrially contaminated sites, is still undergoing study and is uncertain (BEIAS Toxicity Profiles).

\section{Americium -241}

Americium is a completely man-made transuranic element first discovered in 1944. Americium has a radioactive half-life $\left(T_{1 / 2}\right)$ of 458 years and is an alpha emitter with an energy of 5.637 $\mathrm{MeV}$. 


\section{Cesium-137}

Cesium-137, a product of nuclear fission reactions, has a radioactive half-life of 30.2 years. In $\sim 95 \%$ of the radioactive decay, cesium- 137 emits a beta particle with a maximum energy of $0.514 \mathrm{MeV}$ and is transformed to the metastable state of barium-137m. Barium-137m then in turn decays $90 \%$ of the time by emitting a $0.662 \mathrm{MeV}$ gamma-ray. Cesium-137 enters the human body via ingestion and behaves similarly to potassium. It is rapidly and almost completely absorbed from the GI tract and is distributed uniformly throughout the body (ICRP 1990). Because of its relatively short metabolic half-life (110 days), an acute dose of cesium-137 is almost completely excreted in the urine within a year of its initial exposure. However, when considering a potentially long-term (chronic exposure), cesium-137 would always be present in the body.

\section{Cobalt-60}

Cobalt-60 is a man-made isotope produced in nuclear reactors from neutron activation of stable cobalt atoms. These cobalt atoms are present as a corrosion impurity in the water used as the primary reactor coolant of the reactor. Cobalt-60 has a radioactive half-life of 5.27 years and decays by emitting a $0.314 \mathrm{MeV}$ beta particle and two gamma rays exhibiting energies of 1.33 and $1.17 \mathrm{MeV}$. Cobalt enters the environment surrounding the ORR from effluent of the naturally occurring element. It has a half-life of 5.263 years and is a beta and a gamma emitter. It is widely used for medical and industrial purposes.

\section{Plutonium-238,239,240}

Plutonium is a naturally occurring element with several isotopes. Other man-made isotopes are primarily 238, 239, and 240. The half-lives of plutonium 238 and 239 are 86.4 years and 24,390 years, respectively. All three isotopes are alpha emitters with energies of 5.593, 5.244, and $5.255 \mathrm{MeV}$ respectively.

\section{Potassium -40}

Potassium- 40 is a naturally occurring isotope whose half-life is $1.28 \times 10^{9}$ years and is a beta" emitter with an energy of $1.32 \mathrm{MeV}$.

\section{Radium-226,228}

Radium is a naturally occurring radioactive element that exists in several isotopic forms. The radium isotopes are formed from the decay of uranium and thorium. Radium-226 is formed from uranium-238 and uranium-234, and radium-226 has the longest half-life of the radium isotopes (radium-228, radium-224, and radium-223). In general, the activity concentration of radium-226 measured in most soils and rocks is comparable to those of uranium-238 and uranium-234, suggesting that radium does not tend to migrate from either of its uranium precursors under stable conditions. Radium-226 is primarily an alpha and gamma emitter.

Radium has been used as a component of luminous paints for clock and instrument dials. It has also been used in the treatment of cancer, in radiography, and in research. Radium is released 
into the environment in coal fly ash and in uranium mining and processing wastes. The background level of radium in industrial regions in soil is about $8.1 \mathrm{pCi} / \mathrm{g}$. Clays and soil components generally retard the movement of radium in the environment, but acidic processing wastes can enhance its movement. Radium may bioaccumulate in plants and animals, and exposure through the food chain is possible.

Many environmental problems can be directly attributed to the decay products or daughters of radium. The primary daughters are isotopes of radon-a colorless, odorless, radioactive gas. Radon gas can infiltrate basements and water systems, resulting in significant exposure via inhalation pathways.

\section{Strontium-90}

Strontium is a soft, silvery metal and is found in the minerals celestite and strontianite. It is malleable and ductile and is an excellent conductor of electricity. Strontium exists as a number of isotopes. One such isotope is strontium-90 which is a dangerous radioisotope found in the fallout of some nuclear explosions.

Radiostrontium is a fallout hazard because, being chemically similar to calcium, it becomes incorporated into bone where it may damage the bone marrow and induce cancer. It has a radioactive half-life of 29.1 years, and emits a beta particle of $0.546 \mathrm{MeV}$.

Thorium-228, -230, $-232,-234$

Thorium is a naturally occurring, radioactive metal. Small amounts of thorium are present in all rocks, soil, above-ground and underground water, plants, and animals. These small amounts of thorium contribute to the weak background radiation for such substances. Soil commonly contains an average of about $6 \mathrm{ppm}$ of soil. Rocks in some underground mines may also contain thorium in a more concentrated form. After these rocks are mined, thorium is usually concentrated and changes into thorium dioxide or other chemical forms. Thorium-bearing rock that has had most of the thorium removed from it is called "depleted" ore or tailings (ATSDR, 1990g).

Thorium is a metallic element of the actinide series. It exists in several isotopic forms. The isotope thorium-232 is a naturally occurring element that is radioactive. It decays through the emission of a series of alpha and beta particles, gamma radiation, and the formation of daughter products, finally yielding the stable isotope of lead, lead-208. Isotopes thorium-234 and thorium230 are produced during the decay of naturally occurring uranium-238, the isotope thorium-228 during the decay of thorium-232, and the isotopes thorium-231 and thorium-227 during the decay of uranium-235. Of these naturally produced isotopes of thorium, only thorium-232, thorium230 , and thorium- 228 have long enough half-lives to be environmentally significant. More than $99.99 \%$ of natural thorium is thorium-232; the rest is thorium-230 and thorium-228 (ATSDR, 1990g).

Thorium is used to make ceramics, lantern mantles, and metals used in the aerospace industry and in nuclear reactions. Thorium can also be used as a fuel for generating nuclear energy. 
More than 30 years ago, thorium oxides were used in hospitals to make certain kinds of diagnostic X-ray photographs (ATSDR, 1990g).

Because thorium is found almost everywhere, most people in the United States eat some thorium with their food every day. Normally, little of the thorium in lakes, rivers, and oceans gets into the fish or seafood used commercially. More thorium may be found near uncontrolled hazardous waste sites that contain thorium which might not have been disposed of properly. Consequently, people living near one of these sites may be exposed to slightly more thorium as a result of inhaling windblown dust containing thorium or eating food grown in soil contaminated with thorium. Larger-than-normal amounts of thorium might also enter the environment through accidental releases from thorium processing plants (ATSDR, 1990g).

Breathing dust contaminated with thorium is the primary pathway for thorium exposure to the body. A large portion of this dustborne thorium will be eliminated by normal bodily functions (urine/feces); however, a small amount of thorium will be taken up by the blood and subsequently transmitted to the bones. Breathing thorium dust may cause an increased chance of developing lung disease and cancer of the lung or pancreas many years after being exposed. Changes in genetic material have also been shown to occur in workers who breathed thorium dust. Liver diseases and effects on the blood have been found in people injected to take special X rays. Many types of cancer have been shown to occur in these people many years after thorium was injected in their bodies. Since thorium is radioactive and may be stored in bone for a long time, bone cancer is also a potential concern for people exposed to thorium. Animal studies have shown that breathing in thorium may result in lung damage. Other studies in animals suggest drinking massive amounts of thorium can cause death from metal poisoning. The presence of large amounts of thorium in the environment could result in exposure to more hazardous radioactive decay products of thorium, such as radium and thoron, which is an isotope of radon. Thorium is not known to cause birth defects or to affect childbearing abilities (ATSDR 1990g).

\section{Tritium}

Tritium or Hydrogen-3 is a naturally occurring isotope that is used in many areas of science. It has a radioactive half-life of 12.26 years and emits a weak beta particle having $0.0186 \mathrm{MeV}$. It is widely used as a tracer in research and for fusion-based thermonuclear weapons.

\section{Uranium}

Naturally occurring uranium is a lustrous, silver-colored, radioactive, malleable, and ductile metal. Uranium is almost exclusively used as a fuel for nuclear energy; however, it can be used in pigments, ceramics, and photographic chemical processes. The three naturally occurring isotopes of uranium are uranium-238 ( $>99 \%$ by weight), uranium-235 (about $0.72 \%$ by weight), and uranium-234 ( $0.06 \%$ by weight). The half-lives of uranium-234, uranium-235, and uranium238 are $2.4 \times 10^{5}$ years, $7.0 \times 10^{7}$ years and $4.5 \times 10^{9}$ years, respectively. The average concentration of uranium in the Earth's crust is between 2 and 4 parts per million. Some ores contain more concentrated uranium deposits. These deposits are mined and then the percentage of uranium-235 is increased by an enrichment process. The enriched uranium-235 is more radioactive, readily undergoing fission in the presence of thermal neutrons, and therefore is of great use as a nuclear reactor fuel or weapon component. 
Alpha, beta, and gamma radiation are emitted during the decay of uranium. Emission of alpha particles can give rise to other radioactive elements such as thorium-230, radium-226, radon-222, and polonium-218. Radon, which is colorless and odorless, is a significant daughter isotope, and as a gas, it can leak into basements or foundations. Significant exposure by inhalation of radon gas can occur. Deposition of radon and its daughters into lung tissue exposes the lungs to alpha radiation, increasing the probability of cancer. Radium has been known to produce bone sarcomas in radium dial painters resulting in death. The final decay for uranium-238 and uranium-234 is stable lead-206 and the final decay for uranium-235 is stable lead-207 (ATSDR, 1990).

Uranium may be released into the air by natural processes such as volcanic eruption or resuspension of soil. The combustion of fossil fuels is a potential contamination route. Coal and shale oils have varying levels of uranium depending on ore location, and their combustion can concentrate the levels of uranium. Groundwater and surface water releases of uranium are primarily caused by the disposal of uranium mining waste, disposal of nuclear reactor waste, and reactor emissions. Soil naturally contains uranium, but levels may be concentrated by the addition of mining waste and nuclear facility waste. Foods such as, beets and potatoes also contain uranium. These vegetables, along with other root vegetables, contain higher levels of uranium than leafy vegetables. Higher levels of uranium have also been found in cattle that graze near nuclear facilities (ATSDR, 1989h).

Uranium exhibits toxicity through its chemical as well as its radiological properties. The toxicological effects from the ingestion of uranium are the result of the action of uranium as a metal and its radioactive properties. The primary toxic chemical effect is seen in kidney damage. Studies in rabbits, mice, and dogs showed kidney damage in a dose related effect. Exposure to enriched uranium may cause damage to somatic cells resulting in tumors or cause damage to reproductive cells, affecting the next generation. Higher incidence of lung cancer have been observed in uranium mine workers, although this effect is considered to be due to the radiological effects of radon (and its daughter products), which is also present in uranium mines (ATSDR, 1989h).

\subsubsection{Contaminants Evaluated Qualitatively}

\subsubsection{Inorganics}

\section{Aluminum}

Aluminum is a silver-white flexible metal with a vast number of uses. It is poorly absorbed and efficiently eliminated; however, when absorption does occur, aluminum is distributed mainly in bone, liver, testes, kidneys, and brain (ATSDR, 1990a).

Aluminum may be involved in Alzheimer's disease (dialysis dementia) and in Amyotrophic Lateral Sclerosis and Parkinsonism-Dementia Syndromes of Guam (Guam ALS-PD complex) (ATSDR, 1990a). Aluminum content of brain, muscle, and bone increases in Alzheimer's patients. Neurofibrillary tangles (NFTs) are found in patients suffering from aluminum encephalopathy and Alzheimer's disease. Symptoms of "dialysis dementia" include speech disorders, dementia, convulsions, and myoclonus. People of Guam and Rota have an unusually 
high incidence of neurodegenerative diseases. The volcanic soil in the region of Guam where the high incidence of ALS-PD occurs contains high levels of aluminum and manganese. Neurological effects have also been observed in rats orally exposed to aluminum compounds (BEIAS Toxicity Profiles).

The respiratory system appears to be the primary target following inhalation exposure to aluminum. Alveolar proteinosis has been observed in guinea pigs, rats, and hamsters exposed to aluminum powders. Rats and guinea pigs exposed to aluminum chlorohydrate exhibited an increase in alveolar macrophages, increased relative lung weight, and multifocal granulomatous pneumonia (BELAS Toxicity Profiles).

No decrease in reproductive capacity, hormonal abnormalities, or testicular histopathology was observed in male rats exposed to aluminum in drinking water for 90 days. However, male rats exposed to aluminum (as aluminum chloride) via gavage for 6 months exhibited decreased spermatozoa counts and sperm motility, and testicular histological and histochemical changes (BEIAS Toxicity Profiles).

Male rats exposed to drinking water containing aluminum (as aluminum potassium sulfate) for a lifetime exhibited increases in unspecified malignant and nonmalignant tumors, and similarly exposed female mice exhibited an increased incidence of leukemia. Rats and guinea pigs exposed via inhalation to aluminum chlorohydrate developed lung granulomas, while granulomatous foci developed in similarly exposed male hamsters (BEIAS Toxicity Profiles).

\section{Cobalt}

Pure cobalt is a steel-gray, shiny, hard metal. It does not dissolve in water, but some cobalt compounds (substances formed by combining cobalt with other chemicals) do dissolve in water. The metal and other cobalt compounds are mainly used in industry. In the United States, cobalt is mostly used to make alloys (mixtures of metals); as a drier for paint and the porcelain enameling used on steel bathroom fixtures, large appliances and kitchenwares; and in making colored pigments. Small amounts of cobalt are added to or naturally occur in foods. In fact, vitamin B-12 is a cobalt-containing compound that is essential for good health in humans. Cobalt alloys are used to make artificial parts, such as hip and knee joints, that are placed in the human bodies by surgery.

The important natural sources of cobalt in the environment are soil and dust, seawater, volcanic eruptions, and forest fires. The important man-made sources of cobalt in the environment are the byproducts of burning of coal and oil; exhaust from cars, trucks, and aircrafts; industrial processes that use the metal or its compounds; and sewage sludge from cities. Cobalt is found naturally in the soil at about 7 parts per million (ppm). In foods, cobalt is found in varying amounts from $0.01 \mathrm{ppm}$ in corn to about $0.6 \mathrm{ppm}$ in spinach. Drinking water concentrations of cobalt average about 2 parts per billion $(\mathrm{ppb})$.

Cobalt belongs to Group VIII, or the first transitional series, of the periodic classification of elements. There is only one stable isotope of natural cobalt with an atomic mass of 59 . Cobalt60 is a man-made isotope used mainly in the medicinal and weapon industries. 
Cobalt (0.16-1.0 mg cobalt $/ \mathrm{kg})$ has also been used as a treatment for anemia (illness of the blood), particularly in pregnant women, because it causes red blood cells to be produced. Cobalt exposure at normal environmental levels poses no threat to humans.

When too much cobalt is consumed, however, harmful health effects can occur. People exposed to $0.038 \mathrm{mg} \mathrm{cobalt} / \mathrm{m}^{3}$ (about 100,000 times the concentration normally found in air) for 6 hours had trouble breathing. Serious effects on the lungs, including asthma, pneumonia, and wheezing have been found in people exposed to $0.003 \mathrm{mg}$ cobalt $/ \mathrm{m}^{3}$ at work. People exposed to $0.007 \mathrm{mg}$ cobalt $/ \mathrm{m}^{3}$ at work have also developed allergies to cobalt that result in asthma and skin rashes. The general public, however, is not likely to be exposed to the same type or amount of cobalt dust that causes these effects in workers (BEIAS Toxicity Profiles).

In the $1960 \mathrm{~s}$, some breweries added cobalt to beer to stabilize the foam (1.0-1.5 ppm). Some people who drank excessive amounts of beer (8-25 pints/day) experienced serious effects on the heart. In some cases, these effects resulted in death. Vomiting and nausea usually occurred before the effects on the heart were noticed. Effects on the heart were not seen, however, in people with anemia treated with up to $1 \mathrm{mg}$ cobalt $/ \mathrm{kg}$, or in pregnant women with anemia treated with up to $0.6 \mathrm{mg}$ cobalt $/ \mathrm{kg}$. Treatment of the anemic pregnant women with $0.6 \mathrm{mg}$ cobalt $/ \mathrm{kg}$ also did not cure their anemia. Effects on the thyroid were found in people exposed to $0.5 \mathrm{mg}$ cobalt $/ \mathrm{kg}$ for a few weeks. Vision problems were found in one individual following treatment with $1.3 \mathrm{mg}$ cobalt $/ \mathrm{kg}$ for 6 weeks, but this effect has not been seen in other humans or animal studies (BEIAS Toxicity Profiles).

Studies in animals suggest that exposure to high amounts of cobalt during pregnancy might affect the health of the developing fetus. Birth defects, however, have not been found in children born to mothers who were treated with cobalt for anemia during pregnancy. The doses of cobalt used in the animal studies were much higher that the amounts of cobalt to which humans would normally be exposed (BEIAS Toxicity Profiles).

\section{Lead}

Lead $(\mathrm{Pb})$ is a naturally occurring bluish-gray metal that is found in small quantities in the earth's crust. Lead and its compounds can be found in plants and animals that are used for human consumption, as well as in air, drinking water, rivers, lakes, oceans, dust, and soil. Industry is supplied with lead from mined ores or from recycled scrap metal. The primary use of lead is in the manufacture of storage batteries. Lead is also used in the production of ammunition, miscellaneous metal products, and various chemicals, including gasoline additives (ATSDR, 1990d).

The general population's exposure to lead (and its compounds) is from breathing air, drinking water, and consuming foods that contain lead. Another source of exposure is from breathing or ingesting dust or dirt laden with lead. Children, especially those of preschool age, are at a particular risk to lead exposure. Children commonly place their hands, toys and other items in their mouths. Lead-contaminated dirt and dust frequently comes into contact with these items, resulting in a consumption of large amounts of lead. Another source of lead exposure to children is via the ingestion of paint chips (ATSDR, 1990d). 
Lead exposure is particularly dangerous for the developing fetus, because of its sensitivity to lead. Young children are also sensitive to the effects of lead and tend to absorb more of the lead they ingest. A pregnant woman exposed to lead results in a transfer of lead to the fetus, which may cause preterm birth, reduced birth weight, and a decreased intelligence quotient (IQ) in the infant. A decrease in IQ scores and reduced growth in young children may also occur when they are exposed to lead. When middle-aged men are exposed to lead, an increase in blood pressure may also occur. Lead can severely damage the brain and kidneys in adults as well as children when they are exposed to high levels of lead. Additionally, lead exposure at high levels will cause abortion as well as damage to the male reproductive system (ATSDR, 1990d).

\subsubsection{Organics}

\section{Asbestos}

Asbestos is the name applied to a group of six different minerals that occur naturally in the environment. The most common mineral type is white (chrysolite), but others may be blue (crocidolite), gray (anthophyllite), or brown (amosite). These minerals are made up of long, thin fibers that appear somewhat similar to fiberglass. Asbestos fibers are very strong and are resistant to heat and chemicals. Because of these properties, asbestos fibers have been used in a wide range of products, mostly in building materials, friction products, and heat-resistant fabrics. Because the fibers are so resistant to chemicals, they are also very stable in the environment (ATSDR, 1990c).

Studies of asbestos workers show an increased risk of developing two forms of cancer. These two forms are lung cancer and mesothelioma (a cancer of the membrane that surrounds the lung and other organs). There is also evidence of increased risk of cancer of the stomach, intestines, esophagus, pancreas, and kidneys. Inhalation of low doses of asbestos has been linked to a slow accumulation of scar-like tissue in the lungs and surrounding tissue which restricts the normal lung expansion and contraction. This condition is known as asbestosis (ATSDR 1990c).

\section{$\operatorname{Benzo}(g, h, i) p e r y l e n e$}

Benzo[ $[g, h, i]$ perylene, also known as 1,12 -benzoperylene, is a polycyclic aromatic hydrocarbon (PAH) with six aromatic rings. There is no known commercial production or use of benzo[ $g, h, i]$ perylene. It occurs naturally in crude oils and is present ubiquitously in products of incomplete combustion and in coal tar.

\section{Dibenzofuran}

Dibenzofuran, also known as diphenylene oxide, is an double ringed aromatic organic compound with a molecular formula of $\mathrm{C}_{12} \mathrm{H}_{8} \mathrm{O}$. It is found associated with organic residues in petroleum products and incomplete combustion. 


\section{2-Methylnaphthalene}

2-Methylnaphthalene, also known as $\beta$-methylnaphthalene, is an two ringed aromatic organic compound whose molecular formula is $\mathrm{C}_{11} \mathrm{H}_{10}$. It is found associated with organic residues in petroleum products and incomplete combustion as well as coal tar.

\section{Naphthalene}

Naphthalene, a white solid with a characteristic odor of mothballs, is a polycyclic aromatic hydrocarbon composed of two fused benzene rings. The principal end use of naphthalene is as a raw material for the production of phthalic anhydride. It is also used as an intermediate for synthetic resins, celluloid, lampblack, smokeless powder, solvents, and lubricants. Naphthalene is used directly as a moth repellant, insecticide, anthelmintic, and intestinal antiseptic (ATSDR, 1990e).

Naphthalene can be absorbed by the oral, inhalation, and dermal routes of exposure and can cross the placenta in amounts sufficient to cause fetal toxicity. The most commonly observed effect of naphthalene toxicity following acute oral or inhalation exposure in humans is hemolytic anemia associated with decreased hemoglobin and hematocrit values, increased reticulocyte counts, presence of Heinz bodies, and increased serum bilirubin levels (ATSDR, 1990e). Hemolytic anemia has been observed in an infant dermally exposed to naphthalene and in infants whose mothers were exposed to naphthalene during pregnancy ). Infants and individuals having a congenital deficiency of erythrocyte glucose-6-phosphate dehydrogenase are especially susceptible to naphthalene-induced hemolytic anemia (BEIAS Toxicity Profiles).

Acute oral and subchronic inhalation exposure of humans to naphthalene has resulted in neurotoxic effects (confusion, lethargy, listlessness, vertigo), gastrointestinal distress, hepatic effects (jaundice, hepatomegaly, elevated serum enzyme levels), renal effects, and ocular effects (cataracts, optical atrophy). Cataracts have been reported in individuals occupationally exposed to naphthalene (Ghetti and Mariani, 1956) and in rabbits and rats exposed orally to naphthalene. A number of deaths have been reported following intentional ingestion of naphthalene-containing mothballs (ATSDR, 1990e). The estimated lethal dose of naphthalene is $5-15 \mathrm{~g}$ for adults and 2-3 $\mathrm{g}$ for children. Naphthalene is a primary skin irritant and is acutely irritating to the eyes of humans (BEIAS Toxicity Profiles).

Increased mortality, clinical signs of toxicity, kidney and thymus lesions, and signs of anemia were observed in rats treated by gavage with $400 \mathrm{mg} / \mathrm{kg}$ of naphthalene for 13 weeks. No adverse effects occurred at $\leq 50 \mathrm{mg} / \mathrm{kg}$. Transient clinical signs of toxicity were seen in mice exposed by gavage to $53 \mathrm{mg} / \mathrm{kg}$ for 13 weeks. Subchronic oral exposure to $133 \mathrm{mg} / \mathrm{kg} / \mathrm{day}$ for 90 days produced decreased spleen weights in female mice. Reduced numbers of pups/litter were observed when naphthalene was administered orally to pregnant mice (BEIAS Toxicity Profiles).

\section{Phenanthrene}

Phenanthrene is a polycyclic aromatic hydrocarbon (PAH) that can be derived from coal tar. Currently, there is no commercial production or use of this compound. Phenanthrene is 
ubiquitous in the environment as a product of incomplete combustion of fossil fuels and wood and has been identified in ambient air, surface and drinking water, and in foods.

Phenanthrene is absorbed following oral and dermal exposure. Data from structurally related PAHs suggest that phenanthrene would be absorbed from the lungs. Metabolites of phenanthrene identified in in vivo and in vitro studies indicate that metabolism proceeds by epoxidation at the $1-2,3-4$, and $9-10$ carbons, with dihydrodiols as the primary metabolites (BEIAS Toxicity Profiles).

Although a large body of literature exists on the toxicity and carcinogenicity of PAHs, primarily benzo[a]pyrene, toxicity data for phenanthrene are very limited. No human data were available that addressed the toxicity of phenanthrene. Single intraperitoneal injections of phenanthrene produced slight hepatotoxicity in rats. Data regarding the subchronic, chronic, developmental, or reproductive toxicity in experimental animals by any route of exposure could not be located in the available literature.

\subsection{TOXICITY VALUES}

\subsubsection{Toxicity Information for Noncarcinogenic Effects}

The reference dose (RfD) is the toxicity value used to evaluate noncarcinogenic effects resulting from chemical exposure and is an estimate of the daily exposure to the human population, including sensitive subgroups likely to be without appreciable risk of harmful effects. It can be used to estimate a level of exposure at or below which no adverse effects are expected to occur. If the exposure level exceeds this threshold, concern for potential noncarcinoginic/systematic effects might arise. A reference dose, or $\mathrm{RfD}$, is the toxicity value used in evaluating noncarcinogenic effects resulting from exposure at Building 3506. Two types of RfDs are available depending on the exposure route (oral or inhalation), the critical effect (developmental or others), and the length of exposure being evaluated (chronic, subchronic, or single event). A chronic RfD is defined as an estimate of a daily exposure level for the human population, including sensitive subpopulations, that is likely to be without an appreciable risk of deleterious effects during a lifetime. Chronic RfDs are specifically developed to be protective for long-term exposure to a compound. These chronic RfDs are generally used to evaluate the potential noncarcinogenic effects associated with exposure periods between 7 years and a lifetime. Subchronic RfDs may be used to evaluate the potential noncarcinogenic effects associated with short-term exposure periods of two weeks to seven years. Such short-term exposures can result when a particular activity is performed for a limited period of times or when a chemical with a short half-life degrades to negligible concentrations within several months. Therefore, subchronic RfDs are used to evaluate the potential effects on an organ following a single exposure event.

EPA develops RfDs based on the period of contaminant exposure. The toxicity values are derived using U.S.EPA approved methodology. For noncancer health effects, RfDs are derived as a benchmark for the amount of a chemical that can be ingested without an appreciable risk of 
deleterious effects, and similarly, RfCs are derived for the concentrations that can be inhaled without deleterious effects. The basic formula for deriving the RfD and RfC is as follows .

$$
R D / R A C=\frac{N O A E L}{U F \times M F}
$$

where

NOAEL $=$ No-observed adverse effect level

UF = Uncertainty factors

MF = Modifying factor

The NOAEL is selected from human data if possible, but it is more often obtained from studies using laboratory animals. For many noncarcinogenic effects, protective mechanisms are believed to exist that must be overcome before an adverse effect is manifested. Therefore in developing a toxicity value for evaluating noncarcinogenic effects (i.e., RfD), the approach is to identify the upper bound of this tolerance range. Because variability exists in the human population, attempts are made to identify a subthreshold level protective of sensitive individuals in the population. For most chemicals this level can only be estimated; the RfD incorporates uncertainty factors indicating the degree or extrapolation used to derive the estimated values.

Based on extrapolation of uncertainties, there are five possible UFs: (1) human to sensitive human, (2) animal to human, (3) subchronic to chronic toxicity extrapolation, (4) lowest observed adverse effect level (LOAEL) to NOAEL, and (5) incomplete to complete data base. Each of these factors can be 10 , but to avoid the possibility of being too conservative, the EPA has established a maximum UF of 3000 where four areas of uncertainty exist and a maximum of 10,000 for five areas of uncertainty. The MF can be used in addition to UF to account for some aspect of the study that is not covered by the five UFs described above or the MF can be $<1$ which reduces the uncertainty associated with the selected study. The MF most frequently used is 1 which is the default value.

Contaminant RfDs vary depending on the mode of intake (i.e., ingestion, inhalation, or dermal contact). The inhalation $\mathrm{RfD}$ was recently replaced with the inhalation reference concentration $(\mathrm{RfC})$, expressed in units of milligrams per cubic meter $\left(\mathrm{mg} / \mathrm{m}^{3}\right)$. Conversions between the inhalation RfC and RfD are made assuming that humans weigh $70 \mathrm{~kg}$ and breathe at a rate of $20 \mathrm{~m}^{3} /$ day. No RfDs are currently available for the dermal route of exposure. Noncarcinogenic risks associated with dermal exposure were evaluated by extrapolating from an oral RfD to a dermal RfD. This conversion was done by multiplying the oral RfD of each chemical by the percent gastrointestinal absorption efficiency (\%GI) for that chemical. However, the \%GI is known for only a limited amount of chemicals; therefore, for those chemicals where a \%GI is not currently available, $100 \%$ was assumed. Reference values that may be useful for evaluating potential adverse effects associated with oral exposures of shorter duration are known as One-day and Ten-day Health Advisories and these are issued as nonregulatory guidance. Health Advisory values are concentrations of contaminants in drinking water at which adverse health effects would not be expected to occur for an exposure of the specified duration. One-day and Ten-day Health Advisories do not consider any carcinogenic risk associated with the exposure even if the 
compound is a potential carcinogen. Table 4.3 summarizes all the available RfDs along with uncertainty factors, modifying factors.

\subsubsection{Toxicity Information for Carcinogenic Effects}

A slope factor and the accompanying weight-of-evidence cletermination are the toxicity data used to evaluate potential human carcinogenic risks. Carcinogenesis, unlike many noncarcinogenic health effects, is generally thought to be a phenomenon for which risk evaluation based on presumption of a threshold is inappropriate. The hypothesized mechanism for carcinogenesis is referred to as "nonthreshold" because there is believed to be essentially no level of exposure to such a chemical that dose not pose a finite probability, however small, of generating a carcinogenic response. That is, no dose is thought be risk free and therefore in evaluating cancer risks an effect threshold cannot be estimated. Therefore, for carcinogenic effects, EPA uses a two-part evaluation in which the substance is assigned a weight-of-evidence classification, and a slope factor is calculated. In the first step, data are evaluated to determine the likelihood that the agent is a human carcinogen.

Based on this evaluation, EPA gives the agent a weight-of-evidence classification. The second step involves the calculation of a slope factor, which measures the relationship between dose and response. In general, the slope factor is a plausible upper-bound estimate of the probability of a response per unit intake of a chemical over a lifetime. The slope factor is used in this risk assessment to estimate an increased upper-bound lifetime probability of developing cancer as a result of exposure to a particular level of a potential carcinogen (EPA 1989a). Dermal slope factors for each contaminant were adjusted based on that contaminant's oral slope factor. This was done by dividing the oral slop factor by the \%GI. A risk factor of $7.3 \times 10^{-7}$ was used to calculate external exposure risks for the direct measurement samples and the TLD strings (ICRP 1991).

The toxicity values for the contaminants are listed in Tables 4.1 through 4.3. The data presented for each contaminant was taken from Integrated Risk Information System (IRIS) and/or the Health and Environmental Affects Summary Table (HEAST) and are referenced appropriately in each table.

\subsubsection{Chemicals Without EPA Toxicity Values}

Slope factors and RfDs are not currently available for all detected chemicals in Bldg 3506 because their carcinogenicity and/or noncarcinogenic effects have not yet been determined. These chemicals may contribute to carcinogenic and noncarcinogenic effects from exposure to the Building 3506 media, but their effect cannot be quantified at the present time. In addition, for several chemicals, epidemiological studies have indicated that they are not carcinogenic; consequently, these species do not have SFs. 
4-31

Table 4.1. Toxicity values: potential carcinogenic effects contaminants evaluated quantitatively

\begin{tabular}{|c|c|c|c|c|c|c|}
\hline Analyte & CASRN & $\begin{array}{c}\text { ORAL SF } \\
\text { (Risk/pCi) } \\
\text { (mg/kg/day) })^{12}\end{array}$ & $\begin{array}{c}\text { INHALATION SF } \\
\text { (Risk/pCi) } \\
\text { (mg/kg/day) }\end{array}$ & $\begin{array}{c}\text { EXTERNAL } \\
\text { EXPOSURE SF } \\
\text { (Risk/year per pCi/g) }\end{array}$ & $\begin{array}{l}\text { EPA } \\
\text { CLASS }\end{array}$ & $\begin{array}{c}\text { SF } \\
\text { SOURCE }\end{array}$ \\
\hline Acetone & $67-64-1$ & - & - & - & D & HEAST \\
\hline Antimony & $7440-36-0$ & - & - & - & - & HEAST \\
\hline Arsenic & $7440-38-2$ & - & $5.00 E+01$ & - & A & HEAST \\
\hline Barium & $7440-39-3$ & - & - & - & - & HEAST \\
\hline Beryllium & $7440-41-7$ & $4.3 E+00$ & $8.4 E+00$ & - & B 2 & HEAST \\
\hline $\begin{array}{l}\text { Bis(2- } \\
\text { Ethylhexyl)phthalete }\end{array}$ & $117-81-7$ & $1.4 \mathrm{E}-02$ & - & - & B 2 & HEAST \\
\hline Cadmium & $7440-43-9$ & - & $6.1 E+00$ & - & B 1 & HEAST \\
\hline Chromium (VI) & $18540-29-9$ & - & $4.1 E+01$ & - & A & HEAST \\
\hline Cyanide & $57-12-5$ & - & - & - & D & HEAST \\
\hline Manganese & $7439-96-5$ & - & - & - & D & HEAST \\
\hline Mercury & $7439-97-6$ & - & - & - & D & HEAST \\
\hline Nickel & $7440-02-0$ & - & - & - & - & HEAST \\
\hline Selenium & $7782-49-2$ & - & - & - & D & HEAST \\
\hline Vanadium & $7440-62-2$ & - & - & - & - & HEAST \\
\hline Zinc & $7440-66-6$ & - & - & - & D & HEAST \\
\hline $\begin{array}{l}\text { Polychlorinated } \\
\text { Biphenyls (PCBs) }\end{array}$ & $1336-36-3$ & $7.7 E+\infty$ & - & - & B 2 & HEAST \\
\hline
\end{tabular}


4-32

Table 4.1. (continued)

\begin{tabular}{|c|c|c|c|c|c|c|}
\hline Analyte & CASRN & $\begin{array}{c}\text { ORAL SF } \\
\text { (Risk/pCi) } \\
\text { (mg/kg/day) })^{-1}\end{array}$ & $\begin{array}{c}\text { INHALATION SF } \\
\text { (Risk/pCi) } \\
(\mathbf{m g} / \mathbf{k g} / \text { day })^{-1}\end{array}$ & $\begin{array}{c}\text { EXTERNAL } \\
\text { EXPOSURE SF } \\
\text { (Risk/year per pCi/g) }\end{array}$ & $\begin{array}{c}\text { EPA } \\
\text { CLASS }\end{array}$ & $\begin{array}{c}\text { SF } \\
\text { SOURCE }\end{array}$ \\
\hline \multicolumn{7}{|l|}{$\begin{array}{l}\text { Polycyclic Aromatic } \\
\text { Hydrocarbons (PAHs) }\end{array}$} \\
\hline Acenaphthene & $83-32-9$ & - & - & - & - & HEAST \\
\hline Anthracene & $120-12-7$ & - & - & - & D & HEAST \\
\hline Benzo(a)pyrene & $50-32-8$ & $7.3 E+00$ & - & - & B 2 & HEAST \\
\hline Benzo(k)fluoranthene & $207-08-9$ & - & - & - & B 2 & HEAST \\
\hline Carbazole & $86-74-8$ & $2.00 \mathrm{E}-02$ & - & - & B 2 & HEAST \\
\hline Chrysene & $218-01-9$ & - & - & - & B 2 & HEAST \\
\hline Dibenz $(a, h)$ anthracene & $53-70-3$ & - & - & - & B 2 & HEAST \\
\hline Fluoranthene & $206-44-0$ & - & - & - & $\mathbf{D}$ & HEAST \\
\hline Fluorene & $86-73-7$ & - & - & - & $\mathbf{D}$ & HEAST \\
\hline Indeno $(1,2,3-c, \mathrm{~d})$ pyrene & $193-39-5$ & - & - & - & B 2 & HEAST \\
\hline \multicolumn{7}{|l|}{ Radionuclides } \\
\hline Americium-241 & $14596-10-2$ & $2.4 \mathrm{E}-10$ & $3.2 \mathrm{E}-08$ & 4.9E-09 & $\mathbf{A}$ & HEAST \\
\hline Cesium-137 & $10045-97-3$ & $2.8 \mathrm{E}-11$ & $1.9 \mathrm{E}-11$ & 2.0E-06 & $\mathbf{A}$ & HEAST \\
\hline Cobalt- 60 & $10198-40-0$ & $1.5 \mathrm{E}-11$ & $1.5 \mathrm{E}-10$ & $8.6 \mathrm{E}-06$ & A & HEAST \\
\hline Plutonium-238 & $13981-16-3$ & $2.2 \mathrm{E}-10$ & $3.9 \mathrm{E}-08$ & $2.8 \mathrm{E}-11$ & $\mathbf{A}$ & HEAST \\
\hline Plutonium-239 & $15117-48-3$ & $2.3 \mathrm{E}-10$ & $3.8 \mathrm{E}-08$ & $1.7 \mathrm{E}-11$ & $\mathbf{A}$ & HEAST \\
\hline
\end{tabular}


4-33

Table 4.1. (continued)

\begin{tabular}{|c|c|c|c|c|c|c|}
\hline Analyte & CASRN & $\begin{array}{c}\text { ORAL SF } \\
\text { (Risk/pCi) } \\
(\mathbf{m g} / \mathbf{k g} / \text { day })^{-1}\end{array}$ & $\begin{array}{c}\text { INHALATION SF } \\
\text { (Risk/pCi) } \\
\text { (mg/kg/day) }^{-1}\end{array}$ & $\begin{array}{c}\text { EXTERNAL } \\
\text { EXPOSURE SF } \\
\text { (Risk/year per pCi/g) }\end{array}$ & $\begin{array}{c}\text { EPA } \\
\text { CLASS }\end{array}$ & $\begin{array}{c}\text { SF } \\
\text { SOURCE }\end{array}$ \\
\hline Plutonium-240 & $14119-33-6$ & 2.3E-10 & $3.8 \mathrm{E}-08$ & 2.7E-11 & $\mathbf{A}$ & HEAST \\
\hline Potassium-40 & $13966-00-2$ & $1.1 \mathrm{E}-11$ & $7.6 \mathrm{E}-12$ & 5.4E-07 & $\mathbf{A}$ & HEAST \\
\hline Radium-226 & $13982-63-3$ & $1.2 \mathrm{E}-10$ & 3.0E-09 & $6.0 \mathrm{E}-06$ & $\mathbf{A}$ & HEAST \\
\hline Radium-228 & $15262-20-1$ & $1.0 \mathrm{E}-10$ & $6.9 \mathrm{E}-10$ & $2.9 \mathrm{E}-06$ & $\mathbf{A}$ & HEAST \\
\hline Strontium-90 & $10098-97-2$ & $3.6 \mathrm{E}-11$ & $6.2 \mathrm{E}-11$ & $0.0 \mathrm{E}+00$ & $\mathbf{A}$ & HEAST \\
\hline Thorium-228 & $14274-82-9$ & $5.5 \mathrm{E}-11$ & $7.8 \mathrm{E}-06$ & $5.6 \mathrm{E}-06$ & $\mathbf{A}$ & HEAST \\
\hline Thorium-229 & $15594-54-4$ & $8.9 \mathrm{E}-11$ & 7.7E-08 & $6.8 \mathrm{E}-07$ & $\mathbf{A}$ & HEAST \\
\hline Thorium-230 & $14269-63-7$ & $1.3 \mathrm{E}-11$ & $2.9 \mathrm{E}-08$ & $5.4 \mathrm{E}-11$ & A & HEAST \\
\hline Thorium-232 & $7440-29-1$ & $1.2 \mathrm{E}-11$ & $2.8 \mathrm{E}-08$ & $2.6 \mathrm{E}-11$ & A & HEAST \\
\hline Thorium-234 & $15065-10-8$ & $4.0 \mathrm{E}-12$ & 3.2E-11 & $3.5 \mathrm{E}-09$ & A & HEAST \\
\hline Tritium & $10028-17-8$ & $5.4 \mathrm{E}-14$ & $7.8 \mathrm{E}-14$ & $0.0 E+00$ & $\mathbf{A}$ & HEAST \\
\hline Uranium-233 & $13968-55-3$ & $1.6 \mathrm{E}-11$ & 2.7E-08 & $4.2 \mathrm{E}-11$ & $A$ & HEAST \\
\hline Uranium-234 & $13966-29-5$ & $1.6 \mathrm{E}-11$ & $2.6 \mathrm{E}-08$ & $3.0 \mathrm{E}-11$ & $A$ & HEAST \\
\hline Uranium-235 & $15117-96-1$ & $1.6 \mathrm{E}-11$ & $2.5 \mathrm{E}-08$ & $2.4 \mathrm{E}-07$ & $\mathbf{A}$ & HEAST \\
\hline Uranium-238 & $7440-61-1$ & $2.0 \mathrm{E}-11$ & $2.4 \mathrm{E}-08$ & 5.1E-08 & $\mathbf{A}$ & HEAST \\
\hline
\end{tabular}


Table 4.2. Toxicity values: potential carcinogenic effects contaminants evaluated qualitatively

\begin{tabular}{|c|c|c|c|c|c|c|}
\hline Analyte & CASRN & $\begin{array}{c}\text { ORAL SF } \\
\text { (Risk/pCi) } \\
(\mathrm{mg} / \mathrm{kg} / \mathrm{day})^{-1}\end{array}$ & $\begin{array}{c}\text { PNHALATION SF } \\
\text { (Risk/pCi) } \\
(\mathbf{m g} / \mathrm{kg} / \mathrm{day})^{-1}\end{array}$ & $\begin{array}{c}\text { EXTERNAL } \\
\text { EXPOSURE SF } \\
\text { (Risk/year per pCi/g) }\end{array}$ & $\begin{array}{c}\text { EPA } \\
\text { CLASS }\end{array}$ & $\begin{array}{c}\text { SF } \\
\text { SOURCE }\end{array}$ \\
\hline Aluminum & $7429-90-5$ & - & - & - & - & - \\
\hline $\operatorname{Benzo}(g, h, i)$ perylene & $191-24-2$ & - & - & - & D & HEAST \\
\hline Cobalt & - & - & - & - & - & - \\
\hline Dibenofuran & - & - & - & - & - & - \\
\hline Lead & $7439-92-1$ & - & - & - & B 2 & HEAST \\
\hline 2-Methylnaphthalene & - & - & - & - & - & - \\
\hline Naphthalene & $91-20-3$ & - & - & - & $\mathrm{D}$ & HEAST \\
\hline Phenanthrene & $85-01-8$ & - & - & - & D & HEAST \\
\hline
\end{tabular}


Table 4.3. Toxicity values: potential noncarcinogenic effects for Building 3506 COPCs

\begin{tabular}{|c|c|c|c|c|c|c|c|c|c|c|}
\hline ANALYTE & CASRN & $\begin{array}{l}\text { CHRONIC } \\
\text { ORAL RID } \\
\text { (mg/kg/day) }\end{array}$ & $\begin{array}{c}\text { DERMAL } \\
\text { RfD } \\
\text { (mg/kg/day) }\end{array}$ & $\begin{array}{c}\text { INHALATION } \\
\text { RTD } \\
\text { (mg/kg/day) }\end{array}$ & $\begin{array}{c}\text { CONFIDENCE } \\
\text { LEVEL }\end{array}$ & $\mathbf{M F}$ & UF & $\begin{array}{c}\text { RPD } \\
\text { SOURCE }\end{array}$ & $\begin{array}{l}\text { RTD BASIS } \\
\text { (VEHICLE) }\end{array}$ & $\begin{array}{l}\text { CRITICAL } \\
\text { EFFECT }\end{array}$ \\
\hline Acenaphthene & $83-32-9$ & $6.0 \mathrm{E}-02$ & $6.0 \mathrm{E}-02$ & - & Low & 1 & 3000 & IRIS & Oral & $\begin{array}{l}\text { Hepatotoxicity } \\
\text { (mouse) }\end{array}$ \\
\hline Acetone & $67-64-1$ & $1.0 \mathrm{E}-01$ & 8.3E-02 & - & Low & 1 & 1000 & IRIS & Oral & $\begin{array}{c}\text { Kidney } \\
\text { nephrotoxicity and } \\
\text { increased liver and } \\
\text { kidney weights (rat) }\end{array}$ \\
\hline Anthracene & $120-12-7$ & $3.0 \mathrm{E}-01$ & 2.3E-01 & - & Low & 1 & 3000 & IRIS & Oral & $\begin{array}{l}\text { Increased melanosis } \\
\text { incidence }\end{array}$ \\
\hline Antimony & $7440-36-0$ & 4.0E-04 & $8.0 \mathrm{E}-06$ & - & Low & 1 & 1000 & IRIS & Water (Oral) & $\begin{array}{c}\text { Decreased } \\
\text { longevity; } \\
\text { Alteration of blood } \\
\text { chemistry in rats }\end{array}$ \\
\hline Arsenic & $7440-38-2$ & 3.0E-04 & $1.2 \mathrm{E}-04$ & - & Medium & 1 & 100 & IRIS & Occupational & $\begin{array}{c}\text { Keratosis, } \\
\text { Hyperpigmentation }\end{array}$ \\
\hline Barium & $7440-39-3$ & $7.0 \mathrm{E}-02$ & $4.9 \mathrm{E}-03$ & $1.40 \mathrm{E}-04$ & Medium & 1 & 3 & IRIS & Water (Oral) & $\begin{array}{l}\text { Increased blood } \\
\text { pressure; baritosis }\end{array}$ \\
\hline Beryllium & $7440-41-7$ & $5.0 \mathrm{E}-03$ & $5.0 \mathrm{E}-05$ & - & Low & 1 & 100 & IRIS & Oral & $\begin{array}{l}\text { Slightly decreased } \\
\text { body weight (rat) }\end{array}$ \\
\hline $\begin{array}{l}\text { Bis(2- } \\
\text { Ethylhexyl) } \\
\text { phthalate }\end{array}$ & $117-81-7$ & $2.0 \mathrm{E}-02$ & $3.8 \mathrm{E}-03$ & - & Medium & 1 & 1000 & IRIS & Oral & $\begin{array}{c}\text { Increased relative } \\
\text { liver weight (guinea } \\
\text { pig) }\end{array}$ \\
\hline Cadmium & $7440-43-9$ & $1.0 \mathrm{E}-03$ & $\begin{array}{c}1.0 \mathrm{E}-05 \\
.\end{array}$ & - & High & 1 & 10 & IRIS & Oral (Diet) & $\begin{array}{l}\text { Renal toxicity; } \\
\text { osteomalacia, } \\
\text { osteoporosis, and } \\
\text { significant } \\
\text { proteinuria }\end{array}$ \\
\hline
\end{tabular}


4-36

Table 4.3. (continued)

\begin{tabular}{|c|c|c|c|c|c|c|c|c|c|c|}
\hline ANALYTE & CASRN & $\begin{array}{l}\text { CHRONIC } \\
\text { ORAL RfD } \\
\text { (mg/kg/day) }\end{array}$ & $\begin{array}{c}\text { DERMAL } \\
\text { RfD } \\
\text { (mg/kg/day) }\end{array}$ & $\begin{array}{c}\text { INHALATION } \\
\text { RRD } \\
\text { (mg/kg/day) }\end{array}$ & $\begin{array}{c}\text { CONFIDENCE } \\
\text { LEVEL }\end{array}$ & MF & UF & $\begin{array}{c}\text { RAD } \\
\text { SOURCE }\end{array}$ & $\begin{array}{l}\text { RfD BASIS } \\
\text { (VEHICLE) }\end{array}$ & $\begin{array}{c}\text { CRITICAL } \\
\text { EFFECT }\end{array}$ \\
\hline Chromium VI & $18540-29-9$ & $5.0 \mathrm{E}-03$ & $1.0 \mathrm{E}-04$ & - & Low & 1 & 500 & IRIS & Water (Oral) & $\begin{array}{c}\text { Hepato- \& } \\
\text { Nephrotoxicity }\end{array}$ \\
\hline Cyanide & $57-12-5$ & $2.0 \mathrm{E}-02$ & $8.0 \mathrm{E}-03$ & - & Medium & 5 & 100 & IRIS & Oral & $\begin{array}{l}\text { Weight loss, } \\
\text { thyroid effects, and } \\
\text { myelin } \\
\text { degeneration }\end{array}$ \\
\hline $\begin{array}{l}\text { Dimethyl } \\
\text { Phthalate }\end{array}$ & $131-11-3$ & $1.0 \mathrm{E}+01$ & $1.0 E+01$ & - & - & - & 100 & IRIS & Water (Oral) & Kidney effects (rat) \\
\hline Fluoranthene & $206-44-0$ & 4.0E-02 & $1.2 \mathrm{E}-02$ & - & Low & 1 & 3000 & IRIS & Water (Oral) & $\begin{array}{l}\text { Nephrotoxicity; } \\
\text { increased liver } \\
\text { weight, } \\
\text { hematological } \\
\text { aiterations, and } \\
\text { clinical effects } \\
\text { (mouse) }\end{array}$ \\
\hline Fluorene & $86-73-7$ & 4.0E-02 & $4.0 \mathrm{E}-02$ & - & Low & 1 & 3000 & IRIS & Oral & $\begin{array}{l}\text { Decreased RBCs, } \\
\text { packed cell volume, } \\
\text { and hemoglobin } \\
\text { (mouse) }\end{array}$ \\
\hline $\begin{array}{l}\text { Manganese } \\
\text { (Diet) }\end{array}$ & $7439-96-5$ & $1.4 \mathrm{E}-01$ & $5.6 \mathrm{E}-03$ & $1.40 \mathrm{E}-05$ & Medium & 1 & 1 & IRIS & Diet & $\begin{array}{l}\text { Neural Tissue } \\
\text { Damage }\end{array}$ \\
\hline $\begin{array}{l}\text { Mercury, } \\
\text { Inorganic }\end{array}$ & $7439-97-6$ & $3.0 \mathrm{E}-04$ & $3.0 \mathrm{E}-08$ & $8.60 \mathrm{E}-05$ & - & - & 1000 & IRIS & $\begin{array}{c}\text { Oral, } \\
\text { Inhalation }\end{array}$ & $\begin{array}{l}\text { Kidney Damage } \\
\text { Neurotoxicity }\end{array}$ \\
\hline Nickel & $7440-02-0$ & $2.0 \mathrm{E}-02$ & $5.4 \mathrm{E}-03$ & - & Medium & 1 & 300 & IRIS & Oral & $\begin{array}{l}\text { Reduced organ } \\
\text { weight; Decreases } \\
\text { in whole body } \\
\text { weight }\end{array}$ \\
\hline
\end{tabular}


4-37

Table 4.3. (continued)

\begin{tabular}{|c|c|c|c|c|c|c|c|c|c|c|}
\hline ANALYTE & CASRN & $\begin{array}{l}\text { CIIRONIC } \\
\text { ORAL RID } \\
\text { (mg/kg/day) }\end{array}$ & $\begin{array}{c}\text { DERMAL } \\
\text { Rm } \\
\text { (mg/kg/day) }\end{array}$ & $\begin{array}{c}\text { INIAALATION } \\
\text { RRD } \\
\text { (mg/kg/day) }\end{array}$ & $\begin{array}{l}\text { CONFIDENCE } \\
\text { LEVEL }\end{array}$ & MF & UF & $\begin{array}{c}\text { RfD } \\
\text { SOURCE }\end{array}$ & $\begin{array}{l}\text { RfD BASIS } \\
\text { (VEHICLE) }\end{array}$ & $\begin{array}{l}\text { CRITICAL } \\
\text { EFFECT }\end{array}$ \\
\hline Pyrene & $129-00-0$ & $3.0 \mathrm{E}-02$ & $9.3 \mathrm{E}-03$ & - & Low & 1 & 3000 & IRIS & Oral & $\begin{array}{l}\text { Renal tubular } \\
\text { pathology and } \\
\text { decreased kidney } \\
\text { weight (mouse) }\end{array}$ \\
\hline Selenium & $7782-49-2$ & $5.0 \mathrm{E}-03$ & $2.2 \mathrm{E}-03$ & - & High & 1 & 3 & IRIS & Oral & Clinical selenosis \\
\hline Vanadium & $7440-62-2$ & $7.0 \mathrm{E}-03$ & $7.0 \mathrm{E}-05$ & - & Low & 1 & 100 & IRIS & Water (Oral) & $\begin{array}{l}\text { Minor changes in } \\
\text { serum chemistry }\end{array}$ \\
\hline Zinc & $7440-66-6$ & $3.0 \mathrm{E}-01$ & $6.0 \mathrm{E}-02$ & - & Medium & 1 & 3 & $\begin{array}{l}\text { IRIS' } \\
\text { HEAST }\end{array}$ & Gavage & $\begin{array}{l}\text { Hyperactivity; low } \\
\text { body weight }\end{array}$ \\
\hline
\end{tabular}


4-38

THIS PAGE INTENTIONALLY LEFT BLANK 


\section{RISK CHARACTERIZATION}

This risk characterization presents the quantitative estimates of the general magnitude of human health risks posed by contaminants in Building 3506 . Human health risks are evaluated using human exposure results (Sect. 3) and dose-response toxicity information (Sect. 4). In addition, risks are estimated differently for carcinogens and noncarcinogens (systemic toxicants).

For carcinogenic contaminants, risk is expressed in terms of the probability of contracting cancer over a lifetime, over and above the normal background risk. This is the Incremental Lifetime Cancer Risk (ILCR) and is estimated as the product of the estimated exposure to a carcinogen and the contaminant-specific, route-specific slope factor:

where:

$$
\mathrm{ILCR}=\mathrm{SF} \times \mathrm{CDI}
$$

$$
\begin{aligned}
& \text { ILCR }=\text { Incremental lifetime cancer risk (unitless probability) } \\
& \mathrm{SF}=\text { Slope factor (mg/kg-day) } \\
& \mathrm{CDI}=\text { Chronic daily intake (mg/kg-day) }
\end{aligned}
$$

The previous equation is linear and is usually applicable for estimating low level risks (i.e., below estimated risks of 0.01 ). For sites where risks are above 0.01 , an alternative calculation was used:

$$
\mathrm{ILCR}=1-\exp (-\mathrm{CDI} \times \mathrm{SF})
$$

where:

$$
\begin{aligned}
& \text { ILCR = Incremental lifetime cancer risks (unitless probability) } \\
& \exp =\text { the exponential } \\
& \mathrm{CDI}=\text { Chronic daily intake (mg/kg-day) } \\
& \mathrm{SF}=\text { Slope factor (mg/kg-day) }
\end{aligned}
$$

In weighing exposures to potentially carcinogenic compounds, a reasonable level of risk must be identified. EPA specifies a risk range of $10^{-6}$ to $10^{-4}$ associated with the consideration and selection of remedial alternatives for superfund sites (EPA, 1990). The lower end of this range is generally applied to residential risks, while occupational risks may be considered acceptable at the higher end of this range. In the United States, the incidence of cancer occurrence is $3 \times$ $10^{-1}$ or 0.30 (American Cancer Society, 1990). The ILCR (also referred to as excess cancer risk) is defined as the estimated increased risk that occurs over an assumed average lifespan of 70 years as the result of exposure to a specific known carcinogen. Therefore, an incremental lifetime cancer risk of one in one million $\left(1 \times 10^{-6}\right)$ may be interpreted as an increase in the baseline population cancer incidence from 300,000 per million population to 300,001 per million population. Current radiation protection standards for workers include a limit of 5 rem for the annual effective dose equivalent. This dose equates to a 25 -year lifetime risk corresponding to 
$2 \times 10^{-2}$. Similar protection standards for members of the public include a limit of 0.1 rem per year. This dose equates to a 70-year lifetime risk of $4 \times 10^{-3}$.

Noncarcinogenic effects of contaminants are quantified as hazard quotients (HQs). An HQ is the ratio of the estimated daily intake to the allowable daily intake or RfD:

where:

$$
\mathrm{HQ}=\mathrm{CDI} / \mathrm{RfD}
$$

$$
\begin{aligned}
& \mathrm{HQ}=\text { Hazard quotient (unitless) } \\
& \mathrm{CDI}=\text { Chronic daily intake (mg/kg-day) } \\
& \mathrm{RfD}=\text { Reference dose (mg/kg-day) }
\end{aligned}
$$

Because the HQ does not define exposure-response relationships, its numerical value should not be construed as a direct estimate of risk. It is a numerical indication of whether or not acceptable exposure levels are exceeded. As this quotient approaches unity, concern for the potential hazard of the contaminant increases.

In the case of simultaneous exposure to several contaminants, a hazard index (HI) may be calculated to evaluate the potential risk associated with exposure to a mixture by summing the HQs for each contaminant and exposure pathway:

where:

$$
\mathrm{HI}=\mathrm{HQ}_{1}+\mathrm{HQ}_{2}+\mathrm{HQ}_{3}+\ldots
$$

$$
\mathrm{HI}=\text { Hazard index (unitless) }
$$

Mechanistically, it may not be appropriate to sum HQs unless the contaminants that make up the mixture have similar modes of action on the identical organ. Very little synergistic or greater-than-additive interactions among environmental toxicants have been identified in the scientific literature. A number of antagonistic or less than additive interactions are known, and these could be overestimated in an additive model. In general, an HI less than 1 indicates estimated intakes are below the intake that would cause toxic effects.

Excess cancer risk estimates and HQs were calculated for a hypothetical maintenance worker (current), hypothetical S\&M worker (current), and a hypothetical adult trespasser (future) for the ingestion, dermal exposure to chemicals, inhalation, and external exposure to radionuclides pathways as appropriate. The results of the risk characterization are presented in the following text and tables.

\subsection{CURRENT LAND USE}

The following sections will present and discuss the results of the risk characterization for the current land use conditions as represented by the hypothetical maintenance worker and the hypothetical S\&M worker scenarios. Risks are presented for the cell and the east gallery 


\subsubsection{Excess Cancer Risk}

\section{Cell}

\section{Maintenance Worker}

The excess cancer risks associated with the hypothetical maintenance worker for the cell are summarized in Table 5.1. A complete table of results can be found in Appendix E. External exposure to radiation (core samples) from the structure (i.e., walls and floor) presented the dominant risk to maintenance workers at Building 3506 (1.3E-02). This excess cancer risk value is above EPA's target risk range and the risk calculated for ORNL's administrative control limit of $1.5 \mathrm{rem} / \mathrm{yr}$. This value, however, does not exceed the lifetime risk for the radiation protection standard of $5 \mathrm{rem} / \mathrm{yr}$ (2.0E0-2). The risks from all other pathways were insignificant (and within EPA's target risk range) compared to the external exposure pathway (three orders of magnitude less).

The excess cancer risk for this receptor via external exposure was estimated using several different types of data including core samples, direct measurements, and TLD strings. Excess cancer risks estimated for these data types were comparable (risks calculated using the direct measurements and the TLD strings were slightly higher than the core data), which validates the methods used for estimating risks due to external exposure to radionuclides. Using the direct measurement and the TLD data may be a more realistic estimate of the risk because no modelling is necessary to calculate the risk.

The smear samples were collected to indicate how much of the contamination is transferrable (rather than fixed). The core samples represent both the fixed and the transferrable contamination. For the cell, most of the contamination is fixed (approximately 90\%). The concentration of contaminants in the smear samples was calculated as a relative proportion of the contaminants in the core samples. The primary contaminant responsible for the risk from external exposure to radionuclides is cesium-137 (1.6E-03) (Appendix E, Table E-1). As a cross check, risks were calculated using the contaminant-specific smear samples. A risk value of $1.4 \mathrm{E}-03$ was calculated for external exposure using the cesium-137 smear data, indicating that cesium-137 is the major radionuclide of concern for the transferrable contamination in the cell.

Ingestion of the PCB Aroclor-1254 from the sediment presented the dominant risk from chemical contamination to the maintenance worker at Building 3506 (5.5E-04) (Appendix E, Table E-1). This risk value is within EPA's target risk range.

\section{S\&M Worker}

The excess cancer risks estimated for the S\&M worker for the cell are summarized in Table 5.2. A complete table of results can be found in Appendix E. Similar to the maintenance worker, external exposure to radiation (core samples) from the structure (i.e., walls and fioor) presented the dominant risk to the S\&M workers at Building 3506 (1.0E-04). This value is within EPA's target risk range. The risks from all other pathways were insignificant compared to the external exposure pathway (three orders of magnitude less). Risk from external exposure to radionuclides (2.4E-03) was also the dominant risk for the sediment present in the cell. 
$5-4$

Table 5.1. Summary risk characterization results-cancer risk estimates-maintenance worker

\begin{tabular}{|c|c|c|c|c|c|c|c|c|}
\hline \multirow{2}{*}{ Receptor } & \multirow{2}{*}{$\begin{array}{l}\text { Exposure } \\
\text { Pathway }\end{array}$} & \multirow{2}{*}{ Media } & \multirow{2}{*}{ Location } & \multirow{2}{*}{$\begin{array}{c}\text { Total } \\
\text { Pathway } \\
\text { Risk }\end{array}$} & \multicolumn{3}{|c|}{$\begin{array}{l}\text { Total Contaminant } \\
\text { Risk }\end{array}$} & \multirow{2}{*}{$\begin{array}{c}\text { Total } \\
\text { Area } \\
\text { Risk } \\
\text { (Pathways } \\
\text { Summed) }\end{array}$} \\
\hline & & & & & Inorganic & Organic & Radionuclide & \\
\hline Maintenance Worker & Ingestion & Core Samples & Cell & 2.7E-05 & & & & \\
\hline Maintenance Worker & Dermal Exposure & Core Samples & Cell & $8.4 \mathrm{E}-05$ & & & & \\
\hline Maintenance Worker & Inhalation & Core Samples & Cell & $4.8 \mathrm{E}-05$ & & & & \\
\hline Maintenance Worker & External Exposure & Core Samples & Cell & $1.3 \mathrm{E}-02$ & & & & \\
\hline Maintenance Worker Risk & & & & & $9.6 \mathrm{E}-05$ & 2.1E-06 & $1.3 \mathrm{E}-02$ & $1.3 \mathrm{E}-02$ \\
\hline Maintenance Worker & Ingestion & Core Samples & East Gallery & $6.5 \mathrm{E}-06$ & & & & \\
\hline Maintenance Worker & Dermal Exposure & Core Samples & East Gallery & $5.9 \mathrm{E}-06$ & & & & \\
\hline Maintenance Worker & Inhalation & Core Samples & East Gallery & 4.3E-05 & & & & \\
\hline Maintenance Worker & External Exposure & Core Samples & East Gallery & $2.5 E-04$ & & & & \\
\hline Maintenance Worker Risk & & & & & $2.2 \mathrm{E}-605$ & $9.6 \mathrm{E}-06$ & $2.9 E-04$ & $3.1 E-04$ \\
\hline Maintenance Worker & Ingestion & Core Samples & Whole Building & $2.8 \mathrm{E}-04$ & & & & \\
\hline Maintenance Worker & Dermal Exposure & Core Samples & Whole Building & $6.2 \mathrm{E}-04$ & & & & \\
\hline Maintenance Worker & Inhalation & Core Samples & Whole Building & $4.8 \mathrm{E}-05$ & & & & \\
\hline Maintenance Worker & External Exposure & Core Samples & Whole Building & $1.3 \mathrm{E}-02$ & & & & \\
\hline Maintenance Worker Risk & & & & & $6.7 \mathrm{E}-05$ & $9.2 \mathrm{E}-06$ & $1.3 \mathrm{E}-02$ & $1.3 \mathrm{E}-02$ \\
\hline Maintenance Worker & Ingestion & Sediment & Cell & 8.3E-04 & & & & \\
\hline Maintenance Worker & Dermal Exposure & Sediment & Cell & 4.0E-04 & & & & \\
\hline Maintenance Worker & Inhalation & Sediment & Cell & $2.6 \mathrm{E}-07$ & & & & \\
\hline Maintenance Worker & External Exposure & Sediment & Cell & $2.5 E-01$ & & & & \\
\hline
\end{tabular}


5-5

Table 5.1. (continued)

\begin{tabular}{|c|c|c|c|c|c|c|c|c|}
\hline \multirow{2}{*}{ Receptor } & \multirow{2}{*}{$\begin{array}{l}\text { Exposure } \\
\text { Pathway }\end{array}$} & \multirow{2}{*}{ Media } & \multirow{2}{*}{ Location } & \multirow{2}{*}{$\begin{array}{c}\text { Total } \\
\text { Pathway } \\
\text { Risk }\end{array}$} & \multicolumn{3}{|c|}{$\begin{array}{c}\text { Total Contaminant } \\
\text { Risk }\end{array}$} & \multirow{2}{*}{$\begin{array}{c}\text { Total } \\
\text { Area } \\
\text { Risk } \\
\text { (Pathways } \\
\text { Summed) }\end{array}$} \\
\hline & & & & & Inorganic & Organic & Radionuclide & \\
\hline Maintenance Worker Risk & & & & & $1.5 \mathrm{E}-07$ & $9.6 \mathrm{E}-04$ & 2.5E-01 & 2.5E-01 \\
\hline Maintenance Worker & External Exposure & $\begin{array}{c}\text { Direct } \\
\text { Measurement }\end{array}$ & Cell & 4.2E-01 & & & 4.2E-01 & $4.2 \mathrm{E}-01$ \\
\hline Maintenance Worker & External Exposure & $\begin{array}{c}\text { Direct } \\
\text { Measurement }\end{array}$ & East Gallery & $7.8 \mathrm{E}-03$ & & & $7.8 \mathrm{E}-03$ & $7.8 \mathrm{E}-03$ \\
\hline Maintenance Worker & External Exposure & $\begin{array}{c}\text { Direct } \\
\text { Measurement }\end{array}$ & Whole Building & $5.4 \mathrm{E}-01$ & & & $5.4 \mathrm{E}-01$ & $5.4 \mathrm{E}-01$ \\
\hline Maintenance Worker & Ingestion & Smear Samples & Cell & 1.3E-06 & & & & \\
\hline Maintenance Worker & Inhalation & Smear Samples & Cell & $6.3 \mathrm{E}-07$ & & & & \\
\hline Maintenance Worker & Extemal Exposure & Smear Samples & Cell & $1.6 \mathrm{E}-03$ & & & & \\
\hline Maintenance Worker Risk & & & & & & & $1.6 \mathrm{E}-03$ & $1.6 \mathrm{E}-03$ \\
\hline Maintenance Worker & Ingestion & Smear Samples & East Gallery & $2.2 \mathrm{E}-07$ & & & & \\
\hline Maintenance Worker & Inhalation & Smear Samples & East Gallery & $1.8 \mathrm{E}-06$ & & & & \\
\hline Maintenance Worker & External Exposure & Smear Samples & East Gallery & $1.9 \mathrm{E}-04$ & & & & \\
\hline Maintenance Worker Risk & & & & & & & $1.9 \mathrm{E}-04$ & $1.9 \mathrm{E}-04$ \\
\hline Maintenance Worker & Ingestion & Smear Samples & Whole Building & 7.2E-07 & & & & \\
\hline Maintenance Worker & Inhalation & Smear Samples & Whole Building & 7.1E-07 & & & & \\
\hline Maintenance Worker & External Exposure & Smear Samples & Whole Building & $9.2 \mathrm{E}-04$ & & & & \\
\hline Maintenance Worker Risk & ' & & & & & & 9.2E-04 & 9.2E-04 \\
\hline Maintenance Worker & External Exposure & TLD & Cell & $6.7 \mathrm{E}-01$ & & & $6.7 \mathrm{E}-01$ & 6.7E-01 \\
\hline
\end{tabular}




\section{5-6}

Table 5.2. Summary risk characterization results-cancer risk estimates-S\&M worker

\begin{tabular}{|c|c|c|c|c|c|c|c|c|}
\hline \multirow{2}{*}{ Receplor } & \multirow{2}{*}{$\begin{array}{l}\text { Exposure } \\
\text { Pathway }\end{array}$} & \multirow{2}{*}{ Medin } & \multirow{2}{*}{ Location } & \multirow{2}{*}{$\begin{array}{c}\text { Total } \\
\text { Pathway } \\
\text { Risk }\end{array}$} & \multicolumn{3}{|c|}{$\begin{array}{c}\text { Total Contaminent } \\
\text { Rtsk }\end{array}$} & \multirow{2}{*}{$\begin{array}{c}\text { Total } \\
\text { Area } \\
\text { Rilk } \\
\text { (Pathway } \\
\text { Summed) }\end{array}$} \\
\hline & & & & & Inorganic & Organic & Radionuclide & \\
\hline S\&M Worker & Ingestion & Core Samples & Cell & 2.2E-07 & & & & \\
\hline S\&M Worker & Dermal Exposure & Core Sumples & Cell & 6.7E-07 & & & & \\
\hline S\&M Worker & Inhalation & Core Samples & Cell & $3.9 \mathrm{E}-07$ & & & & \\
\hline S\&M Worker & Exterual Exposure & Core Samples & Cell & $1.0 \mathrm{E}-04$ & & & & \\
\hline S\&M Worker Risk & & & & & 7.7E-07 & $1.7 \mathrm{E}-08$ & $1.0 \mathrm{E}-04$ & 1.0E-04 \\
\hline S\&M Worker & Ingestion & Core Samplea & East Gallery & 5.2E-08 & & & & \\
\hline S\&M Worker & Dermal Exposure & Core Samplea & Enst Gallery & 4.7E-08 & & & & \\
\hline S\&M Worker & Inhalation & Core Samples & East Gallery & $3.5 \mathrm{E}-07$ & & & & \\
\hline S\&M Worker & Extemal Exposure & Core Samples & East Gallery & $2.0 \mathrm{E}-06$ & & & & \\
\hline S\&M Worker Risk & & & & & $1.7 \mathrm{E}-08$ & $7.8 \mathrm{E}-08$ & $2.4 \mathrm{E}-06$ & 2.5E-06 \\
\hline S\&M Worker & Ingestion & Core Semples & Whole Building & 2.2E-07 & & & & \\
\hline S\&M Worker & Dermal Exposure & Core Samples & Whole Building & $4.9 \mathrm{E}-07$ & & & & \\
\hline S\&M Worker & Inhalation & Core Samples & Whole Building & $3.9 \mathrm{E}-07$ & & & & \\
\hline S\&M Worker & External Exposure & Core Samples & Whole Building & $1.0 \mathrm{E}-04$ & & & & \\
\hline S\&M Worker Risk & & & & & 5.4E-07 & $7.4 \mathrm{E}-08$ & $1.0 \mathrm{E}-04$ & $1.0 \mathrm{E}-04$ \\
\hline S\&M Worker & Ingestion & Sediment & Cell & $6.7 \mathrm{E} .06$ & & & & \\
\hline S\&M Worker & Dermal Exposure & Sediment & Cell & $3.2 \mathrm{E}-06$ & & & & \\
\hline S\&M Worker & Inhalation & Sediment & Cell & $2.1 \mathrm{E} \cdot 09$ & & & & \\
\hline S\&M Worker & Extemal Exposure & Sediment & Cell & 2.3E-03 & & & & \\
\hline S\&M Worker Risk & & & & & $1.2 \mathrm{E}-09$ & 7.7E-06 & 2.3E-03 & $2.30 \mathrm{E}-03$ \\
\hline
\end{tabular}


5-7

Table 5.2. (continued)

\begin{tabular}{|c|c|c|c|c|c|c|c|c|}
\hline \multirow{2}{*}{ Receptor } & \multirow{2}{*}{$\begin{array}{l}\text { Exposare } \\
\text { Palhway }\end{array}$} & \multirow{2}{*}{ Media } & \multirow{2}{*}{ Loculion } & \multirow{2}{*}{$\begin{array}{l}\text { Total } \\
\text { Pathwray } \\
\text { Risk }\end{array}$} & \multicolumn{3}{|c|}{$\begin{array}{c}\text { Totsl Conteminent } \\
\text { Risk }\end{array}$} & \multirow{2}{*}{$\begin{array}{c}\text { Total } \\
\text { Area } \\
\text { Risk } \\
\text { (Pathwayn } \\
\text { Summed) }\end{array}$} \\
\hline & & & & & Inorganic & Orgenic & Radionuclide & \\
\hline S\&M Worker & External Exposure & $\begin{array}{c}\text { Direct } \\
\text { Measurement }\end{array}$ & Cell & 4.4E-03 & & & 4.4E-03 & 4.4E- 03 \\
\hline S\&M Worker & External Exposure & $\begin{array}{c}\text { Direct } \\
\text { Measurement }\end{array}$ & East Gallery & $6.2 \mathrm{E}-05$ & & & $6.2 \mathrm{E}-05$ & $6.2 \mathrm{E}-05$ \\
\hline S\&M Worker & Extermal Exposure & $\begin{array}{c}\text { Direct } \\
\text { Measurement }\end{array}$ & Whole Building & $6.2 \mathrm{E}-03$ & & & $6.2 \mathrm{E}-03$ & $6.2 \mathrm{E}-03$ \\
\hline S\&M Worker & Ingestion & Smear Samples & Cell & $1.0 \mathrm{E}-08$ & & & & \\
\hline S\&M Worker & Inhalation & Smear Samplea & Cell & $5.1 \mathrm{E}-09$ & & & & \\
\hline S\&M Worker & External Exposure & Smear Samples & Cell & $1.3 \mathrm{E}-05$ & & & & \\
\hline S\&M Worker Risk & & & & & & & $1.3 \mathrm{E}-05$ & $1.3 \mathrm{E}-05$ \\
\hline S\&M Worker & Ingestion & Smear Samples & East Gallery & $1.8 \mathrm{E}-09$ & & . & & \\
\hline S\&M Worker & Inhalation & Smear Samples & East Gallery & $1.4 \mathrm{E}-08$ & & & & \\
\hline S\&M Worker & Extemal Exposure & Smear Samples & East Gallery & $1.5 \mathrm{E}-06$ & & & & \\
\hline S\&M Worker Risk & & & & & & & $1.5 \mathrm{E}-06$ & $1.5 \mathrm{E}-06$ \\
\hline S\&M Worker & Ingeation & Smear Samples & Whole Building & $5.8 \mathrm{E}-09$ & & & & \\
\hline S\&M Worker & Inhalation & Smear Samplea & Whole Building & $5.7 \mathrm{E}-09$ & & & & \\
\hline S\&M Worker & External Exposure & Smear Samples & Whole Building & 7.4E-06 & & & & \\
\hline S\&M Worker Risk & & & & & & & 7.4E-06 & 7.4E-06 \\
\hline S\&M Worker & Extemal Exposure & $\pi D$ & Cell & $8.9 \mathrm{E}-03$ & & & $8.9 E-03$ & $8.9 \mathrm{E}-03$ \\
\hline
\end{tabular}


Similar to the maintenance worker, the risk to the S\&M worker from external exposure was also estimated using several types of data including core sarnples, direct measurements, and TLD strings. The risks were also comparable. The primary contaminant responsible for the risk to the S\&M worker is cesium-137 (see Appendix E, Table E-2).

Ingestion of the PCB Aroclor-1254 from the sediment presented the dominant risk from chemical contamination to the S\&M worker at Building 3506 (4.40E-06) (Appendix E, Table E-2). This value is within EPAs target risk range.

For comparison, the excess cancer risks were calculated for a hypothetical D\&D worker who spends 8 months ( 8 hours per day) in the cell. The excess cancer risk from external exposure to radionuclides was within EPA's target risk range (3.0E-04); however, the excess cancer risk associated with the sediment in the cell did exceed EPA's target risk range (7.0E-03). These risks need to be placed into context because the dose and risk to this receptor would decrease when contaminants are removed from the facility.

\section{East Gallery}

\section{Maintenance Worker}

The greatest excess cancer risks to the maintenance worker in the east gallery are due to external exposure to radionuclides (see Table 5.1). Risks calculated from the core and direct measurements indicate a greater risk, compared to other pathways, due to external exposure to radionuclides (2.5E-04, and $7.8 \mathrm{E}-03$, respectively). The radionuclide contributing the greatest risk via the external exposure pathway (core data) is cesium-137 (see Appendix E, Table E-1).

For the east gallery, approximately $60 \%$ of the contamination is transferrable. The external exposure risk calculated using the cesium-137 smear data is the same as the smear samples proportioned from the core data (1.9E-04 and $1.9 \mathrm{E}-04$, respectively), indicating that the radionuclide of concern for transferrable contamination is cesium-137. These risk values are within EPA's target risk range.

The chemical contaminant responsible for the greatest risk to the maintenance worker is from the PCB Aroclor-1260. Aroclor-1260 was detected in chips of concrete taken from the wall core. Ingestion of this contaminant is the pathway with the highest risk (3.50E-06). Again, this value falls within EPA's target risk range.

\section{S\&M Worker}

As with the maintenance worker, the greatest excess cancer risks to the $S \& M$ worker in the east gallery (core data and direct measurements) are due to external exposure to radionuclides (see Table 5.2). The radionuclide contributing the greatest risk via the external exposure pathway (core data) is that of cesium-137 (see Appendix E, Table E-2).

For the east gallery, approximately $60 \%$ of the contamination is transferrable. The risk due to external exposure for transferrable contamination was $1.5 \mathrm{E}-06$. The primary contaminant 
responsible for this risk is cesium-137. Risks associated with transferrable contamination are within EPA's target risk range.

All chemical contaminants had risk values below EPA's target risk range (see Appendix E, Table E-2).

\section{Whole Building}

\section{Maintenance Worker}

Risks associated with the whole building were calculated to determine what the risk would be to a maintenance worker that will be in both the cell and the east gallery $(1.3 \mathrm{E}-02$, see Table 5.1). Note that the risks from sediment are not included in these results.

Risk to the worker via external exposure from transferrable contamination was from cesium-137 (8.4E-04 for cesium-137 smear data compared to 9.2E-04 for the smear sample data proportioned from the core data).

\section{S\&M Worker}

Risks associated with an S\&M worker who will perform work in the cell and the east gallery were calculated (1.0E-04, see Table 5.2). As with the maintenance worker scenario, the risks associated with the sediment are not included with these results. If the risk due to external exposure to radionuclides present in the sediment from the cell is included, the risk would be $1.9 \mathrm{E}-03$.

For comparison, the excess cancer risks were calculated for a hypothetical D\&D worker who spends 8 months ( 8 hours per day) in the whole building. The excess cancer risk from external exposure to radionuclides was within EPA's target risk range (3.0E-04).

\subsubsection{Noncarcinogenic Effects}

Cell

\section{Maintenance Worker}

While no one HQ exceeded unity for the contaminants in the cell, the HI for the dermal exposure pathway exceeded unity for the contaminants detected in the floor and walls of the cell (see Table 5.3). The HQs for cadmium, mercury, and vanadium (via dermal absorption) had the highest values of the contaminants detected in the floor and walls of the cell. The HQ for the maintenance worker exceeded unity from dermal absorption of mercury from the sediment. 
Table 5.3. Summary risk characterization results-noncancer hazard index estimates-maintenance worker

\begin{tabular}{|c|c|c|c|c|c|c|c|}
\hline \multirow{3}{*}{ Receptor } & \multirow{3}{*}{$\begin{array}{l}\text { Exposure } \\
\text { Pathway }\end{array}$} & \multirow{3}{*}{ Media } & \multirow{3}{*}{ Location } & \multicolumn{4}{|c|}{ Hazard Index } \\
\hline & & & & \multicolumn{2}{|c|}{$\begin{array}{c}\text { Contaminant } \\
\text { Class }\end{array}$} & \multirow{2}{*}{ Pathway } & \multirow{2}{*}{$\begin{array}{c}\text { Total } \\
\text { Area (Pathway } \\
\text { Summed) }\end{array}$} \\
\hline & & & & Inorganic & Organic & & \\
\hline Maintenance Worker & Ingestion & Core Samples & Cell & & & 2.3E-01 & \\
\hline Maintenance Worker & $\begin{array}{c}\text { Dermal } \\
\text { Exposure }\end{array}$ & Core Samples & Cell & & & $1.1 E+\infty$ & \\
\hline Maintenance Worker & Inhalation & Core Samples & Cell & & & 3.1E-02 & \\
\hline Total Inorganic Hazard Index & & & & $1.4 \mathrm{E}+00$ & & & \\
\hline Total Area & & & & & & & $1.4 \mathrm{E}+00$ \\
\hline Maintenance Worker & Ingestion & Core Samples & East Gallery & & & $1.2 \mathrm{E}-02$ & \\
\hline Maintenance Worker & $\begin{array}{c}\text { Dermal } \\
\text { Exposure }\end{array}$ & Core Samples & East Gallery & & & 2.5E-02 & \\
\hline Maintenance Worker & Inhalation & Core Samples & East Gallery & & & 8.5E-04 & \\
\hline Total Inorganic Hazand Index & & & & $3.8 \mathrm{E}-02$ & & & \\
\hline Total Organic Hazard Index & & & & & $2.5 \mathrm{E}-05$ & & \\
\hline Total Area & & & & & & & $3.8 \mathrm{E}-02$ \\
\hline Maintenance Worker & Ingestion & Core Samples & $\begin{array}{l}\text { Whole } \\
\text { Building }\end{array}$ & & & 2.3E-01 & \\
\hline Maintenance Worker & $\begin{array}{c}\text { Dermal } \\
\text { Exposure }\end{array}$ & Core Samples & $\begin{array}{l}\text { Whole } \\
\text { Building }\end{array}$ & & & $1.1 \mathrm{E}+\infty$ & \\
\hline Maintenance Worker & Inhalation & Core Samples & $\begin{array}{l}\text { Whole } \\
\text { Building }\end{array}$ & & & 3.1E-02 & \\
\hline Total Inorganic Hazard Index & & & & $1.4 \mathrm{E}+00$ & & & \\
\hline
\end{tabular}


5-11

Table 5.3. (continued)

\begin{tabular}{|c|c|c|c|c|c|c|c|}
\hline \multirow{3}{*}{ Receptor } & \multirow{3}{*}{$\begin{array}{l}\text { Exposure } \\
\text { Pathway }\end{array}$} & \multirow{3}{*}{ Media } & \multirow{3}{*}{ Location } & \multicolumn{4}{|c|}{ Hazard Index } \\
\hline & & & & \multicolumn{2}{|c|}{$\begin{array}{l}\text { Contaminant } \\
\text { Class }\end{array}$} & \multirow{2}{*}{ Pathway } & \multirow{2}{*}{$\begin{array}{c}\text { Total } \\
\text { Area (Pathways } \\
\text { Summed) }\end{array}$} \\
\hline & & & & Inorganic & Organic & & \\
\hline Total Organic Hazard Index & & & & & 2.5E-05 & & \\
\hline Total Area & & & & & & & $1.4 \mathrm{E}+00$ \\
\hline Maintenance Worker & Ingestion & Sediment & Cell & & & $1.8 \mathrm{E}-01$ & \\
\hline Maintenance Worker & $\begin{array}{l}\text { Dermal } \\
\text { Exposure }\end{array}$ & Sediment & Cell & & & $1.9 \mathrm{E}+01$ & \\
\hline Maintenance Worker & Inhalation & Sediment & Cell & & & 4.2E-03 & \\
\hline Total Inorganic Hazard Index & & & & $2.0 \mathrm{E}+01$ & & & \\
\hline Total Organic Hazard Index & & & & & $2.3 \mathrm{E}-03$ & & \\
\hline Total Area & & & & & & & $2.0 \mathrm{E}+01$ \\
\hline
\end{tabular}




\section{S\&M Worker}

All HQs for exposure to contaminants in the cell were $<1$ (see Table 5.4).

\section{East Gallery}

Maintenance Worker

All HQs for exposure to contaminants in the east gallery were $<1$ (See Table 5.3).

\section{$\underline{\text { S\&M Worker }}$}

All hazard quotients for exposure to contaminants in the east gallery were $<1$ (see Table 5.4).

\section{Whole Building}

Maintenance Worker

The HQ for mercury exceeded unity for the dermal exposure pathway for the whole building. All other HQs for exposure to other contaminants were $<1$ (see Table 5.3).

\section{S\&M Worker}

All HQs for exposure to contaminants in the whole building were $<1$ (see Table 5.4).

\subsection{FUTURE LAND USE}

The following sections will present and discuss the results of the risk characterization for the future land use conditions or the hypothetical resident scenario. This discussion is ordered in the same manner as the discussion of current receptors.

\subsubsection{Excess Cancer Risks}

\section{Cell}

The estimated excess cancer risks for the hypothetical adult trespasser for the cell are summarized in Table 5.5. A complete table of results can be found in Appendix E. External exposure to radiation (core samples) from the structure (i.e., walls and floor) presented the dominant risk to the adult trespasser at Building 3506 (6.2E-02). The risks from the other pathways were two to three orders of magnitude less. Risk from external exposure to 
5-13

Table 5.4. Summary risk characterization results-noncancer hazard index estimates-S\&M worker

\begin{tabular}{|c|c|c|c|c|c|c|c|}
\hline \multirow{3}{*}{ Receptor } & \multirow{3}{*}{$\begin{array}{l}\text { Exposure } \\
\text { Pathway }\end{array}$} & \multirow{3}{*}{ Media } & \multirow{3}{*}{ Location } & \multicolumn{4}{|c|}{ Hazard Index } \\
\hline & & & & \multicolumn{2}{|c|}{$\begin{array}{l}\text { Contaminant } \\
\text { Class }\end{array}$} & \multirow{2}{*}{ Pathway } & \multirow{2}{*}{$\begin{array}{c}\text { Total } \\
\text { Area (Pathways } \\
\text { Summed) }\end{array}$} \\
\hline & & & & Inorganic & Organic & & \\
\hline S\&M Worker & Ingestion & Core Samples & Cell & & & $1.8 \mathrm{E}-03$ & \\
\hline S\&M Worker & $\begin{array}{c}\text { Dermal } \\
\text { Exposure }\end{array}$ & Core Samples & Cell & & & $9.2 \mathrm{E}-03$ & \\
\hline S\&M Worker & Inhalation & Core Samples & Cell & & & $2.5 \mathrm{E}-04$ & \\
\hline Total Inorganic Hazard Index & & & & $1.1 \mathrm{E}-02$ & & & \\
\hline Total Area & & & & & & & $1.1 \mathrm{E}-02$ \\
\hline S\&M Worker & Ingestion & Core Samples & East Gallery & & & $9.4 \mathrm{E}-05$ & \\
\hline S\&M Worker & $\begin{array}{c}\text { Dermal } \\
\text { Exposure }\end{array}$ & Core Samples & East Gallery & & & $2.0 \mathrm{E}-04$ & \\
\hline S\&M Worker & Inhalation & Core Samples & East Gallery & & & $6.8 \mathrm{E}-06$ & \\
\hline Total Inorganic Hazard Index & & & & 3.0E-04 & & & \\
\hline Total Organic Hazard Index & & & & & $2.0 \mathrm{E}-07$ & & \\
\hline Total Area & & & & & & & $3.0 \mathrm{E}-04$ \\
\hline S\&M Worker & Ingestion & Core Samples & $\begin{array}{l}\text { Whole } \\
\text { Building }\end{array}$ & & & $1.8 \mathrm{E}-03$ & \\
\hline S\&M Worker & $\begin{array}{c}\text { Dermal } \\
\text { Exposure }\end{array}$ & Core Samples & $\begin{array}{l}\text { Whole } \\
\text { Building }\end{array}$ & & & $9.0 \mathrm{E}-03$ & \\
\hline S\&M Worker & Inhatảtion & Core Samples & $\begin{array}{l}\text { Whole } \\
\text { Building }\end{array}$ & & & 2.5E-04 & \\
\hline Total Inorganic Hazard Index & & & & $1.1 \mathrm{E}-02$ & & & \\
\hline
\end{tabular}


5-14

Table 5.4. (continued)

\begin{tabular}{|c|c|c|c|c|c|c|c|}
\hline \multirow{3}{*}{ Receptor } & \multirow{3}{*}{$\begin{array}{l}\text { Exposure } \\
\text { Pathway }\end{array}$} & \multirow{3}{*}{ Media } & \multirow{3}{*}{ Location } & \multicolumn{4}{|c|}{ Hazard Index } \\
\hline & & & & \multicolumn{2}{|c|}{$\begin{array}{l}\text { Contaminant } \\
\text { Class }\end{array}$} & \multirow{2}{*}{ Pathway } & \multirow{2}{*}{$\begin{array}{c}\text { Total } \\
\text { Area (Pathway } \\
\text { Summed) }\end{array}$} \\
\hline & & & & Inorganic & Organic & & \\
\hline Total Organic Hazard Index & & & & & $2.0 \mathrm{E}-07$ & & \\
\hline Total Area & & & & & & & $1.1 \mathrm{E}-02$ \\
\hline S\&M Worker & Ingestion & Sediment & Cell & & & $1.4 \mathrm{E}-03$ & \\
\hline S\&M Worker & $\begin{array}{c}\text { Dermal } \\
\text { Exposure }\end{array}$ & Sediment & Cell & & & $1.6 \mathrm{E}-01$ & \\
\hline S\&M Worker & Inhalation & Sediment & Cell & & & $3.4 \mathrm{E}-05$ & \\
\hline Total Inorganic Hazard Index & & & & $1.6 \mathrm{E}-01$ & & & \\
\hline Total Organic Hazard Index & & & & & $1.9 \mathrm{E}-05$ & & \\
\hline Total Area & & & & & & & $1.6 \mathrm{E}-01$ \\
\hline
\end{tabular}


5-15

Table 5.5. Summary risk characterization results-cancer risk estimates-adult trespasser

\begin{tabular}{|c|c|c|c|c|c|c|c|c|}
\hline \multirow[b]{2}{*}{ Receptor } & \multirow[b]{2}{*}{$\begin{array}{l}\text { Exposure } \\
\text { Pathway }\end{array}$} & \multirow[b]{2}{*}{ Media } & \multirow[b]{2}{*}{ Location } & \multirow{2}{*}{$\begin{array}{c}\text { Total } \\
\text { Pathway } \\
\text { Risk }\end{array}$} & \multicolumn{3}{|c|}{$\begin{array}{c}\text { Total Contaminant } \\
\text { Risk }\end{array}$} & \multirow{2}{*}{$\begin{array}{c}\text { Total } \\
\text { Area } \\
\text { Risk } \\
\text { (Pathways } \\
\text { Summed) }\end{array}$} \\
\hline & & & & & $\begin{array}{l}\text { Inorganic } \\
\text { Chemical }\end{array}$ & $\begin{array}{c}\text { Organic } \\
\text { Chemical } \\
\text { Radionuclide }\end{array}$ & & \\
\hline Adult Trespasser & Ingestion & Core Samples & Cell & $9.0 \mathrm{E}-05$ & & & & \\
\hline Adult Trespasser & Dermal Exposure & Core Samples & Cell & $2.4 \mathrm{E}-04$ & & & & \\
\hline Adult Trespasser & Inhalation & Core Samples & Cell & $8.1 E-05$ & & & & \\
\hline Adult Trespasser & $\begin{array}{c}\text { Extermal } \\
\text { Exposure }\end{array}$ & Core Samples & Cell & $6.2 \mathrm{E}-02$ & & & & \\
\hline Adult Trespasser Risk & & & & & 2.8E-04 & 6.5E-06 & $6.2 \mathrm{E}-02$ & $6.2 \mathrm{E}-02$ \\
\hline Aduit Trespasser & Ingestion & Core Samples & $\begin{array}{c}\text { East } \\
\text { Gallery }\end{array}$ & $2.1 \mathrm{E}-05$ & & & & \\
\hline Adult Trespasser & Dermal Exposure & Core Samples & $\begin{array}{c}\text { East } \\
\text { Gallery }\end{array}$ & $1.6 \mathrm{E}-05$ & & & & \\
\hline Adult Trespasser & Inhalation & Core Samples & $\begin{array}{c}\text { East } \\
\text { Gallery }\end{array}$ & $7.3 \mathrm{E}-05$ & & & & \\
\hline Adult Trespasser & $\begin{array}{c}\text { External } \\
\text { Exposure } \\
\end{array}$ & Core Samples & $\begin{array}{c}\text { East } \\
\text { Gallery }\end{array}$ & $1.3 E-03$ & & & & \\
\hline Adult Trespasser Risk & & & & & $6.4 \mathrm{E}-06$ & 3.1E-05 & 1.3E-03 & $1.4 \mathrm{E}-03$ \\
\hline Adult Trespasser & Ingestion & Core Samples & $\begin{array}{c}\text { Whole } \\
\text { Building }\end{array}$ & $9.1 \mathrm{E}-05$ & & & & \\
\hline Adult Trespasser & Dermal Exposure & Core Samples & $\begin{array}{c}\text { Whole } \\
\text { Building }\end{array}$ & $1.8 \mathrm{E}-04$ & & & & \\
\hline Adult Trespasser & Inhalation & Core Samples & $\begin{array}{c}\text { Whole } \\
\text { Building }\end{array}$ & $8.1 \mathrm{E}-05$ & & & & \\
\hline Adult Trespasser & $\begin{array}{l}\text { External } \\
\text { Exposure }\end{array}$ & Core Samples & $\begin{array}{c}\text { Whole } \\
\text { Building }\end{array}$ & $6.2 \mathrm{E}-02$ & & & & \\
\hline
\end{tabular}


5-16

Table 5.5. (continued)

\begin{tabular}{|c|c|c|c|c|c|c|c|c|}
\hline \multirow[b]{2}{*}{ Receptor } & \multirow{2}{*}{$\begin{array}{l}\text { Exposure } \\
\text { Pathway }\end{array}$} & \multirow[b]{2}{*}{ Media } & \multirow{2}{*}{ Location } & \multirow{2}{*}{$\begin{array}{c}\text { Total } \\
\text { Pathway } \\
\text { Risk }\end{array}$} & \multicolumn{3}{|c|}{$\begin{array}{l}\text { Total Contaminant } \\
\text { Risk }\end{array}$} & \multirow{2}{*}{$\begin{array}{c}\text { Total } \\
\text { Area } \\
\text { Risk } \\
\text { (Pathways } \\
\text { Summed) }\end{array}$} \\
\hline & & & & & $\begin{array}{l}\text { Inorganic } \\
\text { Chemical }\end{array}$ & $\begin{array}{c}\text { Chemical } \\
\text { Radionuclide }\end{array}$ & & \\
\hline Adult Trespasser & $\begin{array}{l}\text { External } \\
\text { Exposure }\end{array}$ & Core Samples & $\begin{array}{l}\text { Whole } \\
\text { Building }\end{array}$ & $6.2 \mathrm{E}-02$ & & & & \\
\hline Adult Trespasser Risk & & & & & $2.0 \mathrm{E}-04$ & 2.9E-05 & $\begin{array}{c}6.2 \mathrm{E}- \\
026.2 \mathrm{E}-02\end{array}$ & \\
\hline Adult Trespasser & Ingestion & Sediment & Cell & $2.8 \mathrm{E}-03$ & & & & \\
\hline Adult Trespasser & Dermal Exposure & Sediment & Cell & $1.1 \mathrm{E}-03$ & & & & \\
\hline Adult Trespasser & Inhalation & Sediment & Cell & 4.3E-07 & & & & \\
\hline Adult Trespasser & $\begin{array}{l}\text { External } \\
\text { Exposure }\end{array}$ & Sediment & Cell & $7.6 \mathrm{E}-01$ & & & & \\
\hline Adult Trespasser Risk & & & & & 2.5E-07 & $3.0 \mathrm{E}-03$ & $7.6 \mathrm{E}-01$ & $7.6 \mathrm{E}-01$ \\
\hline Áduit Trespasser & $\begin{array}{r}\text { Externai } \\
\text { Exposure }\end{array}$ & $\begin{array}{c}\text { Direci } \\
\text { Measurement }\end{array}$ & Ceil & $9.4 E-01$ & & & $9.4 \mathrm{E}-0 \mathrm{I}$ & S.AE-01 \\
\hline Adult Trespasser & $\begin{array}{l}\text { External } \\
\text { Exposure }\end{array}$ & $\begin{array}{c}\text { Direct } \\
\text { Measurement }\end{array}$ & $\begin{array}{c}\text { East } \\
\text { gallery }\end{array}$ & $3.8 \mathrm{E}-02$ & & & $3.8 \mathrm{E}-02$ & $3.8 \mathrm{E}-02$ \\
\hline Adult Trespasser & $\begin{array}{l}\text { External } \\
\text { Exposure }\end{array}$ & $\begin{array}{c}\text { Direct } \\
\text { Measurement }\end{array}$ & $\begin{array}{l}\text { Whole } \\
\text { Building }\end{array}$ & $9.8 \mathrm{E}-01$ & & & $9.8 \mathrm{E}-01$ & $9.8 \mathrm{E}-01$ \\
\hline Adult Trespasser & Ingestion & $\begin{array}{c}\text { Smear } \\
\text { Samples }\end{array}$ & Cell & 4.3E-06 & & & & \\
\hline Adult Trespasser & Inhalation & $\begin{array}{c}\text { Smear } \\
\text { Samples }\end{array}$ & Cell & $1.1 \mathrm{E}-06$ & & & & \\
\hline Adult Trespasser & $\begin{array}{r}\text { External } \\
\text { Exposure }\end{array}$ & $\begin{array}{l}\text { Smear } \\
\text { Samples }\end{array}$ & Cell & 8.2E-03 & & & $8.2 \mathrm{E}-03$ & 8.2E-03 \\
\hline & & & & & & & & \\
\hline
\end{tabular}


5-17

Table 5.5. (continued)

\begin{tabular}{|c|c|c|c|c|c|c|c|c|}
\hline \multirow[b]{2}{*}{ Receptor } & \multirow[b]{2}{*}{$\begin{array}{l}\text { Exposure } \\
\text { Pathway }\end{array}$} & \multirow[b]{2}{*}{ Media } & \multirow[b]{2}{*}{ Location } & \multirow[b]{2}{*}{$\begin{array}{c}\text { Total } \\
\text { Pathway } \\
\text { Risk }\end{array}$} & \multicolumn{3}{|c|}{$\begin{array}{l}\text { Total Contaminant } \\
\text { Risk }\end{array}$} & \multirow{2}{*}{$\begin{array}{l}\text { Total } \\
\text { Area } \\
\text { Risk } \\
\text { (Pathways } \\
\text { Summed) }\end{array}$} \\
\hline & & & & & $\begin{array}{l}\text { Inorganic } \\
\text { Chemical }\end{array}$ & $\begin{array}{c}\text { Organic } \\
\text { Chemical } \\
\text { Radionuclide }\end{array}$ & & \\
\hline Adult Trespasser & Ingestion & $\begin{array}{c}\text { Smear } \\
\text { Samples } \\
\end{array}$ & $\begin{array}{c}\text { East } \\
\text { Gallery }\end{array}$ & $7.4 \mathrm{E}-07$ & & & & \\
\hline Adult Trespasser & Inhalation & $\begin{array}{c}\text { Smear } \\
\text { Samples }\end{array}$ & $\begin{array}{c}\text { East } \\
\text { Gallery }\end{array}$ & $3.0 \mathrm{E}-06$ & & & & \\
\hline Adult Trespasser & $\begin{array}{l}\text { External } \\
\text { Exposure }\end{array}$ & $\begin{array}{l}\text { Smear } \\
\text { Samples. }\end{array}$ & $\begin{array}{c}\text { East } \\
\text { Gallery }\end{array}$ & $9.6 \mathrm{E}-04$ & & & & \\
\hline Total Radionuclide Risk & & & & & & & 9.7E-04 & 9.7E-04 \\
\hline Adult Trespasser & Ingestion & $\begin{array}{c}\text { Smear } \\
\text { Samples } \\
\end{array}$ & $\begin{array}{l}\text { Whole } \\
\text { Building }\end{array}$ & $2.4 \mathrm{E}-06$ & & & & \\
\hline Adult Trespasser & Inhalation & $\begin{array}{l}\text { Smear } \\
\text { Samples }\end{array}$ & $\begin{array}{l}\text { Whole } \\
\text { Building }\end{array}$ & $1.2 \mathrm{E}-06$ & & & & \\
\hline Adult Trespasser & $\begin{array}{l}\text { External } \\
\text { Exposure }\end{array}$ & $\begin{array}{l}\text { Smear } \\
\text { Samples }\end{array}$ & $\begin{array}{l}\text { Whole } \\
\text { Building }\end{array}$ & $4.6 \mathrm{E}-03$ & & & $4.6 \mathrm{E}-03$ & $4.6 \mathrm{E}-03$ \\
\hline Adult Trespasser & $\begin{array}{l}\text { External } \\
\text { Exposure }\end{array}$ & TLD & Cell & $1.0 \mathrm{E}+00$ & & & $1.0 \mathrm{E}+00$ & $1.0 \mathrm{E}+00$ \\
\hline
\end{tabular}




\section{$5-18$}

radionuclides was also the dominant risk for the sediment present in the cell (7.6E-01). Risk from external exposure estimated from the other data types (direct measurements and TLD strings) were comparable to the risk calculated form the core samples. These risk values exceed EPA's target risk range, as well as the risk for the ORNL administrative control limit (6.0E-03) and the risk for the radiation protection standard (2E-02). As was the case with the current receptors, the contaminant responsible for the risk to the adult trespasser is cesium-137 (see Appendix E, Table E-5).

For the cell, most of the contamination is fixed (approximately 90\%). A risk value of 2.7E-03 was calculated for external exposure using the cesium-137 smear data. This is equivalent to the risk calculated for the smear samples, proportioned from core data $(8.3 \mathrm{E}-03)$, indicating that cesium-137 is the major radionuclide of concern.

Ingestion of the PCB Aroclor-1254 from the sediment presented the dominant risk from chemical contamination to the trespasser at Building 3506 (1.9E-03; see Appendix E, Table E-5). This risk value exceeds EPA's target risk range.

\section{East Gallery}

The greatest excess cancer risks to the adult trespasser in the east gallery are due to external exposure to radionuclides (see Table 5.5). Risks calculated from the core and direct measurements indicate a greater risk, compared to other pathways, from external exposure to radionuclides (1.3E-03, and $3.8 \mathrm{E}-02$, respectively). For the east gallery, approximately $60 \%$ of the contamination is transferrable. These risk values are above EPA's target risk range.

The radionuclide contributing the greatest risk via the external exposure pathway is cesium-137 (see Appendix E). The external exposure risk calculated using the cesium-137 smear data is again comparable to the smear samples (risks of 7.1E-04 and 9.6E-04, respectively), indicating that the radionuclide of concern for transferrable contamination is cesium-137.

Ingestion of the PCB Aroclor-1260 presented the dominant risk from chemical contamination to the adult trespasser at Building 3506 (1.2E-05).

\section{Whole Building}

Risks associated with the whole building (6.2E-02, Table 5.5) were calculated to determine what the risk would be to the adult trespasser who might be in both the cell and the east gallery. Note that the risks from sediment are not included in these results.

Risk to the adult trespasser via external exposure from transferrable contamination was from cesium-137 (3.4E-03 for cesium-137 smear data compared to 4.6E-03 for the smear sample data).

Ingestion of the PCB Aroclor-1260 presented the dominant risk from chemical contamination to the adult trespasser (1.2E-05). 


\subsubsection{Noncarcinogenic Effects}

\section{Cell}

The HQ (dermal pathway) for cadmium exceeded unity (see Table 5.6). Other contaminants (mercury and vanadium) had HQs, although not exceeding unity, which contributed to the HI. The HQ for this receptor also exceeded unity from the dermal pathway from exposure to mercury from the sediment.

\section{East Gallery}

All HQs for exposure to contaminants in the east gallery were $<1$.

\section{Whole Building}

The HQ (dermal pathway) for cadmium exceeded unity (see Table 5.6). Other contaminants (mercury and vanadium) had HQs, although not exceeding unity, which contributed to the HI.

\subsection{SUMMARY OF RISK CHARACTERIZATION}

Excess cancer risks due to external exposure to radionuclides (specifically cesium-137) exceeded the range of concern set by EPA $\left(10^{-6}\right.$ to $\left.10^{-4}\right)$ for the hypothetical maintenance worker and hypothetical adult trespasser. However, it should be noted that current radiation protection standards for workers include a limit of $5 \mathrm{rem}$ for the annual effective dose equivalent. Moreover, ORNL has imposed an annual limit of $1.5 \mathrm{rem}$. A $5 \mathrm{rem}$ annual dose equates to a 25-year lifetime risk corresponding to $2 \times 10^{-2}$.

Excess cancer risks calculated (from sediment in cell) for the adult trespasser for other pathways, specifically dermal and ingestion, also exceeded EPA's range of concern. However, it should be noted that the risks associated from these pathways assume that the entire contaminant concentration is available for exposure; therefore, these risks are only realistic if the walls and floors are disturbed (as might be the case during D\&D activities) or totally disintegrate. Even with the assumption and associated uncertainty that the fixed contamination is immediately available for exposure to the receptors, the risks associated with these pathways are not the main contributors to the risk in this risk assessment.

Noncarcinogenic hazards to the maintenance worker and the adult trespasser are primarily associated with the mercury contained in the sediment. These hazards are likely because the sediment is available for exposure to the receptors. There are, however, hazards associated with the contaminants in the cell walls and floor, but these hazards are less likely to occur. Release

of the contaminants from the walls and floors must occur to expose the receptors to the contaminants. 
Table 5.6. Summary risk characterization results-noncancer hazard index estimates-adult trespasser

\begin{tabular}{|c|c|c|c|c|c|c|c|}
\hline \multirow{3}{*}{ Receptor } & \multirow{3}{*}{$\begin{array}{l}\text { Exposure } \\
\text { Pathway }\end{array}$} & \multirow{3}{*}{ Media } & \multirow{3}{*}{ Location } & \multicolumn{4}{|c|}{ Hazard Index } \\
\hline & & & & \multicolumn{2}{|c|}{$\begin{array}{l}\text { Contaminant } \\
\text { Class }\end{array}$} & \multirow{2}{*}{ Pathway } & \multirow{2}{*}{$\begin{array}{l}\text { Total } \\
\text { Area (pathways } \\
\text { Summed) }\end{array}$} \\
\hline & & & & Inorganic & Organic & & \\
\hline Adult Trespasser & Ingestion & Core Samples & Cell & & & $6.4 \mathrm{E}-01$ & \\
\hline Adult Trespasser & $\begin{array}{l}\text { Dermal } \\
\text { Exposure }\end{array}$ & Core Samples & Cell & & & $2.7 \mathrm{E}+\infty$ & \\
\hline Adult Trespasser & Inhalation & Core Samples & Cell & & & 4.3E-02 & \\
\hline Total Inorganic Hazard Index & & & & $3.4 \mathrm{E}+00$ & & & \\
\hline Total Area & & & & & & & $3.4 \mathrm{E}+\infty 0$ \\
\hline Adult Trespasser & Ingestion & Core Samples & East Gallery & & & 3.3E-02 & \\
\hline Adult Trespasser & $\begin{array}{l}\text { Dermal } \\
\text { Exposure }\end{array}$ & Core Samples & East Gallery & & & $6.0 \mathrm{E}-02$ & \\
\hline Adult Trespasser & Inhalation & Core Samples & East Gallery & & & $1.2 \mathrm{E}-03$ & \\
\hline Total Inorganic Hazard Index & & & & $9.4 \mathrm{E}-02$ & & & \\
\hline Total Organic Hazard Index & & & & & $6.2 \mathrm{E}-05$ & & \\
\hline Total Area & & & & & & & $9.4 \mathrm{E}-02$ \\
\hline Adult Trespasser & Ingestion & Core Samples & $\begin{array}{l}\text { Whole } \\
\text { Building }\end{array}$ & & & $6.4 \mathrm{E}-01$ & \\
\hline Adult Trespasser & $\begin{array}{l}\text { Dermal } \\
\text { Exposure }\end{array}$ & Core Samples & $\begin{array}{l}\text { Whole } \\
\text { Building }\end{array}$ & & & $2.7 E+\infty$ & \\
\hline Adult Trespasser & Inhalation & Core Samples & $\begin{array}{l}\text { Whole } \\
\text { Building }\end{array}$ & & & 4.3E-02 & \\
\hline Total Inorganic Hazard Index & & & & $3.3 E+00$ & & & \\
\hline
\end{tabular}


5-21

Table 5.6. (continued)

\begin{tabular}{|c|c|c|c|c|c|c|c|}
\hline \multirow{3}{*}{ Receplor } & \multirow{3}{*}{$\begin{array}{l}\text { Exposure } \\
\text { Pathway }\end{array}$} & \multirow{3}{*}{ Media } & \multirow{3}{*}{ Location } & \multicolumn{4}{|c|}{ Hazard Index } \\
\hline & & & & \multicolumn{2}{|c|}{$\begin{array}{l}\text { Contaminant } \\
\text { Class }\end{array}$} & \multirow{2}{*}{ Pathway } & \multirow{2}{*}{$\begin{array}{c}\text { Total } \\
\text { Area (pathways } \\
\text { Summed) }\end{array}$} \\
\hline & & & & Inorganic & Organic & & \\
\hline Total Organic Hazand Index & & & & & $6.2 \mathrm{E}-05$ & & \\
\hline Total Area & & & & & & & $3.3 E+\infty 0$ \\
\hline Adult Trespasser & Ingestion & Sediment & Cell & & & $5.0 \mathrm{E}-01$ & \\
\hline Adult Trespasser & $\begin{array}{l}\text { Dermal } \\
\text { Exposure }\end{array}$ & Sediment & Cell & & & 4.6E+01 & \\
\hline Adult Trespasser & Inhalation & Sediment & Cell & & & $5.9 \mathrm{E}-03$ & \\
\hline Total Inorganic Hazard Index & & & & 4.7E+01 & & & \\
\hline Total Organic Hazard Index & & & & & $5.9 \mathrm{E}-03$ & & \\
\hline Total Area & & & & & & & $4.7 \mathrm{E}+01$ \\
\hline
\end{tabular}


Adequate data were unavailable to quantify the risks associated with several contaminants (see Table 2.2). Risks from asbestos are expected to be negligible because asbestos exists in only the putty surrounding the windows and is considered nonfriable. Risks associated with lead from paint could be a potential hazard if chips of paint are ingested or if dust created from the paint is inhaled.

A risk evaluation is an integrated evaluation of historical, contaminant, analytical, environmental, demographic, and toxicological data that are as site-specific as possible. All of the uncertainties associated with each step of the risk evaluation process impact the risk characterization. To minimize the effects of uncertainties in the evaluation, each step is biased toward health-protective estimations. Because each step builds on the previous one, this biased approach should more than compensate for risk evaluation uncertainties. These calculations do not represent currently existing or expected future exposure or health risks. Rather, they are estimates of potential risks only if all of the conservative assumptions are realized.

\section{Risk Perspective}

To place the results presented in this risk assessment in perspective, one should consider the probability of an individual's developing cancer from unavoidable exposure to naturally occurring background radiation. The lifetime risk of cancer incidence from background radiation in the general population is approximately 1E-02 (Martin Marietta Energy Systems, 1993). This value is 100 times greater than the upperbound $\left(10^{-4}\right)$ of EPA's target risk range. In addition, Table 5.7 lists activities that increase the chance of cancer fatality by $1 E-06$. The reader should note that these values are for fatal cancers, not the incidence of cancer.

Table 5.7. Risks that increase the chance of death by one part in one million (1E-06)

\begin{tabular}{|l|l|}
\hline \multicolumn{1}{|c|}{ Activity } & \multicolumn{1}{c|}{ Cause of death } \\
\hline Smoking 1.4 cigarettes & Cancer, heart disease \\
\hline Flying 6000 miles by jet & Cancer caused by cosmic radiation \\
\hline $\begin{array}{l}\text { Living 2 months in average stone or } \\
\text { brick building }\end{array}$ & Cancer from natural radioactivity \\
\hline Eating 40 tablespoons of peanut butter & Liver cancer caused by aflatoxin B \\
\hline
\end{tabular}

Taken from Wilson, 1979

\subsection{EVALUATION OF UNCERTAINTY}

\subsubsection{Uncertainties and Assumptions for Chemical Risk Evaluation}

Risk evaluation as a scientific activity is subject to uncertainty with regard to both risk evaluations in general (Table 5.8) and site-specific risk evaluations (Table 5.9).

The toxicological parameters used to quantify potential risk to a receptor include SFs for carcinogens and RfDs for noncarcinogenic chemicals. These values are generally derived from 
Table 5.8. Site-specific uncertainty factors

\begin{tabular}{lll}
\hline Uncertainty Factor & \multicolumn{1}{c}{ Effect of Uncertainty } & \multicolumn{1}{c}{ Comment } \\
\hline $\begin{array}{l}\text { Metals analysis for } \\
\text { total metals only. }\end{array}$ & May overestimate risks. & $\begin{array}{l}\text { Did not distinguish between valences or speciation. } \\
\text { Assumed the metal was present in its most toxic form. }\end{array}$ \\
$\begin{array}{l}\text { Not all chemicals at } \\
\text { the site have toxicity } \\
\text { values. }\end{array}$ & May underestimate risks. & These chemicals are not addressed quantitatively. \\
$\begin{array}{l}\text { Exposure } \\
\text { assumptions. }\end{array}$ & $\begin{array}{l}\text { May over- or underestimate } \\
\text { risks. }\end{array}$ & $\begin{array}{l}\text { Assumptions regarding media intake, population } \\
\text { characteristics, and exposure patterns may not } \\
\text { characterize exposures. }\end{array}$ \\
$\begin{array}{l}\text { Exposures assumed } \\
\text { constant. }\end{array}$ & $\begin{array}{l}\text { May over- or underestimate } \\
\text { risks. }\end{array}$ & $\begin{array}{l}\text { Does not account for fate, transport, or transfer that } \\
\text { may alter concentration. }\end{array}$ \\
\hline
\end{tabular}


Table 5.8. Site-specific uncertainty factors

\begin{tabular}{lll}
\hline \multicolumn{1}{c}{ Uncertainty Factor } & \multicolumn{1}{c}{ Effect of Uncertainty } & \multicolumn{1}{c}{ Comment } \\
\hline $\begin{array}{l}\text { Assumed an average } \\
\text { distribution of contamination } \\
\text { based on data two data points } \\
\text { for the floors and walls from } \\
\text { core samples. }\end{array}$ & $\begin{array}{l}\text { May over- or } \\
\text { underestimate risks. }\end{array}$ & $\begin{array}{l}\text { Sections of the floors and walls not sampled may } \\
\text { have either higher or lower levels of } \\
\text { contamination. }\end{array}$ \\
$\begin{array}{l}\text { Assumed distribution of } \\
\text { radionuclides in smear } \\
\text { samples was same as core } \\
\text { data from floors and walls }\end{array}$ & $\begin{array}{l}\text { May over- or } \\
\text { underestimate risks. }\end{array}$ & $\begin{array}{l}\text { Transferrable contamination may not contain the } \\
\text { same radionuclides as the floors and walls }\end{array}$ \\
$\begin{array}{l}\text { Exposure assumptions. } \\
\text { May over- or } \\
\text { underestimate risks. }\end{array}$ & $\begin{array}{l}\text { Assumptions regarding media intake, population } \\
\text { characteristics, and exposure patterns may not } \\
\text { characterize exposures. }\end{array}$ \\
Exposure scenarios & May overestimate risks. & $\begin{array}{l}\text { Assumed that contaminants were available to } \\
\text { receptor from wa!ls and floors }\end{array}$ \\
Exposures assumed constant. & $\begin{array}{l}\text { May over- or } \\
\text { underestimate risks. }\end{array}$ & $\begin{array}{l}\text { Does not account for fate, transport, or transfer } \\
\text { that may alter concentration. Did not account for } \\
\text { decay of radionuclides }\end{array}$ \\
\hline
\end{tabular}


laboratory animal studies. The overriding uncertainties associated with the use of laboratory animal studies are:

- the extrapolation of toxic effects observed at the high doses necessary to conduct animal studies to predict effects that might occur at the much lower, relevant doses and

- the extrapolation from toxic effects in animals to toxic effects in man (i.e., responses of animals may be different from responses of man).

The EPA has derived SFs using a weight-of-evidence approach to studies in the scientific literature. The SFs represent the upper 95th percent confidence limits on the slope of the dose response curve for carcinogenic responses. Because cancer potentency factors represent the upper limits of the slope of the line, the use of SFS is more likely to overestimate the actual risk rather than underestimate it.

Uncertainties also arise in the development of the RfDs used to characterize noncarcinogenic effects. These reference values are derived using studies in humans or animals by identifying the no observable effects level (NOAEL). Two basic types of uncertainty arise. The first is related to the extrapolation from toxic effects seen at high doses to predict effects at the low doses encountered by the receptors. The second involves extrapolation from effects in animals to effects in man. Each of these is offset by the uncertainty factor, which is actually a product of as many as five separate factors, each intended to account for one type of uncertainty. The lowest observable adverse effects level (LOAEL) or NOAEL is then divided by this composite uncertainty factor. The uncertainty factors usually range from 10 to 10,000 . The five types of uncertainty included in the assignment of the uncertainty factor are (each representing an uncertainty factor of 5 to 10 ):

- Sensitive subpopulations in the general population

- Extrapolation from animals to humans

- Extrapolation from subchronic study to a chronic estimate

- Extrapolation from an LOAEL to an NOAEL

- Additional uncertainties in the critical study used in setting the RfD.

\subsubsection{Assumptions}

The major assumptions used in this assessment are:

- Contaminant concentrations remain constant over the exposure period.

- The building will be allowed to decay in place without decontamination activities being performed.

- Selected intake rates and population characteristics (weight, lifespan, and activities) are representative of the potentially exposed populations. 
- All intake of contaminants is from site-related exposure media, and no other sources contribute to the receptor's health risk.

\subsubsection{Uncertainties in Radiological Risk Evaluation}

The risk of increased incidence of cancer or of fatal cancer: from exposure to low-level radiation is estimated by applying a risk factor to either the radiation dose or the radionuclide intake. Regardless of the type of risk factor used, the same basic uncertainties remain. The uncertainties are related to the model used for determining the health effects of radiation exposure.

The model most frequently used for determining risk of radiation exposure is the linear nonthreshold model. This model assumes that there is some increased risk for any increment of radiation exposure, there being no threshold below which effects are not seen. This is the most conservative model for evaluating radiation risk. It uses data from high dose radiation exposures (such as from the survivors of the atomic bomb) and extrapolates risk from these high exposures to the low level-environment or occupational dose range.

\subsection{CONCLUSIONS}

The results of the BRE for Building 3506 indicate that long-term exposures to contaminated media inside the building pose a potential health risk. While it is unlikely that adult trespasser exposures will ever occur at this facility, these exposures were evaluated to provide an upperbound estimate of the potential human health risks. Current exposures to contaminated media at Building 3506 indicate that worker protection is necessary to ensure worker safety. Future health risks from exposures to contaminants are estimated to be above levels of concern as set by EPA. 


\section{REFERENCES}

American Cancer Society. 1990. Cancer Facts and Figures-1990. New York, 31 pp.

ATSDR (Agency for Toxic Substances and Disease Registry). 1988a. Toxicological Profile for Beryllium. Prepared by Syracuse Research Corporation under Contract 68-C8-0004. U.S. Public Health Service. ATSDR/TP-88/09.

ATSDR (Agency for Toxic Substances and Disease Registry). 1988b. Toxicological Profile for Nickel. ATSDR/U.S. Public Health Service, ATSDR/TP-88/19.

ATSDR (Agency for Toxic Substances and Disease Registry). 1989a. Toxicological Profile for Arsenic.Agency for Toxic Substances and Disease Registry, U.S. Public Health Service, Atlanta, GA. ATSDR/TP-88/02.

ATSDR (Agency for Toxic Substances and Disease Registry). 1989b. Toxicological profile for Cadmium. ATSDR/U.S. Public Health Service, ATSDR/TP-88/08.

ATSDR (Agency for Toxic Substances and Disease Registry). 1989c. Toxicological Profile for Chromium. Prepared by Syracuse Research Corporation under Contract 68-C8-0004. U.S. Public Health Service. ATSDR/TP-88/10.

ATSDR (Agency for Toxic Substances and Disease Registry). 1989d. Toxicological Profile for Cyanide. ATSDR/TP-88/12; PB90-162058. Prepared by Syracuse Research Corporation for ATSDR, U.S. Public Health Service, under Contract No. 68-C8-0004.

ATSDR (Agency for Toxic Substances and Disease Registry). 1989e. Toxicological Profile for Mercury. ATSDR/U.S. Public Health Service.

ATSDR (Agency for Toxic Substances and Disease Registry). 1989f. Toxicological profile for Plutonium. United States Department of Health and Human Services, Public Health Service. Atlanta, GA.

ATSDR (Agency for Toxic Substances and Disease Registry). 1989g. Toxicological Profile for Selenium. Agency for Toxic Substances and Disease Registry, U.S. Public Health Service, Atlanta GA.

ATSDR (Agency for Toxic Substances and Disease Registry). 1989h. Toxicological Profile for Uranium, U.S. Public Health Service, Washington, D. C., October.

ATSDR (Agency for Toxic Substances and Disease Registry). 1989i. Toxicological Profile for Zinc. Agency for Toxic Substances and Disease Registry, U.S. Public Health Service, Atlanta, GA. 121 pp. ATSDR/TP-89-25.

ATSDR (Agency for Toxic Substances and Disease Registry). 1990a. Toxicological Profile for Aluminum. U.S. Department of Health and Human Services. Public Health Service.

ATSDR (Agency for Toxic Substances and Disease Registry). 1990b. Antimony. ATSDR/U.S. Public Health Service, DRAFT. 
ATSDR (Agency for Toxic Substances and Disease Registry). 1990c. Toxicological Profile for Asbestos, TP-90-04, (ATSDR), U.S. Public Health Service, Washington, D. C., December.

ATSDR (Agency for Toxic Substances and Disease Registry). 1990d. DRAFT. Toxicological Profile for Lead. United States Department of Health and Human Services, Public Health Service. Atlanta, ATSDR.

ATSDR (Agency for Toxic Substances and Disease Registry). 1990e. Toxicological Profile for Polycyclic Aromatic Hydrocarbons. Acenaphthene, Acenaphthylene, Anthracene, Benzo(a)anthracene, Benzo(a)pyrene, Benzo(b)fluoranthene, Benzo(g,i,h)perylene, Benzo(k)fluoranthene, Chrysene, Dibenzo(a,h)anthracene, Fluoranthene, Fluorene, Indeno(1,2,3-c,d)pyrene, Phenanthrene, Pyrene. Prepared by Clement International Corporation, under Contract No. 205-88-0608. ATSDR/TP-90-20.

ATSDR (Agency for Toxic Substances and Disease Registry). 1990f. Toxicological Profile for Radon. United States Department of Health and Human Services, Public Health Service. Atlanta, ATSDR.

ATSDR (Agency for Toxic Substances and Disease Registry). 1990g. Toxicological Profile for Thorium. TP-90-25. United States Department of Health \& Human Services, Public Health Service, Atlanta, GA.

ATSDR (Agency for Toxic Substances and Disease Registry). 1990h. Toxicological Profile for Vanadium. Prepared by Clement Associates, Inc., under Contract 205-88-0608. Agency for Toxic Substances and Disease Registry, U.S. Public Health Service, Atlanta, GA. Draft report, October 1990.

ATSDR (Agency for Toxic Substances and Disease Registry). 1991. Draft Update Toxicological Profile for Selected PCBs (Aroclor-1260, -1254, -1242, -1232, -1221, and -1016). United States Department of Health and Human Services, Public Health Service. Atlanta: ATSDR.

ATSDR (Agency for Toxic Substances and Disease Registry). 1993. Toxicological Profile for Polychlorinated Biphenyls. U.S. Department of Health \& Human Services, Public Health Service, April.

Bechtel, 1992. Preliminary Risk Assessment Report for Waste Area Grouping 1 at Oak Ridge National Laboratory, Oak Ridge, Tennessee.

Bechtel, 1993. Site Characterization Plan for Decontamination and Decommissioning of Buildings 3506 and 3515 at Oak Ridge National Laboratory, Oak Ridge, Tennessee. ORNL/ER/sub/87-99053/69, Oak Ridge, Tennessee.

Bechtel, 1994. Site Characterization Report for Building 3506 at Oak Ridge National Laboratory, Oak Ridge Tennessee.

Beems, R.B. and L. van Beek. 1985. Short-term (6-week) oral toxicity study of selenium in Syrian hamsters. Fd. Chem. Toxicol. 23: 945-947.

BEIAS (Biomedical and Environmental Information Analysis Section). 1993. Toxicity Profiles for Contaminants of Concern on the Oak Ridge Reservation. (ES/ER/TM-77). Oak Ridge, TN. 
DOE (United States Department of Energy). 1992. Federal Facility Agreement for the Oak Ridge Reservation, DOE/OR-1014, Department of Energy-Oak Ridge Reservation, Oak Ridge, Tennessee.

Ganther, H.E. and C.A. Baumann. 1962. Selenium metabolism. II. Modifying effects of sulfate. J. Nutr. 77: 408-414.

Ghetti, G. and L. Mariani. 1956. [Eye changes due to naphthalene]. Med. Lav. 47: 533-538.

Harr, J.R., J.F. Bone, I.J. Tinsley, P.H. Weswig and R.S. Yamamoto. 1967. Selenium toxicity in rats. II. Histopathology. In: Selenium in Biomedicine, O.H. Muth, ed., First Int. Symp., Oregon State University, 1966. Avi Publ., Westport, CT. pp. 153-178.

ICRP (International Commission on Radiological Protection). 1991. 1990 Recommendations of the International Commission on Radiological Protection. ICRP Publication NO. 60. Pergamon Press, Inc. New York, NY.

Martin Marietta Energy Systems, Inc., 1992a, Baseline Design Report for Decontamination and Decommissioning (D\&D) of Building 9201-4 at the Y-12 Plant, Oak Ridge, Tennessee, Y/EN4798, Oak Ridge, Tennessee.

Martin Marietta Energy Systems, Inc., 1992b, Oak Ridge Reservation Site Management Plan for the Environmental Restoration Program, DOE/OR-1001/R2, Environmental Restoration Division, Oak Ridge, Tennessee.

Martin Marietta Energy Systems, Inc., 1993, Final Report on the Background Soil Characterization Project at the Oak Ridge Reservation, Oak Ridge, Tennessee, DOE/OR/011175/VI.

NCRP (National Council on Radiation Protection). 1977. Environmental Radiation Measurements. NCRP Report No. 50. Washington, D.C.

NRC (National Research Council). 1989. Recommended Dietary Allowances. 10th ed. National Academy Press, Washington, D.C.

ORNL (Oak Ridge National Laboratory). 1988. Limiting Values of Radionuclides Intake and Air Concentrations and Dose Conversion Factors for Inhalation, Submersion, and Ingestion, Federal Guidance Report No. 11, Oak Ridge, Tennessee.

Radian, 1993. Remedial Investigation Report Feasibility Study For The Gunite and Associated Tanks Operable Unit At Waste Area Grouping 1.

Schroeder, H.A. 1967. Effects of selenate, selenite and tellurite on the growth and early survival of mice and rats. J. Nutr. 92: 334-338.

Tinsley, I.J., J.R. Harr, J.F. Bone, P.H. Weswig and R.S. Yamamoto. 1967. Selenium toxicity in rats. I. Growth and longevity. In: Selenium in Biomedicine, O.H. Muth, ed., First Int. Symp., Oregon State University, 1966. Avi Publishing Co., Inc.: Westport, CT. pp. 141-152. 
United States Department of Commerce, Bureau of the Census. 1991. 1990 Census of Population and Housing Public Law 94-171 Data, Washington, D.C.

United States Environmental Protection Agency. 1988. Guidance for Conducting Remedial Investigations and Feasibility Studies under CERCLA. EPA/540/G-89-004, Office of Emergency and Remedial Response, Washington, D.C.

United States Environmental Protection Agency. 1989a. Risk Assessment Guidance for Superfund: Human Health Evaluation Manual, Part A, Interim Final. EPA/540/1-89/002, Office of Emergency and Remedial Response, Washington, D.C.

United States Environmental Protection Agency. 1989b. Risk Assessment Guidance for Superfund: Volume II, Environmental Evaluation Manual, EPA/540/1-89/001, Office of Emergency and Remedial Response, Washington, D.C.

United States Environmental Protection Agency. 1989c. Exposure Factors Handbook. EPA/600/8-89/043, Office of Health and Environmental Assessment, Washington, D.C.

United States Environmental Protection Agency. 1990. National Oil and Hazardous Substance Pollution Contingency Plan. Federal Register 55(46): pp. 8666-8865.

United States Environmental Protection Agency. 1991. Risk Assessment Guidance for Superfund: Volume I - Human Health Evaluation Manual, Part B, Development of Risk-Based Preliminary Remediation Goals. Publication 9285.7-01B, Office of Emergency and Remedial Response.

Wilson, R. 1979. Technol. Rev. 81: 40-46. 


\section{APPENDIX A}

Characterization Data Set for Building 3506 
THIS PAGE INTENTIONALLY LEFT BLANK 
Table A.1. Analytical detects for organic compounds for Building 3506

\begin{tabular}{|c|c|c|c|c|c|c|c|c|c|}
\hline \multirow{2}{*}{$\frac{\text { Location ID }}{\text { Sample ID }}$} & \multicolumn{3}{|c|}{$\begin{array}{c}72.5 B 001 \\
\text { (East Gallery Floor) }\end{array}$} & \multicolumn{2}{|c|}{$\begin{array}{c}72.5 B 002 \\
\text { (East Gallery Wall) }\end{array}$} & \multirow{2}{*}{$\begin{array}{c}\begin{array}{c}\text { 72.SB003 } \\
\text { (Cell Wall) }\end{array} \\
04044\end{array}$} & \multicolumn{2}{|c|}{$\begin{array}{c}\text { 72.SB004 } \\
\text { (Cell Floor) }\end{array}$} & \multirow{2}{*}{$\begin{array}{c}\begin{array}{c}\text { 72.SD001 } \\
\text { (Cell Floor) }\end{array} \\
03934^{d}\end{array}$} \\
\hline & 03936 & 03943 & 03933 & $03944^{a}$ & 04237 & & $\begin{array}{c}\text { 04045, } \\
\text { Composite }^{b}\end{array}$ & $\begin{array}{c}\text { 04045, Hot } \\
\text { Interval }^{c}\end{array}$ & \\
\hline Sample Type & $\begin{array}{l}\text { QC/Equip. } \\
\text { Rinsate }\end{array}$ & Concrete Core & Soil & $\begin{array}{l}\text { Concrete } \\
\text { Core }\end{array}$ & $\begin{array}{l}\text { Concrete } \\
\text { Chips }\end{array}$ & $\begin{array}{c}\text { Concrete } \\
\text { Core }\end{array}$ & $\begin{array}{l}\text { Concrete } \\
\text { Core }\end{array}$ & $\begin{array}{c}\text { Concrete } \\
\text { Core }\end{array}$ & $\begin{array}{c}\text { Misc. } \\
\text { Sediment }\end{array}$ \\
\hline Units & $\mu \mathrm{g} / \mathrm{L}$ & $\mathrm{mg} / \mathrm{kg}$ & $\mathrm{mg} / \mathrm{kg}$ & $\mathrm{mg} / \mathrm{kg}$ & $\mathrm{mg} / \mathrm{kg}$ & $\mathrm{mg} / \mathrm{kg}$ & $\mathrm{mg} / \mathrm{kg}$ & $\mathrm{mg} / \mathrm{kg}$ & $\mathrm{mg} / \mathrm{kg}$ \\
\hline \multicolumn{10}{|c|}{ VOCs } \\
\hline Acetone & NA & NA & & NA & NA & NA & NA & NA & $0.09 \mathrm{~J}$ \\
\hline \multicolumn{10}{|c|}{ VOC TICs (No. of TICs) } \\
\hline Unknown & NA & NA & & NA & NA & NA & NA & NA & $0.019 \mathrm{NJ}(1)$ \\
\hline \multicolumn{10}{|c|}{ BNAES } \\
\hline Acenaphthene & & & & & & & & & $0.96 \mathrm{~J}$ \\
\hline Anthracene & & & & & & & & & $1.9 \mathrm{~J}$ \\
\hline Benzo(a)pyrene & & & & & & & & & $5.7 \mathrm{~J}$ \\
\hline Benzo(g,h,i)perylene & & & & & & & & & $1.1 \mathrm{~J}$ \\
\hline Benzo(k)fluoranthene & & & & & & & & & $46 \mathrm{~J}$ \\
\hline Carbazole & & & & & & & & & $2 \mathrm{~J}$ \\
\hline Chrysene & & & & & & & & & $19 \mathrm{~J}$ \\
\hline Dibenz(a,h)anthracene & & & & & & & & & $1.5 \mathrm{~J}$ \\
\hline Dibenzofuran & & & & & & & & & $0.51 \mathrm{~J}$ \\
\hline Fluoranthene & & & & & & & & & $27 \mathrm{~J}$ \\
\hline Fluorene & & & & & & & & & $0.85 \mathrm{~J}$ \\
\hline Indeno(1,2,3-cd)pyrene & & . & & & & & & & $9.6 \mathrm{~J}$ \\
\hline
\end{tabular}


Table A.1 (continued)

\begin{tabular}{|c|c|c|c|c|c|c|c|c|c|}
\hline \multirow{2}{*}{$\frac{\text { Location ID }}{\text { Sample ID }}$} & \multicolumn{3}{|c|}{$\begin{array}{c}72.5 B 001 \\
\text { (East Gallery Flomr) }\end{array}$} & \multicolumn{2}{|c|}{$\begin{array}{c}72 . \mathrm{SBO02} \\
\text { (East Gallery Wall) }\end{array}$} & \multirow{2}{*}{$\begin{array}{c}\begin{array}{c}72 . S B 003 \\
\text { (Cell Wall) }\end{array} \\
04044\end{array}$} & \multicolumn{2}{|c|}{$\begin{array}{l}\text { 72.SB004 } \\
\text { (Cell Flocor) }\end{array}$} & \multirow{2}{*}{$\begin{array}{c}\begin{array}{c}\text { 72.SD001 } \\
\text { (Cell Floor) }\end{array} \\
03934^{d}\end{array}$} \\
\hline & 03936 & 03943 & 03933 & $03944^{a}$ & 04237 & & $\begin{array}{c}\text { 04045, } \\
\text { Composite }^{b}\end{array}$ & $\begin{array}{l}\text { 04045, Hot } \\
\text { Interval }^{c}\end{array}$ & \\
\hline Sample Type & $\begin{array}{l}\text { QC/Equip. } \\
\text { Rinsate }\end{array}$ & Concrete Core & Soil & $\begin{array}{c}\text { Concrete } \\
\text { Core }\end{array}$ & $\begin{array}{l}\text { Concrete } \\
\text { Chips }\end{array}$ & $\begin{array}{c}\text { Concrete } \\
\text { Core }\end{array}$ & $\begin{array}{l}\text { Concrete } \\
\text { Core }\end{array}$ & $\begin{array}{c}\text { Concrete } \\
\text { Core }\end{array}$ & $\begin{array}{l}\text { Misc. } \\
\text { Sediment }\end{array}$ \\
\hline Units & $\mu \mathrm{g} / \mathrm{L}$ & $\mathrm{mg} / \mathrm{kg}$ & $\mathrm{mg} / \mathrm{kg}$ & $\mathrm{mg} / \mathrm{kg}$ & $\mathrm{mg} / \mathrm{kg}$ & $\mathrm{mg} / \mathrm{kg}$ & $\mathrm{mg} / \mathrm{kg}$ & $\mathrm{mg} / \mathrm{kg}$ & $\mathrm{mg} / \mathrm{kg}$ \\
\hline 2-Methylnaphthalene & & & & & $0.089 \mathrm{~J}$ & & & & $0.27 \mathrm{~J}$ \\
\hline Naphthalene & & & & & $0.053 \mathrm{~J}$ & & & & $0.42 \mathrm{~J}$ \\
\hline Phenanthrene & & & & & $0.24 \mathrm{~J}$ & & & & $17 \mathrm{~J}$ \\
\hline Pyrene & & & & & & & & & $22 \mathrm{~J}$ \\
\hline Dimethyl phthalate & & & & & $0.055 \mathrm{~J}$ & & & & \\
\hline Di-n-butylphthalate & $3 \mathrm{~J}$ & & & & & & & & \\
\hline Bis(2-ethylhexyl)phthalate & $4 \mathrm{~J}$ & & & & $0.24 \mathrm{~J}$ & & & & $2.1 \mathrm{~J}$ \\
\hline \multicolumn{10}{|c|}{ BNAE TTCs (No. of T7Cs) } \\
\hline Dimethyl phenanthrene isomer & & & & & $0.37 \mathrm{NJ}(1)$ & & & & \\
\hline Hexanedioic acid ester & & & & & $5.1 \mathrm{NJ}(1)$ & & & & \\
\hline 4-Hydroxy-4-methyl-2-pentanone & & $530 \mathrm{R}(1)$ & & & & $2,900 \mathrm{NJ}(1)$ & $2,900 \mathrm{NJ}(1)$ & $2,500 \mathrm{NJ}(1)$ & \\
\hline Methyl phenanthrene isomer & & & & & $0.68 \mathrm{NJ}(2)$ & & & & \\
\hline Oxygenated hydrocarbon & & & & & & $3.5 \mathrm{NJ}(1)$ & & & \\
\hline Substituted benzene & & & & & $0.44 \mathrm{NJ}(1)$ & & & & \\
\hline Unknown & & & & & & & & & $0.81 \mathrm{NJ}(1)$ \\
\hline Unknown alcohol & & & $0.82 \mathrm{NJ}(1)$ & & & $62 \mathrm{NJ}(1)$ & $39 \mathrm{NJ}(1)$ & & \\
\hline Unknown alkane & & & & & $1.12 \mathrm{NJ}(3)$ & $1 \mathrm{NJ}(1)$ & $2.5 \mathrm{NJ}(1)$ & $2.7 \mathrm{NJ}(2)$ & \\
\hline Unknown alkene & & $106 R(2)^{\prime}$ & & $70.6 \mathrm{NJ}(2)$ & & $3.1 \mathrm{NJ}(1)$ & & & \\
\hline
\end{tabular}


Table A.1 (continued)

\begin{tabular}{|c|c|c|c|c|c|c|c|c|c|}
\hline \multirow{2}{*}{$\begin{array}{l}\text { Location ID } \\
\text { Sample ID }\end{array}$} & \multicolumn{3}{|c|}{$\begin{array}{c}\text { 72.SBOO1 } \\
\text { (East Gallery Floor) }\end{array}$} & \multicolumn{2}{|c|}{$\begin{array}{c}72 . \mathrm{SBO02} \\
\text { (East Gallery Wall) }\end{array}$} & \multirow{2}{*}{$\begin{array}{c}\begin{array}{c}\text { 72.SB003 } \\
\text { (Cell Wall) }\end{array} \\
04044\end{array}$} & \multicolumn{2}{|c|}{$\begin{array}{c}\text { 72.SB004 } \\
\text { (Cell Floor) }\end{array}$} & \multirow{2}{*}{$\begin{array}{c}\begin{array}{c}72.5 D 001 \\
\text { (Cell Floor) }\end{array} \\
03934^{d}\end{array}$} \\
\hline & 03936 & 03943 & 03933 & $03944^{a}$ & 04237 & & $\begin{array}{c}04045, \\
\text { Composite }\end{array}$ & $\begin{array}{l}\text { 04045, Hot } \\
\text { Interval }\end{array}$ & \\
\hline Sample Type & $\begin{array}{l}\text { QC/Equip. } \\
\text { Rinsate }\end{array}$ & Concrete Core & Soil & $\begin{array}{c}\text { Concrete } \\
\text { Core }\end{array}$ & $\begin{array}{c}\text { Concrete } \\
\text { Chips }\end{array}$ & $\begin{array}{c}\text { Concrete } \\
\text { Core }\end{array}$ & $\begin{array}{c}\text { Concrete } \\
\text { Core }\end{array}$ & $\begin{array}{l}\text { Concrete } \\
\text { Core }\end{array}$ & $\begin{array}{l}\text { Misc. } \\
\text { Sediment }\end{array}$ \\
\hline Units & $\mu g / L$ & $\mathrm{mg} / \mathrm{kg}$ & $\mathrm{mg} / \mathrm{kg}$ & $\mathrm{mg} / \mathrm{kg}$ & $\mathrm{mg} / \mathrm{kg}$ & $\mathrm{mg} / \mathrm{kg}$ & $\mathrm{mg} / \mathrm{kg}$ & $\mathrm{mg} / \mathrm{kg}$ & $\mathbf{m g} / \mathbf{k g}$ \\
\hline Unknown carboxylic acid ester & & & $1.1 \mathrm{NJ}(1)$ & & $3.2 \mathrm{NJ}(1)$ & & & & \\
\hline Unknown cycloalkene & & & & $57 \mathrm{NJ}(1)$ & & & & $4.8 \mathrm{NJ}(1)$ & \\
\hline Unknown hydrocarbons & $12 \mathrm{NJ}(3)$ & $1,736.3 \mathrm{R}(17)^{\mathrm{r}}$ & $6.192 \mathrm{NJ}(6)$ & $\begin{array}{r}12,637.1 \\
\mathrm{NJ}(11)\end{array}$ & $0.75 \mathrm{NJ}(2)$ & $379.4 \mathrm{NJ}(13)$ & $337.2 \mathrm{NJ}(12)$ & $\begin{array}{l}392.89 \\
N J(15)\end{array}$ & \\
\hline Unknown ketone & & & & & $0.88 \mathrm{NJ}(1)$ & $127.4 \mathrm{NJ}(3)$ & $131 \mathrm{NJ}(2)$ & $131 \mathrm{NJ}(2)$ & \\
\hline Unknown PCB & & & & $26.1 \mathrm{NJ}(6)$ & $1.46 \mathrm{NJ}(4)$ & & & & $29.1 \mathrm{NJ}(18)$ \\
\hline \multicolumn{10}{|c|}{ Pesticides/PCBs } \\
\hline Aroclor-1254 & & 0.37 & & $25 \mathrm{R}^{z}$ & $1.7 \mathrm{~J}$ & $0.11 \mathrm{~J}$ & $0.34 \mathrm{~J}$ & $0.66 \mathrm{~J}$ & 410 \\
\hline Aroclor-1260 & & 0.11 & 0.011 & $31 \mathbf{R}^{8}$ & $2.6 \mathrm{~J}$ & $0.13 \mathrm{~J}$ & $0.57 \mathrm{~J}$ & $1.14 \mathrm{~J}$ & \\
\hline Heptachlor & $4.7 \mathrm{~J}$ & & & & & & & & \\
\hline
\end{tabular}

ample 03944 was reanalyzed for BNAEs because the surrogates were diluted out of the first sample. The BNAE results are for the reanalyzed sample.

${ }^{b}$ Composite core from cell floor using intervals of 0 to 6.5 in., 10.5 to 19.5 in., and 19.5 to 24 in.

" "Hot" core interval from 6.5 to 10.5 in.

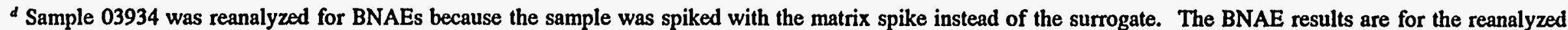

sample, even though it is outside the holding times.

- The result is the sum of the TICs. The number of TICs is shown in parentheses.

$\checkmark$ BNAE values rejected due to the severe matrix effect; most of the surrogates and all of the spike recoveries were outside the required limits.

- PCB values rejected because all surrogates for the original run and dilution were diluted out.

Note: no organic analysis was performed for sample 03935 (soil sample from 72.SB004) due to the limited sample size.

Note: "NA" indicates that analyses were not conducted; a blank space indicates that the analyte was not detected. 
Table A.2. Analytical results for metals and cyanide for Building 3506

\begin{tabular}{|c|c|c|c|c|c|c|c|c|c|}
\hline Location ID & \multicolumn{3}{|c|}{$\begin{array}{l}\text { 72.SB001 } \\
\text { (East Gallery Floor) }\end{array}$} & \multirow{2}{*}{$\begin{array}{c}\begin{array}{c}72.5 B 002 \\
\text { (East Gallery } \\
\text { Wall) }\end{array} \\
03944\end{array}$} & \multirow{2}{*}{$\begin{array}{c}\begin{array}{c}72 . S B 003 \\
\text { (Cell Wall) }\end{array} \\
04044\end{array}$} & \multicolumn{2}{|c|}{$\begin{array}{l}\text { 72.SB004 } \\
\text { (Cell Floor) }\end{array}$} & \multirow{2}{*}{$\begin{array}{c}\begin{array}{c}72 . \text { SD001 } \\
\text { (Cell } \\
\text { Floor) }\end{array} \\
03934\end{array}$} & \multirow{3}{*}{$\begin{array}{l}\text { RCRA Toxicity } \\
\text { Characteristic } \\
\text { Equivalent Limits } \\
\text { for Solids }\end{array}$} \\
\hline Sample ID & 03936 & 03943 & 03933 & & & $\begin{array}{c}\text { 04045a, } \\
\text { Composite }\end{array}$ & $\begin{array}{l}04045^{b}, \text { Hot } \\
\text { Interval }\end{array}$ & & \\
\hline Sample Type & $\begin{array}{l}\text { QC/Equip. } \\
\text { Rinsate }\end{array}$ & $\begin{array}{c}\text { Concrete } \\
\text { Core }\end{array}$ & Soil & $\begin{array}{l}\text { Concrete } \\
\text { Core }\end{array}$ & $\begin{array}{c}\text { Concrete } \\
\text { Core }\end{array}$ & $\begin{array}{l}\text { Concrete } \\
\text { Core }\end{array}$ & $\begin{array}{c}\text { Concrete } \\
\text { Core }\end{array}$ & $\begin{array}{l}\text { Misc. } \\
\text { Sediment }\end{array}$ & \\
\hline Units & $\mu \mathrm{g} / \mathrm{L}$ & $\mathrm{mg} / \mathrm{kg}$ & $\mathrm{mg} / \mathrm{kg}$ & $\mathrm{mg} / \mathrm{kg}$ & $\mathrm{mg} / \mathrm{kg}$ & $\mathrm{mg} / \mathrm{kg}$ & $\mathrm{mg} / \mathrm{kg}$ & $\mathrm{mg} / \mathrm{kg}$ & $\mathbf{m g} / \mathbf{k g}$ \\
\hline
\end{tabular}

\begin{tabular}{|c|c|c|c|c|c|c|c|c|c|}
\hline \multicolumn{10}{|c|}{ RCRA Metals } \\
\hline Arsenic & $<1.3$ & $2.6 \mathrm{~J}$ & $1.1 \mathrm{~J}$ & $3.0 \mathrm{~J}$ & $2.5 \mathrm{~J}$ & $2.6 \mathrm{~J}$ & $2.9 \mathrm{~J}$ & $21.5 \mathrm{~J}$ & 100 \\
\hline Barium & $<2.6$ & 21.4 & 52.4 & 19.3 & 722 & 26.9 & 22.5 & 120 & 2,000 \\
\hline Cadmium & $<1.3$ & 4.1 & $<0.25$ & 5.1 & $8 \%$ & $5.8 \mathrm{~J}$ & $3.6 \mathrm{~J}$ & 60 聯 & 20 \\
\hline Chromium & 3.3 & 8.3 & 17.5 & 8.2 & \% & 13.1 & 13.2 & 1. & 100 \\
\hline Lead & $<2.5$ & 7.2 & 17.1 & 4.7 & 2.7 & 7.1 & 6.0 & 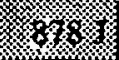 & 100 \\
\hline Mercury & $<0.2$ & $<0.14$ & \%x & $<0.09$ & 0.34 & 0.05 & 0.07 & x & 4 \\
\hline Selenium & $<3.1$ & $<2.7$ & $<0.66$ & $<2.4$ & $<0.65$ & $<3.2$ & $<0.64$ & $0.87 \mathrm{~J}$ & 20 \\
\hline Silver & $<2.6$ & $<0.74$ & $1.4 \mathrm{~J}$ & $<0.69$ & $<26$ & $<0.73$ & $<0.78$ & $<1.9$ & 100 \\
\hline
\end{tabular}

Other TAL Metals

\begin{tabular}{|c|c|c|c|c|c|c|c|c|c|}
\hline Aluminum & 174 & 4,080 & 9,590 & 4,820 & 147,000 & 4,540 & 4,570 & 9,530 & NA \\
\hline Antimony & $<12.9$ & $<1.8$ & $<2.3$ & $<1.7$ & $<64.6 \mathrm{R}^{d}$ & $<1.8 \mathrm{R}^{d}$ & $<1.9 \mathrm{R}^{d}$ & $36.2 \mathrm{~J}$ & NA \\
\hline Beryllium & $<0.2$ & 0.4 & 0.75 & $<0.23$ & 17.6 & 0.42 & 0.53 & $<0.95$ & $\mathbf{N A}$ \\
\hline Calcium & $<250$ & 215,000 & 16,300 & 257,000 & $8,340,000$ & 261,000 & 239,000 & 20,300 & $\mathbf{N A}$ \\
\hline Cobalt & $<2.6$ & 3.2 & 9.6 & 2.0 & 68.8 & 2.3 & 2.7 & $<25.1$ & $\mathbf{N A}$ \\
\hline Copper & $<13.3$ & 10.8 & 14.2 & 7.5 & 378 & 9.8 & 9.6 & 385 & NA \\
\hline
\end{tabular}


Table A.2 (continued)

\begin{tabular}{|c|c|c|c|c|c|c|c|c|c|}
\hline \multirow{2}{*}{$\begin{array}{l}\text { Location ID } \\
\text { Sample ID }\end{array}$} & \multicolumn{3}{|c|}{$\begin{array}{c}\text { 72.SB001 } \\
\text { (East Gallery Floor) }\end{array}$} & \multirow{2}{*}{$\begin{array}{c}\begin{array}{c}72 . \mathrm{SB} 002 \\
\text { (East Gallery } \\
\text { Wall) }\end{array} \\
\\
03944\end{array}$} & \multirow{2}{*}{$\begin{array}{c}\begin{array}{c}72 . S B 003 \\
\text { (Cell Wall) }\end{array} \\
04044 \\
\end{array}$} & \multicolumn{2}{|c|}{$\begin{array}{l}\text { 72.SB004 } \\
\text { (Cell Floor) }\end{array}$} & \multirow{2}{*}{$\begin{array}{c}\begin{array}{c}72 . \text { SD001 } \\
\text { (Cell } \\
\text { Floor) }\end{array} \\
\\
03934 \\
\end{array}$} & \multirow{3}{*}{$\begin{array}{l}\text { RCRA Toxicity } \\
\text { Characteristic } \\
\text { Equivalent Limits } \\
\text { for Solids }\end{array}$} \\
\hline & 03936 & 03943 & 03933 & & & $\begin{array}{l}04045^{a} \\
\text { Composite }\end{array}$ & $\begin{array}{l}04045^{b}, \text { Hot } \\
\text { Interval }\end{array}$ & & \\
\hline Sample Type & $\begin{array}{c}\text { QC/Equip. } \\
\text { Rinsate }\end{array}$ & $\begin{array}{l}\text { Concrete } \\
\text { Core }\end{array}$ & Soil & $\begin{array}{l}\text { Concrete } \\
\text { Core }\end{array}$ & $\begin{array}{l}\text { Concrete } \\
\text { Core }\end{array}$ & $\begin{array}{l}\text { Concrete } \\
\text { Core }\end{array}$ & $\begin{array}{l}\text { Concrete } \\
\text { Core }\end{array}$ & $\begin{array}{c}\text { Misc. } \\
\text { Sediment }\end{array}$ & \\
\hline Units & $\mu \mathrm{g} / \mathrm{L}$ & $\mathrm{mg} / \mathrm{kg}$ & $\mathrm{mg} / \mathrm{kg}$ & $\mathrm{mg} / \mathrm{kg}$ & $\mathrm{mg} / \mathrm{kg}$ & $\mathrm{mg} / \mathrm{kg}$ & $\mathrm{mg} / \mathrm{kg}$ & $\mathrm{mg} / \mathrm{kg}$ & $\mathrm{mg} / \mathrm{kg}$ \\
\hline Iron & 106 & 5,770 & 25,500 & $5,690 \mathrm{~J}$ & 176,000 & 5,290 & 5,120 & 110,000 & NA \\
\hline Magnesium & $<56.4$ & 36,800 & 5,110 & 42,300 & $1,390,000$ & 37,900 & 38,700 & 7,930 & NA \\
\hline Manganese & $<6.1$ & 261 & 348 & $265 \mathrm{~J}$ & 9,530 & 281 & 274 & 1,310 & NA \\
\hline Nickel & $<3.4$ & 5.3 & 12.6 & 6.0 & 138 & 6.8 & 6.6 & 169 & NA \\
\hline Potassium & 94.4 & 829 & 1,710 & 1,030 & 33,700 & 792 & 756 & 822 & NA \\
\hline Sodium & 264 & $309 \mathrm{~J}$ & $<152$ & $<303$ & $7,050 \mathrm{~J}$ & $221 \mathrm{~J}$ & 214 & 133 & NA \\
\hline Thallium & $<3.8$ & $<0.75$ & $<0.93$ & $<0.76$ & $<0.48$ & $<0.47$ & $<0.48$ & $<0.46$ & NA \\
\hline Vanadium & $<5.5$ & 5.7 & 18.7 & 9.1 & 240 & 9.0 & 9.1 & $<28.7$ & NA \\
\hline Zinc & 16.8 & 737 & 60.2 & 979 & $34,900 \mathrm{~J}$ & $1,000 \mathrm{~J}$ & $687 \mathrm{~J}$ & $7,420 \mathrm{~J}$ & NA \\
\hline \multicolumn{10}{|c|}{ TAL Cyanide } \\
\hline Cyanide & $<10$ & $<0.48$ & $<0.63$ & $<0.52 \mathrm{R}^{e}$ & $<0.5$ & $<0.53$ & $<0.51$ & $0.58 \mathrm{~J}$ & NA \\
\hline
\end{tabular}

a Composite core from cell floor using intervals of 0 to 6.5 in., 10.5 to 19.5 -in., and 19.5 to 24 in.

b "Hot" interval from 6.5 to 10.5 in.

- The RCRA equivalent limits are derived from values in 40 CFR 261.24; the maximum concentration of contaminants for the toxicity characteristic, listed by EPA in 40 CFR 261.24 in units of $\mathrm{mg} / \mathrm{L}$, was multiplied by a factor of 20 to obtain equivalent limits for solids in units of mg/kg.

'Antimony values rejected because they are biased low, as indicated by an interference check source and the negative results in the preparation blank.

- Cyanide value rejected because cyanide holding time was missed by 15 days.

Note 1: Samples 03960 (trip blank), 04237 (concrete chips), 03935 (soil), and 04047 (trip blank) did not undergo metals and cyanide analyses.

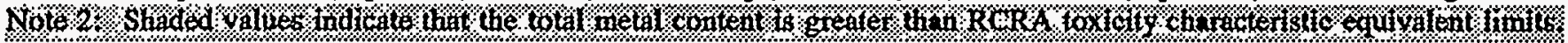


Table A.3. Chemical sampling summary for WAG 1 soil borings near Building 3506

\begin{tabular}{|c|c|c|c|c|c|c|c|c|c|c|c|}
\hline \multirow[b]{2}{*}{ Location } & \multirow[b]{2}{*}{$\begin{array}{c}\text { Distance from } \\
\text { Bldg. } 3506^{a}\end{array}$} & \multirow[b]{2}{*}{$\begin{array}{l}\text { Total Depth } \\
\text { (ft BGS } \text { BG }^{b}\end{array}$} & \multirow[b]{2}{*}{$\begin{array}{l}\text { Interval } \\
\text { (ft) }\end{array}$} & \multirow[b]{2}{*}{$\begin{array}{l}\text { Sample } \\
\text { No. }\end{array}$} & \multicolumn{7}{|c|}{ Chemical Analyses } \\
\hline & & & & & VOCs & BNAEs & $\begin{array}{l}\text { Pest./ } \\
\text { PCBs }\end{array}$ & Metals & Cyanide & Sulfide & TOC \\
\hline 01.SB196 & $18 \mathrm{ft}$ south & 6.4 & $0-2$ & 2203 & $\mathrm{x}$ & $\mathbf{x}$ & $\mathbf{x}$ & $\mathbf{X}$ & $\mathbf{X}$ & & \\
\hline \multirow[t]{4}{*}{$01 . \mathrm{SB} 197$} & \multirow[t]{4}{*}{$8 \mathrm{ft}$ north } & \multirow[t]{4}{*}{8.8} & $2-4$ & 2293 & $\mathbf{x}$ & $\mathbf{X}$ & $\mathbf{x}$ & $\mathbf{X}$ & $\mathbf{x}$ & $\mathrm{X}$ & $\mathbf{x}$ \\
\hline & & & $2-4$ & $2294^{c}$ & $\mathbf{x}$ & $\mathbf{x}$ & $\mathbf{x}$ & $\mathrm{x}$ & $\mathrm{x}$ & $\mathbf{x}$ & $\mathrm{X}$ \\
\hline & & & $6-8$ & 2308 & $\mathbf{x}$ & $\mathrm{x}$ & & & & & \\
\hline & & & $8-8.8$ & 2329 & $\mathbf{X}$ & $\mathbf{x}$ & & $\mathbf{x}$ & & & \\
\hline
\end{tabular}

a For location 01.SB196, the ORNL grid coordinates are 21963 (northing) and 30804 (easting); for location 01.SB197, the coordinates are 22021 (northing) and 30794 (easting).

$\checkmark$ BGS, below ground surface.

c Sample 2294 is a duplicate to sample 2293. 
Table A.4. Analytical detects for organic compounds in WAG 1 soil borings

\begin{tabular}{|c|c|c|c|c|c|}
\hline Location ID & $01 . S B 196$ & \multicolumn{4}{|c|}{ 01.SB197 } \\
\hline Sample ID & 2203 & 2293 & $\begin{array}{c}2294 \\
\text { (duplicate) }\end{array}$ & 2308 & 2329 \\
\hline Interval & 0 to $2 \mathrm{ft}$ & 2 to $4 \mathrm{ft}$ & 2 to $4 \mathrm{ft}$ & 6 to $8 \mathrm{ft}$ & 8 to $8.8 \mathrm{ft}$ \\
\hline Units & $\mathrm{mg} / \mathrm{kg}$ & $\mathrm{mg} / \mathrm{kg}$ & $\mathrm{mg} / \mathrm{kg}$ & $\mathrm{mg} / \mathrm{kg}$ & $\mathrm{mg} / \mathrm{kg}$ \\
\hline \multicolumn{6}{|c|}{ VOCs } \\
\hline Carbon disulfide & & 0.013 & 0.016 & & \\
\hline \multicolumn{6}{|c|}{$B N A E S$} \\
\hline Chrysene & & & 0.086 & & \\
\hline Fluoranthene & $0.041 \mathrm{~J}$ & & & & \\
\hline Butylbenzylphthalate & & 0.14 & & & \\
\hline Di-n-butylphthalate & & & & & $0.11 \mathrm{~J}$ \\
\hline Bis(2-ethylhexyl)phthalate & $0.14 \mathrm{~J}$ & & & & \\
\hline
\end{tabular}

Note (1): Sample 2308 was not analyzed for pesticides/PCBs.

Note (2): VOC and BNAE TICs were not reported in the WAG 1 data base and are therefore not included in this table.

Note (3): Blanks indicate that the analyte was not detected. 
Table A.5. Analytical results for metals and cyanide in WAG 1 soil borings

\begin{tabular}{|c|c|c|c|c|c|}
\hline Location ID & 01.SB196 & \multicolumn{3}{|c|}{ 01.SB197 } & \multirow{3}{*}{$\begin{array}{l}\text { RCRA Toxicity } \\
\text { Characteristic } \\
\text { Equivalent Limits } \\
\text { for Solids }\end{array}$} \\
\hline Sample ID & 2203 & 2293 & $\begin{array}{c}2294 \\
\text { (duplicate) }\end{array}$ & 2329 & \\
\hline Interval & 0 to $2 \mathrm{ft}$ & 2 to $4 \mathrm{ft}$ & 2 to $4 \mathrm{ft}$ & 8 to $8.8 \mathrm{ft}$ & \\
\hline Units & $\mathrm{mg} / \mathrm{kg}$ & $\mathrm{mg} / \mathrm{kg}$ & $\mathrm{mg} / \mathrm{kg}$ & $\mathrm{mg} / \mathrm{kg}$ & $\mathrm{mg} / \mathrm{kg}$ \\
\hline \multicolumn{6}{|c|}{ RCRA Metals } \\
\hline Arsenic & 1.7 & $9.9 \mathrm{~J}$ & $8.4 \mathrm{~J}$ & 6.1 & 100 \\
\hline Barium & 48 & 63.7 & 50 & 93.3 & 2,000 \\
\hline Cadmium & $<0.67$ & $<0.75$ & $<0.72$ & $<0.64$ & 20 \\
\hline Chromium & 13 & $20.5 \mathrm{~J}$ & $14 \mathrm{~J}$ & 13.5 & 100 \\
\hline Lead & 25 & $27 \mathrm{~J}$ & $28.3 \mathrm{~J}$ & $42 \mathrm{R}$ & 100 \\
\hline Mercury & $<0.11$ & $0.17 \mathrm{~J}$ & $0.43 \mathrm{~J}$ & $<0.12$ & 4 \\
\hline Selenium & $<0.87 \mathrm{R}$ & $<0.75 R$ & $<0.72 \mathrm{R}$ & $<3.4$ & 20 \\
\hline Silver & $<0.89$ & $<0.5$ & $<0.48$ & $<0.43$ & 100 \\
\hline \multicolumn{6}{|c|}{ Other TAL Metals } \\
\hline Aluminum & 10,200 & 15,800 & 13,400 & 19,600 & NA \\
\hline Antimony & $<3.8$ & $<3.2 \mathrm{R}$ & $<3.1 \mathrm{R}$ & $<2.8 \mathrm{R}$ & NA \\
\hline Beryllium & 0.44 & $1.1 \mathrm{~J}$ & $1.1 \mathrm{~J}$ & 1.1 & NA \\
\hline Calcium & $42,800 \mathrm{~J}$ & 9,470 & 7,860 & $51,500 \mathrm{~J}$ & NA \\
\hline Cobalt & 8.1 & $16.1 \mathrm{~J}$ & $10.6 \mathrm{~J}$ & $17.7 \mathrm{~J}$ & $\mathrm{NA}$ \\
\hline Copper & 14.7 & $3.7 \mathrm{~J}$ & $3.3 \mathrm{~J}$ & $30.1 \mathrm{~J}$ & NA \\
\hline Iron & 15,300 & 24,600 & 23,800 & 28,000 & NA \\
\hline Magnesium & $25,900 \mathrm{~J}$ & 2,490 & 2,010 & 5,100 & NA \\
\hline Manganese & $371 \mathrm{~J}$ & $1,080 \mathrm{~J}$ & $847 \mathrm{~J}$ & 1,210 & NA \\
\hline Nickel & 12.1 & $11.8 \mathrm{~J}$ & $10.8 \mathrm{~J}$ & 20.6 & NA \\
\hline Potassium & 1,490 & 1,030 & 955 & 2,170 & NA \\
\hline Sodium & 54.4 & $<69.9$ & $<67.1$ & 94.1 & NA \\
\hline Thallium & $<0.22$ & $<0.75$ & $<0.72$ & $<3.4$ & NA \\
\hline Vanadium & 19.3 & $31.3 \mathrm{~J}$ & $26.5 \mathrm{~J}$ & $21.7 \mathrm{~J}$ & NA \\
\hline Zinc & 43.3 & $21.3 \mathrm{~J}$ & $19.5 \mathrm{~J}$ & 50.4 & NA \\
\hline
\end{tabular}

TAL Cyanide

\begin{tabular}{l|l|l|l|l|l}
\hline Cyanide & $<5.7$ & $<0.38$ & $<0.39$ & NA & NA \\
\hline
\end{tabular}




\section{Table A.5 (continued)}

a The RCRA equivalent limits are derived from values in 40 CFR 261.24; the maximum concentration of contaminants for the toxicity characteristic, listed by EPA in 40 CFR 261.24 in units of $\mathrm{mg} / \mathrm{L}$, was multiplied by a factor of 20 to obtain equivalent limits for solids in units of $\mathrm{mg} / \mathrm{kg}$.

Note (1): Sample 2308 (location 01.SB197, interval 6-8 ft) was not analyzed for metals and cyanide; sample 2329 (location 01.SB197, interval 8-8.8 ft) was not analyzed for cyanide. 


\begin{tabular}{|c|c|c|c|c|c|c|c|c|c|c|c|c|c|}
\hline \multirow[t]{2}{*}{-1} & & & & & & & & & & & & & $\mathbf{L}_{\mathbf{d}_{\mathbf{s}}}$ \\
\hline & & & & & & & & & & & & & $4 I_{6 \pi}$ \\
\hline \multirow[t]{7}{*}{ I } & $L \cdot 1$ & $\$ 9^{\circ} 0$ & $\boldsymbol{I}$ & $60^{\circ} \mathrm{b}$ & $9^{\circ} 0 \varepsilon$ & I & $90^{\circ} \varepsilon$ & $9^{\circ} \mathrm{SZ}$ & $\Omega$ & & $6.62 I$ & $9 I^{\circ} 0$ & urn!!!ు \\
\hline & & & & & & & & & & & & & $0 \mathrm{O}_{0 \theta}$ \\
\hline & $E^{\prime} I$ & $1 \cdot 8$ & m & & $1 t^{\circ} 0$ & $\Omega$ & & SS'0 & $\mathrm{fn}$ & & $28^{\circ} 0$ & $9 E^{\circ} 0$ & ${ }^{1} S_{06}$ \\
\hline & & & & $82^{\circ} 0$ & $86^{\circ} \mathrm{I}$ & & $\$ 20$ & $\angle E^{\circ} O$ & & & & 6.0 & $\mathbf{B}_{8 x z}$ \\
\hline & & & & & & & & & & & & 59.0 & घy \\
\hline & $00 \% 1$ & $8 E^{\circ} S$ & & $9 L^{\prime} 1$ & I6.0E & & $65^{\circ} 0$ & $t I^{\circ} S$ & & & & $29^{\circ} 6$ & $\mathbf{X}_{0 b}$ \\
\hline & $15^{\circ} 0$ & $6 L \cdot 2 Z$ & & $\angle E^{\prime} O$ & $82^{\circ} 02$ & & $6 \varepsilon^{\circ} 0$ & SO'SI & $\Omega$ & & $b^{\circ} \mathrm{Cl}$ & $\mathrm{SI} 0$ & $\mathbf{S O}_{L \varepsilon I}$ \\
\hline \multirow[t]{2}{*}{1} & OE' 9 & $\varepsilon^{\prime} 9 t$ & I & $06^{\circ} 6$ & $2 \cdot 16$ & f & $I L$ & $t+5 s$ & $\mathrm{In}$ & & $\tau \varepsilon^{\circ} 0$ & $69^{\circ} S I$ & B]әg SSOID \\
\hline & $D E^{\circ} 0$ & $\angle L O O$ & & $8 t^{\circ} 0$ & $b^{\circ} I$ & & $62^{\circ} 0$ & $29^{\circ} 0$ & $\Omega$ & & $\angle I^{\circ} O$ & $6^{\circ} 0$ & $\Omega_{8 \varepsilon z}$ \\
\hline \multirow[t]{2}{*}{$\Omega$} & & $\angle 1^{\circ} O$ & $\Omega$ & & $90^{\circ} 0$ & In & & $90 \%$ & $\Omega$ & & $81^{\circ} 0$ & II'0 & $\Omega_{s \pi}$ \\
\hline & $9 E^{\circ} 0$ & 6.0 & & $\$ 0$ & $I^{\circ} I$ & & SE 0 & $08^{\circ} 0$ & $\Omega$ & & $\angle I^{\circ} 0$ & $88^{\circ} 0$ & $\Omega_{\text {pqukz }}$ \\
\hline \multirow[t]{2}{*}{ I } & $\$ 20$ & $E t^{\circ} 0$ & & $29^{\circ} 0$ & $L \cdot 1$ & I & 220 & 620 & $\mathbf{d}$ & 60.0 & $90^{\circ} 0^{-}$ & $E I^{\prime} I$ & ULz⿰丿 \\
\hline & $E t^{\circ} 0$ & $00^{\circ} \mathrm{I}$ & & $\angle O$ & $0: 2$ & & $E S^{\circ} 0$ & $0 Z \cdot I$ & $\mathbf{d}$ & $60 \%$ & $90^{\circ} 0^{-}$ & $\angle I^{\circ} I$ & LLorz \\
\hline \multirow[t]{2}{*}{ I } & $0 \varepsilon^{\circ} 0$ & $95^{\circ} 0$ & I & $09 \%$ & $09^{\circ} I$ & $\boldsymbol{I}$ & EE. 0 & ES'O & $\mathbf{y}$ & $28^{\circ} 0$ & $9 L^{\circ} 0$ & $\nabla L E$ & $\mathrm{YL}_{8 \pi z}$ \\
\hline & & & & $91^{\circ} 0$ & $\angle 6^{\circ} 0$ & & $\$ I^{\circ} 0$ & $9 \varepsilon^{\circ} 0$ & & & & $\$ 9^{\circ} 0$ & $\mathrm{Bd}_{9 x}$ \\
\hline $\mathrm{In}$ & & $6 \varepsilon^{\circ} 0$ & $\mathrm{In}$ & $S Z 0$ & 620 & $\mathbf{X}$ & SZO & $90^{\circ} 0$ & & $\angle E^{\circ} O$ & $\angle 9^{\circ} 0$ & & $n_{d}{ }_{8 z}$ \\
\hline In & & $6 \varepsilon^{\circ} 0$ & I & sto & $S L O$ & $\mathbf{y}$ & 00.0 & $00^{\circ} 0$ & $\Omega$ & & 120 & & $n_{\text {dorer }}$ \\
\hline I & 620 & $05^{\circ} 0$ & I & $62^{\circ} 0$ & $69^{\circ} 1$ & & & & & $L \cdot S$ & $\varepsilon Z \cdot S Z$ & & ur $_{\text {In }}$ \\
\hline I & $I^{\circ} \varepsilon$ & $8^{\circ} t$ & ᄃ & $\mathrm{OI}^{\circ} \mathrm{S}$ & $\boldsymbol{b} \boldsymbol{t} \boldsymbol{I}$ & I & $8^{\circ} \varepsilon$ & 8.21 & $\mathrm{\rho} \Omega$ & 6.0 & $t 2 \cdot 0$ & $E 8^{\circ} I Z$ & $\begin{array}{l}\text { Budit } \\
\text { ssoID }\end{array}$ \\
\hline 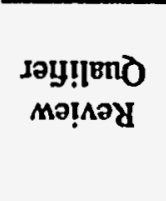 & 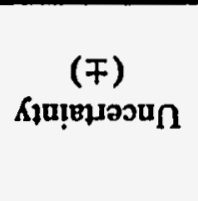 & $\begin{array}{c}(8 / !) d) \\
\text { uo!jeม! } \\
\text {-uอouo }\end{array}$ & 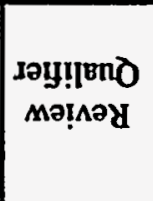 & 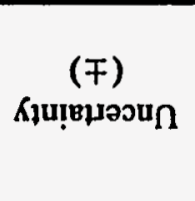 & 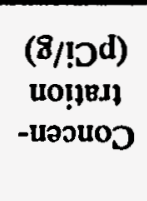 & 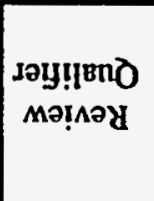 & 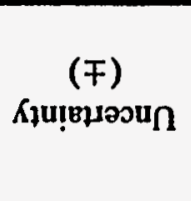 & $\begin{array}{c}\left(\boldsymbol{g} / ! \mathrm{O}^{\mathrm{d}}\right) \\
\text { uo!!вd! } \\
\text {-uวouoว }\end{array}$ & 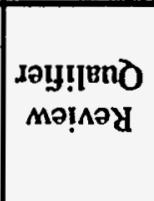 & 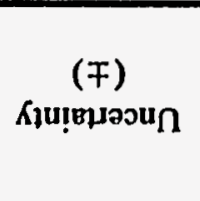 & $\begin{array}{c}\text { (T/!od) } \\
\text { uo!̣en } \\
\text {-นองuoว }\end{array}$ & 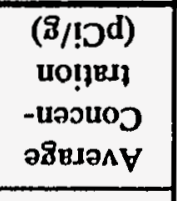 & \\
\hline & 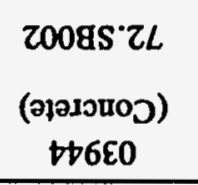 & & & $\begin{array}{c}\text { I008S`てL } \\
\text { (I!OS) } \\
\text { EE6EO }\end{array}$ & & & 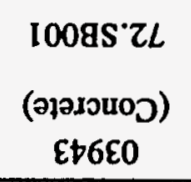 & & & $\begin{array}{c}\text { Jesu!̣ ba D } \\
9 E 6 E 0\end{array}$ & & 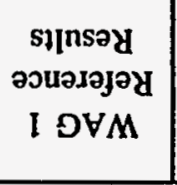 & 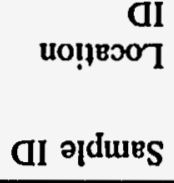 \\
\hline
\end{tabular}

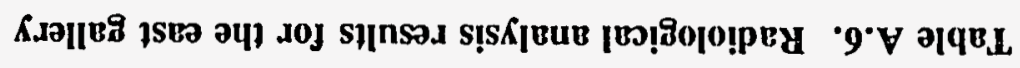




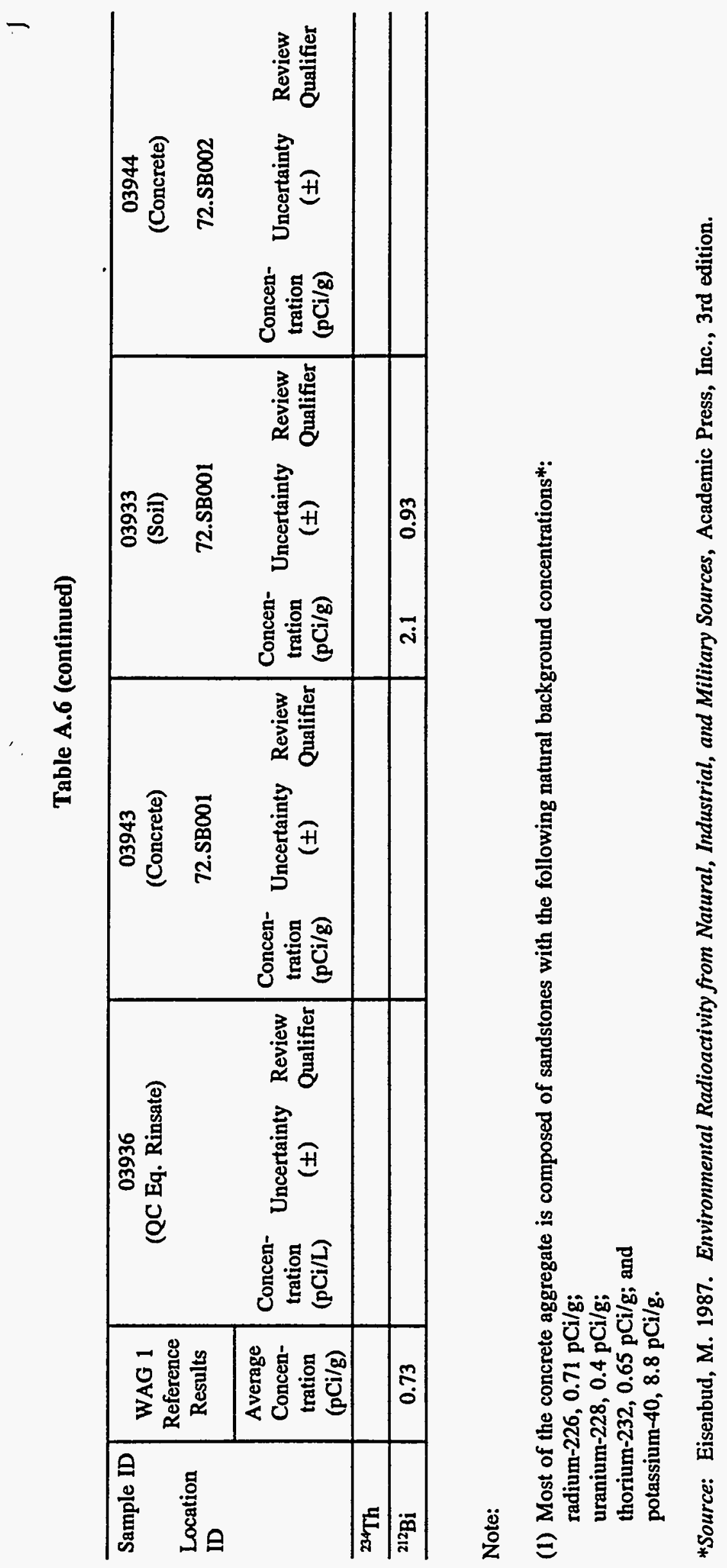


Table A.7 Radiological analysis results for the cell area

\begin{tabular}{|c|c|c|c|c|c|c|c|c|c|c|c|c|c|c|c|c|}
\hline \multirow{2}{*}{$\begin{array}{l}\text { Sample } \\
\text { ID } \\
\text { Location } \\
\text { ID }\end{array}$} & \multirow{2}{*}{$\begin{array}{c}\text { WAG 1 } \\
\text { Reference } \\
\text { Results } \\
\text { Average } \\
\text { Concen- } \\
\text { tration } \\
\text { (pCi/g) }\end{array}$} & \multicolumn{3}{|c|}{04044 (Concrele) } & \multicolumn{3}{|c|}{$\begin{array}{c}04045 \text { (Concrete) } \\
\text { (Container 3) } \\
72.5 B 004\end{array}$} & \multicolumn{3}{|c|}{$\begin{array}{c}04045 \text { (Concrete) } \\
\text { (Container 9) } \\
72.5 B 004\end{array}$} & \multicolumn{3}{|c|}{03935 (Soil) } & \multicolumn{3}{|c|}{$\begin{array}{c}03934 \text { (Sediment) } \\
72 . \text { SD001 }\end{array}$} \\
\hline & & $\begin{array}{l}\text { Concen- } \\
\text { tration } \\
(\mathrm{pCi} / \mathrm{g})\end{array}$ & $\begin{array}{c}\text { Uncertainty } \\
( \pm)\end{array}$ & $\begin{array}{l}\text { Review } \\
\text { Qualifier }\end{array}$ & $\begin{array}{l}\text { Concen- } \\
\text { tration } \\
(\mathrm{pCi} / \mathrm{g})\end{array}$ & $\begin{array}{c}\text { Uncertainty } \\
( \pm)\end{array}$ & $\begin{array}{l}\text { Review } \\
\text { Qualifier }\end{array}$ & $\begin{array}{l}\text { Concen- } \\
\text { tration } \\
(\mathrm{pCi} / \mathrm{g})\end{array}$ & $\begin{array}{c}\text { Uncertainty } \\
( \pm)\end{array}$ & $\begin{array}{l}\text { Review } \\
\text { Qualifier }\end{array}$ & $\begin{array}{l}\text { Concen- } \\
\text { tration } \\
(\mathrm{pCi} / \mathrm{g})\end{array}$ & $\begin{array}{c}\text { Uncertainty } \\
( \pm)\end{array}$ & $\begin{array}{l}\text { Review } \\
\text { Qualifier }\end{array}$ & $\begin{array}{l}\text { Concen- } \\
\text { tration } \\
(\mathrm{pCi} / \mathrm{g})\end{array}$ & $\begin{array}{l}\text { Uncertainty } \\
( \pm)\end{array}$ & $\begin{array}{l}\text { Review } \\
\text { Qualifier }\end{array}$ \\
\hline $\begin{array}{l}\text { Gross } \\
\text { Alpha }\end{array}$ & 21.83 & 3.80 & 3.30 & $\mathbf{J}$ & 0.0 & 12.90 & J & 1.7 & 13.60 & J & 13.6 & 4.70 & J & 1330 & 305 & $\mathbf{J}$ \\
\hline${ }^{211} \mathrm{Am}$ & & & & & & & & & & & 0.40 & 0.29 & J & 7.9 & & $\mathbf{U}$ \\
\hline $239 / 240 \mathrm{Pu}$ & & 0.48 & & UJ & 2.0 & 1.0 & J & 4.0 & 1.8 & $\mathbf{J}$ & 0.13 & & UJ & 35.40 & 7.80 & \\
\hline${ }^{238} \mathrm{Pu}$ & & 0.64 & & UJ & 0.0 & 0.14 & $\mathbf{R}$ & 0.45 & 0.44 & $\mathbf{J}$ & 0.03 & & UJ & 1.16 & 0.42 & J \\
\hline${ }^{226} \mathrm{Ra}$ & 0.65 & 0.401 & 0.17 & & & & & & & & 0.64 & 0.19 & & & & \\
\hline${ }^{228} \mathrm{Th}$ & 3.74 & 0.84 & 0.57 & J & 0.33 & & UJ & 0.38 & 0.27 & $\mathbf{J}$ & 1.40 & 0.50 & J & 0.54 & 0.22 & \\
\hline${ }^{230} \mathrm{Th}$ & 1.17 & 1.40 & 0.77 & J & 1.10 & 0.53 & & 1.20 & 0.56 & & 1.70 & 0.58 & & 0.04 & & UJ \\
\hline${ }^{232} \mathrm{Th}$ & 1.13 & 0.87 & 0.56 & $\mathbf{J}$ & 0.35 & 0.27 & J & 0.52 & 0.32 & $\mathbf{J}$ & 1.40 & 0.52 & $\mathbf{J}$ & 0.55 & 0.22 & \\
\hline $233234 \mathrm{U}$ & 0.88 & 0.49 & 0.27 & & 1.10 & 0.45 & & 1.30 & 0.49 & & 0.96 & 0.31 & & 3.43 & 0.84 & \\
\hline${ }^{235} \mathrm{U}$ & 0.11 & 0.07 & & $\mathbf{U}$ & 0.06 & & $\mathbf{U}$ & 0.12 & 0.11 & & 0.04 & & $\mathbf{U}$ & 0.16 & & $\mathbf{U}$ \\
\hline${ }^{238} \mathrm{U}$ & 0.9 & 0.62 & 0.31 & & 0.92 & 0.38 & & 1.70 & 0.61 & & 0.87 & 0.29 & & 2.24 & 0.59 & \\
\hline $\begin{array}{l}\text { Gross } \\
\text { Beta } \\
\end{array}$ & 15.69 & 53.6 & 6.6 & J & 1997 & 20.8 & J & 2464.7 & 26.3 & J & 63.7 & 8.80 & $\mathbf{J}$ & 20200 & 4040 & \\
\hline${ }^{137} \mathrm{Cs}$ & 0.15 & 34.54 & 0.66 & & 1535.0 & 4.0 & & 1375.0 & 3.0 & & 11.53 & 0.40 & & 30800 & 3400 & \\
\hline${ }^{40} \mathrm{~K}$ & 9.62 & 5.28 & 1.20 & & 4.76 & 1.12 & & 3.47 & 0.91 & & 19.59 & 2.08 & & 9.43 & 6.19 & \\
\hline${ }^{226} \mathrm{Ra}$ & 0.65 & & & & & & & & & & & & & & & \\
\hline${ }^{228} \mathrm{Ra}$ & 0.9 & & & & & & & & & & 1.04 & 0.34 & & & & \\
\hline${ }^{\infty} \mathrm{Sr}$ & 0.36 & 0.34 & & $\mathbf{U}$ & 12.8 & 1.9 & & 11.6 & 1.5 & & 0.41 & & UJ & 97.5 & 9.81 & \\
\hline${ }^{60} \mathrm{Co}$ & & & & & 0.25 & 0.09 & & 0.13 & 0.07 & & & & & 3.43 & 0.63 & \\
\hline
\end{tabular}


Table A.7 (continued)

\begin{tabular}{|c|c|c|c|c|c|c|c|c|c|c|c|c|c|c|c|c|}
\hline \multirow{2}{*}{$\begin{array}{l}\text { Sample } \\
\text { ID } \\
\text { Location } \\
\text { ID }\end{array}$} & \multirow{2}{*}{$\begin{array}{c}\text { WAG } 1 \\
\text { Reference } \\
\text { Results } \\
\text { Average } \\
\text { Concen- } \\
\text { tration } \\
\text { (pCi/g) }\end{array}$} & \multirow[b]{2}{*}{$\begin{array}{l}\text { Concen- } \\
\text { Iration } \\
(\mathrm{pCi} / \mathrm{g})\end{array}$} & \multicolumn{2}{|c|}{4044 (Concrete) } & \multicolumn{3}{|c|}{$\begin{array}{c}04045 \text { (Concrete) } \\
\text { (Container 3) } \\
72.5 B 004\end{array}$} & \multicolumn{3}{|c|}{$\begin{array}{c}04045 \text { (Concrete) } \\
\text { (Container 9) } \\
72.5 B 004\end{array}$} & \multicolumn{3}{|c|}{$\begin{array}{c}03935 \text { (Soil) } \\
72.5 B 004\end{array}$} & \multicolumn{3}{|c|}{03934 (Sediment) } \\
\hline & & & $\begin{array}{l}\text { Uncertainty } \\
\text { (土) }\end{array}$ & $\begin{array}{l}\text { Review } \\
\text { Qualifier }\end{array}$ & $\begin{array}{l}\text { Concen- } \\
\text { tration } \\
(\mathrm{pCi} / \mathrm{g})\end{array}$ & $\begin{array}{c}\text { Uncertainty } \\
( \pm)\end{array}$ & $\begin{array}{l}\text { Review } \\
\text { Qualifier }\end{array}$ & $\begin{array}{l}\text { Concen- } \\
\text { tration } \\
(\mathrm{pCi} / \mathrm{g})\end{array}$ & $\begin{array}{c}\text { Uncertainty } \\
\text { (土) }\end{array}$ & $\begin{array}{l}\text { Review } \\
\text { Qualifier }\end{array}$ & $\begin{array}{l}\text { Concen- } \\
\text { tration } \\
(\mathrm{pCi} / \mathrm{g})\end{array}$ & $\begin{array}{l}\text { Uncertainty } \\
( \pm)\end{array}$ & $\begin{array}{l}\text { Review } \\
\text { Qualifier }\end{array}$ & $\begin{array}{l}\text { Concen- } \\
\text { tration } \\
(\mathrm{pCi} / \mathrm{g})\end{array}$ & $\begin{array}{l}\text { Uncertainty } \\
\qquad( \pm)\end{array}$ & $\begin{array}{l}\text { Review } \\
\text { Qualifier }\end{array}$ \\
\hline Tritium & 0.16 & 21.1 & 2.78 & J & 0.86 & 1.65 & $\mathbf{J}$ & 2.04 & 2.73 & J & & & & 1.00 & 0.16 & \\
\hline${ }^{29} \mathrm{Th}$ & & & & & & & & & & & & & & 18.70 & 2.90 & \\
\hline${ }^{145} \mathrm{Pm}$ & & & & & 87.2 & 5.14 & & 16.9 & 13.6 & & & & & & & \\
\hline${ }^{234} \mathrm{Th}$ & & 6.31 & 2.90 & & & & & & & & & & & & & \\
\hline${ }^{212} \mathrm{Bi}$ & 0.73 & & & & & & & & & & & & & & & \\
\hline
\end{tabular}

Note:

(1) Most of the concrete aggregate is composed of sandstones with the following natural background concentrations*:

radium-226, $0.71 \mathrm{pCi} / \mathrm{g}$;

uranium-228, $0.4 \mathrm{pCi} / \mathrm{g}$;

thorium-232, $0.65 \mathrm{pCi} / \mathrm{g}$; and

potassium-40, 8.8 $\mathrm{pCi} / \mathrm{g}$.

*Source: Eisenbud, M. 1987. Environmental Radioactivity from Natural, Industrial, and Military Sources, Academic Press, Inc., 3rd edition. 
Table A.8. Radionuclide concentrations in WAG 1 soil samples taken near Building 3506

\begin{tabular}{|c|c|c|c|c|c|c|c|c|c|c|c|c|}
\hline \multirow{3}{*}{$\begin{array}{l}\text { Sample ID } \\
\text { Location } \\
\text { ID }\end{array}$} & & \multicolumn{3}{|c|}{2293} & \multicolumn{3}{|c|}{$2294^{a}$} & \multicolumn{3}{|c|}{2329} \\
\hline & \multicolumn{3}{|c|}{ 01.SB196 } & \multicolumn{3}{|c|}{$01 . S B 197$} & \multicolumn{3}{|c|}{ 01.SB197 } & \multicolumn{3}{|c|}{ 01.SB197 } \\
\hline & $\begin{array}{c}\text { Concen- } \\
\text { tration } \\
(\mathrm{pCi} / \mathrm{g})\end{array}$ & $\begin{array}{c}\text { Uncertainty } \\
( \pm)\end{array}$ & $\begin{array}{l}\text { Review } \\
\text { Qualifier }\end{array}$ & $\begin{array}{c}\text { Concen- } \\
\text { tration } \\
(\mathrm{pCi} / \mathrm{g})\end{array}$ & $\begin{array}{c}\text { Uncertainty } \\
( \pm)\end{array}$ & $\begin{array}{l}\text { Review } \\
\text { Qualifier }\end{array}$ & $\begin{array}{c}\text { Concen- } \\
\text { tration } \\
(\mathrm{pCi} / \mathrm{g})\end{array}$ & $\begin{array}{l}\text { Uncertainty } \\
( \pm)\end{array}$ & $\begin{array}{l}\text { Review } \\
\text { Qualifier }\end{array}$ & $\begin{array}{l}\text { Concen- } \\
\text { tration } \\
(\mathrm{pCi} / \mathrm{g})\end{array}$ & $\begin{array}{l}\text { Uncertainty } \\
( \pm)\end{array}$ & $\begin{array}{l}\text { Review } \\
\text { Qualifier }\end{array}$ \\
\hline $\begin{array}{l}\text { Gross } \\
\text { Alpha }\end{array}$ & 48 & 22.2 & & 30.5 & 30.5 & & 85.5 & 30.5 & & 10.2 & 10.1 & \\
\hline Gross Beta & 77.9 & 15 & & 37.3 & 9.1 & & 101.2 & 18.4 & & 16.1 & 6.6 & \\
\hline $\begin{array}{l}\text { Total } \\
\text { Strontium }\end{array}$ & 0.12 & 0.3 & UJ & 2.83 & 0.6 & & 2.76 & 0.59 & & 6.73 & 0.99 & \\
\hline${ }^{3} \mathrm{H}$ & 0.11 & 0.02 & & 0.13 & 0.01 & & 0.2 & 0.02 & & 0.14 & 0.02 & \\
\hline${ }^{137} \mathrm{Cs}$ & 3.94 & 0.35 & & 0.89 & 0.2 & & 1.3 & 0.18 & & & & \\
\hline${ }^{40} \mathrm{~K}$ & 25 & 3.2 & & 9.66 & 2.36 & & 6.9 & 2.01 & & 11.3 & 2.1 & \\
\hline${ }^{212} \mathrm{~Pb}$ & 1.02 & 0.22 & & 0.85 & 0.25 & & 0.73 & 0.19 & & 1.01 & 0.24 & \\
\hline${ }^{228} \mathbf{R a}$ & 1.75 & 0.34 & & 0.93 & 0.45 & & 1.09 & 0.41 & & 1.41 & 0.51 & \\
\hline${ }^{206} \mathrm{Tl}$ & 0.96 & 0.45 & & 0.72 & 0.36 & & 0.89 & 0.34 & & 1.39 & 0.36 & \\
\hline${ }^{206} \mathrm{Ra}$ & 0.59 & 0.29 & & 0.43 & 0.26 & & 0.54 & 0.23 & $\mathbf{J}$ & 0.43 & 0.18 & \\
\hline${ }^{214} \mathrm{~Pb}$ & 0.46 & 0.3 & & 0.75 & 0.25 & & 0.65 & 0.2 & & & & \\
\hline${ }^{238} U$ & 6.48 & 0.92 & $\mathbf{J}$ & 1.53 & 0.32 & $\mathbf{J}$ & 39.2 & 9.2 & & 4.1 & 3.38 & \\
\hline${ }^{235} \mathrm{U}$ & 0.14 & 0.08 & $\mathbf{J}$ & 0.06 & 0.05 & $\mathbf{J}$ & 0.59 & 0.89 & UJ & & & \\
\hline${ }^{234} \mathrm{U}$ & 1.91 & 0.37 & $\mathbf{J}$ & 0.35 & 0.24 & $\mathbf{J}$ & 9.06 & 3.65 & & & & \\
\hline${ }^{90} \mathrm{Tc}$ & 0.32 & 0.43 & UJ & & & & & & & & & \\
\hline${ }^{232} \mathrm{Th}$ & 1.33 & 0.28 & $\mathbf{J}$ & 1.2 & 0.28 & $\mathbf{J}$ & 1.19 & 0.34 & $\mathbf{J}$ & 1.41 & 0.51 & \\
\hline $230 \mathrm{Th}$ & 1.05 & 0.24 & J & 0.91 & 0.24 & J & 0.93 & 0.29 & $\mathbf{J}$ & & & \\
\hline${ }^{208} \mathrm{Th}$ & 1.45 & 0.29 & $\mathbf{J}$ & 1.37 & 0.31 & $\mathrm{~J}$ & 0.96 & 0.37 & & 0.97 & 0.23 & \\
\hline${ }^{214} \mathrm{Bi}$ & & & & 0.45 & 0.26 & & & & & & & \\
\hline${ }^{228} \mathrm{AC}$ & & & & & & & 1.09 & 0.41 & & & & \\
\hline
\end{tabular}

aDuplicate of sample 2293. 
Table A.9. Location-specific measurement results for the east gallery

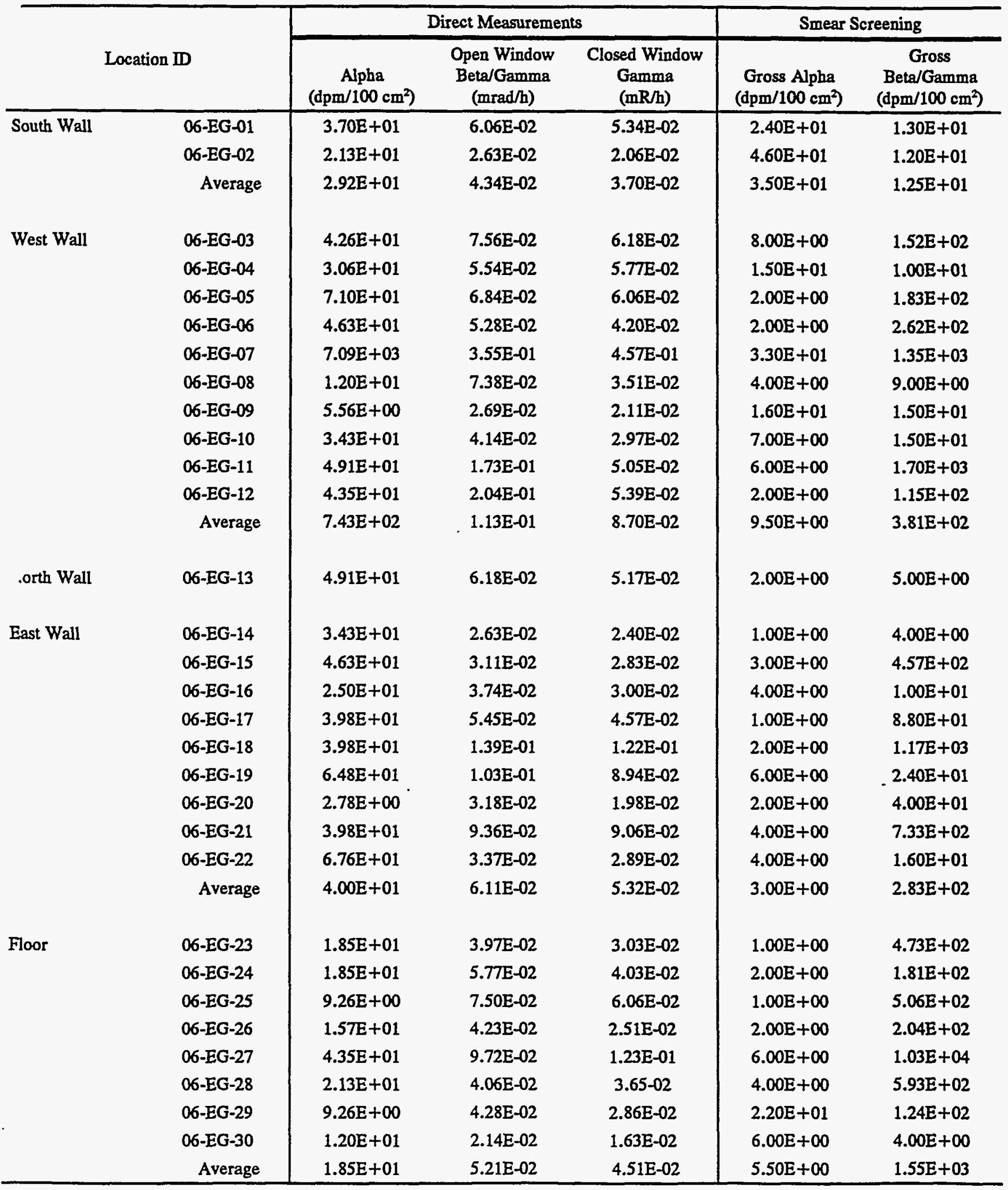


Table A.10. CSL gamma spectroscopy results for east gallery smears

\begin{tabular}{|c|c|c|c|}
\hline \multicolumn{2}{|c|}{ Location ID } & \multirow{2}{*}{$\frac{\begin{array}{c}{ }^{137} \mathrm{Cs} /{ }^{137 m} \mathrm{Ba} \\
\left(\mu \mathrm{Ci} / 1\left(10 \mathrm{~cm}^{2}\right)\right.\end{array}}{7.72 \mathrm{E}-06}$} & \multirow{2}{*}{$\begin{array}{c}{ }^{60} \mathrm{Co} \\
\left(\mu \mathrm{Ci} / 100 \mathrm{~cm}^{2}\right)\end{array}$} \\
\hline South Wall & 06-EG-01 & & \\
\hline & 06-EG-02 & 8.49]E106 & \\
\hline \multirow[t]{9}{*}{ West Wall } & $06-E G-03$ & $3.80 \mathrm{E}-05$ & \\
\hline & 06-EG-04 & $8.88 \mathrm{E}-06$ & \\
\hline & 06-EG-05 & $4.98 \mathrm{E}-05$ & \\
\hline & 06-EG-06 & $1.08 \mathrm{E}-04$ & \\
\hline & 06-EG-07 & $1.89 \mathrm{E}-0.4$ & \\
\hline & 06-EG-08 & $9.07 E-06$ & \\
\hline & 06-EG-10 & $9.84 E-06$ & \\
\hline & 06-EG-11 & $3.64 \mathrm{E}-0.4$ & $1.46 \mathrm{E}-05$ \\
\hline & 06-EG-12 & $3.26 \mathrm{E}-05$ & $1.53 E-05$ \\
\hline North Wall & 06-EG-13 & *k & \\
\hline \multirow[t]{7}{*}{ East Wall } & 06-EG-15 & $3.78 E-05$ & $1.19 E-05$ \\
\hline & 06-EG-16 & * & $1.37 \mathrm{E}-05$ \\
\hline & 06-EG-17 & $4.03 E-05$ & \\
\hline & 06-EG-18 & $5.03 E-04$ & \\
\hline & 06-EG-19 & $1.33 E-05$ & \\
\hline & 06-EG-20 & $1.76 \mathrm{E}-05$ & \\
\hline & 06-EG-21 & $3.02 \mathrm{E}-04$ & \\
\hline \multirow[t]{8}{*}{ Floor } & $06-E G-23$ & $2.00 \mathrm{E}-04$ & \\
\hline & 06-EG-24 & $7.35 \mathrm{E}-05$ & $8.69 E-06$ \\
\hline & 06-EG-25 & $2.28 \mathrm{E}-04$ & \\
\hline & 06-EG-26 & $7.78 \mathrm{E}-05$ & \\
\hline & 06-EG-27 & $1.38 \mathrm{E}-03$ & \\
\hline & 06-EG-28 & $2.19 \mathrm{E}-04$ & \\
\hline & 06-EG-29 & $4.94 \mathrm{E}-05$ & \\
\hline & 06-EG-30 & * & \\
\hline
\end{tabular}

*Not detected or not measured. 
Table A.11. Slit scanning results for east gallery floor core (location 72.SB001)

\begin{tabular}{cccc}
\hline $\begin{array}{c}\text { Core } \\
\begin{array}{c}\text { Position } \\
\text { (in.) }\end{array}\end{array}$ & $\begin{array}{c}\text { Region of } \\
\text { Interest } \\
\text { (counts) }^{\text {and }}\end{array}$ & $\begin{array}{c}\text { Area Bkg. } \\
\text { (counts) }^{a}\end{array}$ & $\begin{array}{c}\text { Measured } \\
\text { Activity vs. Bkg. } \\
\text { (nCi/cm }{ }^{3} \text { ) }\end{array}$ \\
\hline 0.25 & 18 & 3.12 & Bkg. level \\
0.5 & 22 & 7.12 & Bkg. level \\
0.75 & 12.05 & -2.83 & Bkg. level \\
1 & 19 & 4.12 & Bkg. level \\
1.25 & 34 & 19.12 & 1.9 \\
1.5 & 32 & 17.12 & 1.7 \\
1.75 & 19.5 & 4.62 & Bkg. level \\
2 & 29.5 & 14.62 & 1.4 \\
2.25 & 29.5 & 14.62 & 1.4 \\
2.5 & 19.5 & 4.62 & Bkg. level \\
3.5 & 7.5 & -7.38 & Bkg. level \\
4.5 & 22.5 & 7.62 & Bkg. level \\
5.5 & 14.5 & -0.38 & Bkg. level \\
6.5 & 8.5 & -6.38 & Bkg. level \\
7.5 & 20 & 5.12 & Bkg. level \\
8.5 & 16.5 & 1.62 & Bkg. level \\
\hline
\end{tabular}

${ }^{a}$ Counts are for 4 minutes; core was rotated $90^{\circ}$ each minute.

${ }^{b}$ Area background was measured to be 15 counts per $4 \mathrm{~min}$. in the region of interest.

If ROI counts were less than $\mathrm{L}_{C}=\mathrm{CTS}_{\mathrm{B}}+2 \sigma_{\mathrm{B}}$,

where $\sigma_{B}=\sqrt{\text { CTS }_{B}}$,

then the measurement was assumed to be at or below the area background level. Here, $L_{c}=23$ counts per 4 min. 
Table A.12. Slit scanning results for east gallery wall core (location 72.SBO0.2)

\begin{tabular}{|c|c|c|c|}
\hline $\begin{array}{c}\text { Core } \\
\text { Position } \\
\text { (in.) }\end{array}$ & $\begin{array}{c}\text { Region of } \\
\text { Interest (counts) }\end{array}$ & $\begin{array}{l}\text { Area Blig. } \\
\text { (counts) }\end{array}$ & $\begin{array}{c}\text { Measured } \\
\text { Activity vs. Bkg. } \\
\left(\mathrm{nCi} / \mathrm{cm}^{3}\right)\end{array}$ \\
\hline 0.25 & 37.5 & 22.62 & 2.2 \\
\hline 0.5 & 67.5 & 52.62 & 5.2 \\
\hline 0.75 & 47 & 32.12 & 3.2 \\
\hline 1 & 50.5 & 35.62 & 3.5 \\
\hline 1.25 & 36.5 & 21.62 & 2.1 \\
\hline 1.5 & 27 & 12.12 & 1.2 \\
\hline 1.75 & 49 & 34.12 & 3.4 \\
\hline 2. & 53 & 38.12 & 3.8 \\
\hline 2.25 & 57 & 42.12 & 4.1 \\
\hline 2.5 & 43 & 28.12 & 2.8 \\
\hline 3.19 & 55 & 40.12 & 3.9 \\
\hline 4.19 & 58 & 43.12 & 4.2 \\
\hline 5.19 & 66 & 51.12 & 5.0 \\
\hline 6.19 & 65.5 & 50.62 & 5.0 \\
\hline 7.19 & 54.5 & 39.62 & 3.9 \\
\hline 8.19 & 76 & 61.12 & 6.0 \\
\hline 9.19 & 84.5 & 69.62 & 6.9 \\
\hline 10.19 & 57.5 & 42.62 & 4.2 \\
\hline 11.19 & 56 & 41.12 & 4.0 \\
\hline 12.19 & 49.5 & 34.62 & 3.4 \\
\hline 13.19 & 51.5 & 36.62 & 3.6 \\
\hline 14.19 & 52 & 37.12 & 3.7 \\
\hline 15.19 & 49.5 & 34.62 & 3.4 \\
\hline 16.19 & 47 & 32.12 & 3.2 \\
\hline 17.19 & 51.5 & 36.6 .2 & 3.6 \\
\hline 18.19 & 52.5 & 37.6 .2 & 3.7 \\
\hline 19.19 & 48.5 & 33.6 .2 & 3.3 \\
\hline 20.19 & 48 & 33.12 & 3.3 \\
\hline 21.19 & 61 & 46.12 & 4.5 \\
\hline 21.44 & 53 & 38.12 & 3.8 \\
\hline 21.69 & 41 & 26.12 & 2.6 \\
\hline 21.94 & 53 & 38.12 & 3.8 \\
\hline 22.19 & 67.5 & 52.62 & 5.2 \\
\hline 22.44 & 56 & 41.12 & 4.0 \\
\hline 22.69 & 39.5 & 24.62 & 2.4 \\
\hline 22.94 & 50.5 & 35.62 & 3.5 \\
\hline 23.19 & 67.5 & 52.62 & 5.2 \\
\hline 23.44 & 58 & 43.12 & 4.2 \\
\hline 23.69 & 59.5 & 44.62 & 4.4 \\
\hline
\end{tabular}




\section{Table A.12 (continued)}

${ }^{\circ}$ Counts are for 4 minutes; core was rotated $90^{\circ}$ each minute.

${ }^{b}$ Area background was measured to be 15 counts per $4 \mathrm{~min}$. in the region of interest.

If ROI counts were less than $\mathrm{L}_{C}=\mathrm{CTS}_{\mathrm{B}}+2 \sigma_{\mathrm{B}}$,

where $\sigma_{B}=\sqrt{\text { CTS }_{B}}$,

then the measurement was assumed to be at or below the area background level. Here, $L_{c}=23$ counts per $4 \mathrm{~min}$. 
Table A.13. Location-specific measurement results for the north portion of the vell

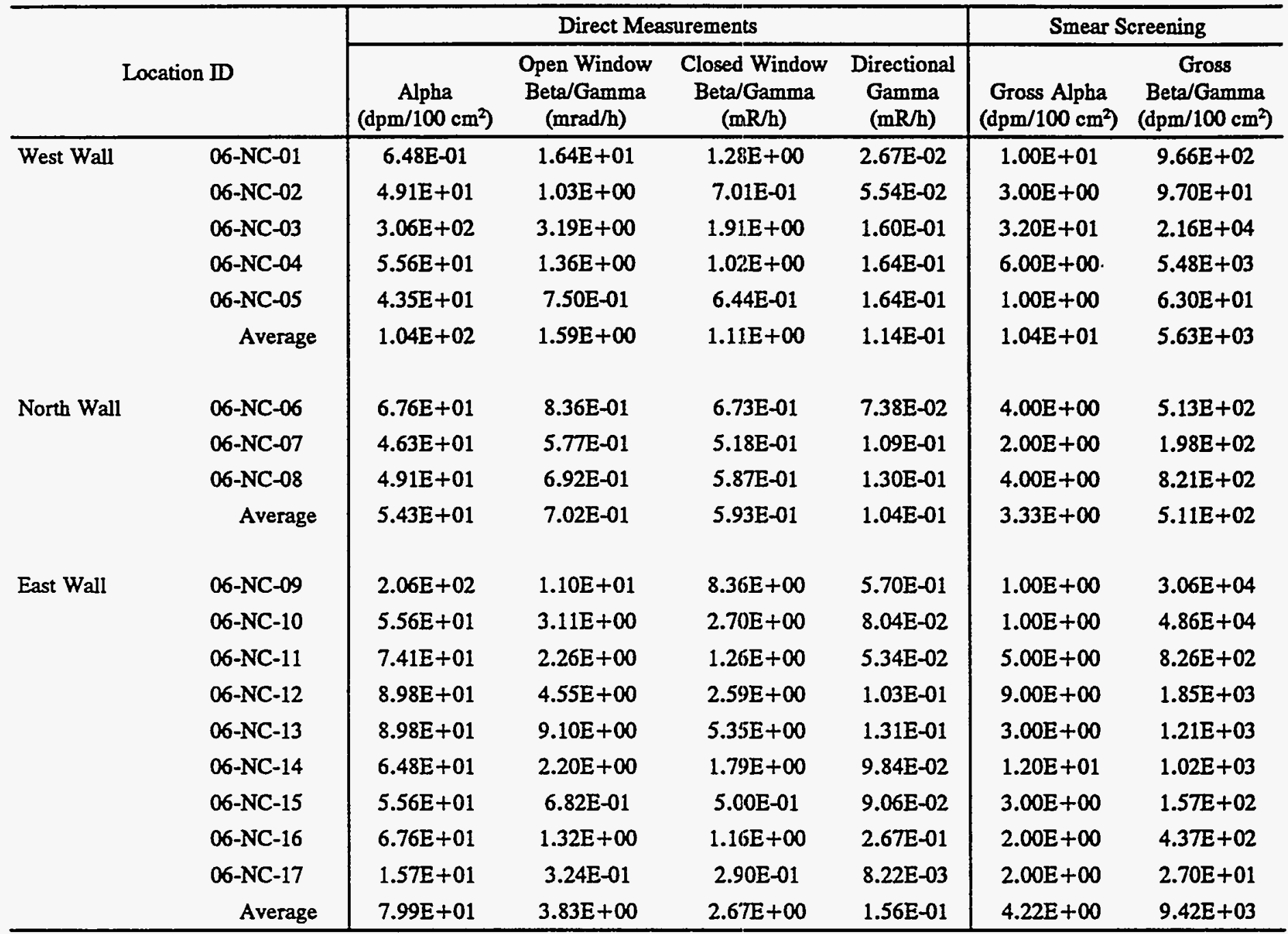


Table A.14. Location-specific measurement results for the south portion of the cell

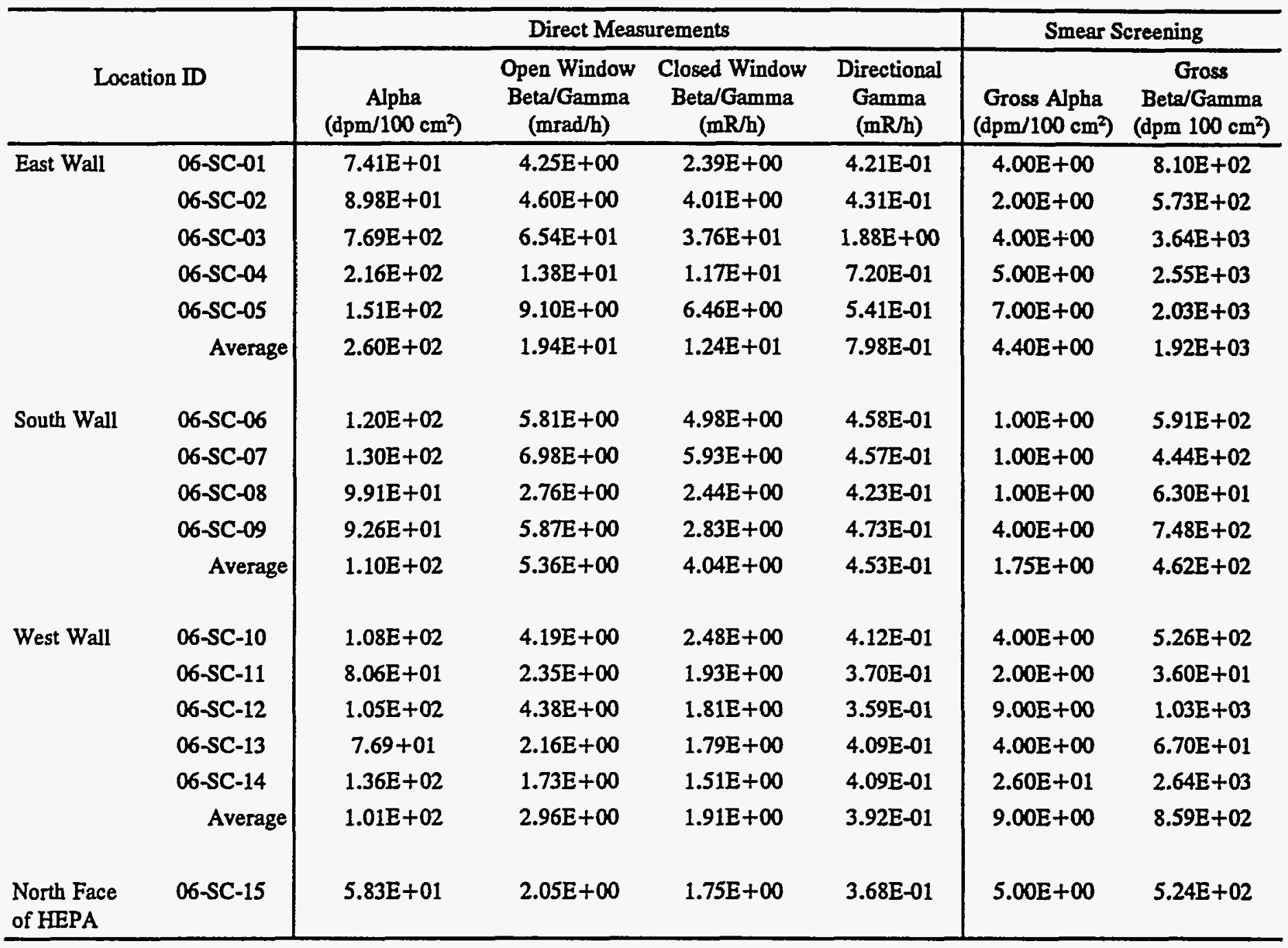


Table A.15. CSL gamma spectroscopy results for north cell area smears

\begin{tabular}{|c|c|c|c|}
\hline \multicolumn{2}{|c|}{ Location ID } & $\begin{array}{c}{ }^{137} \mathrm{Cs} /{ }^{1377} \mathrm{Ba} \\
\left(\mu \mathrm{Ci} / 100 \mathrm{~cm}^{2}\right)\end{array}$ & $\begin{array}{c}{ }^{206} \mathrm{Ra} \\
\left(\mu \mathrm{Ci} / 100 \mathrm{~cm}^{2}\right)\end{array}$ \\
\hline \multirow[t]{5}{*}{ West Wall } & $06-\mathrm{NC}-01$ & $4.33-04$ & \\
\hline & $06-\mathrm{NC}-02$ & $3.22 \mathrm{E}-1) 5$ & $7.08 \mathrm{E}-5$ \\
\hline & $06-\mathrm{NC}-03$ & $5.21 E-103$ & \\
\hline & $06-\mathrm{NC}-04$ & $1.29 \mathrm{E}-03$ & \\
\hline & $06-\mathrm{NC}-05$ & 2.68E- -15 & \\
\hline \multirow[t]{3}{*}{ North Wall } & $06-\mathrm{NC}-06$ & $2.30 \mathrm{E}-24$ & \\
\hline & $06-\mathrm{NC}-07$ & $8.80 \mathrm{E}-105$ & \\
\hline & 06-NC-08 & $3.47 \mathrm{E}-04$ & \\
\hline \multirow[t]{9}{*}{ East Wall } & $06-\mathrm{NC}-09$ & 8.35E- 03 & \\
\hline & $06-\mathrm{NC}-10$ & $1.29 \mathrm{E}-102$ & \\
\hline & $06-\mathrm{NC}-11$ & $3.75 \mathrm{E}-124$ & \\
\hline & $06-\mathrm{NC}-12$ & 8.13E-144 & \\
\hline & $06-N C-13$ & $5.28 \mathrm{E}-04$ & \\
\hline & 06-NC-14 & $3.76 \mathrm{E}-124$ & \\
\hline & 06-NC-15 & $7.35 \mathrm{E}-05$ & \\
\hline & $06-N C-16$ & 2.01E-104 & \\
\hline & $06-N C-17$ & & \\
\hline
\end{tabular}

Note: Blanks indicate no data. 
Table A.16. CSL gamma spectroscopy results for south cell area smears

\begin{tabular}{lc|c}
\hline \multicolumn{1}{c|}{ Location ID } & $\begin{array}{c}{ }^{137} \mathrm{Cs} /{ }^{137 m} \mathrm{Ba} \\
(\mu \mathrm{Ci} / \mathrm{smear})\end{array}$ \\
\hline East Wall & $06-\mathrm{SC}-01$ & $3.58 \mathrm{E}-04$ \\
& $06-\mathrm{SC}-02$ & $2.44 \mathrm{E}-04$ \\
& $06-\mathrm{SC}-03$ & $1.45 \mathrm{E}-03$ \\
& $06-\mathrm{SC}-04$ & $1.10 \mathrm{E}-03$ \\
& $06-\mathrm{SC}-05$ & $8.85 \mathrm{E}-04$ \\
South Wall & $06-\mathrm{SC}-06$ & \\
& $06-\mathrm{SC}-07$ & $2.35 \mathrm{E}-04$ \\
& $06-\mathrm{SC}-08$ & $1.95 \mathrm{E}-04$ \\
& $06-\mathrm{SC}-09$ & $2.59 \mathrm{E}-05$ \\
& & $3.22 \mathrm{E}-04$ \\
West Wall & $06-\mathrm{SC}-10$ & $2.16 \mathrm{E}-04$ \\
& $06-\mathrm{SC}-11$ & $1.35 \mathrm{E}-05$ \\
& $06-\mathrm{SC}-12$ & $4.46 \mathrm{E}-04$ \\
North Face & $06-\mathrm{SC}-13$ & $2.61 \mathrm{E}-05$ \\
of HEPA & $06-\mathrm{SC}-14$ & $1.21 \mathrm{E}-04$ \\
& & \\
& $06-\mathrm{SC}-15$ & $2.14 \mathrm{E}-04$ \\
& &
\end{tabular}


Table A.17. CSL strontium-90 analysis results for cell area smears

\begin{tabular}{c|cc|c}
\hline \multicolumn{2}{|c|}{ Location ID } & & $\begin{array}{c}{ }^{9} \text { Sr Activity } \\
\left(\mu \mathrm{Ci} / 100 \mathrm{~cm}^{2}\right)\end{array}$ \\
\hline North Cell Area & West Wall & $06-\mathrm{NC}-03$ & $7.69 \mathrm{E}-06$ \\
& & $06-\mathrm{NC}-04$ & $2.58 \mathrm{E}-05$ \\
& \multirow{3}{*}{ East Wall } & $06-\mathrm{NC}-09$ & $2.81 \mathrm{E}-05$ \\
& & $06-\mathrm{NC}-10$ & $1.29 \mathrm{E}-05$ \\
& & $06-\mathrm{NC}-12$ & $4.96 \mathrm{E}-06$ \\
& & $06-\mathrm{NC}-13$ & $3.57 \mathrm{E}-06$ \\
& & $06-\mathrm{SC}-03$ & $3.25 \mathrm{E}-06$ \\
& \multirow{4}{*}{ East Wall } & $06-\mathrm{SC}-04$ & $2.29 \mathrm{E}-06$ \\
& & $06-\mathrm{SC}-05$ & $3.68 \mathrm{E}-06$ \\
& & $06-\mathrm{SC}-14$ & $6.84 \mathrm{E}-06$ \\
\hline
\end{tabular}


Table A.18. Slit scanning results for cell area wall core (location 72.SBO03)

\begin{tabular}{|c|c|c|c|}
\hline $\begin{array}{c}\text { Core } \\
\text { Position } \\
\text { (in.) }\end{array}$ & $\begin{array}{l}\text { Region of Interest } \\
\text { (ROI) (counts) }\end{array}$ & $\begin{array}{l}\text { ROI Bkg. } \\
\text { (counts) }\end{array}$ & $\begin{array}{l}\text { Activity } \\
\left(\mathrm{nCi} / \mathrm{cm}^{3}\right)\end{array}$ \\
\hline 0.25 & 304.5 & 289.62 & 28.5 \\
\hline 0.5 & 210 & 195.12 & 19.2 \\
\hline 0.75 & 105 & 90.12 & 8.9 \\
\hline 1 & 75.5 & 60.62 & 6.0 \\
\hline 1.25 & 27 & 12.12 & 1.2 \\
\hline 1.5 & 23 & 8.12 & 0.8 \\
\hline 1.75 & 23.5 & 8.62 & 0.8 \\
\hline 2 & 23 & 8.12 & 0.8 \\
\hline 3 & 1.5 & -13.38 & Bkg. level \\
\hline 4 & 20.5 & 5.62 & 0.6 \\
\hline 6 & 0 & -14.88 & Bkg. level \\
\hline 8 & 14 & -0.88 & Bkg. level \\
\hline 10 & 26.5 & 11.62 & 1.1 \\
\hline 12 & 9 & -5.88 & Bkg. level \\
\hline 14 & 12.5 & -2.38 & Bkg. level \\
\hline 16 & 14 & -0.88 & Bkg. level \\
\hline 18 & 10 & -4.88 & Bkg. level \\
\hline 20 & 10.5 & -4.38 & Bkg. level \\
\hline 21.62 & 33 & 18.12 & 1.8 \\
\hline 21.87 & 10.5 & -4.38 & Bkg. level \\
\hline 22.12 & 3.5 & -11.38 & Bkg. level \\
\hline 22.37 & 13 & -1.88 & Bkg. level \\
\hline 23.62 & 25.5 & 10.62 & 1.0 \\
\hline 23.87 & 12 & -2.88 & Bkg. level \\
\hline 24.12 & 12.5 & -2.38 & Bkg. level \\
\hline 24.37 & 10 & -4.88 & Bkg. level \\
\hline 24.62 & 22 & 7.12 & 0.7 \\
\hline
\end{tabular}


Table A.19. Slit scanning results for cell area floor core (location 72.SB00-4)

\begin{tabular}{|c|c|c|c|c|}
\hline & $\begin{array}{c}\text { Core } \\
\text { Position } \\
\text { (in.) }\end{array}$ & $\begin{array}{l}\text { Region of } \\
\text { Interest } \\
\text { (counts) }\end{array}$ & $\begin{array}{l}\text { ROI Bkg. } \\
\text { (counts) }\end{array}$ & $\begin{array}{c}\text { Activity } \\
\left(\mathrm{nCi} / \mathrm{cm}^{3}\right)\end{array}$ \\
\hline \multirow[t]{20}{*}{ Piece 1} & 0.25 & 39 & 24.12 & 2.4 \\
\hline & 0.5 & 25.5 & 10.62 & 1.0 \\
\hline & 0.75 & 10 & -4.88 & Bkg. level \\
\hline & 1 & 19 & 4.12 & 0.4 \\
\hline & 1.25 & 11 & -3.88 & Bkg. level \\
\hline & 1.5 & 19.5 & 4.62 & 0.5 \\
\hline & 1.75 & 13 & -1.88 & Bkg. level \\
\hline & 2 & 21 & 6.12 & 0.6 \\
\hline & 2.5 & 17 & 2.12 & 0.2 \\
\hline & 3.25 & 17.5 & 2.62 & 0.3 \\
\hline & 4 & 9.5 & -5.38 & Bkg. level \\
\hline & 4.5 & 23.5 & 8.62 & 0.8 \\
\hline & 4.75 & 30 & 15.12 & 1.5 \\
\hline & 5 & 24 & 9.12 & 0.9 \\
\hline & 5.25 & 18 & 3.12 & 0.3 \\
\hline & 5.5 & 5 & -9.88 & Bkg. level \\
\hline & 5.75 & 33.5 & 18.62 & 1.8 \\
\hline & 6 & 21.5 & 6.62 & 0.7 \\
\hline & 6.25 & 32.5 & 17.62 & 1.7 \\
\hline & 6.5 & 54.5 & 39.62 & 3.9 \\
\hline \multirow[t]{15}{*}{ Piece 2} & 6.75 & 131 & 116.12 & 11.4 \\
\hline & 7 & 115.5 & 100.62 & 9.9 \\
\hline & 7.25 & 72 & 57.12 & 5.6 \\
\hline & 7.5 & 92 & 77.12 & 7.6 \\
\hline & 7.75 & 85.5 & 70.62 & 7.0 \\
\hline & 8 & 73 & 58.12 & 5.7 \\
\hline & 8.25 & 74 & 59.12 & 5.8 \\
\hline & 8.5 & 88.5 & 73.62 & 7.2 \\
\hline & 8.75 & 123.5 & 108.62 & 10.7 \\
\hline & 9 & 195 & 180.12 & 17.7 \\
\hline & 9.25 & 479 & 464.12 & 45.7 \\
\hline & 9.5 & 910.5 & 895.62 & 88.2 \\
\hline & 9.75 & 334.5 & 319.62 & 31.5 \\
\hline & 10 & 196 & 181.12 & 17.8 \\
\hline & 10.25 & 116.5 & 101.62 & 10.0 \\
\hline
\end{tabular}


Table A.19 (continued)

\begin{tabular}{|c|c|c|c|c|}
\hline & $\begin{array}{c}\text { Core } \\
\text { Position } \\
\text { (in.) }\end{array}$ & $\begin{array}{l}\text { Region of } \\
\text { Interest } \\
\text { (counts) }\end{array}$ & $\begin{array}{l}\text { ROI Bkg. } \\
\text { (counts) }\end{array}$ & $\begin{array}{c}\text { Activity } \\
\left(\mathrm{nCi} / \mathrm{cm}^{3}\right)\end{array}$ \\
\hline \multirow{19}{*}{ Piece 3} & 10.5 & 54 & 39.12 & 3.9 \\
\hline & 10.75 & 167.5 & 152.62 & 15.0 \\
\hline & 11 & 215.5 & 200.62 & 19.7 \\
\hline & 11.25 & 213.5 & 198.62 & 19.6 \\
\hline & 11.5 & 205.5 & 190.62 & 18.8 \\
\hline & 11.75 & 159 & 144.12 & 14.2 \\
\hline & 12 & 148.5 & 133.62 & 13.2 \\
\hline & 12.25 & 130 & 115.12 & 11.3 \\
\hline & 12.5 & 116.5 & 101.62 & 10.0 \\
\hline & 13.5 & 128.5 & 113.62 & 11.2 \\
\hline & 14.5 & 99.5 & 84.62 & 8.3 \\
\hline & 15.5 & 114.5 & 99.62 & 9.8 \\
\hline & 16.5 & 108.5 & 93.62 & 9.2 \\
\hline & 17.5 & 125 & 110.12 & 10.8 \\
\hline & 18.5 & 113 & 98.12 & 9.7 \\
\hline & 18.75 & 106.5 & 91.62 & 9.0 \\
\hline & 19 & 120.5 & 105.62 & 10.4 \\
\hline & 19.25 & 118 & 103.12 & 10.2 \\
\hline & 19.5 & 113 & 98.12 & 9.7 \\
\hline \multirow[t]{10}{*}{ Piece 4} & 19.75 & 83.5 & 68.62 & 6.8 \\
\hline & 20 & 38.5 & 23.62 & 2.3 \\
\hline & 20.25 & 41.5 & 26.62 & 2.6 \\
\hline & 20.5 & 36.5 & 21.62 & 2.1 \\
\hline & 21.5 & 44 & 29.12 & 2.9 \\
\hline & 22.75 & 38 & 23.12 & 2.3 \\
\hline & 23 & 40.5 & 25.62 & 2.5 \\
\hline & 23.25 & 33 & 18.12 & 1.8 \\
\hline & 23.5 & 48 & 33.12 & 3.3 \\
\hline & 23.75 & 47 & 32.12 & 3.2 \\
\hline \multirow[t]{6}{*}{ Piece 5} & 24 & 80 & 65.12 & 6.4 \\
\hline & 24.25 & 85.5 & 70.62 & 7.0 \\
\hline & 24.5 & 92 & 77.12 & 7.6 \\
\hline & 24.75 & 110.5 & 95.62 & 9.4 \\
\hline & 25.75 & 84.5 & 69.62 & 6.9 \\
\hline & 26.75 & 104.5 & 89.62 & 8.8 \\
\hline
\end{tabular}


Table A.19 (continued)

\begin{tabular}{c|c|c|c}
\hline $\begin{array}{c}\text { Core } \\
\text { Position } \\
\text { (in.) }\end{array}$ & $\begin{array}{c}\text { Region of } \\
\text { Interest } \\
\text { (counts) }\end{array}$ & $\begin{array}{c}\text { ROI Bkg. } \\
\text { (counts) }\end{array}$ & $\begin{array}{c}\text { Activity } \\
\left(\mathrm{nCi} / \mathrm{cm}^{3}\right)\end{array}$ \\
\hline 28.75 & 108 & 93.12 & 9.2 \\
31.75 & 106.5 & 91.62 & 9.0 \\
35.75 & 96 & 81.12 & 8.0 \\
38.75 & 93.5 & 78.62 & 7.7 \\
40.75 & 118 & 103.12 & 10.2 \\
42.75 & 114.5 & 99.62 & 9.8 \\
43.75 & 75 & 60.12 & 5.9 \\
44.75 & 133 & 118.12 & 11.6 \\
45.5 & 120.5 & 105.62 & 10.4 \\
45.75 & 84 & 69.12 & 6.8 \\
46 & 80.5 & 65.62 & 6.5 \\
46.25 & 44 & 29.12 & 2.9 \\
46.5 & 51 & 36.12 & 3.6 \\
46.75 & 72 & 57.12 & 5.6 \\
\hline
\end{tabular}


Table A.20. Analytical results for TLD strings in the cell

\begin{tabular}{|c|c|c|c|}
\hline String No. & $\begin{array}{l}\text { Elev from } \\
\text { Bottom (ft) }\end{array}$ & $\begin{array}{l}\mathrm{H}_{3} \text { Rate } \\
\text { (mrem/h) }\end{array}$ & $\begin{array}{l}\mathrm{H}_{\mathrm{d}} \text { Rate } \\
(\mathrm{mrem} / \mathrm{h})\end{array}$ \\
\hline \multirow[t]{10}{*}{1} & 14.5 & 0.5 & 0.4 \\
\hline & 12.5 & 0.6 & 0.6 \\
\hline & 10.5 & 0.6 & 0.5 \\
\hline & 8.5 & 0.6 & 0.6 \\
\hline & 6.5 & 0.7 & 0.9 \\
\hline & 4.5 & 0.9 & 0.7 \\
\hline & 3.5 & 1.4 & 0.9 \\
\hline & 2.5 & 2.1 & 1.0 \\
\hline & 1.5 & 3.1 & 1.2 \\
\hline & 0.5 & 2.8 & 1.2 \\
\hline \multirow[t]{10}{*}{2} & 14.5 & 1.6 & 1.4 \\
\hline & 12.5 & 2.0 & 1.6 \\
\hline & 10.5 & 2.4 & 2.3 \\
\hline & 8.5 & 2.3 & 1.9 \\
\hline & 6.5 & 2.8 & 2.7 \\
\hline & 4.5 & 2.4 & 2.0 \\
\hline & 3.5 & 2.5 & 2.1 \\
\hline & 2.5 & 2.8 & 2.2 \\
\hline & 1.5 & 4.5 & 2.8 \\
\hline & 0.5 & 5.2 & 2.7 \\
\hline \multirow[t]{10}{*}{3} & 14.5 & 8.9 & 5.9 \\
\hline & 12.5 & 98.3 & 16.7 \\
\hline & 10.5 & 70.2 & 18.4 \\
\hline & 8.5 & 90.4 & 24.2 \\
\hline & 6.5 & 78.8 & 19.3 \\
\hline & 4.5 & 47.9 & 12.4 \\
\hline & 3.5 & 32.5 & 9.1 \\
\hline & 2.5 & 33.1 & 7.8 \\
\hline & 1.5 & 35.5 & 7.0 \\
\hline & 0.5 & 12.0 & 4.8 \\
\hline \multirow[t]{4}{*}{4} & 14.5 & 3.4 & 3.4 \\
\hline & 12.5 & 4.6 & 4.6 \\
\hline & 10.5 & 5.8 & 5.6 \\
\hline & 8.5 & 5.8 & 5.7 \\
\hline
\end{tabular}


Table A.20 (continued)

\begin{tabular}{c|c|c|c}
\hline String No. & $\begin{array}{c}\text { Elev from } \\
\text { Bottom }(\mathrm{ft})\end{array}$ & $\begin{array}{c}\mathrm{H}_{\mathrm{b}} \text { lRate } \\
(\mathrm{mrem} / \mathrm{h})\end{array}$ & $\begin{array}{c}\mathrm{H}_{\mathrm{d}} \text { Rate } \\
(\mathrm{mrem} / \mathrm{h})\end{array}$ \\
\hline & 6.5 & 4.9 & 5.2 \\
& 4.5 & 4.1 & 3.7 \\
& 3.5 & 4.1 & 4.1 \\
& 2.5 & 4.0 & 4.0 \\
& 1.5 & 4.7 & 3.5 \\
& 0.5 & 3.1 & 2.5 \\
\hline
\end{tabular}


APPENDIX B

Radiation and Concentration Equations 
THIS PAGE INTENTIONALLY LEFT BLANK 


\section{Conversion of dpm to $\mathrm{pCi}$}

\section{Radiation and Concentration Equations}

conversion of dpm to $\mathrm{pCi}$

$$
\frac{d p m}{100 \mathrm{~cm}^{2}} \times \frac{p C i}{22 d \mathrm{dm}}=\frac{p C i}{100 \mathrm{~cm}^{2}}
$$

conversion of pounds per cubic feet to kilograms per cubic centimeter

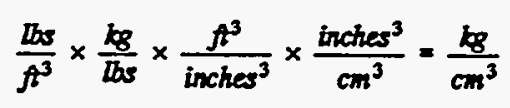

conversion of square feet to square centimeters

$$
f^{2} \times \frac{144 \text { inches }}{f^{2}} \times \frac{6.452 \mathrm{~cm}^{2}}{i n c h e s^{2}}=c \mathrm{~m}^{2}
$$

derivation of $\mathrm{pCi}$ per kilogram from $\mathrm{pCi}$ and volume of substrate

$$
P C l \times\left(\mathrm{cm}^{3} \times \frac{\mathrm{kg}}{\mathrm{Cm}}\right)^{-1}=\frac{p C i}{\mathrm{~g}}
$$


THIS PAGE INTENTIONALLY LEFT BLANK 
APPENDIX C

Summary Statistics 
THIS PAGE INTENTIONALLY LEFT BLANK 
Table C-1. Summary Statistics for Data used in BRE

\begin{tabular}{|c|c|c|c|c|c|c|c|c|c|}
\hline DATA & LOCATION & CONTAMINANT & MIN_DET & MAX_DET & DIST & MEAN & UCL95' & MAX_NOND & UNITS \\
\hline Core & Cell & Aluminum & $4.54 \mathrm{E}+03$ & $1.47 \mathrm{E}+05$ & $\mathbf{N}$ & $7.58 \mathrm{E}+04$ & $5.25 \mathrm{E}+05$ & & MG/KG \\
\hline Core & Cell & Arsenic & $2.50 \mathrm{E}+00$ & $2.60 \mathrm{E}+\infty 0$ & $\mathbf{N}$ & $2.55 \mathrm{E}+00$ & $2.87 E+\infty$ & & MG/KG \\
\hline Core & Cell & Barium & $2.69 \mathrm{E}+01$ & $7.22 \mathrm{E}+02$ & $\mathbf{N}$ & $3.74 \mathrm{E}+02$ & $2.57 \mathrm{E}+03$ & & $\mathbf{M G / K G}$ \\
\hline Core & Cell & Beryllium & $4.20 \mathrm{E}-01$ & $1.76 \mathrm{E}+01$ & $\mathbf{N}$ & $9.01 \mathrm{E}+00$ & $6.32 \mathrm{E}+01$ & & $\mathbf{M G / K G}$ \\
\hline Core & Cell & Cadmium & $5.80 \mathrm{E}+00$ & $1.80 \mathrm{E}+02$ & $\mathbf{N}$ & $9.29 \mathrm{E}+01$ & $6.43 E+02$ & & MG/KG \\
\hline Core & Cell & Calcium & $2.61 E+05$ & $8.34 E+06$ & $\mathbf{N}$ & $4.30 \mathrm{E}+06$ & $2.98 \mathrm{E}+07$ & & MG/KG \\
\hline Core & Cell & Chromium & $1.31 \mathrm{E}+01$ & $1.96 \mathrm{E}+02$ & $\mathbf{N}$ & $1.05 \mathrm{E}+02$ & $6.82 \mathrm{E}+02$ & & MG/KG \\
\hline Core & Cell & Cobalt & $2.30 \mathrm{E}+00$ & $6.88 \mathrm{E}+01$ & $\mathbf{N}$ & $3.56 \mathrm{E}+01$ & $2.45 E+02$ & & MG/KG \\
\hline Core & Cell & Copper & $9.80 \mathrm{E}+00$ & $3.78 \mathrm{E}+02$ & $\mathbf{N}$ & $1.94 \mathrm{E}+02$ & $1.36 \mathrm{E}+03$ & & $\mathbf{M G / K G}$ \\
\hline Core & Cell & Cyanide & & & NT & $5.15 \mathrm{E}-01$ & & $5.30 \mathrm{E}-01$ & MG/KG \\
\hline Core & Cell & Iron & $5.29 E+03$ & $1.76 \mathrm{E}+05$ & $\mathbf{N}$ & $9.06 \mathrm{E}+04$ & $6.30 \mathrm{E}+05$ & & MG/KG \\
\hline Core & Cell & Lead & $2.70 \mathrm{E}+00$ & $7.10 \mathrm{E}+\infty 0$ & $\mathbf{N}$ & $4.90 \mathrm{E}+00$ & $1.88 \mathrm{E}+01$ & & MG/KG \\
\hline Core & Cell & Magnesium & $3.79 \mathrm{E}+04$ & $1.39 \mathrm{E}+06$ & $\mathbf{N}$ & $7.14 \mathrm{E}+05$ & $4.98 \mathrm{E}+06$ & & MG/KG \\
\hline Core & Cell & Manganese & $2.81 \mathrm{E}+02$ & $9.53 \mathrm{E}+03$ & $\mathbf{N}$ & $4.91 E+03$ & $3.41 \mathrm{E}+04$ & & MG/KG \\
\hline Core & Cell & Mereury & $5.00 \mathrm{E}-02$ & $3.40 \mathrm{E}-01$ & $\mathbf{N}$ & $1.95 \mathrm{E}-01$ & $1.11 \mathrm{E}+00$ & & MG/KG \\
\hline Core & Cell & Nickel & $6.80 \mathrm{E}+00$ & $1.38 \mathrm{E}+02$ & $\mathbf{N}$ & $7.24 \mathrm{E}+01$ & $4.87 \mathrm{E}+02$ & & MG/KG \\
\hline Core & Cell & Potansium & $7.92 \mathrm{E}+02$ & $3.37 E+04$ & $\mathbf{N}$ & $1.72 \mathrm{E}+04$ & $1.21 \mathrm{E}+05$ & & MG/KG \\
\hline Core & Cell & Selenium & & & NT & $1.93 \mathrm{E}+\infty 0$ & & $3.20 \mathrm{E}+00$ & MG/KG \\
\hline
\end{tabular}

= minimum detection

$b=$ maximum detection

$c=$ distribution: $N=$ Normal $;=L=$ Log Normal

$d=95 \%$ upper confidence limit

$\circ=$ maximum nondetection 


\begin{tabular}{|c|c|c|c|c|c|c|c|c|c|}
\hline DATA & LOCATION & CONTAMINANT & MIN_DET & MAX_DET ${ }^{b}$ & DIST & MEAN & UCL95' & MAX_NOND & UNIrS \\
\hline Core & Cell & Silver & & & NT & $1.34 \mathrm{E}+01$ & & $2.60 \mathrm{E}+01$ & $\mathrm{MG} / \mathrm{KG}$ \\
\hline Core & Cell & Sodium & $2.21 E+02$ & $7.05 E+03$ & $\mathbf{N}$ & $3.64 \mathrm{E}+03$ & $2.52 \mathrm{E}+04$ & & $\mathrm{MG} / \mathrm{KG}$ \\
\hline Core & Cell & Thallium & & & $\mathrm{NT}$ & 4.75E-01 & & $4.80 \mathrm{E}-01$ & $\mathbf{M G} / \mathbf{K G}$ \\
\hline Core & Cell & Vanadium & $9.00 \mathrm{E}+\infty 0$ & $2.40 E+02$ & $\mathbf{N}$ & $1.25 \mathrm{E}+02$ & $8.54 \mathrm{E}+02$ & & MG/KG \\
\hline Core & Cell & Zine & $1.00 E+03$ & $3.49 \mathrm{E}+04$ & $\mathbf{N}$ & $1.80 \mathrm{E}+04$ & $1.25 E+05$ & & $\mathrm{MG} / \mathrm{KG}$ \\
\hline Core & Cell & Aroclor- 1254 & $1.10 \mathrm{E}-01$ & $3.40 \mathrm{E}-01$ & $\mathbf{N}$ & $2.25 \mathrm{E}-01$ & $9.51 \mathrm{E}-01$ & & $\mathrm{MG} / \mathrm{KG}$ \\
\hline Core & Cell & Aroclor- 1260 & $1.30 \mathrm{E}-01$ & $5.70 \mathrm{E}-01$ & $\mathbf{N}$ & $3.50 \mathrm{E}-01$ & $1.74 E+\infty$ & & $\mathrm{MG} / \mathrm{KG}$ \\
\hline Core & Cell & $137 \mathrm{Cs}$ & $3.45 E+01$ & $1.38 \mathrm{E}+03$ & $\mathbf{N}$ & $7.05 \mathrm{E}+02$ & $4.94 \mathrm{E}+03$ & & PCI/G \\
\hline Core & Cell & $226 \mathrm{Ra}$ & 4.01E-01 & $4.01 \mathrm{E}-01$ & $\mathrm{~N}$ & $4.01 \mathrm{E}-01$ & $4.01 \mathrm{E}-01$ & & PCI/G \\
\hline Core & Cell & $228 \mathrm{Th}$ & $3.80 \mathrm{E}-01$ & $8.40 \mathrm{E}-01$ & $\mathbf{N}$ & $6.10 \mathrm{E}-01$ & $2.06 \mathrm{E}+00$ & & PCI/G \\
\hline Core & Cell & $230 \mathrm{Th}$ & $1.20 \mathrm{E}+\infty 0$ & $1.40 \mathrm{E}+\infty 0$ & $\mathbf{N}$ & $1.30 E+\infty$ & $1.93 \mathrm{E}+00$ & & PCI/G \\
\hline Core & Cell & $232 \mathrm{Th}$ & $5.20 \mathrm{E}-01$ & $8.70 \mathrm{E}-01$ & $\mathbf{N}$ & $6.95 \mathrm{E}-01$ & $1.80 \mathrm{E}+\infty 0$ & & PCI/G \\
\hline Core & Cell & $233 / 234 \mathrm{U}$ & $4.90 \mathrm{E}-01$ & $1.30 \mathrm{E}+00$ & $\mathbf{N}$ & 8.95E-01 & $3.45 \mathrm{E}+\infty$ & & PCI/G \\
\hline Core & Cell & $234 T h$ & $6.31 \mathrm{E}+00$ & $6.31 \mathrm{E}+00$ & $\mathbf{N}$ & $6.31 E+\infty 0$ & $6.31 E+\infty 0$ & & $\mathrm{PCI} / \mathrm{G}$ \\
\hline Core & Cell & $235 \mathrm{U}$ & $1.20 \mathrm{E}-01$ & $1.20 \mathrm{E}-01$ & $\mathbf{N}$ & $9.50 \mathrm{E}-02$ & 2.53E-01 & $7.00 \mathrm{E}-02$ & PCI/G \\
\hline Core & Cell & 238Pu & $4.50 \mathrm{E}-01$ & $4.50 \mathrm{E}-01$ & $\mathbf{N}$ & $5.45 \mathrm{E}-01$ & $1.14 \mathrm{E}+\infty$ & $6.40 \mathrm{E}-01$ & PCI/ \\
\hline Core & Cell & $238 \mathrm{U}$ & $6.20 \mathrm{E}-01$ & $1.70 \mathrm{E}+\infty 0$ & $\mathbf{N}$ & $1.16 \mathrm{E}+\infty 0$ & $4.57 \mathrm{E}+00$ & & PCI/G \\
\hline Core & Cell & 239/240Pu & $4.00 \mathrm{E}+00$ & $4.00 \mathrm{E}+00$ & $\mathbf{N}$ & $2.24 \mathrm{E}+00$ & $1.34 \mathrm{E}+01$ & $4.80 \mathrm{E}-01$ & PCI/G \\
\hline Core & Cell & $40 \mathrm{~K}$ & $3.47 \mathrm{E}+00$ & $5.28 \mathrm{E}+\infty 0$ & $\mathbf{N}$ & $4.38 \mathrm{E}+00$ & $1.01 \mathrm{E}+01$ & & PCL/G \\
\hline Core & Cell & $60 \mathrm{Co}$ & $1.30 \mathrm{E}-01$ & $1.30 \mathrm{E}-01$ & $\mathbf{N}$ & $1.30 \mathrm{E}-01$ & $1.30 \mathrm{E}-01$ & & PCI/G \\
\hline
\end{tabular}

$\mathrm{a}=$ minimum detection

$b=$ maximum detection

$c=$ distribution: $\mathbf{N}=$ Normal; $\mathbf{L}=\log$ Normal

$d=95 \%$ upper confidence limit

${ }^{\circ}=$ maximum nondetection 
Table C-1. Cont.

\begin{tabular}{|c|c|c|c|c|c|c|c|c|c|}
\hline DATA & LOCATION & CONTAMINANT & MIN_DET & MAX_DET' & DIST & MEAN & UCL95' & MAX_NOND & UNITS \\
\hline Core & Cell & $90 \mathrm{sr}$ & $1.16 \mathrm{E}+01$ & $1.16 \mathrm{E}+01$ & $\mathbf{N}$ & $5.97 \mathrm{E}+\infty 0$ & $4.15 \mathrm{E}+01$ & $3.40 \mathrm{E}-01$ & PCI/G \\
\hline Core & Cell & Tritium & $2.04 \mathrm{E}+00$ & $2.11 \mathrm{E}+01$ & $\mathbf{N}$ & $1.16 \mathrm{E}+01$ & $7.17 \mathrm{E}+01$ & & PCUG \\
\hline Cone & East Gallery & Aluminum & $4.08 \mathrm{E}+03$ & $4.82 \mathrm{E}+03$ & $\mathbf{N}$ & $4.45 \mathrm{E}+03$ & $6.79 \mathrm{E}+03$ & & $\mathbf{M G / K G}$ \\
\hline Core & East Gallery & Antimony & & & NT & $1.75 E+00$ & & $1.80 \mathrm{E}+00$ & MG/KG \\
\hline Core & East Gallery & Arsenic & $2.60 \mathrm{E}+00$ & $3.00 \mathrm{E}+\infty$ & $\mathbf{N}$ & $2.80 \mathrm{E}+00$ & $4.06 \mathrm{E}+00$ & & MG/KG \\
\hline Core & East Gallery & Barium & $1.93 \mathrm{E}+01$ & $2.14 \mathrm{E}+01$ & $\mathbf{N}$ & $2.04 \mathrm{E}+01$ & $2.70 \mathrm{E}+01$ & & MG/KG \\
\hline Core & East Gallery & Beryllium & $4.00 \mathrm{E}-01$ & $4.00 \mathrm{E}-01$ & $\mathbf{N}$ & $3.15 \mathrm{E}-01$ & 8.52E-01 & $2.30 \mathrm{E}-01$ & MO/KG \\
\hline Core & East Gallery & Cadmium & $4.10 \mathrm{E}+00$ & $5.10 \mathrm{E}+\infty$ & $\mathbf{N}$ & $4.60 \mathrm{E}+00$ & $7.76 \mathrm{E}+00$ & & MG/KG \\
\hline Core & East Gallery & Calcium & $2.15 E+05$ & $2.57 \mathrm{E}+05$ & $\mathbf{N}$ & $2.36 \mathrm{E}+05$ & $3.69 \mathrm{E}+05$ & & MG/KG \\
\hline Core & East Gallery & Chromium & $8.20 \mathrm{E}+00$ & $8.30 \mathrm{E}+\infty 0$ & $\mathbf{N}$ & $8.25 E+00$ & 8.57E+00 & & MG/KG \\
\hline Core & East Gallery & Cobalt & $2.00 \mathrm{E}+00$ & $3.20 \mathrm{E}+00$ & $\mathbf{N}$ & $2.60 \mathrm{E}+00$ & $6.39 \mathrm{E}+\infty$ & & $\mathbf{M G / K G}$ \\
\hline Core & East Gallery & Copper & $7.50 \mathrm{E}+00$ & $1.08 \mathrm{E}+01$ & $\mathbf{N}$ & $9.15 \mathrm{E}+\infty$ & $1.96 \mathrm{E}+01$ & & MG/KG \\
\hline Core & East Gallery & Cyanide & & & NT & $4.80 \mathrm{E}-01$ & & $4.80 \mathrm{E}-01$ & MG/KG \\
\hline Core & East Gallery & Iron & $5.69 \mathrm{E}+03$ & $5.77 \mathrm{E}+03$ & $\mathbf{N}$ & $5.73 \mathrm{E}+03$ & $5.98 \mathrm{E}+03$ & & MG/KG \\
\hline Core & East Gallery & Lead & $4.70 \mathrm{E}+00$ & $7.20 \mathrm{E}+00$ & $\mathbf{N}$ & $5.95 \mathrm{E}+00$ & $1.38 \mathrm{E}+01$ & & MG/KG \\
\hline Core & East Gallery & Magnesium & $3.68 \mathrm{E}+04$ & $4.23 \mathrm{E}+04$ & $\mathbf{N}$ & $3.96 \mathrm{E}+04$ & $5.69 \mathrm{E}+04$ & & MG/KG \\
\hline Core & East Gallery & Manganese & $2.61 \mathrm{E}+02$ & $2.65 \mathrm{E}+02$ & $\mathbf{N}$ & $2.63 \mathrm{E}+02$ & $2.76 \mathrm{E}+02$ & & MG/KG \\
\hline Core & East Gallery & Mencury & & & NT & $1.15 \mathrm{E}-01$ & & $1.40 \mathrm{E}-01$ & MG/KG \\
\hline
\end{tabular}

- = minimum detection

$b=$ maximum detection

c= distribution: $N=$ Normal $;=$ Log Normal

$d=95 \%$ upper confidence limit

$\circ=$ maximum nondetection 
Table C-1. Cont.

\begin{tabular}{|c|c|c|c|c|c|c|c|c|c|}
\hline DATA & LOCATION & CONTAMINANT & MIN_DET & MAX_DET & DIST & MEAN & UCL954 & MAX_NOND & UNrTS \\
\hline Core & Esat Gallery & Nickel & $5.30 \mathrm{E}+\infty$ & $6.00 \mathrm{E}+00$ & $\mathbf{N}$ & $5.65 \mathrm{E}+00$ & $7.86 \mathrm{E}+\infty 0$ & & MO/KG \\
\hline Core & East Gallery & Potassium & $8.29 E+02$ & $1.03 \mathrm{E}+03$ & $\mathbf{N}$ & $9.30 \mathrm{E}+02$ & $1.56 \mathrm{E}+03$ & & MG/KG \\
\hline Core & East Gallery & Selenium & & & NT & $2.55 \mathrm{E}+00$ & & $2.70 \mathrm{E}+00$ & MG/KG \\
\hline Core & East Gallery & Silver & & & NT & $7.15 E-01$ & & $7.40 \mathrm{E}-01$ & MG/KG \\
\hline Core & East Gallery & Sodium & $3.09 \mathrm{E}+02$ & $3.09 \mathrm{E}+02$ & $\mathbf{N}$ & $3.06 \mathrm{E}+02$ & $3.25 \mathrm{E}+02$ & $3.03 E+02$ & MG/KG \\
\hline Cone & East Gallery & Thallium & & & NT & $7.55 \mathrm{E}-01$ & & $7.60 \mathrm{E}-01$ & MG/KG \\
\hline Core & East Gallery & Vanadium & $5.70 \mathrm{E}+00$ & $9.10 \mathrm{E}+00$ & $\mathbf{N}$ & $7.40 \mathrm{E}+\infty 0$ & $1.81 E+01$ & & $\mathbf{M G} / \mathbf{K G}$ \\
\hline Core & East Gallery & Zinc & $7.37 E+02$ & $9.79 E+02$ & $\mathbf{N}$ & $8.58 \mathrm{E}+02$ & $1.62 E+03$ & & MG/KG \\
\hline Core & East Gallery & 2-Methylnaphthalene & $8.90 \mathrm{E}-02$ & $8.90 \mathrm{E}-02$ & NT & $8.90 \mathrm{E}-02$ & & & MG/KG \\
\hline Core & East Ġallery & Aroclor- 1254 & $3.70 \mathrm{E}-01$ & $1.70 \mathrm{E}+00$ & $\mathbf{N}$ & $8.13 \mathrm{E}-01$ & $2.11 E+00$ & & MG/KG \\
\hline Core & East Gallery & Aroclor-1260 & $1.10 \mathrm{E}-01$ & $2.60 \mathrm{E}+00$ & $\mathbf{N}$ & $9.40 \mathrm{E}-01$ & $3.36 \mathrm{E}+\infty 0$ & & $\mathbf{M G / K G}$ \\
\hline Core & East Gallery & Bis(2-ethylhexyl)phthalate & $2.40 \mathrm{E}-01$ & $2.40 \mathrm{E}-01$ & NT & $2.40 \mathrm{E}-01$ & & & MG/KG \\
\hline Core & East Gallery & Dimethyl phthalate & $5.50 \mathrm{E}-02$ & $5.50 \mathrm{E}-02$ & NT & $5.50 \mathrm{E}-02$ & & & MG/KG \\
\hline Core & East Gallery & Naphthalene & $5.30 \mathrm{E}-02$ & $5.30 \mathrm{E}-02$ & NT & $5.30 \mathrm{E}-02$ & & & MG/KG \\
\hline Core & East Gallery & Phenanthrene & $2.40 \mathrm{E}-01$ & $2.40 \mathrm{E}-01$ & NT & $2.40 \mathrm{E}-01$ & & & MG/KG \\
\hline Core & East Gallery & $137 \mathrm{Cs}$ & $1.51 E+01$ & $2.28 \mathrm{E}+01$ & $\mathbf{N}$ & $1.89 \mathrm{E}+01$ & $4.34 \mathrm{E}+01$ & & PCVG \\
\hline Core & East Gallery & $226 \mathrm{Ra}$ & $3.60 \mathrm{E}-01$ & $3.60 \mathrm{E}-01$ & $\mathbf{N}$ & $3.60 \mathrm{E}-01$ & $3.60 \mathrm{E}-01$ & & PCI/G \\
\hline Cone & Eant Gallery & $228 R=$ & $3.70 \mathrm{E}-01$ & $3.70 \mathrm{E}-01$ & $\mathbf{N}$ & $3.70 \mathrm{E}-01$ & $3.70 \mathrm{E}-01$ & & PCI/G \\
\hline
\end{tabular}

$=$ minimum detection

$b=$ maximum detection

$c=$ distribution: $N=$ Normal; $L=L o g$ Normal

$d=95 \%$ upper confidence limit

$\circ=$ maximum nondetection 
Table C-1. Cont.

\begin{tabular}{|c|c|c|c|c|c|c|c|c|c|}
\hline DATA & LOCATION & CONTAMINANT & MIN_DET & MAX_DET & DIST $^{\mathbf{C}}$ & MEAN & UCL95" & MAX_NOND & UNITS \\
\hline Core & East Gallery & 228Th & $5.30 \mathrm{E}-01$ & $5.60 \mathrm{E}-01$ & $\mathbf{N}$ & $5.45 \mathrm{E}-01$ & $6.40 \mathrm{E}-01$ & & PCI/G \\
\hline Core & East Gallery & $230 \mathrm{Th}$ & $1.00 \mathrm{E}+00$ & $1.20 \mathrm{E}+\infty 0$ & $\mathbf{N}$ & $1.10 \mathrm{E}+00$ & $1.73 \mathrm{E}+00$ & & PCL/G \\
\hline Core & East Gallery & $232 \mathrm{Th}$ & $2.90 \mathrm{E}-01$ & 4.30E-01 & $\mathbf{N}$ & $3.60 \mathrm{E}-01$ & 8.02E-01 & & $\mathbf{P C I} / \mathbf{G}$ \\
\hline Core & East Gallery & $233 / 234 \mathrm{U}$ & $8.00 \mathrm{E}-01$ & $9.00 \mathrm{E}-01$ & $\mathbf{N}$ & $8.50 \mathrm{E}-01$ & $1.17 \mathrm{E}+\infty$ & & PCI/G \\
\hline Core & East Gallery & $235 \mathrm{U}$ & & & $\mathrm{NT}$ & $1.15 \mathrm{E}-01$ & & $1.70 \mathrm{E}-01$ & PCL/G \\
\hline Core & East Gallery & 238Pu & & & NT & $3.90 \mathrm{E}-01$ & & $3.90 \mathrm{E}-01$ & PCI/G \\
\hline Core & East Gallery & $238 \mathrm{U}$ & $6.20 \mathrm{E}-01$ & $7.70 \mathrm{E}-01$ & $\mathbf{N}$ & $6.95 \mathrm{E}-01$ & $1.17 \mathrm{E}+00$ & & PCL/G \\
\hline Core & Eam Gallery & 239/240Pu & & & $\mathrm{NT}$ & $3.90 \mathrm{E}-01$ & & $3.90 \mathrm{E}-01$ & PCI/G \\
\hline Core & East Gallery & $241 \mathrm{Am}$ & $5.00 \mathrm{E}-01$ & $5.00 \mathrm{E}-01$ & $\mathbf{N}$ & $5.00 \mathrm{E}-01$ & $5.00 \mathrm{E}-01$ & & PCI/G \\
\hline Core & East Gallery & $40 \mathrm{~K}$ & $5.14 E+00$ & $5.38 \mathrm{E}+\infty 0$ & $\mathbf{N}$ & $5.26 \mathrm{E}+00$ & $6.02 \mathrm{E}+00$ & & PCL/G \\
\hline Core & East Gallery & $90 \mathrm{Sr}$ & $8.10 \mathrm{E}+\infty 0$ & $8.10 \mathrm{E}+\infty$ & $\mathbf{N}$ & $4.33 \mathrm{E}+\infty 0$ & $2.82 \mathrm{E}+01$ & $5.50 \mathrm{E}-01$ & PCI/G \\
\hline Core & East Gallery & Tritium & $6.50 \mathrm{E}-01$ & $2.56 \mathrm{E}+01$ & $\mathbf{N}$ & $1.31 E+01$ & $9.19 \mathrm{E}+01$ & & $\mathrm{PCI} / \mathrm{G}$ \\
\hline Sediment & Cell & Aluminum & $9.53 E+03$ & $9.53 \mathrm{E}+03$ & NT & $9.53 E+03$ & & & MG/KG \\
\hline Sediment & Cell & Antimony & $3.62 \mathrm{E}+01$ & $3.62 \mathrm{E}+01$ & NT & $3.62 \mathrm{E}+01$ & & & MG/KG \\
\hline Sediment & Cell & Arsenic & $2.15 E+01$ & $2.15 \mathrm{E}+01$ & NT & $2.15 \mathrm{E}+01$ & & & MG/KG \\
\hline Sediment & Cell & Barium & $1.20 \mathrm{E}+02$ & $1.20 \mathrm{E}+02$ & NT & $1.20 \mathrm{E}+02$ & & & MG/KG \\
\hline Sediment & Cell & Beryllium & & & NT & 9.50E-01 & & $9.50 \mathrm{E}-01$ & MG/KG \\
\hline Sediment & Cell & Cadmium & $6.01 E+01$ & $6.01 E+01$ & NT & $6.01 \mathrm{E}+01$ & & & MG/KG \\
\hline
\end{tabular}

= minimum detection

$b=$ maximum detection

$c=$ distribution: $N=$ Normal $;=$ Log Normal

$d=95 \%$ upper confidence limit

$\circ=$ maximum nondetection 
Table C-1. Cont.

\begin{tabular}{|c|c|c|c|c|c|c|c|c|c|}
\hline DATA & LOCATION & CONTAMINANT & MIN_DET & MAX_DET ${ }^{b}$ & DIST & MEAN & UCL95 & MAX_NOND & uNITS \\
\hline Sediment & Cell & Calcium & $2.03 \mathrm{E}+04$ & $2.03 E+04$ & NT & $2.03 E+04$ & & & MG/KG \\
\hline Sediment & Cell & Chromium & $1.89 \mathrm{E}+02$ & $1.89 \mathrm{E}+02$ & NT & $1.89 \mathrm{E}+02$ & & & MG/KG \\
\hline Sediment & Cell & Cobalt & & & NT & $2.51 \mathrm{E}+01$ & & $2.51 E+01$ & MG/KG \\
\hline Sediment & Cell & Copper & $3.85 \mathrm{E}+02$ & $3.85 \mathrm{E}+02$ & NT & $3.85 \mathrm{E}+02$ & & & MG/KG \\
\hline Sediment & Cell & Cyanide & $5.80 \mathrm{E}-01$ & $5.80 \mathrm{E}-01$ & NT & $5.80 \mathrm{E}-01$ & & & MG/KG \\
\hline Sediment & Cell & Iron & $1.10 \mathrm{E}+05$ & $1.10 \mathrm{E}+05$ & $\mathrm{NT}$ & $1.10 \mathrm{E}+05$ & & & MG/KG \\
\hline Sediment & Cell & Lead & $8.78 \mathrm{E}+02$ & $8.78 E+02$ & NT & $8.78 \mathrm{E}+02$ & & & $\mathbf{M G / K G}$ \\
\hline Sediment & Cell & Magnesium & $7.93 \mathrm{E}+03$ & $7.93 \mathrm{E}+03$ & NT & $7.93 \mathrm{E}+03$ & & & MG/KG \\
\hline Sediment & Cell & Manganese & $1.31 \mathrm{E}+03$ & $1.31 \mathrm{E}+03$ & NT & $1.31 E+03$ & & & MG/KG \\
\hline Sediment & Cell & Mercury & $1.87 \mathrm{E}+01$ & $1.87 \mathrm{E}+01$ & NT & $1.87 \mathrm{E}+01$ & & & MG/KG \\
\hline Sediment & Cell & Nickel & $1.69 \mathrm{E}+02$ & $1.69 \mathrm{E}+02$ & NT & $1.69 \mathrm{E}+02$ & & & MG/KG \\
\hline Sediment & Cell & Potassium & $8.22 E+02$ & $8.22 E+02$ & NT & $8.22 E+02$ & & & MG/KG \\
\hline Sediment & Cell & Selenium & 8.70E-01 & 8.70E-01 & NT & 8.70E-01 & & & MG/KG \\
\hline Sediment & Cell & Silver & & & NT & $1.90 \mathrm{E}+00$ & & $1.90 \mathrm{E}+\infty 0$ & MG/KG \\
\hline Sediment & Cell & Sodium & $1.33 \mathrm{E}+02$ & $1.33 \mathrm{E}+02$ & NT & $1.33 E+02$ & & & $\mathrm{MG} / \mathrm{KG}$ \\
\hline Sediment & Cell & Thallium & & & NT & $4.60 \mathrm{E}-01$ & & $4.60 \mathrm{E}-01$ & $\mathbf{M G / K G}$ \\
\hline Sediment & Cell & Vanadium & & & NT & $2.87 \mathrm{E}+01$ & & $2.87 E+01$ & $\mathrm{MG} / \mathrm{KG}$ \\
\hline Sediment & Cell & Zinc & $7.42 E+03$ & $7.42 \mathrm{E}+03$ & NT & $7.42 \mathrm{E}+03$ & & & MG/KG \\
\hline
\end{tabular}

$=$ minimum detection

$b=$ maximum detection

' = distribution: $\mathrm{N}=$ Normal; $\mathrm{L}=\mathrm{Log}$ Normal

$d=95 \%$ upper confidence limit

$\circ=$ maximum nondetection 
Table C-1. Cont.

\begin{tabular}{|c|c|c|c|c|c|c|c|c|c|}
\hline DATA & LOCATION & CONTAMINANT & MIN_DET & MAX_DET ${ }^{b}$ & DIST & MEAN & UCL954 & MAX_NOND & UNITS \\
\hline Sedimene & Cell & 2-Methylnaphthalene & $2.70 \mathrm{E}-01$ & $2.70 \mathrm{E}-01$ & NT & $2.70 \mathrm{E}-01$ & & & MG/KG \\
\hline Sedimene & Cell & Acenaphthene & $9.60 \mathrm{E}-01$ & $9.60 \mathrm{E}-01$ & NT & $9.60 \mathrm{E}-01$ & & & $\mathbf{M G / K G}$ \\
\hline Sediment & Cell & Acetone & $9.00 \mathrm{E}-02$ & $9.00 \mathrm{E}-02$ & $\mathrm{NT}$ & $9.00 \mathrm{E}-02$ & & & MG/KG \\
\hline Sediment & Cell & Anthracene & $1.90 \mathrm{E}+00$ & $1.90 \mathrm{E}+\infty$ & NT & $1.90 \mathrm{E}+00$ & & & $\mathbf{M G / K G}$ \\
\hline Sedimen & Cell & Aroclor-1254 & $4.10 \mathrm{E}+02$ & $4.10 \mathrm{E}+02$ & NT & $4.10 \mathrm{E}+02$ & & & MG/KG \\
\hline Sediment & Cell & Benzo(a)pyrene & $5.70 \mathrm{E}+00$ & $5.70 \mathrm{E}+\infty 0$ & NT & $5.70 \mathrm{E}+00$ & & & MG/KG \\
\hline Sedimene & Cell & "Benzo(g,h,i)perylene" & $1.10 \mathrm{E}+00$ & $1.10 \mathrm{E}+\infty 0$ & NT & $1.10 \mathrm{E}+\infty$ & & & MG/KG \\
\hline Sediment & Cell & Benzo(k) lluoranthene & $4.60 \mathrm{E}+01$ & $4.60 \mathrm{E}+01$ & NT & $4.60 \mathrm{E}+01$ & & & MG/KG \\
\hline Sedimene & Cell & Bis(2-ethylhexyl)phthalate & $2.10 \mathrm{E}+\infty$ & $2.10 \mathrm{E}+00$ & NT & $2.10 \mathrm{E}+00$ & & & MG/KG \\
\hline Sedimene & Cell & Carbazole & $2.00 \mathrm{E}+\infty$ & $2.00 \mathrm{E}+00$ & NT & $2.00 \mathrm{E}+00$ & & & MG/KG \\
\hline Sediment & Cell & Chrysene & $1.90 \mathrm{E}+01$ & $1.90 \mathrm{E}+01$ & NT & $1.90 \mathrm{E}+01$ & & & MG/KG \\
\hline Sediment & Cell & "Dibenz (a,h)anthracene" & $1.50 \mathrm{E}+\infty 0$ & $1.50 \mathrm{E}+00$ & NT & $1.50 \mathrm{E}+\infty$ & & & MG/KG \\
\hline Sediment & Cell & Dibenzofuran & $5.10 \mathrm{E}-01$ & $5.10 \mathrm{E}-01$ & $\mathrm{NT}$ & $5.10 \mathrm{E}-01$ & & & MG/KG \\
\hline Sedimene & Cell & Fluoranthene & $2.70 \mathrm{E}+01$ & $2.70 \mathrm{E}+01$ & $\mathrm{NT}$ & $2.70 \mathrm{E}+01$ & & & MG/KG \\
\hline Sediment & Cell & Fluorene & $8.50 \mathrm{E}-01$ & $8.50 \mathrm{E}-01$ & $\mathrm{NT}$ & $8.50 \mathrm{E}-01$ & & & MG/KG \\
\hline Sedimene & Cell & "Indeno(1,2,3-cd)pyrene" & $9.60 \mathrm{E}+00$ & $9.60 \mathrm{E}+00$ & NT & $9.60 \mathrm{E}+\infty 0$ & & & MG/KG \\
\hline Sedimene & Cell & Naphthalene & $4.20 \mathrm{E}-01$ & $4.20 \mathrm{E}-01$ & NT & $4.20 \mathrm{E}-01$ & & & MG/KG \\
\hline Sediment & Cell & Phenanthrene & $1.70 \mathrm{E}+01$ & $1.70 \mathrm{E}+01$ & NT & $1.70 \mathrm{E}+01$ & & & MG/KG \\
\hline
\end{tabular}

" = minimum detection

$b=$ maximum detection

' = distribution: $N=$ Normal $;=L$ Log Normal

$d=95 \%$ upper confidence limit

${ }^{\circ}=$ maximum nondetection 
Table C-1. Cont.

\begin{tabular}{|c|c|c|c|c|c|c|c|c|c|}
\hline DATA & LOCATION & CONTAMINANT & MIN_DET & MAX_DET & DIST & MEAN & UCL954 & MAX_NOND & UNITS \\
\hline Sedimen & Cell & Pyrene & $2.20 \mathrm{E}+01$ & $2.20 \mathrm{E}+01$ & NT & $2.20 \mathrm{E}+01$ & & & MG/KG \\
\hline Sediment & Cell & $137 \mathrm{Cs}_{\mathrm{s}}$ & $3.08 \mathrm{E}+04$ & $3.08 \mathrm{E}+04$ & NT & $3.08 E+04$ & & & PCUG \\
\hline Sedimene & Cell & $228 \mathrm{Th}$ & $5.40 \mathrm{E}-01$ & $5.40 \mathrm{E}-01$ & $\mathrm{NT}$ & $5.40 \mathrm{E}-01$ & & & PCI/G \\
\hline Sediment & Cell & $229 \mathrm{Th}$ & $1.87 E+01$ & $1.87 \mathrm{E}+01$ & NT & $1.87 \mathrm{E}+01$ & & & PCU/G \\
\hline Sedimen & Cell & $230 \mathrm{Th}$ & & & NT & 4.00E-02 & & $4.00 \mathrm{E}-02$ & PCU/G \\
\hline Sediment & Cell & $232 \mathrm{Th}$ & $5.50 \mathrm{E}-01$ & $5.50 \mathrm{E}-01$ & NT & 5.50E-01 & & & PCI/G \\
\hline Sediment & Cell & $233 / 234 U$ & $3.43 E+\infty 0$ & $3.43 \mathrm{E}+\infty$ & NT & $3.43 E+\infty$ & & & PCI/G \\
\hline Sediment & Cell & $235 \mathrm{U}$ & & & NT & $1.60 \mathrm{E}-01$ & & $1.60 \mathrm{E}-01$ & PCUG \\
\hline Sedimene & Cell & 238Pu & $1.16 \mathrm{E}+\infty 0$ & $1.16 \mathrm{E}+\infty$ & $\mathrm{NT}$ & $1.16 \mathrm{E}+\infty$ & & & PCI/G \\
\hline Sedimene & Cell & $238 \mathrm{U}$ & $2.24 \mathrm{E}+\infty 0$ & $2.24 \mathrm{E}+\infty$ & $\mathrm{NT}$ & $2.24 E+\infty 0$ & & & $\mathbf{P C L / G}$ \\
\hline Sediment & Cell & 239/240Pu & $3.54 \mathrm{E}+01$ & $3.54 \mathrm{E}+01$ & $\mathrm{NT}$ & $3.54 \mathrm{E}+01$ & & & PCI/G \\
\hline Sediment & Cell & $241 \mathrm{Am}$ & & & NT & $7.90 \mathrm{E}+00$ & & $7.90 \mathrm{E}+\infty 0$ & PCI/G \\
\hline Sedimene & Cell & $40 \mathrm{~K}$ & $9.43 \mathrm{E}+00$ & $9.43 \mathrm{E}+00$ & NT & $9.43 E+00$ & & & PCI/G \\
\hline Sediment & Cell & $60 \mathrm{Co}$ & $3.43 E+\infty$ & $3.43 \mathrm{E}+00$ & NT & $3.43 \mathrm{E}+00$ & & & PCI/G \\
\hline Sediment & Cell & $90 \mathrm{Sr}$ & $9.75 \mathrm{E}+01$ & $9.75 \mathrm{E}+01$ & NT & $9.75 \mathrm{E}+01$ & & & PCL/G \\
\hline Sedimene & Cell & Tritium & $1.00 \mathrm{E}+00$ & $1.00 \mathrm{E}+00$ & NT & $1.00 \mathrm{E}+00$ & & & $\mathrm{PCl} / \mathrm{G}$ \\
\hline Core & Whole Building & Aluminum & $4.08 E+03$ & $1.47 \mathrm{E}+05$ & L & $3.29 E+04$ & $1.56 \mathrm{E}+05$ & & MG/KG \\
\hline Core & Whole Building & Antimony & $3.62 \mathrm{E}+01$ & $3.62 \mathrm{E}+01$ & L & $2.22 E+01$ & $2.53 E+02$ & $1.80 \mathrm{E}+\infty 0$ & MG/KG \\
\hline
\end{tabular}

${ }^{a}=$ minimum detection

$b=$ maximum detection

$c=$ distribution: $N=$ Normal $L=$ Log Normal

$d=95 \%$ upper confidence limit

$\circ=$ maximum nondetection 
Table C-1. Cont.

\begin{tabular}{|c|c|c|c|c|c|c|c|c|c|}
\hline DATA & LOCATION & CONTAMINANT & MIN_DET & MAX_DET ${ }^{+}$ & DIST & MEAN & UCL95' & MAX_NOND & UNIrS \\
\hline Core & Whole Building & Arzenic & $2.50 \mathrm{E}+00$ & $2.15 E+01$ & $\mathbf{L}$ & $6.28 \mathrm{E}+00$ & $1.40 \mathrm{E}+01$ & & MG/KG \\
\hline Core & Whole Building & Barium & $1.93 \mathrm{E}+01$ & $7.22 \mathrm{E}+02$ & $\mathbf{L}$ & $2.09 \mathrm{E}+02$ & $1.06 \mathrm{E}+03$ & & MG/KG \\
\hline Core & Whole Building & Beryllium & $4.00 \mathrm{E}-01$ & $1.76 \mathrm{E}+01$ & $\mathbf{L}$ & $1.33 E+00$ & $7.52 \mathrm{E}+\infty 0$ & $9.50 \mathrm{E}-01$ & MG/KG \\
\hline Core & Whole Building & Cadmium & $4.10 \mathrm{E}+00$ & $1.80 \mathrm{E}+02$ & $\mathbf{L}$ & $7.31 E+01$ & $4.81 \mathrm{E}+02$ & & MG/KG \\
\hline Core & Whole Building & Calcium & $2.03 \mathrm{E}+04$ & $8.34 E+06$ & $\mathbf{L}$ & $3.02 \mathrm{E}+06$ & $4.50 \mathrm{E}+07$ & & MG/KG \\
\hline Core & Whole Building & Chromium & $8.20 \mathrm{E}+00$ & $1.96 \mathrm{E}+02$ & $\mathbf{L}$ & $1.25 \mathrm{E}+02$ & $7.37 \mathrm{E}+02$ & & MG/KG \\
\hline Core & Whole Building & Cobalt & $2.00 \mathrm{E}+00$ & $6.88 \mathrm{E}+01$ & $\mathbf{L}$ & $1.05 \mathrm{E}+01$ & $3.30 \mathrm{E}+01$ & $2.51 \mathrm{E}+01$ & MG/KG \\
\hline Core & Whole Building & Copper & $7.50 \mathrm{E}+\infty 0$ & $3.85 \mathrm{E}+02$ & $\mathbf{L}$ & $3.29 \mathrm{E}+02$ & $3.94 \mathrm{E}+03$ & & MG/KG \\
\hline Core & Whole Building & Cyanide & $5.80 \mathrm{E}-01$ & $5.80 \mathrm{E}-01$ & $\mathbf{L}$ & $5.23 \mathrm{E}-01$ & $5.60 \mathrm{E}-01$ & $5.30 \mathrm{E}-01$ & $\mathbf{M G / K G}$ \\
\hline Core & Whole Building & Imon & $5.29 \mathrm{E}+03$ & $1.76 \mathrm{E}+05$ & $\mathbf{N}$ & $6.06 \mathrm{E}+04$ & $1.36 \mathrm{E}+05$ & & $\mathbf{M G / K G}$ \\
\hline Core & Whole Building & Lead & $2.70 \mathrm{E}+\infty 0$ & $8.78 \mathrm{E}+02$ & $\mathbf{L}$ & $2.20 \mathrm{E}+02$ & $4.98 \mathrm{E}+03$ & & MG/KG \\
\hline Core & Whole Building & Magnesium & $7.93 \mathrm{E}+03$ & $1.39 \mathrm{E}+06$ & $\mathbf{L}$ & $3.56 \mathrm{E}+05$ & $3.29 \mathrm{E}+06$ & & MG/KG \\
\hline Core & Whole Building & Manganese & $2.61 \mathrm{E}+02$ & $9.53 E+03$ & $\mathbf{L}$ & $2.61 E+03$ & $1.36 \mathrm{E}+04$ & & MG/KG \\
\hline Core & Whole Building & Mercury & $5.00 \mathrm{E}-02$ & $1.87 \mathrm{E}+01$ & $\mathbf{L}$ & $5.84 \mathrm{E}-01$ & $6.52 \mathrm{E}+\infty 0$ & $1.40 \mathrm{E}-01$ & MG/KG \\
\hline Core & Whole Building & Nickel & $5.30 \mathrm{E}+00$ & $1.69 \mathrm{E}+02$ & $\mathbf{N}$ & $6.50 \mathrm{E}+01$ & $1.43 \mathrm{E}+02$ & & MG/KG \\
\hline Core & Whole Building & Potassium & $7.92 \mathrm{E}+02$ & $3.37 \mathrm{E}+04$ & $\mathbf{L}$ & $6.92 \mathrm{E}+03$ & $4.02 \mathrm{E}+04$ & & MG/KG \\
\hline Core & Whole Building & Selenium & $8.70 \mathrm{E}-01$ & $8.70 \mathrm{E}-01$ & $\mathbf{N}$ & $1.96 \mathrm{E}+00$ & $3.05 E+00$ & $3.20 \mathrm{E}+\infty$ & MG/KG \\
\hline Core & Whole Building & Silver & & & NT & $6.01 E+\infty 0$ & & $2.60 \mathrm{E}+01$ & MG/KG \\
\hline
\end{tabular}

= minimum detection

$b=$ maximum detection

'= distribution: $N=$ Normal; $L=$ Log Normal

$d=95 \%$ upper confidence limit

${ }^{\circ}=$ maximum nondetection 
Table C-1. Cont.

\begin{tabular}{|c|c|c|c|c|c|c|c|c|c|}
\hline DATA & LOCATION & CONTAMINANT & MIN_DET & MAX_DET ${ }^{b}$ & DIST & MEAN & UCL95' & MAX_NOND & UNITS \\
\hline Core & Whole Building & Sodium & $1.33 \mathrm{E}+02$ & $7.05 \mathrm{E}+03$ & $\mathbf{L}$ & $7.95 \mathrm{E}+02$ & $2.82 \mathrm{E}+03$ & $3.03 E+02$ & MG/KG \\
\hline Core & Whole Building & Thallium & & & NT & $5.84 \mathrm{E}-01$ & & $7.60 \mathrm{E}-01$ & MO/KG \\
\hline Core & Whole Building & Vanadium & $5.70 \mathrm{E}+00$ & $2.40 \mathrm{E}+02$ & $\mathbf{L}$ & $3.13 \mathrm{E}+01$ & $1.01 E+02$ & $2.87 E+01$ & MG/KG \\
\hline Core & Whole Building & Zinc & $7.37 E+02$ & $3.49 \mathrm{E}+04$ & $\mathbf{L}$ & $1.16 \mathrm{E}+04$ & $7.17 \mathrm{E}+04$ & & MG/KG \\
\hline Core & Whole Building & 2-Methylnaphthalene & $8.90 \mathrm{E}-02$ & $2.70 \mathrm{E}-01$ & $\mathbf{N}$ & $1.80 \mathrm{E}-01$ & $7.51 \mathrm{E}-01$ & & MG/KG \\
\hline Core & Whole Bujlding & Acenaphthene & $9.60 \mathrm{E}-01$ & $9.60 \mathrm{E}-01$ & $\mathrm{NT}$ & $9.60 \mathrm{E}-01$ & & & MG/KG \\
\hline Core & Whole Building & Acctone & $9.00 \mathrm{E}-02$ & $9.00 \mathrm{E}-02$ & NT & $9.00 \mathrm{E}-02$ & & & MG/KG \\
\hline Core & Whole Building & Anthracene & $1.90 \mathrm{E}+00$ & $1.90 \mathrm{E}+00$ & NT & $1.90 \mathrm{E}+00$ & & & MG/KG \\
\hline Core & Whole Building & Araclor-1254 & $1.10 \mathrm{E}-01$ & $4.10 \mathrm{E}+02$ & $\mathbf{L}$ & $1.03 \mathrm{E}+02$ & $8.14 \mathrm{E}+03$ & & MG/KG \\
\hline Core & Whole Building & Aroclor-1260 & $1.10 \mathrm{E}-01$ & $2.60 \mathrm{E}+\infty$ & $\mathbf{L}$ & $7.88 \mathrm{E}-01$ & $3.13 E+\infty$ & & MG/KG \\
\hline Core & Whole Building & Benzo(a)pyrene & $5.70 \mathrm{E}+00$ & $5.70 \mathrm{E}+\infty$ & NT & $5.70 \mathrm{E}+00$ & & & MG/KG \\
\hline Core & Whole Building & "Benzo $(g, h, i)$ perylene" & $1.10 \mathrm{E}+00$ & $1.10 \mathrm{E}+\infty$ & NT & $1.10 \mathrm{E}+\infty 0$ & & & $\mathrm{MG} / \mathrm{KG}$ \\
\hline Core & Whole Building & Benzo(k)fluoranthene & $4.60 \mathrm{E}+01$ & $4.60 E+01$ & $\mathrm{NT}$ & $4.60 \mathrm{E}+01$ & & & MG/KG \\
\hline Core & Whole Building & Bis(2-ethylhexyl)phthalate & $2.40 \mathrm{E}-01$ & $2.10 \mathrm{E}+\infty 0$ & $\mathbf{N}$ & $1.17 \mathrm{E}+00$ & $7.04 \mathrm{E}+00$ & & MG/KG \\
\hline Core & Whole Building & Carbazole & $2.00 \mathrm{E}+00$ & $2.00 \mathrm{E}+\infty 0$ & NT & $2.00 \mathrm{E}+\infty 0$ & & & MG/KG \\
\hline Core & Whole Building & Chrysene & $1.90 \mathrm{E}+01$ & $1.90 \mathrm{E}+01$ & $\mathbf{N T}$ & $1.90 \mathrm{E}+01$ & & & MG/KG \\
\hline Core & Whole Building & "Dibenz(a,h)anthracene" & $1.50 \mathrm{E}+00$ & $1.50 \mathrm{E}+00$ & NT & $1.50 \mathrm{E}+\infty 0$ & & & $\mathrm{MG} / \mathrm{KG}$ \\
\hline Core & Whole Building & Dibenzofuran & $5.10 \mathrm{E}-01$ & $5.10 \mathrm{E}-01$ & $\mathrm{NT}$ & $5.10 \mathrm{E}-01$ & & & MG/KG \\
\hline
\end{tabular}

$=$ minimum detection

$b=$ maximum detection

$c=$ distribution: $N=$ Normal $;=\log$ Normal

$\mathrm{d}=95 \%$ upper confidence limit

$\circ=$ maximum nondetection 
Table C-1. Cont.

\begin{tabular}{|c|c|c|c|c|c|c|c|c|c|}
\hline DATA & LOCATION & CONTAMINANT & MIN_DET & MAX_DET & DIST & MEAN & UCL954 & MAX_NOND & unIrs \\
\hline Core & Whole Building & Dimethyl phthalate & $5.50 \mathrm{E}-02$ & $5.50 \mathrm{E}-02$ & $\mathrm{NT}$ & $5.50 \mathrm{E}-02$ & & & $\mathbf{M G / K G}$ \\
\hline Core & Whole Building & Fluoranthene & $2.70 \mathrm{E}+01$ & $2.70 \mathrm{E}+01$ & NT & $2.70 E+01$ & & & MG/KG \\
\hline Cone & Whole Building & Fluorene & $8.50 \mathrm{E}-01$ & $8.50 \mathrm{E}-01$ & NT & $8.50 \mathrm{E}-01$ & & & MG/KG \\
\hline Core & Whole Building & "Indeno(1,2,3-cd)pyrene" & $9.60 \mathrm{E}+00$ & $9.60 \mathrm{E}+00$ & $\mathrm{NT}$ & $9.60 \mathrm{E}+00$ & & & MG/KG \\
\hline Core & Whole Building & Naphthalene & $5.30 \mathrm{E}-02$ & $4.20 \mathrm{E}-01$ & $\mathbf{N}$ & 2.37E-01 & $1.40 \mathrm{E}+00$ & & MG/KG \\
\hline Core & Whole Building & Phenanthrene & $2.40 \mathrm{E}-01$ & $1.70 \mathrm{E}+01$ & $\mathrm{~N}$ & 8.62E+ $+\infty$ & $6.15 E+01$ & & MG/KG \\
\hline Core & Whole Building & Pyrene & $2.20 \mathrm{E}+01$ & $2.20 \mathrm{E}+01$ & NT & $2.20 \mathrm{E}+01$ & & & MG/KG \\
\hline Core & Whole Building & $137 \mathrm{Cs}$ & $1.51 \mathrm{E}+01$ & $3.08 \mathrm{E}+04$ & $\mathbf{L}$ & $5.06 \mathrm{E}+04$ & $1.53 \mathrm{E}+07$ & & PCI/G \\
\hline Core & Whole Building & $226 \mathrm{Ra}$ & 3.60E-01 & 4.01E-01 & $\mathbf{N}$ & 3.81E-01 & $4.08 \mathrm{E}-01$ & & PCI/G \\
\hline Core & Whole Building & 228Ra & $3.70 \mathrm{E}-01$ & $3.70 \mathrm{E}-01$ & $\mathbf{N}$ & $3.70 \mathrm{E}-01$ & $3.70 \mathrm{E}-01$ & & PCI/G \\
\hline Core & Whole Building & $228 \mathrm{Th}$ & $3.80 \mathrm{E}-01$ & $8.40 \mathrm{E}-01$ & $\mathbf{L}$ & 5.74E-01 & $7.09 \mathrm{E}-01$ & & PCUG \\
\hline Core & Whole Building & $229 \mathrm{Th}$ & $1.87 \mathrm{E}+01$ & $1.87 \mathrm{E}+01$ & $\mathrm{Nr}$ & $1.87 \mathrm{E}+01$ & & & PCI/G \\
\hline Core & Whole Building & 230Th & $1.00 \mathrm{E}+\infty$ & $1.40 \mathrm{E}+\infty 0$ & $\mathbf{N}$ & $9.68 \mathrm{E}-01$ & $1.48 \mathrm{E}+\infty 0$ & $4.00 \mathrm{E}-02$ & $\mathrm{PCI} / \mathrm{G}$ \\
\hline Core & Whole Building & $232 \mathrm{Th}$ & $2.90 \mathrm{E}-01$ & $8.70 \mathrm{E}-01$ & $\mathbf{L}$ & $5.41 \mathrm{E}-01$ & 7.32E-01 & & $\mathrm{PCD} / \mathrm{G}$ \\
\hline Core & Whole Building & $233 / 234 \mathrm{U}$ & $4.90 \mathrm{E}-01$ & $3.43 \mathrm{E}+\infty$ & $\mathbf{L}$ & $1.43 \mathrm{E}+\infty$ & $2.58 \mathrm{E}+\infty$ & & PCVG \\
\hline Core & Whole Building & $234 \mathrm{Th}$ & $6.31 \mathrm{E}+00$ & $6.31 \mathrm{E}+\infty$ & $\mathbf{N}$ & $6.31 E+\infty 0$ & $6.31 E+\infty 0$ & & PCI/G \\
\hline Core & Whole Building & $235 \mathrm{U}$ & $1.20 \mathrm{E}-01$ & $1.20 \mathrm{E}-01$ & $\mathbf{N}$ & $1.16 \mathrm{E}-01$ & $1.64 \mathrm{E}-01$ & $1.70 \mathrm{E}-01$ & PCI/G \\
\hline Core & Whole Building & 238Pu & $4.50 \mathrm{E}-01$ & $1.16 \mathrm{E}+\infty$ & $\mathcal{L}$ & $6.26 \mathrm{E}-01$ & $1.15 \mathrm{E}+\infty$ & $6.40 \mathrm{E}-01$ & PCV/G \\
\hline
\end{tabular}

- = minimum detection

$\mathrm{b}=$ maximum detection

$c=$ distribution: $N=$ Normal; $L=L o g$ Normal

$d=95 \%$ upper confidence limit

${ }^{\circ}=$ maximum nondetection 
Table C-1. Cont.

\begin{tabular}{|c|c|c|c|c|c|c|c|c|c|}
\hline DATA & LOCATION & CONTAMINANT & MIN_DET & MAX_DET & DIST & MEAN & UCL954 & MAX_NOND & UNITS \\
\hline Core & Whole Building & $238 U$ & $6.20 \mathrm{E}-01$ & $2.24 \mathrm{E}+\infty 0$ & L & $1.23 \mathrm{E}+\infty 0$ & $1.98 \mathrm{E}+00$ & & PCU/G \\
\hline Core & Whole Building & $239 / 240 \mathrm{Pu}$ & $4.00 \mathrm{E}+00$ & $3.54 \mathrm{E}+01$ & $\mathbf{L}$ & $3.40 \mathrm{E}+00$ & $6.59 \mathrm{E}+01$ & $4.80 \mathrm{E}-01$ & PCUG \\
\hline Core & Whole Building & $241 \mathrm{Am}$ & $5.00 \mathrm{E}-01$ & $5.00 \mathrm{E}-01$ & $\mathbf{N}$ & $2.97 \mathrm{E}+00$ & $1.02 \mathrm{E}+01$ & $7.90 \mathrm{E}+00$ & PCI/G \\
\hline Core & Whole Building & $40 \mathrm{~K}$ & $3.47 \mathrm{E}+00$ & $9.43 \mathrm{E}+00$ & $\mathbf{L}$ & $5.80 \mathrm{E}+\infty 0$ & $7.59 \mathrm{E}+00$ & & PCI/G \\
\hline Core & Whole Building & $60 \mathrm{Co}$ & $1.30 \mathrm{E}-01$ & $3.43 E+00$ & $\mathbf{N}$ & $1.23 \mathrm{E}+00$ & $4.44 \mathrm{E}+00$ & & PCUG \\
\hline Core & Whole Building & $90 S r$ & $8.10 \mathrm{E}+\infty 0$ & $9.75 E+01$ & $\underline{L}$ & $9.62 \mathrm{E}+\infty 0$ & $1.24 \mathrm{E}+02$ & $5.50 \mathrm{E}-01$ & PCUG \\
\hline Core & Whole Building & Tritium & $6.50 \mathrm{E}-01$ & $2.56 \mathrm{E}+01$ & $\mathbf{L}$ & $1.64 \mathrm{E}+01$ & $1.09 \mathrm{E}+02$ & & PCU/G \\
\hline Direct & Cell & Beta-Gamma & 4.05E-01 & $2.05 \mathrm{E}+01$ & $\mathbf{L}$ & $5.68 \mathrm{E}+00$ & $8.09 \mathrm{E}+00$ & & mrem/hr \\
\hline Direct & Cell & Gamma & 7.13E-01 & $5.36 \mathrm{E}+01$ & $\mathbf{L}$ & $4.95 E+00$ & $6.95 \mathrm{E}+00$ & & $\mathrm{mrem} / \mathrm{hr}$ \\
\hline Direct & East Ġallery & Beta-Gamma & $2.67 E-02$ & $4.44 \mathrm{E}-01$ & $\mathbf{L}$ & $9.05 \mathrm{E}-02$ & $1.12 \mathrm{E}-01$ & & $\mathrm{mrem} / \mathrm{hr}$ \\
\hline Direct & East Gallery & Gamma & 2.32E-02 & $6.51 \mathrm{E}-01$ & L & $\cdot 8.03 E-02$ & $1.01 \mathrm{E}-01$ & & $\mathrm{mrem} / \mathrm{hr}$ \\
\hline Direct & Whole Building & Beta-Gamma & $2.67 \mathrm{E}-02$ & $2.05 \mathrm{E}+01$ & $\mathbf{L}$ & $5.04 \mathrm{E}+00$ & $1.11 \mathrm{E}+01$ & & mrem/hr \\
\hline Direct & Whole Building & Gamma & $2.32 \mathrm{E}-02$ & $5.36 \mathrm{E}+01$ & $\mathbf{L}$ & $4.56 \mathrm{E}+00$ & $1.02 \mathrm{E}+01$ & & $\mathrm{mrem} / \mathrm{hr}$ \\
\hline Smear & Cell & $137 \mathrm{Cs}_{8}$ & $1.43 E+02$ & $1.43 E+02$ & & $1.43 E+02$ & & & PCVG \\
\hline Smear & Cell & $226 \mathrm{Ra}$ & $5.27 E-03$ & $5.27 \mathrm{E}-03$ & & $5.27 \mathrm{E}-03$ & . & & $\mathbf{P C I} / \mathrm{G}$ \\
\hline Smear & Cell & $228 \mathrm{Th}$ & $1.10 \mathrm{E}-02$ & $1.10 \mathrm{E}-02$ & & $1.10 \mathrm{E}-02$ & & & $\mathrm{PCI} / \mathrm{G}$ \\
\hline Smear & Cell & $230 \mathrm{Th}$ & $1.84 \mathrm{E}-02$ & $1.84 \mathrm{E}-02$ & & $1.84 \mathrm{E}-02$ & & & PCI/G \\
\hline Smear & Cell & $232 \mathrm{Th}$ & $1.14 \mathrm{E}-02$ & $1.14 \mathrm{E}-02$ & & $1.14 \mathrm{E}-02$ & & & $P C L / G$ \\
\hline
\end{tabular}

$=$ minimum detection

$b=$ maximum detection

c= distribution: $N=$ Normal $;=$ Log Normal

$d=95 \%$ upper confidence limit

$\circ=$ maximum nondetection 
Table C-1. Cont.

\begin{tabular}{|c|c|c|c|c|c|c|c|c|c|}
\hline DATA & LOCATION & CONTAMINANT & MIN_DET & MAX_DET & DIST & MEAN & UCL9S4 & MAX_NOND & UNITS \\
\hline Smear & Cell & $233 / 234 \mathrm{U}$ & $1.71 \mathrm{E}-02$ & $1.71 E-02$ & & $1.71 \mathrm{E}-02$ & & & PCL/G \\
\hline Smear & Cell & $235 \mathrm{U}$ & $1.58 \mathrm{E}-03$ & $1.58 \mathrm{E}-03$ & & $1.58 \mathrm{E}-03$ & & & PCI/G \\
\hline Smear & Cell & $238 \mathrm{Pu}$ & $8.40 \mathrm{E}-03$ & $8.40 E-03$ & & $8.40 \mathrm{E}-03$ & & & PCI/G \\
\hline Smear & Cell & $238 \mathrm{U}$ & $2.23 \mathrm{E}-02$ & $2.23 E-02$ & & $2.23 \mathrm{E}-02$ & & & PCL/G \\
\hline Smear & Cell & $239 / 240 \mathrm{Pu}$ & $5.25 E-02$ & $5.25 \mathrm{E}-02$ & & $5.25 \mathrm{E}-02$ & & & PCUG \\
\hline Smear & Cell & $40 \mathrm{~K}$ & $5.50 \mathrm{E}-01$ & $5.50 \mathrm{E}-01$ & & $5.50 \mathrm{E}-01$ & & & PCL/G \\
\hline Smear & Cell & $60 \mathrm{Co}$ & $1.35 \mathrm{E}-02$ & $1.35 \mathrm{E}-02$ & & $1.35 \mathrm{E}-02$ & & & PCL/G \\
\hline Smear & Cell & $90 \mathrm{Sr}$ & $1.21 \mathrm{E}+00$ & $1.21 \mathrm{E}+\infty$ & & $1.21 \mathrm{E}+00$ & & & PCUG \\
\hline Smear & East Gallery & 137Cs & $1.53 \mathrm{E}+01$ & $1.53 \mathrm{E}+01$ & & $1.53 \mathrm{E}+01$ & & & PCL/G \\
\hline Smear & East Gallery & $226 \mathrm{Ra}$ & $1.46 \mathrm{E}-02$ & $1.46 \mathrm{E}-02$ & & $1.46 \mathrm{E}-02$ & & & PCUG \\
\hline Smear & East Gallery & $228 \mathrm{Ra}$ & $2.42 \mathrm{E}-01$ & 2.42E-01 & & 2.42E-01 & & & PCL/G \\
\hline Smear & East Gallery & 228Th & $2.26 \mathrm{E}-02$ & $2.26 \mathrm{E}-02$ & & $2.26 \mathrm{E}-02$ & & & PCI/G \\
\hline Smear & East Gallery & 230Th & $4.85 \mathrm{E}-02$ & $4.85 \mathrm{E}-02$ & & $4.85 \mathrm{E}-02$ & & & PCI/G \\
\hline Smear & East Gallery & $232 \mathrm{Th}$ & $1.74 \mathrm{E}-02$ & $1.74 \mathrm{E}-02$ & & $1.74 \mathrm{E}-02$ & & & PCL/G \\
\hline Smear & East Gallery & $233 / 234 U$ & $3.64 \mathrm{E}-02$ & $3.64 \mathrm{E}-02$ & & 3.64E-02 & & & PCI/G \\
\hline Smear & East Gallery & $235 \mathrm{U}$ & $2.43 \mathrm{E}-03$ & $2.43 \mathrm{E}-03$ & & $2.43 \mathrm{E}-03$ & & & PCI/G \\
\hline Smear & East Gallery & $238 \mathrm{Pu}$ & $1.58 \mathrm{E}-02$ & $1.58 \mathrm{E}-02$ & & $1.58 \mathrm{E}-02$ & & & PCVG \\
\hline Smear & East Gallery & $238 U$ & $2.83 E-02$ & $2.83 E-02$ & & $2.83 \mathrm{E}-02$ & & & PCI/G \\
\hline
\end{tabular}

$\therefore=$ minimum detection

$b=$ maximum detection

$c=$ distribution: $N=$ Normal; $L=L o g$ Normal

$d=95 \%$ upper confidence limit

$\circ=$ maximum nondetection 
Table C-1. Cont.

\begin{tabular}{|c|c|c|c|c|c|c|c|c|c|}
\hline DATA & LOCATION & CONTAMINANT & MIN_DET & MAX_DET & DIST & MEAN & UCL954 & MAX_NOND & UNITS \\
\hline Smear & East Gallery & $239 / 240 \mathrm{Pu}$ & $1.58 \mathrm{E}-02$ & $1.58 \mathrm{E}-02$ & & $1.58 \mathrm{E}-02$ & & & $\mathbf{P C I} / \mathbf{G}$ \\
\hline Smear & East Gallery & $241 \mathrm{Am}$ & $2.02 \mathrm{E}-02$ & $2.02 \mathrm{E}-02$ & & $2.02 \mathrm{E}-02$ & & & PCI/G \\
\hline Smear & East Gallery & $40 \mathrm{~K}$ & $3.61 E+00$ & $3.61 \mathrm{E}+\infty 0$ & & $3.61 \mathrm{E}+\infty 0$ & & & PCU/G \\
\hline Smear & East Gallery & $90 \mathrm{sr}$ & $5.44 \mathrm{E}+\infty 0$ & $5.44 \mathrm{E}+\infty 0$ & & $5.44 \mathrm{E}+\infty 0$ & & & PCI/G \\
\hline Smear & Whole Building & $137 \mathrm{Cs}_{\mathrm{s}}$ & $8.03 E+01$ & $8.03 E+01$ & & $8.03 E+01$ & & & PCD/G \\
\hline Smear & Whole Building & $226 \mathrm{Ra}$ & $5.92 \mathrm{E}-03$ & $5.92 \mathrm{E}-03$ & & $5.92 \mathrm{E}-03$ & & & PCI/G \\
\hline Smear & Whole Building & 228Ra & $2.82 \mathrm{E}-02$ & $2.82 \mathrm{E}-02$ & & $2.82 \mathrm{E}-02$ & & & PCI/G \\
\hline Smear & Whole Building & $228 \mathrm{Th}$ & $1.13 \mathrm{E}-02$ & $1.13 \mathrm{E}-02$ & & $1.13 \mathrm{E}-02$ & & & PCI/G \\
\hline Smear & Whole Building & $230 \mathrm{Th}$ & $2.01 \mathrm{E}-02$ & $2.01 \mathrm{E}-02$ & & $2.01 \mathrm{E}-02$ & & & PCI/G \\
\hline Smear & Whole Building & $232 \mathrm{Th}$ & $1.14 \mathrm{E}-02$ & $1.14 \mathrm{E}-02$ & & $1.14 \mathrm{E}-02$ & & & PCI/G \\
\hline Smear & Whole Building & $233 / 234 U$ & $1.77 \mathrm{E}-02$ & $1.77 \mathrm{E}-02$ & & $1.77 \mathrm{E}-02$ & & & PCI/G \\
\hline Smear & Whole Building & $235 U$ & $1.77 \mathrm{E}-03$ & $1.77 \mathrm{E}-03$ & & $1.77 \mathrm{E}-03$ & & & PCL/G \\
\hline Smear & Whole Building & 238Pu & $9.45 \mathrm{E}-03$ & $9.45 \mathrm{E}-03$ & & $9.45 \mathrm{E}-03$ & & & PCI/G \\
\hline Smear & Whole Building & $238 U$ & $2.51 \mathrm{E}-02$ & $2.51 \mathrm{E}-02$ & & $2.51 \mathrm{E}-02$ & & & PCU/G \\
\hline Smear & Whole Building & 239/240Pu & $5.79 \mathrm{E}-02$ & $5.79 \mathrm{E}-02$ & & $5.79 \mathrm{E}-02$ & . & & PCI/G \\
\hline Smear & Whole Building & $241 \mathrm{Am}$ & $7.38 \mathrm{E}-03$ & $7.38 \mathrm{E}-03$ & & $7.38 \mathrm{E}-03$ & & & PCI/G \\
\hline Smear & Whole Building & $40 K$ & $4.22 \mathrm{E}-01$ & $4.22 \mathrm{E}-01$ & & $4.22 \mathrm{E}-01$ & & & PCI/G \\
\hline Smear & Whole Building & $60 \mathrm{Co}$ & $1.02 \mathrm{E}-02$ & $1.02 \mathrm{E}-02$ & & $1.02 \mathrm{E}-02$ & & & PCI/G \\
\hline
\end{tabular}

$a$ = minimum detection

$b=$ maximum detection

$c=$ distribution: $N=$ Normal; $L=$ Log Normal

$d=95 \%$ upper confidence limit

$\circ=$ maximum nondetection 
Table C-1. Cont.

\begin{tabular}{|l|l|l|l|l|l|l|l|l|l||}
\hline \hline DATA & LOCATION & CONTAMINANT & MIN_DET & MAX_DET & DIST & MEAN & UCL95` & MAX_NOND & UNITS \\
\hline Smear & Whole Building & $90 S \mathrm{Sr}$ & $9.10 \mathrm{E}-01$ & $9.10 \mathrm{E}-01$ & & $9.10 \mathrm{E}-01$ & & & PCI/G \\
\hline TLD & Cell & Bela & $5.00 \mathrm{E}-01$ & $9.83 \mathrm{E}+01$ & L & $1.36 \mathrm{E}+01$ & $2.34 \mathrm{E}+01$ & & $\mathrm{mrem} / \mathrm{hr}$ \\
\hline TLD & Cell & Gamma & $4.00 \mathrm{E}-01$ & $2.42 \mathrm{E}+01$ & L & $5.07 \mathrm{E}+00$ & $7.12 \mathrm{E}+00$ & & $\mathrm{mrem} / \mathrm{hr}$ \\
\hline
\end{tabular}

\footnotetext{
$a=$ minimum detection

$b=$ maximum detection

${ }^{c}=$ distribution: $\mathrm{N}=$ Normal $\mathrm{L}=\mathrm{L}$ g Normal

$d=95 \%$ upper confidence limit

${ }^{\circ}=$ maximum nondetection
} 
THIS PAGE INTENTIONALLY LEFT BLANK 
APPENDIX D

Exposure Assessment Results 
THIS PAGE INTENTIONALLY LEFT BLANK 
Table D-1. Maintenance Worker Exposure Concentrations and Chronic Daily Intake Values for Core Samples and Sediment

\begin{tabular}{|c|c|c|c|c|c|c|c|c|}
\hline \multirow{2}{*}{ Receptor } & \multirow{2}{*}{ Media } & \multirow{2}{*}{ Location } & \multirow[b]{2}{*}{$\begin{array}{l}\text { Exposure } \\
\text { Pathway }\end{array}$} & \multirow[b]{2}{*}{$\begin{array}{c}\text { Contaminant } \\
\text { Class }\end{array}$} & \multirow{2}{*}{ Contaminant } & \multirow[b]{2}{*}{$\begin{array}{l}\text { Exposure Point } \\
\text { Concentration" }\end{array}$} & \multicolumn{2}{|c|}{ Chronic Daily Intake } \\
\hline & & & & & & & $\begin{array}{l}\text { Carcinogenic } \\
\text { Effects }\end{array}$ & $\begin{array}{c}\text { Noncarcinogenic } \\
\text { Effects }\end{array}$ \\
\hline Maintenance Worker & Core & Cell & Ingestion & Inorganics & Arsenic & $2.6 \mathrm{E}+\infty 0$ & $4.5 \mathrm{E}-07$ & $1.3 \mathrm{E}-06$ \\
\hline Maintenance Worker & Core & Cell & Ingestion & Inorganics & Barium & $7.2 \mathrm{E}+02$ & $1.3 E-04$ & $3.5 E-04$ \\
\hline Maintenance Worker & Core & Cell & Ingestion & Inorganic: & Beryllium & $1.8 \mathrm{E}+01$ & 3.1E-06 & $8.6 \mathrm{E}-06$ \\
\hline Maintenance Worker & Core & Cell & Ingestion & Inorganics & Cadmium & $1.8 \mathrm{E}+02$ & 3.1E-05 & $8.8 \mathrm{E}-05$ \\
\hline Maintenance Worker & Core & Cell & Ingestion & Inorganics & Chromium VI & $2.0 \mathrm{E}+02$ & 3.4E-05 & $9.6 \mathrm{E}-05$ \\
\hline Maintenance Worker & Core & Cell & Ingestion & Inorganics & Manganese & $9.5 \mathrm{E}+03$ & $1.7 \mathrm{E}-03$ & $4.7 \mathrm{E}-03$ \\
\hline Maintenance Worker & Core & Cell & Ingestion & Inorganics & Mercury & $3.4 \mathrm{E}-01$ & $5.9 \mathrm{E}-08$ & $1.7 \mathrm{E}-07$ \\
\hline Maintenance Worker & Core & Cell & Ingestion & Inorganics & Nickel & $1.4 \mathrm{E}+02$ & $2.4 \mathrm{E}-05$ & $6.8 \mathrm{E}-05$ \\
\hline Maintenance Worker & Core & Cell & Ingestion & Inorganics & Vanadium & $2.4 \mathrm{E}+02$ & 4.2E-05 & $1.2 \mathrm{E}-04$ \\
\hline Maintenance Worker & Core & Cell & Ingestion & Inorganics & Zinc & $3.5 \mathrm{E}+04$ & $6.1 E-03$ & $1.7 \mathrm{E}-02$ \\
\hline Maintenance Worker & Core & Cell & Ingestion & Organics & Aroclor-1254 & $3.4 \mathrm{E}-01$ & $5.9 \mathrm{E}-08$ & $1.7 \mathrm{E}-07$ \\
\hline Maintenance Worker & Core & Cell & Ingestion & Organics & Aroclor-1260 & 5.7E-01 & $1.0 \mathrm{E}-07$ & $2.8 \mathrm{E}-07$ \\
\hline Maintenance Worker & Core & Cell & Ingestion & Radionuclides & ${ }^{137} \mathrm{Cs}$ & $1.4 \mathrm{E}+03$ & $4.3 \mathrm{E}+05$ & \\
\hline Maintenance Worker & Core & Cell & Ingestion & Radionuclides & ${ }^{226} \mathrm{Ra}$ & $4.0 \mathrm{E}-01$ & $1.3 \mathrm{E}+02$ & \\
\hline Maintenance Worker & Core & Cell & Ingestion & Radionuclides & ${ }^{220} \mathrm{Th}$ & $8.4 \mathrm{E}-01$ & $2.6 \mathrm{E}+02$ & \\
\hline Maintenance Worker & Core & Cell & Ingestion & Radionuclides & ${ }^{230} \mathrm{Th}$ & $1.4 \mathrm{E}+\infty$ & $4.4 \mathrm{E}+02$ & \\
\hline Maintenance Worker & Core & Cell & Ingestion & Radionuclides & ${ }^{232} \mathrm{Th}$ & 8.7E-01 & $2.7 E+02$ & \\
\hline Maintenance Worker & Core & Cell & Ingestion & Radionuclides & ${ }^{233 n} \mathrm{U}$ & $1.3 \mathrm{E}+\infty 0$ & $4.1 E+02$ & \\
\hline Maintenance Worker & Core & Cell & Ingestion & Radionuclides & ${ }^{234} \mathrm{Th}$ & $6.3 \mathrm{E}+00$ & $2.0 \mathrm{E}+03$ & \\
\hline
\end{tabular}

- Units are $\mathrm{mg} / \mathrm{kg}$ for inorganics and organics; $\mathrm{pCi} / \mathrm{g}$ for radionuclides

b Units are $\mathrm{mg} / \mathrm{kg}$-day for inorganics and organics; pCi-day for radionuclides 


\begin{tabular}{|c|c|c|c|c|c|c|c|c|}
\hline \multirow[b]{2}{*}{ Receptor } & \multirow[b]{2}{*}{ Media } & \multirow[b]{2}{*}{ Location } & \multirow[b]{2}{*}{$\begin{array}{l}\text { Exposure } \\
\text { Pathway }\end{array}$} & \multirow[b]{2}{*}{$\begin{array}{c}\text { Contaminant } \\
\text { Class }\end{array}$} & \multirow[b]{2}{*}{ Contaminant } & \multirow[b]{2}{*}{$\begin{array}{l}\text { Exposure Point } \\
\text { Concentration" }\end{array}$} & \multicolumn{2}{|c|}{ Chronic Daily Intake } \\
\hline & & & & & & & $\begin{array}{l}\text { Carcinogenic } \\
\text { Effects }\end{array}$ & $\begin{array}{c}\text { Noncarcinogenic } \\
\text { Effects }\end{array}$ \\
\hline Maintenance Worker & Core & Cell & Ingestion & Radionuclides & ${ }^{23 S} U$ & $1.2 \mathrm{E}-01$ & $3.8 \mathrm{E}+01$ & \\
\hline Maintenance Worker & Core & Cell & Ingestion & Radionuclides & ${ }^{238} \mathrm{Pu}$ & $4.5 \mathrm{E}-01$ & $1.4 \mathrm{E}+02$ & \\
\hline Maintenance Worker & Core & Cell & Ingestion & Radionuclides & ${ }^{244} \mathrm{U}$ & $1.7 \mathrm{E}+00$ & $5.3 \mathrm{E}+02$ & \\
\hline Maintenance Worker & Core & Cell & Ingestion & Radionuclides & ${ }^{2501220} \mathrm{Pu}$ & $4.0 \mathrm{E}+\infty 0$ & $1.3 \mathrm{E}+03$ & \\
\hline Maintenance Worker & Core & Cell & Ingestion & Radionuclides & ${ }^{40} \mathrm{~K}$ & $5.3 \mathrm{E}+\infty 0$ & $1.7 \mathrm{E}+03$ & \\
\hline Maintenance Worker & Core & Cell & Ingestion & Radionuclides & ${ }^{\infty} \mathrm{Co}$ & 1.3E-01 & $4.1 \mathrm{E}+01$ & \\
\hline Maintenance Worker & Core & Cell & Ingestion & Radionuclides & ${ }^{\infty} \mathrm{Sr}$ & $1.2 \mathrm{E}+01$ & $3.6 \mathrm{E}+03$ & \\
\hline Maintenance Worker & Core & Cell & Ingestion & Radionuclides & Tritium & $2.1 E+01$ & $6.6 \mathrm{E}+03$ & \\
\hline Maintenance Worker & Core & Cell & 2-Dermal & Inorganics & Arsenic & $2.6 \mathrm{E}+00$ & $2.8 \mathrm{E}-08$ & $7.9 \mathrm{E}-08$ \\
\hline Maintenance Worker & Core & Cell & 2-Dermal & Inorganics & Barium & $7.2 \mathrm{E}+02$ & $7.9 \mathrm{E}-06$ & $2.2 \mathrm{E}-05$ \\
\hline Maintenance Worker & Core & Cell & 2-Dermal & Inorganics & Beryllium & $1.8 \mathrm{E}+01$ & $1.9 \mathrm{E}-07$ & $5.4 \mathrm{E}-07$ \\
\hline Maintenance Worker & Core & Cell & 2-Dermal & Inorganics & Cadmium & $1.8 \mathrm{E}+02$ & $2.0 \mathrm{E}-06$ & $5.5 \mathrm{E}-06$ \\
\hline Maintenance Worker & Core & Cell & 2-Dermal & Inorganics & Chromium VI & $2.0 \mathrm{E}+02$ & 2.1E-06 & $6.0 \mathrm{E}-06$ \\
\hline Maintenance Worker & Core & Cell & 2-Dermal & Inorganics & Manganese & $9.5 \mathrm{E}+03$ & $1.0 \mathrm{E}-04$ & $2.9 \mathrm{E}-04$ \\
\hline Maintenance Worker & Core & Cell & 2-Dermal & Inorganics & Mercury & $3.4 \mathrm{E}-01$ & $3.7 \mathrm{E}-09$ & $1.0 \mathrm{E}-08$ \\
\hline Maintenance Worker & Core & Cell & 2-Dermal & Inorganics & Nickel & $1.4 \mathrm{E}+02$ & $1.5 \mathrm{E}-06$ & $4.2 \mathrm{E}-06$ \\
\hline Maintenance Worker & Core & Cell & 2-Dermal & Inorganics & Vanadium & $2.4 \mathrm{E}+02$ & $2.6 \mathrm{E}-06$ & $7.3 \mathrm{E}-06$ \\
\hline Maintenance Worker & Core & Cell & 2-Dermal & Inorganics & Zinc & $3.5 \mathrm{E}+04$ & $3.8 \mathrm{E}-04$ & $1.1 \mathrm{E}-03$ \\
\hline Maintenance Worker & Core & Cell & Dermal & Organics & Aroclor-1254 & $3.4 \mathrm{E}-01$ & 3.7E-08 & $1.0 \mathrm{E}-07$ \\
\hline Maintenance Worker & Core & Cell & Dermal & Organics & Aroclor-1260 & 5.7E-01 & $6.2 \mathrm{E}-08$ & $1.7 \mathrm{E}-07$ \\
\hline Maintenance Worker & Core & Cell & Inhalation & Inorganics & Arsenic & $2.6 \mathrm{E}+00$ & $4.2 \mathrm{E}-11$ & $1.2 \mathrm{E}-10$ \\
\hline
\end{tabular}

- Units are $\mathrm{mg} / \mathrm{kg}$ for inorganics and organics; $\mathrm{pCi} / \mathrm{g}$ for radionuclides

- Units are mg/kg-day for inorganics and organics; pCi-day for radionuclides 


\begin{tabular}{|c|c|c|c|c|c|c|c|c|}
\hline \multirow{2}{*}{ Receptor } & \multirow{2}{*}{ Media } & \multirow{2}{*}{ Location } & \multirow{2}{*}{$\begin{array}{l}\text { Exposure } \\
\text { Pathway }\end{array}$} & \multirow{2}{*}{$\begin{array}{l}\text { Contaminant } \\
\text { Class }\end{array}$} & \multirow{2}{*}{ Contaminant } & \multirow{2}{*}{$\begin{array}{l}\text { Exposure Point } \\
\text { Concentration" }\end{array}$} & \multicolumn{2}{|c|}{ Chronic Daily Intake } \\
\hline & & & & & & & $\begin{array}{c}\text { Carcinogenic } \\
\text { Erfects }\end{array}$ & $\begin{array}{l}\text { Noncarcinogenic } \\
\text { Effects }\end{array}$ \\
\hline Maintenance Worker & Core & Cell & Inhalation & Inorganics & Barium & $7.2 \mathrm{E}+02$ & $1.2 \mathrm{E}-08$ & 3.3E-08 \\
\hline Maintenance Worker & Core & Cell & Inhalation & Inorganics & Beryllium & $1.8 \mathrm{E}+01$ & $2.9 \mathrm{E}-10$ & 8.0E-10 \\
\hline Maintenance Worker & Core & Cell & Inhalation & Inorganics & Cadmium & $1.8 E+02$ & $2.9 \mathrm{E}-09$ & 8.2E-09 \\
\hline Maintenance Worker & Core & Cell & Inhalation & Inorganiç: & Chromium VI & $2.0 \mathrm{E}+02$ & $3.2 \mathrm{E}-09$ & $9.0 \mathrm{E}-09$ \\
\hline Maintenance Worker & Core & Cell & Inhalation & Inorganics & Manganese & $9.5 E+03$ & $1.6 \mathrm{E}-07$ & 4.4E-07 \\
\hline Maintenance Worker & Core & Cell & Inhalation & Inorganics & Mencury & $3.4 \mathrm{E}-01$ & $5.5 \mathrm{E}-12$ & $1.6 \mathrm{E}-11$ \\
\hline Maintenance Worker & Core & Cell & Inhalation & Inorganics & Nickel & $1.4 \mathrm{E}+02$ & 2.3E-09 & $6.3 E-09$ \\
\hline Maintenance Worker & Core & Cell & Inhalation & Inorganics & Vanadium & $2.4 \mathrm{E}+02$ & $3.9 \mathrm{E}-09$ & $1.1 \mathrm{E}-08$ \\
\hline Maintenance Worker & Core & Cell & Inhalation & Inorganics & Zinc & $3.5 \mathrm{E}+0.4$ & $5.7 E-07$ & $1.6 \mathrm{E}-06$ \\
\hline Maintenance Worker & Core & Cell & Inhalation & Organics & Aroclor- 1254 & $3.4 \mathrm{E}-01$ & $5.5 \mathrm{E}-12$ & $1.6 \mathrm{E}-11$ \\
\hline Maintenance Worker & Core & Cell & Inhalation & Organics & Anoclor-1260 & $5.7 \mathrm{E}-01$ & $9.3 \mathrm{E}-12$ & $2.6 \mathrm{E}-11$ \\
\hline Maintenance Worker & Core & Cell & Inhalation & Radionuclides & ${ }^{157} \mathrm{Cs}$ & $1.4 E+03$ & $4.0 \mathrm{E}+01$ & \\
\hline Maintenance Worker & Core & Cell & Inhalation & Radionuclides & ${ }^{206} \mathbf{R a}$ & $4.0 \mathrm{E}-01$ & $1.2 \mathrm{E}-02$ & \\
\hline Maintenance Worker & Core & Cell & Inhalation & Radionuclides & ${ }^{20} \mathrm{Th}$ & $8.4 \mathrm{E}-01$ & $2.5 \mathrm{E}-02$ & \\
\hline Maintenance Worker & Core & Cell & Inhalation & Radionuclides & ${ }^{230} \mathrm{Th}$ & $1.4 \mathrm{E}+\infty 0$ & 4.1E-02 & \\
\hline Maintenance Worker & Core & Cell & Inhalation & Radionuclides & ${ }^{25} \mathrm{Th}$ & 8.7E-01 & $2.5 \mathrm{E}-02$ & \\
\hline Maintenance Worker & Core & Cell & Inhalation & Radionuclides & ${ }^{233 / 24} U$ & $1.3 E+\infty$ & $3.8 \mathrm{E}-02$ & \\
\hline Maintenance Worker & Core & Cell & Inhalation & Radionuclides & ${ }^{28} \mathrm{Th}$ & $6.3 E+00$ & $1.8 \mathrm{E}-01$ & \\
\hline Maintenance Worker & Core & Cell & Inhalation & Radionuclides & ${ }^{235} U$ & $1.2 \mathrm{E}-01$ & $3.5 \mathrm{E}-03$ & \\
\hline Maintenance Worker & Core & Cell & Inhalation & Radionuclides & ${ }^{238} \mathrm{Pu}$ & 4.5E-01 & $1.3 \mathrm{E}-02$ & \\
\hline Maintenance Worker & Core & Cell & Inhalation & Radionuclides & ${ }^{2 \times} U$ & $1.7 \mathrm{E}+00$ & $5.0 \mathrm{E}-02$ & \\
\hline
\end{tabular}

- Units are $\mathrm{mg} / \mathrm{kg}$ for inorganics and organics; $\mathrm{pCi} / \mathrm{g}$ for radionuclides

- Units are mg/kg-day for inorganics and organics; pCi-day for radionuclides 


\begin{tabular}{|c|c|c|c|c|c|c|c|c|}
\hline \multirow{2}{*}{ Receptor } & \multirow{2}{*}{ Media } & \multirow{2}{*}{ Location } & \multirow{2}{*}{$\begin{array}{l}\text { Exposure } \\
\text { Pathway }\end{array}$} & \multirow{2}{*}{$\begin{array}{l}\text { Contaminant } \\
\text { Class }\end{array}$} & \multirow{2}{*}{ Contaminant } & \multirow{2}{*}{$\begin{array}{l}\text { Exposure Point } \\
\text { Concentration" }\end{array}$} & \multicolumn{2}{|c|}{ Chronic Daily Intake } \\
\hline & & & & & & & $\begin{array}{c}\text { Carcinogenic } \\
\text { Effects } \\
\end{array}$ & $\begin{array}{c}\text { Noncarcinogenic } \\
\text { Efrects }\end{array}$ \\
\hline Maintenance Worker & Core & Cell & Inhalation & Radionuclides & ${ }^{299220} \mathrm{Pu}$ & $4.0 \mathrm{E}+00$ & $1.2 \mathrm{E}-01$ & \\
\hline Maintenance Worker & Core & Cell & Inhalation & Radionuclides & ${ }^{10} \mathrm{~K}$ & $5.3 \mathrm{E}+\infty 0$ & 1.5E-01 & \\
\hline Maintenance Worker & Core & Cell & Inhalation & Radionuclides & ${ }^{\infty} \mathrm{Co}$ & $1.3 \mathrm{E}-01$ & $3.8 \mathrm{E}-03$ & \\
\hline Maintenance Worker & Core & Cell & Inhalation & Radionuclides & ${ }^{90} \mathrm{Sr}$ & $1.2 \mathrm{E}+01$ & $3.4 \mathrm{E}-01$ & \\
\hline Maintenance Worker & Core & Cell & Inhalation & Radionuclides & Tritium & $2.1 E+01$ & $6.2 \mathrm{E}-01$ & \\
\hline Maintenance Worker & Core & Cell & External Exposure & Radionuclides & ${ }^{157} \mathrm{Cs}$ & $1.4 \mathrm{E}+03$ & $6.3 \mathrm{E}+03$ & \\
\hline Maintenance Worker & Core & Cell & External Exposure & Radionuclides & ${ }^{200} \mathrm{Ra}$ & $4.0 \mathrm{E}-01$ & $1.8 \mathrm{E}+00$ & \\
\hline Maintenance Worker & Core & Cell & External Exposure & Radionuclides & ${ }^{220} \mathrm{Th}$ & 8.4E-01 & $3.8 \mathrm{E}+\infty 0$ & \\
\hline Maintenance Worker & Core & Cell & External Exposure & Radionuclides & ${ }^{230} \mathrm{Th}$ & $1.4 E+\infty 0$ & $6.4 E+\infty$ & \\
\hline Maintenance Worker. & Core & Cell & External Exposure & Radionuclides & ${ }^{232} \mathrm{Th}$ & 8.7E-01 & $4.0 \mathrm{E}+00$ & \\
\hline Maintenance Worker & Core & Cell & External Exposure & Radionuclides & ${ }^{233234} \mathrm{U}$ & $1.3 \mathrm{E}+00$ & $5.9 E+\infty$ & \\
\hline Maintenance Worker & Core & Cell & External Exposure & Radionuclides & ${ }^{234} \mathrm{Th}$ & $6.3 \mathrm{E}+00$ & $2.9 \mathrm{E}+01$ & \\
\hline Maintenance Worker & Core & Cell & External Exposure & Radionuclides & ${ }^{25 S} \mathbf{U}$ & $1.2 \mathrm{E}-01$ & $5.5 \mathrm{E}-01$ & \\
\hline Maintenance Worker & Core & Cell & External Exposure & Radionuclides & ${ }^{25 s} \mathrm{Pu}$ & $4.5 \mathrm{E}-01$ & $2.1 \mathrm{E}+\infty$ & \\
\hline Maintenance Worker & Core & Cell & External Exposure & Radionuclides & ${ }^{238} \mathrm{U}$ & $1.7 E+\infty 0$ & $7.8 \mathrm{E}+00$ & \\
\hline Maintenance Worker & Core & Cell & External Exposure & Radionuclides & ${ }^{299210} \mathrm{Pu}$ & $4.0 \mathrm{E}+00$ & $1.8 E+01$ & \\
\hline Maintenance Worker & Core & Cell & External Exposure & Radionuclides & ${ }^{10} \mathrm{~K}$ & $5.3 E+\infty$ & $2.4 E+01$ & \\
\hline Maintenance Worker & Core & Cell & External Exposure & Radionuclides & ${ }^{60} \mathrm{Co}$ & $1.3 \mathrm{E}-01$ & $5.9 \mathrm{E}-01$ & \\
\hline Maintenance Worker & Core & Cell & External Exposure & Radionuclides & ${ }^{90} \mathrm{Sr}$ & $1.2 \mathrm{E}+01$ & $5.3 \mathrm{E}+01$ & \\
\hline Maintenance Worker & Core & Cell & External Exposure & Radionuclides & Tritium & $2.1 \mathrm{E}+01$ & $9.6 \mathrm{E}+01$ & \\
\hline Maintenance Worker & Core & East Gallery & Ingestion & Inorganics & Arsenic & $3.0 \mathrm{E}+\infty 0$ & $5.2 \mathrm{E}-07$ & $1.5 \mathrm{E}-06$ \\
\hline
\end{tabular}

- Units are $\mathrm{mg} / \mathrm{kg}$ for inorganics and organics; $\mathrm{pCi} / \mathrm{g}$ for radionuclides

- Units are mg/kg-day for inorganics and organics; pCi-day for radionuclides 


\begin{tabular}{|c|c|c|c|c|c|c|c|c|}
\hline \multirow{2}{*}{ Receptor } & \multirow{2}{*}{ Media } & \multirow{2}{*}{ Location } & \multirow{2}{*}{$\begin{array}{l}\text { Exposure } \\
\text { Pathway }\end{array}$} & \multirow[b]{2}{*}{$\begin{array}{l}\text { Contaminant } \\
\text { Class }\end{array}$} & \multirow{2}{*}{ Contaminant } & \multirow[b]{2}{*}{$\begin{array}{l}\text { Exposure Point } \\
\text { Concentration" }\end{array}$} & \multicolumn{2}{|c|}{ Chronic Daily Intake } \\
\hline & & & & & & & $\begin{array}{l}\text { Carcinogenic } \\
\text { Efrects }\end{array}$ & $\begin{array}{c}\text { Noncarcinogenic } \\
\text { Effects }\end{array}$ \\
\hline Maintenance Worker & Core & East Gallery & Ingestion & Inorganics & Barium & $2.1 \mathrm{E}+01$ & 3.7E-06 & $1.0 \mathrm{E}-05$ \\
\hline Maintenance Worker & Core & East Gallery & Ingestion & Inorganics & Beryllium & $4.0 \mathrm{E}-01$ & $7.0 \mathrm{E}-08$ & $2.0 \mathrm{E}-07$ \\
\hline Maintenance Worker & Core & East Gallery & Ingestion & Inorganics & Cadmium & $5.1 \mathrm{E}+00$ & $8.9 \mathrm{E}-07$ & $2.5 \mathrm{E}-06$ \\
\hline Maintenance Worker & Core & East Gallery & Ingestion & Inorganics & Chromium VI & $8.3 \mathrm{E}+00$ & $1.5 \mathrm{E}-06$ & $4.1 \mathrm{E}-06$ \\
\hline Maintenance Worker & Core & East Gallery & Ingestion & Inorganics & Manganese & $2.7 \mathrm{E}+02$ & $4.6 \mathrm{E}-05$ & $1.3 \mathrm{E}-04$ \\
\hline Maintenance Worker & Core & East Gallery & Ingestion & Inorganics & Nickel & $6.0 \mathrm{E}+00$ & $1.0 \mathrm{E}-06$ & $2.9 \mathrm{E}-06$ \\
\hline Maintenance Worker & Core & East Gallery & Ingestion & Inorganics & Vanadium & $9.1 \mathrm{E}+00$ & $1.6 \mathrm{E}-06$ & 4.5E-06 \\
\hline Maintenance Worker & Core & Enst Gallery & Ingestion & Inorganics & Zine & $9.8 \mathrm{E}+02$ & $1.7 E-04$ & $4.8 \mathrm{E}-04$ \\
\hline Maintenance Worker & Core & East Gallery & Ingestion & Organics & Aroclor-1254 & $1.7 E+\infty 0$ & $3.0 \mathrm{E}-07$ & $8.3 E-07$ \\
\hline Maintenance Worker & Core & East Gallery & Ingestion & Organics & Aroclor-1260 & $2.6 \mathrm{E}+00$ & $4.5 \mathrm{E}-07$ & $1.3 \mathrm{E}-06$ \\
\hline Maintenance Worker & Core & East Gallery & Ingestion & Organics & Bis(2-ethythexyl)phthalate & $2.4 \mathrm{E}-01$ & 4.2E-08 & $1.2 \mathrm{E}-07$ \\
\hline Maintenance Worker & Core & East Gallery & Ingestion & Organics & Dimethyl phthalate & $5.5 \mathrm{E}-02$ & $9.6 \mathrm{E}-09$ & 2.7E-08 \\
\hline Maintenance Worker & Core & East Gallery & Ingestion & Radionuclides & ${ }^{197} \mathrm{Cs}$ & $2.3 E+01$ & $7.1 \mathrm{E}+03$ & \\
\hline Maintenance Worker & Core & East Gallery & Ingestion & Radionuclides & ${ }^{226} \mathrm{Ra}$ & $3.6 \mathrm{E}-01$ & $1.1 E+02$ & \\
\hline Maintenance Worker & Core & East Gallery & Ingestion & Radionuclides & ${ }^{2 n} \mathrm{Ra}$ & $3.7 \mathrm{E}-01$ & $1.2 \mathrm{E}+02$ & \\
\hline Maintenance Worker & Core & East Gallery & Ingestion & Radionuclides & ${ }^{20} \mathrm{Th}$ & $5.6 \mathrm{E}-01$ & $1.8 \mathrm{E}+02$ & \\
\hline Maintenance Worker & Core & East Gallery & Ingestion & Radionuclides & ${ }^{250} \mathrm{Th}$ & $1.2 \mathrm{E}+00$ & $3.8 \mathrm{E}+02$ & \\
\hline Maintenance Worker & Core & East Gallery & Ingestion & Radionuclides & ${ }^{230} \mathrm{Th}$ & $4.3 \mathrm{E}-01$ & $1.3 \mathrm{E}+02$ & \\
\hline Maintenance Worker & Core & East Gallery & Ingestion & Radionuclides & ${ }^{233234} \mathrm{U}$ & $9.0 \mathrm{E}-01$ & $2.8 \mathrm{E}+02$ & \\
\hline Maintenance Worker & Core & East Gallery & Ingestion & Radionuclides & $238 \mathrm{U}$ & 7.7E-01 & $2.4 E+02$ & \\
\hline Maintenance Worker & Core & East Gallery & Ingestion & Radionuclides & ${ }^{211} \mathrm{Am}$ & $5.0 \mathrm{E}-01$ & $1.6 \mathrm{E}+02$ & \\
\hline
\end{tabular}

- Units are $\mathrm{mg} / \mathrm{kg}$ for inorganics and organics; $\mathrm{pCi} / \mathrm{g}$ for radionuclides

- Units are $\mathrm{mg} / \mathrm{kg}$-day for inorganics and organics; pCi-day for radionuclides 


\begin{tabular}{|c|c|c|c|c|c|c|c|c|}
\hline \multirow{2}{*}{ Receptor } & \multirow{2}{*}{ Media } & \multirow{2}{*}{ location } & \multirow{2}{*}{$\begin{array}{l}\text { Exposure } \\
\text { Pathway }\end{array}$} & \multirow[b]{2}{*}{$\begin{array}{c}\text { Contaminant } \\
\text { Class }\end{array}$} & \multirow{2}{*}{ Contaminant } & \multirow{2}{*}{$\begin{array}{l}\text { Exposure Point } \\
\text { Concentration" }\end{array}$} & \multicolumn{2}{|c|}{ Chronic Daily Intake } \\
\hline & & & & & & & $\begin{array}{l}\text { Carcinogenic } \\
\text { Efrects }\end{array}$ & $\begin{array}{l}\text { Noncarcinogenic } \\
\text { Effects }\end{array}$ \\
\hline Maintenance Worker & Core & East Gallery & Ingestion & Radionuclides & ${ }^{+0} \mathrm{~K}$ & $5.4 E+\infty 0$ & $1.7 \mathrm{E}+03$ & \\
\hline Maintenance Worker & Core & East Gallery & Ingestion & Radionuclides & ${ }^{90} \mathrm{Sr}$ & $8.1 E+\infty$ & $2.5 E+03$ & \\
\hline Maintenance Worker & Core & East Gallery & Ingestion & Radionuclides & Tritium & $2.6 \mathrm{E}+01$ & $8.0 \mathrm{E}+03$ & \\
\hline Maintenance Worker & Core & East Gallery & Dermal & Inorganics & Arsenic & $3.0 \mathrm{E}+\infty 0$ & 3.3E-08 & $9.2 \mathrm{E}-08$ \\
\hline Maintenance Worker & Core & East Gallery & Dermal & Inorganics & Barium & $2.1 E+01$ & $2.3 \mathrm{E}-07$ & $6.5 \mathrm{E}-07$ \\
\hline Maintenance Worker & Core & East Gallery & Dermal & Inorganics & Beryilium & $4.0 \mathrm{E}-01$ & $4.4 \mathrm{E}-09$ & $1.2 \mathrm{E}-08$ \\
\hline Maintenance Worker & Core & East Gallery & Dermal & Inorganics & Cadmium & $5.1 \mathrm{E}+00$ & $5.6 \mathrm{E}-08$ & $1.6 \mathrm{E}-07$ \\
\hline Maintenance Worker & Core & East Gallery & Dermal & Inorganics & Chromium VI & $8.3 E+00$ & $9.0 \mathrm{E}-08$ & $2.5 \mathrm{E}-07$ \\
\hline Maintenance Worker & Core & East Gallery & Dermal & Inorganics & Manganese & $2.7 \mathrm{E}+02$ & $2.9 \mathrm{E}-06$ & $8.1 \mathrm{E}-06$ \\
\hline Maintenance Worker & Core & East Gallery & Dermal & Inorganics & Nickel & $6.0 \mathrm{E}+00$ & $6.5 \mathrm{E}-08$ & $1.8 \mathrm{E}-07$ \\
\hline Maintenance Worker & Core & East Gallery & Dermal & Inorganics & Vanadium & $9.1 \mathrm{E}+00$ & $9.9 \mathrm{E}-08$ & $2.8 \mathrm{E}-07$ \\
\hline Maintenance Worker & Core & East Gallery & Dermal & Inorganics & Zinc & $9.8 \mathrm{E}+02$ & $1.1 \mathrm{E}-05$ & $3.0 \mathrm{E}-05$ \\
\hline Maintenance Worker & Core & East Gallery & Dermal & Organics & Aroclor-1254 & $1.7 \mathrm{E}+\infty 0$ & $1.9 \mathrm{E}-07$ & $5.2 \mathrm{E}-07$ \\
\hline Maintenance Worker & Core & East Gallery & Dermal & Organics & Aroclor- 1260 & $2.6 \mathrm{E}+00$ & $2.8 \mathrm{E}-07$ & $7.9 \mathrm{E}-07$ \\
\hline Maintenance Worker & Core & East Gallery & Dermal & Organics & Bis(2-ethylhexyl)phthalate & 2.4E-01 & $2.6 \mathrm{E}-08$ & $7.3 \mathrm{E}-08$ \\
\hline Maintenance Worker & Core & East Gallery & Dermal & Organics & Dimethyl phthalate & $5.5 \mathrm{E}-02$ & $6.0 \mathrm{E}-09$ & $1.7 \mathrm{E}-08$ \\
\hline Maintenance Worker & Core & East Gallery & Inhalation & Inorganics & Arsenic & $3.0 \mathrm{E}+00$ & $4.9 \mathrm{E}-11$ & $1.4 \mathrm{E}-10$ \\
\hline Maintenance Worker & Core & East Gallery & Inhalation & Inorganics & Barium & $2.1 \mathrm{E}+01$ & $3.5 \mathrm{E}-10$ & $9.8 \mathrm{E}-10$ \\
\hline Maintenance Worker & Core & East Gallery & Inhalation & Inorganics & Beryllium & $4.0 \mathrm{E}-01$ & $6.5 \mathrm{E}-12$ & $1.8 \mathrm{E}-11$ \\
\hline Maintenance Worker & Core & East Gallery & Inhalation & Inorganics & Cadmium & $5.1 \mathrm{E}+00$ & 8.3E-11 & $2.3 \mathrm{E}-10$ \\
\hline Maintenance Worker & Core & East Gallery & Inhalation & Inorganics & Chromium VI & $8.3 E+00$ & $1.4 \mathrm{E}-10$ & $3.8 \mathrm{E}-10$ \\
\hline
\end{tabular}

- Units are $\mathrm{mg} / \mathrm{kg}$ for inorganics and organics; $\mathrm{pCi} / \mathrm{g}$ for radionuclides

- Units are mg/kg-day for inorganics and organics; pCi-day for radionuclides 


\begin{tabular}{|c|c|c|c|c|c|c|c|c|}
\hline \multirow{2}{*}{ Receptor } & \multirow{2}{*}{ Media } & \multirow{2}{*}{ Location } & \multirow{2}{*}{$\begin{array}{l}\text { Exposure } \\
\text { Pathway }\end{array}$} & \multirow{2}{*}{$\begin{array}{c}\text { Contaminant } \\
\text { Class }\end{array}$} & \multirow{2}{*}{ Contaminant } & \multirow{2}{*}{$\begin{array}{l}\text { Exposure Point } \\
\text { Concentration" }\end{array}$} & \multicolumn{2}{|c|}{ Chronic Daily Intake } \\
\hline & & & & & & & $\begin{array}{c}\text { Carcinogenic } \\
\text { Efrects } \\
\end{array}$ & $\begin{array}{c}\text { Noncarcinogenic } \\
\text { Effects }\end{array}$ \\
\hline Maintenance Worker & Core & East Gallery & Inhalation & Inorganics & Manganese & $2.7 E+02$ & 4.3E-09 & $1.2 \mathrm{E}-08$ \\
\hline Maintenance Worker & Core & East Gallery & Inhalation & Inorganics & Nickel & $6.0 \mathrm{E}+\infty$ & $9.8 \mathrm{E}-11$ & $2.7 \mathrm{E}-10$ \\
\hline Maintenance Worker & Core & East Gallery & Inhalation & Inorganics & Vanadium & $9.1 \mathrm{E}+\infty$ & $1.5 \mathrm{E}-10$ & $4.2 \mathrm{E}-10$ \\
\hline Maintenance Worker & Core & East Gallery & Inhalation & Inorganics & Zinc & $9.8 \mathrm{E}+02$ & $1.6 \mathrm{E}-08$ & 4.5E-08 \\
\hline Maintenance Worker & Core & East Gallery & Inhalation & Organics & Aroclor-1254 & $1.7 E+\infty$ & $2.8 \mathrm{E}-11$ & $7.8 \mathrm{E}-11$ \\
\hline Maintenance Worker & Core & East Gallery & Inhalation & Organics & Aroclor- 1260 & $2.6 \mathrm{E}+00$ & $4.2 \mathrm{E}-11$ & $1.2 \mathrm{E}-10$ \\
\hline Maintenance Worker & Core & East Gallery & Inhalation & Organics & Bis(2-ethylhexyl)phthalate & $2.4 \mathrm{E}-01$ & $3.9 \mathrm{E}-12$ & $1.1 \mathrm{E}-11$ \\
\hline Maintenance Worker & Core & East Gallery & Inhalation & Organics & Dimethyl phthalate & $5.5 \mathrm{E}-02$ & $9.0 \mathrm{E}-13$ & 2.5E-12 \\
\hline Maintenance Worker & Core & East Gallery & Inhalation & Radionuclides & ${ }^{137} \mathrm{C} 8$ & $2.3 E+01$ & $6.6 \mathrm{E}-01$ & \\
\hline Maintenance Worker & Core & East Gallery & Inhalation & Radionuclides & ${ }^{206} \mathbf{R a}$ & $3.6 \mathrm{E}-01$ & $1.1 \mathrm{E}-02$ & \\
\hline Maintenance Worker & Core & East Gallery & Inhalation & Radionuclides & ${ }^{2 n} \mathrm{Ra}$ & $3.7 E-01$ & $1.1 E-02$ & \\
\hline Maintenance Worker & Core & East Gallery & Inhalation & Radionuclides & $20 \mathrm{Th}$ & $5.6 \mathrm{E}-01$ & $1.6 \mathrm{E}-02$ & \\
\hline Maintenance Worker & Core & East Gallery & Inhalation & Radionuclides & ${ }^{230} \mathrm{Th}$ & $1.2 \mathrm{E}+00$ & $3.5 \mathrm{E}-02$ & \\
\hline Maintenance Worker & Core & East Gallery & Inhalation & Radionuclides & ${ }^{232} \mathrm{Th}$ & 4.3E-01 & $1.3 \mathrm{E}-02$ & \\
\hline Maintenance Worker & Core & East Gallery & Inhalation & Radionuclides & ${ }^{23323} \mathrm{U}$ & $9.0 \mathrm{E}-01$ & $2.6 \mathrm{E}-02$ & \\
\hline Maintenance Worker & Core & East Gallery & Inhalation & Radionuclides & ${ }^{239} \mathrm{U}$ & 7.7E-01 & $2.2 \mathrm{E}-02$ & \\
\hline Maintenance Worker & Core & East Gallery & Inhalation & Radionuclides & ${ }^{2 n !} \mathrm{Am}$ & $5.0 \mathrm{E}-01$ & $1.5 \mathrm{E}-02$ & \\
\hline Maintenance Worker & Core & East Gallery & Inhalation & Radionuclider & ${ }^{10} \mathrm{~K}$ & $5.4 \mathrm{E}+00$ & $1.6 \mathrm{E}-01$ & \\
\hline Maintenance Worker & Core & East Gallery & Inhalation & Radionuclides & ${ }^{\infty} \mathrm{Sr}$ & $8.1 E+\infty$ & 2.4E-01 & \\
\hline Maintenance Worker & Core & East Gallery & Inhalation & Radionuclides & Tritium & $2.6 \mathrm{E}+01$ & 7.5E-01 & \\
\hline Maintenance Worker & Core & East Gallery & External Exposure & Radionuclides & ${ }^{17} \mathrm{Cs}$ & $2.3 E+01$ & $1.0 \mathrm{E}+02$ & \\
\hline
\end{tabular}

- Units are mg/kg for inorganics and organics; $\mathrm{pCi} / \mathrm{g}$ for radionuclides

- Units are mg/kg-day for inorganics and organics; pCi-day for radionuclides 


\begin{tabular}{|c|c|c|c|c|c|c|c|c|}
\hline \multirow{2}{*}{ Receptor } & \multirow{2}{*}{ Media } & \multirow{2}{*}{ Location } & \multirow{2}{*}{$\begin{array}{l}\text { Exposure } \\
\text { Pathway }\end{array}$} & \multirow{2}{*}{$\begin{array}{c}\text { Contaminant } \\
\text { Class }\end{array}$} & \multirow{2}{*}{ Contaminant } & \multirow{2}{*}{$\begin{array}{l}\text { Exposure Point } \\
\text { Concentration" }\end{array}$} & \multicolumn{2}{|c|}{ Chronic Daily Intake } \\
\hline & & & & & & & $\begin{array}{c}\text { Carcinogenic } \\
\text { Efrects }\end{array}$ & $\begin{array}{c}\text { Noncarcinogenic } \\
\text { Effects }\end{array}$ \\
\hline Maintenance Worker & Core & Eant Gallery & External Exposure & Radionuclides & ${ }^{20 x} \mathrm{Ra}$ & $3.6 \mathrm{E}-01$ & $1.6 \mathrm{E}+\infty$ & \\
\hline Maintenance Worker & Core & East Gallery & External Exposure & Radionuclides & ${ }^{22 x} \mathrm{Ra}$ & $3.7 \mathrm{E}-01$ & $1.7 \mathrm{E}+\infty$ & \\
\hline Maintenance Worker & Core & East Gallery & External Exposure & Radionuclides & ${ }^{220} \mathrm{Th}$ & $5.6 \mathrm{E}-01$ & $2.6 \mathrm{E}+00$ & \\
\hline Maintenance Worker & Core & East Gallery & External Exposure & Radionuclides & ${ }^{200} \mathrm{Th}$ & $1.2 \mathrm{E}+00$ & $5.5 E+\infty 0$ & \\
\hline Maintenance Worker & Core & East Gallery & External Exposure & Radionuclides & ${ }^{250} \mathrm{Th}$ & 4.3E-01 & $2.0 \mathrm{E}+\infty 0$ & \\
\hline Maintenance Worker & Core & East Gallery & Extemal Exposure & Radionuclides & ${ }^{2312354} \mathrm{U}$ & $9.0 \mathrm{E}-01$ & $4.1 \mathrm{E}+00$ & \\
\hline Maintenance Worker & Core & East Gallery & External Exposure & Radionuclides & ${ }^{230} \mathrm{U}$ & 7.7E-01 & $3.5 \mathrm{E}+\infty$ & \\
\hline Maintenance Worker & Core & Esst Gallery & External Exposure & Radionuclides & ${ }^{211} \mathrm{Am}$ & $5.0 \mathrm{E}-01$ & $2.3 \mathrm{E}+\infty$ & \\
\hline Maintenance Worker & Cone & East Gallery & External Exposure & Radionuclides & ${ }^{10} \mathrm{~K}$ & $5.4 E+00$ & $2.5 E+01$ & \\
\hline Maintenance Worker & Core & East Gallery & External Exposure & Radionuclides & ${ }^{90} \mathrm{Sr}$ & $8.1 E+00$ & $3.7 \mathrm{E}+01$ & \\
\hline Maintenance Worker & Core & East Gallery & External Exposure & Radionuclides & Tritium & $2.6 \mathrm{E}+01$ & $1.2 \mathrm{E}+02$ & \\
\hline Maintenance Worker & Core & Sediment & Ingestion & Inorganics & Antimony & $3.6 \mathrm{E}+01$ & $6.3 \mathrm{E}-06$ & $1.8 \mathrm{E}-05$ \\
\hline Maintenance Worker & Core & Sediment & Ingestion & Inorganics & Arsenic & $2.2 \mathrm{E}+01$ & $3.8 \mathrm{E}-06$ & 1.1E-05 \\
\hline Maintenance Worker & Core & Sediment & Ingestion & Inorganics & Barium & $1.2 \mathrm{E}+02$ & 2.1E-05 & $5.9 \mathrm{E}-05$ \\
\hline Maintenance Worker & Core & Sediment & Ingestion & Inorganics & Cadmium & $6.0 \mathrm{E}+01$ & $1.1 \mathrm{E}-05$ & $2.9 \mathrm{E}-05$ \\
\hline Maintenance Worker & Core & Sediment & Ingestion & Inorganics & Chromium VI & $1.9 \mathrm{E}+02$ & $3.3 \mathrm{E}-05$ & $9.2 \mathrm{E}-05$ \\
\hline Maintenance Worker & Core & Sediment & Ingestion & Inorganics & Cyanide & $5.8 \mathrm{E}-01$ & $1.0 \mathrm{E}-07$ & $2.8 \mathrm{E}-07$ \\
\hline Maintenance Worker & Core & Sediment & Ingestion & Inorganics & Manganese & $1.3 \mathrm{E}+03$ & 2.3E-04 & 6.4E-04 \\
\hline Maintenance Worker & Core & Sediment & Ingestion & Inorganics & Mercury & $1.9 \mathrm{E}+01$ & $3.3 \mathrm{E}-06$ & 9.1E-06 \\
\hline Maintenance Worker & Core & Sediment & Ingestion & Inorganics & Nickel & $1.7 \mathrm{E}+02$ & $3.0 \mathrm{E}-05$ & 8.3E-05 \\
\hline Maintenance Worker & Core & Sediment & Ingestion & Inorganics & Selenium & 8.7E-01 & $1.5 \mathrm{E}-07$ & 4.3E-07 \\
\hline
\end{tabular}

- Units are $\mathrm{mg} / \mathrm{kg}$ for inorganics and organics; $\mathrm{pCi} / \mathrm{g}$ for radioniclides

- Units are mg/kg-day for inorganics and organics; pCi-day for radionuclides 


\begin{tabular}{|c|c|c|c|c|c|c|c|c|}
\hline \multirow{2}{*}{ Receptor } & \multirow{2}{*}{ Media } & \multirow{2}{*}{ Location } & \multirow{2}{*}{$\begin{array}{l}\text { Exposure } \\
\text { Pathway }\end{array}$} & \multirow{2}{*}{$\begin{array}{l}\text { Contaminant } \\
\text { Class }\end{array}$} & \multirow{2}{*}{ Contaminant } & \multirow[b]{2}{*}{$\begin{array}{l}\text { Exposure Point } \\
\text { Concentration" }\end{array}$} & \multicolumn{2}{|c|}{ Chronic Daily Intake } \\
\hline & & & & & & & $\begin{array}{c}\text { Carcinogenic } \\
\text { Effects }\end{array}$ & $\begin{array}{c}\text { Noncarcinogenic } \\
\text { Errects }\end{array}$ \\
\hline Maintenance Worker & Core & Sediment & Ingestion & Inorganica & Zine & $7.4 E+03$ & 1.3E-03 & $3.6 \mathrm{E}-03$ \\
\hline Maintenance Worker & Core & Sediment & Ingestion & Organics & Aceraphthene & 9.6E-01 & 1.7E-07 & 4.7E-07 \\
\hline Maintenance Worker & Core & Sediment & Ingestion & Organics & Acetone & 9.0E-02 & $1.6 \mathrm{E}-08$ & 4.4E-08 \\
\hline Maintenance Worker & Core & Sediment & Ingestion & Organics & Anthracene & $1.9 \mathrm{E}+00$ & 3.3E-07 & 9.3E-07 \\
\hline Maintenance Worker & Core & Sediment & Ingestion & Organics & Aroclor-1254 & $4.1 \mathrm{E}+02$ & 7.2E-05 & $2.0 \mathrm{E}-04$ \\
\hline Maintenance Worker & Core & Sediment & Ingestion & Organics & Benzo(a)pyrene & $5.7 E+00$ & $1.0 \mathrm{E}-06$ & $2.8 \mathrm{E}-06$ \\
\hline Maintenance Worker & Core & Sediment & Ingestion & Organics & Benzo(k)fluoranthene & $4.6 \mathrm{E}+01$ & 8.0E-06 & 2.3E-05 \\
\hline Maintenance Worker & Core & Sediment & Ingestion & Organica & Bis(2-ethylhexyl)phthalate & $2.1 E+00$ & 3.7E-07 & $1.0 \mathrm{E}-06$ \\
\hline Maintenance Worker & Core & Sediment & Ingestion & Organics & Carbazole & $2.0 \mathrm{E}+00$ & $3.5 \mathrm{E}-07$ & 9.8E-07 \\
\hline Maintenance Worker & Core & Sediment & Ingestion & Organics & Chrysene & $1.9 \mathrm{E}+01$ & 3.3E-06 & $9.3 E-06$ \\
\hline Maintenance Worker & Core & Sediment & Ingestion & Organics & Dibenz $(\mathrm{a}, \mathrm{h})$ anthracene & $1.5 \mathrm{E}+\infty 0$ & 2.6E-07 & 7.3E-07 \\
\hline Maintenance Worker & Core & Sediment & Ingestion & Organicı & Fluoranthene & $2.7 \mathrm{E}+01$ & 4.7E-06 & $1.3 \mathrm{E}-05$ \\
\hline Maintenance Worker & Core & Sediment & Ingestion & Organics & Fluorene & 8.5E-01 & $1.5 \mathrm{E}-07$ & $4.2 \mathrm{E}-07$ \\
\hline Maintenance Worker & Core & Sediment & Ingeation & Organic & Indeno $(1,2,3-c d)$ pyrene & $9.6 \mathrm{E}+00$ & $1.7 \mathrm{E}-06$ & 4.7E-06 \\
\hline Maintenance Worker & Core & Sediment & Ingestion & Organica & Pyrene & $2.2 E+01$ & 3.8E-06 & $1.1 \mathrm{E}-05$ \\
\hline Maintenance Worker & Core & Sediment & Ingestion & Radionuclides & ${ }^{17} \mathrm{Cs}$ & $3.1 \mathrm{E}+04$ & $9.6 \mathrm{E}+06$ & \\
\hline Maintenance Worker & Core & Sediment & Ingestion & Radionuclides & ${ }^{n * T h}$ & 5.4E-01 & $1.7 \mathrm{E}+02$ & \\
\hline Maintenance Worker & Core & Sediment & Ingestion & Radionuclides & 229Th & $1.9 \mathrm{E}+01$ & $5.8 E+03$ & \\
\hline Maintenance Worker & Core & Sediment & Ingestion & Radionuclides & ${ }^{232} \mathrm{Th}$ & $.5 .5 E-01$ & $1.7 \mathrm{E}+02$ & \\
\hline Maintenance Worker & Core & Sediment & Ingestion & Radionuclides & $23223 \mathrm{U}$ & $3.4 E+00$ & $1.1 E+03$ & \\
\hline Maintenance Worker & Core & Sediment & Ingestion & Radionuclides & ${ }^{238} \mathrm{Pu}$ & $1.2 \mathrm{E}+00$ & $3.6 \mathrm{E}+02$ & \\
\hline
\end{tabular}

- Units are mg/kg for inorganics and organics; $\mathrm{pCi} / \mathrm{g}$ for radionuclides

- Units are mg/kg-day for inorganica and organics; pCi-day for radionuclides 


\begin{tabular}{|c|c|c|c|c|c|c|c|c|}
\hline \multirow{2}{*}{ Receptor } & \multirow{2}{*}{ Media } & \multirow{2}{*}{ Location } & \multirow{2}{*}{$\begin{array}{l}\text { Exposure } \\
\text { Pathway }\end{array}$} & \multirow{2}{*}{$\begin{array}{c}\text { Contaminant } \\
\text { Class }\end{array}$} & \multirow{2}{*}{ Contaminant } & \multirow{2}{*}{$\begin{array}{l}\text { Exposure Point } \\
\text { Concentration" }\end{array}$} & \multicolumn{2}{|c|}{ Chronic Daily Intake" } \\
\hline & & & & & & & $\begin{array}{c}\text { Carcinogenic } \\
\text { Erfects }\end{array}$ & $\begin{array}{c}\text { Noncarcinogenic } \\
\text { Effects }\end{array}$ \\
\hline Maintenance Worker & Core & Sediment & Ingestion & Radionuclidea & ${ }^{230} \mathrm{U}$ & $2.2 \mathrm{E}+00$ & $7.0 \mathrm{E}+02$ & \\
\hline Maintenance Worker & Core & Sediment & Ingestion & Radionuclides & ${ }^{2597200} \mathrm{Pu}$ & $3.5 \mathrm{E}+01$ & $1.1 \mathrm{E}+04$ & \\
\hline Maintenance Worker & Core & Sediment & Ingestion & Radionuclides & ${ }^{10} \mathrm{~K}$ & $9.4 \mathrm{E}+00$ & $2.9 \mathrm{E}+03$ & \\
\hline Maintenance Worker & Core & Sediment & Ingeation & Radionuclides & ${ }^{\infty} \mathrm{Co}_{0}$ & $3.4 \mathrm{E}+00$ & $1.1 \mathrm{E}+03$ & \\
\hline Maintenance Worker & Cone & Sediment & Ingestion & Radionuclides & ${ }^{\infty} \mathrm{Sr}$ & $9.8 \mathrm{E}+01$ & $3.0 \mathrm{E}+04$ & \\
\hline Maintenance Worker & Core & Sediment & Ingestion & Radionuclides & Tritium & $1.0 \mathrm{E}+00$ & $3.1 \mathrm{E}+02$ & \\
\hline Maintenance Worker & Core & Sediment & Dermal & Inorganics & Antimony & $3.6 \mathrm{E}+01$ & 3.9E-07 & $1.1 \mathrm{E}-06$ \\
\hline Maintenance Worker & Core & Sediment & Dermal & Inorganics & Arsenic & $2.2 \mathrm{E}+01$ & 2.3E-07 & $6.6 \mathrm{E}-07$ \\
\hline Maintenance Worker & Core & Sediment & Dermal & Inorganics & Barium & $1.2 \mathrm{E}+02$ & $1.3 \mathrm{E}-06$ & $3.7 \mathrm{E}-06$ \\
\hline Maintenance Worker & Core & Sediment & Dermal & Inorganics & Cadmium & $6.0 \mathrm{E}+01$ & $6.6 \mathrm{E}-07$ & $1.8 \mathrm{E}-06$ \\
\hline Maintenance Worker & Core & Sediment & Dermal & Inorganics & Chromium VI & $1.9 \mathrm{E}+02$ & $2.1 \mathrm{E}-06$ & $5.8 \mathrm{E}-06$ \\
\hline Maintenance Worker & Core & Sediment & Dermal & Inorganics & Cyanide & $5.8 \mathrm{E}-01$ & $6.3 \mathrm{E}-09$ & $1.8 \mathrm{E}-08$ \\
\hline Miaintenance Workér & Core & Sediment & Derma! & Inorgenice & Manganese & $1.3 E+03$ & $1.4 \mathrm{E}-05$ & $4.0 \mathrm{E}-05$ \\
\hline Maintenance Worker & Core & Sediment & Dermal & Inorganics & Mercury & $1.9 \mathrm{E}+01$ & $2.0 \mathrm{E}-07$ & $5.7 \mathrm{E}-07$ \\
\hline Maintenance Worker & Core & Sediment & Dermal & Inorganics & Nicke! & $1.7 \mathrm{E}+02$ & $1.8 \mathrm{E}-06$ & $5.2 \mathrm{E}-06$ \\
\hline Maintenance Worker & Core & Sediment & Dermal & Inorganics & Selenium & 8.7E-01 & 9.5E-09 & $2.7 \mathrm{E}-08$ \\
\hline Maintenance Worker & Core & Sediment & Dermal & Inorganics & Zine & $7.4 E+03$ & 8.1E-05 & 2.3E-04 \\
\hline Maintenance Worker & Core & Sediment & Dermal & Organics & Acenaphthene & $9.6 \mathrm{E}-01$ & $1.0 \mathrm{E}-07$ & $2.9 \mathrm{E}-07$ \\
\hline Maintenance Worker & Core & Sediment & Dermal & Organics & Acetone & $9.0 \mathrm{E}-02$ & $9.8 \mathrm{E}-09$ & $2.7 E-08$ \\
\hline Maintenance Worker & Core & Sediment & Dermal & Organics & Anthracene & $1.9 E+\infty 0$ & 2.1E-07 & $5.8 \mathrm{E}-07$ \\
\hline Maintenance Worker & Core & Sediment & Dermal & Organics & Aroclor-1254 & $4.1 E+02$ & $4.5 E-05$ & $1.3 \mathrm{E}-04$ \\
\hline
\end{tabular}

- Units are $\mathrm{mg} / \mathrm{kg}$ for inorganics and organics; $\mathrm{pCi} / \mathrm{g}$ for radionuclides

- Units are $\mathrm{mg} / \mathrm{kg}$-day for inorganics and organics; $\mathrm{pCi}$-day for radionuclides 


\begin{tabular}{|c|c|c|c|c|c|c|c|c|}
\hline \multirow{2}{*}{ Receptor } & \multirow{2}{*}{ Media } & \multirow{2}{*}{ Location } & \multirow{2}{*}{$\begin{array}{l}\text { Exposure } \\
\text { Pathway }\end{array}$} & \multirow{2}{*}{$\begin{array}{c}\text { Contaminant } \\
\text { Class }\end{array}$} & \multirow{2}{*}{ Contaminant } & \multirow[b]{2}{*}{$\begin{array}{l}\text { Exposure Point } \\
\text { Concentration" }\end{array}$} & \multicolumn{2}{|c|}{ Chronic Daily Intoket } \\
\hline & & & & & & & $\begin{array}{c}\text { Carcinogenic } \\
\text { Effects }\end{array}$ & $\begin{array}{c}\text { Noncarcinogenic } \\
\text { Efrects }\end{array}$ \\
\hline Maintenance Worker & Core & Sediment & Dermal & Organics & Benzo(a)pyrene & $5.7 E+\infty 0$ & 6.2E- -07 & 1.7E-06 \\
\hline Maintenance Worker & Core & Sediment & Dermal & Organica & Benzo(k)fluoranthene & $4.6 \mathrm{E}+01$ & 5.0E-06 & $1.4 E-05$ \\
\hline Maintenance Worker & Core & Sediment & Dermal & Organics & Bir(2-ethylhexyl)phthalate & $2.1 \mathrm{E}+\infty 0$ & 2.3E-07 & $6.4 \mathrm{E}-07$ \\
\hline Maintenance Worker & Core & Sediment & Dermal & Organica & Carbezole & $2.0 \mathrm{E}+\infty 0$ & 2.2E-07 & 6.1E-07 \\
\hline Maintenance Worker & Core & Sediment & Dermal & Organics & Chryecne & $1.9 E+01$ & 2.1E-06 & $5.8 \mathrm{E}-06$ \\
\hline Maintenance Worker & Core & Sediment & Dermal & Organica & Dibenz $(\mathrm{a}, \mathrm{h})$ anthracene & $1.5 \mathrm{E}+\infty 0$ & $1.6 \mathrm{E}-07$ & $4.6 \mathrm{E}-07$ \\
\hline Maintenance Worker & Core & Sediment & Dermal & Organics & Fluoranthene & $2.7 \mathrm{E}+01$ & $2.9 \mathrm{E}-06$ & 8.2E-06 \\
\hline Maintenance Worker & Core & Sediment & Dermal & Organics & Fluorene & 8.5E-01 & 9.3E- -08 & 2.6E-07 \\
\hline Maintenance Worker & Core & Sediment & Dermal & Organics & Indeno(1,2,3-cd)pyrene & $9.6 \mathrm{E}+00$ & $1.0 \mathrm{E}-06$ & $2.9 \mathrm{E}-06$ \\
\hline Maintenance Worker & Core & Sediment & Dermal & Organics & Pyrene & $2.2 \mathrm{E}+01$ & $2.4 \mathrm{E}-06$ & $6.7 E-06$ \\
\hline Maintenance Worker & Core & Sediment & Inhalation & Inorganics & Antimony & $3.6 \mathrm{E}+01$ & $5.9 \mathrm{E}-10$ & $1.7 \mathrm{E}-09$ \\
\hline Maintenance Worker & Core & Sediment & Inhalation & Inorganics & Arsenic & $2.2 \mathrm{E}+01$ & $3.5 \mathrm{E}-10$ & 9.8E-10 \\
\hline Maintenance Worker & Core & Sediment & Inhalation & Inorganics & Barium & $1.2 \mathrm{E}+02$ & 2.0E-09 & 5.5E-09 \\
\hline Maintenance Worker & Core & Sediment & Inhalation & Inorganics & Cadmium & $6.0 \mathrm{E}+01$ & $9.8 \mathrm{E}-10$ & 2.7E-09 \\
\hline Maintenance Worker & Core & Sediment & Inhalation & Inorganics & Chromium VI & $1.9 \mathrm{E}+02$ & 3.1E-09 & 8.6E-09 \\
\hline Maintenance Worker & Core & Sediment & Inhalation & Inorganics & Cyanide & $5.8 \mathrm{E}-01$ & $9.5 \mathrm{E}-12$ & $2.6 \mathrm{E}-11$ \\
\hline Maintenance Worker & Core & Sediment & Inhalation & Inorganics & Manganese & $1.3 E+03$ & 2.1E-08 & $6.0 \mathrm{E}-08$ \\
\hline Maintenance Worker & Core & Sediment & Inhalation & Inorganics & Mercury & $1.9 E+01$ & $3.1 \mathrm{E}-10$ & $8.5 E-10$ \\
\hline Maintenance Worker & Core & Sediment & Inhalation & Inorganics & Nickel & $1.7 E+02$ & $2.8 \mathrm{E}-09$ & 7.7E-09 \\
\hline Maintenance Worker & Core & Sediment & Inhalation & Inorganics & Selenium & 8.7E-01 & $1.4 \mathrm{E}-11$ & $4.0 \mathrm{E}-11$ \\
\hline Maintenance Worker & Core & Sediment & Inhalation & Inorganic: & Zinc & $7.4 E+03$ & 1.2E-07 & 3.4E-07 \\
\hline
\end{tabular}

- Units are $\mathrm{mg} / \mathrm{kg}$ for inorganics and organics; $\mathrm{pCi} / \mathrm{g}$ for radionuclides

- Units are mg/kg-day for inorganics and onganics; pCi-day for radionuclides 
sวp!|

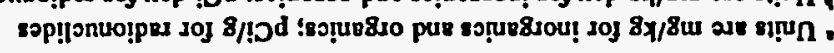

\begin{tabular}{|c|c|c|c|c|c|c|c|c|}
\hline & $20-35^{\prime} 9$ & $00+3 \tau^{\prime} \tau$ & $\mathbf{n}_{\mathrm{sxz}}$ & sop!|onuo!pry & uo!ाрएषчuा & jusu!pos & 250J & 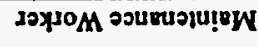 \\
\hline & $20-3 b^{*} \varepsilon$ & $00+32 \cdot I$ & $n_{d_{n} z z}$ & sop!!onuo!̣pey & 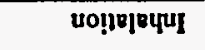 & נuวu!pos & 2100 & 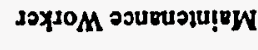 \\
\hline & $10-90^{\circ} I$ & $00+3 b^{\circ} \varepsilon$ & $\bigcap_{\text {rezkiz }}$ & sop!!onuo!pry & 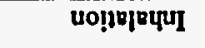 & มนจน!̣อS & 2100 & JPX10 $M$ Psurusiu!̣W \\
\hline & $20-399^{\circ} 1$ & $10.95 \%$ & YLoxz & sop!|จnuo!pey & 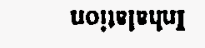 & juow!̣ps & 2100 & JPYIOM Poน"uอIu!̣W \\
\hline & I0-95's & $10+96^{\circ} I$ & นщ622 & epp!|onuo!pzy & uo!ng|⿰口丩पा & zusu!pos & 2100 & JoxpoM osurusju!̣"W \\
\hline & $20-39 \cdot 1$ & 10-3t's & पhex & sop!|ønuọpey & 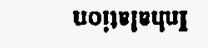 & Iuourtpos & 2100 & 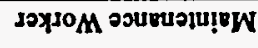 \\
\hline & $20+90^{\circ} 6$ & $60+3 I^{\prime} \varepsilon$ & $P_{L S I}$ & sop!!onuo!pry & 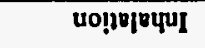 & juวu!̣ps & 2100 & 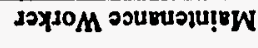 \\
\hline $60-90^{\circ} \mathrm{I}$ & Ol-g9' $\varepsilon$ & $10+3 \tau^{\prime} z$ & PUDSKd & 8ง!นष810 & นo!̣p|Bपया & juou!pos & 2100 & JOXIOM osuruolu!pW \\
\hline $0 i-\overline{s i p} \div \dot{v}$ & $0 i-399^{\circ} i$ & $00+599^{\circ}$ & 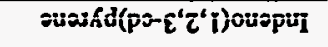 & 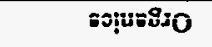 & 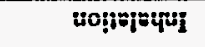 & jusubpes & 200 & 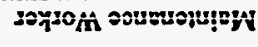 \\
\hline II-96. $\mathcal{E}$ & II-9t'I & 10-95'8 & oussonly & sร̣นะ8ม & 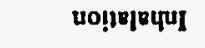 & juourppos & 2100 & Joy10M องumuoju!̣W \\
\hline $60-9 \tau^{\prime} 1$ & $01-356$ & $10+3 L \cdot \tau$ & 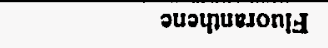 & sรกนอ8ม & 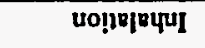 & נuจน!̣pPS & 2105 & 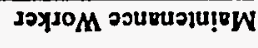 \\
\hline $11-36.9$ & $\| 1-9+2$ & $00+3 S^{*} 1$ & 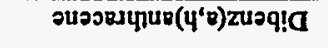 & 8อเ̣นษ8ิง & นo!!|ק|вนุा & jusutp? & 2503 & JOYIOM POURUPJu!EW \\
\hline $01-3<\cdot 8$ & OI-GI'E & $10+96 \cdot 1$ & วนวВКธบว & รงกุe8so & 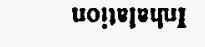 & guouয!pos & 2100 & 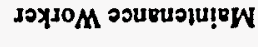 \\
\hline$I I-9 I^{\cdot} 6$ & $I I-\Xi \varepsilon \mathcal{E}$ & $00+90^{\circ} \tau$ & ग0Z"q10ว & sọue8so & uo!!p|ำuI & juouịpos & 2300 & 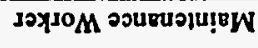 \\
\hline II-99.6 & II-gat $\boldsymbol{t}^{\circ} \boldsymbol{\varepsilon}$ & $00+3 I^{\prime} \tau$ & 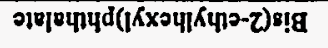 & รง̣นए88 & 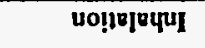 & נusuบ!pจS & 2505 & 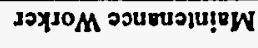 \\
\hline $60-91 \%$ & $01-3 S^{\prime} L$ & $10+396$ & әuวหpuedonj(y) ozusg & 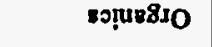 & иo!̣p|вчuा & juวu!̣pos & asos & 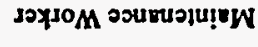 \\
\hline $01-99^{\prime} Z$ & II-GE.6 & $00+3 L^{\prime} S$ & su2skd(v)ozurg & sọue8so & uo!!ฑ|вนนI & มusu!̣pos & 2305 & 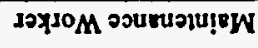 \\
\hline $80-36.1$ & $60-3 L \cdot 9$ & $20+31+$ & BSZI-10poasy & sọ̣u8810 & uo!ाण|вцण & นuจu!pes & 2300 & soxjoM ग0urupju!nW \\
\hline$I I-3 L \cdot 8$ & $\| I^{\circ} \varepsilon$ & $00+96^{\circ} I$ & 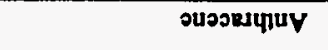 & sọuะ8so & uo!!|ण|в१ग] & มนวu!pos & 2500 & soyjoM องนpus|u!̣W \\
\hline 21310 & ZI-GS'I & $20-30.6$ & suopov & sง!uष810 & 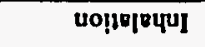 & นนวน!pวs & 2100 & JoxjoM Dourusiu!ew \\
\hline $11-3016$ & II-99. I & $10-39.6$ & 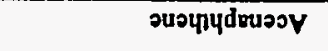 & sọur8so & 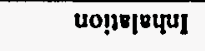 & มนวแ!̣ps & 2100 & 10Y10 M osumuslu!!W \\
\hline 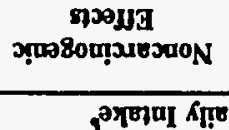 & 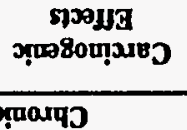 & 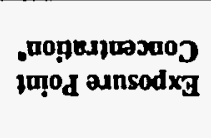 & 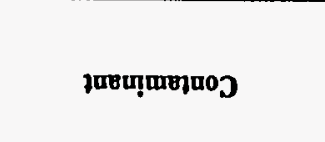 & 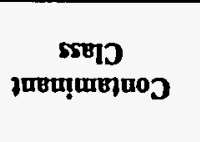 & $\begin{array}{l}\text { Rymqued } \\
\text { ansodxy }\end{array}$ & 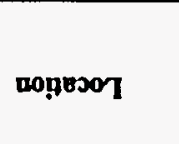 & 甲!pow & s0ydaxoy \\
\hline
\end{tabular}




\begin{tabular}{|c|c|c|c|c|c|c|c|c|}
\hline \multirow{2}{*}{ Receptor } & \multirow{2}{*}{ Media } & \multirow{2}{*}{ Location } & \multirow[b]{2}{*}{$\begin{array}{l}\text { Exposure } \\
\text { Pathway }\end{array}$} & \multirow[b]{2}{*}{$\begin{array}{l}\text { Contaminant } \\
\text { Class }\end{array}$} & \multirow{2}{*}{ Contaminant } & \multirow[b]{2}{*}{$\begin{array}{l}\text { Exposure Point } \\
\text { Concentration" }\end{array}$} & \multicolumn{2}{|c|}{ Chronic Daily Intake } \\
\hline & & & & & & & $\begin{array}{c}\text { Carcinogenic } \\
\text { Efrects }\end{array}$ & $\begin{array}{c}\text { Noncarcinogenic } \\
\text { Effects }\end{array}$ \\
\hline Maintenance Worker & Core & Sediment & Inhalation & Radionuclides & ${ }^{2592000 \mathrm{Pu}}$ & $3.5 \mathrm{E}+01$ & $1.0 E+\infty$ & \\
\hline Maintenance Worker & Core & Sediment & Inhalation & Radionuclides & ${ }^{*} \mathrm{~K}$ & $9.4 E+\infty$ & $2.8 \mathrm{E}-01$ & \\
\hline Maintenance Worker & Core & Sediment & Inhalation & Radionuclide: & ${ }^{\infty} \mathrm{Co}$ & $3.4 \mathrm{E}+\infty$ & $1.0 \mathrm{E}-01$ & \\
\hline Maintenance Worker & Core & Sediment & Inhalation & Radionuclides & ${ }^{\circ} \mathrm{Sr}$ & $9.8 \mathrm{E}+01$ & $2.8 \mathrm{E}+\infty$ & \\
\hline Maintenance Worker & Core & Sediment & Inhalation & Radionuclides & Tritium & $1.0 \mathrm{E}+\infty$ & $2.9 \mathrm{E}-02$ & \\
\hline Maintenance Worker & Core & Sediment & External Exposure & Radionuclides & ${ }^{15} \mathrm{Cs}$ & $3.1 E+04$ & $1.4 \mathrm{E}+05$ & \\
\hline Maintenance Worker & Core & Sediment & External Exposure & Radionuclides & ${ }^{20} \mathrm{Th}$ & $5.4 \mathrm{E}-01$ & $2.5 \mathrm{E}+00$ & \\
\hline Maintenance Worker & Core & Sediment & External Exposure & Radionuclides & $229 \mathrm{Th}$ & $1.9 \mathrm{E}+01$ & $8.5 E+01$ & \\
\hline Maintenance Worker & Core & Sediment & External Exposure & Radionuclides & ${ }^{233} \mathrm{Th}$ & $5.5 \mathrm{E}-01$ & $2.5 E+\infty$ & \\
\hline Maintenance Worker & Core & Sediment & External Exposure & Radionuclides & ${ }^{231234} U$ & $3.4 \mathrm{E}+\infty 0^{\circ}$ & $1.6 \mathrm{E}+01$ & \\
\hline Maintenance Worker & Core & Sediment & External Exposure & Radionuclides & ${ }^{2{ }^{2} \mathrm{Pu}}$ & $1.2 \mathrm{E}+\infty$ & $5.3 \mathrm{E}+\infty$ & \\
\hline Maintenance Worker & Core & Sediment & External Exposure & Radionuclides & ${ }^{234} \mathrm{U}$ & $2.2 E+\infty$ & $1.0 \mathrm{E}+01$ & \\
\hline Maintenance Worker & Core & Sediment & External Exposure & Radionuclides & ${ }^{2598200} \mathrm{Pu}$ & 3.5E+01 & $1.6 \mathrm{E}+02$ & \\
\hline Maintenance Worker & Core & Sediment & External Exposure & Radionuclides & ${ }^{\infty} \mathrm{K}$ & $9.4 E+\infty$ & $4.3 \mathrm{E}+01$ & \\
\hline Maintenance Worker & Core & Sediment & External Exposure & Radionuclides & ${ }^{\infty} \mathrm{Co}$ & $3.4 E+\infty 0$ & $1.6 \mathrm{E}+01$ & \\
\hline Maintenance Worker & Core & Sediment & External Exposure & Radionuclides & ${ }^{x} \mathrm{Sr}$ & $9.8 \mathrm{E}+01$ & 4.5E +02 & \\
\hline Maintenance Worker & Core & Sediment & External Exposure & Radionuclides & Tritium & $1.0 \mathrm{E}+00$ & $4.6 \mathrm{E}+\infty$ & \\
\hline Maintenance Worker & Core & Whole Building & Ingestion & Inorganics & Aruenic & $2.9 \mathrm{E}+00$ & $5.0 \mathrm{E}-07$ & $1.4 \mathrm{E}-06$ \\
\hline Maintenance Worker & Core & Whole Building & Ingestion & Inorganics & Barium & $7.2 \mathrm{E}+02$ & $1.3 \mathrm{E}-04$ & 3.5E-04 \\
\hline Maintenance Worker & Core & Whole Building & Ingestion & Inorganics & Beryllium & $1.2 \mathrm{E}+01$ & $2.2 \mathrm{E}-06$ & $6.0 \mathrm{E}-06$ \\
\hline Maintenance Worker & Core & Whole Building & Ingestion & Inorganics & Cadmium & $1.8 \mathrm{E}+02$ & $3.1 \mathrm{E}-05$ & $8.8 \mathrm{E}-05$ \\
\hline
\end{tabular}

- Units are $\mathrm{mg} / \mathrm{kg}$ for inorganics and organics; $\mathrm{pCi} / \mathrm{g}$ for radionuclides

- Units are mg/kg-day for inorganics and organics; pCi-day for radionuclides 


\begin{tabular}{|c|c|c|c|c|c|c|c|c|}
\hline \multirow{2}{*}{ Receptor } & \multirow{2}{*}{ Media } & \multirow{2}{*}{ Location } & \multirow{2}{*}{$\begin{array}{l}\text { Exposure } \\
\text { Pathway }\end{array}$} & \multirow{2}{*}{$\begin{array}{c}\text { Contaminant } \\
\text { Class }\end{array}$} & \multirow{2}{*}{ Contaminant } & \multirow{2}{*}{$\begin{array}{l}\text { Exposure Point } \\
\text { Concentration" }\end{array}$} & \multicolumn{2}{|c|}{ Chronic Daily Intake } \\
\hline & & & & & & & $\begin{array}{l}\text { Carcinogenic } \\
\text { Efrects }\end{array}$ & $\begin{array}{c}\text { Noncarcinogenic } \\
\text { Effects }\end{array}$ \\
\hline Maintenance Worker & Core & Whole Building & Ingestion & Inorganics & Chromium VI & $2.0 \mathrm{E}+02$ & $3.4 \mathrm{E}-05$ & $9.6 \mathrm{E}-05$ \\
\hline Maintenance Worker & Core & Whole Building & Ingestion & Inorganics & Manganese & $9.5 E+03$ & $1.7 \mathrm{E}-03$ & 4.7E-03 \\
\hline Maintenance Worker & Core & Whole Building & Ingestion & Inorganics & Mercury & 3.2E-01 & $5.6 \mathrm{E}-08$ & $1.6 \mathrm{E}-07$ \\
\hline Maintenance Worker & Core & Whole Building & Ingestion & Inorganics & Nickel & $1.4 \mathrm{E}+02$ & $2.4 \mathrm{E}-05$ & $6.8 \mathrm{E}-05$ \\
\hline Maintenance Worker & Core & Whole Building & Ingestion & Inorganica & Vanadium & $2.4 \mathrm{E}+02$ & $4.2 \mathrm{E}-05$ & $1.2 \mathrm{E}-04$ \\
\hline Maintenance Worker & Core & Whole Building & Ingestion & Inorganics & Zinc & $3.5 \mathrm{E}+0.4$ & $6.1 \mathrm{E}-03$ & $1.7 \mathrm{E}-02$ \\
\hline Maintenance Worker & Core & Whole Building & Ingestion & Organics & Aroclor-1254 & $1.5 \mathrm{E}+\infty 0$ & 2.5E-07 & $7.1 \mathrm{E}-07$ \\
\hline Maintenance Worker & Core & Whole Building & Ingestion & Organics & Aroclor- 1260 & $2.6 \mathrm{E}+\infty 0$ & 4.5E-07 & 1.3E-06 \\
\hline Maintenance Worker & Core & Whole Building & Ingestion & Organics & Bis(2-ethylhexyl)phthalate & $2.4 \mathrm{E}-01$ & $4.2 \mathrm{E}-08$ & $1.2 \mathrm{E}-07$ \\
\hline Maintenance Worker & Core & Whole Building & Ingestion & Organics & Dimethyl phthalate & $5.5 \mathrm{E}-02$ & $9.6 \mathrm{E}-09$ & 2.7E-08 \\
\hline Maintenance Worker & Core & Whole Building & Ingestion & Radionuclides & ${ }^{197} \mathrm{Ca}$ & $1.4 \mathrm{E}+03$ & $4.3 \mathrm{E}+05$ & \\
\hline Maintenance Worker & Core & Whole Building & Ingestion & Radionuclides & ${ }^{206} \mathrm{Ra}$ & $4.0 \mathrm{E}-01$ & $1.3 E+02$ & \\
\hline Maintenance Worker & Core & Whole Builiding & ingestion & R̃adionuciides & $\mathbf{n}_{\mathbf{R a}}$ & $3.7 E-01$ & $1.2 E+82$ & \\
\hline Maintenance Worker & Core & Whole Building & Ingestion & Radionuclides & ${ }^{2 n} \mathrm{Th}$ & 7.7E-01 & $2.4 E+02$ & \\
\hline Maintenance Worker & Core & Whole Building & Ingestion & Radionuclides & ${ }^{250} \mathrm{Th}$ & $1.4 \mathrm{E}+00$ & $4.4 E+02$ & \\
\hline Maintenance Worker & Core & Whole Building & Ingestion & Radionuclides & ${ }^{20} \mathrm{Th}$ & $8.0 \mathrm{E}-01$ & $2.5 E+02$ & \\
\hline Mainlenance Worker & Core & Whole Building & Ingestion & Radionuclides & ${ }^{2312 \pi} \mathrm{U}$ & $1.3 \mathrm{E}+00$ & $4.0 \mathrm{E}+02$ & \\
\hline Maintenance Worker & Core & Whole Building & Ingestion & Radionuclides & ${ }^{231} \mathrm{Th}$ & $6.3 E+\infty 0$ & $2.0 \mathrm{E}+03$ & \\
\hline Maintenance Worker & Core & Whole Building & Ingestion & Radionuclides & ${ }^{231} U$ & $1.2 \mathrm{E}-01$ & $3.8 E+01$ & \\
\hline Maintenance Worker & Core & Whole Building & Ingestion & Radionuclides & ${ }^{23} \mathrm{Pu}$ & $4.5 \mathrm{E}-01$ & $1.4 E+02$ & \\
\hline Maintenance Worker & Core & Whole Building & Ingestion & Radionuelides & ${ }^{23} \mathrm{U}$ & $1.4 \mathrm{E}+\infty$ & $4.5 \mathrm{E}+02$ & \\
\hline
\end{tabular}

- Units are $\mathrm{mg} / \mathrm{kg}$ for inorganics and organics; $\mathrm{pCi} / \mathrm{g}$ for radionuclides

- Units are mg/kg-day for inorganics and organics; pCi-day for radionuclides 


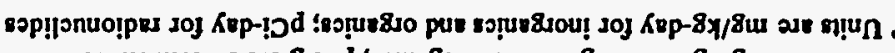
sop!

\begin{tabular}{|c|c|c|c|c|c|c|c|c|}
\hline OIユีE"I & $I I-3 L L^{\circ}$ & . $00+362$ & o!̣osey & อง̣uv\&sou & प0!ाच|च्भण & 8u!PI!ng ग०นM & 2105 & 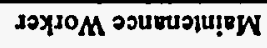 \\
\hline $80-3 L \cdot 1$ & $60-30.9$ & 20-35"s & 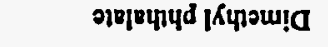 & sอ̣นะ8นO & prumad & 8u!pl!ng गочм & 2100 & JoxisoM osuruopu!̣pW \\
\hline $80-\exists E L L$ & $80-39^{\prime} Z$ & $10-9+2$ & 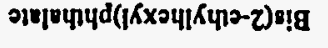 & sก̣uะ\&\&o & fruwsa & 8u!Pl!ng गочМ & 2100 & 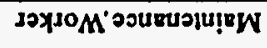 \\
\hline$\angle 0.96 . \mathrm{L}$ & $20-38^{\prime} Z$ & $00+39 \cdot 2$ & 092I-Jojoas & 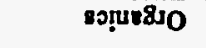 & ןвumea & 8u!P!!ng ग104M & 210J & JOY10M องumus|u!̣W \\
\hline$\angle 0-3 t^{\circ} t$ & LO- $99^{\circ} I$ & $00+3 S^{\prime} 1$ & tSZI-J010a1Y & sp̣uv\&o & ןrusrd & 8u!pl!ng श애 & axos & 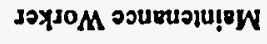 \\
\hline E0-3I'I & to-38. $\varepsilon$ & $60+\pi S \cdot \varepsilon$ & จu!Z & 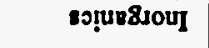 & imusa & 8u!pl!ng ग०४पM & 2100 & 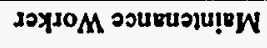 \\
\hline $90 \mathrm{gE} L$ & $90-39 \cdot 2$ & $z 0+a+2$ & un!pvus & sọur\&oul & Imu=a & 8u!pI!ng ग0ษM & 2505 & 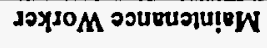 \\
\hline $90-32 \%$ & $90-3 S^{\prime} I$ & $20+9 r^{\circ} \cdot 1$ & |PXI!N & sọ̣ur8fouI & ןвurea & 8u!pl!ng गоㄴ⿻ & 2105 & 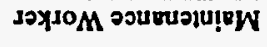 \\
\hline $60-3<\cdot 6$ & $60-\mathrm{gS} \cdot \mathrm{E}$ & $10-3 T^{\prime} E$ & SInฆsวW & จ गุนe\&sou & 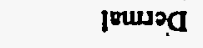 & 8u!p!!ng ग०ЧM & 2300 & 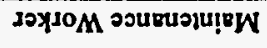 \\
\hline $10-36 . \tau$ & $10-30.1$ & $20+35.6$ & rsour81ury & sง̣ur8siouI & ןвured & 8u!pI!ng गочM & 2305 & 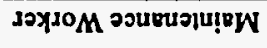 \\
\hline $90-90.9$ & $90-31 \cdot 2$ & $20+30 \tau$ & 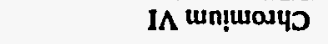 & 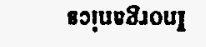 & ןвurea & 8u!pI!ng गочM & 210ग & 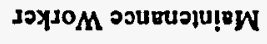 \\
\hline 90-3S'S & $90-90 \% 2$ & $20+981$ & un!̣pes & sọue8souI & ןoured & 8u!pj!ng गочM & 250ว & 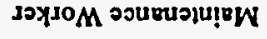 \\
\hline$\angle 0-38^{\circ} \varepsilon$ & $\angle 0-\exists \varepsilon^{\prime} I$ & $10+3 z \cdot 1$ & un!̣!ार्गg & 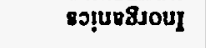 & frumed & 8u!pl!ng गочм & 2500 & 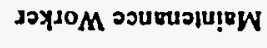 \\
\hline so-az'z & $90-96 . \mathrm{L}$ & $z 0+3 z \cdot L$ & unழฺgg & mọuष8souI & joursa & 8u!pl!ng गочM & 2505 & 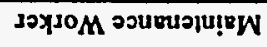 \\
\hline $80-3 L^{\circ} 8$ & 80-GI'E & $00+36 \cdot \tau$ & गฺบขอมY & 8s!̣u\&\&10 & ןвw2 & 8u!pl!̣ng गочM & 2100 & 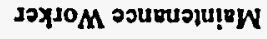 \\
\hline & $60+30^{\circ} 8$ & $10+39 \cdot \tau$ & un!ฺ!ป & sop!|Pnuo!pry & บo!̣เมอ8u1 & 8u!pl!ng गочм & 210ว & 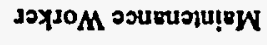 \\
\hline & $60+39^{\circ} \varepsilon$ & $10+\exists 2 \cdot 1$ & ${ }^{J} S_{\infty 6}$ & sop!|onuo!pry & บo!̣เอจ8นป & 8u!pl!ng ๆ००ЧМ & 2100 & 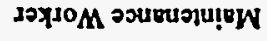 \\
\hline & $10+3 I^{\circ} t$ & I0-3E'I & $0 D_{\infty}$ & :sp!|onuo!̣pry & uọ̣เอ8นI & 8u!pl!ng ग04M & 210ग & 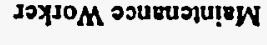 \\
\hline & $\varepsilon 0+\mathbf{g} \ell \cdot \mathbf{l}$ & $00+3 b^{\circ} s$ & $\mathrm{X}_{0 r}$ & sop!|’nuolpry & 40!1898นI & 8u!pl!ng ग04M & 200 & 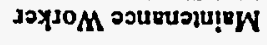 \\
\hline & $20+391$ & $10-30^{\circ} \mathrm{s}$ & wV & spp!|onuo!p»y & 40!ฺหร8) I & 8u!pl!ng गочм & 2100 & JOXIOM DouruכJu!nW \\
\hline & $\varepsilon 0+3 \varepsilon \cdot 1$ & $00+30^{\circ} t$ & $n_{d_{\text {ondest }}}$ & sop!|गnuo!pry & บọ̣คo8บ I & Su!P|!ng गоนM & 2105 & JoXIOM Dourusju!nW \\
\hline 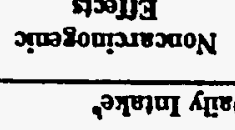 & 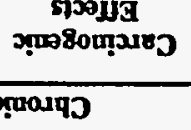 & 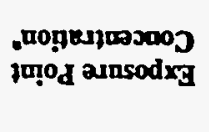 & 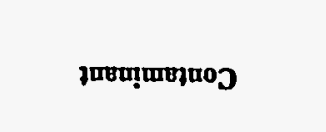 & 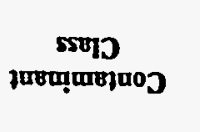 & $\begin{array}{l}\text { REMqDed } \\
\text { 2unsodxy }\end{array}$ & nopgero7 & ت!paw & دopdaวay \\
\hline
\end{tabular}




\begin{tabular}{|c|c|c|c|c|c|c|c|c|}
\hline \multirow{2}{*}{ Receptor } & \multirow{2}{*}{ Media } & \multirow{2}{*}{ Location } & \multirow{2}{*}{$\begin{array}{l}\text { Exposure } \\
\text { Pathway }\end{array}$} & \multirow{2}{*}{$\begin{array}{c}\text { Contaminant } \\
\text { Class }\end{array}$} & \multirow{2}{*}{ Contaminant } & \multirow[b]{2}{*}{$\begin{array}{l}\text { Exposure Point } \\
\text { Concentration" }\end{array}$} & \multicolumn{2}{|c|}{ Chronic Daily Intake } \\
\hline & & & & & & & $\begin{array}{l}\text { Carcinogenic } \\
\text { Errects }\end{array}$ & $\begin{array}{c}\text { Noncarcinogenic } \\
\text { Effects }\end{array}$ \\
\hline Maintenance Worker & Core & Whole Building & Inhalation & Inorganics & Barium & $7.2 \mathrm{E}+02$ & $1.2 \mathrm{E}-08$ & 3.3E-08 \\
\hline Maintenance Worker & Core & Whole Building & Inhalation & Inorganics & Beryllium & $1.2 \mathrm{E}+01$ & 2.0E-10 & $5.6 \mathrm{E}-10$ \\
\hline Maintenance Worker & Core & Whole Building & Inhalation & Inorganics & Cadmium & $1.8 \mathrm{E}+02$ & $2.9 \mathrm{E}-09$ & $8.2 \mathrm{E}-09$ \\
\hline Maintenance Worker & Core & Whole Building & Inhalation & Inorganics & Chromium VI & $2.0 \mathrm{E}+02$ & $3.2 \mathrm{E}-09$ & $9.0 \mathrm{E}-09$ \\
\hline Maintenance Worker & Core & Whole Building & Inhalation & Inorganics & Manganese & $9.5 \mathrm{E}+03$ & $1.6 \mathrm{E}-07$ & $4.4 \mathrm{E}-07$ \\
\hline Maintenance Worker & Core & Whole Building & Inhalation & Inorganics & Mereury & $3.2 \mathrm{E}-01$ & $5.2 \mathrm{E}-12$ & $1.5 \mathrm{E}-11$ \\
\hline Maintenance Worker & Core & Whole Building & Inhalation & Inorganics & Nickel & $1.4 \mathrm{E}+02$ & 2.3E-09 & $6.3 E-09$ \\
\hline Maintenance Worker & Core & Whole Building & Inhalation & Inorganics & Vanadium & $2.4 \mathrm{E}+02$ & $3.9 \mathrm{E}-09$ & $1.1 \mathrm{E}-08$ \\
\hline Maintenance Worker & Core & Whole Building & Inhalation & Inorganics & Zinc & $3.5 E+04$ & 5.7E-07 & $1.6 \mathrm{E}-06$ \\
\hline Maintenance Worker & Core & Whole Building & Inhalation & Organics & Aroclor-1254 & $1.5 \mathrm{E}+00$ & $2.4 \mathrm{E}-11$ & $6.6 \mathrm{E}-11$ \\
\hline Maintenance Worker & Core & Whole Building & Inhalation & Organics & Aroclor-1260 & $2.6 \mathrm{E}+00$ & $4.2 \mathrm{E}-11$ & $1.2 \mathrm{E}-10$ \\
\hline Maintenance Worker & Core & Whole Building & Inhalation & Organics & Bis(2-ethylhexyl)phthalate & 2.4E-01 & $3.9 \mathrm{E}-12$ & $1.1 \mathrm{E}-11$ \\
\hline Miaintenance Worker & Core & Whoie Euuilaing & innaiation & Orgànics & Dimethyi phitutaiate & $5.5 \mathrm{E}-02$ & 9.0E-13 & $2.5 \mathrm{E}-12$ \\
\hline Maintenance Worker & Core & Whole Building & Inhalation & Radionuclides & ${ }^{13} \mathrm{Cs}$ & $1.4 \mathrm{E}+03$ & $4.0 \mathrm{E}+01$ & \\
\hline Maintenance Worker & Core & Whole Building & Inhalation & Radionuclides & ${ }^{20 x} \mathrm{Ra}$ & 4.0E-01 & $1.2 \mathrm{E}-02$ & \\
\hline Maintenance Worker & Core & Whole Building & Inhalation & Radionuclides & $20 \mathrm{Ra}$ & $3.7 \mathrm{E}-01$ & $1.1 \mathrm{E}-02$ & \\
\hline Maintenance Worker & Core & Whole Building & Inhalation & Radionuclides & ${ }^{2 x} \mathrm{Th}$ & 7.7E-01 & $2.2 \mathrm{E}-02$ & \\
\hline Maintenance Worker & Core & Whole Building & Inhalation & Radionuclides & ${ }^{200} \mathrm{Th}$ & $1.4 \mathrm{E}+00$ & 4.1E-02 & \\
\hline Maintenance Worker & Core & Whole Building & Inhalation & Radionuclides & ${ }^{250} \mathrm{Th}$ & $8.0 \mathrm{E}-01$ & $2.3 \mathrm{E}-02$ & \\
\hline Maintenance Worker & Core & Whole Building & Inhalation & Radionuclides & ${ }^{238 / 24} \mathrm{U}$ & $1.3 \mathrm{E}+\infty$ & $3.7 \mathrm{E}-02$ & \\
\hline Maintenance Worker & Core & Whole Building & Inhalation & Radionuclides & ${ }^{23} \mathrm{Th}$ & $6.3 E+00$ & $1.8 \mathrm{E}-01$ & \\
\hline
\end{tabular}

- Unita are $\mathrm{mg} / \mathrm{kg}$ for inorganics and organics; $\mathrm{pCi} / \mathrm{g}$ for radionuclides

- Units are $\mathrm{mg} / \mathrm{kg}$-day for inorganics and organics; pCi-day for radionuclides 


\begin{tabular}{|c|c|c|c|c|c|c|c|c|}
\hline \multirow{2}{*}{ Receptor } & \multirow{2}{*}{ Medin } & \multirow{2}{*}{ Location } & \multirow{2}{*}{$\begin{array}{l}\text { Exposure } \\
\text { Pathway }\end{array}$} & \multirow{2}{*}{$\begin{array}{l}\text { Conteminant } \\
\text { Class }\end{array}$} & \multirow{2}{*}{ Contaminant } & \multirow{2}{*}{$\begin{array}{l}\text { Exposure Point } \\
\text { Concentration" }\end{array}$} & \multicolumn{2}{|c|}{ Chronic Daily Intoke" } \\
\hline & & & & & & & $\begin{array}{c}\text { Cancinogenic } \\
\text { Efrects }\end{array}$ & $\begin{array}{c}\text { Noncarcinogenic } \\
\text { Effects }\end{array}$ \\
\hline Maintenance Worker & Core & Whole Building & Inhalation & Radionuclide: & ${ }^{233} \mathrm{U}$ & $1.2 \mathrm{E}-01$ & $3.5 \mathrm{E}-03$ & \\
\hline Maintenance Worker & Core & Whole Building & Inhalation & Radionuclides & ${ }^{204} \mathrm{Pu}$ & $4.5 E-01$ & $1.3 \mathrm{E}-02$ & \\
\hline Maintenance Worker & Core & Whole Building & Inhalation & Radionuclides & ${ }^{23 \mathrm{U}}$ & $1.4 \mathrm{E}+\infty$ & $4.2 \mathrm{E}-02$ & \\
\hline Maintenance Worker & Core & Whole Building & Inhalation & Radionuclides & ${ }^{299200 \mathrm{Pu}}$ & $4.0 \mathrm{E}+\infty 0$ & $1.2 \mathrm{E}-01$ & \\
\hline Maintenance Worker & Core & Whole Building & Inhalation & Radionuclides & ${ }^{211} \mathrm{Am}$ & $5.0 \mathrm{E}-01$ & $1.5 \mathrm{E}-02$ & \\
\hline Maintenance Worker & Core & Whole Building & Inhalation & Radionuclides & ${ }^{+\infty} \mathrm{K}$ & $5.4 E+\infty$ & $1.6 \mathrm{E}-01$ & \\
\hline Maintenance Worker & Core & Whole Building & Inhalation & Radionuclides & ${ }^{\infty} \mathrm{Co}$ & $1.3 \mathrm{E}-01$ & $3.8 \mathrm{E}-03$ & \\
\hline Maintenance Worker & Core & Whole Building & Inhalation & Radionuclides & ${ }^{x} \mathrm{Sr}$ & $1.2 \mathrm{E}+01$ & $3.4 \mathrm{E}-01$ & \\
\hline Maintenance Worker & Core & Whole Building & Inhalation & Radionuclides & Tritium & $2.6 \mathrm{E}+01$ & 7.5E-01 & \\
\hline Maintenance Worker & Core & Whole Building & External Exposure & Radionuclides & ${ }^{19} \mathrm{Cs}$ & $1.4 \mathrm{E}+03$ & $6.3 E+03$ & \\
\hline Maintenance Worker & Core & Whole Building & External Exposure & Radionuclides & ${ }^{22 \times R_{a}}$ & $4.0 \mathrm{E}-01$ & $1.8 \mathrm{E}+\infty$ & \\
\hline Maintenance Worker & Core & Whole Building & External Exposure & Radionuclides & ${ }^{n}{ }_{\mathrm{Ra}}$ & 3.7E-01 & $1.7 E+\infty$ & \\
\hline Maintenance Worker & Core & Whole Building & External Exposure & Radionuelides & ${ }^{20} \mathrm{Th}$ & $7.7 \mathrm{E}-01$ & $3.5 E+\infty$ & \\
\hline Maintenance Worker & Core & Whole Building & External Exposure & Radionuclides & ${ }^{250} \mathrm{Th}$ & $1.4 \mathrm{E}+00$ & $6.4 \mathrm{E}+00$ & \\
\hline Maintenance Worker & Core & Whole Building & External Exposure & Radionuclides & ${ }^{25} \mathrm{Th}$ & $8.0 \mathrm{E}-01$ & $3.7 \mathrm{E}+\infty$ & \\
\hline Maintensnce Worker & Core & Whole Building & External Exposure & Radionuclides & ${ }^{232324} U$ & $1.3 \mathrm{E}+\infty$ & $5.8 \mathrm{E}+00$ & \\
\hline Maintenance Worker & Core & Whole Building & External Exposure & Radionuclides & ${ }^{254} \mathrm{Th}$ & $6.3 \mathrm{E}+00$ & $2.9 \mathrm{E}+01$ & \\
\hline Maintenance Worker & Core & Whole Building & Extermal Exposure & Radionuclide: & ${ }^{239} \mathrm{U}$ & $1.2 \mathrm{E}-01$ & $5.5 \mathrm{E}-01$ & \\
\hline Maintenance Worker & Core & Whole Building & External Exposure & Radionuclides & ${ }^{23} \mathrm{Pu}$ & $4.5 \mathrm{E}-01$ & $2.1 E+00$ & \\
\hline Maintenance Worker & Core & Whole Building & External Exposure & Radionuclides & ${ }^{280} \mathrm{U}$ & $1.4 E+\infty 0$ & $6.5 E+\infty$ & \\
\hline Maintenance Worker & Core & Whole Building & External Exposure & Radionuclides & ${ }^{259820} \mathrm{Pu}$ & $4.0 \mathrm{E}+00$ & $1.8 \mathrm{E}+01$ & \\
\hline
\end{tabular}

- Units are $\mathrm{mg} / \mathrm{kg}$ for inorganics and organics; $\mathrm{pCi} / \mathrm{g}$ for radionuclides

- Units are mg/kg-day for inorganica and organics; pCi-day for radionuclides 


\begin{tabular}{|c|c|c|c|c|c|c|c|c|}
\hline \multirow{2}{*}{ Receptor } & \multirow{2}{*}{ Media } & \multirow{2}{*}{ Location } & \multirow[b]{2}{*}{$\begin{array}{l}\text { Exposure } \\
\text { Pathway }\end{array}$} & \multirow[b]{2}{*}{$\begin{array}{c}\text { Contaminant } \\
\text { Class }\end{array}$} & \multirow[b]{2}{*}{ Contaminant } & \multirow[b]{2}{*}{$\begin{array}{l}\text { Exposure Point } \\
\text { Concentrations }\end{array}$} & \multicolumn{2}{|c|}{ Chronic Daily Intaket } \\
\hline & & & & & & & $\begin{array}{l}\text { Carcinogenic } \\
\text { Erfocts }\end{array}$ & $\begin{array}{c}\text { Noncarcinogenic } \\
\text { Effects }\end{array}$ \\
\hline Mainlenance Worker & Core & Whole Building & Extemal Exposure & Radionuclides & ${ }^{211} \mathrm{Am}$ & S.0E-01 & $2.3 \mathrm{E}+00$ & \\
\hline Maintenance Worker & Core & Whole Building & External Exposure & Radionuclides & ${ }^{\infty} \mathrm{K}$ & $5.4 \mathrm{E}+00$ & $2.5 \mathrm{E}+01$ & \\
\hline Maintenance Worker & Core & Whole Building & External Exposure & Radionuclides & ${ }^{\infty} \mathrm{Co}$ & 1.3E-01 & $5.9 \mathrm{E}-01$ & \\
\hline Mainlenasce Worker & Core & Whole Building & Extermal Exposure & Radionuclides & ${ }^{\infty} \mathrm{Sr}$ & $1.2 \mathrm{E}+01$ & $5.3 \mathrm{E}+01$ & \\
\hline Maintenance Worker & Core & Whole Building & External Exposure & Radionuclides & Tritium & $2.6 \mathrm{E}+01$ & $1.2 \mathrm{E}+02$ & \\
\hline
\end{tabular}


Table D-2. Maintenance Worker Exposure Concentrations and Chronic Lifetime Exposures for Direct Measurement Samples

\begin{tabular}{|c|c|c|c|c|c|c|c|c|}
\hline \multirow[b]{2}{*}{ Receptor } & \multirow[b]{2}{*}{ Media } & \multirow[b]{2}{*}{ Location } & \multirow[b]{2}{*}{ Exposure Palhway } & \multirow[b]{2}{*}{$\begin{array}{l}\text { Contaminant } \\
\text { Class }\end{array}$} & \multirow[b]{2}{*}{ Contaminant } & \multirow{2}{*}{$\begin{array}{c}\text { Exposure } \\
\text { Point } \\
\text { Concentration }\end{array}$} & \multicolumn{2}{|c|}{ Chronic Lifetime Exposure" } \\
\hline & & & & & & & $\begin{array}{c}\text { Carcinogenic } \\
\text { Effects }\end{array}$ & $\begin{array}{c}\text { Noncarcinogenic } \\
\text { Erfects }\end{array}$ \\
\hline Maintenance Worker & Direct & Cell & Extemal Exposure & Radionuclides & Beta-Gamma & $8.1 E+\infty 0$ & $4.0 \mathrm{E}+05$ & \\
\hline Maintenance Worker & Direct & Cell & External Exposure & Radionuclides & Gamma & $7.0 \mathrm{E}+00$ & $3.5 E+05$ & \\
\hline Maintenance Worker & Direct & East Gallery & External Exposure & Radionuclides & Beta-Gamma & 1.1E-01 & $5.6 \mathrm{E}+03$ & \\
\hline Maintenance Worker & Direct & East Gallery & External Exposure & Radionuclides & Gamma & $1.0 \mathrm{E}-01$ & $5.0 \mathrm{E}+03$ & \\
\hline Maintenance Worker & Direct & Whole Building & External Exposure & Radionuclides & Beta-Gamma & $1.1 E+01$ & $5.6 \mathrm{E}+05$ & \\
\hline Maintenance Worker & Direct & Whole Building & Extemal Exposure & Radionuclides & Gamma & $1.0 \mathrm{E}+01$ & $5.1 E+05$ & \\
\hline
\end{tabular}

- Untis are mrem

Units are mrem 
THIS PAGE INTENTIONALLY LEFT BLANK 
Table D-3 Maintenance Worker Exposure Concentrations and Chronic Daily Intakes from Smear Samples

\begin{tabular}{|c|c|c|c|c|c|c|c|c|}
\hline \multirow{2}{*}{ Receptor } & \multirow{2}{*}{ Media } & \multirow{2}{*}{ Location } & \multirow{2}{*}{ Exposure Pathway } & \multirow{2}{*}{$\begin{array}{c}\text { Contominant } \\
\text { Class }\end{array}$} & \multirow{2}{*}{ Contaminant } & \multirow{2}{*}{$\begin{array}{c}\text { Exposure } \\
\text { Point } \\
\text { Concentration }\end{array}$} & \multicolumn{2}{|c|}{$\begin{array}{c}\text { Chronic Daily } \\
\text { Intake" }\end{array}$} \\
\hline & & & & & & & $\begin{array}{c}\text { Carcinogenic } \\
\text { Effects }\end{array}$ & $\begin{array}{c}\text { Noncarcinogenic } \\
\text { Effects }\end{array}$ \\
\hline Maintenance Worker & Smear & Cell & Ingestion & Radionuclides & ${ }^{17} \mathrm{Cs}$ & $1.4 E+02$ & $4.5 E+04$ & \\
\hline Maintenance Worker & Smear & Cell & Ingestion & Radionuclides & ${ }^{2 x} \mathrm{Ra}$ & $5.3 \mathrm{E}-03$ & $1.6 \mathrm{E}+00$ & \\
\hline Maintenance Worker & Smear & Cell & Ingestion & Radionuclides & ${ }^{20} \mathrm{Th}$ & 1.1E-02 & $3.4 \mathrm{E}+00$ & \\
\hline Maintenance Worker & Smear & Cell & Ingestion & Radionuclides & ${ }^{250} \mathrm{Th}$ & $1.8 \mathrm{E}-02$ & $5.8 E+00$ & \\
\hline Maintenance Worker & Smear & Cell & Ingestion & Radionuclides & ${ }^{200} \mathrm{Th}$ & $1.1 \mathrm{E}-02$ & $3.6 \mathrm{E}+00$ & \\
\hline Maintenance Worker & Smear & Cell & Ingestion & Radionuclides & ${ }^{23 s} 2 \pi x$ & $1.7 \mathrm{E}-02$ & $5.3 \mathrm{E}+\infty 0$ & \\
\hline Maintenance Worker & Smear & Cell & Ingestion & Radionuclides & ${ }^{231} \mathrm{U}$ & $1.6 \mathrm{E}-03$ & 4.9E-01 & \\
\hline Maintenance Worker & Smear & Cell & Ingestion & Radionuclides & ${ }^{23} \mathrm{Pu}$ & 8.4E-03 & $2.6 \mathrm{E}+\infty 0$ & \\
\hline Maintenance Worker & Smear & Cell & Ingestion & Radionuclides & ${ }^{230} \mathrm{U}$ & $2.2 \mathrm{E}-02$ & $7.0 \mathrm{E}+\infty 0$ & \\
\hline Maintenance Worker & Smear & Cell & Ingestion & Radionuclides & ${ }^{23912200} \mathrm{Pu}$ & 5.3E-02 & $1.6 \mathrm{E}+01$ & \\
\hline Maintenance Worker & Smear & Cell & Ingestion & Radionuclides & ${ }^{40} \mathrm{~K}$ & 5.5E-01 & $1.7 E+02$ & \\
\hline Maintenance Worker & Smear & Cell & Ingestion & Radionuclides & ${ }^{60} \mathrm{Co}$ & $1.4 \mathrm{E}-02$ & $4.2 \mathrm{E}+00$ & \\
\hline Maintenance Worker & Smear & Cell & Ingestion & Radionuclides & ${ }^{\infty} \mathrm{Sr}$ & $1.2 \mathrm{E}+\infty$ & $3.8 \mathrm{E}+02$ & \\
\hline Maintenance Worker & Smear & Cell & Inhalation & Radionuclides & ${ }^{137} \mathrm{Ca}$ & $1.4 \mathrm{E}+02$ & $4.2 E+\infty$ & \\
\hline Maintenance Worker & Smear & Cell & Inhalation & Radionuclides & ${ }^{20 x} \mathrm{Ra}$ & 5.3E-03 & $1.5 \mathrm{E}-04$ & \\
\hline Maintenance Worker & Smear & Cell & Inhalation & Radionuclides & ${ }^{20} \mathrm{Th}$ & $1.1 \mathrm{E}-02$ & $3.2 E-04$ & \\
\hline Maintenance Worker & Smear & Cell & Inhalation & Radionuclides & ${ }^{200} \mathrm{Th}$ & $1.8 \mathrm{E}-02$ & 5.4E-04 & \\
\hline
\end{tabular}




\begin{tabular}{|c|c|c|c|c|c|c|c|c|}
\hline \multirow[b]{2}{*}{ Receptor } & \multirow[b]{2}{*}{ Media } & \multirow[b]{2}{*}{ Location } & \multirow[b]{2}{*}{ Exposure Pathway } & \multirow{2}{*}{$\begin{array}{l}\text { Contaminant } \\
\text { Class }\end{array}$} & \multirow[b]{2}{*}{ Contaminant } & \multirow{2}{*}{$\begin{array}{c}\text { Exposure } \\
\text { Point } \\
\text { Concentration" }\end{array}$} & \multicolumn{2}{|c|}{$\begin{array}{c}\text { Chronic Daily } \\
\text { Intake }\end{array}$} \\
\hline & & & & & & & $\begin{array}{l}\text { Carcinogenic } \\
\text { Effects }\end{array}$ & $\begin{array}{c}\text { Noncancinogenic } \\
\text { Erfects }\end{array}$ \\
\hline Maintenance Worker & Smear & Cell & Inhalation & Radionuclides & ${ }^{20} \mathrm{Th}$ & $1.1 E-02$ & 3.3E-04 & \\
\hline Maintenance Worker & Smear & Cell & Inhalation & Radionuclides & ${ }^{233 / 29} U$ & $1.7 \mathrm{E}-02$ & $5.0 \mathrm{E}-04$ & \\
\hline Maintenance Worker & Smear & Cell & Inhalation & Radionuclides & ${ }^{21} U$ & $1.6 \mathrm{E}-03$ & $4.6 \mathrm{E}-05$ & \\
\hline Maintenance Worker & Smear & Cell & Inhalation & Radionuclides & ${ }^{238} \mathrm{Pu}$ & 8.4E-03 & $2.5 \mathrm{E}-04$ & \\
\hline Maintenance Worker & Smear & Cell & Inhalation & Radionuclides & ${ }^{2 \times} \mathrm{U}$ & $2.2 \mathrm{E}-02$ & $6.5 \mathrm{E}-04$ & \\
\hline Maintenance Worker & Smear & Cell & Inhalation & Radionuclides & ${ }^{259200} \mathrm{Pu}$ & $5.3 \mathrm{E}-02$ & $1.5 \mathrm{E}-03$ & \\
\hline Maintenance Worker & Smear & Cell & Inhalation & Radionuclides & ${ }^{40} \mathrm{~K}$ & $5.5 \mathrm{E}-01$ & $1.6 \mathrm{E}-02$ & \\
\hline Maintenance Worker & Smear & Cell & Inhalation & Radionuclides & ${ }^{60} \mathrm{Co}$ & $1.4 \mathrm{E}-02$ & $3.9 \mathrm{E}-04$ & \\
\hline Maintenance Worket & Smear & Cell & Inhalation & Radionuclides & ${ }^{\infty} \mathrm{Sr}$ & $1.2 \mathrm{E}+00$ & $3.5 \mathrm{E}-02$ & \\
\hline Maintenance Worker & Smear & Cell & External Exposure & Radionuclides & ${ }^{17} \mathrm{Cs}$ & $1.4 \mathrm{E}+02$ & $8.2 E+02$ & \\
\hline Maintenance Worker & Smear & Cell & External Exposure & Radionuclides & ${ }^{2 \times} \mathrm{Ra}$ & 5.3E-03 & $3.0 \mathrm{E}-02$ & \\
\hline Maintenance Worker & Smear & Cell & External Exposure & Radionuclides & ${ }^{220} \mathrm{Th}$ & $1.1 \mathrm{E}-02$ & $6.3 \mathrm{E}-02$ & \\
\hline Maintenance Worker & Smear & Cell & External Exposure & Radionuclides & ${ }^{230} \mathrm{Th}$ & $1.8 \mathrm{E}-02$ & $1.1 \mathrm{E}-01$ & \\
\hline Maintenance Worker & Smear & Cell & External Exposure & Radionuclides & ${ }^{2 x} \mathrm{Th}$ & $1.1 E-02$ & $6.5 E-02$ & \\
\hline Maintenance Worker & Smear & Cell & External Exposure & Radionuclides & $238254 U$ & $1.7 \mathrm{E}-02$ & $9.8 \mathrm{E}-02$ & \\
\hline Maintenance Worker & Smear & Cell & External Exposure & Radionuclides & ${ }^{205} U$ & $1.6 \mathrm{E}-03$ & $9.0 \mathrm{E}-03$ & \\
\hline Maintenance Worker & Smear & Cell & External Exposure & Radionuclides & ${ }^{22} \mathrm{Pu}$ & 8.4E-03 & 4.8E-02 & \\
\hline Maintenance Worker & Smear & Cell & External Exposure & Radionuclides & ${ }^{29} \mathrm{U}$ & $2.2 \mathrm{E}-02$ & $1.3 \mathrm{E}-01$ & \\
\hline Maintenance Worker & Smear & Cell & Extemal Exposure & Radionuclides & ${ }^{250200} \mathrm{Pu}$ & $5.3 \mathrm{E}-02$ & $3.0 \mathrm{E}-01$ & \\
\hline
\end{tabular}

- Units are pCi/g

- Units are pCi-day 


\begin{tabular}{|c|c|c|c|c|c|c|c|c|}
\hline \multirow[b]{2}{*}{ Receptor } & \multirow[b]{2}{*}{ Medin } & \multirow{2}{*}{ Location } & \multirow{2}{*}{ Exposure Pathway } & \multirow{2}{*}{$\begin{array}{l}\text { Contaminant } \\
\text { Class }\end{array}$} & \multirow[b]{2}{*}{ Contaminant } & \multirow{2}{*}{$\begin{array}{c}\text { Exposure } \\
\text { Point } \\
\text { Concentration" }\end{array}$} & \multicolumn{2}{|c|}{$\begin{array}{l}\text { Chronic Daily } \\
\text { Intake }\end{array}$} \\
\hline & & & & & & & $\begin{array}{l}\text { Carcinogenic } \\
\text { Efrects }\end{array}$ & $\begin{array}{l}\text { Noncarcinogenic } \\
\text { Effects }\end{array}$ \\
\hline Maintenance Worker & Smear & Cell & Extemal Exposure & Radionuclides & ${ }^{*} \mathrm{~K}$ & 5.5E-01 & $3.1 E+\infty 0$ & \\
\hline Maintenance Worker & Smear & Cell & Extemal Exposure & Radionuclides & ${ }^{\infty} \mathrm{Co}$ & $1.4 \mathrm{E}-02$ & 7.7E-02 & \\
\hline Maintenance Worker & Smear & Cell & External Exposure & Radionuclides & ${ }^{\infty} \mathrm{Sr}$ & $1.2 E+\infty$ & $6.9 E+00$ & \\
\hline Maintenance Worker & Smear & East Gallery & Ingestion & Radionuclides & ${ }^{206} \mathrm{Ra}$ & $1.5 \mathrm{E}-02$ & $4.6 \mathrm{E}+\infty 0$ & \\
\hline Maintenance Worker & Smear & East Gallery & Ingestion & Radionuclides & ${ }^{2 n} \mathrm{Ra}$ & $2.4 \mathrm{E}-01$ & $7.6 \mathrm{E}+01$ & \\
\hline Maintenance Worker & Smear & East Gallery & Ingestion & Radionuclides & ${ }^{20} \mathrm{Th}$ & $2.3 E-02$ & $7.1 \mathrm{E}+00$ & \\
\hline Maintenance Worker & Smear & East Gallery & Ingestion & Radionuclides & ${ }^{200} \mathrm{Th}$ & $4.9 \mathrm{E}-02$ & $1.5 \mathrm{E}+01$ & \\
\hline Maintenance Worker & Smear & East Gallery & Ingestion & Radionuclides & ${ }^{29} \mathrm{Pu}$ & $1.6 \mathrm{E}-02$ & $4.9 E+00$ & \\
\hline Maintenance Worker & Smear & East Gallery & Ingestion & Radionuclides & ${ }^{2 N H} \mathrm{U}$ & $2.8 \mathrm{E}-02$ & $8.8 \mathrm{E}+\infty 0$ & \\
\hline Maintenance Worker & Smear & East Gallery & Ingestion & Radionuclides & ${ }^{250210} \mathrm{Pu}$ & $1.6 \mathrm{E}-02$ & $4.9 E+\infty 0$ & \\
\hline Maintenance Worker & Smear & East Gallery & Ingestion & Radionuclides & ${ }^{21} \mathrm{Am}$ & $2.0 \mathrm{E}-02$ & $6.3 E+\infty$ & \\
\hline Maintenance Worker & Smear & East Gallery & Ingeution & Radionuclides & ${ }^{\infty} \mathrm{K}$ & $3.6 \mathrm{E}+\infty 0$ & $1.1 E+03$ & \\
\hline Maintenance Worker & Smear & East Gallery & Ingeation & Radionuclide: & ${ }^{\circ} \mathrm{Sr}$ & $5.4 \mathrm{E}+00$ & $1.7 E+03$ & \\
\hline Maintenance Worker & Smear & East Gallery & Inhalation & Radionuclides & ${ }^{19} \mathrm{Ca}$ & $1.5 \mathrm{E}+01$ & 4.5E-01 & \\
\hline Maintenance Worker & Smear & Eaut Gallery & Inhalation & Radionuclides & ${ }^{n_{R}}$ & $1.5 \mathrm{E}-02$ & 4.3E-04 & \\
\hline
\end{tabular}

\section{- Units are $\mathrm{pCi} / \mathrm{g}$}

- Units are pCi-day 


\begin{tabular}{|c|c|c|c|c|c|c|c|c|}
\hline \multirow[b]{2}{*}{ Receptor } & \multirow{2}{*}{ Media } & \multirow{2}{*}{ Location } & \multirow[b]{2}{*}{ Exposure Pathway } & \multirow{2}{*}{$\begin{array}{c}\text { Contaminant } \\
\text { Class }\end{array}$} & \multirow[b]{2}{*}{ Contaminant } & \multirow{2}{*}{$\begin{array}{c}\text { Exposure } \\
\text { Point } \\
\text { Concentration" }\end{array}$} & \multicolumn{2}{|c|}{$\begin{array}{c}\text { Chronic Daily } \\
\text { Intake }\end{array}$} \\
\hline & & & & & & & $\begin{array}{c}\text { Carcinogenic } \\
\text { Errects }\end{array}$ & $\begin{array}{c}\text { Noncarcinogenic } \\
\text { Efrocts }\end{array}$ \\
\hline Maintenance Worker & Smear & East Gallery & Inhalation & Radionuclides & ${ }^{n+R a}$ & 2.4E-01 & $7.1 \mathrm{E}-03$ & \\
\hline Maintenance Worker & Smear & East Gallery & Inhalation & Radionuclides & ${ }^{2 n} \mathrm{Th}$ & $2.3 \mathrm{E}-02$ & $6.6 \mathrm{E}-04$ & \\
\hline Maintenance Worker & Smear & East Gallery & Inhalation & Radionuclides & ${ }^{200} \mathrm{Th}$ & $4.9 \mathrm{E}-02$ & $1.4 \mathrm{E}-03$ & \\
\hline Maintenance Worker & Smear & Enst Gallery & Inhalation & Radionuclides & ${ }^{232} \mathrm{Th}$ & $1.7 \mathrm{E}-02$ & $5.1 \mathrm{E}-0.4$ & \\
\hline Maintenance Worker & Smear & East Gallery & Inhalation & Radionuclides & ${ }^{23} 32 \times 3 \mathrm{U}$ & $3.6 \mathrm{E}-02$ & $1.1 \mathrm{E}-03$ & \\
\hline Maintenance Worker & Smear & East Gallery & Inhalation & Radionuclides & ${ }^{23} \mathrm{U}$ & $2.4 \mathrm{E}-03$ & $7.1 \mathrm{E}-05$ & \\
\hline Maintenance Worker & Smear & East Gallery & Inhalation & Radionuclides & ${ }^{231} \mathrm{Pu}$ & $1.6 \mathrm{E}-02$ & $4.6 \mathrm{E}-04$ & \\
\hline Maintenance Worker & Smear & East Gallery & Inhalation & Radionuclides & ${ }^{231} \mathrm{U}$ & $2.8 \mathrm{E}-02$ & 8.3E-04 & \\
\hline Maintenance Worker & Smear & East Gallery & Inhalation & Radionuclides. & ${ }^{25012200} \mathrm{Pu}$ & $1.6 \mathrm{E}-02$ & $4.6 \mathrm{E}-04$ & \\
\hline Maintenance Worker & Smear & East Gallery & Inhalation & Radionuclides & ${ }^{2 n 1} \mathrm{Am}$ & $2.0 \mathrm{E}-02$ & $5.9 \mathrm{E}-04$ & \\
\hline Maintenance Worker & Smear & East Gallery & Inhalation & Radionuclides & ${ }^{\infty} \mathrm{K}$ & $3.6 \mathrm{E}+\infty 0$ & $1.1 \mathrm{E}-01$ & \\
\hline Maintenance Worker & Smear & East Gallery & Inhalation & Radionuclides & ${ }^{\infty} \mathrm{Sr}$ & $5.4 \mathrm{E}+\infty 0$ & $1.6 \mathrm{E}-01$ & \\
\hline Maintenance Worker & Smear & East Gallery & External Exposure & Radionuclides & ${ }^{15} \mathrm{Cs}$ & $1.5 E+01$ & $8.7 E+01$ & \\
\hline Maintenance Worker & Smear & East Gallery & External Exposure & Radionuclides & ${ }^{206} \mathrm{Ra}$ & $1.5 \mathrm{E}-02$ & 8.3E-02 & \\
\hline Maintenance Worker & Smear & East Gallery & External Exposure & Radionuclides & ${ }^{20} \mathrm{Ra}$ & $2.4 \mathrm{E}-01$ & $1.4 \mathrm{E}+00$ & \\
\hline Maintenance Worker & Smear & East Gallery & External Exposure & Radionuclides & ${ }^{200} \mathrm{Th}$ & 2.3E-02 & $1.3 \mathrm{E}-01$ & \\
\hline Maintenance Worker & Smear & East Gallery & External Exposure & Radionuclides & ${ }^{200} \mathrm{Th}$ & $4.9 \mathrm{E}-02$ & $2.8 \mathrm{E}-01$ & \\
\hline Maintenance Worker & Smear & East Gallery & External Exposure & Radionuclides & ${ }^{203} \mathrm{Th}$ & $1.7 \mathrm{E}-02$ & $9.9 \mathrm{E}-02$ & \\
\hline Maintenance Worker & Smear & East Gallery & External Exposure & Radionuclides & ${ }^{23} 325 \mathrm{U}$ & $3.6 \mathrm{E}-02$ & $2.1 E-01$ & \\
\hline
\end{tabular}

- Units are pCi/g

- Units are pCi-day 


\begin{tabular}{|c|c|c|c|c|c|c|c|c|}
\hline \multirow[b]{2}{*}{ Receptor } & \multirow[b]{2}{*}{ Media } & \multirow[b]{2}{*}{ Location } & \multirow[b]{2}{*}{ Exposure Pathway } & \multirow{2}{*}{$\begin{array}{c}\text { Contaminant } \\
\text { Class }\end{array}$} & \multirow[b]{2}{*}{ Contaminant } & \multirow{2}{*}{$\begin{array}{c}\text { Exposure } \\
\text { Point } \\
\text { Concentration" }\end{array}$} & \multicolumn{2}{|c|}{$\begin{array}{c}\text { Chronic Daily } \\
\text { Intake }\end{array}$} \\
\hline & & & & & & & $\begin{array}{c}\text { Carcinogenic } \\
\text { Effects }\end{array}$ & $\begin{array}{c}\text { Noncarcinogenic } \\
\text { Effects }\end{array}$ \\
\hline Maintenance Worker & Smear & East Gallery & External Exposure & Radionuclides & ${ }^{23 S} \mathbf{U}$ & $2.4 E-03$ & $1.4 \mathrm{E}-02$ & \\
\hline Maintenance Worker & Smear & East Gallery & External Exposure & Radionuclides & ${ }^{23 s} \mathrm{Pu}$ & $1.6 \mathrm{E}-02$ & $9.0 \mathrm{E}-02$ & \\
\hline Maintenance Worker & Smear & East Gallery & External Exposure & Radionuclides & ${ }^{2 \times} \mathrm{U}$ & $2.8 \mathrm{E}-02$ & $1.6 \mathrm{E}-01$ & \\
\hline Maintenance Worker & Smear & East Gallery & External Exposure & Radionuclides & ${ }^{2502200} \mathrm{Pu}$ & $1.6 \mathrm{E}-02$ & $9.0 \mathrm{E}-02$ & \\
\hline Maintenance Worker & Smear & East Gallery & External Exposure & Radionuclides & ${ }^{\prime \prime} \mathrm{Am}$ & $2.0 \mathrm{E}-02$ & $1.2 \mathrm{E}-01^{\prime}$ & \\
\hline Maintenance Worker & Smear & East Gallery & Extemal Exposure & Radionuclides & ${ }^{\infty} \mathrm{K}$ & $3.6 E+00$ & $2.1 E+01$ & \\
\hline Maintenance Worker & Smear & East Gallery & External Exposure & Radionuclides & ${ }^{\infty} \mathrm{Sr}$ & $5.4 E+\infty 0$ & $3.1 E+01$ & \\
\hline Maintenance Worker & Smear & Whole Building & Ingestion & Radionuclides & ${ }^{17} \mathrm{Cs}$ & $8.0 E+01$ & $2.5 E+04$ & \\
\hline Maintenance Worker & Smear & Whole Building & Ingestion & Radionuclides & ${ }^{2 \times} \mathrm{Ra}$ & $5.9 \mathrm{E}-03$ & $1.9 \mathrm{E}+00$ & \\
\hline Maintenance Worker & Smear & Whole Building & Ingestion & Radionuclides & 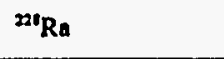 & $2.8 E-02$ & $8.8 E+00$ & \\
\hline Maintenance Worker & Smear & Whole Building & Ingestion & Radionuelides & ${ }^{20} \mathrm{Th}$ & 1.1E-02 & $3.5 E+00$ & \\
\hline Maintenance Worker & Smear & Whole Building & Ingestion & Radionuelides & ${ }^{200} \mathrm{Th}$ & $2.0 \mathrm{E}-02$ & $6.3 E+00$ & \\
\hline Maintenance Worker & Smear & Whole Building & Ingestion & Radionuclides & ${ }^{28} \mathrm{Th}$ & 1.1E-02 & $3.6 \mathrm{E}+00$ & \\
\hline Maintenance Worker & Smear & Whole Building & Ingestion & Radionuclides & ${ }^{23 \times 24} \mathrm{U}$ & $1.8 \mathrm{E}-02$ & $5.5 E+\infty$ & \\
\hline Maintenance Worker & Smear & Whole Building & Ingestion & Radionuclides & ${ }^{23} \mathbf{U}$ & $1.8 \mathrm{E}-03$ & $5.5 \mathrm{E}-01$ & \\
\hline Maintenance Worker & Smear & Whole Building & Ingestion & Radionuclides & ${ }^{234} \mathrm{Pu}$ & $9.5 \mathrm{E}-03$ & $3.0 \mathrm{E}+00$ & \\
\hline Maintenance Worker & Smear & Whole Building & Ingestion & Radionuclides & ${ }^{240} \mathrm{U}$ & 2.5E-02 & $7.8 E+\infty 0$ & \\
\hline Maintenance Worker & Smear & Whole Building & Ingevtion & Radionuelides & ${ }^{201200} \mathrm{Pu}$ & 5.8E-02 & $1.8 \mathrm{E}+01$ & \\
\hline Maintenance Worker & Smear & Whole Building & Ingertion & Radionuclides & ${ }^{211} \mathrm{Am}$ & $7.4 \mathrm{E}-03$ & $2.3 \mathrm{E}+\infty$ & \\
\hline
\end{tabular}

- Units are pCi/g

- Units are pCi-day 


\begin{tabular}{|c|c|c|c|c|c|c|c|c|}
\hline \multirow{2}{*}{ Receptor } & \multirow{2}{*}{ Media } & \multirow{2}{*}{ Location } & \multirow{2}{*}{ Exposure Pathway } & \multirow{2}{*}{$\begin{array}{l}\text { Contaminant } \\
\text { Class }\end{array}$} & \multirow[b]{2}{*}{ Contaminant } & \multirow{2}{*}{$\begin{array}{c}\text { Exposure } \\
\text { Point } \\
\text { Concentration }\end{array}$} & \multicolumn{2}{|c|}{$\begin{array}{c}\text { Chronic Daily } \\
\text { Intake }\end{array}$} \\
\hline & & & & & & & $\begin{array}{l}\text { Carcinogenic } \\
\text { Erfects }\end{array}$ & $\begin{array}{c}\text { Noncarcinogenic } \\
\text { Erfects }\end{array}$ \\
\hline Maintenance Worker & Smear & Whole Building & Ingestion & Radionuclide: & ${ }^{\infty} \mathrm{K}$ & $4.2 \mathrm{E}-01$ & $1.3 \mathrm{E}+02$ & \\
\hline Maintenance Worker & Smear & Whole Building & Ingestion & Radionuclides & ${ }^{\infty} \mathrm{Co}$ & $1.0 \mathrm{E}-02$ & $3.2 E+\infty 0$ & \\
\hline Maintenance Worker & Smear & Whole Building & Ingestion & Radionuclides & ${ }^{\infty} \mathrm{Sr}$ & $9.1 \mathrm{E}-01$ & $2.8 \mathrm{E}+02$ & \\
\hline Maintenance Worker & Smear & Whole Building & Inhalation & Radionuclides & ${ }^{19} \mathrm{Cr}$ & $8.0 \mathrm{E}+01$ & $2.3 \varepsilon+\infty$ & \\
\hline Maintenance Worker & Smear & Whole Building & Inhalation & Radionuclides & ${ }^{2 \times} \mathrm{Ra}$ & $5.9 \mathrm{E}-03$ & 1.7E-04 & \\
\hline Maintenance Worker & Smear & Whole Building & Inhalation & Radionuclides & ${ }^{n} \mathbf{R a}$ & $2.8 \mathrm{E}-02$ & 8.2E-04 & \\
\hline Maintenance Worker & Smear & Whole Building & Inhalation & Radionuclides & ${ }^{n}$ Th & $1.1 \mathrm{E}-02$ & 3.3E-0.4 & \\
\hline Maintenance Worker & Smear & Whole Building & Inhalation & Radionuclides & ${ }^{200} \mathrm{Th}$ & $2.0 \mathrm{E}-02$ & $5.9 \mathrm{E}-04$ & \\
\hline Maintenance Worker & Smear & Whole Building & Inhalation & Radionuclides & ${ }^{20} \mathrm{Th}$ & $1.1 \mathrm{E}-02$ & 3.3E-04 & \\
\hline Maintenance Worker & Smear & Whole Building & Inhalation & Radionuclides & ${ }^{233234} U$ & $1.8 \mathrm{E}-02$ & $5.2 \mathrm{E}-04$ & \\
\hline Maintenance Worker & Smear & Whole Building & Inhalation & Radionuclides & ${ }^{23} \mathrm{U}$ & $1.8 \mathrm{E}-03$ & $5.2 \mathrm{E}-05$ & \\
\hline Maintenance Worker & Smear & Whole Building & Inhalation & Radionuclides & ${ }^{230} \mathrm{Pu}$ & $9.5 \mathrm{E}-03$ & $2.8 \mathrm{E}-04$ & \\
\hline Maintenance Worker & Smear & Whole Building & Inhalation & Radionuelides & ${ }^{2310} \mathrm{U}$ & $2.5 E-02$ & $7.3 \mathrm{E}-04$ & \\
\hline Maintenance Worker & Smear & Whole Building & Inhalation & Radionuclides & ${ }^{2391200} \mathrm{Pu}$ & $5.8 \mathrm{E}-02$ & $1.7 E-03$ & \\
\hline Maintenance Worker & Smear & Whole Building & Inhalation & Radionuclides & ${ }^{211} \mathrm{Am}$ & $7.4 \mathrm{E}-03$ & 2.2E-04 & \\
\hline Maintenance Worker & Smear & Whole Building & Inhalation & Radionuclidea & ${ }^{+0} \mathrm{~K}$ & $4.2 \mathrm{E}-01$ & $1.2 \mathrm{E}-02$ & \\
\hline Maintenance Worker & Smear & Whole Building & Inhalation & Radionuclides & ${ }^{\infty} \mathrm{Co}$ & $1.0 \mathrm{E}-02$ & $3.0 \mathrm{E}-04$ & \\
\hline Mainlenance Worker & Smear & Whole Building & Inhalation & Radionuclides & ${ }^{\infty} \mathrm{Sr}$ & 9.1E-01 & 2.7E-02 & \\
\hline Maintenance Worker & Smear & Whole Building & External Exposure & Radionuclide: & ${ }^{27} \mathrm{Cs}$ & $8.0 \mathrm{E}+01$ & $4.6 \mathrm{E}+02$ & \\
\hline
\end{tabular}

- Units are pCi/g

- Units are pCi-day 


\begin{tabular}{|c|c|c|c|c|c|c|c|c|}
\hline \multirow[b]{2}{*}{ Receptor } & \multirow[b]{2}{*}{ Media } & \multirow[b]{2}{*}{ Location } & \multirow[b]{2}{*}{ Exposure Pathway } & \multirow{2}{*}{$\begin{array}{c}\text { Contaminant } \\
\text { Cless }\end{array}$} & \multirow[b]{2}{*}{ Contaminant } & \multirow{2}{*}{$\begin{array}{c}\text { Exposure } \\
\text { Point } \\
\text { Concentration" }\end{array}$} & \multicolumn{2}{|c|}{$\begin{array}{c}\text { Chronic Daily } \\
\text { Intake" }\end{array}$} \\
\hline & & & & & & & $\begin{array}{l}\text { Cercinogenic } \\
\text { Effects }\end{array}$ & $\begin{array}{c}\text { Noncancinogenic } \\
\text { Efrects }\end{array}$ \\
\hline Maintenance Worker & Smear & Whole Building & External Exposure & Radionuclides & ${ }^{2 \times} \mathrm{Ra}$ & $5.9 \mathrm{E}-03$ & $3.4 \mathrm{E}-02$ & r \\
\hline Maintenance Worker & Smear & Whole Building & External Exposure & Radionuclides & ${ }^{2 x} \mathrm{Ra}$ & $2.8 \mathrm{E}-02$ & $1.6 \mathrm{E}-01$ & \\
\hline Maintenance Worker & Smear & Wholc Building & External Exposure & Radionuclides & $29 T h$ & $1.1 \mathrm{E}-02$ & $6.4 E-02$ & \\
\hline Maintenance Worker & Smear & Whole Building & External Exposure & Radionuclides & ${ }^{200} \mathrm{Th}$ & $2.0 \mathrm{E}-02$ & $1.1 \mathrm{E}-01$ & \\
\hline Maintenance Worker & Smear & Whole Building & External Exposure & Radionuclides & ${ }^{25} \mathrm{Th}$ & $1.1 E-02$ & $6.5 \mathrm{E}-02$ & \\
\hline Maintenance Worker & Smear & Whole Building & External Exposure & Radionuclides & ${ }^{23 s} 2 \pi \mathrm{U}$ & $1.8 \mathrm{E}-02$ & $1.0 \mathrm{E}-01$ & \\
\hline Maintenance Worker & Smear & Whole Building & External Exposure & Radionuclides & ${ }^{23 S U}$ & $1.8 \mathrm{E}-03$ & $1.0 \mathrm{E}-02$ & \\
\hline Maintenance Worker & Smear & Whole Building & External Exposure & Radionuclides & ${ }^{23 \mathrm{Pus}}$ & $9.5 E-03$ & $5.4 E-02$ & \\
\hline Maintenance Worker & Smear & Whole Building & External Exposure & Radionuclides & ${ }^{28} \mathrm{U}$ & 2.5E-02 & $1.4 \mathrm{E}-01$ & \\
\hline Maintenance Worker & Smear & Whole Building & External Exposure & Radionuclides & ${ }^{298200} \mathrm{Pu}$ & $5.8 \mathrm{E}-02$ & 3.3E-01 & \\
\hline Maintenance Worker & Smear & Whole Building & Extemal Exposure & Radionuclides & ${ }^{211} \mathrm{Am}$ & $7.4 \mathrm{E}-03$ & $4.2 \mathrm{E}-02$ & \\
\hline Maintenance Worker & Smear & Whole Building & External Exposure & Radionuclides & ${ }^{+\infty} \mathrm{K}$ & $4.2 \mathrm{E}-01$ & $2.4 E+\infty 0$ & \\
\hline Maintenance Worker & Smear & Whole Building & External Exposure & Radionuclides & ${ }^{60} \mathrm{Co}$ & $1.0 \mathrm{E}-02$ & $5.8 \mathrm{E}-02$ & \\
\hline Maintenance Worker & Smear & Whole Building & External Exposure & Radionuclides & ${ }^{90} \mathrm{Sr}$ & 9.1E-01 & $5.2 \mathrm{E}+00$ & \\
\hline
\end{tabular}

- Units are $\mathrm{pCi} / \mathrm{g}$

- Units are pCi-day 
THIS PAGE INTENTIONALLY LEFT BLANK 


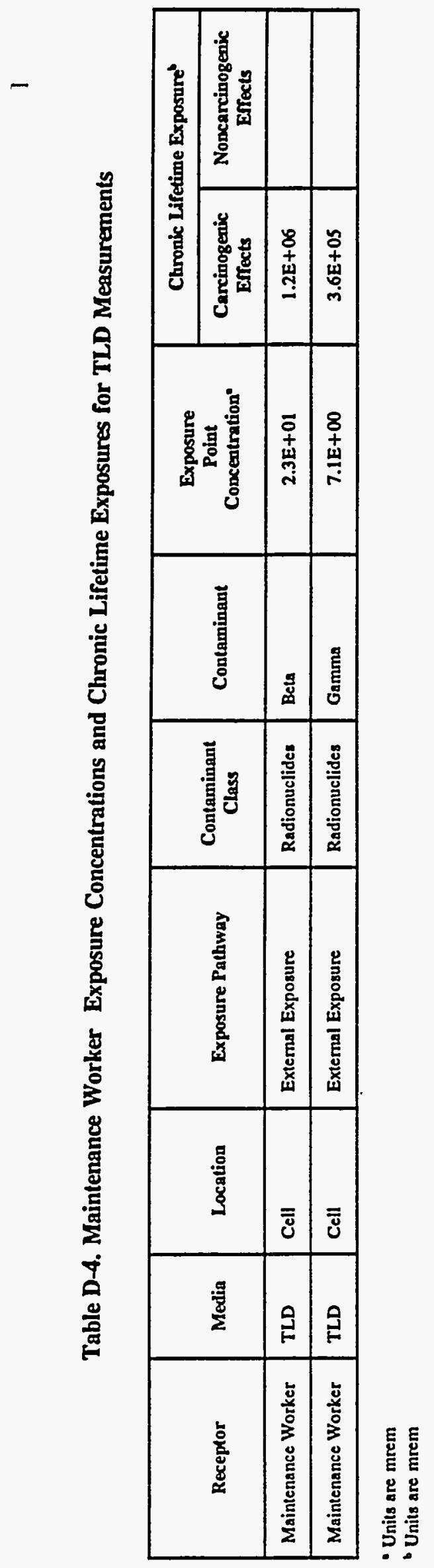


THIS PAGE INTENTIONALLY LEFT BLANK 
Table D-5. S\&M Worker Exposure Concentrations and Chronic Daily Intake Values for Core Samples and Sediment

\begin{tabular}{|c|c|c|c|c|c|c|c|c|}
\hline \multirow{2}{*}{ Receptor } & \multirow{2}{*}{ Medin } & \multirow{2}{*}{ Location } & \multirow[b]{2}{*}{$\begin{array}{l}\text { Exposure } \\
\text { Pathway }\end{array}$} & \multirow[b]{2}{*}{$\begin{array}{c}\text { Contaminant } \\
\text { Class }\end{array}$} & \multirow{2}{*}{ Contaminant } & \multirow[b]{2}{*}{$\begin{array}{l}\text { Exposure Point } \\
\text { Concentration" }\end{array}$} & \multicolumn{2}{|c|}{ Chronic Daily Intake } \\
\hline & & & & & & & $\begin{array}{c}\text { Cencinogenic } \\
\text { Efrects } \\
\end{array}$ & $\begin{array}{c}\text { Noncarcinogenic } \\
\text { Efrects }\end{array}$ \\
\hline S\&M Worker & Core & Cell & Ingestion & Inorganics & Arsenic & $2.6 \mathrm{E}+00$ & $3.6 \mathrm{E}-09$ & $1.0 \mathrm{E}-08$ \\
\hline S\&M Worker & Core & Cell & Ingestion & Inorganic: & Barium & $7.2 E+02$ & $1.0 \mathrm{E}-06$ & $2.8 \mathrm{E}-06$ \\
\hline S\&M Worker & Core & Cell & Ingestion & Inorganics & Beryllium & $1.8 \mathrm{E}+01$ & 2.5E-08 & $6.9 \mathrm{E}-08$ \\
\hline S\&M Worker & Core & Cell & Ingestion & Inorganic: & Cadmium & $1.8 \mathrm{E}+02$ & 2.5E-07 & $7.0 \mathrm{E}-07$ \\
\hline S\&M Worker & Core & Cell & Ingestion & Inorganics & Chromium VI & $2.0 \mathrm{E}+02$ & 2.7E-07 & $7.7 \mathrm{E}-07$ \\
\hline S\&M Worker & Core & Cell & Ingestion & Inorganics & Manganese & $9.5 E+03$ & 1.3E-05 & 3.7E-05 \\
\hline S\&M Worker & Core & Cell & Ingestion & Inorganics & Mercury & $3.4 \mathrm{E}-01$ & $4.8 \mathrm{E}-10$ & $1.3 \mathrm{E}-09$ \\
\hline S\&M Worker & Core & Cell & Ingestion & Inorganic: & Nickel & $1.4 \mathrm{E}+02$ & $1.9 \mathrm{E}-07$ & $5.4 \mathrm{E}-07$ \\
\hline S\&M Worker & Core & Cell & Ingestion & Inorganics & Vanadium & $2.4 E+02$ & $3.4 \mathrm{E}-07$ & $9.4 \mathrm{E}-07$ \\
\hline S\&M Worker & Core & Cell & Ingestion & Inorganics & Zinc & $3.5 E+04$ & 4.9E-05 & $1.4 \mathrm{E}-04$ \\
\hline S\&M Worker & Core & Cell & Ingestion & Organics & Aroclor-1254 & $3.4 \mathrm{E}-01$ & $4.8 \mathrm{E}-10$ & $1.3 \mathrm{E}-09$ \\
\hline S\&M Worker & Core & Cell & Ingestion & Organics & Aroclor- 1260 & $5.7 \mathrm{E}-01$ & $8.0 \mathrm{E}-10$ & $2.2 \mathrm{E}-09$ \\
\hline S\&M Worker & Core & Cell & Ingestion & Radionuclide: & ${ }^{17} \mathrm{Cs}$ & $1.4 \mathrm{E}+03$ & $3.4 E+03$ & \\
\hline S\&M Worker & Core & Cell & Ingestion & Radionuclides & ${ }^{2 x_{R a}}$ & $4.0 \mathrm{E}-01$ & $1.0 \mathrm{E}+00$ & \\
\hline S\&M Worker & Core & Cell & Ingestion & Radionuclides & ${ }^{2 x} \mathrm{Th}$ & 8.4E-01 & $2.1 E+00$ & \\
\hline S\&M Worker & Core & Cell & Ingestion & Radionuclides & ${ }^{250} \mathrm{Th}$ & $1.4 E+\infty 0$ & $3.5 E+\infty 0$ & \\
\hline S\&M Worker & Core & Cell & Ingestion & Radionuclides & ${ }^{20} \mathrm{Th}$ & 8.7E-01 & $2.2 \mathrm{E}+00$ & \\
\hline S\&M Worker & Core & Cell & Ingeation & Radionuclides & 2มs2s $\mathrm{U}$ & $1.3 \mathrm{E}+00$ & $3.3 E+00$ & \\
\hline S\&M Worker & Core & Cell & Ingestion & Radionuclides & 2siTh & $6.3 E+00$ & $1.6 \mathrm{E}+01$ & \\
\hline
\end{tabular}

- Units are $\mathrm{mg} / \mathrm{kg}$ for inorganics and organics; $\mathrm{pCi} / \mathrm{g}$ for radionuclides

- Units are mg/kg-day for inorganics and organics; $\mathrm{pCi}$-day for radionuclides 


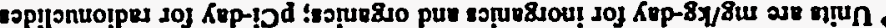

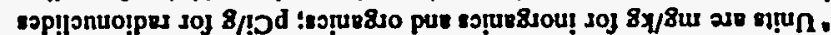

\begin{tabular}{|c|c|c|c|c|c|c|c|c|}
\hline$\varepsilon 1-9 S^{\prime} 6$ & $\varepsilon I-\exists b^{\circ} \varepsilon$ & $00+39 \cdot 2$ & 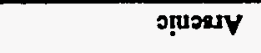 & 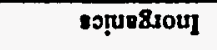 & 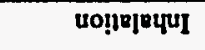 & IIPJ & 2100 & د5X10M WrS \\
\hline $60-31.1$ & $0 \mathrm{I}-30 \mathrm{~S}$ & $10-3<\cdot S$ & 0921-נolpary & sต̣ue\&ిo & prumad & וקיכ & 2100 & Iจף10M WX8S \\
\hline ot-ge'8 & $01-30^{\circ} \varepsilon$ & $10-3 b^{\prime} \varepsilon$ & tSZI-Jopoos & 85̣u8810 & pounsa & $\| P \supset$ & 200 & I5X10M Wrs \\
\hline $90-35.8$ & $90-30^{\circ} \varepsilon$ & $60+3 S^{\circ} \varepsilon$ & su! & 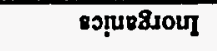 & faured & IIPO & 2500 & دOYנOM WrS \\
\hline $80-36.5$ & $80-3 I^{\prime} Z$ & $20+3 b^{\circ} \tau$ & 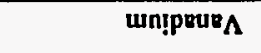 & sp̣ut8810u & pewrog & IIPJ & 2100 & มоఖ10M WrS \\
\hline $80-a b \cdot \varepsilon$ & $80-a \tau^{\prime} 1$ & $20+3 b^{\circ} 1$ & PPX!N & sọue8souI & fousod & IIיכ & 2200 & 10Y10M WrS \\
\hline II-JE'8 & II-g0' $\varepsilon$ & I0-gb' $\varepsilon$ & Sinouow & sọur\&souI & 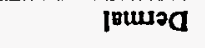 & $\| P O$ & 2503 & IOYIOM WPS \\
\hline $90-\mathrm{GE} \cdot Z$ & LO- $9 E \cdot 8$ & ع0+35: 6 & วsวuะ8ในeพ & 8ง!̣u88sou| & Iвuшว & $\| 100$ & 2100 & دоҮगоM WrS \\
\hline 80-28: & so-aL! & $20+90.2$ & 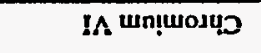 & cọuอ8sou! & !вน世ว & !!อ2 & 2100 & 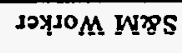 \\
\hline $80-966$ & $80-39^{\circ} 1$ & $20+38^{\circ} 1$ & แก!̣upgo & sọue810uI & prussa & $\| 100$ & 2100 & ЈәҮ10M N78 \\
\hline $60-3 \varepsilon^{\prime} b$ & $60-\exists S^{\prime} 1$ & $10+38^{\circ} 1$ & un!!|K1อg & sọuv8soul & ןвuчsa & $\| P 0$ & 2100 & دO¥10M W8S \\
\hline$\angle 0^{-98} \cdot \mid$ & $80^{-G \varepsilon^{*} 9}$ & $20+\exists \tau L L$ & un!̣eg & 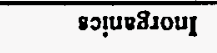 & pв世上2 & ॥Pग & 2100 & J0X10 M WrgS \\
\hline $01-\exists \varepsilon^{\prime} 9$ & $01-\exists \varepsilon^{\prime} Z$ & $00+39 \cdot z$ & ṣuossy & ะọuv8ิเouI & ןвயะ⿰ & \|PJ & 2503 & دoXj10M WrS \\
\hline & $10+9 \varepsilon^{\circ} S$ & $10+a I \cdot \tau$ & un!!!I & sop!|jnuo!pry & 40!185814I & IIPכ & 2503 & 30X10 M W78S \\
\hline & $10+96 \cdot 2$ & $10+3 \tau^{\prime} 1$ & ${ }^{1} S_{06}$ & sop!|गnuo!ped & นо!ฺsว8นuI & $\|$ ॥ग & 2500 & 20X10M Wr8S \\
\hline & $10-\Xi \varepsilon^{\prime} \varepsilon$ & $10-9 \varepsilon^{\prime} !$ & 050 & sรp!!onuo!pey & 40!!sร8) & $\| P \supset$ & 2200 & $30 \times 10 \mathrm{M} \mathrm{W}$ WS \\
\hline & $10+9 \varepsilon^{\prime} 1$ & $00+\exists \varepsilon^{\prime} S$ & $\mathrm{H}_{0 \mathrm{t}}$ & sop!|pnuo!pry & 40!!8584I & $\| \odot 0$ & 2500 & J0×10M W8S \\
\hline & $10+30.1$ & $00+30^{\circ} 6$ & ndonesere & sop!|pnuo!pry & 40!!s & ॥Pग & 2303 & $20 \times 10 M$ WrS \\
\hline & $00+3 \varepsilon^{\prime} t$ & $00+\exists L^{\prime} \mathbf{I}$ & $n_{x z}$ & sop!!onuo!pry & uo!̣อP8นI & $\| P D$ & 2305 & sэУנоM WrS \\
\hline & $00+3 l^{\prime} I$ & 10-gs"t & $n_{d x z}$ & sop!|onuo!pry & 40!!80841 & $\| 100$ & 2303 & ION10M WrS \\
\hline & $1030^{\circ} \varepsilon$ & $10-32 \cdot 1$ & $\mathrm{n}_{\mathrm{sx} z}$ & sop!|onuo!pry & น0!!8ว8นI & $\| 100$ & 2300 & JoXjoM WrS \\
\hline 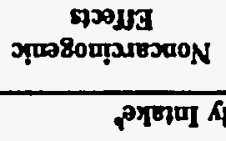 & 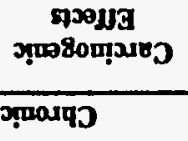 & 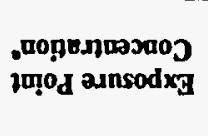 & 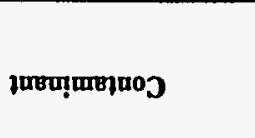 & 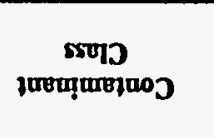 & $\begin{array}{l}\text { KEMqRed } \\
\text { ansodxg }\end{array}$ & uop̣esot & ยฺpow & Loxdaxy \\
\hline
\end{tabular}




\begin{tabular}{|c|c|c|c|c|c|c|c|c|}
\hline \multirow{2}{*}{ Roceptor } & \multirow{2}{*}{ Media } & \multirow{2}{*}{ Location } & \multirow{2}{*}{$\begin{array}{l}\text { Exposure } \\
\text { Pathway }\end{array}$} & \multirow{2}{*}{$\begin{array}{l}\text { Contaminant } \\
\text { Class }\end{array}$} & \multirow{2}{*}{ Contaminant } & \multirow{2}{*}{$\begin{array}{l}\text { Exposure Point } \\
\text { Concentration" }\end{array}$} & \multicolumn{2}{|c|}{ Chranic Daily Intake" } \\
\hline & & & & & & & $\begin{array}{c}\text { Carcinogenic } \\
\text { Efrects }\end{array}$ & $\begin{array}{c}\text { Noncancinogenic } \\
\text { Efrects }\end{array}$ \\
\hline S\&M Worker & Core & Cell & Inhalation & Inorganics & Barium & $7.2 \mathrm{E}+02$ & 9.4E-11 & 2.6E-10 \\
\hline S\&M Worker & Core & Cell & Inhalation & Inorganics & Beryllium & $1.8 \mathrm{E}+01$ & $2.3 \mathrm{E}-12$ & 6.4E-12 \\
\hline S\&M Worker & Core & Cell & Inhalation & Inorganics & Cadmium & $1.8 \mathrm{E}+02$ & $2.3 E-11$ & $6.6 \mathrm{E}-11$ \\
\hline S\&M Worker & Core & Cell & Inhalation & Inorganics & Chromium VI & $2.0 \mathrm{E}+02$ & 2.6E-11 & $7.2 \mathrm{E}-11$ \\
\hline S\&M Worker & Core & Cell & Inhalation & Inorganics & Manganese & $9.5 \mathrm{E}+03$ & $1.2 \mathrm{E}-09$ & $3.5 \mathrm{E}-09$ \\
\hline S\&M Worker & Core & Cell & Inhalation & Inorganics & Mencury & $3.4 \mathrm{E}-01$ & 4.4E-14 & 1.2E-13 \\
\hline S\&M Worker & Core & Cell & Inhalation & Inorganice & Nickel & $1.4 \mathrm{E}+02$ & $1.8 \mathrm{E}-11$ & $5.0 \mathrm{E}-11$ \\
\hline S\&M Worker & Core & Cell & Inhalation & Inorganics & Vanadium & $2.4 \mathrm{E}+02$ & $3.1 \mathrm{E}-11$ & $8.8 \mathrm{E}-11$ \\
\hline S\&M Worker & Core & Cell & Inhalation & Inorganics & Zinc & $3.5 E+04$ & $4.6 \mathrm{E}-09$ & $1.3 \mathrm{E}-08$ \\
\hline S\&M Worker & Core & Cell & Inhalation & Organics & Aroclor-1254 & $3.4 \mathrm{E}-01$ & 4.4E-14 & $1.2 \mathrm{E}-13$ \\
\hline S\&M Worker & Core & Cell & Inhalation & Organics & Aroclor- 1260 & $5.7 \mathrm{E}-01$ & 7.4E-14 & 2.1E-13 \\
\hline S\&M Worker & Core & Cell & Inhalation & Radionuclides & ${ }^{15} \mathrm{Cs}$ & $1.4 E+03$ & $3.2 \mathrm{E}-01$ & \\
\hline S\&M Worker & Core & Cell & Inhalation & Radionuclides & ${ }^{20 \times R a}$ & $4.0 \mathrm{E}-01$ & $9.4 \mathrm{E}-05$ & \\
\hline S\&M Worker & Core & Cell & Inhalation & Radionuclides & ${ }^{20} \mathrm{Th}$ & 8.4E-01 & $2.0 \mathrm{E}-04$ & \\
\hline S\&M Worker & Core & Cell & Inhalation & Radionuclides & ${ }^{200} \mathrm{Th}$ & $1.4 \mathrm{E}+\infty 0$ & 3.3E-04 & \\
\hline S\&M Worker & Core & Cell & Inhalation & Radionuclides & ${ }^{25} \mathrm{Th}$ & 8.7E-01 & $2.0 \mathrm{E}-04$ & \\
\hline S\&M Worker & Core & Cell & Inhalation & Radionuclides & ${ }^{23823} \mathrm{U}$ & $1.3 E+\infty$ & $3.0 \mathrm{E}-04$ & \\
\hline S\&M Worker & Core & Cell & Inhalation & Radionuclides & ${ }^{24} \mathrm{Th}$ & $6.3 \mathrm{E}+\infty$ & $1.5 \mathrm{E}-03$ & \\
\hline S\&M Worker & Core & Cell & Inhalation & Radionuclides & ${ }^{231} \mathrm{U}$ & $1.2 \mathrm{E}-01$ & $2.8 \mathrm{E}-05$ & \\
\hline S\&M Worker & Core & Cell & Inhalation & Radionuclides & ${ }^{28} \mathrm{Pu}$ & 4.5E-01 & 1.1E-04 & \\
\hline S\&M Worker & Core & Cell & Inhalation & Radionuclides & $2 x U$ & $1.7 E+\infty 0$ & $4.0 \mathrm{E}-04$ & \\
\hline
\end{tabular}

- Units are $\mathrm{mg} / \mathrm{kg}$ for inorganics end organics; $\mathrm{pCi} / \mathrm{g}$ for radionuclides

- Units are $\mathrm{mg} / \mathrm{kg}$-day for inorganics and organics; $\mathrm{PCi}$-day for radionuclides 


\begin{tabular}{|c|c|c|c|c|c|c|c|c|}
\hline \multirow{2}{*}{ Receptor } & \multirow{2}{*}{ Media } & \multirow{2}{*}{ Location } & \multirow{2}{*}{$\begin{array}{l}\text { Exposure } \\
\text { Pathway }\end{array}$} & \multirow{2}{*}{$\begin{array}{l}\text { Contaminant } \\
\text { Class }\end{array}$} & \multirow{2}{*}{ Contaminant } & \multirow{2}{*}{$\begin{array}{l}\text { Exposure Point } \\
\text { Concentration" }\end{array}$} & \multicolumn{2}{|c|}{ Chronic Daily Intake" } \\
\hline & & & & & & & $\begin{array}{c}\text { Carcinogenic } \\
\text { Efrocts }\end{array}$ & $\begin{array}{c}\text { Noncarcinogenic } \\
\text { Effects }\end{array}$ \\
\hline S\&M Worker & Core & Cell & Inhalation & Radionuclides & ${ }^{290210} \mathrm{Pu}$ & $4.0 \mathrm{E}+\infty 0$ & 9.3E-04 & \\
\hline S\&M Worker & Core & Cell & Inhalation & Radionuclides & ${ }^{\infty} \mathrm{K}$ & $5.3 E+\infty$ & $1.2 \mathrm{E}-03$ & \\
\hline S\&M Worker & Core & Cell & Inhalation & Radionuclides & ${ }^{60} \mathrm{Co}$ & $1.3 \mathrm{E}-01$ & $3.0 \mathrm{E}-05$ & \\
\hline S\&M Worker & Core & Cell & Inhalation & Radionuclides & ${ }^{x} \mathrm{Sr}$ & $1.2 \mathrm{E}+01$ & 2.7E-03 & \\
\hline S\&M Worker & Core & Cell & Inhalation & Radionuclides & Tritium & $2.1 \mathrm{E}+01$ & $4.9 \mathrm{E}-03$ & \\
\hline S\&M Worker & Core & Cell & External Exposure & Radionuclides & "s: & $1.4 \mathrm{E}+03$ & $5.0 \mathrm{E}+01$ & \\
\hline S\&M Worker & Core & Cell & External Exposure & Radionuclides & ${ }^{20} \mathrm{Ra}$ & 4.0E-01 & $1.5 \mathrm{E}-02$ & \\
\hline S\&M Worker & Core & Cell & External Exposure & Radionuclides & ${ }^{20} \mathrm{Th}$ & $8.4 \mathrm{E}-01$ & 3.1E-02 & \\
\hline S\&M Worker & Core & Cell & External Exposure & Radionuclides & ${ }^{250} \mathrm{Th}$ & $1.4 \mathrm{E}+00$ & $5.1 \mathrm{E}-02$ & \\
\hline S\&M Worker & Core & Cell & External Exposure & Radionuclides & ${ }^{2 n} \mathrm{Th}$ & 8.7E-01 & $3.2 \mathrm{E}-02$ & \\
\hline S\&M Worker & Core & Cell & External Exposure & Radionuclides & ${ }^{239} 324 \mathrm{U}$ & $1.3 \mathrm{E}+00$ & 4.7E-02 & \\
\hline S\&M Worker & Core & Cell & External Exposure & Radionuclides & ${ }^{23} \mathrm{Th}$ & $6.3 \mathrm{E}+00$ & 2.3E-01 & \\
\hline SâM Worker & Cote & Cell & Extemal Expozure & Radioniucliứ: & ${ }^{239} \mathrm{U}$ & $1.2 \mathrm{E}-01$ & $4.45-03$ & \\
\hline S\&M Worker & Core & Cell & External Exposure & Radionuclides & ${ }^{201} \mathrm{Pu}$ & 4.5E-01 & $1.6 \mathrm{E}-02$ & \\
\hline S\&M Worker & Core & Cell & External Exposure & Radionuclide: & ${ }^{230} \mathrm{U}$ & $1.7 \mathrm{E}+\infty 0$ & $6.2 \mathrm{E}-02$ & \\
\hline S\&M Worker & Core & Cell & External Exposure & Radionuclides & ${ }^{258200} \mathrm{Pu}$ & $4.0 \mathrm{E}+00$ & $1.5 \mathrm{E}-01$ & \\
\hline S\&M Worker & Core & Cell & External Exposure & Radionuclides & ${ }^{10} \mathrm{~K}$ & $5.3 \mathrm{E}+00$ & $1.9 \mathrm{E}-01$ & \\
\hline S\&M Worker & Core & Cell & External Exposure & Radionuclides & ${ }^{80} \mathrm{Co}$ & $1.3 \mathrm{E}-01$ & $4.7 \mathrm{E}-03$ & \\
\hline S\&M Worker & Core & Cell & Extermal Exposure & Radionuclides & ${ }^{\infty} \mathrm{Sr}$ & $1.2 \mathrm{E}+01$ & $4.2 \mathrm{E}-01$ & \\
\hline S\&M Worker & Core & Cell & Extemal Exposure & Radionuclides & Tritium & $2.1 E+01$ & 7.7E-01 & \\
\hline S\&M Worker & Core & East Gallery & Ingestion & Inorganics & Artenic & $3.0 \mathrm{E}+00$ & $4.2 \mathrm{E}-09$ & $1.2 \mathrm{E}-08$ \\
\hline
\end{tabular}

- Units are mg/kg for inorganics and organics; $\mathrm{pCi} / \mathrm{g}$ for radionuclides

- Units are mg/kg-day for inorganics and organica; $\mathrm{pCi}$-day for radionuclides 


\begin{tabular}{|c|c|c|c|c|c|c|c|c|}
\hline \multirow{2}{*}{ Receptor } & \multirow{2}{*}{ Media } & \multirow{2}{*}{ Location } & \multirow{2}{*}{$\begin{array}{l}\text { Exposure } \\
\text { Pathway }\end{array}$} & \multirow{2}{*}{$\begin{array}{l}\text { Contaminant } \\
\text { Class }\end{array}$} & \multirow{2}{*}{ Contaminant } & \multirow{2}{*}{$\begin{array}{l}\text { Exposure Point } \\
\text { Concentration" }\end{array}$} & \multicolumn{2}{|c|}{ Chronic Daily Intake" } \\
\hline & & & & & & & $\begin{array}{c}\text { Cancinogenic } \\
\text { Effects }\end{array}$ & $\begin{array}{l}\text { Noncercinogenic } \\
\text { Enfects }\end{array}$ \\
\hline S\&M Worker & Core & East Gallery & Ingestion & Inorganics & Barium & $2.1 E+01$ & 3.0E-08 & 8.4E-08 \\
\hline S\&M Worker & Core & East Gallery & Ingestion & Inorganics & Beryllium & $4.0 \mathrm{E}-01$ & $5.6 \mathrm{E}-10$ & $1.6 \mathrm{E}-09$ \\
\hline S\&M Worker & Core & East Gallery & Ingestion & Inorganic» & Cadmium & $5.1 \mathrm{E}+\infty$ & 7.1E-09 & $2.0 \mathrm{E}-08$ \\
\hline S\&M Worker & Core & East Gallery & Ingestion & Inorganic» & Chromium VI & $8.3 E+\infty$ & $1.2 \mathrm{E}-08$ & $3.2 E-08$ \\
\hline S\&M Worker & Core & East Gallery & Ingestion & Inorganics & Manganese & $2.7 E+02$ & 3.7E-07 & $1.0 \mathrm{E}-06$ \\
\hline S\&M Worker & Core & East Gallery & Ingestion & Inorganics & Nickel & $6.0 \mathrm{E}+\infty 0$ & 8.4E-09 & 2.3E-08 \\
\hline S\&M Worker & Core & East Gallery & Ingestion & Inorganics & Vanadium & $9.1 \mathrm{E}+\infty$ & 1.3E-08 & $3.6 \mathrm{E}-08$ \\
\hline S\&M Worker & Core & East Gallery & Ingestion & Inorganics & Zinc & $9.8 \mathrm{E}+02$ & $1.4 \mathrm{E}-06$ & $3.8 \mathrm{E}-06$ \\
\hline S\&M Worker & Core & East Gallery & Ingestion & Organics & Aroclor-1254 & $1.7 \mathrm{E}+00$ & 2.4E-09 & 6.7E-09 \\
\hline S\&M Worker & Core & East Gallery & Ingestion & Organics & Aroclor-1260 & $2.6 \mathrm{E}+00$ & 3.6E-09 & $1.0 \mathrm{E}-08$ \\
\hline S\&M Worker & Core & East Gallery & Ingestion & Organics & Bis(2-ethylhexyl)phthalate & $2.4 \mathrm{E}-01$ & 3.4E-10 & $9.4 \mathrm{E}-10$ \\
\hline S\&M Worker & Core & East Gallery & Ingestion & Organics & Dimethyl phthalate & $5.5 \mathrm{E}-02$ & 7.7E-11 & $2.2 \mathrm{E}-10$ \\
\hline S\&M Worker & Core & East Gallery & Ingestion & Radionuclides & ${ }^{15} \mathrm{Cs}$ & $2.3 \mathrm{E}+01$ & $5.7 \mathrm{E}+01$ & \\
\hline S\&M Worker & Core & East Gallery & Ingestion & Radionuclides & ${ }^{20} \mathrm{Ra}$ & 3.6E-01 & $9.0 \mathrm{E}-01$ & \\
\hline S\&M Worker & Core & East Gallery & Ingestion & Radionuclides & ${ }^{20} \mathrm{Ra}$ & 3.7E-01 & 9.3E-01 & \\
\hline S\&M Worker & Core & East Gallery & Ingestion & Radionuclides & $2 n \mathrm{Th}$ & $5.6 \mathrm{E}-01$ & $1.4 E+\infty 0$ & \\
\hline S\&M Worker & Core & East Gallery & Ingestion & Radionuclides & ${ }^{200} \mathrm{Th}$ & $1.2 \mathrm{E}+\infty$ & $3.0 E+\infty$ & \\
\hline S\&M Worker & Core & East Gallery & Ingestion & Radionuclides & ${ }^{20} \mathrm{Th}$ & 4.3E-01 & $1.1 E+\infty 0$ & \\
\hline S\&M Worker & Core & Eant Gallery & Ingestion & Radionuclides & ${ }^{231234} \mathrm{U}$ & $9.0 \mathrm{E}-01$ & $2.3 E+\infty 0$ & \\
\hline S\&M Worker & Core & Eant Gallery & Ingextion & Radionuclides & 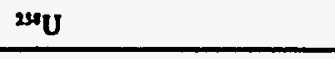 & 7.7E-01 & $1.9 \mathrm{E}+00$ & \\
\hline S\&M Worker & Core & East Gallery & Ingertion & Radionuclides & ${ }^{22} \mathrm{Am}$ & S.0E-01 & $1.3 E+\infty$ & \\
\hline
\end{tabular}

- Units are $\mathrm{mg} / \mathrm{kg}$ for inorganics and organica; $\mathrm{pCi} / \mathrm{g}$ for radionuclides

- Units are $m g / k_{g}$-day for inorganics and organica; pCi-day for radionuclides 


\begin{tabular}{|c|c|c|c|c|c|c|c|c|}
\hline \multirow{2}{*}{ Receptor } & \multirow{2}{*}{ Media } & \multirow{2}{*}{ Location } & \multirow{2}{*}{$\begin{array}{l}\text { Exposure } \\
\text { Pathway }\end{array}$} & \multirow{2}{*}{$\begin{array}{l}\text { Contaminant } \\
\text { Class }\end{array}$} & \multirow{2}{*}{ Contaminant } & \multirow{2}{*}{$\begin{array}{l}\text { Exposure Point } \\
\text { Concentration" }\end{array}$} & \multicolumn{2}{|c|}{ Chronic Daily Intake } \\
\hline & & & & & & & $\begin{array}{c}\text { Carcinogenic } \\
\text { Effects }\end{array}$ & $\begin{array}{c}\text { Noncarcinogenic } \\
\text { Effects }\end{array}$ \\
\hline S\&M Worker & Core & East Gallery & Ingestion & Radionuclides & ${ }^{\infty} \mathrm{K}$ & $5.4 E+00$ & $1.3 \mathrm{E}+01$ & \\
\hline S\&M Worker & Core & East Gallery & Ingestion & Radionuclides & ${ }^{\infty} \mathrm{Sr}$ & $8.1 E+\infty 0$ & $2.0 \mathrm{E}+01$ & \\
\hline S\&M Worker & Core & East Gallery & Ingestion & Radionuclides & Tritium & $2.6 \mathrm{E}+01$ & $6.4 E+01$ & \\
\hline S\&M Worker & Core & East Gallery & Dermal & Inorganics & Arsenic & $3.0 \mathrm{E}+00$ & $2.6 \mathrm{E}-10$ & $7.3 \mathrm{E}-10$ \\
\hline S\&M Worker & Core & East Gallery & Dermal & Inorganics & Barium & $2.1 E+01$ & $1.9 \mathrm{E}-09$ & $5.2 \mathrm{E}-09$ \\
\hline S\&M Worker & Core & East Gallery & Dermal & Inorganics & Beryllium & $4.0 \mathrm{E}-01$ & 3.5E-11 & $9.8 \mathrm{E}-11$ \\
\hline S\&M Worker & Core & East Gallery & Dermal & Inorganics & Cadmium & $5.1 \mathrm{E}+00$ & 4.4E-10 & $1.2 \mathrm{E}-09$ \\
\hline S\&M Worker & Core & East Gallery & Dermal & Inorganics & Chromium VI & $8.3 \mathrm{E}+00$ & $7.2 \mathrm{E}-10$ & 2.0E-09 \\
\hline S\&M Worker & Core & East Gallery & Dermal & Inorganics & Manganese & $2.7 E+02$ & $2.3 E-08$ & $6.5 \mathrm{E}-08$ \\
\hline S\&M Worker & Core & East Gallery & Dermal & Inorganics & Nickel & $6.0 \mathrm{E}+00$ & $5.2 \mathrm{E}-10$ & $1.5 \mathrm{E}-09$ \\
\hline S\&M Worker & Core & East Gallery & Dermal & Inorganics & Vanadium & $9.1 \mathrm{E}+\infty 0$ & $7.9 \mathrm{E}-10$ & $2.2 E-09$ \\
\hline S\&M Worker & Core & East Gallery & Dermal & Inorganics & Zinc & $9.8 \mathrm{E}+02$ & 8.5E-08 & $2.4 \mathrm{E}-07$ \\
\hline Såmi Worker & Core & Easi Gaiicry & Dermai & Organics & Arocior-i254 & i.7E+00 & i.5E-09 & 4.2E-09 \\
\hline S\&M Worker & Core & East Gallery & Dermal & Organics & Aroclor- 1260 & $2.6 E+\infty 0$ & 2.3E-09 & $6.3 \mathrm{E}-09$ \\
\hline S\&M Worker & Core & East Gallery & Dermal & Organics & Bis(2-ethylhexyl)phthalate & $2.4 \mathrm{E}-01$ & $2.1 \mathrm{E}-10$ & $5.9 \mathrm{E}-10$ \\
\hline S\&M Worker & Core & East Gallery & Dermal & Organics & Dimethyl phthalate & $5.5 \mathrm{E}-02$ & $4.8 \mathrm{E}-11$ & $1.3 \mathrm{E}-10$ \\
\hline S\&M Worker & Core & East Gallery & Inhalation & Inorganics & Arsenic & $3.0 \mathrm{E}+\infty 0$ & $3.9 \mathrm{E}-13$ & $1.1 \mathrm{E}-12$ \\
\hline S\&M Worker & Core & East Gallery & Inhalation & Inorganics & Barium & $2.1 \mathrm{E}+01$ & $2.8 \mathrm{E}-12$ & $7.8 \mathrm{E}-12$ \\
\hline S\&M Worker & Core & East Gallery & Inhalation & Inorganics & Beryllium & $4.0 \mathrm{E}-01$ & $5.2 \mathrm{E}-14$ & $1.5 \mathrm{E}-13$ \\
\hline S\&M Worker & Core & East Gallery & Inhalation & Inorganics & Cadmium & $5.1 E+\infty 0$ & $6.7 \mathrm{E}-13$ & $1.9 \mathrm{E}-12$ \\
\hline S\&M Worker & Core & East Gallery & Inhalation & Inorganics & Chromium VI & $8.3 E+\infty 0$ & $1.1 \mathrm{E}-12$ & $3.0 \mathrm{E}-12$ \\
\hline
\end{tabular}

- Units are $\mathrm{mg} / \mathrm{kg}$ for inorganics and organica; $\mathrm{pCi} / \mathrm{g}$ for radionuclides

- Units are $\mathrm{mg} / \mathrm{kg}$-day for inorganics and organicu; pCi-day for radionuclides 


\begin{tabular}{|c|c|c|c|c|c|c|c|c|}
\hline \multirow{2}{*}{ Receptor } & \multirow{2}{*}{ Media } & \multirow{2}{*}{ Location } & \multirow{2}{*}{$\begin{array}{l}\text { Exposure } \\
\text { Pathwray }\end{array}$} & \multirow{2}{*}{$\begin{array}{l}\text { Contaminant } \\
\text { Class }\end{array}$} & \multirow{2}{*}{ Conteminant } & \multirow{2}{*}{$\begin{array}{l}\text { Exposure Point } \\
\text { Concentration" }\end{array}$} & \multicolumn{2}{|c|}{ Chronic Daily Intake' } \\
\hline & & & & & & & $\begin{array}{c}\text { Carcinogenic } \\
\text { Effocts }\end{array}$ & $\begin{array}{c}\text { Noncarcinogenic } \\
\text { Effects }\end{array}$ \\
\hline S\&M Worker & Core & East Gallery & Inhalation & Inorganick & Manganese & $2.7 E+02$ & 3.5E-11 & $9.7 \mathrm{E}-11$ \\
\hline S\&M Worker & Core & Eart Gallery & Inhalation & Inorganics & Nickel & $6.0 E+\infty$ & $7.8 \mathrm{E}-13$ & $2.2 \mathrm{E}-12$ \\
\hline S\&M Worker & Core & East Gallery & Inhalation & Inorganics & Vanadium & $9.1 \mathrm{E}+\infty$ & $=1.2 \mathrm{E}-12$ & 3.3E-12 \\
\hline S\&M Worker & Core & Eant Gallery & Inhalation & Inorganics & Zine & $9.8 \mathrm{E}+02$ & $1.3 \mathrm{E}-10$ & $3.6 \mathrm{E}-10$ \\
\hline S\&M Worker & Core & East Gallery & Inhalation & Organics & Aroclor-1254 & $1.7 E+\infty$ & $2.2 \mathrm{E}-13$ & $6.2 \mathrm{E}-13$ \\
\hline S\&M Worker & Core & East Gallery & Inhalation & Organics & Aroclor- 1260 & $2.6 \mathrm{E}+00$ & $3.4 \mathrm{E}-13$ & 9.5E-13 \\
\hline S\&M Worker & Core & Eant Gallery & Inhalation & Organica & Bis(2-ethylhexyl)phthalate & $2.4 \mathrm{E}-01$ & $3.1 \mathrm{E}-14$ & $8.8 \mathrm{E}-14$ \\
\hline S\&M Worker & Core & East Gallery & Inhatation & Organics & Dimethyl phthalate & $5.5 \mathrm{E}-02$ & 7.2E-15 & $2.0 \mathrm{E}-14$ \\
\hline S\&M Worker & Core & East Gallery & Inhalation & Radionuclides & ${ }^{137} \mathrm{Cs}$ & $2.3 E+01$ & $5.3 \mathrm{E}-03$ & \\
\hline S\&M Worker & Core & East Gallery & Inhalation & Radionuclides & ${ }^{2 \times}{ }_{R a}$ & $3.6 \mathrm{E}-01$ & 8.4E-05 & \\
\hline S\&M Worker & Core & East Gallery & Inhalation & Radionuclides & ${ }^{n n_{R}}$ & $3.7 \mathrm{E}-01$ & $8.6 \mathrm{E}-05$ & \\
\hline S\&M Worker & Core & East Gallery & Inhalation & Radionuclides & ${ }^{20} \mathrm{Th}$ & $5.6 \mathrm{E}-01$ & $1.3 \mathrm{E}-04$ & \\
\hline S\&M Worker & Core & East Gallery & Inhalation & Radionuclides & ${ }^{200} \mathrm{Th}$ & $1.2 E+\infty$ & $2.8 \mathrm{E}-04$ & \\
\hline S\&M Worker & Core & East Gallery & Inhalation & Radionuclides & ${ }^{20.0} \mathrm{Th}$ & 4.3E-01 & $1.0 \mathrm{E}-04$ & \\
\hline S\&M Worker & Core & East Gallery & Inhalation & Radionuclides & ${ }^{23825} \mathrm{U}$ & $9.0 \mathrm{E}-01$ & 2.1E-04 & \\
\hline S\&M Worker & Core & East Gallery & Inhalation & Radionuclides & ${ }^{240} \mathrm{U}$ & 7.7E-01 & $1.8 \mathrm{E}-04$ & \\
\hline S\&M Worker & Core & East Gallery & Inhalation & Radionuclides & ${ }^{211} \mathrm{Am}$ & $5.0 \mathrm{E}-01$ & $1.2 \mathrm{E}-04$ & \\
\hline S\&M Worker & Core & East Gallery & Inhalation & Radionuclides & ${ }^{\infty} \mathrm{K}$ & $5.4 \mathrm{E}+\infty$ & $1.3 \mathrm{E}-03$ & \\
\hline S\&M Worker & Core & East Gallery & Inhalation & Radionuclides & ${ }^{N} \mathrm{Sr}$ & $8.1 E+00$ & $1.9 \mathrm{E}-03$ & \\
\hline S\&M Worker & Core & Eant Gallery & Inhalation & Radionuclides & Tritium & $2.6 \mathrm{E}+01$ & $6.0 \mathrm{E}-03$ & \\
\hline S\&M Worker & Core & Eant Gallery & External Exposure & Radionuclides & ${ }^{15} \mathrm{C}_{2}$ & 2.3E+01 & 8.3E-01 & \\
\hline
\end{tabular}

- Units are $\mathrm{mg} / \mathrm{kg}$ for inorganics and organics; $\mathrm{pCi} / \mathrm{g}$ for radionuclides

- Units are $\mathrm{mg} / \mathrm{kg}$-day for inorganics and organici; pCi-day for radionuclides 


\begin{tabular}{|c|c|c|c|c|c|c|c|c|}
\hline \multirow{2}{*}{ Receptor } & \multirow{2}{*}{ Media } & \multirow{2}{*}{ Location } & \multirow{2}{*}{$\begin{array}{l}\text { Exposure } \\
\text { Pathway }\end{array}$} & \multirow{2}{*}{$\begin{array}{c}\text { Contaminant } \\
\text { Class }\end{array}$} & \multirow{2}{*}{ Contaminant } & \multirow[b]{2}{*}{$\begin{array}{l}\text { Exposure Point } \\
\text { Concentration" }\end{array}$} & \multicolumn{2}{|c|}{ Chronic Daily Intake" } \\
\hline & & & & & & & $\begin{array}{c}\text { Carcinogenic } \\
\text { Erfects }\end{array}$ & $\begin{array}{l}\text { Noncancinogenic } \\
\text { Effects }\end{array}$ \\
\hline S\&M Worker & Core & East Gallery & External Exposure & Radionuclide: & ${ }^{2 x} \mathbf{R a}$ & $3.6 \mathrm{E}-01$ & $1.3 E-02$ & \\
\hline S\&M Worker & Core & East Gallery & External Exposure & Radionuclides & ${ }^{20} R_{n}$ & $3.7 \mathrm{E}-01$ & $1.4 \mathrm{E}-02$ & \\
\hline S\&M Worker & Core & East Gallery & External Exposure & Radionuclides & n'Th & S.6E-01 & $2.0 \mathrm{E}-02$ & \\
\hline S\&M Worker & Core & East Gallery & External Exposure & Radionuclides & ${ }^{200} \mathrm{Th}$ & $1.2 \mathrm{E}+00$ & $4.4 \mathrm{E}-02$ & \\
\hline S\&M Worker & Core & East Gallery & Extemal Exposure & Radionuclides & $200 \mathrm{Th}$ & 4.3E-01 & $1.6 \mathrm{E}-02$ & \\
\hline S\&M Worker & Core & East Gallery & External Exposure & Radionuclides & ${ }^{23 \times 25} U$ & 9.0E-01 & 3.3E-02 & \\
\hline S\&M Worker & Core & East Gallery & External Exposure & Radionuclides & ${ }^{22 H} \mathrm{U}$ & 7.7E-01 & $2.8 \mathrm{E}-02$ & \\
\hline S\&M Worker & Core & East Gallery & Extemal Exposure & Radionuclides & ${ }^{2 N 1} \mathrm{Am}$ & $5.0 \mathrm{E}-01$ & $1.8 \mathrm{E}-02$ & \\
\hline S\&M Worker & Core & East Gallery & External Exposure & Radionuclides & ${ }^{+0} \mathrm{~K}$ & $5.4 \mathrm{E}+00$ & $2.0 \mathrm{E}-01$ & \\
\hline S\&M Worker & Core & East Gallery & External Exposure & Radionuclides & ${ }^{90} \mathrm{Sr}$ & $8.1 \mathrm{E}+00$ & $3.0 \mathrm{E}-01$ & \\
\hline S\&M Worker & Core & East Gallery & Extermal Exposure & Radionuclides & Tritium & $2.6 \mathrm{E}+01$ & $9.4 \mathrm{E}-01$ & \\
\hline S\&M Worker & Core & Sediment & Ingestion & Inorganics & Antimony & $3.6 \mathrm{E}+01$ & $5.1 \mathrm{E}-08$ & $1.4 \mathrm{E}-07$ \\
\hline S\&M Worker & Core & Sediment & ingestion & inorganics & Ârsenic & $2.2 \mathrm{E}+0 \mathrm{i}$ & $3.0 \mathrm{E}-06$ & 0.4E-00 \\
\hline S\&M Worker & Core & Sediment & Ingestion & Inorganics & Barium & $1.2 \mathrm{E}+02$ & $1.7 \mathrm{E}-07$ & $4.7 \mathrm{E}-07$ \\
\hline S\&M Worker & Core & Sediment & Ingestion & Inorganics & Cadmium & $6.0 \mathrm{E}+01$ & 8.4E-08 & 2.4E-07 \\
\hline S\&M Worker & Core & Sediment & Ingestion & Inorganics & Chromium VI & $1.9 \mathrm{E}+02$ & $2.6 \mathrm{E}-07$ & 7.4E-07 \\
\hline S\&M Worker & Core & Sediment & Ingestion & Inorganics & Cyanide & $5.8 \mathrm{E}-01$ & 8.1E-10 & 2.3E-09 \\
\hline S\&M Worker & Core & Sediment & Ingestion & Inorganics & Manganese & $1.3 E+03$ & $1.8 \mathrm{E}-06$ & 5.1E-0S \\
\hline S\&M Worker & Core & Sediment & Ingestion & Inorganics & Mercury & $1.9 \mathrm{E}+01$ & $2.6 \mathrm{E}-08$ & 7.3E-08 \\
\hline S\&M Worker & Core & Sediment & Ingestion & Inorganics & Nickel & $1.7 \mathrm{E}+02$ & 2.4E-07 & $6.6 \mathrm{E}-07$ \\
\hline S\&M Worker & Core & Sediment & Ingention & Inorganics & Selenium & 8.7E-01 & $1.2 \mathrm{E}-09$ & $3.4 \mathrm{E}-09$ \\
\hline
\end{tabular}

- Units are $\mathrm{mg} / \mathrm{kg}$ for inorganics and organics; $\mathrm{pCi} / \mathrm{g}$ for radionuclides

- Units are mg/kg-day for inorganics and organics; pCi-day for radionuclides 


\begin{tabular}{|c|c|c|c|c|c|c|c|c|}
\hline \multirow{2}{*}{ Receptor } & \multirow{2}{*}{ Media } & \multirow{2}{*}{ Location } & \multirow{2}{*}{$\begin{array}{l}\text { Exposure } \\
\text { Pathway }\end{array}$} & \multirow{2}{*}{$\begin{array}{l}\text { Contaminant } \\
\text { Cless }\end{array}$} & \multirow{2}{*}{ Contaminant } & \multirow[b]{2}{*}{$\begin{array}{l}\text { Exposure Point } \\
\text { Concentration" }\end{array}$} & \multicolumn{2}{|c|}{ Chronic Deily Intakeb } \\
\hline & & & & & & & $\begin{array}{c}\text { Carcinogenic } \\
\text { Efrects }\end{array}$ & $\begin{array}{c}\text { Noncarcinogenic } \\
\text { ENfocts }\end{array}$ \\
\hline S\&M Worker & Core & Sediment & Ingertion & Inorganics & Zine & $7.4 \mathrm{E}+03$ & $1.0 \mathrm{E}-05$ & $2.9 \mathrm{E}-05$ \\
\hline S\&M Worker & Core & Sediment & Ingestion & Organics & Acensaphthene & $9.6 \mathrm{E}-01$ & $1.3 \mathrm{E}-09$ & $3.8 \mathrm{E}-09$ \\
\hline S\&M Worker & Core & Sediment & Ingestion & Organics & Acetone & $9.0 \mathrm{E}-02$ & $\sim 1.3 \mathrm{E}-10$ & 3.5E-10 \\
\hline S\&M Worker & Core & Sediment & Ingertion & Organics & Anthracene & $1.9 \mathrm{E}+\infty 0$ & 2.7E-09 & $7.4 \mathrm{E}-09$ \\
\hline S\&M Worker & Core & Sediment & Ingeation & Organice & Aroclor-1254 & $4.1 E+02$ & 5.7E-07 & $1.6 \mathrm{E}-06$ \\
\hline S\&M Worker & Core & Sediment & Ingestion & Organics & Benzo(a)pyrene & $5.7 E+\infty$ & $8.0 \mathrm{E}-09$ & 2.2E-08 \\
\hline S\&M Worker & Core & Sediment & Ingestion & Organice & Benzo(k) fluoranthene & $4.6 \mathrm{E}+01$ & $6.4 \mathrm{E}-08$ & $1.8 \mathrm{E}-07$ \\
\hline S\&M Worker & Core & Sediment & Ingestion & Organics & Bis(2-ethylhexyl)phthalate & $2.1 E+\infty$ & 2.9E-09 & 8.2E-09 \\
\hline S\&M Worker & Core & Sediment & Ingestion & Organics & Carbazole & $2.0 E+\infty$ & $2.8 \mathrm{E}-09$ & $7.8 \mathrm{E}-09$ \\
\hline S\&M Worker & Core & Sediment & Ingestion & Organics & Chrysene & $1.9 \mathrm{E}+01$ & 2.7E-08 & 7.4E-08 \\
\hline S\&M Worker & Core & Sediment & Ingestion & Organics & Dibenz $(\mathbf{a}, \mathbf{h})$ anthracene & $1.5 \mathrm{E}+\infty 0$ & $2.1 \mathrm{E}-09$ & $5.9 \mathrm{E}-09$ \\
\hline S\&M Worker & Core & Sediment & Ingestion & Organics & Fluoranthene & $2.7 E+01$ & 3.8E-08 & 1.1E-07 \\
\hline S\&M Worker & Core & Sediment & Ingestion & Organics & Fluorene & $8.5 \mathrm{E}-01$ & $1.2 \mathrm{E}-09$ & 3.3E-09 \\
\hline S\&M Worker & Core & Sediment & Ingettion & Organics & Indeno(1,2,3-cd)pyrene & $9.6 E+\infty$ & $1.3 \mathrm{E}-08$ & $3.8 \mathrm{E}-08$ \\
\hline S\&M Worker & Core & Sediment & Ingeution & Organics & Pyrene & $2.2 E+01$ & 3.1E-08 & $8.6 \mathrm{E}-08$ \\
\hline S\&M Worker & Core & Sediment & Ingestion & Radionuclides & ${ }^{18} \mathrm{Ca}$ & $3.1 \mathrm{E}+04$ & $7.7 E+04$ & \\
\hline S\&M Worker & Core & Sediment & Ingeation & Radionuclides & ${ }^{20} \mathrm{Th}$ & $5.4 \mathrm{E}-01$ & $1.4 E+\infty 0$ & \\
\hline S\&M Worker & Core & Sediment & Ingestion & Radionuclides & ${ }^{29} \mathrm{Th}$ & $1.9 E+01$ & $4.7 \mathrm{E}+01$ & \\
\hline S\&M Worker & Core & Sediment & Ingestion & Radionuclides & ${ }^{200} \mathrm{Th}$ & 5.5E-01 & $1.4 \mathrm{E}+\infty$ & \\
\hline S\&M Worker & Core & Sediment & Ingertion & Radionuclides & ${ }^{23324} \mathrm{U}$ & $3.4 E+\infty$ & $8.6 \mathrm{E}+\infty$ & \\
\hline S\&M Worker & Core & Sediment & Ingertion & Radionuclides & ${ }^{25} \mathrm{Pu}$ & $1.2 E+\infty 0$ & $2.9 \mathrm{E}+\infty$ & \\
\hline
\end{tabular}

- Units are $\mathrm{mg} / \mathrm{kg}$ for inorganicu and onganics; $\mathrm{pCi} / \mathrm{g}$ for radionuclides

- Units are $\mathrm{mg} / \mathrm{kg}$-day for inorganice and organics; pCi-day for radionuclides 


\begin{tabular}{|c|c|c|c|c|c|c|c|c|}
\hline \multirow{2}{*}{ Receptor } & \multirow{2}{*}{ Media } & \multirow{2}{*}{ Location } & \multirow{2}{*}{$\begin{array}{l}\text { Exposure } \\
\text { Pathway }\end{array}$} & \multirow{2}{*}{$\begin{array}{c}\text { Contaminant } \\
\text { Class }\end{array}$} & \multirow{2}{*}{ Contaminant } & \multirow[b]{2}{*}{$\begin{array}{l}\text { Exposure Point } \\
\text { Concentration" }\end{array}$} & \multicolumn{2}{|c|}{ Chromic Daily Intake' } \\
\hline & & & & & & & $\begin{array}{c}\text { Carcinogenic } \\
\text { Erfects }\end{array}$ & $\begin{array}{c}\text { Noncarcinogenic } \\
\text { Erfects }\end{array}$ \\
\hline S\&M Worker & Core & Sediment & Ingertion & Radionuclide: & ${ }^{2 M U} \mathrm{U}$ & $2.2 E+\infty 0$ & $5.6 \mathrm{E}+\infty$ & \\
\hline S\&M Worker & Core & Sediment & Ingestion & Radionuclides & ${ }^{200200} \mathrm{Pu}$ & $3.5 E+01$ & $8.9 E+01$ & \\
\hline S\&M Worker & Core & Sediment & Ingertion & Radionuclides & ${ }^{\infty} \mathrm{K}$ & $9.4 \mathrm{E}+00$ & $2.4 \mathrm{E}+01$ & \\
\hline S\&M Worker & Core & Sediment & Ingeation & Radionuclides & ${ }^{\infty} \mathrm{Co}$ & $3.4 E+00$ & $8.6 E+00$ & \\
\hline S\&M Worker & Core & Sediment & Ingertion & Radionuclides & ${ }^{\infty} \mathrm{Sr}$ & $9.8 \mathrm{E}+01$ & $2.4 \mathrm{E}+02$ & \\
\hline S\&M Worker & Core & Sediment & Ingestion & Radionuclides & Tritium & $1.0 \mathrm{E}+\infty$ & $2.5 \mathrm{E}+00$ & \\
\hline S\&M Worker & Core & Sediment & Dermal & Inorganics & Antimony & $3.6 \mathrm{E}+01$ & $3.2 \mathrm{E}-09$ & $8.8 \mathrm{E}-09$ \\
\hline S\&M Worker & Core & Sediment & Dermal & Inorganics & Arsenic & $2.2 E+01$ & $1.9 \mathrm{E}-09$ & 5.3E-09 \\
\hline S\&M Worker & Core & Sediment & Dermal & Inorganics & Barium & $1.2 \mathrm{E}+02$ & $1.0 \mathrm{E}-08$ & 2.9E-08 \\
\hline S\&M Worker & Core & Sediment & Dermal & Inorganics & Cadmium & $6.0 E+01$ & $5.2 \mathrm{E}-09$ & $1.5 \mathrm{E}-08$ \\
\hline S\&M Worker & Core & Sediment & Dermal & Inorganics & Chromium VI & $1.9 \mathrm{E}+02$ & $1.6 \mathrm{E}-08$ & $4.6 \mathrm{E}-08$ \\
\hline S\&M Worker & Core & Sediment & Dermal & Inorganics & Cyanide & $5.8 \mathrm{E}-01$ & $5.1 \mathrm{E}-11$ & $1.4 \mathrm{E}-10$ \\
\hline SQM Woiker & Cote & Sediment & Derma! & Inorganice & Manganese & $1.3 E \div 03$ & 1.1E-07 & 3.2E- 07 \\
\hline S\&M Worker & Core & Sediment & Dermal & Inorganics & Mercury & $1.9 \mathrm{E}+01$ & $1.6 \mathrm{E}-09$ & $4.6 \mathrm{E}-09$ \\
\hline S\&M Worker & Core & Sediment & Dermal & Inorganics & Nickel & $1.7 \mathrm{E}+02$ & $1.5 \mathrm{E}-08$ & 4.1E-08 \\
\hline S\&M Worker & Core & Sediment & Dermal & Inorganics & Selenium & 8.7E-01 & $7.6 \mathrm{E}-11$ & $2.1 \mathrm{E}-10$ \\
\hline S\&M Worker & Core & Sediment & Dermal & Inorganics & Zinc & $7.4 E+03$ & 6.5E-07 & $1.8 \mathrm{E}-06$ \\
\hline S\&M Worker & Core & Sediment & Dermal & Organics & Acenaphthene & $9.6 \mathrm{E}-01$ & $8.4 \mathrm{E}-10$ & $2.3 E-09$ \\
\hline S\&M Worker & Core & Sediment & Dermal & Organics & Acetone & $9.0 \mathrm{E}-02$ & $7.9 \mathrm{E}-11$ & $2.2 \mathrm{E}-10$ \\
\hline S\&M Worker & Core & Sediment & Dermal & Organics & Anthracene & $1.9 \mathrm{E}+00$ & $1.7 \mathrm{E}-09$ & $4.6 \mathrm{E}-09$ \\
\hline S\&M Worker & Core & Sediment & Dermal & Organics & Aroclor-1254 & $4.1 \mathrm{E}+02$ & 3.6E-07 & $1.0 \mathrm{E}-06$ \\
\hline
\end{tabular}

- Units are $\mathrm{mg} / \mathrm{kg}$ for inorganics and organics; $\mathrm{pCi} / \mathrm{g}$ for radionuclides

- Units are $\mathrm{mg} / \mathrm{kg}$-day for inorganics and organics; $\mathrm{PCi}$-day for radionuclides 


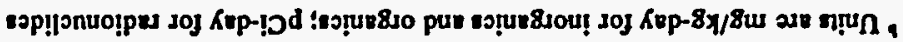
esp!| กnuotpes soj 8/!

\begin{tabular}{|c|c|c|c|c|c|c|c|c|}
\hline $60-3 L \cdot 2$ & $0 \mathrm{I} \cdot \mathrm{AL} \cdot 6$ & $\varepsilon 0+3 b^{\circ} L$ & su!z & sต̣นu\&sou & 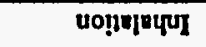 & zuou! & 2505 & JOYNOM WPS \\
\hline$\varepsilon I-\exists \tau^{\circ} \varepsilon$ & Eโ-มI' & $10-9<8$ & wnก̣uगps & sọ̣u8suour & 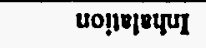 & Iuวu!pəS & 2ros & JoY10M WPS \\
\hline II-GZ'9 & II-gz' & $20+3<\cdot 1$ & IDXIN & sp̣uestoul & 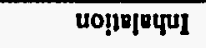 & IUวแ! & 2100 & JoY10M WPS \\
\hline $2\left[-38^{\prime} 9\right.$ & $\tau I-9 b^{\circ} \tau$ & $10+\exists 6^{\prime} 1$ & SInנSOW & 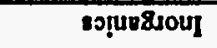 & 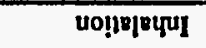 & นนวแ!p?S & 2105 & J0X10M WPS \\
\hline 0I-98' & $01-3 L \cdot 1$ & $\mathfrak{\varepsilon 0 + \exists \varepsilon ^ { \prime } !}$ & ocour8urw & sộ881ou & uo!̣p|quा & ในจน!pos & 210J & IOX10M WPSS \\
\hline$\varepsilon I-g I \cdot \tau$ & †I-39: L & $10-98^{\circ} \mathrm{S}$ & วp!̣uRKo & 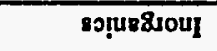 & นo!̣p|xนuा & 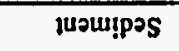 & 2100 & 30X10M WrSS \\
\hline $11-9699$ & II-GS' 2 & $20+36.1$ & In un!usตร & sง!ur81oul & 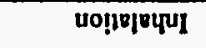 & IUจน!pวS & 2100 & I0X10M WroS \\
\hline II-az' & $21-98 . L$ & $10+30^{\circ} 9$ & un!̣upro & sọue8soul & uo!!ᄆㅁำ & jusu!pos & 2100 & IOX10M WPSS \\
\hline $11-a b^{\circ}$ & II-99. I & $20+3 \tau \cdot 1$ & unupg & sọ!ur8souI & 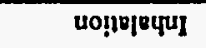 & Juจนu!pos & 2100 & JoxioM WPSS \\
\hline $21-96.2$ & ZI-38. & $10+3 \tau \cdot \tau$ & ग़िวมY & sọur\&ouI & uo!|미ำu| & ןนรแ!pวs & 2100 & JOX10M WPSS \\
\hline II-GE'I & $2 I-g L \cdot b$ & $10+39 \cdot \varepsilon$ & Kuow!uYY & cọue8souI & 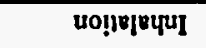 & נนэน!pPS & 2100 & JPX10M W8S \\
\hline $80-3 t^{\circ} S$ & $80-36^{\circ} I$ & $10+92 \cdot 2$ & ouss $K_{d}$ & รง!นเ810 & joursa & נนэแ!pगS & 2100 & JPX10M WPSS \\
\hline 80-3ह' & $60-3 b^{\prime} 8$ & $00+39 \cdot 6$ & 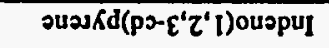 & sọue8so & poused & นuวu!!pos & 2200 & JכX10M WrSS \\
\hline $60-31 \cdot 2$ & $0 t-3+L$ & $10-35^{\circ} 8$ & จuxionly & sọun8so & pruwg & jusulpos & 2500 & JoYIOM WrS \\
\hline $80-39.9$ & $80-3 b^{\prime} Z$ & $10+\exists L \cdot \tau$ & PUP4) & 8ง̣ue8so & ןвшга & Iuวu!pəs & 2roग & JOX10M WPS \\
\hline $60-\exists L \cdot \varepsilon$ & 60-3E'I & $00+35 \cdot 1$ & 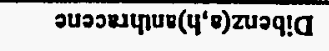 & 80140810 & fouked & jusu!pos & 2105 & JPXJOM WPSS \\
\hline $80-39^{\circ} 6$ & $80-3 L^{\circ} I$ & $10+36.1$ & 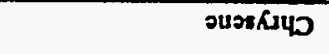 & sง̣นะ8\&O & Iruisa & jusu!pəs & 2100 & JOXIOM WRS \\
\hline $60-36.6$ & $60-3<\div 1$ & $00+30^{\circ} \tau$ & गิ0z"qus & 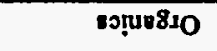 & Ionura & IUวu!pos & 2100 & JOXJOM WPS \\
\hline $60-31 \% 5$ & $60-38^{\circ} 1^{12}$ & $00+31 \cdot 2$ & 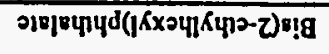 & รง̣บ48ะO & fourex & jusu!pos & 2000 & IPYIIOM NPS \\
\hline$\angle O A I \cdot T$ & $80-90 \%$ & $10+39^{\circ} 6$ & susquureng(y)ozrog & sọ̣u\&80 & 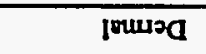 & jusw!pos & 2100 & मOXY10M WPS \\
\hline $80-36^{\circ} I$ & $60-90^{\circ} 5$ & $00+3 L \cdot S$ & oussאd(v)ozupg & sọ़นะిO & Ioumpa & กันu!p?S & 2105 & JOXIOM WPS \\
\hline 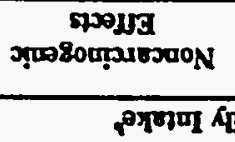 & 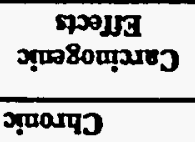 & 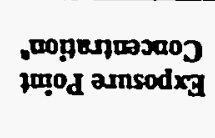 & 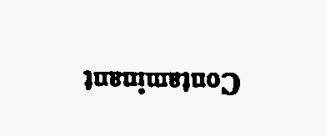 & 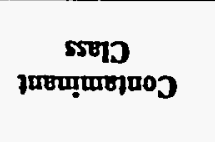 & $\begin{array}{l}\text { REmpled } \\
\text { aunsodxg }\end{array}$ & topg8007 & ๒трэพ & soxdaxoy \\
\hline
\end{tabular}




\begin{tabular}{|c|c|c|c|c|c|c|c|c|}
\hline \multirow{2}{*}{ Receptor } & \multirow{2}{*}{ Media } & \multirow{2}{*}{ Location } & \multirow{2}{*}{$\begin{array}{l}\text { Exposure } \\
\text { Pathway }\end{array}$} & \multirow{2}{*}{$\begin{array}{l}\text { Contaminant } \\
\text { Class }\end{array}$} & \multirow{2}{*}{ Contaminant } & \multirow{2}{*}{$\begin{array}{l}\text { Exposure Point } \\
\text { Concentration" }\end{array}$} & \multicolumn{2}{|c|}{ Chronic Daily Intake" } \\
\hline & & & & & & & $\begin{array}{c}\text { Carcinogenic } \\
\text { Erfects }\end{array}$ & $\begin{array}{c}\text { Noncarcinogenic } \\
\text { Efrects }\end{array}$ \\
\hline S\&M Worker & Core & Sediment & Inhalation & Onganic: & Acenaphthene & 9.6E-01 & $1.3 \mathrm{E}-13$ & $3.5 \mathrm{E}-13$ \\
\hline S\&M Worker & Core & Sediment & Inhalation & Organic: & Acetone & $9.0 \mathrm{E}-02$ & $1.2 \mathrm{E}-14$ & 3.3E-14 \\
\hline S\&M Worker & Core & Sediment & Inhalation & Organice & Anthracene & $1.9 \mathrm{E}+00$ & $2.5 \mathrm{E}-13$ & $6.9 \mathrm{E}-13$ \\
\hline S\&M Worker & Core & Sediment & Inhalation & Organic: & Aroclor-1254 & $4.1 \mathrm{E}+02$ & $5.4 \mathrm{E}-11$ & $1.5 \mathrm{E}-10$ \\
\hline S\&M Worker & Core & Sediment & Inhalation & Organics & Benzo(a)pyrene & $5.7 E+00$ & $7.4 \mathrm{E}-13$ & $2.1 \mathrm{E}-12$ \\
\hline S\&M Worker & Core & Sediment & Inhalation & Organics & Benzo(k)fluoranthene & $4.6 \mathrm{E}+01$ & $6.0 \mathrm{E}-12$ & $1.7 \mathrm{E}-11$ \\
\hline S\&M Worker & Core & Sediment & Inhalation & Organics & Bis(2-ethylhexyl)phthalate & $2.1 \mathrm{E}+00$ & $2.7 \mathrm{E}-13$ & 7.7E-13 \\
\hline S\&M Worker & Core & Sediment & Inhalation & Organica & Carbazole & $2.0 \mathrm{E}+00$ & $2.6 \mathrm{E}-13$ & $7.3 \mathrm{E}-13$ \\
\hline S\&M Worker & Core & Sediment & Inhalation & Organica & Chrysene & $1.9 \mathrm{E}+01$ & $2.5 \mathrm{E}-12$ & $6.9 \mathrm{E}-12$ \\
\hline S\&M Worker & Core & Sediment & Inhalation & Organics & Dibenz(a,h)anthracene & $1.5 E+00$ & $2.0 \mathrm{E}-13$ & $5.5 \mathrm{E}-13$ \\
\hline S\&M Worker & Core & Sediment & Inhalation & Organics & Fluoranthene & $2.7 E+01$ & $3.5 \mathrm{E}-12$ & $9.9 \mathrm{E}-12$ \\
\hline S\&M Worker & Core & Sediment & Inhalation & Organics & Fluorene & $8.5 \mathrm{E}-01$ & $1.1 \mathrm{E}-13$ & $3.1 \mathrm{E}-13$ \\
\hline Sầìi Worker & Core & Sédiment & Intatation & Oiganics & Indeno(1,2,3-ed)pyrene & $9.6 E+00$ & $1.3 \mathrm{E}-12$ & $3.5 \mathrm{E}-12$ \\
\hline S\&M Worker & Core & Sediment & Inhalation & Organice & Pyrene & $2.2 E+01$ & $2.9 \mathrm{E}-12$ & $8.0 \mathrm{E}-12$ \\
\hline S\&M Worker & Core & Sediment & Inhalation & Radionuclides & ${ }^{157} \mathrm{Cs}$ & $3.1 \mathrm{E}+04$ & $7.2 \mathrm{E}+\infty 0$ & \\
\hline S\&M Worker & Core & Sediment & Inhalation & Radionuclides & noTh & $5.4 E-01$ & $1.3 \mathrm{E}-04$ & \\
\hline S\&M Worker & Core & Sediment & Inhalation & Radionuclides & ${ }^{20} \mathrm{Th}$ & $1.9 \mathrm{E}+01$ & 4.4E- 03 & \\
\hline S\&M Worker & Core & Sediment & Inhalation & Radionuclides & ${ }^{25} \mathrm{Th}$ & 5.5E-01 & $1.3 \mathrm{E}-04$ & \\
\hline S\&M Worker & Core & Sediment & Inhalation & Radionuclides & $23234 \mathrm{U}$ & $3.4 E+00$ & 8.0E-04 & \\
\hline S\&M Worker & Core & Sediment & Inhalation & Radionuclides & ${ }^{2 x} \mathrm{Pu}$ & $1.2 \mathrm{E}+00$ & 2.7E-04 & \\
\hline S\&M Worker & Core & Sediment & Inhalation & Radionuclide: & ${ }^{200} \mathrm{U}$ & $2.2 \mathrm{E}+00$ & $5.2 \mathrm{E}-04$ & \\
\hline
\end{tabular}

- Units are $\mathrm{mg} / \mathrm{kg}$ for inorganice and organics; $\mathrm{pCi} / \mathrm{g}$ for radionuclides

- Units are $\mathrm{mg} / \mathrm{kg}$-day for inorganics and organica; PCi-day for radionuclides 


\begin{tabular}{|c|c|c|c|c|c|c|c|c|}
\hline \multirow{2}{*}{ Receptor } & \multirow{2}{*}{ Media } & \multirow{2}{*}{ Location } & \multirow{2}{*}{$\begin{array}{l}\text { Exposure } \\
\text { Pathway }\end{array}$} & \multirow{2}{*}{$\begin{array}{c}\text { Contaminant } \\
\text { Class }\end{array}$} & \multirow{2}{*}{ Contaminant } & \multirow[b]{2}{*}{$\begin{array}{l}\text { Exposure Point } \\
\text { Concentration" }\end{array}$} & \multicolumn{2}{|c|}{ Chronic Daily Intake } \\
\hline & & & & & & & $\begin{array}{c}\text { Carcinogenic } \\
\text { Ertects }\end{array}$ & $\begin{array}{c}\text { Noncarcinogenic } \\
\text { Effects }\end{array}$ \\
\hline S\&M Worker & Core & Sediment & Inhalation & Radionuclides & ${ }^{203200} \mathrm{Pu}$ & $3.5 E+01$ & 8.3E-03 & \\
\hline S\&M Worker & Core & Sediment & Inhalation & Radionuclides & ${ }^{\infty} \mathrm{K}$ & $9.4 E+\infty 0$ & $2.2 E-03$ & \\
\hline S\&M Worker & Core & Sediment & Inhalation & Radionuclides & ${ }^{\infty} \mathrm{Co}$ & $3.4 E+\infty$ & ' 8.0E-04 & \\
\hline S\&M Worker & Core & Sediment & Inhalation & Radionuclides & ${ }^{\infty} S_{r}$ & $9.8 E+01$ & $2.3 E-02$ & \\
\hline S\&M Worker & Cone & Sediment & Inhalation & Radionuclides & Tritium & $1.0 \mathrm{E}+00$ & 2.3E-04 & \\
\hline S\&M Worker & Core & Sediment & External Exposure & Radionuclides & ${ }^{n} \mathrm{Cs}$ & $3.1 \mathrm{E}+04$ & $1.1 \mathrm{E}+03$ & \\
\hline S\&M Worker & Core & Sediment & External Exposure & Radionuclides & ${ }^{20}$ Th & $5.4 \mathrm{E}-01$ & $2.0 \mathrm{E}-02$ & \\
\hline S\&M Worker & Core & Sediment & External Exposure & Radionuclides & ${ }^{20} \mathrm{Th}$ & $1.9 \mathrm{E}+01$ & $6.8 \mathrm{E}-01$ & \\
\hline S\&M Worker & Core & Sediment & External Exposure & Radionuclides & ${ }^{2 n} \mathrm{Th}$ & $5.5 \mathrm{E}-01$ & $2.0 \mathrm{E}-02$ & \\
\hline S\&M Worker & Core & Sediment & External Exposure & Radionuclides & ${ }^{203254} \mathrm{U}$ & $3.4 \mathrm{E}+\infty 0$ & $1.3 \mathrm{E}-01$ & \\
\hline S\&M Worker & Core & Sediment & Extemal Exposure & Radionuclides & ${ }^{2 x} \mathrm{Pu}$ & $1.2 \mathrm{E}+00$ & $4.2 \mathrm{E}-02$ & \\
\hline S\&M Worker & Core & Sediment & External Exposure & Radionuclides & ${ }^{228} \mathrm{U}$ & $2.2 \mathrm{E}+00$ & $8.2 \mathrm{E}-02$ & \\
\hline S\&M Worker & Core & Sediment & External Exposure & Radionuclides & ${ }^{2982000} \mathrm{Pu}$ & $3.5 E+01$ & $1.3 \mathrm{E}+\infty$ & \\
\hline S\&M Worker & Core & Sediment & External Exposure & Radionuclides & ${ }^{10} \mathrm{~K}$ & $9.4 E+\infty 0$ & $3.4 E-01$ & \\
\hline S\&M Worker & Core & Sediment & External Exposure & Radionuclides & ${ }^{\infty} \mathrm{Co}$ & $3.4 \mathrm{E}+\infty 0$ & $1.3 \mathrm{E}-01$ & \\
\hline S\&M Worker & Core & Sediment & Extemal Exposure & Radionuclides & ${ }^{\infty} \mathrm{Sr}$ & $9.8 \mathrm{E}+01$ & $3.6 \mathrm{E}+00$ & \\
\hline S\&M Worker & Core & Sediment & External Exposure & Radionuclides & Tritium & $1.0 \mathrm{E}+00$ & 3.7E-02 & \\
\hline S\&M Worker & Core & $\begin{array}{l}\text { Whole } \\
\text { Building }\end{array}$ & Ingestion & Inorganics & Arsenic & $2.9 \mathrm{E}+00$ & $4.0 \mathrm{E}-09$ & $1.1 \mathrm{E}-08$ \\
\hline S\&M Worker & Core & $\begin{array}{l}\text { Whole } \\
\text { Building }\end{array}$ & Ingextion & Inorganica & Barium & $7.2 \mathrm{E}+02$ & $1.0 \mathrm{E}-06$ & $2.8 \mathrm{E}-06$ \\
\hline
\end{tabular}

- Units are $\mathrm{mg} / \mathrm{kg}$ for inorganics and organics; $\mathrm{pCi} / \mathrm{g}$ for radionuclides

- Units are $\mathrm{mg} / \mathrm{kg}$-day for inorganics and organics; $\mathrm{pCi}$-day for radionuclides 


\begin{tabular}{|c|c|c|c|c|c|c|c|c|}
\hline \multirow{2}{*}{ Receptor } & \multirow{2}{*}{ Media } & \multirow{2}{*}{ Location } & \multirow{2}{*}{$\begin{array}{l}\text { Exposure } \\
\text { Pathway }\end{array}$} & \multirow{2}{*}{$\begin{array}{l}\text { Contaminant } \\
\text { Class }\end{array}$} & \multirow{2}{*}{ Contaminant } & \multirow[b]{2}{*}{$\begin{array}{l}\text { Exposure Point } \\
\text { Concentration" }\end{array}$} & \multicolumn{2}{|c|}{ Chronic Daily Intakeh } \\
\hline & & & & & & & $\begin{array}{l}\text { Carcinogenic } \\
\text { Erfects }\end{array}$ & $\begin{array}{l}\text { Noncarcinogenic } \\
\text { Effects }\end{array}$ \\
\hline S\&M Worker & Core & $\begin{array}{l}\text { Whole } \\
\text { Building }\end{array}$ & Ingestion & Inorganics & Beryllium & $1.2 \mathrm{E}+01$ & 1.7E-08 & $4.8 \mathrm{E}-08$ \\
\hline S\&M Worker & Core & $\begin{array}{l}\text { Whole } \\
\text { Building }\end{array}$ & Ingestion & Inorganics & Cadmium & $1.8 E+02$ & $2.5 \mathrm{E}-07$ & $7.0 \mathrm{E}-07$ \\
\hline S\&M Worker & Core & $\begin{array}{l}\text { Whole } \\
\text { Building }\end{array}$ & Ingestion & Inorganics & Chromium VI & $2.0 \mathrm{E}+02$ & 2.7E-07 & 7.7E-07 \\
\hline S\&M Worker & Core & $\begin{array}{l}\text { Whole } \\
\text { Building }\end{array}$ & Ingestion & Inorganics & Manganese & $9.5 E+03$ & $1.3 \mathrm{E}-05$ & 3.7E-05 \\
\hline S\&M Worker & Core & $\begin{array}{l}\text { Whole } \\
\text { Building }\end{array}$ & Ingestion & Inorganics & Mercury & 3.2E-01 & 4.4E-10 & $1.2 \mathrm{E}-09$ \\
\hline S\&M Worker & Core & $\begin{array}{l}\text { Whole } \\
\text { Building }\end{array}$ & Ingestion & Inorganics & Nickel & $1.4 E+02$ & $1.9 \mathrm{E}-07$ & $5.4 E-07$ \\
\hline S\&M Worker & Core & $\begin{array}{l}\text { Whole } \\
\text { Building }\end{array}$ & Ingestion & Inorganics & Vanadium & $2.4 E+02$ & 3.4E-07 & 9.4E-07 \\
\hline S\&M Worker & Core & $\begin{array}{l}\text { Whole } \\
\text { Building }\end{array}$ & Ingestion & Inorganics & Zinc & $3.5 E+04$ & 4.9E-05 & $1.4 \mathrm{E}-0.4$ \\
\hline S\&M Worker & Core & $\begin{array}{l}\text { Whole } \\
\text { Building }\end{array}$ & Ingestion & Organics & Aroclor-1254 & $1.5 \mathrm{E}+\infty$ & $2.0 \mathrm{E}-09$ & $5.7 \mathrm{E}-09$ \\
\hline S\&M Worker & Core & $\begin{array}{l}\text { Whole } \\
\text { Building }\end{array}$ & Ingestion & Organics & Aroclor-1260 & $2.6 \mathrm{E}+00$ & $3.6 \mathrm{E}-09$ & $1.0 \mathrm{E}-08$ \\
\hline S\&M Worker & Core & $\begin{array}{l}\text { Whole } \\
\text { Building }\end{array}$ & Ingestion & Organics & Bis(2-ethylhexyl)phthalate & $2.4 \mathrm{E}-01$ & 3.4E-10 & $9.4 \mathrm{E}-10$ \\
\hline S\&M Worker & Core & $\begin{array}{l}\text { Whole } \\
\text { Building }\end{array}$ & Ingestion & Organics & Dimethyl phthalate & S.5E-02 & 7.7E-11 & 2.2E-10 \\
\hline S\&M Worker & Core & $\begin{array}{l}\text { Whole } \\
\text { Building }\end{array}$ & Ingestion & Radionuclides & ${ }^{19} \mathrm{Cr}$ & $1.4 E+03$ & $3.4 \mathrm{E}+03$ & \\
\hline
\end{tabular}

- Units are $\mathrm{mg} / \mathrm{kg}$ for inorganics and organics; $\mathrm{pCi} / \mathrm{g}$ for radionuclides

- Units are mg/kg-day for inorganics and organics; pCi-day for radionuclides 


\begin{tabular}{|c|c|c|c|c|c|c|c|c|}
\hline \multirow{2}{*}{ Receptor } & \multirow{2}{*}{ Medin } & \multirow{2}{*}{ Location } & \multirow{2}{*}{$\begin{array}{l}\text { Exposure } \\
\text { Pathway }\end{array}$} & \multirow{2}{*}{$\begin{array}{c}\text { Contaminant } \\
\text { Cless }\end{array}$} & \multirow{2}{*}{ Conteminant } & \multirow{2}{*}{$\begin{array}{l}\text { Exposure Point } \\
\text { Concentration" }\end{array}$} & \multicolumn{2}{|c|}{ Chronic Daily Intoke } \\
\hline & & & & & & & $\begin{array}{l}\text { Cancinogenic } \\
\text { Effocts }\end{array}$ & $\begin{array}{c}\text { Noncarcinogenic } \\
\text { Effects }\end{array}$ \\
\hline S\&M Worker & Core & $\begin{array}{l}\text { Whole } \\
\text { Building }\end{array}$ & Ingertion & Radionuclides & ${ }^{20} \mathrm{Ra}$ & $4.0 \mathrm{E}-01$ & $1.0 \mathrm{E}+\infty$ & \\
\hline S\&M Worker & Core & $\begin{array}{l}\text { Whole } \\
\text { Building }\end{array}$ & Ingestion & Radionuclide: & ${ }^{20} \mathrm{Ra}$ & 3.7E-01 & 9.3E-01 & \\
\hline S\&M Worker & Core & $\begin{array}{l}\text { Whole } \\
\text { Building }\end{array}$ & Ingestion & Radionuclides & ${ }^{20} \mathrm{Th}$ & 7.7E-01 & $1.9 E+\infty$ & \\
\hline S\&M Worker & Core & $\begin{array}{l}\text { Whole } \\
\text { Building }\end{array}$ & Ingestion & Radionuclides & ${ }^{200} \mathrm{Th}$ & $1.4 \mathrm{E}+\infty 0$ & $3.5 E+00$ & \\
\hline S\&M Worker & Core & $\begin{array}{l}\text { Whole } \\
\text { Building }\end{array}$ & Ingestion & Radionuclides & ${ }^{200} \mathrm{Th}$ & $8.0 \mathrm{E}-01$ & $2.0 \mathrm{E}+00$ & \\
\hline S\&M Worker & Core & $\begin{array}{l}\text { Whole } \\
\text { Building }\end{array}$ & Ingestion & Radionuclides & ${ }^{23525} U$ & $1.3 E+\infty 0$ & $3.2 \mathrm{E}+00$ & \\
\hline S\&M Worker & Core & $\begin{array}{l}\text { Whole } \\
\text { Building }\end{array}$ & Ingestion & Radionuclides & ${ }^{2 s+} \mathrm{Th}$ & $6.3 E+\infty 0$ & $1.6 \mathrm{E}+01$ & \\
\hline S\&M Worker & Core & $\begin{array}{l}\text { Whole } \\
\text { Building }\end{array}$ & Ingestion & Radionuclides & 2su & $1.2 \mathrm{E}-01$ & $3.0 \mathrm{E}-01$ & \\
\hline S\&M Worker & Core & $\begin{array}{l}\text { Whole } \\
\text { Building }\end{array}$ & Ingestion & Radionuclides & ${ }^{25} \mathrm{Pu}$ & 4.5E-01 & $1.1 E+\infty$ & \\
\hline S\&M Worker & Core & $\begin{array}{l}\text { Whole } \\
\text { Building }\end{array}$ & Ingestion & Radionuclides & ${ }^{2 \mathrm{NO} U}$ & $1.4 E+00$ & $3.6 \mathrm{E}+\infty$ & \\
\hline S\&M Worker & Core & $\begin{array}{l}\text { Whole } \\
\text { Building }\end{array}$ & Ingestion & Radionuclides & ${ }^{2098200} \mathrm{Pu}$ & $4.0 \mathrm{E}+\infty 0$ & $1.0 E+01$ & \\
\hline S\&M Worker & Core & $\begin{array}{l}\text { Whole } \\
\text { Building }\end{array}$ & Ingestion & Radionuclides & ${ }^{211} \mathrm{Am}$ & $5.0 \mathrm{E}-01$ & $1.3 E+\infty$ & \\
\hline S\&M Worker & Core & $\begin{array}{l}\text { Whole } \\
\text { Building }\end{array}$ & Ingertion & Radionuclides & ${ }^{*} \mathrm{~K}$ & $5.4 E+\infty 0$ & $1.3 E+01$ & \\
\hline
\end{tabular}

- Units are mg/kg for inorganics and organics; $\mathrm{pCi} / \mathrm{g}$ for radioniuclides

- Units are mg/kg-day for inorganics and organics; $\mathrm{pCi}$-day for radionuclides 


\begin{tabular}{|c|c|c|c|c|c|c|c|c|}
\hline \multirow{2}{*}{ Receptor } & \multirow{2}{*}{ Media } & \multirow{2}{*}{ Location } & \multirow{2}{*}{$\begin{array}{l}\text { Exposure } \\
\text { Palhway }\end{array}$} & \multirow{2}{*}{$\begin{array}{c}\text { Contaminant } \\
\text { Class }\end{array}$} & \multirow{2}{*}{ Contaminant } & \multirow{2}{*}{$\begin{array}{l}\text { Exposure Point } \\
\text { Concentration" }\end{array}$} & \multicolumn{2}{|c|}{ Chronic Daily Intake } \\
\hline & & & & & & & $\begin{array}{l}\text { Carcinogenic } \\
\text { Erfects }\end{array}$ & $\begin{array}{l}\text { Noncarcinogenic } \\
\text { Effects }\end{array}$ \\
\hline S\&M Worker & Core & $\begin{array}{l}\text { Whole } \\
\text { Building }\end{array}$ & Ingestion & Radionuclides & ${ }^{\infty} \mathrm{Co}$ & $1.3 \mathrm{E}-01$ & $3.3 \mathrm{E}-01$ & \\
\hline S\&M Worker & Core & $\begin{array}{l}\text { Whole } \\
\text { Building }\end{array}$ & Ingestion & Radionuclides & ${ }^{\infty} \mathrm{Sr}$ & $1.2 \mathrm{E}+01$ & $2.9 E+01$ & \\
\hline S\&M Worker & Core & $\begin{array}{l}\text { Whole } \\
\text { Building }\end{array}$ & Ingestion & Radionuclides & Tritium & $2.6 \mathrm{E}+01$ & $6.4 E+01$ & \\
\hline S\&M Worker & Core & $\begin{array}{l}\text { Whole } \\
\text { Building }\end{array}$ & Dermol & Inorganics & Arsenic & $2.9 E+00$ & $2.5 \mathrm{E}-10$ & $7.0 \mathrm{E}-10$ \\
\hline S\&M Worker & Core & $\begin{array}{l}\text { Whole } \\
\text { Building }\end{array}$ & Dermal & Inorganics & Barium & $7.2 E+02$ & $6.3 \mathrm{E}-08$ & $1.8 \mathrm{E}-07$ \\
\hline S\&M Worker & Core & $\begin{array}{l}\text { Whole } \\
\text { Building }\end{array}$ & Dermal & Inorganics & Beryllium & $1.2 \mathrm{E}+01$ & $1.1 \mathrm{E}-09$ & $3.0 \mathrm{E}-09$ \\
\hline S\&M Worker & Core & $\begin{array}{l}\text { Whole } \\
\text { Building }\end{array}$ & Dermal & Inorganics & Cadmium & $1.8 E+02$ & $1.6 \mathrm{E}-08$ & 4.4E-08 \\
\hline S\&M Worker & Core & $\begin{array}{l}\text { Whole } \\
\text { Building }\end{array}$ & Dermal & Inorganics & Chromium VI & $2.0 \mathrm{E}+02$ & 1.7E-08 & $4.8 \mathrm{E}-08$ \\
\hline S\&M Worker & Core & $\begin{array}{l}\text { Whole } \\
\text { Building }\end{array}$ & Dermal & Inorganics & Manganese & $9.5 \mathrm{E}+03$ & 8.3E-07 & $2.3 \mathrm{E}-06$ \\
\hline S\&M Worker & Core & $\begin{array}{l}\text { Whole } \\
\text { Building }\end{array}$ & Dermal & Inorganics & Mereury & $3.2 \mathrm{E}-01$ & $2.8 \mathrm{E}-11$ & $7.8 \mathrm{E}-11$ \\
\hline S\&M Worker & Core & $\begin{array}{l}\text { Whole } \\
\text { Building }\end{array}$ & Dermal & Inorganics & Nickel & $1.4 E+02$ & $1.2 \mathrm{E}-08$ & $3.4 \mathrm{E}-08$ \\
\hline S\&M Worker & Core & $\begin{array}{l}\text { Whole } \\
\text { Building }\end{array}$ & Dermal & Inorganics & Vanadium & $2.4 E+02$ & 2.1E-08 & $5.9 \mathrm{E}-08$ \\
\hline S\&M Worker & Core & $\begin{array}{l}\text { Whole } \\
\text { Building }\end{array}$ & Dermal & Inorganics & Zinc & $3.5 E+04$ & $3.0 \mathrm{E}-06$ & 8.5E-06 \\
\hline
\end{tabular}

- Unile are $\mathrm{mg} / \mathrm{kg}$ for inorganics and organics; $\mathrm{pCi} / \mathrm{g}$ for radionuclides

- Units are $\mathrm{mg} / \mathrm{kg}$-day for inorganics and organics; pCi-day for radionuclides 


\begin{tabular}{|c|c|c|c|c|c|c|c|c|}
\hline \multirow{2}{*}{ Receptor } & \multirow{2}{*}{ Media } & \multirow{2}{*}{ Location } & \multirow{2}{*}{$\begin{array}{l}\text { Exposure } \\
\text { Pathway }\end{array}$} & \multirow{2}{*}{$\begin{array}{c}\text { Contaminant } \\
\text { Class }\end{array}$} & \multirow{2}{*}{ Contamingnt } & \multirow[b]{2}{*}{$\begin{array}{l}\text { Exposure Point } \\
\text { Concentration" }\end{array}$} & \multicolumn{2}{|c|}{ Chronic Daily Intake } \\
\hline & & & & & & & $\begin{array}{c}\text { Carcinogenic } \\
\text { Effects }\end{array}$ & $\begin{array}{c}\text { Noncarcinogenic } \\
\text { Entects }\end{array}$ \\
\hline S\&M Worker & Core & $\begin{array}{l}\text { Whole } \\
\text { Building }\end{array}$ & Dermal & Organics & Aroclor-1254 & $1.5 E+\infty 0$ & $1.3 E-09$ & 3.5E-09 \\
\hline S\&M Worker & Core & $\begin{array}{l}\text { Whole } \\
\text { Building }\end{array}$ & Dermal & Organics & Aroclor- 1260 & $2.6 \mathrm{E}+\infty 0$ & 2.3E-09 & $6.3 \mathrm{E}-09$ \\
\hline S\&M Worker & Core & $\begin{array}{l}\text { Whole } \\
\text { Building }\end{array}$ & Dermal & Organics & Bis(2-ethylhexyl)phthalate & $2.4 \mathrm{E}-01$ & $2.1 E-10$ & $5.9 \mathrm{E}-10$ \\
\hline S\&M Worker & Core & $\begin{array}{l}\text { Whole } \\
\text { Building }\end{array}$ & Dermal & Organics & Dimethyl phthalate & $5.5 E-02$ & $4.8 \mathrm{E}-11$ & $1.3 \mathrm{E}-10$ \\
\hline S\&M Worker & Core & $\begin{array}{l}\text { Whole } \\
\text { Building }\end{array}$ & Inhalation & Inonganics & Arsenic & $2.9 \mathrm{E}+00$ & $3.7 E-13$ & $1.0 \mathrm{E}-12$ \\
\hline S\&M Worker & Core & $\begin{array}{l}\text { Whole } \\
\text { Building }\end{array}$ & Inhalation & Inorganics & Barium & $7.2 \mathrm{E}+02$ & 9.4E-11 & $2.6 \mathrm{E}-10$ \\
\hline S\&M Worker & Cone & $\begin{array}{l}\text { Whole } \\
\text { Building }\end{array}$ & Inhalation & Inorganics & Beryllium & $1.2 \mathrm{E}+01$ & $1.6 \mathrm{E}-12$ & $4.5 \mathrm{E}-12$ \\
\hline S\&M Worker & Core & $\begin{array}{l}\text { Whole } \\
\text { Building }\end{array}$ & Inhalation & Inorganics & Cadmium & $1.8 \mathrm{E}+02$ & 2.3E-11 & $6.6 \mathrm{E}-11$ \\
\hline S\&M Worker & Core & $\begin{array}{l}\text { Whole } \\
\text { Building }\end{array}$ & Inhalation & Inorganics & Chromium VI & $2.0 \mathrm{E}+02$ & $2.6 \mathrm{E}-11$ & $7.2 \mathrm{E}-11$ \\
\hline S\&M Worker & Core & $\begin{array}{l}\text { Whole } \\
\text { Building }\end{array}$ & Inhalation & Inorganics & Manganese & $9.5 E+03$ & $1.2 \mathrm{E}-09$ & $3.5 \mathrm{E}-09$ \\
\hline S\&M Worker & Core & $\begin{array}{l}\text { Whole } \\
\text { Building }\end{array}$ & Inhalation & Inorganics & Mercury & $3.2 \mathrm{E}-01$ & $4.1 \mathrm{E}-14$ & $1.2 \mathrm{E}-13$ \\
\hline S\&M Worker & Core & $\begin{array}{l}\text { Whole } \\
\text { Building }\end{array}$ & Inhalation & Inorganica & Nickel & $1.4 \mathrm{E}+02$ & $1.8 \mathrm{E}-11$ & $5.0 \mathrm{E}-11$ \\
\hline S\&M Worker & Core & $\begin{array}{l}\text { Whole } \\
\text { Building }\end{array}$ & Inhalation & Inorganics & Vanadium & $2.4 E+02$ & $3.1 \mathrm{E}-11$ & $8.8 \mathrm{E}-11$ \\
\hline
\end{tabular}

- Units are $\mathrm{mg} / \mathrm{kg}$ for inorganics and organics; $\mathrm{pCi} / \mathrm{g}$ for radionucliden

- Unils are $\mathrm{mg} / \mathrm{kg}$-day for inorganics and organics; $\mathrm{pCi}$-day for radionuclides 


\begin{tabular}{|c|c|c|c|c|c|c|c|c|}
\hline \multirow{2}{*}{ Receptor } & \multirow{2}{*}{ Media } & \multirow{2}{*}{ Location } & \multirow{2}{*}{$\begin{array}{l}\text { Exposure } \\
\text { Pathway }\end{array}$} & \multirow{2}{*}{$\begin{array}{l}\text { Contaminant } \\
\text { Class }\end{array}$} & \multirow{2}{*}{ Contaminant } & \multirow{2}{*}{$\begin{array}{l}\text { Exposure Point } \\
\text { Concentration" }\end{array}$} & \multicolumn{2}{|c|}{ Chronic Deily Intake } \\
\hline & & & & & & & $\begin{array}{c}\text { Cancinogenic } \\
\text { Erfects }\end{array}$ & $\begin{array}{c}\text { Noncarcinogenic } \\
\text { Efrects }\end{array}$ \\
\hline S\&M Worker & Core & $\begin{array}{l}\text { Whole } \\
\text { Building }\end{array}$ & Inhalation & Inorganic: & Zinc & $3.5 E+04$ & $4.6 \mathrm{E}-09$ & $1.3 E-08$ \\
\hline S\&M Worker & Core & $\begin{array}{l}\text { Whole } \\
\text { Building }\end{array}$ & Inhalation & Organics & Aroclor-1254 & $1.5 E+00$ & $1.9 \mathrm{E}-13$ & $5.3 \mathrm{E}-13$ \\
\hline S\&M Worker & Core & $\begin{array}{l}\text { Whole } \\
\text { Building }\end{array}$ & Inhalation & Organics & Aroclor- 1260 & $2.6 E+00$ & 3.4E-13 & $9.5 \mathrm{E}-13$ \\
\hline S\&M Worker & Core & $\begin{array}{l}\text { Whole } \\
\text { Building }\end{array}$ & Inhalation & Organics & Bis(2-ethylhexyl)phthalate & 2.4E-01 & $3.1 \mathrm{E}-14$ & $8.8 \mathrm{E}-14$ \\
\hline S\&M Worker & Core & $\begin{array}{l}\text { Whole } \\
\text { Building }\end{array}$ & Inhalation & Organics & Dimethyl phthalate & 5.5E-02 & 7.2E-15 & $2.0 \mathrm{E}-14$ \\
\hline S\&M Worker & Core & $\begin{array}{l}\text { Whole } \\
\text { Building }\end{array}$ & Inhalation & Radionuclides & ${ }^{151} \mathrm{C}$ & $1.4 \mathrm{E}+03$ & $3.2 \mathrm{E}-01$ & \\
\hline S\&M Worker & Core & $\begin{array}{l}\text { Whole } \\
\text { Building }\end{array}$ & Inhalation & Radionuclides & ${ }^{20 \times} \mathrm{Ra}$ & $4.0 \mathrm{E}-01$ & $9.4 \mathrm{E}-05$ & \\
\hline S\&M Worker & Core & $\begin{array}{l}\text { Whole } \\
\text { Building }\end{array}$ & Inhalation & Radionuclides & ${ }^{2 a} \mathrm{Ra}$ & 3.7E-01 & 8.6E-05 & \\
\hline S\&M Worker & Core & $\begin{array}{l}\text { Whole } \\
\text { Building }\end{array}$ & Inhalation & Radionuclides & ${ }^{200}$ Th & $7.7 \mathrm{E}-01$ & $1.8 \mathrm{E}-04$ & \\
\hline S\&M Worker & Core & $\begin{array}{l}\text { Whole } \\
\text { Building }\end{array}$ & Inhalation & Radionuclides & ${ }^{200} \mathrm{Th}$ & $1.4 \mathrm{E}+\infty$ & 3.2E-04 & \\
\hline S\&M Worker & Core & $\begin{array}{l}\text { Whole } \\
\text { Building }\end{array}$ & Inhalation & Radionuclides & ${ }^{20} \mathrm{Th}$ & $8.0 \mathrm{E}-01$ & $1.9 \mathrm{E}-04$ & \\
\hline S\&M Worker & Core & $\begin{array}{l}\text { Whole } \\
\text { Building }\end{array}$ & Inhalation & Radionuclides & ${ }^{233} 3 \mathrm{H} \mathrm{U}$ & $1.3 \mathrm{E}+\infty 0$ & $3.0 \mathrm{E}-04$ & \\
\hline S\&M Worker & Core & $\begin{array}{l}\text { Whole } \\
\text { Building }\end{array}$ & Inhalation & Radionuclides & ${ }^{284} \mathrm{Th}$ & $6.3 E+00$ & $1.5 E-03$ & \\
\hline
\end{tabular}

- Units are mg/kg for inorganics and organics; $\mathrm{pCi} / \mathrm{g}$ for radionuclides

- Units are mg/kg-day for inorganics and organics; pCi-day for radionuclides 


\begin{tabular}{|c|c|c|c|c|c|c|c|c|}
\hline \multirow{2}{*}{ Receptor } & \multirow{2}{*}{ Media } & \multirow{2}{*}{ Location } & \multirow{2}{*}{$\begin{array}{l}\text { Exposure } \\
\text { Pathway }\end{array}$} & \multirow{2}{*}{$\begin{array}{l}\text { Contaminant } \\
\text { Cless }\end{array}$} & \multirow{2}{*}{ Contaminant } & \multirow[b]{2}{*}{$\begin{array}{l}\text { Exposure Point } \\
\text { Concentration" }\end{array}$} & \multicolumn{2}{|c|}{ Chronic Daily Intake" } \\
\hline & & & & & & & $\begin{array}{l}\text { Carcinogenic } \\
\text { Effocts }\end{array}$ & $\begin{array}{l}\text { Noncarcinogenic } \\
\text { Effects }\end{array}$ \\
\hline S\&M Worker & Cone & $\begin{array}{l}\text { Whole } \\
\text { Building }\end{array}$ & Inhalation & Radionuclides & ${ }^{225} U$ & $1.2 \mathrm{E}-01$ & $2.8 \mathrm{E}-05$ & \\
\hline S\&M Worker & Core & $\begin{array}{l}\text { Whole } \\
\text { Building }\end{array}$ & Inhalation & Radionuclides & ${ }^{228} \mathrm{~Pa}$ & 4.5E-01 & 1.1E-04 & \\
\hline S\&M Worker & Core & $\begin{array}{l}\text { Whole } \\
\text { Building }\end{array}$ & Inhalation & Radionuclides & ${ }^{2 M} \mathrm{U}$ & $1.4 E+\infty 0$ & 3.3E-04 & \\
\hline S\&M Worker & Core & $\begin{array}{l}\text { Whole } \\
\text { Building }\end{array}$ & Inhalation & Radionuclides & ${ }^{258250} \mathrm{Pu}$ & $4.0 E+\infty$ & $9.3 \mathrm{E}-04$ & \\
\hline S\&M Worker & Core & $\begin{array}{l}\text { Whole } \\
\text { Building }\end{array}$ & Inhalation & Radionuclides & ${ }^{211} \mathrm{Am}$ & $5.0 \mathrm{E}-01$ & $1.2 \mathrm{E}-04$ & \\
\hline S\&M Worker & Core & $\begin{array}{l}\text { Whole } \\
\text { Building }\end{array}$ & Inhalation & Radionuclides & $40 \mathrm{~K}$ & $5.4 E+00$ & $1.3 \mathrm{E}-03$ & \\
\hline S\&M Worker & Core & $\begin{array}{l}\text { Whole } \\
\text { Building }\end{array}$ & Inhalation & Radionuclides & ${ }^{\infty} \mathrm{Co}$ & $1.3 \mathrm{E}-01$ & 3.0E-05 & \\
\hline S\&M Worker & Core & $\begin{array}{l}\text { Whole } \\
\text { Building }\end{array}$ & Inhalation & Radionuclides & ${ }^{\infty} \mathrm{Sr}$ & $1.2 E+01$ & 2.7E-03 & \\
\hline S\&M Worker & Core & $\begin{array}{l}\text { Whole } \\
\text { Building }\end{array}$ & Inhalation & Radionuclides & Tritium & $2.6 \mathrm{E}+01$ & $6.0 \mathrm{E}-03$ & \\
\hline S\&M Worker & Core & $\begin{array}{l}\text { Whole } \\
\text { Building }\end{array}$ & External Exposure & Radionuclides & ${ }^{19} \mathrm{Cs}$ & $1.4 \mathrm{E}+03$ & $5.0 E+01$ & \\
\hline S\&M Worker & Core & $\begin{array}{l}\text { Whole } \\
\text { Building }\end{array}$ & Extemal Exposure & Radionuclides & ${ }^{20} \mathrm{Ra}$ & $4.0 \mathrm{E}-01$ & $1.5 \mathrm{E}-02$ & \\
\hline S\&M Worker & Core & $\begin{array}{l}\text { Whole } \\
\text { Building }\end{array}$ & External Exposure & Radionuclides & ${ }^{20} \mathrm{Ra}$ & $3.7 \mathrm{E}-01$ & $1.4 \mathrm{E}-02$ & \\
\hline S\&M Worker & Core & $\begin{array}{l}\text { Whole } \\
\text { Building }\end{array}$ & External Exposure & Radionuclides & ${ }^{200} \mathrm{Th}$ & 7.7E-01 & $2.8 \mathrm{E}-02$ & \\
\hline
\end{tabular}

Units are $\mathrm{mg} / \mathrm{kg}$ for inorganics and organics; $\mathrm{pCi} / \mathrm{g}$ for radionuclides

- Units are $\mathrm{mg} / \mathrm{kg}$-day for inorganics and organics; $\mathrm{pCi}$-day for radionuclides 


\begin{tabular}{|c|c|c|c|c|c|c|c|c|}
\hline \multirow{2}{*}{ Receptor } & \multirow{2}{*}{ Media } & \multirow{2}{*}{ Location } & \multirow{2}{*}{$\begin{array}{l}\text { Exposure } \\
\text { Pathway }\end{array}$} & \multirow{2}{*}{$\begin{array}{l}\text { Contaminant } \\
\text { Class }\end{array}$} & \multirow{2}{*}{ Contaminant } & \multirow{2}{*}{$\begin{array}{l}\text { Exposure Point } \\
\text { Concentration" }\end{array}$} & \multicolumn{2}{|c|}{ Chronic Daily Intake } \\
\hline & & & & & & & $\begin{array}{c}\text { Carcinogenic } \\
\text { Effects }\end{array}$ & $\begin{array}{c}\text { Noncarcinogenic } \\
\text { Effects }\end{array}$ \\
\hline S\&M Worker & Core & $\begin{array}{l}\text { Whole } \\
\text { Building }\end{array}$ & External Exposure & Radionuclides & ${ }^{200} \mathrm{Th}$ & $1.4 \mathrm{E}+00$ & S.1E-02 & \\
\hline S\&M Worker & Core & $\begin{array}{l}\text { Whole } \\
\text { Building }\end{array}$ & External Exposure & Radionuclides & ${ }^{200} \mathrm{Th}$ & 8.0E-01 & $2.9 \mathrm{E}-02$ & \\
\hline S\&M Worker & Core & $\begin{array}{l}\text { Whole } \\
\text { Building }\end{array}$ & Extemal Exposure & Radionuclides & ${ }^{232} 2324 U$ & $1.3 E+\infty 0$ & $4.6 \mathrm{E}-02$ & \\
\hline S\&M Worker & Core & $\begin{array}{l}\text { Whole } \\
\text { Building }\end{array}$ & Extemal Exposure & Radionuclides & ${ }^{234} \mathrm{Th}$ & $6.3 E+00$ & 2.3E-01 & \\
\hline S\&M Worker & Core & $\begin{array}{l}\text { Whole } \\
\text { Building }\end{array}$ & External Exposure & Radionuclides & ${ }^{235} \mathrm{U}$ & $1.2 \mathrm{E}-01$ & 4.4E-03 & \\
\hline S\&M Worker & Core & $\begin{array}{l}\text { Whole } \\
\text { Building }\end{array}$ & External Exposure & Radionuclides & ${ }^{22} \mathrm{Pu}$ & $4.5 \mathrm{E}-01$ & $1.6 \mathrm{E}-02$ & \\
\hline S\&M Worker & Core & $\begin{array}{l}\text { Whole } \\
\text { Building }\end{array}$ & External Exposure & Radionuclides & ${ }^{23 t} U$ & $1.4 \mathrm{E}+\infty 0$ & $5.2 \mathrm{E}-02$ & \\
\hline S\&M Worker & Core & $\begin{array}{l}\text { Whole } \\
\text { Building }\end{array}$ & External Exposure & Radionuclides & ${ }^{2595200} \mathrm{Pu}$ & $4.0 \mathrm{E}+00$ & $1.5 \mathrm{E}-01$ & \\
\hline S\&M Worker & Core & $\begin{array}{l}\text { Whole } \\
\text { Building }\end{array}$ & External Exposure & Radionuclides & ${ }^{211} \mathrm{Am}$ & S.0E-01 & $1.8 \mathrm{E}-02$ & \\
\hline S\&M Worker & Core & $\begin{array}{l}\text { Whole } \\
\text { Building }\end{array}$ & External Exposure & Radionuclides & ${ }^{+\infty} \mathrm{K}$ & $5.4 E+00$ & $2.0 \mathrm{E}-01$ & \\
\hline S\&M Worker & Core & $\begin{array}{l}\text { Whole } \\
\text { Building }\end{array}$ & External Exposure & Radionuclides & ${ }^{\infty} \mathrm{Co}$ & $1.3 \mathrm{E}-01$ & $4.7 \mathrm{E}-03$ & \\
\hline S\&M Worker & Core & $\begin{array}{l}\text { Whole } \\
\text { Building }\end{array}$ & External Exposure & Radionuclides & ${ }^{\infty} \mathrm{Sr}$ & $1.2 \mathrm{E}+01$ & $4.2 \mathrm{E}-01$ & \\
\hline S\&M Worker & Core & $\begin{array}{l}\text { Whole } \\
\text { Building }\end{array}$ & External Exposure & Radionuclide: & Tritium & $2.6 \mathrm{E}+01$ & 9.4E-01 & \\
\hline
\end{tabular}

- Units are $\mathrm{mg} / \mathrm{kg}$ for inorganica and organics; $\mathrm{pCi} / \mathrm{g}$ for radionuclides

- Units are mg/kg-day for inorganics and organics; pCi-day for radionuclides 
Table D-6. S\&M Worker Exposure Concentrations and Chronic Lifetime Exposures for Direct Measurement Samples

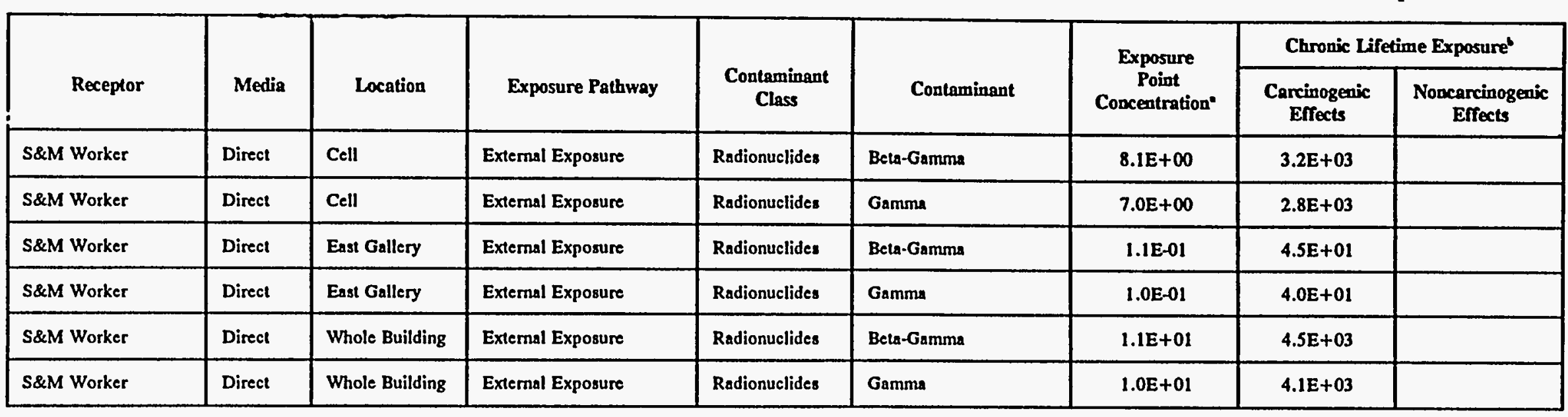

- Untis are mrem

Units are mrem 
THIS PAGE INTENTIONALLY LEFT BLANK 
Table D-7. S\&M Worker Exposure Concentrations and Chronic Daily Intakes from Smear Samples

\begin{tabular}{|c|c|c|c|c|c|c|c|c|}
\hline \multirow{2}{*}{ Receptor } & \multirow{2}{*}{ Media } & \multirow{2}{*}{ Location } & \multirow{2}{*}{ Exposure Pathway } & \multirow{2}{*}{$\begin{array}{c}\text { Contaminant } \\
\text { Class }\end{array}$} & \multirow{2}{*}{ Contaminant } & \multirow{2}{*}{$\begin{array}{l}\text { Exposure Point } \\
\text { Concentration" }\end{array}$} & \multicolumn{2}{|c|}{ Chronic Daily Intake } \\
\hline & & & & & & & $\begin{array}{c}\text { Carcinogenic } \\
\text { Efrects }\end{array}$ & $\begin{array}{c}\text { Noncarcinogenic } \\
\text { Effects }\end{array}$ \\
\hline S\&M Worker & Smear & Cell & Ingestion & Radionuclides & ${ }^{17} \mathrm{Cs}$ & $1.4 \mathrm{E}+02$ & $3.6 \mathrm{E}+02$ & \\
\hline S\&M Worker & Smear & Cell & Ingertion & Radionuclide: & ${ }^{200} \mathrm{Ra}$ & $5.3 \mathrm{E}-03$ & $1.3 \mathrm{E}-02$ & \\
\hline S\&M Worker & Smear & Cell & Ingeution & Radionuclides & neth & 1.1E-02 & $2.8 \mathrm{E}-02$ & \\
\hline S\&M Worker & Smear & Cell & Ingertion & Radionuclides & ${ }^{200} \mathrm{Th}$ & $1.8 \mathrm{E}-02$ & $4.6 \mathrm{E}-02$ & \\
\hline S\&M Worker & Smear & Cell & Ingertion & Radionuclides & ${ }^{20} \mathrm{Th}$ & 1.1E-02 & $2.9 \mathrm{E}-02$ & \\
\hline S\&M Worker & Smear & Cell & Ingestion & Radionuclides & ${ }^{231234} \mathrm{U}$ & $1.7 \mathrm{E}-02$ & $4.3 \mathrm{E}-02$ & \\
\hline S\&M Worker & Smear & Cell & Ingestion & Radionuclides & ${ }^{219} \mathrm{U}$ & $1.6 \mathrm{E}-03$ & $4.0 \mathrm{E}-03$ & \\
\hline S\&M Worker & Smear & Cell & Ingestion & Radionuclides & ${ }^{25} \mathrm{Pu}$ & 8.4E-03 & $2.1 \mathrm{E}-02$ & \\
\hline S\&M Worker & Smear & Cell & Ingestion & Radionuclides & ${ }^{250} \mathrm{U}$ & $2.2 \mathrm{E}-02$ & $5.6 \mathrm{E}-02$ & \\
\hline S\&M Worker & Smear & Cell & Ingestion & Radionuclides & ${ }^{295200} \mathrm{Pu}$ & $5.3 \mathrm{E}-02$ & $1.3 \mathrm{E}-01$ & \\
\hline S\&M Worker & Smear & Cell & Ingestion & Radionuclides & ${ }^{\infty} \mathrm{K}$ & S.SE-01 & $1.4 E+\infty 0$ & \\
\hline S\&M Worker & Smear & Cell & Ingestion & Radionuclides & ${ }^{\infty} \mathrm{Co}$ & $1.4 \mathrm{E}-02$ & 3.4E-02 & \\
\hline S\&M Worker & Smear & Cell & Ingestion & Radionuclide: & ${ }^{\infty} \mathrm{Sr}$ & $1.2 \mathrm{E}+\infty 0$ & $3.0 \mathrm{E}+00$ & \\
\hline S\&M Worker & Smear & Cell & Inhalation & Radionuclides & ${ }^{{ }^{n} \mathrm{Cs}}$ & $1.4 \mathrm{E}+02$ & $3.3 \mathrm{E}-02$ & \\
\hline S\&M Worker & Smear & Cell & Inhalation & Radionuclides & ${ }^{200} \mathrm{Ra}$ & $5.3 \mathrm{E}-03$ & $1.2 \mathrm{E}-06$ & \\
\hline S\&M Worker & Smear & Cell & Inhalation & Radionuclide: & 20Th & $1.1 \mathrm{E}-02$ & $2.6 \mathrm{E}-06$ & \\
\hline S\&M Worker & Smear & Cell & Inhalation & Radionuclides & ${ }^{200} \mathrm{Th}$ & $1.8 \mathrm{E}-02$ & $4.3 \mathrm{E}-06$ & \\
\hline S\&M Worker & Smear & Cell & Inhalation & Radionuclides & ${ }^{2 n} \mathrm{Th}$ & 1.1E-02 & $2.7 \mathrm{E}-06$ & \\
\hline S\&M Worker & Smear & Cell & Inhalation & Radionuclides & ${ }^{232323} U$ & $1.7 E-02$ & $4.0 \mathrm{E}-06$ & \\
\hline
\end{tabular}

- Units are $\mathrm{pCi} / \mathrm{g}$

- Units are pCi-day 


\begin{tabular}{|c|c|c|c|c|c|c|c|c|}
\hline \multirow{2}{*}{ Receptor } & \multirow{2}{*}{ Media } & \multirow{2}{*}{ Location } & \multirow{2}{*}{ Exposure Pathway } & \multirow{2}{*}{$\begin{array}{l}\text { Contaminant } \\
\text { Class }\end{array}$} & \multirow{2}{*}{ Contaminant } & \multirow[b]{2}{*}{$\begin{array}{l}\text { Exposure Point } \\
\text { Concentration" }\end{array}$} & \multicolumn{2}{|c|}{ Chronic Daily Intake" } \\
\hline & & & & & & & $\begin{array}{c}\text { Carcinogenic } \\
\text { Erfects }\end{array}$ & $\begin{array}{l}\text { Noncarcinogenic } \\
\text { Erfects }\end{array}$ \\
\hline S\&M Worker & Smear & Cell & Inhalation & Radionuclides & ${ }^{239} \mathrm{U}$ & $1.6 \mathrm{E}-03$ & 3.7E-07 & \\
\hline S\&M Worker & Smear & Cell & Inhalation & Radionuclides & ${ }^{280} \mathrm{Pu}$ & 8.4E-03 & $2.0 \mathrm{E}-06$ & \\
\hline S\&M Worker & Smear & Cell & Inhalation & Radionuclides & ${ }^{200} U$ & $2.2 E-02$ & $5.2 \mathrm{E}-06$ & \\
\hline S\&M Worker & Smear & Cell & Inhalation & Radionuclides & ${ }^{251200} \mathrm{Pu}$ & $5.3 \mathrm{E}-02$ & $1.2 \mathrm{E}-05$ & \\
\hline S\&M Worker & Smear & Cell & Inhalation & Radionuclides & ${ }^{+\infty} \mathrm{K}$ & 5.5E-01 & $1.3 \mathrm{E}-04$ & \\
\hline S\&M Worker & Smear & Cell & Inhalation & Radionuclides & ${ }^{\infty} \mathrm{Co}$ & $1.4 \mathrm{E}-02$ & $3.2 \mathrm{E}-06$ & \\
\hline S\&M Worker & Smear & Cell & Inhalation & Radionuclides & ${ }^{*} \mathrm{Sr}$ & $1.2 \mathrm{E}+00$ & $2.8 \mathrm{E}-04$ & \\
\hline S\&M Worker & Smear & Cell & External Exposure & Radionuclides & ${ }^{17} \mathrm{Cs}$ & $1.4 \mathrm{E}+02$ & $6.5 E+\infty 0$ & \\
\hline S\&M Worker & Smear & Cell & External Exposure & Radionuclides & ${ }^{2 \infty} \mathrm{Ra}$ & $5.3 \mathrm{E}-03$ & 2.4E-04 & \\
\hline S\&M Worker & Smear & Cell & External Exposure & Radionuclides & ${ }^{n+} \mathrm{Th}$ & $1.1 \mathrm{E}-02$ & $5.0 \mathrm{E}-04$ & \\
\hline S\&M Worker & Smear & Cell & Extemal Exposure & Radionuclides & ${ }^{200} \mathrm{Th}$ & $1.8 \mathrm{E}-02$ & 8.4E-04 & \\
\hline S\&M Worker & Smear & Cell & External Exposure & Radionuclides & ${ }^{2 x} \mathrm{Th}$ & $1.1 \mathrm{E}-02$ & $5.2 \mathrm{E}-04$ & \\
\hline SĒîi Wioricer & Smear & Ceii & Extermai Exposure & Radionuchiứes & 2382sy & $1.7 E-02$ & $7.8 E-04$ & \\
\hline S\&M Worker & Smear & Cell & External Exposure & Radionuclides & ${ }^{20 s} \mathrm{U}$ & $1.6 \mathrm{E}-03$ & 7.2E-05 & \\
\hline S\&M Worker & Smear & Cell & External Exposure & Radionuclides & ${ }^{2 s e} \mathrm{Pu}$ & $8.4 \mathrm{E}-03$ & $3.8 \mathrm{E}-04$ & \\
\hline S\&M Worker & Smear & Cell & External Exposure & Radionuclides & ${ }^{2 n} \mathrm{U}$ & $2.2 \mathrm{E}-02$ & $1.0 \mathrm{E}-03$ & \\
\hline S\&M Worker & Smear & Cell & External Exposure & Radionuclides & ${ }^{2080200} \mathrm{Pu}$ & $5.3 E-02$ & 2.4E-03 & \\
\hline S\&M Worker & Smear & Cell & External Exposure & Radionuclides & ${ }^{10} \mathrm{~K}$ & S.SE-01 & $2.5 E-02$ & \\
\hline S\&M Worker & Smear & Cell & Extemal Exposure & Radionuclides & ${ }^{60} \mathrm{Co}$ & $1.4 \mathrm{E}-02$ & $6.2 \mathrm{E}-04$ & \\
\hline S\&M Worker & Smear & Cell & External Exposure & Radionuclides & ${ }^{*} \mathrm{Sr}$ & $1.2 \mathrm{E}+00$ & S.SE-02 & \\
\hline S\&M Worker & Smear & East Gallery & Ingestion & Radionuclides & ${ }^{317} \mathrm{Cs}$ & $1.5 \mathrm{E}+01$ & $3.8 \mathrm{E}+01$ & \\
\hline
\end{tabular}

- Units are pCi/g

- Units are pCi-day 
Kxp-!วd aะ ข!̣u , $8 /$ :

\begin{tabular}{|c|c|c|c|c|c|c|c|c|}
\hline & LO-GL'S & $60-3 t^{\prime} \tau$ & $\mathbf{n}_{s c t}$ & epp!|onuo!pwd & บ०|!ा|ำण & 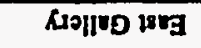 & sEows & J2710M Wช9 \\
\hline & $90-958$ & $20-39^{\circ} \mathrm{E}$ & $\mathbf{n}_{\text {rezsiz }}$ & esp!|onuo!pry & 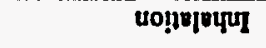 & 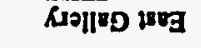 & soows & JPYIOM WPS \\
\hline & $90-31.6$ & $20-9 L^{\circ} t$ & $\boldsymbol{u L}_{2 \pi z}$ & esp!lonuo!pry & 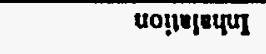 & Ralleg Irxg & ivous & $20 \times 10 M$ Wrs \\
\hline & SO-AI'I & $20-96.6$ & HLort & eop!!onuo!pzy & น0!ाข|вนण & אगाषD भBष & sosurs & JOYIOM WPSS \\
\hline & $90-3 E ' S$ & $20-9 \varepsilon \cdot \tau$ & $4 L_{0 \pi}$ & sop!|onuo!pry & 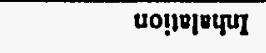 & Kगाएळ $4 \mathrm{~g}$ & disouls & دоצ10M W88 \\
\hline & so-39's & $10-3 b \cdot 2$ & volsc & sop!|onuo!pry & 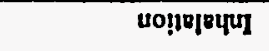 & SPIIPD ISVG & IRows & 20Y10M WrS \\
\hline & $90-96^{\circ} E$ & $20-95^{\circ} 1$ & vargr & eəp!|’nuo!p:y & 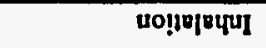 & 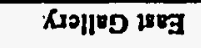 & stows & soyjoM WPS \\
\hline & E0-39.' $\mathcal{E}$ & $10+3 s^{*} 1$ & $D_{L s t}$ & sop!|jnuotpey & 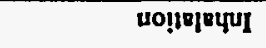 & GPIIED IREg & seous & دOY10M WrS \\
\hline & $10+3 b^{\circ} 1$ & $00+36 \cdot s$ & ${ }^{\prime} S_{\infty}$ & spp!|गnuo!pry & นo!n8จ8น & 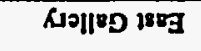 & Ivouns & 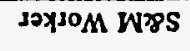 \\
\hline & $00+30^{\circ} 6$ & $00+39^{\circ} \varepsilon$ & $\mathbf{X X}_{00}$ & sop!|onuo!ppy & uo!!sอ84บ & 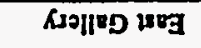 & sEows & I0Y10M WPS \\
\hline & $20-91$ 's & $20-90 \cdot 2$ & $u_{v} v_{i n}$ & sop!|⿰nuo!pry & นo!̣8จ8บ & 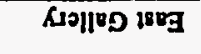 & dovws & دәఖ10 M WrS \\
\hline & $20-30 \%$ & $20-39^{\circ} 1$ & rdowassz & eop!|pnuo!pry & บo!̣co8ใบ & KAग|RD ISBg & 30ous & Jay10M WrS \\
\hline & $20-91 \cdot 2$ & $20-98^{\circ} \tau$ & $\mathbf{n}_{\mathbf{s z z}}$ & esp!|юnuo!̣pey & บ0!̣เร8ิน & Kมा|ण 280 & Joows & JOY10M WrS \\
\hline & $20-30^{\circ} b$ & $20-99^{\circ} 1$ & $n_{d}$ isz & sop!|onuo!ped & 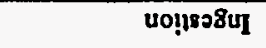 & К」गाएD I8ख్ & spows & IOYJOM WrS \\
\hline & $80-3 I^{\prime} 9$ & $\varepsilon 0-\exists \boldsymbol{t}^{\circ} \tau$ & $\mathbf{n}_{s i z}$ & sop!|Pnuo!pry & น0!ฺ8ว8บ & Кગગ|एD น8ช & doכws & I0Y10M WrS \\
\hline & $20-31 \% 6$ & 20.59. $\varepsilon$ & n nzrssz $_{2}$ & opp!|จnuo!̣py & นo!̣ตร8บบ & AIग|PD LRB & sopus & IOYIOM WPS \\
\hline & $20-96 \cdot t$ & $20.36 \cdot 1$ & $4 I_{a x z}$ & eop!!onuo!pzy & บo!̣เฉร8บบ & 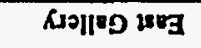 & LoगUS & IOXJoM WrS \\
\hline & IO-JZ:I & $20-366^{\circ}$ & thoxz & sop!|onuo!pry & นo!̣sอ8นI & 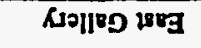 & joous & JoY10M WPS \\
\hline & wo-sL's & $20-3 \varepsilon^{\prime} \tau$ & $4 L+a x$ & spp!|rnuo!pry & 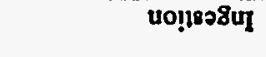 & KIगIPD ISBg & drows & 1әY10M Wrs \\
\hline & $10.91 \% 9$ & $10-3+2$ & $\log _{\text {sza }}$ & Isp!|jnuo!pry & uọุ15อ8บ & 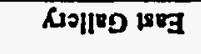 & sosurs & 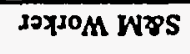 \\
\hline & $\boldsymbol{z O}-\mathbf{I L} \mathcal{E}$ & 20-9s. I & $y_{x a}$ & sэp!|คnuoupry & uot!sอ8บบ & KगाएD & soows & 20¥10M W85 \\
\hline 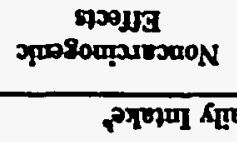 & פ70011 & 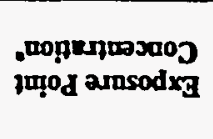 & 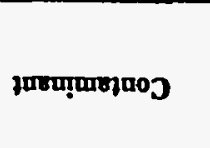 & 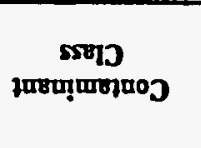 & fEMqTEd ansodxg & nop̣so7 & ๒ฺ̣ & 10xdaxoy \\
\hline
\end{tabular}




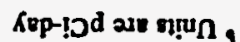
$8 / !$ d $^{\mathrm{d}}$ as mụ

\begin{tabular}{|c|c|c|c|c|c|c|c|c|}
\hline & $20+30^{\circ} \tau$ & $10+30.8$ & $D_{\Delta s i}$ & sop!|ønuo!pry & 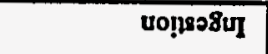 & 8u!PI!ng गО४M & Josus & JOYIOM WPS \\
\hline & $10-a s z$ & $00+3 b \cdot s$ & ${ }^{1} S_{\infty}$ & sop!|onuo!prd & 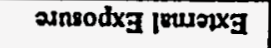 & SNOाED म8. & sesurs & JOXIOM NPS \\
\hline & $10-39 \cdot 1$ & $00+39^{\circ} \varepsilon$ & $\mathbf{X}_{00}$ & ePp!|юnuo!ped & ansodxG Imus2xg & CगIPD & sosus & JOXJOM WrS \\
\hline & to-az:6 & $20-30 \cdot 2$ & urvin $_{\text {in }}$ & sop!|pnuotpqy & asnsodxg ן"uว)xg & KIOाIDD IS8G & desuls & JoyjoM WrSS \\
\hline & $50 \pi 2 \cdot L$ & $20-39+i$ & ndoteser & sop!|onuo!pry & 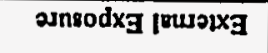 & KJOIIPD 100 & svouns & دo¥10M WPS \\
\hline & EO-JE' I & $20-98^{\circ} 2$ & $\Omega_{s s z}$ & sop!|onuo!pey & asnsodxg ןruəxxg & APा|णD ISPg & seows & $20 \times 10 M$ NखSS \\
\hline & $t 0-\exists Z \cdot L$ & $20-39 \cdot 1$ & $n_{d_{k z}}$ & ssp!|onuo!̣pry & 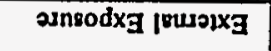 & KAगाएా 280 & sosus & I0X10M W'8S \\
\hline & to-3III & $60-3 b^{\prime} 2$ & $\Omega_{s s t}$ & esp!|onuo!pry & 2unsodxヨ lpusplx & 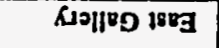 & snows & دOX10M WrS \\
\hline & $60-3 ! \div !$ & $70-39^{\circ} \varepsilon$ & nnzwst & อn!!pnuo!p?y & sunrodx & 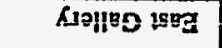 & sqüus & 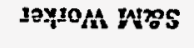 \\
\hline & $50-96 \cdot L$ & $20-9 L^{\prime} I$ & LI. $a z$ & sop!|จnuo!pzy & annsodxg louspXg & אIगाषיפ I8Bg & soous & J0X10M WrS \\
\hline & $\varepsilon 0-\exists \tau \cdot \tau$ & $20-36^{\circ} t$ & $4 L_{0 x t}$ & sop!|юnuo!pry & 2unsodxg InusolX3 & KJग|IיD ISEg & sosurs & JOYIOM WrS \\
\hline & $60-30^{\circ} I$ & $20-a \varepsilon^{\prime} 2$ & $4 L_{\text {sez }}$ & sop!jonuo!pry & aunsodxg Imwesxg & AsP|leD 2s89 & deours & JOY10M WrS \\
\hline & $20-311$ & $10-3 b 2$ & $8 y_{n z z}$ & eop!|pnuo!pey & 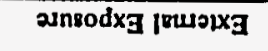 & CNO\|PD ISPG & Josurs & J2X10M WrSS \\
\hline & $50-36.9$ & $20-351$ & $y_{x x z}$ & sop!joneo!ped & 25nsodxg IEw)LXg & 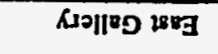 & dEous & JOYNOM WPS \\
\hline & $10-90^{\circ} \mathrm{L}$ & $10+\exists S^{\circ} I$ & $D_{L L I}$ & ep!!pnuo!pry & 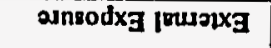 & SIOIIPD 280 & dosurs & נכУJOM WrS \\
\hline & EO-GE" I & $\infty 0+3 b^{\circ} \cdot 5$ & ${ }^{\perp} S_{\infty}$ & esp!|วnuo!pry & 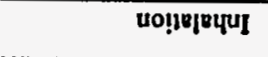 & KJPIIDD $40 \mathrm{~g}$ & Josws & IOX10M WPS \\
\hline & $50-368$ & $00+39 \cdot \varepsilon$ & $\mathrm{X}_{000}$ & op!|วnuo!pry & 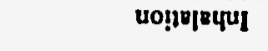 & 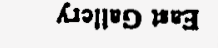 & Irotus & มכশ10M WrS \\
\hline & $90-3<\div$ & $20-30 \cdot \tau$ & urvine & sop!|jnuolpey & 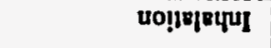 & 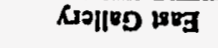 & dertus & IOX10M WrS \\
\hline & $90-3 / \cdot \varepsilon$ & $20-39 \cdot 1$ & $n_{\text {doreser }}$ & -op!!onuo!pry & uo!̣vㄹน & 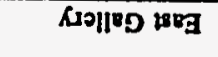 & sosurs & JOX10M Wros \\
\hline & $90-39.9$ & $20-38 \cdot \tau$ & $\Omega_{x z}$ & esp!|onuo!pry & U0!!미나미 & Кนगाषפ 280 & seous & $20 \times 10 \mathrm{M}$ WPS \\
\hline & $90-3 L \cdot \varepsilon$ & $20-991$ & $n_{\text {dere }}$ & sop!!onuo!pwd & 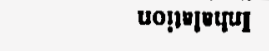 & 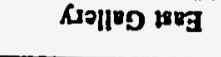 & swows & JO¥10M WPS \\
\hline 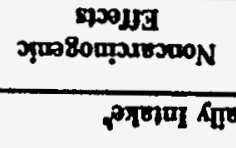 & 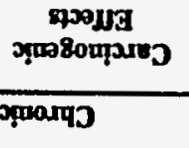 & 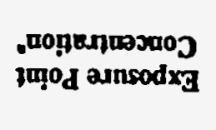 & 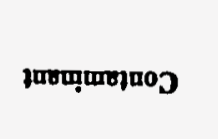 & 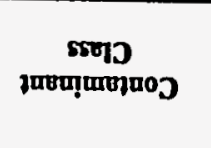 & AEMqIEd ansodxg & uopeso7 & עpow & $10 x d \partial 30 y$ \\
\hline
\end{tabular}




\begin{tabular}{|c|c|c|c|c|c|c|c|c|}
\hline \multirow{2}{*}{ Receptor } & \multirow{2}{*}{ Medin } & \multirow{2}{*}{ Location } & \multirow{2}{*}{ Exposure Pathway } & \multirow{2}{*}{$\begin{array}{l}\text { Contaminant } \\
\text { Class }\end{array}$} & \multirow{2}{*}{ Contaminant } & \multirow{2}{*}{$\begin{array}{l}\text { Exposure Point } \\
\text { Concentration" }\end{array}$} & \multicolumn{2}{|c|}{ Chronic Daily Intake } \\
\hline & & & & & & & $\begin{array}{l}\text { Carcinogenic } \\
\text { Effects }\end{array}$ & $\begin{array}{c}\text { Noncarcinogenic } \\
\text { Effects }\end{array}$ \\
\hline S\&M Worker & Smear & Whole Building & Ingertion & Radionuclides & ${ }^{20} R_{a}$ & $5.9 \mathrm{E}-03$ & $1.5 \mathrm{E}-02$ & \\
\hline S\&M Worker & Smear & Whole Building & Ingertion & Radionuclides & ${ }^{2 x} \mathrm{R}$ & 2.8E-02 & 7.1E-02 & \\
\hline S\&M Worker & Smear & Whole Building & Ingertion & Radionuclides & ${ }^{2 n} \mathrm{Th}$ & $1.1 E-02$ & $=m 2.8 \mathrm{E}-02$ & \\
\hline S\&M Worker & Smear & Whole Building & Ingestion & Radionuclides & ${ }^{200} \mathrm{Th}$ & $2.0 \mathrm{E}-02$ & $5.0 \mathrm{E}-02$ & \\
\hline S\&M Worker & Smear & Whole Building & Ingestion & Radionuclides & ${ }^{20} \mathrm{Th}$ & $1.1 E-02$ & $2.9 \mathrm{E}-02$ & \\
\hline S\&M Worker & Smear & Whole Building & Ingestion & Radionuclides & ${ }^{231254} \mathrm{U}$ & $1.8 \mathrm{E}-02$ & 4.4E-02 & \\
\hline S\&M Worker & Smear & Whole Building & Ingestion & Radionuclides & ${ }^{23} \mathrm{U}$ & $1.8 \mathrm{E}-03$ & 4.4E-03 & \\
\hline S\&M Worker & Smear & Whole Building & Ingestion & Radionuclides & ${ }^{2 s} \mathrm{Pu}$ & $9.5 \mathrm{E}-03$ & 2.4E-02 & \\
\hline S\&M Worker & Smear & Whole Building & Ingestion & Radionuclides & ${ }^{200} \mathrm{U}$ & 2.5E-02 & $6.3 \mathrm{E}-02$ & \\
\hline S\&M Worker & Smear & Whole Building & Ingestion & Radionuclides & ${ }^{299210} \mathrm{Pu}$ & $5.8 \mathrm{E}-02$ & $1.4 \mathrm{E}-01$ & \\
\hline S\&M Worker & Smear & Whole Building & Ingestion & Radionuclides & ${ }^{213} \mathrm{Am}$ & 7.4E-03 & $1.8 \mathrm{E}-02$ & \\
\hline S\&M Worker & Smear & Whole Building & Ingestion & Radionuclides & ${ }^{+0} \mathrm{~K}$ & 4.2E-01 & $1.1 E+\infty$ & \\
\hline S\&M Worker & Smear & Whole Building & Ingestion & Radionuclides & ${ }^{60} \mathrm{Co}$ & $1.0 \mathrm{E}-02$ & $2.6 \mathrm{E}-02$ & \\
\hline S\&M Worker & Smear & Whole Building & Ingestion & Radionuclides & ${ }^{\infty} \mathrm{Sr}$ & $9.1 \mathrm{E}-01$ & $2.3 E+\infty$ & \\
\hline S\&M Worker & Smear & Whole Building & Inhalation & Radionuclides & ${ }^{137} \mathrm{Cs}$ & $8.0 \mathrm{E}+01$ & $1.9 \mathrm{E}-02$ & \\
\hline S\&M Worker & Smear & Whole Building & Inhalation & Radionuclides & ${ }^{25}{ }_{\mathrm{Ra}}$ & $5.9 \mathrm{E}-03$ & $1.4 \mathrm{E}-06$ & \\
\hline S\&M Worker & Smear & Whole Building & Inhalation & Radionuclides & ${ }^{22} \mathrm{Ra}$ & $2.8 \mathrm{E}-02$ & $6.6 \mathrm{E}-06$ & \\
\hline S\&M Worker & Smear & Whole Building & Inhalation & Radionuclides & ${ }^{200} \mathrm{Th}$ & $1.1 \mathrm{E}-02$ & $2.6 \mathrm{E}-06$ & \\
\hline S\&M Worker & Smear & Whole Building & Inhalation & Radionuclides & ${ }^{200} \mathrm{Th}$ & $2.0 \mathrm{E}-02$ & 4.7E-06 & \\
\hline S\&M Worker & Smear & Whole Building & Inhalation & Radionuclides & ${ }^{200} \mathrm{Th}$ & $1.1 \mathrm{E}-02$ & 2.7E-06 & \\
\hline S\&M Worker & Smear & Whole Building & Inhalation & Radionuclides & ${ }^{292524} U$ & $1.8 \mathrm{E}-02$ & 4.1E-06 & \\
\hline
\end{tabular}

- Units are $\mathrm{pCi} / \mathrm{g}$

- Units are pCi-day 


\begin{tabular}{|c|c|c|c|c|c|c|c|c|}
\hline \multirow{2}{*}{ Receptor } & \multirow{2}{*}{ Media } & \multirow{2}{*}{ Location } & \multirow{2}{*}{ Exposure Pathway } & \multirow[b]{2}{*}{$\begin{array}{l}\text { Contaminant } \\
\text { Class }\end{array}$} & \multirow{2}{*}{ Contaminant } & \multirow[b]{2}{*}{$\begin{array}{l}\text { Exposure Point } \\
\text { Concentration" }\end{array}$} & \multicolumn{2}{|c|}{ Chronic Daily Intake" } \\
\hline & & & & & & & $\begin{array}{l}\text { Carcinogenic } \\
\text { Effocts }\end{array}$ & $\begin{array}{l}\text { Noncarcinogenic } \\
\text { Effects }\end{array}$ \\
\hline S\&M Worker & Smear & Whole Building & Inhalation & Radionuclides & ${ }^{209} \mathrm{U}$ & $1.8 \mathrm{E}-03$ & $4.1 E-07$ & \\
\hline S\&M Worker & Smear & Whole Building & Inhualation & Radionuclides & ${ }^{2 \times} \mathrm{Pu}$ & 9.5E-03 & 2.2E-06 & \\
\hline S\&M Worker & Smear & Whole Building & Inhalation & Radionuclides & ${ }^{298} \mathrm{U}$ & $2.5 \mathrm{E}-02$ & $5.9 \mathrm{E}-06$ & \\
\hline S\&M Worker & Smear & Whole Building & Inhalation & Radionuclides & ${ }^{258250} \mathrm{Pu}$ & $5.8 E-02$ & $1.4 \mathrm{E}-0 \mathrm{~S}$ & \\
\hline S\&M Worker & Smear & Whole Building & Inhalation & Radionuclides & ${ }^{211} \mathrm{Am}$ & 7.4E-03 & $1.7 \mathrm{E}-06$ & \\
\hline S\&M Worker & Smear & Whole Building & Inhalation & Radionuclides & ${ }^{\infty} \mathrm{K}$ & $4.2 \mathrm{E}-01$ & 9.8E-05 & \\
\hline S\&M Worker & Smear & Whole Building & Inhalation & Radionuclides & ${ }^{\infty} \mathrm{Co}$ & $1.0 \mathrm{E}-02$ & $2.4 E-06$ & \\
\hline S\&M Worker & Smear & Whole Building & Inhalation & Radionuclides & ${ }^{\infty} \mathrm{Sr}$ & 9.1E-01 & 2.1E-04 & \\
\hline S\&M Worker & Smear & Whole Building & External Exposure & Radionuclides & ${ }^{197} \mathrm{Cs}$ & $8.0 \mathrm{E}+01$ & $3.7 E+00$ & \\
\hline S\&M Worker & Smear & Whole Building & External Exposure & Radionuclides & ${ }^{20} \mathrm{Ra}$ & $5.9 \mathrm{E}-03$ & 2.7E-04 & \\
\hline S\&M Worker & Smear & Whole Building & External Exposure & Radionuclides & ${ }^{n !} \mathbf{R a}$ & 2.8E-02 & $1.3 \mathrm{E}-03$ & \\
\hline S\&M Worker & Smear & Whole Building & Extemal Exposure & Radionuclides & ${ }^{20} \mathrm{Th}$ & $1.1 \mathrm{E}-02$ & $5.2 \mathrm{E}-04$ & \\
\hline SẾĩ Ẅorker & Smear & Wrnoic Ëuiiding & Exicmai Exposuri & R̃àdionuciiá̛è & ${ }^{250} \mathrm{Th}$ & $2.0 \mathrm{E}-02$ & S.2E-04 & \\
\hline S\&M Worker & Smear & Whole Building & External Exposure & Radionuclides & ${ }^{2 n} \mathrm{Th}$ & $1.1 \mathrm{E}-02$ & $5.2 \mathrm{E}-04$ & \\
\hline S\&M Worker & Smear & Whole Building & External Exposure & Radionuclides & 23s25 U & $1.8 \mathrm{E}-02$ & 8.1E-04 & \\
\hline S\&M Worker & Smear & Whole Building & External Exposure & Radionuclides & ${ }^{2 s s} \mathrm{U}$ & $1.8 \mathrm{E}-03$ & 8.1E-05 & \\
\hline S\&M Worker & Smear & Whole Building & External Exposure & Radionuclides & ${ }^{230} \mathrm{Pu}$ & 9.5E-03 & 4.3E-04 & \\
\hline S\&M Worker & Smear & Whole Building & External Exposure & Radionuclides & ${ }^{234} \mathrm{U}$ & $2.5 \mathrm{E}-02$ & $1.1 E-03$ & \\
\hline S\&M Worker & Smear & Whole Building & External Exposure & Redionuclides & ${ }^{290200 \mathrm{Pu}}$ & $5.8 \mathrm{E}-02$ & $2.6 \mathrm{E}-03$ & \\
\hline S\&M Worker & Smear & Whole Building & External Exposure & Radionuclides & ${ }^{211} \mathrm{Am}$ & $7.4 \mathrm{E}-03$ & 3.1E-04 & \\
\hline S\&M Worker & Smear & Whole Building & External Exposure & Radionuclides & ${ }^{\infty} \mathrm{K}$ & $4.2 \mathrm{E}-01$ & $1.9 \mathrm{E}-02$ & \\
\hline
\end{tabular}

- Units are pCi/g

- Units are pCi-day 


\begin{tabular}{|c|c|c|c|c|c|c|c|c|}
\hline \multirow{2}{*}{ Receptor } & \multirow{2}{*}{ Medin } & \multirow{2}{*}{ Location } & \multirow{2}{*}{ Exposure Pathway } & \multirow{2}{*}{$\begin{array}{c}\text { Contaminant } \\
\text { Class }\end{array}$} & \multirow{2}{*}{ Contaminant } & \multirow{2}{*}{$\begin{array}{l}\text { Exposure Point } \\
\text { Concentration" }\end{array}$} & \multicolumn{2}{|c|}{ Chronic Daily Intakes } \\
\hline & & & & & & & $\begin{array}{l}\text { Carcinogenic } \\
\text { Efrects }\end{array}$ & $\begin{array}{c}\text { Noocarcinogenic } \\
\text { Effects }\end{array}$ \\
\hline S\&M Worker & Smear & Whole Building & Extemal Exposure & Radionuclides & ${ }^{\infty} \mathrm{Co}$ & $1.0 \mathrm{E}-02$ & 4.7E-04 & \\
\hline S\&M Worker & Smear & Whole Building & External Exposure & Radionuclides & ${ }^{\infty} \mathrm{Sr}$ & 9.1E-01 & $4.2 E-02$ & \\
\hline
\end{tabular}


THIS PAGE INTENTIONALILY LEFT BLANK 
Table D-8. S\&M Worker Exposure Concentrations and Chronic Lifetime Exposures for TLD Measurements

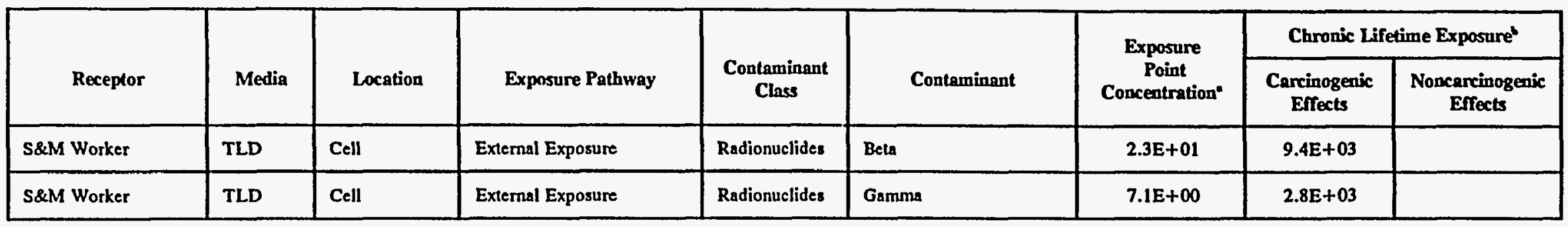

- Units are mrem

- Units are mrem 
THIS PAGE INTENTIONALLY LEFT BLANK 
Table D-9. Adult Trespasser Exposure Concentrations and Chronic Daily Intake Values from Core Samples and Sediment

\begin{tabular}{|c|c|c|c|c|c|c|c|c|}
\hline \multirow{2}{*}{ Receptor } & \multirow{2}{*}{ Media } & \multirow{2}{*}{ Location } & \multirow{2}{*}{ Exposure Pathway } & \multirow{2}{*}{$\begin{array}{c}\text { Contaminant } \\
\text { Class }\end{array}$} & \multirow{2}{*}{ Contaminant } & \multirow{2}{*}{$\begin{array}{l}\text { Exposure Point } \\
\text { Concentration" }\end{array}$} & \multicolumn{2}{|c|}{ Chronic Daily Intake } \\
\hline & & & & & & & $\begin{array}{c}\text { Carcinogenic } \\
\text { Effects }\end{array}$ & $\begin{array}{c}\text { Noncarcinogenic } \\
\text { Effects }\end{array}$ \\
\hline Adult Trespasser & Core & Cell & Ingestion & Inorganics & Arsenic & $2.6 \mathrm{E}+\infty$ & 1.5E-06 & $3.6 \mathrm{E}-06$ \\
\hline Adult Trespasser & Core & Cell & Ingestion & Inorganics & Barium & $7.2 E+02$ & 4.2E-04 & 9.9E-04 \\
\hline Adult Trespasser & Core & Cell & Ingestion & Inorganics & Beryllium & $1.8 \mathrm{E}+01$ & $1.0 \mathrm{E}-05$ & 2.4E-05 \\
\hline Adult Trespasser & Core & Cell & Ingeation & Inorganics & Cadmium & $1.8 \mathrm{E}+02$ & 1.1E-04 & $2.5 \mathrm{E}-04$ \\
\hline Adult Trespasser & Core & Cell & Ingestion & Inorganics & Chromium VI & $2.0 \mathrm{E}+02$ & 1.2E-0.4 & 2.7E-04 \\
\hline Adult Trespasser & Core & Cell & Ingestion & Inorganic: & Manganese & $9.5 E+03$ & $5.6 \mathrm{E}-03$ & $1.3 \mathrm{E}-02$ \\
\hline Adult Trespasser & Core & Cell & Ingestion & Inorganics & Mencury & $3.4 \mathrm{E}-01$ & $2.0 \mathrm{E}-07$ & 4.7E-07 \\
\hline Adult Trespasser & Core & Cell & Ingestion & Inorganics & Nickel & $1.4 E+02$ & 8.1E-05 & $1.9 \mathrm{E}-04$ \\
\hline Adult Trespasser & Core & Cell & Ingestion & Inorganics & Vanadium & $2.4 \mathrm{E}+02$ & 1.4E-04 & 3.3E-04 \\
\hline Adult Trespasser & Core & Cell & Ingestion & Inorganics & Zinc & $3.5 \mathrm{E}+04$ & $2.0 \mathrm{E}-02$ & $4.8 \mathrm{E}-02$ \\
\hline Adult Trespasser & Core & Cell & Ingestion & Organics & Aroclor-1254 & $3.4 \mathrm{E}-01$ & 2.0E-07 & 4.7E-07 \\
\hline Adult Trespasser & Core & Cell & Ingestion & Organics & Aroclor- 1260 & 5.7E-01 & 3.3E-07 & $7.8 \mathrm{E}-07$ \\
\hline Adult Trespasser & Core & Cell & Ingestion & Radionuclides & ${ }^{17} \mathrm{Cs}$ & $1.4 \mathrm{E}+03$ & $1.4 \mathrm{E}+06$ & \\
\hline Adult Trespasser & Core & Cell & Ingestion & Radionuclides & ${ }^{20 \times a}$ & $4.0 \mathrm{E}-01$ & $4.2 \mathrm{E}+02$ & \\
\hline Adult Trespasser & Core & Cell & Ingestion & Radionuclides & ${ }^{2 n} \mathrm{Th}$ & $8.4 \mathrm{E}-01$ & $8.8 E+02$ & \\
\hline Adult Trespasser & Core & Cell & Ingestion & Radionuclides & ${ }^{200} \mathrm{Th}$ & $1.4 \mathrm{E}+00$ & $1.5 \mathrm{E}+03$ & \\
\hline Adult Trespasser & Core & Cell & Ingestion & Radionuclides & ${ }^{202} \mathrm{Th}$ & 8.7E-01 & $9.1 \mathrm{E}+02$ & \\
\hline Adult Trespasser & Core & Cell & Ingeation & Radionuclides & ${ }^{2 \mathrm{~S} / 2 \mathrm{SH}} \mathrm{U}$ & $1.3 \mathrm{E}+\infty$ & $1.4 E+03$ & \\
\hline Adult Trespaser & Core & Cell & Ingeution & Radionuclides & ${ }^{24} \mathrm{Th}$ & $6.3 E+\infty 0$ & $6.6 E+03$ & \\
\hline
\end{tabular}

- Units are mg/kg for inorganics and organics; $\mathrm{pCi} / \mathrm{g}$ for radionuclides

- Units are mg/kg-day for inorganics and organics; pCi-day for radionuclides 


\begin{tabular}{|c|c|c|c|c|c|c|c|c|}
\hline \multirow{2}{*}{ Receptor } & \multirow{2}{*}{ Media } & \multirow{2}{*}{ Location } & \multirow{2}{*}{ Exposure Pathway } & \multirow{2}{*}{$\begin{array}{c}\text { Contaminant } \\
\text { Class }\end{array}$} & \multirow{2}{*}{ Contaminant } & \multirow{2}{*}{$\begin{array}{l}\text { Exposure Point } \\
\text { Concentration" }\end{array}$} & \multicolumn{2}{|c|}{ Chronic Daily Intoket } \\
\hline & & & & & & & $\begin{array}{c}\text { Cancinogenic } \\
\text { Erfects }\end{array}$ & $\begin{array}{c}\text { Noncarcinogenic } \\
\text { Erfects }\end{array}$ \\
\hline Adult Trespasser & Core & Cell & Ingestion & Radionuclides & ${ }^{2 s s} \mathrm{U}$ & $1.2 \mathrm{E}-01$ & $1.3 E+02$ & \\
\hline Adult Trespasser & Core & Cell & Ingestion & Radionuclides & ${ }^{24} \mathrm{Pu}$ & 4.5E-01 & $4.7 E+02$ & \\
\hline Adult Trespasser & Core & Cell & Ingestion & Radionuclides & ${ }^{234} \mathrm{U}$ & $1.7 E+00$ & $1.8 \mathrm{E}+03$ & \\
\hline Adult Trespasser & Core & Cell & Ingestion & Radionuclides & ${ }^{2535200 \mathrm{Pu}}$ & $4.0 \mathrm{E}+00$ & $4.2 \mathrm{E}+03$ & \\
\hline Adult Trespasser & Core & Cell & Ingestion & Radionuclides & ${ }^{4} \mathrm{~K}$ & $5.3 E+00$ & $5.5 E+03$ & \\
\hline Adult Trespasser & Core & Cell & Ingestion & Radionuclides & ${ }^{\infty} \mathrm{Co}$ & $1.3 \mathrm{E}-01$ & $1.4 \mathrm{E}+02$ & \\
\hline Adult Trespasser & Core & Cell & Ingestion & Radionuclides & ${ }^{\infty} \mathrm{Sr}$ & $1.2 \mathrm{E}+01$ & $1.2 \mathrm{E}+04$ & \\
\hline Adult Trespasser & Core & Cell & Ingestion & Radionuclides & Tritium & 2.1E+01 & $2.2 \mathrm{E}+04$ & \\
\hline Adult Trespasser & Core & Cell & Dermal & Inorganics & Arsenic & $2.6 \mathrm{E}+\infty 0$ & 8.1E-08 & $1.9 \mathrm{E}-07$ \\
\hline Adult Trespasser & Core & Cell & Dermal & Inorganics & Barium & $7.2 \mathrm{E}+02$ & $2.2 \mathrm{E}-05$ & $5.2 \mathrm{E}-05$ \\
\hline Adult Trespasser & Core & Cell & Dermal & Inorganics & Beryllium & $1.8 \mathrm{E}+0 \mathrm{t}$ & 5.5E-07 & $1.3 \mathrm{E}-06$ \\
\hline Adult Trespasser & Core & Cell & Dermal & Inorganics & Cadmium & $1.8 \mathrm{E}+02$ & $5.6 \mathrm{E}-06$ & 1.3E-05 \\
\hline Adult Treopazsci & Core & Cell & Demal & Inorganics & Chromium VI & $2.0 E+02$ & 6.1E-Q & $1.45-05$ \\
\hline Adult Trespasser & Core & Cell & Dermal & Inorganics & Manganese & $9.5 E+03$ & $3.0 \mathrm{E}-04$ & $6.9 \mathrm{E}-04$ \\
\hline Adult Trespasser & Core & Cell & Dermal & Inorganics & Mercury & $3.4 \mathrm{E}-01$ & $1.1 \mathrm{E}-08$ & $2.5 \mathrm{E}-08$ \\
\hline Adult Trespasser & Core & Cell & Dermal & Inorganics & Nickel & $1.4 \mathrm{E}+02$ & 4.3E-06 & $1.0 \mathrm{E}-05$ \\
\hline Adult Trespasser & Core & Cell & Dermal & Inorganics & Vanadium & $2.4 \mathrm{E}+02$ & $7.5 \mathrm{E}-06$ & 1.7E-05 \\
\hline Adult Trespasser & Core & Cell & Dermal & Inorganics & Zine & $3.5 E+04$ & $1.1 \mathrm{E}-03$ & $2.5 \mathrm{E}-03$ \\
\hline Adult Trespasser & Core & Cell & Dermal & Organics & Aroclor-1254 & $3.4 \mathrm{E}-01$ & $1.1 \mathrm{E}-07$ & $2.5 \mathrm{E}-07$ \\
\hline Adult Trespasser & Core & Cell & Dermal & Organics & Aroclor-1260 & $5.7 \mathrm{E}-01$ & $1.8 \mathrm{E}-07$ & 4.1E-07 \\
\hline Adult Trespasser & Core & Cell & Inhalation & Inorganics & Arsenic & $2.6 \mathrm{E}+00$ & $7.1 E-11$ & $1.7 \mathrm{E}-10$ \\
\hline
\end{tabular}

- Units are $\mathrm{mg} / \mathrm{kg}$ for inorganics and organics; $\mathrm{pCi} / \mathrm{g}$ for radionuclides

- Units are mg/kg-day for inorganics and organicn; pCi-day for radionuclides 


\begin{tabular}{|c|c|c|c|c|c|c|c|c|}
\hline \multirow{2}{*}{ Receptor } & \multirow{2}{*}{ Media } & \multirow{2}{*}{ Location } & \multirow{2}{*}{ Exposare Pathway } & \multirow{2}{*}{$\begin{array}{c}\text { Contaminant } \\
\text { Class }\end{array}$} & \multirow{2}{*}{ Contaminant } & \multirow{2}{*}{$\begin{array}{l}\text { Exposure Point } \\
\text { Concentration" }\end{array}$} & \multicolumn{2}{|c|}{ Chronic Daily Intaket } \\
\hline & & & & & & & $\begin{array}{c}\text { Cancinogenic } \\
\text { Enfects }\end{array}$ & $\begin{array}{c}\text { Noncarcinogenic } \\
\text { Effects }\end{array}$ \\
\hline Adult Trespasser & Core & Cell & Inhalation & Inorganic: & Barium & $7.2 \mathrm{E}+02$ & $2.0 \mathrm{E}-08$ & $4.6 \mathrm{E}-08$ \\
\hline Adult Trespasser & Core & Cell & Inhalation & Inorganics & Beryllium & $1.8 \mathrm{E}+01$ & $4.8 \mathrm{E}-10$ & 1.1E-09 \\
\hline Adult Trespasser & Core & Cell & Inhalation & Inorganics & Cadmium & $1.8 \mathrm{E}+02$ & $\quad 4.9 \mathrm{E}-09$ & $1.2 \mathrm{E}-08$ \\
\hline Adult Trespasser & Core & Cell & Inhalation & Inorganics & Chromium VI & $2.0 \mathrm{E}+02$ & $5.4 \mathrm{E}-09$ & $1.3 \mathrm{E}-08$ \\
\hline Adult Trespasser & Core & Cell & Inhalation & Inorganics & Manganese & $9.5 E+03$ & $2.6 \mathrm{E}-07$ & $6.1 \mathrm{E}-07$ \\
\hline Adult Trespasser & Core & Cell & Inhalation & Inorganics & Mercury & $3.4 \mathrm{E}-01$ & $9.3 \mathrm{E}-12$ & $2.2 \mathrm{E}-11$ \\
\hline Adult Trespasser & Core & Cell & Inhalation & Inorganics & Nickel & $1.4 \mathrm{E}+02$ & $3.8 \mathrm{E}-09$ & $8.8 \mathrm{E}-09$ \\
\hline Adult Trespasser & Core & Cell & Inhalation & Inorganice & Vanadium & $2.4 \mathrm{E}+02$ & $6.6 \mathrm{E}-09$ & $1.5 \mathrm{E}-08$ \\
\hline Adult Trespasser & Core & Cell & Inhalation & Inorganics & Zine & $3.5 E+04$ & 9.6E-07 & $2.2 \mathrm{E}-06$ \\
\hline Adult Trespasser & Core & Cell & Inhalation & Organics & Aroclor-1254 & $3.4 \mathrm{E}-01$ & 9.3E-12 & $2.2 \mathrm{E}-11$ \\
\hline Adult Trespasser & Core & Cell & Inhalation & Organics & Aroclor- 1260 & 5.7E-01 & $1.6 \mathrm{E}-11$ & $3.6 \mathrm{E}-11$ \\
\hline Adull Trespasser & Core & Cell & Inhalation & Radionuclides & ${ }^{17} \mathrm{Cs}$ & $1.4 \mathrm{E}+03$ & $6.7 \mathrm{E}+01$ & \\
\hline Adult Trespasser & Core & Cell & Inhalation & Radionuclides & ${ }^{205} \mathbf{R a}$ & $4.0 \mathrm{E}-01$ & $2.0 \mathrm{E}-02$ & \\
\hline Adult Trespasser & Core & Cell & Inhalation & Radionuclides & ${ }^{20} \mathrm{Th}$ & $8.4 \mathrm{E}-01$ & $4.1 \mathrm{E}-02$ & \\
\hline Adull Trespasser & Core & Cell & Inhalation & Radionuclides & ${ }^{250} \mathrm{Th}$ & $1.4 \mathrm{E}+00$ & $6.9 \mathrm{E}-02$ & \\
\hline Adult Trespasser & Core & Cell & Inhalation & Radionuclide: & ${ }^{25} \mathrm{Th}$ & 8.7E-01 & $4.3 \mathrm{E}-02$ & \\
\hline Adult Trespasser & Core & Cell & Inhalation & Radionuclide: & ${ }^{23} 3 / 2 \times$ & $1.3 \mathrm{E}+00$ & $6.4 \mathrm{E}-02$ & \\
\hline Adult Trespasser & Core & Cell & Inhalation & Radionuclides & ${ }^{23} \mathrm{Th}$ & $6.3 \mathrm{E}+00$ & $3.1 \mathrm{E}-01$ & \\
\hline Adult Trespasser & Core & Cell & Inhalation & Radionuclides & ${ }^{231} \mathrm{U}$ & $1.2 \mathrm{E}-01$ & $5.9 \mathrm{E}-03$ & \\
\hline Adult Trespasser & Core & Cell & Inhalation & Radionuclides & ${ }^{231} \mathrm{Pu}$ & $4.5 \mathrm{E}-01$ & $2.2 \mathrm{E}-02$ & \\
\hline Adult Trespasser & Core & Cell & Inhalation & Radionuclides & ${ }^{230} U$ & $1.7 E+\infty 0$ & 8.3E-02 & \\
\hline
\end{tabular}

- Units are $\mathrm{mg} / \mathrm{kg}$ for inorganics and organics; $\mathrm{pCi} / \mathrm{g}$ for radionuclides

- Units are $\mathrm{mg} / \mathrm{kg}$-day for inorganics and organica; pCi-day for redionuclides 


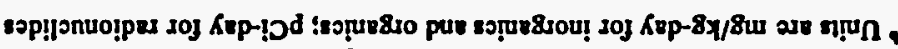
sop!|’nuo!pes soj 8/!

\begin{tabular}{|c|c|c|c|c|c|c|c|c|}
\hline $9031 \%$ & $90-98^{\circ} 1$ & $00+30^{\circ} \varepsilon$ & 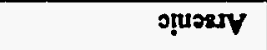 & 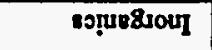 & นo!̣คว8น & KIOIएD I80G & 200 & LORendesI I UnPV \\
\hline & $20+96^{\circ} 6$ & $10+31 \cdot 2$ & wn!̣!̣I & sp!|jnuo!pry & ansodxg jpuropxg & \|IDO & 2100 & Isesedsarl unpY \\
\hline & $20+3 L \cdot 2$ & $10+32^{\circ} 1$ & ${ }^{I} S_{\infty 6}$ & sop!|ønuo!pry & 2unsodxg /EusolXg & IIग0 & 200 & 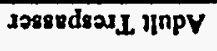 \\
\hline & $00+\exists 0^{\circ} \varepsilon$ & $|0-\exists E \cdot|$ & $\circ \supset_{0 \phi}$ & sop!|эnuo!pey & annsodxg jpurojxg & $\| 100$ & 200 & Lossedsal L unpY \\
\hline & $20+32 \cdot 1$ & $00+3 \varepsilon^{\circ} S$ & $\mathbf{X}_{0+}$ & epp!|วnuo!pry & 2unsodx马 ןนwอIXg & IIPS & 2305 & Josezds2I L UnpV \\
\hline & $10+32 \cdot 6$ & $00+30^{\circ} 6$ & $n_{d_{\text {oreace }}}$ & sop!|गnuo!pry & annsodxg jourojxg & IIPO & 2003 & Iossudsay J JupV \\
\hline & $10+36^{\circ} \varepsilon$ & $00+3<\cdot I$ & $\mathbf{n}_{\text {orq }}$ & sop!!onuo!pry & 2unsodxg [ซuspixg & IIPS & 2503 & Jossods21L נInPY \\
\hline & $10+30.1$ & $10-35 \%$ & $n_{d_{\text {orzz }}}$ & sәp!|ønuo!pvy & a)nsodxg IrWoLXg & IIPS & 200 & Jossedsarl unpV \\
\hline & $00+38 \cdot \tau$ & $\bar{i} 0 \bar{a} c^{\prime} i$ & $\mathrm{n}_{\mathrm{s} s t}$ & 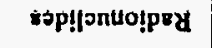 & 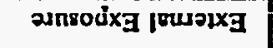 & IIPO & 200 & 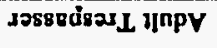 \\
\hline & $20+951$ & $00+3 E^{*} 9$ & $4 L_{k z}$ & sop!|ønuo!pry & ansodxg I"uwovxg & $\| \oplus 0$ & 2100 & J08sedsas I JnPY \\
\hline & $10+30^{\circ} \varepsilon$ & $00+\exists \varepsilon^{\prime} I$ & $\mathrm{n}_{\text {rezsiz }}$ & sop!|jnuo!ppy & 2snsodxg [vusolxg & \|थכ & มू० & 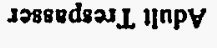 \\
\hline & $10+30^{\circ} 2$ & $10-36 \cdot 8$ & $\mathbf{H L}_{\mathrm{tx} e}$ & sop!|onuo!pry & 2Insodx J IRuPLX & Iथ0כ| & 210 & J2888ds21 L J JnPY \\
\hline & $10+3 \tau \cdot \varepsilon$ & $00+9+1$ & ULxt & sop!|คnuo!̣pey & ansodxg [pusopg & IIPכ & 2103 & Issgedes_ I UnpY \\
\hline & $10+36 \cdot 1$ & $10-96 \cdot 8$ & $4 L_{6 \pi}$ & sop!|jonuo!pry & ansodx I ןWUOLX & Iיכ & 2100 & JPrgedsax I JnPY \\
\hline & $00+32 \cdot 6$ & $10-30.6$ & $\mathbf{y}_{x a x}$ & sop!!onuo!pry & ansodx $\mathrm{g}$ | & כיכון & 2sos. & JPsspds21 I IInPY \\
\hline & $t 0+3 \tau^{\prime} \varepsilon$ & $\varepsilon 0+3 \boldsymbol{\bullet}^{\circ} \mathbf{I}$ & $\nu_{\Delta s 1}$ & eวp!|วnuo!pyy & asnsodx I IEwopxg & $\|$ Iיכ & 2100 & 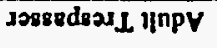 \\
\hline & $00+30^{\circ} I$ & $10+31 \cdot 2$ & un!!uฺ I & sop!jonuo!pry & นo!̣|р|вนU] & IIP & 2soग & IFrevds21 IInPY \\
\hline & $10-9 L ' S$ & $10+\exists 2 \cdot 1$ & ${ }^{2} S_{\infty}$ & esp!|onuo!pry & uo!̣p|вчप1 & IIPO & 2005 & Joserdosil UInPY \\
\hline & $\varepsilon 0-3 b^{\prime} 9$ & IO-AE' I & $0 D_{\infty}$ & eวp!|эnuo!pry & 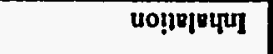 & $\| P 0$ & 2500 & JPSERdsaIL unpY \\
\hline & $10-392$ & $00+9 € \cdot s$ & $\mathrm{SH}_{00}$ & sop!!onuo!pry & uo!ाр|पया & $\| \boldsymbol{D O}$ & 2003 & 208sedsad I UnpY \\
\hline & $10-90 \cdot \tau$ & $00+30^{\circ}$ & ndorecort & esp!pnuo!pry & 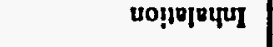 & $\|$ IP & 250ग & Isserdsas L UInPV \\
\hline 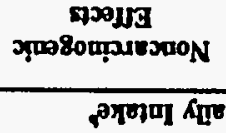 & 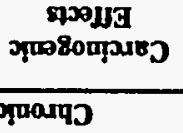 & 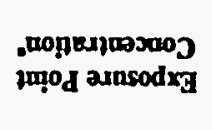 & 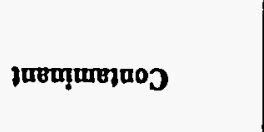 & 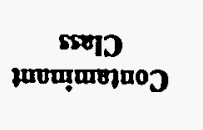 & SOMqPBd ansodxg & uopisoon & ซฺค & دoxdə30y \\
\hline
\end{tabular}




\begin{tabular}{|c|c|c|c|c|c|c|c|c|}
\hline \multirow{2}{*}{ Receptor } & \multirow{2}{*}{ Media } & \multirow{2}{*}{ Location } & \multirow{2}{*}{ Exposure Pathway } & \multirow{2}{*}{$\begin{array}{l}\text { Contaminant } \\
\text { Class }\end{array}$} & \multirow{2}{*}{ Contaminant } & \multirow{2}{*}{$\begin{array}{l}\text { Exposure Point } \\
\text { Concentration" }\end{array}$} & \multicolumn{2}{|c|}{ Chronic Daily Intake } \\
\hline & & & & & & & $\begin{array}{l}\text { Carcinogenic } \\
\text { Effects }\end{array}$ & $\begin{array}{l}\text { Noncarcinogenic } \\
\text { Effects }\end{array}$ \\
\hline Adult Trespasser & Core & Earr Gallery & Ingestion & Inorganics & Barium & $2.1 E+01$ & 1.3E-05 & 2.9E-05 \\
\hline Adult Trespasser & Core & East Gallery & Ingestion & Inorganics & Beryllium & 4.0E-01 & $2.3 E-07$ & 5.5E-07 \\
\hline Adult Trespasser & Core & East Gallery & Ingertion & Inorganics & Cadmium & $5.1 E+\infty$ & $3.0 \mathrm{E}-06$ & $7.0 \mathrm{E}-06$ \\
\hline Adult Trespasser & Core & East Gallery & Ingestion & Inorganics & Chromium VI & $8.3 E+\infty 0$ & 4.9E-06 & $1.1 \mathrm{E}-05$ \\
\hline Adult Trespasser & Core & East Gallery & Ingestion & Inorganice & Manganere & $2.7 E+02$ & $1.6 \mathrm{E}-04$ & $3.6 \mathrm{E}-04$ \\
\hline Adult Trespasser & Core & East Gallery & Ingestion & Inorganics & Nickel & $6.0 \mathrm{E}+00$ & $3.5 E-06$ & $8.2 \mathrm{E}-06$ \\
\hline Adult Trespasser & Core & East Gallery & Ingestion & Inorganics & Vanadium & $9.1 \mathrm{E}+\infty$ & $5.3 \mathrm{E}-06$ & $1.2 \mathrm{E}-0 \mathrm{~S}$ \\
\hline Adult Trespasser & Core & East Gallery & Ingestion & Inorganics & Zine & $9.8 \mathrm{E}+02$ & 5.7E-04 & $1.3 \mathrm{E}-03$ \\
\hline Adult Trespasser & Cone & East Gallery & Ingestion & Organica & Aroclor-1254 & $1.7 \mathrm{E}+00$ & $1.0 \mathrm{E}-06$ & 2.3E-06 \\
\hline Adult Trespasser & Core & East Gallery & Ingestion & Organics & Aroclor-1260 & $2.6 \mathrm{E}+\infty$ & $1.5 E-06$ & $3.6 \mathrm{E}-06$ \\
\hline Adult Trespasser & Core & East Gallery & Ingestion & Organics & Bis(2-ethylhexyl)phthalate & $2.4 \mathrm{E}-01$ & $1.4 \mathrm{E}-07$ & 3.3E-07 \\
\hline Adult Trespasser & Core & East Gallery & Ingestion & Organica & Dimethyl phthalate & 5.5E-02 & 3.2E-08 & 7.5E-08 \\
\hline Adult Trespasser & Core & East Gallery & Ingestion & Radionuclider & ${ }^{17} \mathrm{Cs}$ & $2.3 E+01$ & $2.4 E+04$ & \\
\hline Adull Trespasser & Core & East Gallery & Ingestion & Redionuclides & ${ }^{2 \times} \mathrm{Ra}$ & $3.6 \mathrm{E}-01$ & $3.8 \mathrm{E}+02$ & \\
\hline Adull Trespasser & Core & East Gallery & Ingestion & Radionuclides & ${ }^{n a_{R a}}$ & 3.7E-0t & $3.9 E+02$ & \\
\hline Adult Trespasser & Core & East Gallery & Ingestion & Radionuclides & ${ }^{20} \mathrm{Th}$ & $5.6 \mathrm{E}-01$ & $5.9 E+02$ & \\
\hline Adult Trespasser & Core & East Gallery & Ingestion & Radionuclides & ${ }^{200} \mathrm{Th}$ & $1.2 E+\infty$ & $1.3 E+03$ & \\
\hline Adult Trespasser & Core & East Gallery & Ingestion & Radionuclides & ${ }^{25} \mathrm{Th}$ & 4.3E-01 & $4.5 \mathrm{E}+02$ & \\
\hline Adule Trespasser & Core & East Gallery & Ingestion & Radionuclides & ${ }^{23 s 2 s} U$ & 9.0E-01 & $9.5 \mathrm{E}+02$ & \\
\hline Adult Trespasser & Core & East Gallery & Ingestion & Radionuclides & ${ }^{28} U$ & 7.7E-01 & $8.1 E+02$ & \\
\hline Adult Trespasser & Core & Eaxt Gallery & Ingertion & Radionuclides & ${ }^{2{ }^{\prime}} \mathrm{Am}$ & $5.0 \mathrm{E}-01$ & $5.3 E+02$ & \\
\hline
\end{tabular}

- Units are $\mathrm{mg} / \mathrm{kg}$ for inorganics and organics; $\mathrm{pCi} / \mathrm{g}$ for radionuclides

- Units are mg/kg-day for inorganics and organics; pCi-day for radionuclides 


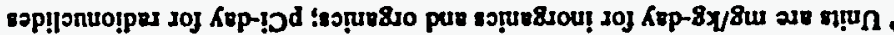

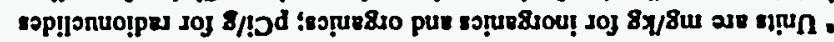

\begin{tabular}{|c|c|c|c|c|c|c|c|c|}
\hline OI-GE'S & OI-AEZ & $00+\exists \varepsilon: 8$ & In wn!̣ayy & sọur8sou & uo!̣v|pчuा & SUग|PD भorg & 2100 & IFrendes] IInPY \\
\hline 0โ-รย์ $\varepsilon$ & $0 I-\mathbf{g} b^{*} \mathrm{I}$ & $00+g l ' s$ & un!̣upro & sp̣un8sou & uo!̣v|84ण & KJग|rD $180 \mathrm{~g}$ & 200 & Js8sedsal I ynpY \\
\hline II- $99^{\circ} \tau$ & $\left|I-A I^{\top}\right|$ & $10-30^{\circ}$ & wn!||Kงอg & sọungsouI & uo!!p|вquI & KIOIIDD 280 & 2200 & I98sedsad L. UnPY \\
\hline $60-9 b^{\circ} 1$ & $0 \mathrm{I}-36 . \mathrm{S}$ & $10+9 I^{\prime} z$ & unụeg & sง!̣u810u & uo!ाp|вцй & 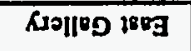 & 200 & IOSspdsaI I UnPY \\
\hline $01-96.1$ & $1 t-a Z^{\prime} 8$ & $00+30^{\circ} \varepsilon$ & ग̣usssy & 8ง!̣น8souI & U0!ाק|в४यI & 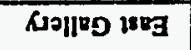 & 2100 & JPssedsas L JupV \\
\hline $80-30^{\circ} 6$ & $80-9 L \cdot 1$ & $20.35 \%$ & 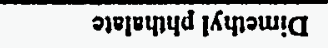 & sọue20 & ןruwra & SIVIPD 280 & 200 & 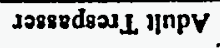 \\
\hline$\angle 0^{-} \mathbf{B} L^{\circ} \mathbf{I}$ & 80-95:L & $10-3 b^{\circ} 2$ & 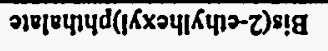 & รง!นะ810 & Iвurea & אगाएD 1809 & 2100 & Jossuds2II $\mid \mathrm{npV}$ \\
\hline $90-36.1$ & LO-GI'8 & $00+99 \cdot 2$ & $0921-3010017$ & 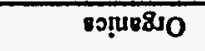 & forusa & KLIIPD ISRG & 2100 & IOsseds2xI Unp \\
\hline sag?! & LOAES & $00+a ! \cdot !$ & $4521-20 ! 021 y$ & 8อุug?10 & fruvea & Geffpe 18吗 & 202 & IọssodsarI yุnpy \\
\hline SO-AI. $\mathrm{L}$ & SO- $\exists 0^{\circ} \varepsilon$ & $20+98 \cdot 6$ & งu!Z & sọuegioul & |вщшश्व & KIO|णD $250 \mathrm{~g}$ & 2100 & JossedsadL נ]npV \\
\hline LO-39.9 & $20-38 \cdot \tau$ & $00+31 \cdot 6$ & un!pruv $\Lambda$ & 8ง!̣uR8souI & ןвurग्व & KIDIPS $180 \mathrm{~g}$ & 2100 & sossedsax $\perp$ JInp \\
\hline LO-36'b & $\angle 0-36 \cdot \mid$ & $00+30.9$ & PYO!N & 80!1น8810uI & poused & KUIIPD IS & 2100 & Lossedsos L Unp \\
\hline $50-36 \cdot 1$ & $90-32 \cdot 8$ & $20+3 L \cdot \tau$ & orour8unW & 8ง̣uอ8ి10uI & Irrured & KLग|"D 18Rg & 2005 & 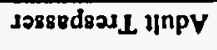 \\
\hline$\angle 0^{-30^{\circ} 9}$ & $20-39 \cdot 2$ & $00+\exists \varepsilon^{\prime} 8$ & 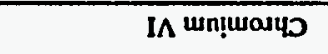 & sọur8soul & prus? & Aมग|"D I8Rg & 2soग & J28sedsos $L$ Unpy \\
\hline$\angle O-G \angle \cdot E$ & $20-39 \cdot 1$ & $00+3 I \cdot s$ & 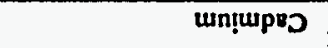 & sọue8soul & 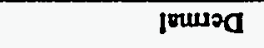 & 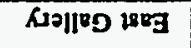 & 200 & 1058eds21 I UnpV \\
\hline $80-36 \cdot 2$ & $80-3 \tau^{\circ} 1$ & $10-30^{\circ} \mathrm{b}$ & un!!|Kuog & sọup810uT & poursa & 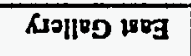 & 200 & Iosszdeas L unpy \\
\hline $90^{\circ}-19^{\circ} \mathrm{I}$ & $\angle O-G L^{\prime} 9$ & $10+9 I \%$ & unupg & sग̣ur\&souI & gruwed & KUगाPD भBg & 2005 & JPssedsed I UnpY \\
\hline $20-32: 2$ & $80-3 E^{\circ} 6$ & $00+30^{\circ} \varepsilon$ & 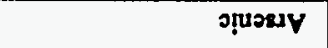 & งอ̣ue8souI & jouwsa & KIDIPD 198G & 2003 & J288zdB2J $\perp$ UnPV \\
\hline & $60+3 L 2$ & $10+99^{\circ} 2$ & un!!!̣ L & sop!|onuo!ped & to|̣รso8uI & 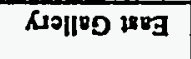 & 2100 & Jrsegdes L Jnpy \\
\hline & $60+358$ & $00+3 I^{\prime} \cdot 8$ & ${ }^{2} S_{\infty s}$ & eop!|onuotpry & น0!̣เรอ8นI & 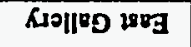 & 2503 & JPsepds2JI InPY \\
\hline & $\mathrm{EO}+39^{\prime} \mathrm{S}$ & $00+3 b^{\circ} s$ & $\mathrm{X}_{0 r}$ & ep!||กnuo!pyy & uọ!so8uI & KUગા"D 200马 & asoj & dFsendeaj I JnPY \\
\hline 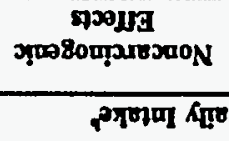 & 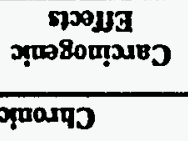 & 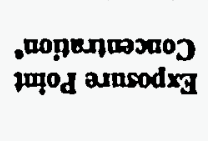 & 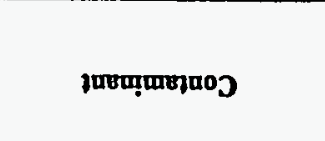 & 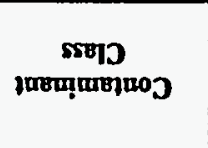 & kempled ansodxg & aog6sot & v!paW & دохdวээy \\
\hline
\end{tabular}




\begin{tabular}{|c|c|c|c|c|c|c|c|c|}
\hline \multirow{2}{*}{ Receptor } & \multirow{2}{*}{ Media } & \multirow{2}{*}{ Location } & \multirow{2}{*}{ Exposure Pathway } & \multirow{2}{*}{$\begin{array}{c}\text { Contaminant } \\
\text { Class }\end{array}$} & \multirow{2}{*}{ Contaminant } & \multirow[b]{2}{*}{$\begin{array}{l}\text { Exposure Point } \\
\text { Concentration" }\end{array}$} & \multicolumn{2}{|c|}{ Chronic Daily Intake } \\
\hline & & & & & & & $\begin{array}{c}\text { Carcinogenic } \\
\text { Efrects }\end{array}$ & $\begin{array}{c}\text { Noncarcinogenic } \\
\text { Effects }\end{array}$ \\
\hline Adult Trespasser & Core & Ennt Gallery & Inhalation & Inorganica & Manganese & $2.7 \mathrm{E}+02$ & $7.3 E-09$ & $1.7 E-08$ \\
\hline Adult Trespasser & Core & East Gallery & Inhalation & Inorganics & Nickel & $6.0 \mathrm{E}+00$ & $1.6 \mathrm{E}-10$ & 3.8E-10 \\
\hline Adult Trespasser & Core & East Gallery & Inhalation & Inorganics & Vanadium & $9.1 E+\infty 0$ & $-\quad 2.5 \mathrm{E}-10$ & $5.8 \mathrm{E}-10$ \\
\hline Adult Trespasser & Core & East Gallery & Inhalation & Inorganics & Zinc & $9.8 \mathrm{E}+02$ & 2.7E-08 & $6.3 \mathrm{E}-08$ \\
\hline Adult Trespasser & Core & East Gallery & Inhalation & Organics & Aroclor-1254 & $1.7 \mathrm{E}+\infty$ & $4.7 \mathrm{E}-11$ & $1.1 \mathrm{E}-10$ \\
\hline Adult Trespasser & Core & East Gallery & Inhalation & Organics & Aroclor- 1260 & $2.6 E+\infty$ & $7.1 \mathrm{E}-11$ & $1.7 \mathrm{E}-10$ \\
\hline Adult Trespasser & Core & East Gallery & Inhalation & Organics & Bis(2-ethylhexyi)phthalate & $2.4 \mathrm{E}-01$ & $6.6 \mathrm{E}-12$ & $1.5 \mathrm{E}-11$ \\
\hline Adult Trespasser & Core & East Gallery & Inhalation & Organics & Dimethyl phthalate & $5.5 \mathrm{E}-02$ & $1.5 \mathrm{E}-12$ & $3.5 \mathrm{E}-12$ \\
\hline Adult Trespasser & Core & East Gallery & Inhalation & Radionuclides & ${ }^{n+} \mathrm{Cs}$ & $2.3 \mathrm{E}+01$ & $1.1 \mathrm{E}+00$ & \\
\hline Adult Trespasser & Core & East Gallery & Inhalation & Radionuclides & ${ }^{2 x} \mathrm{Ra}$ & $3.6 \mathrm{E}-01$ & $1.8 \mathrm{E}-02$ & \\
\hline Adult Trespasser & Core & East Gallery & Inhalation & Radionuclides & ${ }^{n_{\mathbf{R}}}$ & 3.7E-01 & $1.8 \mathrm{E}-02$ & \\
\hline Adult Trespasser & Core & East Gallery & Inhalation & Radionuclides & ${ }^{2 x} \mathrm{Th}$ & $5.6 \mathrm{E}-01$ & 2.7E-02 & \\
\hline Adult Trespasser & Core & East Gallery & Inhalation & Radionuclides & ${ }^{200} \mathrm{Th}$ & $1.2 \mathrm{E}+00$ & $5.9 \mathrm{E}-02$ & \\
\hline Adult Trespasser & Core & East Gallery & Inhalation & Radionuclides & ${ }^{2 n} \mathrm{Th}$ & 4.3E-01 & 2.1E-02 & \\
\hline Adult Trespasser & Core & East Gallery & Inhalation & Radionuclides & ${ }^{23325} \mathrm{U}$ & $9.0 \mathrm{E}-01$ & $4.4 \mathrm{E}-02$ & \\
\hline Adult Trespasser & Core & East Gallery & Inhalation & Radionuclides & ${ }^{23+} \mathrm{U}$ & 7.7E-01 & $3.8 \mathrm{E}-02$ & \\
\hline Adult Trespasser & Core & East Gallery & Inhalation & Radionuclides & ${ }^{2 n 1} \mathrm{Am}$ & $5.0 \mathrm{E}-01$ & 2.5E-02 & \\
\hline Adult Trespasser & Core & East Gallery & Inhalation & Radionuclides & ${ }^{\infty} \mathrm{K}$ & $5.4 \mathrm{E}+00$ & 2.6E-01 & \\
\hline Adult Trespasser & Core & Enst Gallery & Inhalation & Radionuclides & ${ }^{\infty} \mathrm{Sr}$ & $8.1 \mathrm{E}+00$ & $4.0 \mathrm{E}-01$ & \\
\hline Adult Trespasser & Core & Enat Gallery & Inhalation & Radionuclides & Tritium & $2.6 \mathrm{E}+01$ & $1.3 \mathrm{E}+00$ & \\
\hline Adult Trespasser & Core & Eant Gallery & Extemal Exposure & Radionuclides & ${ }^{n} \mathrm{C}$. & $2.3 E+01$ & $5.2 E+02$ & \\
\hline
\end{tabular}

- Unils are $\mathrm{mg} / \mathrm{kg}$ for inorganics and organica; $\mathrm{pCi} / \mathrm{g}$ for radionuclides

- Units are mg/kg-day for inorganics and organics; pCi-day for radionuclides 


\begin{tabular}{|c|c|c|c|c|c|c|c|c|}
\hline \multirow{2}{*}{ Receptor } & \multirow{2}{*}{ Metia } & \multirow{2}{*}{ Location } & \multirow{2}{*}{ Exposure Pathway } & \multirow{2}{*}{$\begin{array}{l}\text { Contaminant } \\
\text { Class }\end{array}$} & \multirow{2}{*}{ Contaminant } & \multirow{2}{*}{$\begin{array}{l}\text { Exposure Point } \\
\text { Concentration" }\end{array}$} & \multicolumn{2}{|c|}{ Chronic Daily Intake' } \\
\hline & & & & & & & $\begin{array}{c}\text { Carcinogenic } \\
\text { Effects }\end{array}$ & $\begin{array}{c}\text { Noncarcinogenic } \\
\text { Erfects }\end{array}$ \\
\hline Adult Trespanser & Coro & Eant Gallery & External Exposure & Radionuclides & ${ }^{20} \mathrm{Re}$ & $3.6 \mathrm{E}-01$ & $8.3 \mathrm{E}+00$ & \\
\hline Adull Trespasser & Core & East Gallery & External Exposure & Radionuclides & ${ }^{21} \mathrm{Ra}$ & 3.7E-01 & $8.5 E+00$ & \\
\hline Adult Trespasser & Core & East Gallery & External Exposure & Radionuclides & $220 \mathrm{Th}$ & $5.6 \mathrm{E}-01$ & $1.3 \mathrm{E}+01$ & \\
\hline Adult Trespasser & Core & East Gallery & External Exposure & Radionuclides & ${ }^{200} \mathrm{Th}$ & $1.2 \mathrm{E}+00$ & $2.8 \mathrm{E}+01$ & \\
\hline Adult Trespasser & Core & Eaxt Gallery & Extermal Exposure & Radionuclides & ${ }^{20} \mathrm{Th}$ & 4.3E-01 & $9.9 \mathrm{E}+00$ & \\
\hline Adult Trespasser & Core & East Gallery & External Exposure & Radionuclides & 23252MU & $9.0 \mathrm{E}-01$ & $2.1 E+01$ & \\
\hline Adull Trespasser & Core & East Gallery & Extemal Exposure & Radionuclides & ${ }^{230} \mathrm{U}$ & 7.7E-01 & $1.8 \mathrm{E}+01$ & \\
\hline Adult Trespasser & Core & East Gallery & External Exposure & Radionuclides & ${ }^{211} \mathrm{Am}$ & $5.0 \mathrm{E}-01$ & $1.2 \mathrm{E}+01$ & \\
\hline Adult Trespasser & Core & East Gallery & External Exposure & Radionuclides & ${ }^{\infty} \mathrm{K}$ & $5.4 E+\infty 0$ & $1.2 \mathrm{E}+02$ & \\
\hline Adult Trespasser & Core & East Gallery & External Exposure & Radionuclides & ${ }^{\infty} \mathrm{Sr}$ & $8.1 E+\infty$ & $1.9 \mathrm{E}+02$ & \\
\hline Adult Trespasser & Core & East Gallery & Extemal Exposure & Radionuclides & Tritium & $2.6 \mathrm{E}+01$ & $5.9 \mathrm{E}+02$ & \\
\hline Adult Trespasser & Core & Sediment & Ingestion & Inorganics & Antimony & $3.6 \mathrm{E}+01$ & 2.1E-05 & $5.0 \mathrm{E}-05$ \\
\hline Áuiuit Trespasser & Core & Sediment & ingesion & inorganics & Ârsenic & 2..2̌E+ôt & i. $3 E-05$ & 2. $.9 \bar{E}-0 \overline{5}$ \\
\hline Adult Trespasser & Core & Sediment & Ingestion & Inorganics & Barium & $1.2 \mathrm{E}+02$ & $7.0 \mathrm{E}-05$ & $1.6 \mathrm{E}-04$ \\
\hline Adult Trespasser & Core & Sediment & Ingestion & Inorganics & Cadmium & $6.0 \mathrm{E}+01$ & 3.5E-05 & 8.2E-05 \\
\hline Adult Trespasser & Core & Sediment & Ingestion & Inorganics & Chromium VI & $1.9 \mathrm{E}+02$ & 1.1E-04 & $2.6 \mathrm{E}-04$ \\
\hline Adult Trespasser & Core & Sediment & Ingestion & Inorganics & Cyanide & $5.8 \mathrm{E}-01$ & $3.4 \mathrm{E}-07$ & $7.9 \mathrm{E}-07$ \\
\hline Adult Trespasser & Core & Sediment & Ingestion & Inorganics & Manganese & $1.3 E+03$ & 7.7E-04 & $1.8 \mathrm{E}-03$ \\
\hline Adult Trespasser & Core & Sediment & Ingestion & Inorganics & Mereury & $1.9 \mathrm{E}+0 \mathrm{I}$ & 1.1E-05 & $2.6 \mathrm{E}-05$ \\
\hline Adult Trespasser & Core & Sediment & Ingestion & Inorganics & Nickel & $1.7 E+02$ & $9.9 \mathrm{E}-05$ & 2.3E-04 \\
\hline Adult Trespasser & Core & Sediment & Ingestion & Inorganice & Selenium & 8.7E-01 & 5.1E-07 & $1.2 \mathrm{E}-06$ \\
\hline
\end{tabular}

- Units are $\mathrm{mg} / \mathrm{kg}$ for inorganics and organics; $\mathrm{pCi} / \mathrm{g}$ for radionuclides

- Units are mg/kg-day for inorganics and organica; pCi-day for radionuclides 


\begin{tabular}{|c|c|c|c|c|c|c|c|c|}
\hline \multirow{2}{*}{ Receptor } & \multirow{2}{*}{ Media } & \multirow{2}{*}{ Location } & \multirow{2}{*}{ Exposure Pathway } & \multirow{2}{*}{$\begin{array}{l}\text { Contaminent } \\
\text { Class }\end{array}$} & \multirow{2}{*}{ Contaminant } & \multirow{2}{*}{$\begin{array}{l}\text { Exposure Point } \\
\text { Concentration" }\end{array}$} & \multicolumn{2}{|c|}{ Chromic Deily Intake' } \\
\hline & & & & & & & $\begin{array}{l}\text { Carcinogenic } \\
\text { Efrects }\end{array}$ & $\begin{array}{c}\text { Noncarcinogenic } \\
\text { Effects }\end{array}$ \\
\hline Adull Trespasser & Core & Sediment & Ingettion & Inorganics & Zine & 7.4E+03 & 4.4E-03 & $1.0 \mathrm{E}-02$ \\
\hline Adult Trespasser & Core & Sediment & Ingestion & Organics & Acenaphthene & $9.6 \mathrm{E}-01$ & $5.6 \mathrm{E}-07$ & $1.3 E-06$ \\
\hline Adult Trespasser & Core & Sediment & Ingestion & Organics & Acetone & $9.0 \mathrm{E}-02$ & 5.3E-08 & $1.2 E-07$ \\
\hline Adult Trespasser & Core & Sediment & Ingestion & Organicı & Anthracene & $1.9 E+\infty$ & $1.1 \mathrm{E}-06$ & $2.6 \mathrm{E}-06$ \\
\hline Adult Trespasser & Core & Sediment & Ingestion & Organice & Aroclor-1254 & $4.1 E+02$ & 2.4E-04 & $5.6 \mathrm{E}-04$ \\
\hline Adult Trespasser & Core & Sediment & Ingestion & Organica & Benzo(a)pyrene & $5.7 \mathrm{E}+\infty$ & 3.3E-06 & $7.8 \mathrm{E}-06$ \\
\hline Adult Trespasser & Core & Sediment & Ingestion & Organics & Benzo(k)fluoranthene & $4.6 \mathrm{E}+01$ & 2.7E-05 & $6.3 \mathrm{E}-05$ \\
\hline Adult Trespasser & Core & Sediment & Ingestion & Organics & Bis(2-ethylhexyl)phthalate & $2.1 E+\infty$ & $1.2 \mathrm{E}-06$ & $2.9 \mathrm{E}-06$ \\
\hline Adult Trespasser & Core & Sediment & Ingestion & Organice & Carbazole & $2.0 E+\infty$ & $1.2 \mathrm{E}-06$ & 2.7E-06 \\
\hline Adult Trespasser & Core & Sediment & Ingestion & Organics & Chrysene & $1.9 E+01$ & $1.1 \mathrm{E}-05$ & $2.6 \mathrm{E}-05$ \\
\hline Adult Trespasser & Core & Sediment & Ingestion & Organics & Dibenz (a,h)anthracene & $1.5 \mathrm{E}+00$ & $8.8 \mathrm{E}-07$ & 2.1E-06 \\
\hline Adult Trespasser & Core & Sediment & Ingestion & Organics & Fluoranthene & $2.7 E+01$ & $1.6 \mathrm{E}-05$ & 3.7E-05 \\
\hline Adult Trespasser & Core & Sediment & Ingestion & Organics & Fiuorene & $8.5 \mathrm{E}-01$ & S.0E-07 & $1.2 \mathrm{E}-06$ \\
\hline Adult Trespasser & Core & Sediment & Ingestion & Organics & Indeno(1,2,3-ed)pyrene & $9.6 E+\infty$ & $5.6 \mathrm{E}-06$ & $1.3 \mathrm{E}-05$ \\
\hline Adult Trespasser & Core & Sediment & Ingestion & Organics & Pyrene & $2.2 \mathrm{E}+01$ & $1.3 \mathrm{E}-05$ & $3.0 \mathrm{E}-05$ \\
\hline Adult Trespasser & Core & Sediment & Ingestion & Radionuclides & ${ }^{10} \mathrm{Ca}$ & $3.1 \mathrm{E}+04$ & $3.2 \mathrm{E}+07$ & \\
\hline Adult Trespasser & Core & Sediment & Ingestion & Radionuclides & ${ }^{n 9} \mathrm{Th}$ & $5.4 \mathrm{E}-01$ & $5.7 \mathrm{E}+02$ & \\
\hline Adult Trespasser & Core & Sediment & Ingestion & Radionuclide: & ${ }^{209} \mathrm{Th}$ & $1.9 \mathrm{E}+01$ & $2.0 E+04$ & \\
\hline Adult Trespasser & Core & Sediment & Ingertion & Radionuclides & ${ }^{2 x} \mathrm{Th}$ & 5.5E-01 & $5.8 \mathrm{E}+02$ & \\
\hline Adult Trespasser & Core & Sediment & Ingestion & Radionuclides & ${ }^{23824} \mathrm{U}$ & $3.4 \mathrm{E}+\infty 0$ & $3.6 \mathrm{E}+03$ & \\
\hline Adult Trespasser & Core & Sediment & Ingertion & Radionuclides & ${ }^{24} \mathrm{Pu}$ & $1.2 E+\infty$ & $1.2 E+03$ & \\
\hline
\end{tabular}

- Units are $\mathrm{mg} / \mathrm{kg}$ for inorganics and organics; $\mathrm{pCi} / \mathrm{g}$ for radionuclides

- Units are mg/kg-day for inorganics and organics; pCi-day for madionuclides 


\begin{tabular}{|c|c|c|c|c|c|c|c|c|}
\hline \multirow{2}{*}{ Receptor } & \multirow{2}{*}{ Media } & \multirow{2}{*}{ Location } & \multirow{2}{*}{ Exposure Pathway } & \multirow[b]{2}{*}{$\begin{array}{c}\text { Contaminant } \\
\text { Class }\end{array}$} & \multirow{2}{*}{ Contaminant } & \multirow[b]{2}{*}{$\begin{array}{l}\text { Exposure Point } \\
\text { Concentration" }\end{array}$} & \multicolumn{2}{|c|}{ Chronic Daily Intake } \\
\hline & & & & & & & $\begin{array}{c}\text { Carcinogenic } \\
\text { Effects }\end{array}$ & $\begin{array}{c}\text { Noncarcinogenic } \\
\text { Effects }\end{array}$ \\
\hline Adull Trespasser & Core & Sediment & Ingeation & Radionuclides & ${ }^{23 H} \mathrm{U}$ & $2.2 \mathrm{E}+00$ & $2.4 E+03$ & \\
\hline Adull Trespasser & Core & Sediment & Ingestion & Radionuclides & ${ }^{200210} \mathrm{Pu}$ & $3.5 E+01$ & $3.7 E+04$ & \\
\hline Adull Trespasser & Core & Sediment & Ingestion & Radionuclides & ${ }^{40} \mathrm{~K}$ & $9.4 \mathrm{E}+\infty 0$ & $9.9 E+03$ & \\
\hline Adult Trespasser & Core & Sediment & Ingeation & Radionuclides & ${ }^{\infty} \mathrm{Co}$ & $3.4 E+\infty$ & $3.6 \mathrm{E}+03$ & \\
\hline Adult Trespasser & Core & Sediment & Ingestion & Radionuclides & ${ }^{\infty} \mathrm{Sr}$ & $9.8 E+01$ & $1.0 \mathrm{E}+05$ & \\
\hline Adult Trespasser & Core & Sediment & Ingestion & Radionuclides & Tritium & $1.0 \mathrm{E}+\infty 0$ & $1.1 E+03$ & \\
\hline Adult Trespasser & Core & Sediment & Dermal & Inorganics & Antimony & $3.6 \mathrm{E}+01$ & 1.1E-05 & $2.6 \mathrm{E}-06$ \\
\hline Adult Trespasser & Core & Sediment & Dermal & Inorganics & Arsenic & $2.2 \mathrm{E}+01$ & $6.7 E-07$ & $1.6 \mathrm{E}-06$ \\
\hline Adult Trespasser & Core & Sediment & Dermal & Inorganics & Barium & $1.2 \mathrm{E}+02$ & 3.7E-06 & 8.7E-06 \\
\hline Adult Trespasser & Core & Sediment & Dermal & Inorganics & Cadmium & $6.0 \mathrm{E}+01$ & $1.9 \mathrm{E}-06$ & $4.4 \mathrm{E}-06$ \\
\hline Adult Trespasser & Core & Sediment & Dermal & Inorganics & Chromium VI & $1.9 \mathrm{E}+02$ & 5.9E-06 & $1.4 \mathrm{E}-05$ \\
\hline Adult Trespasser & Core & Sediment & Dermal & Inorganics & Cyanide & $5.8 \mathrm{E}-01$ & $1.8 \mathrm{E}-08$ & $4.2 \mathrm{E}-08$ \\
\hline Adull Trespeseer & Core & Sediment & Derme! & Inorganics & Manganese & $1,3 \mathrm{E}+03$ & 4.1E-0.5 & $9.5 \mathrm{E}-0.5$ \\
\hline Adult Trespasser & Core & Sediment & Dermal & Inorganics & Mercury & $1.9 \mathrm{E}+01$ & 5.8E-07 & $1.4 \mathrm{E}-06$ \\
\hline Adult Trespasser & Core & Sediment & Dermal & Inorganica & Nickel & $1.7 \mathrm{E}+02$ & 5.3E-06 & $1.2 \mathrm{E}-05$ \\
\hline Adult Trespasser & Core & Sediment & Dermal & Inorganics & Selenium & $8.7 E-01$ & 2.7E-08 & $6.3 \mathrm{E}-08$ \\
\hline Adult Trespasser & Core & Sediment & Dermal & Inorganice & Zinc & $7.4 \mathrm{E}+03$ & 2.3E-04 & $5.4 \mathrm{E}-04$ \\
\hline Adult Trespasser & Core & Sediment & Dermal & Organice & Acenaphthene & $9.6 \mathrm{E}-01$ & $3.0 \mathrm{E}-07$ & $7.0 \mathrm{E}-07$ \\
\hline Adult Trespasser & Core & Sediment & Dermal & Organics & Acetone & $9.0 \mathrm{E}-02$ & $2.8 \mathrm{E}-08$ & 6.SE-08 \\
\hline Adult Trespasser & Core & Sediment & Dermal & Organics & Anthracene & $1.9 E+\infty 0$ & $5.9 \mathrm{E}-07$ & $1.4 \mathrm{E}-06$ \\
\hline Adult Trespasuer & Core & Sediment & Dermal & Organic: & Aroclor-1254 & $4.1 E+02$ & $1.3 \mathrm{E}-04$ & $3.0 \mathrm{E}-04$ \\
\hline
\end{tabular}

- Units are $m g / \mathrm{kg}_{\mathrm{g}}$ for inorganics and organics; $\mathrm{pCi} / \mathrm{g}$ for radionuclides

- Units are $\mathrm{mg} / \mathrm{kg}$-day for inorganics and organics; $\mathrm{pCi}$-day for radionuclides 


\begin{tabular}{|c|c|c|c|c|c|c|c|c|}
\hline \multirow{2}{*}{ Receptor } & \multirow{2}{*}{ Media } & \multirow{2}{*}{ Location } & \multirow{2}{*}{ Expostre Pathrray } & \multirow{2}{*}{$\begin{array}{l}\text { Conteminant } \\
\text { Class }\end{array}$} & \multirow{2}{*}{ Contaminant } & \multirow{2}{*}{$\begin{array}{l}\text { Exposure Point } \\
\text { Concentration" }\end{array}$} & \multicolumn{2}{|c|}{ Chronic Daily Intake" } \\
\hline & & & & & & & $\begin{array}{c}\text { Carcinogenic } \\
\text { Effects }\end{array}$ & $\begin{array}{c}\text { Noncarcinogenic } \\
\text { Effects }\end{array}$ \\
\hline Adult Trespasser & Core & Sediment & Dermal & Organics & Benzo(x)pyrene & $5.7 E+\infty$ & $1.8 \mathrm{E}-06$ & 4.1E-06 \\
\hline Adult Trespasser & Core & Sediment & Dermal & Organics & Benzo(k)fluoranthene & $4.6 \mathrm{E}+01$ & 1.4E-05 & 3.3E-05 \\
\hline Adult Trespasser & Core & Sediment & Dermal & Onganics & Bir(2-ethylhexyl)phthalare & $2.1 E+\infty$ & $6.5 \mathrm{E}-07$ & $1.5 \mathrm{E}-06$ \\
\hline Adult Trespasser & Core & Sediment & Dermal & Organics & Carbazole & $2.0 E+\infty$ & $6.2 \mathrm{E}-07$ & $1.5 \mathrm{E}-06$ \\
\hline Adull Trespasser & Core & Sediment & Dermal & Organice & Chrysene & $1.9 \mathrm{E}+01$ & $5.9 \mathrm{E}-06$ & $1.4 \mathrm{E}-05$ \\
\hline Adult Trespasser & Core & Sediment & Dermal & Organics & Dibenz $(a, h)$ anthracene & $1.5 E+\infty$ & 4.7E-07 & 1.1E-06 \\
\hline Adull Trespasser & Core & Sediment & Dermal & Organics & Fluoranthene & $2.7 E+01$ & 8.4E-06 & $2.0 \mathrm{E}-05$ \\
\hline Adull Trespasser & Core & Sediment & Dermal & Organics & Fluorene & 8.5E-01 & $2.6 \mathrm{E}-07$ & $6.2 \mathrm{E}-07$ \\
\hline Adull Trespasser & Core & Sediment & Dermal & Organics & Indeno(1,2,3-cd)pyrene & $9.6 \mathrm{E}+00$ & $3.0 \mathrm{E}-06$ & $7.0 \mathrm{E}-06$ \\
\hline Adull Trespasser & Core & Sediment & Dermal & Organics & Pyrene & $2.2 \mathrm{E}+01$ & $6.8 \mathrm{E}-06$ & $1.6 \mathrm{E}-05$ \\
\hline Adult Trespasser & Core & Sediment & Inhalation & Inorganics & Antimony & $3.6 \mathrm{E}+01$ & 9.9E-10 & 2.3E-09 \\
\hline Adull Trespasser & Core & Sediment & Inhalation & Inorganics & Arsenic & $2.2 E+01$ & $5.9 \mathrm{E}-10$ & $1.4 \mathrm{E}-09$ \\
\hline Adult Trespasser & Core & Sediment & Inhalation & Inorganics & Barium & $1.2 \mathrm{E}+02$ & 3.3E-09 & 7.7E-09 \\
\hline Adull Trespasser & Core & Sediment & Inhalation & Inorganica & Cadmium & $6.0 \mathrm{E}+01$ & 1.6E-09 & $3.8 \mathrm{E}-09$ \\
\hline Adult Trespasser & Core & Sediment & Inhalation & Inorganics & Chromium VI & $1.9 E+02$ & 5.2E-09 & $1.2 \mathrm{E}-08$ \\
\hline Adull Trespasser & Core & Sediment & Inhalation & Inorganics & Cyanide & S.8E-01 & $1.6 \mathrm{E}-11$ & 3.7E-11 \\
\hline Adult Trespasser & Core & Sediment & Inhalation & Inorganics & Manganese & $1.3 E+03$ & 3.6E-08 & 8.4E-08 \\
\hline Adult Trespasser & Core & Sediment & Inhalation & Inorganics & Mercury & $1.9 \mathrm{E}+01$ & 5.1E-10 & $1.2 \mathrm{E}-09$ \\
\hline Adult Trespasser & Core & Sediment & Inhalation & Inorganic: & Nickel & $1.7 E+02$ & 4.6E-09 & 1.1E-08 \\
\hline Adult Trespasser & Core & Sediment & Inhalation & Inorganice & Selenium & 8.7E-01 & 2.4E-11 & $5.6 \mathrm{E}-11$ \\
\hline Adult Trespaszer & Core & Sediment & Inhalation & Inorgenic: & Zinc & $7.4 E+03$ & 2.0E-07 & 4.7E-07 \\
\hline
\end{tabular}

- Units are $\mathrm{mg} / \mathrm{kg}$ for inorgenics and organica; $\mathrm{pCi} / \mathrm{g}$ for radionuclides

- Units are $\mathrm{mg} / \mathrm{kg}$-day for inorganics and organics; $\mathrm{pCi}$-day for radionuclides 


\begin{tabular}{|c|c|c|c|c|c|c|c|c|}
\hline \multirow{2}{*}{ Receptor } & \multirow{2}{*}{ Media } & \multirow{2}{*}{ Location } & \multirow{2}{*}{ Exposure Pathway } & \multirow{2}{*}{$\begin{array}{c}\text { Contaminant } \\
\text { Class }\end{array}$} & \multirow{2}{*}{ Contaminant } & \multirow{2}{*}{$\begin{array}{l}\text { Exposure Point } \\
\text { Concentration" }\end{array}$} & \multicolumn{2}{|c|}{ Chronic Daily Inteke" } \\
\hline & & & & & & & $\begin{array}{l}\text { Carcinogenic } \\
\text { Errects }\end{array}$ & $\begin{array}{c}\text { Noncarcinogenic } \\
\text { Effects }\end{array}$ \\
\hline Adult Trespasser & Core & Sediment & Inhalation & Organics & Acenaphthene & $9.6 \mathrm{E}-01$ & $2.6 \mathrm{E}-11$ & $6.1 E-11$ \\
\hline Adult Trespasser & Core & Sediment & Inhalation & Organics & Acetone & $9.0 \mathrm{E}-02$ & $2.5 E-12$ & $5.8 \mathrm{E}-12$ \\
\hline Adult Trespasser & Core & Sediment & Inhalation & Organics & Anthracene & $1.9 E+00$ & $5.2 \mathrm{E}-11$ & $1.2 \mathrm{E}-10$ \\
\hline Adult Trespanser & Core & Sediment & Inhalation & Organic: & Aroclor-1254 & $4.1 E+02$ & $1.1 E-08$ & $2.6 \mathrm{E}-08$ \\
\hline Adult Trespasser & Core & Sediment & Inhalation & Organics & Benzo(a)pyrene & $5.7 E+\infty 0$ & $1.6 \mathrm{E}-10$ & $3.6 \mathrm{E}-10$ \\
\hline Adult Trespasser & Core & Sediment & Inhalation & Organics & Benzo(k)fluoranthene & $4.6 \mathrm{E}+01$ & $1.3 \mathrm{E}-09$ & $2.9 \mathrm{E}-09$ \\
\hline Adult Trespasser & Core & Sediment & Inhalation & Organies & Bis(2-ethylhexyl)phthalate & $2.1 E+\infty 0$ & $5.8 \mathrm{E}-11$ & $1.3 \mathrm{E}-10$ \\
\hline Adult Trespasser & Core & Sediment & Inhalation & Organics & Carbazole & $2.0 \mathrm{E}+\infty 0$ & $5.5 \mathrm{E}-11$ & $1.3 \mathrm{E}-10$ \\
\hline Adult Trespasser & Core & Sediment & Inhalation & Organics & Chrysene & $1.9 \mathrm{E}+01$ & $5.2 \mathrm{E}-10$ & $1.2 \mathrm{E}-09$ \\
\hline Adult Trespasser & Core & Sediment & Inhalation & Organic: & Dibenz $(\mathrm{a}, \mathrm{h})$ anthracene & $1.5 E+00$ & $4.1 \mathrm{E}-11$ & $9.6 \mathrm{E}-11$ \\
\hline Adult Trespasser & Core & Sediment & Inhalation & Organics & Fluoranthene & $2.7 E+01$ & $7.4 \mathrm{E}-10$ & $1.7 \mathrm{E}-09$ \\
\hline Adult Trespasser & Core & Sediment & Inhalation & Organica & Fluorene & 8.5E-01 & $2.3 \mathrm{E}-11$ & 5.4E-11 \\
\hline Adult Trespasser & Core & Sediment & Inhaiation & Organics & indieno(i, $\hat{\imath}, \hat{3}$-cá)pyrene & ร..6E+ôt & 2.6́E-io & 6.iE-io \\
\hline Adult Trespasser & Core & Sediment & Inhalation & Organice & Pyrene & $2.2 \mathrm{E}+01$ & $6.0 \mathrm{E}-10$ & $1.4 \mathrm{E}-09$ \\
\hline Adult Trespasser & Core & Sediment & Inhalation & Radionuclides & ${ }^{15} \mathrm{Cs}$ & $3.1 E+04$ & $1.5 E+03$ & . \\
\hline Adult Trespasser & Core & Sediment & Inhalation & Radionuclides & ${ }^{220} \mathrm{Th}$ & $5.4 \mathrm{E}-01$ & $2.6 \mathrm{E}-02$ & \\
\hline Adult Trespasser & Cone & Sediment & Inhalation & Radionuclides & ${ }^{20} \mathrm{Th}$ & $1.9 E+01$ & $9.2 \mathrm{E}-01$ & \\
\hline Adult Trespasser & Core & Sediment & Inhalation & Radionuclides & ${ }^{200} \mathrm{Th}$ & 5.5E-01 & $2.7 \mathrm{E}-02$ & \\
\hline Adult Trespasser & Core & Sediment & Inhalation & Radionuclides & ${ }^{23 \times 23} U$ & $3.4 E+\infty 0$ & $1.7 \mathrm{E}-01$ & \\
\hline Adult Trespasser & Cone & Sediment & Inhalation & Radionuclides & ${ }^{2 s} \mathrm{Pu}$ & $1.2 \mathrm{E}+00$ & 5.7E-02 & \\
\hline Adult Trespasser & Core & Sediment & Inhalation & Radionuclides & ${ }^{2 N} U$ & $2.2 E+\infty 0$ & 1.1E-01 & \\
\hline
\end{tabular}

- Units are $\mathrm{mg} / \mathrm{kg}$ for inorganics and organics; $\mathrm{pCi} / \mathrm{g}$ for radionuclides

- Units are mg/kg-day for inorganics and organicr; pCi-day for radionuclides 


\begin{tabular}{|c|c|c|c|c|c|c|c|c|}
\hline \multirow{2}{*}{ Receptor } & \multirow{2}{*}{ Media } & \multirow{2}{*}{ Location } & \multirow{2}{*}{ Exposure Pathway } & \multirow[b]{2}{*}{$\begin{array}{l}\text { Contaminant } \\
\text { Class }\end{array}$} & \multirow{2}{*}{ Contaminant } & \multirow[b]{2}{*}{$\begin{array}{l}\text { Exposure Point } \\
\text { Concentration" }\end{array}$} & \multicolumn{2}{|c|}{ Chronic Daily Intoke" } \\
\hline & & & & & & & $\begin{array}{l}\text { Carcinogenic } \\
\text { Effects }\end{array}$ & $\begin{array}{l}\text { Noncarcinogenic } \\
\text { Effects }\end{array}$ \\
\hline Adult Trespasser & Core & Sediment & Inhalation & Radionuclides & ${ }^{200520} \mathrm{Pu}$ & $3.5 \mathrm{E}+01$ & $1.7 \mathrm{E}+\infty 0$ & \\
\hline Adult Trespasser & Core & Sedimemt & Inhalation & Radionuclides & ${ }^{\infty} \mathrm{K}$ & $9.4 E+00$ & $4.6 \mathrm{E}-01$ & \\
\hline Adult Trespasser & Core & Sediment & Inhalation & Radionuclides & ${ }^{\infty} \mathrm{Co}$ & $3.4 \mathrm{E}+00$ & 1.7E-01 & . \\
\hline Adult Trespasser & Core & Sediment & Inhalation & Radionuclides & ${ }^{\circ} \mathrm{Sr}$ & $9.8 \mathrm{E}+01$ & $4.8 E+\infty 0$ & \\
\hline Adult Trespasser & Core & Sediment & Inhalation & Radionuclides & Tritium & $1.0 \mathrm{E}+00$ & $4.9 \mathrm{E}-02$ & \\
\hline Adult Trespasser & Core & Sediment & External Exposure & Radionuclides & ${ }^{19} \mathrm{Cs}$ & $3.1 E+04$ & $7.1 \mathrm{E}+05$ & \\
\hline Adult Trespasser & Core & Sediment & External Exposure & Radionuclides & ${ }^{20} \mathrm{Th}$ & $5.4 \mathrm{E}-01$ & $1.2 E+01$ & \\
\hline Adult Trespasser & Core & Sediment & External Exposure & Radionuclides & ${ }^{n T h}$ & $1.9 \mathrm{E}+01$ & $4.3 \mathrm{E}+02$ & \\
\hline Adult Trespasser & Core & Sediment & Extermal Exposure & Radionuclides & ${ }^{2 x} \mathrm{Th}$ & 5.5E-01 & $1.3 E+01$ & \\
\hline Adult Trespasser & Core & Sediment & External Exposure & Radionuelides & $2 \sin 23 \mathrm{U}$ & $3.4 \mathrm{E}+00$ & $2.9 E+01$ & \\
\hline Adult Trespasser & Core & Sediment & External Exposure & Radionuclides & ${ }^{28 \mathrm{Pu}}$ & $1.2 \mathrm{E}+00$ & $2.7 \mathrm{E}+01$ & - \\
\hline Adult Trespasser & Core & Sediment & External Exposure & Radionuclides & ${ }^{238} \mathrm{U}$ & $2.2 \mathrm{E}+00$ & $5.2 \mathrm{E}+01$ & \\
\hline Adult Trespasser & Core & Sediment & External Exposure & Radionuclides & ${ }^{2398200 \mathrm{Pu}}$ & $3.5 \mathrm{E}+01$ & $8.1 E+02$ & \\
\hline Adult Trespasser & Core & Sediment & External Exposure & Radionuclides & ${ }^{\infty} \mathrm{K}$ & $9.4 E+00$ & $2.2 \mathrm{E}+02$ & \\
\hline Adult Trespasser & Core & Sediment & External Exposure & Radionuclides & ${ }^{\infty} \mathrm{Co}$ & $3.4 \mathrm{E}+\infty 0$ & $7.9 \mathrm{E}+01$ & \\
\hline Adult Trespasser & Core & Sediment & External Exposure & Radionuclides & ${ }^{\circ} \mathrm{Sr}$ & $9.8 \mathrm{E}+01$ & $2.2 \mathrm{E}+03$ & \\
\hline Adult Trespasser & Cone & Sediment & External Exposure & Radionuclides & Tritium & $1.0 \mathrm{E}+00$ & $2.3 \mathrm{E}+01$ & \\
\hline Adull Trespasser & Core & Whole Building & Ingestion & Inorganics & Arsenic & $2.9 \mathrm{E}+00$ & $1.7 E-06$ & $3.9 \mathrm{E}-06$ \\
\hline Adult Trespasser & Core & Whole Building & Ingestion & Inorganics & Barium & $7.2 E+02$ & 4.2E-04 & 9.9E-04 \\
\hline Adult Trespasser & Core & Whole Building & Ingestion & Inorganics & Beryllium & $1.2 \mathrm{E}+01$ & 7.2E-06 & $1.7 \mathrm{E}-05$ \\
\hline Adult Trespasser & Core & Whole Building & Ingestion & Inorganic: & Cadmium & $1.8 \mathrm{E}+02$ & 1.1E-04 & 2.5E-04 \\
\hline
\end{tabular}

- Units are $\mathrm{mg} / \mathrm{kg}$ for inorganics and organics; $\mathrm{pCi} / \mathrm{g}$ for radionuclides

- Units are mg/kg-day for inorganics and organics; $\mathrm{pCi}$-day for radionuclides 


\begin{tabular}{|c|c|c|c|c|c|c|c|c|}
\hline \multirow{2}{*}{ Receptor } & \multirow{2}{*}{ Medin } & \multirow{2}{*}{ Location } & \multirow{2}{*}{ Exposure Pathway } & \multirow{2}{*}{$\begin{array}{l}\text { Contaminant } \\
\text { Class }\end{array}$} & \multirow{2}{*}{ Contaminant } & \multirow[b]{2}{*}{$\begin{array}{l}\text { Exposure Point } \\
\text { Concentration" }\end{array}$} & \multicolumn{2}{|c|}{ Chronic Daily Intake" } \\
\hline & & & & & & & $\begin{array}{c}\text { Carcinogenic } \\
\text { Effects }\end{array}$ & $\begin{array}{c}\text { Noncarcinosenic } \\
\text { Efrects }\end{array}$ \\
\hline Adult Trespasser & Core & Whole Building & Ingestion & Inorganic: & Chromium VI & $2.0 \mathrm{E}+02$ & $1.2 \mathrm{E}-04$ & 2.7E-04 \\
\hline Adult Trespasser & Core & Whole Building & Ingestion & Inorganice & Manganese & $9.5 E+03$ & $5.6 \mathrm{E}-03$ & $1.3 \mathrm{E}-02$ \\
\hline Adult Trespasser & Core & Whole Building & Ingertion & Inorganics & Mercury & $3.2 \mathrm{E}-01$ & $1.9 \mathrm{E}-07$ & 4.4E-07 \\
\hline Adult Trespasser & Core & Whole Building & Ingestion & Inorganics & Nickel & $1.4 E+02$ & 8.1E-0S & $1.9 \mathrm{E}-04$ \\
\hline Adult Trespasser & Core & Whole Building & Ingestion & Inorganic: & Vanadium & $2.4 E+02$ & $1.4 \mathrm{E}-04$ & 3.3E-04 \\
\hline Adult Trespasser & Core & Whole Building & Ingestion & Inorganics & Zinc & $3.5 \mathrm{E}+04$ & $2.0 \mathrm{E}-02$ & $4.8 \mathrm{E}-02$ \\
\hline Adult Trespasser & Core & Whole Building & Ingestion & Organics & Aroclor-1254 & $1.5 E+\infty 0$ & 8.5E-07 & 2.0E-06 \\
\hline Adult Trespasser & Core & Whole Building & Ingestion & Organic: & Aroclor- 1260 & $2.6 \mathrm{E}+00$ & $1.5 \mathrm{E}-06$ & $3.6 \mathrm{E}-06$ \\
\hline Adull Trespasser & Core & Whole Building & Ingestion & Organics & Bis(2-ethylhexyl)phthalate & 2.4E-01 & $1.4 \mathrm{E}-07$ & 3.3E-07 \\
\hline Adult Trespasser & Core & Whole Building & Ingestion & Organics & Dimethyl phthalate & $5.5 \mathrm{E}-02$ & $3.2 \mathrm{E}-08$ & $7.5 \mathrm{E}-08$ \\
\hline Adult Trespasser & Core & Whole Building & Ingestion & Radionuclides & ${ }^{19} \mathrm{Cs}$ & $1.4 E+03$ & $1.4 E+06$ & \\
\hline Adult Trespasser & Core & Whole Building & Ingestion & Radionuclides & ${ }^{20} \mathrm{Ra}$ & $4.0 \mathrm{E}-01$ & $4.2 \mathrm{E}+02$ & \\
\hline Aduil Trespasser & Core & Whoic Eutiiding & ingestion & Kaadionuciides & $\dddot{m}_{\mathbf{K a}}$ & 3.7E-0̂i & $3.9 E+02$ & \\
\hline Adult Trespasser & Core & Whole Building & Ingestion & Radionuclides & 2nTh & 7.7E-01 & $8.1 E+02$ & \\
\hline Adult Trespasser & Core & Whole Building & Ingestion & Radionuclides & ${ }^{250} \mathrm{Th}$ & $1.4 E+\infty$ & $1.5 E+03$ & \\
\hline Adult Trespasser & Core & Whole Building & Ingestion & Radionuclides & ${ }^{2 n} \mathrm{Th}$ & $8.0 \mathrm{E}-01$ & $8.4 E+02$ & \\
\hline Adult Trespasser & Core & Whole Building & Ingestion & Radionuclides & $23 \times 24 \mathrm{U}$ & $1.3 \mathrm{E}+00$ & $1.3 E+03$ & \\
\hline Adult Trespasser & Core & Whole Building & Ingestion & Radionuclides & ${ }^{24} \mathrm{Th}$ & $6.3 E+00$ & $6.6 \mathrm{E}+03$ & \\
\hline Adult Trespasser & Core & Whole Building & Ingestion & Radionuclides & ${ }^{23 s} \mathrm{U}$ & $1.2 \mathrm{E}-01$ & $1.3 \mathrm{E}+02$ & \\
\hline Adult Trespasser & Core & Whole Building & Ingestion & Radionuclides & ${ }^{213} \mathrm{Pu}$ & 4.5E-01 & 4.7E+02 & \\
\hline Adult Trespasser & Core & Whole Building & Ingertion & Radionuclides & ${ }^{250} \mathrm{U}$ & $1.4 E+00$ & $1.5 E+03$ & \\
\hline
\end{tabular}

- Units are $\mathrm{mg} / \mathrm{kg}$ for inorganics and organics; $\mathrm{pCi} / \mathrm{g}$ for radionuclides

- Units are mg/kg-day for inorganics and organics; pCi-day for radionuclides 


\begin{tabular}{|c|c|c|c|c|c|c|c|c|}
\hline \multirow{2}{*}{ Receptor } & \multirow{2}{*}{ Metia } & \multirow{2}{*}{ Location } & \multirow{2}{*}{ Exposure Pathway } & \multirow[b]{2}{*}{$\begin{array}{l}\text { Contaminant } \\
\text { Cless }\end{array}$} & \multirow{2}{*}{ Conteminant } & \multirow[b]{2}{*}{$\begin{array}{l}\text { Exposure Point } \\
\text { Concentration" }\end{array}$} & \multicolumn{2}{|c|}{ Cbronic Daily Intaket } \\
\hline & & & & & & & $\begin{array}{l}\text { Cancinogeric } \\
\text { Effects }\end{array}$ & $\begin{array}{l}\text { Noncancinogenic } \\
\text { Effects }\end{array}$ \\
\hline Adult Trespasser & Core & Whole Building & Ingestion & Radionuclides & ${ }^{2502500 \mathrm{Pu}}$ & $4.0 \mathrm{E}+\infty$ & $4.2 E+03$ & \\
\hline Adult Treapasser & Core & Whole Building & Ingestion & Radionuclides & ${ }^{2 \mu_{1} \mathrm{Am}}$ & $5.0 \mathrm{E}-01$ & $5.3 E+02$ & \\
\hline Adult Trespasser & Core & Whole Building & Ingestion & Radionuclides & ${ }^{\infty} \mathrm{K}$ & $5.4 \mathrm{E}+\infty$ & $5.6 \mathrm{E}+03$ & \\
\hline Adult Trespasser & Core & Whole Building & Ingention & Radionuclides & ${ }^{\infty} \mathrm{Co}$ & $1.3 \mathrm{E}-01$ & $1.4 \mathrm{E}+02$ & \\
\hline Adult Trespauser & Core & Whole Building & Ingestion & Radionuclides & ${ }^{\infty} \mathrm{Sr}$ & $1.2 E+01$ & $1.2 E+04$ & \\
\hline Adult Trespasser & Core & Whole Building & Ingestion & Radionuclides & Tritium & $2.6 \mathrm{E}+01$ & $2.7 E+04$ & \\
\hline Adult Trespasser & Core & Whole Building & Dermal & Inorganics & Arsenic & $2.9 \mathrm{E}+00$ & $8.9 \mathrm{E}-08$ & 2.1E-07 \\
\hline Adult Trespasser & Core & Whole Building & Dermal & Inorganics & Barium & $7.2 \mathrm{E}+02$ & $2.2 E-05$ & $5.2 E-05$ \\
\hline Adult Trespasser & Core & Whole Building & Dermal & Inorganics & Beryllium & $1.2 \mathrm{E}+01$ & 3.8E-07 & $9.0 \mathrm{E}-07$ \\
\hline Adult Trespasser & Core & Whole Building & Dermal & Inorganics & Cadmium & $1.8 \mathrm{E}+02$ & $5.6 \mathrm{E}-06$ & $1.3 \mathrm{E}-05$ \\
\hline Adult Trespasser & Core & Whole Building & Dermal & Inorganics & Chromium VI & $2.0 \mathrm{E}+02$ & $6.1 E-06$ & 1.4E-05 \\
\hline Adult Trespasser & Core & Whole Building & Dermal & Inorganics & Manganese & $9.5 E+03$ & $3.0 \mathrm{E}-04$ & $6.9 \mathrm{E}-04$ \\
\hline Adult Trespasser & Core & Whole Building & Dermal & Inorganics & Mercury & 3.2E-01 & $9.9 \mathrm{E}-09$ & $2.3 \mathrm{E}-08$ \\
\hline Adult Trespasser & Core & Whole Building & Dermal & Inorganice & Nickel & $1.4 E+02$ & 4.3E-06 & 1.0E-05 \\
\hline Adult Trespasser & Core & Whole Building & Dermal & Inorganica & Vanadium & $2.4 E+02$ & $7.5 \mathrm{E}-06$ & $1.7 \mathrm{E}-05$ \\
\hline Adult Trespasser & Core & Whole Building & Dermal & Inorganics & Zine & 3.5E+04 & 1.1E-03 & 2.5E-03 \\
\hline Adult Trespasser & Core & Whole Building & Dermal & Organics & Aroclor-1254 & $1.5 \mathrm{E}+\infty$ & 4.5E-07 & $1.1 E-06$ \\
\hline Adult Trespasser & Core & Whole Building & Dermal & Organick & Aroclor-1260 & $2.6 \mathrm{E}+00$ & 8.1E-07 & $1.9 \mathrm{E}-06$ \\
\hline Adult Trespasser & Core & Whole Building & Dermal & Organice & Bis(2-ethylhexyl)phthalate & 2.4E-01 & 7.5E-08 & 1.7E-07 \\
\hline Adult Treapasser & Core & Whole Building & Dermal & Organice & Dimethyl phthalate & 5.5E-02 & $1.7 \mathrm{E}-08$ & $4.0 \mathrm{E}-08$ \\
\hline Adult Treapanser & Core & Whole Building & Inhalation & Inorganic: & Arsenic & $2.9 \mathrm{E}+\infty 0$ & $7.8 \mathrm{E}-11$ & $1.8 \mathrm{E}-10$ \\
\hline
\end{tabular}

- Units are mg/kg for inorganics and organiç; $\mathrm{pCi} / \mathrm{g}$ for radionuclides

- Units are mg/kg-day for inorganics and organics; pCi-day for radionuclides 


\begin{tabular}{|c|c|c|c|c|c|c|c|c|}
\hline \multirow{2}{*}{ Receptor } & \multirow{2}{*}{ Media } & \multirow{2}{*}{ Location } & \multirow{2}{*}{ Exposure Pathway } & \multirow{2}{*}{$\begin{array}{l}\text { Contaminant } \\
\text { Class }\end{array}$} & \multirow{2}{*}{ Contaminant } & \multirow[b]{2}{*}{$\begin{array}{l}\text { Exposure Point } \\
\text { Concentration" }\end{array}$} & \multicolumn{2}{|c|}{ Chronic Daily Intake" } \\
\hline & & & & & & & $\begin{array}{l}\text { Carcinogenic } \\
\text { Erfects }\end{array}$ & $\begin{array}{c}\text { Noncancinogenic } \\
\text { Effects }\end{array}$ \\
\hline Adult Trespasser & Core & Whole Building & Inhalation & Inorganica & Barium & $7.2 \mathrm{E}+02$ & $2.0 \mathrm{E}-08$ & $4.6 \mathrm{E}-08$ \\
\hline Adult Trespasser & Core & Whole Building & Inhalation & Inorganic: & Beryllium & $1.2 \mathrm{E}+01$ & $3.4 \mathrm{E}-10$ & $7.9 \mathrm{E}-10$ \\
\hline Adult Trespasser & Core & Whole Building & Inhalation & Inorganics & Cadmium & $1.8 E+02$ & $4.9 \mathrm{E}-09$ & $1.2 \mathrm{E}-08$ \\
\hline Adult Trespasser & Core & Whole Building & Inhalation & Inorganics & Chromium VI & $2.0 E+02$ & $5.4 \mathrm{E}-09$ & $1.3 \mathrm{E}-08$ \\
\hline Adult Trespasser & Core & Whole Building & Inhalation & Inorganics & Manganese & $9.5 \mathrm{E}+03$ & $2.6 \mathrm{E}-07$ & $6.1 E-07$ \\
\hline Adult Trespasser & Core & Whole Building & Inhalation & Inorganics & Mencury & $3.2 \mathrm{E}-01$ & 8.7E-12 & $2.0 \mathrm{E}-11$ \\
\hline Adult Trespasser & Core & Whole Building & Inhalation & Inorganics & Nickel & $1.4 \mathrm{E}+02$ & $3.8 \mathrm{E}-09$ & $8.8 \mathrm{E}-09$ \\
\hline Adult Trespasser & Core & Whole Building & Inhalation & Inorganics & Vanadium & $2.4 \mathrm{E}+02$ & $6.6 \mathrm{E}-09$ & $1.5 \mathrm{E}-08$ \\
\hline Adult Trespasser & Core & Whole Building & Inhalation & Inorganics & Zinc & $3.5 \mathrm{E}+04$ & 9.6E-07 & $2.2 \mathrm{E}-06$ \\
\hline Adult Trespasser & Core & Whole Building & Inhalation & Organics & Aroclor-1254 & $1.5 E+00$ & $4.0 \mathrm{E}-11$ & $9.3 \mathrm{E}-11$ \\
\hline Adult Trespasser & Core & Whole Building & Inhalation & Organics & Aroclor -1260 & $2.6 E+00$ & $7.1 \mathrm{E}-11$ & $1.7 \mathrm{E}-10$ \\
\hline Adult Trespasser . & Core & Whole Building & Inhalation & Organic: & Bis(2-ethylhexyl)phthalate & 2.4E-01 & $6.6 \mathrm{E}-12$ & $1.5 \mathrm{E}-11$ \\
\hline 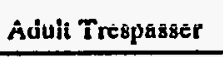 & Core & Whole Building & Intatation & Organica & Dimethy! phthalate & 5.55 .02 & $2.55-12$ & $3.55-12$ \\
\hline Adult Trespasser & Core & Whole Building & Inhalation & Radionuclides & ${ }^{137} \mathrm{Cs}$ & $1.4 \mathrm{E}+03$ & $6.7 \mathrm{E}+01$ & \\
\hline Adult Trespasser & Core & Whole Building & Inhalation & Radionuclides & ${ }^{2 x} \mathrm{Ra}$ & $4.0 \mathrm{E}-01$ & $2.0 \mathrm{E}-02$ & \\
\hline Adult Trespasser & Core & Whole Building & Inhalation & Radionuclides & ${ }^{20} \mathbf{R a}$ & 3.7E-01 & $1.8 \mathrm{E}-02$ & \\
\hline Adult Trespasser & Core & Whole Building & Inhalation & Radionuclides & ${ }^{2 n} \mathrm{Th}$ & 7.7E-01 & $3.8 \mathrm{E}-02$ & \\
\hline Adult Trespasser & Core & Whole Building & Inhalation & Radionuclides & ${ }^{200} \mathrm{Th}$ & $1.4 \mathrm{E}+00$ & $6.8 \mathrm{E}-02$ & \\
\hline Adult Trespasser & Core & Whole Building & Inhalation & Radionuclides & ${ }^{2 x} \mathrm{Th}$ & $8.0 \mathrm{E}-01$ & $3.9 \mathrm{E}-02$ & \\
\hline Adult Trespanser & Core & Whole Building & Inhalation & Radionuclides & 235234 U & $1.3 E+\infty$ & $6.2 \mathrm{E}-02$ & \\
\hline Adult Trespasser & Core & Whole Building & Inhalation & Radionuclides & ${ }^{204} \mathrm{Th}$ & $6.3 E+\infty 0$ & 3.1E-01 & \\
\hline
\end{tabular}

- Unitu are $\mathrm{mg} / \mathrm{kg}$ for inorganics and organics; $\mathrm{pCi} / \mathrm{g}$ for radionuclides

- Units are $\mathrm{mg} / \mathrm{kg}$-day for inorganics and organics; $\mathrm{pCi}$-day for radionuclides 


\begin{tabular}{|c|c|c|c|c|c|c|c|c|}
\hline \multirow{2}{*}{ Receptor } & \multirow{2}{*}{ Media } & \multirow{2}{*}{ Location } & \multirow{2}{*}{ Exposure Pathway } & \multirow{2}{*}{$\begin{array}{l}\text { Contaminant } \\
\text { Class }\end{array}$} & \multirow{2}{*}{ Contaminent } & \multirow{2}{*}{$\begin{array}{l}\text { Exposure Point } \\
\text { Concentration" }\end{array}$} & \multicolumn{2}{|c|}{ "Chronic Daily Intake" } \\
\hline & & & & & & & $\begin{array}{c}\text { Carcinogenic } \\
\text { Enfocts }\end{array}$ & $\begin{array}{c}\text { Noncarcinogenic } \\
\text { Effects }\end{array}$ \\
\hline Adult Trespasser & Core & Whole Building & Inhalation & Radionuclides & ${ }^{239} \mathrm{U}$ & $1.2 \mathrm{E}-01$ & $5.9 \mathrm{E}-03$ & \\
\hline Adult Trespasser & Core & Whole Building & Inhalation & Radionuclides & ${ }^{2 \times} \mathrm{Pu}$ & 4.5E-01 & 2.2E-02 & \\
\hline Adult Trespasser & Core & Whole Building & Inhalation & Radionuclides & ${ }^{230} \mathrm{U}$ & $1.4 E+\infty$ & $\therefore \quad 7.0 \mathrm{E}-02$ & \\
\hline Adult Trespasser & Core & Whole Building & Inhalation & Radionuclides & ${ }^{209200} \mathrm{Pu}$ & $4.0 \mathrm{E}+00$ & $2.0 \mathrm{E}-01$ & \\
\hline Adult Trespasser & Core & Whole Building & Inhalation & Radionuclides & ${ }^{211} \mathrm{Am}$ & $5.0 \mathrm{E}-01$ & $2.5 \mathrm{E}-02$ & \\
\hline Adult Trespasser & Core & Whole Building & Inhalation & Radionuclides & ${ }^{\infty} \mathrm{K}$ & $5.4 E+00$ & 2.6E-01 & \\
\hline Adull Trespasser & Core & Whole Building & Inhalation & Radionuclides & ${ }^{\infty} \mathrm{Co}$ & $1.3 \mathrm{E}-0 \mathrm{~L}$ & $6.4 \mathrm{E}-03$ & \\
\hline Adull Trespasser & Core & Whole Building & Inhalation & Radionuclides & ${ }^{90} \mathrm{Sr}$ & $1.2 \mathrm{E}+01$ & 5.7E-01 & \\
\hline Adult Trespasser & Core & Whole Building & Inhalation & Radionuclides & Tritium & $2.6 \mathrm{E}+01$ & $1.3 \mathrm{E}+\infty 0$ & \\
\hline Adult Trespasser & Core & Whole Building & External Exposure & Radionuclides & ${ }^{1 n} \mathrm{Cs}$ & $1.4 E+03$ & $3.2 \mathrm{E}+04$ & \\
\hline Adult Trespasser & Core & Whole Building & Extemal Exposure & Radionuclides & ${ }^{28} \mathrm{Ra}$ & $4.0 \mathrm{E}-01$ & $9.2 \mathrm{E}+00$ & \\
\hline Adult Trespasser & Core & Whole Building & External Exposure & Radionuclides & ${ }^{220} \mathrm{Ra}$ & 3.7E-01 & $8.5 \mathrm{E}+00$ & \\
\hline Adult Trespasser & Core & Whole Building & External Exposure & Radionuclides & ${ }^{230} \mathrm{Th}$ & 7.7E-01 & $1.8 \mathrm{E}+01$ & \\
\hline Adult Trespasser & Core & Whole Building & External Exposure & Radionuclides & ${ }^{250} \mathrm{Th}$ & $1.4 E+00$ & $3.2 \mathrm{E}+01$ & \\
\hline Adult Trespasser & Core & Whole Building & External Exposure & Radionuclides & ${ }^{2 n} \mathrm{Th}$ & $8.0 \mathrm{E}-01$ & $1.8 \mathrm{E}+01$ & \\
\hline Adult Trespasser & Core & Whole Building & External Exposure & Radionuclides & ${ }^{233} 32 \times \mathrm{U}$ & $1.3 \mathrm{E}+00$ & $2.9 \mathrm{E}+01$ & \\
\hline Adult Trespasser & Core & Whole Building & External Exposure & Radionuclides & ${ }^{24} \mathrm{Th}$ & $6.3 \mathrm{E}+00$ & $1.5 E+02$ & \\
\hline Adult Trespasser & Core & Whole Building & External Exposure & Radionuclides & ${ }^{235} \mathrm{U}$ & $1.2 \mathrm{E}-01$ & $2.8 \mathrm{E}+00$ & \\
\hline Adult Trespasser & Core & Whole Building & Extemal Exposure & Radionuclides & 2sepu & $4.5 E-01$ & $1.0 \mathrm{E}+01$ & \\
\hline Adult Trespasser & Core & Whole Building & External Exposure & Radionuclides & $288 \mathrm{U}$ & $1.4 \mathrm{E}+00$ & $3.3 \mathrm{E}+01$ & \\
\hline Adult Trespanser & Core & Whole Building & External Expoxure & Radionuclides & ${ }^{251250} \mathrm{Pu}$ & $4.0 \mathrm{E}+00$ & $9.2 \mathrm{E}+01$ & \\
\hline
\end{tabular}

- Units are mg/kg for inorganics and organics; $\mathrm{pCi} / \mathrm{g}$ for radionuclides

- Units are $\mathrm{mg} / \mathrm{kg}$-day for inorganics and organicu; $\mathrm{pCi}$-day for radionuclides 


\begin{tabular}{|c|c|c|c|c|c|c|c|c|}
\hline \multirow{2}{*}{ Receptor } & \multirow{2}{*}{ Medin } & \multirow{2}{*}{ Location } & \multirow{2}{*}{ Exposure Pathway } & \multirow{2}{*}{$\begin{array}{l}\text { Contaminant } \\
\text { Class }\end{array}$} & \multirow{2}{*}{ Contaminant } & \multirow{2}{*}{$\begin{array}{l}\text { Exposure Point } \\
\text { Concentration" }\end{array}$} & \multicolumn{2}{|c|}{ Chronic Daily Intaket } \\
\hline & & & & & & & $\begin{array}{c}\text { Carcinogenic } \\
\text { Effects }\end{array}$ & $\begin{array}{c}\text { Noncarcinogenic } \\
\text { Effects }\end{array}$ \\
\hline Adult Trespasser & Core & Whole Building & Extermal Exposure & Radionuclides & ${ }^{21:} \mathrm{Am}$ & 5.0E-01 & $1.2 \mathrm{E}+01$ & \\
\hline Adult Trespasser & Core & Whole Building & Extermal Exposure & Radionuclides & ${ }^{\infty} \mathrm{K}$ & $5.4 \mathrm{E}+00$ & $1.2 \mathrm{E}+02$ & \\
\hline Adult Trespascer & Core & Whole Building & Extemal Exposure & Radionuclides & ${ }^{\infty} \mathrm{Co}$ & $1.3 \mathrm{E}-01$ & $3.0 \mathrm{E}+\infty 0$ & \\
\hline Adult Trespasser & Core & Whole Building & External Exposure & Radionuclides & ${ }^{\infty} \mathrm{Sr}$ & $1.2 \mathrm{E}+01$ & $2.7 \mathrm{E}+02$ & \\
\hline Adult Trespasser & Core & Whole Building & Extemal Exposure & Radionuclides & Tritium & $2.6 E+01$ & $5.9 E+02$ & \\
\hline
\end{tabular}


Table D-10. Adult Trespasser Exposure Concentrations and Chronic Lifetime Exposures for Direct Measurement Samples

\begin{tabular}{|c|c|c|c|c|c|c|c|c|}
\hline \multirow[b]{2}{*}{ Receplor } & \multirow[b]{2}{*}{ Media } & \multirow[b]{2}{*}{ Location } & \multirow[b]{2}{*}{ Exposure Pathway } & \multirow[b]{2}{*}{$\begin{array}{l}\text { Contaminant } \\
\text { Class }\end{array}$} & \multirow[b]{2}{*}{ Contaminant } & \multirow{2}{*}{$\begin{array}{l}\text { Exposure Point } \\
\text { Concentration" }\end{array}$} & \multicolumn{2}{|c|}{ Chronic Lifetime Exposure } \\
\hline & & & & & & & $\begin{array}{c}\text { Cancinogenic } \\
\text { Effects }\end{array}$ & $\begin{array}{c}\text { Noncarcinogenic } \\
\text { Effects }\end{array}$ \\
\hline Adult Trespasser & Direct & Cell & Externsl Exposure & Radionuclides & Bela-Gamma & $8.1 E+\infty$ & $2.0 \mathrm{E}+06$ & \\
\hline Adult Trespasser & Direct & Cell & External Exposure & Radionuclides & Gamma & $7.0 \mathrm{E}+\infty 0$ & $1.8 \mathrm{E}+06$ & \\
\hline Adult Trespasser & Direct & East Gallery & Extemal Exposure & Radionuclides & Beta-Gamma & $1.1 \mathrm{E}-01$ & $2.8 \mathrm{E}+04$ & \\
\hline Adult Trespasser & Direct & East Gallery & External Exposure & Radionuclides & Gamma & $1.0 \mathrm{E}-01$ & $2.5 E+04$ & \\
\hline Adult Trespasser & Direct & Whole Building & External Exposure & Radionuclides & Beta-Gamma & $1.1 E+01$ & $2.8 E+06$ & \\
\hline Adull Trespasser & Direct & Whole Building & External Exposure & Radionuclides & Gamma & $1.0 \mathrm{E}+01$ & $2.6 \mathrm{E}+06$ & \\
\hline
\end{tabular}

-Units are mrem

Units are mrem 
THIS PAGE INTENTIONALLY LEFT BLANK 
Table D-11. Adult Trespasser Exposure Concentrations and Chronic Daily Intakes for Smear Samples

\begin{tabular}{|c|c|c|c|c|c|c|c|c|}
\hline \multirow{2}{*}{ Receptor } & \multirow{2}{*}{ Media } & \multirow{2}{*}{ Location } & \multirow{2}{*}{ Exposure Pathway } & \multirow{2}{*}{ Contaminant Class } & \multirow{2}{*}{ Contaminant } & \multirow{2}{*}{$\begin{array}{l}\text { Exposure Point } \\
\text { Concentration" }\end{array}$} & \multicolumn{2}{|c|}{ Chronic Daily Intake" } \\
\hline & & & & & & & $\begin{array}{c}\text { Carcinogenic } \\
\text { Efrects }\end{array}$ & $\begin{array}{c}\text { Noncarcinogenic } \\
\text { Effects }\end{array}$ \\
\hline Adull Trespasser & Smear & Cell & Ingestion & Radionuclides & ${ }^{17} \mathrm{Cs}$ & $1.4 \mathrm{E}+02$ & $1.5 \mathrm{E}+05$ & \\
\hline Adult Trespasser & Smear & Cell & Ingestion & Radionuclides & ${ }^{2} R_{R}$ & 5.3E-03 & $5.5 E+00$ & \\
\hline Adult Trespasser & Smear & Cell & Ingestion & Radionuclider & ${ }^{n \cdot T h}$ & $1.1 \mathrm{E}-02$ & $1.2 \mathrm{E}+01$ & \\
\hline Adult Trespasser & Smear & Cell & Ingestion & Radionuclides & ${ }^{200} \mathrm{Th}$ & $1.8 \mathrm{E}-02$ & $1.9 \mathrm{E}+01$ & \\
\hline Adult Trespasser & Smear & Cell & Ingestion & Radionuclides & ${ }^{200} \mathrm{Th}$ & $1.1 \mathrm{E}-02$ & $1.2 \mathrm{E}+01$ & \\
\hline Adult Trespasser & Smear & Cell & Ingestion & Radionuclides & $23 \times 240$ & $1.7 \mathrm{E}-02$ & $1.8 \mathrm{E}+01$ & \\
\hline Adult Trespasser & Smear & Cell & Ingestion & Radionuclides & ${ }^{293} U$ & $1.6 \mathrm{E}-03$ & $1.7 \mathrm{E}+00$ & \\
\hline Adult Trespasser & Smear & Cell & Ingestion & Radionuclides & ${ }^{234} \mathrm{Pu}$ & $8.4 \mathrm{E}-03$ & $8.8 E+\infty$ & \\
\hline Adull Trespasser & Smear & Cell & Ingestion & Radionuclides & ${ }^{2 N} U$ & $2.2 \mathrm{E}-02$ & $2.3 \mathrm{E}+01$ & \\
\hline Adult Trespasser & Smear & Cell & Ingestion & Radionuclides & ${ }^{250210} \mathrm{Pu}$ & $5.3 \mathrm{E}-02$ & $5.5 \mathrm{E}+01$ & \\
\hline Adult Trespasser & Smear & Cell & Ingestion & Radionuclides & ${ }^{\infty} \mathrm{K}$ & $5.5 \mathrm{E}-01$ & $5.8 \mathrm{E}+02$ & \\
\hline Adult Trespasser & Smear & Cell & Ingestion & Radionuclides & ${ }^{\infty} \mathrm{Co}$ & $1.4 \mathrm{E}-02$ & $1.4 \mathrm{E}+01$ & \\
\hline Adult Trespasser & Smear & Cell & Ingestion & Radionuclides & ${ }^{\infty} \mathrm{Sr}$ & $1.2 \mathrm{E}+00$ & $1.3 E+03$ & \\
\hline Adull Trespasser & Smear & Cell & Inhalation & Radionuclides & ${ }^{15} \mathrm{C}$ & $1.4 \mathrm{E}+02$ & $7.0 \mathrm{E}+00$ & \\
\hline Adult Trespasser & Smear & Cell & Inhalation & Radionuclides & ${ }^{2 \infty} x_{\mathrm{R}}$ & $5.3 \mathrm{E}-03$ & $2.6 \mathrm{E}-04$ & \\
\hline Adull Trespasser & Smear & Cell & Inhalation & Radionuclides & nth & $1.1 \mathrm{E}-02$ & $5.4 \mathrm{E}-04$ & \\
\hline Adult Trespasser & Smear & Cell & Inhalation & Radionuclides & ${ }^{200} \mathrm{Th}$ & $1.8 \mathrm{E}-02$ & 9.0E-04 & \\
\hline Adull Trespasser & Smear & Cell & Inhalation & Radionuclides & ${ }^{200} \mathrm{Th}$ & $1.1 \mathrm{E}-02$ & $5.6 \mathrm{E}-04$ & \\
\hline Adult Trespasser & Smear & Cell & Inhalation & Radionuclides & 231234 U & 1.7E-02 & 8.4E-04 & \\
\hline
\end{tabular}




\begin{tabular}{|c|c|c|c|c|c|c|c|c|}
\hline \multirow{2}{*}{ Receptor } & \multirow{2}{*}{ Media } & \multirow{2}{*}{ Location } & \multirow{2}{*}{ Exposure Pathway } & \multirow{2}{*}{ Contaminant Class } & \multirow{2}{*}{ Contaminant } & \multirow{2}{*}{$\begin{array}{l}\text { Exposure Point } \\
\text { Concentration" }\end{array}$} & \multicolumn{2}{|c|}{ Chronic Daily Intake } \\
\hline & & & & & & & $\begin{array}{c}\text { Carcinogenic } \\
\text { Effects }\end{array}$ & $\begin{array}{c}\text { Noncarcinogenic } \\
\text { Efrects }\end{array}$ \\
\hline Adult Trespasser & Smear & Cell & Inhalation & Radionuclides & ${ }^{23 S} \mathrm{U}$ & $1.6 \mathrm{E}-03$ & 7.7E-05 & \\
\hline Adult Trespasser & Smear & Cell & Inhalation & Radionuclides & ${ }^{23} \mathrm{Pu}$ & $8.4 E-03$ & 4.1E-04 & \\
\hline Adult Trespasser & Smear & Cell & Inhalation & Radionuclides & ${ }^{2 M} U$ & $2.2 \mathrm{E}-02$ & $1.1 \mathrm{E}-03$ & \\
\hline Adull Trespasser & Smear & Cell & Inhalation & Radionuclides & ${ }^{2998200} \mathrm{Pu}$ & S.3E-02 & $2.6 \mathrm{E}-03$ & \\
\hline Adull Treapasser & Smear & Cell & Inhalation & Radionuclides & ${ }^{40} \mathrm{~K}$ & S.5E-01 & $2.7 E-02$ & \\
\hline Adult Trespasser & Smear & Cell & Inhalation & Radionuclides & ${ }^{\infty} \mathrm{Co}$ & $1.4 \mathrm{E}-02$ & 6.6E-04 & \\
\hline Adult Trespasser & Smear & Cell & Inhalation & Radionuclides & ${ }^{x} \mathrm{Sr}$ & $1.2 \mathrm{E}+00$ & $5.9 \mathrm{E}-02$ & \\
\hline Adult Trespasser & Smear & Cell & External Exposure & Radionuclides & ${ }^{15} \mathrm{C}$ & $1.4 E+02$ & $4.1 E+03$ & \\
\hline Adult Trespasser & Smear & Cell & External Exposure & Radionuclides & ${ }^{20 x} \mathrm{Ra}$ & $5.3 \mathrm{E}-03$ & $1.5 \mathrm{E}-01$ & \\
\hline Adult Trespasser & Smear & Cell & External Exposure & Radionuclides & ${ }^{20} \mathrm{Th}$ & $1.1 \mathrm{E}-02$ & $3.2 \mathrm{E}-01$ & \\
\hline Adult Trespasser & Smear & Cell & External Exposure & Radionuclides & ${ }^{200} \mathrm{Th}$ & $1.8 \mathrm{E}-02$ & 5.3E-01 & \\
\hline Adult Trespasser & Smear & Cell & Extemal Exposure & Radionuclides & ${ }^{2 x} \mathrm{Th}$ & $1.1 \mathrm{E}-02$ & 3.3E-01 & \\
\hline Adult Trespasser & Smear & Cell & External Exposure & Radionuclides & ${ }^{23} 312 \times \mathrm{U}$ & $1.7 \mathrm{E}-02$ & $4.9 \mathrm{E}-01$ & \\
\hline Adult Trespasser & Smear & Cell & External Exposure & Radionuclides & ${ }^{231} \mathrm{U}$ & $1.6 \mathrm{E}-03$ & 4.5E-02 & \\
\hline Adult Trespasser & Smear & Cell & External Exposure & Radionuclides & ${ }^{234} \mathrm{Pu}$ & 8.4E-03 & $2.4 E-01$ & \\
\hline Adult Trespasser & Smear & Cell & External Exposure & Radionuclides & ${ }^{2 N O}$ & 2.2E-02 & $6.4 \mathrm{E}-01$ & \\
\hline Adult Trespasser & Smear & Cell & External Exposure & Radionuclides & ${ }^{290210} \mathrm{Pu}$ & 5.3E-02 & $1.5 \mathrm{E}+00$ & \\
\hline Adult Trespasser & Smear & Cell & Extermal Exposure & Radionuclides & ${ }^{4} \mathrm{~K}$ & 5.5E-01 & $1.6 \mathrm{E}+01$ & \\
\hline Adull Trespasser & Smear & Cell & External Exposure & Radionuclide: & ${ }^{\infty} \mathrm{Co}$ & $1.4 \mathrm{E}-02$ & $3.9 \mathrm{E}-01$ & \\
\hline Adult Trespasser & Smear & Cell & Extemal Exposure & Radionuclides & ${ }^{\infty} \mathrm{Sr}$ & $1.2 \mathrm{E}+\infty$ & $3.5 E+01$ & \\
\hline Adult Trespasser & Smear & East Gallery & Ingestion & Radionuclides & ${ }^{17} \mathrm{Cs}$ & $1.5 \mathrm{E}+01$ & $1.6 \mathrm{E}+04$ & \\
\hline
\end{tabular}

- Units are pCi/g

- Unito are pCi-day 


\begin{tabular}{|c|c|c|c|c|c|c|c|c|}
\hline \multirow{2}{*}{ Receptor } & \multirow{2}{*}{ Media } & \multirow{2}{*}{ Location } & \multirow{2}{*}{ Exposure Pathway } & \multirow{2}{*}{ Contaminant Class } & \multirow{2}{*}{ Contaminant } & \multirow{2}{*}{$\begin{array}{l}\text { Exposure Point } \\
\text { Concentration" }\end{array}$} & \multicolumn{2}{|c|}{ Chronic Daily Intake } \\
\hline & & & & & & & $\begin{array}{l}\text { Carcinogenic } \\
\text { Enfects }\end{array}$ & $\begin{array}{c}\text { Noncarcinogenix } \\
\text { Efrects }\end{array}$ \\
\hline Adult Trespasser & Smear & East Gallery & Ingestion & Radionuclides & $x_{R s}$ & $1.5 \mathrm{E}-02$ & $1.5 E+01$ & \\
\hline Adult Trespasser & Smear & East Gallery & Ingestion & Radionuclider & ${ }^{2} r_{R}$ & 2.4E-01 & $2.5 E+02$ & \\
\hline Adult Trespasser & Smear & Ean Gallery & Ingestion & Radionuclides & ${ }^{200} \mathrm{Th}$ & $2.3 \mathrm{E}-02$ & $2.4 E+01$ & \\
\hline Adult Trespasser & Smear & East Gallery & Ingestion & Radionuclides & ${ }^{200} \mathrm{Th}$ & $4.9 \mathrm{E}-02$ & $5.1 E+01$ & \\
\hline Adult Trespasser & Smear & East Gallery & Ingestion & Radionuclideः & ${ }^{200} \mathrm{Th}$ & $1.7 \mathrm{E}-02$ & $1.8 \mathrm{E}+01$ & \\
\hline Adult Trespasser & Smear & East Gallery & Ingestion & Radionuelides & $23325 \mathrm{U}$ & $3.6 \mathrm{E}-02$ & $3.8 \mathrm{E}+01$ & \\
\hline Adult Trespasser & Smear & East Gallery & Ingestion & Radionuclides & ${ }^{233} \mathrm{U}$ & $2.4 \mathrm{E}-03$ & $2.6 \mathrm{E}+00$ & \\
\hline Adult Trespasser & Smear & East Gallery & Ingestion & Radionuclides & ${ }^{23} \mathrm{Pu}$ & $1.6 \mathrm{E}-02$ & $1.7 E+01$ & \\
\hline Adult Trespasser & Smear & East Gallery & Ingestion & Radionuclides & $280 \mathrm{U}$ & $2.8 \mathrm{E}-02$ & $3.0 \mathrm{E}+01$ & \\
\hline Adult Trespasser & Smear & East Gallery & Ingestion & Radionuclides & ${ }^{259200} \mathrm{Pu}$ & $1.6 \mathrm{E}-02$ & $1.7 E+01$ & \\
\hline Adult Trespasser & Smear & East Gallery & Ingestion & Radionuclides & ${ }^{211} \mathrm{Am}$ & $2.0 \mathrm{E}-02$ & $2.1 E+01$ & \\
\hline Adult Trespasser & Smear & East Gallery & Ingestion & Radionuclides & ${ }^{+1} \mathrm{~K}$ & $3.6 \mathrm{E}+\infty$ & $3.8 \mathrm{E}+03$ & \\
\hline Adult Trespasser & Smear & East Gallery & Ingestion & Radionuclides & ${ }^{90} \mathrm{Sr}$ & $5.4 E+\infty$ & $5.7 E+03$ & \\
\hline Adult Trespasser & Smear & East Gallery & Inhalation & Radionuclides & ${ }^{157} \mathrm{Cs}$ & $1.5 E+01$ & $7.5 \mathrm{E}-01$ & \\
\hline Adult Trespasser & Smear & East Gallery & Inhalation & Radionuclides & ${ }^{20 \times} \mathrm{Ra}$ & $1.5 \mathrm{E}-02$ & $7.2 \mathrm{E}-04$ & \\
\hline Adult Trespasser & Smear & East Gallery & Inhalation & Radionuclides & ${ }^{200} \mathrm{Ra}$ & $2.4 \mathrm{E}-01$ & $1.2 \mathrm{E}-02$ & \\
\hline Adult Trespasser & Smear & East Gallery & Inhalation & Radionuclides & ${ }^{200} \mathrm{Th}$ & $2.3 \mathrm{E}-02$ & $1.1 \mathrm{E}-03$ & \\
\hline Adult Trespasser & Smear & East Gallery & Inhalation & Radionuclider & ${ }^{200} \mathrm{Th}$ & $4.9 \mathrm{E}-02$ & 2.4E-03 & \\
\hline Adult Trespasser & Smear & East Gallery & Inhalation & Radionuclides & ${ }^{200} \mathrm{Th}$ & $1.7 \mathrm{E}-02$ & 8.5E-04 & \\
\hline Adult Trespasser & Smear & Eart Gallery & Inhalation & Radionuclides & ${ }^{23} 3252 \mathrm{U}$ & $3.6 \mathrm{E}-02$ & $1.8 \mathrm{E}-03$ & \\
\hline Adull Trespasser & Smear & East Gallery & Inhalation & Radionuclider & ${ }^{235} \mathrm{U}$ & 2.4E-03 & 1.2E-04 & \\
\hline
\end{tabular}

- Units are $\mathrm{pCi} / \mathrm{g}$

- Units are pCi-day 


\begin{tabular}{|c|c|c|c|c|c|c|c|c|}
\hline \multirow{2}{*}{ Receptor } & \multirow{2}{*}{ Media } & \multirow{2}{*}{ Location } & \multirow{2}{*}{ Exposure Pathway } & \multirow{2}{*}{ Contaminant Class } & \multirow{2}{*}{ Contaminant } & \multirow{2}{*}{$\begin{array}{l}\text { Exposure Point } \\
\text { Concentration" }\end{array}$} & \multicolumn{2}{|c|}{ Chronic Daily Intake" } \\
\hline & & & & & & & $\begin{array}{l}\text { Carcinogenic } \\
\text { Errects }\end{array}$ & $\begin{array}{l}\text { Noncarcinogenic } \\
\text { Effects }\end{array}$ \\
\hline Adult Trespasser & Smear & East Gallery & Inhalation & Radionuclides & ${ }^{20} \mathrm{Pu}$ & $1.6 \mathrm{E}-02$ & 7.7E-04 & \\
\hline Adult Trespasser & Smear & Eant Gallery & Inhalation & Radionuclides & $280 \mathrm{U}$ & $2.8 \mathrm{E}-02$ & $1.4 \mathrm{E}-03$ & \\
\hline Adult Trespasser & Smear & East Gallery & Inhalation & Radionuclides & ${ }^{209250} \mathrm{Pu}$ & $1.6 \mathrm{E}-02$ & 7.7E-04 & \\
\hline Adult Trespasser & Smear & Ean Gallery & Inhalation & Radionuclide: & "uAm & $2.0 \mathrm{E}-02$ & $9.9 \mathrm{E}-04$ & \\
\hline Adult Trespasser & Smear & Eant Gallery & Inhalation & Radionuclides & ${ }^{\infty} \mathrm{K}$ & $3.6 \mathrm{E}+00$ & $1.8 \mathrm{E}-01$ & \\
\hline Adult Trespasser & Smear & East Gallery & Inhalation & Radionuclides & ${ }^{\infty} \mathrm{Sr}$ & $5.4 \mathrm{E}+00$ & 2.7E-01 & \\
\hline Adult Trespasser & Smear & Eant Gallery & External Exposure & Radionuclides & ${ }^{19} \mathrm{Cs}$ & $1.5 \mathrm{E}+01$ & $4.4 E+02$ & \\
\hline Adult Trespasser & Smear & East Gallery & External Exposure & Radionuclides & ${ }^{2 x} \mathrm{Ra}$ & $1.5 \mathrm{E}-02$ & $4.2 \mathrm{E}-01$ & \\
\hline Adult Trespasser & Smear & Eant Gallery & External Exposure & Radionuclides & ${ }^{20} \mathrm{Ra}$ & 2.4E-01 & $7.0 \mathrm{E}+00$ & \\
\hline Adult Trespasser & Smear & East Gallery & External Exposure & Radionuclides & nnth & 2.3E-02 & $6.5 \mathrm{E}-01$ & \\
\hline Adult Trespasser & Smear & East Gallery & External Exposure & Radionuclides & ${ }^{200} \mathrm{Th}$ & $4.9 \mathrm{E}-02$ & $1.4 \mathrm{E}+00$ & \\
\hline Adull Trespasser & Smear & East Gallery & External Exposure & Radionuclides & ${ }^{2 x} \mathrm{Th}$ & $1.7 \mathrm{E}-02$ & $5.0 \mathrm{E}-01$ & \\
\hline Adult Trespasser & Smear & East Gallery & External Exposure & Radionuclides & ${ }^{231 / 254} \mathrm{U}$ & $3.6 \mathrm{E}-02$ & $1.0 \mathrm{E}+\infty 0$ & \\
\hline Adult Trespasser & Smear & East Gallery & External Exposure & Radionuclides & ${ }^{23 S U}$ & $2.4 \mathrm{E}-03$ & $7.0 \mathrm{E}-02$ & \\
\hline Adull Trespasser & Smear & East Gallery & External Exposure & Radionuclides & ${ }^{250} \mathrm{Pu}$ & $1.6 \mathrm{E}-02$ & 4.5E-01 & \\
\hline Adull Trespasser & Smear & East Gallery & External Exposure & Radionuclides & ${ }^{230} \mathrm{U}$ & $2.8 \mathrm{E}-02$ & $8.1 E-01$ & \\
\hline Adult Trespasser & Smear & Eant Gallery & External Exposure & Radionuclides & $298210 \mathrm{Pu}$ & $1.6 \mathrm{E}-02$ & 4.5E-01 & \\
\hline Adult Trespasser & Smear & Eant Gallery & External Exposure & Radionuclides & ${ }^{211} \mathrm{Am}$ & $2.0 \mathrm{E}-02$ & $5.8 \mathrm{E}-01$ & \\
\hline Adult Trespasser & Smear & Eant Gallery & Extermal Exposure & Radionuclides & ${ }^{\infty} \mathrm{K}$ & $3.6 \mathrm{E}+\infty$ & $1.0 \mathrm{E}+02$ & \\
\hline Adult Trespasser & Smear & East Gallery & External Exposure & Radionuclides & ${ }^{\circ} \mathrm{Sr}$ & $5.4 E+\infty 0$ & $1.6 \mathrm{E}+02$ & \\
\hline Adult Trespasser & Smear & Whole Building & Ingestion & Radionuclide: & ${ }^{15} \mathrm{Cs}$ & $8.0 \mathrm{E}+0 \mathrm{I}$ & $8.4 E+04$ & \\
\hline
\end{tabular}

- Units are $\mathrm{pCi} / \mathrm{g}$

- Units are pCi-day 


\begin{tabular}{|c|c|c|c|c|c|c|c|c|}
\hline \multirow{2}{*}{ Receptor } & \multirow{2}{*}{ Media } & \multirow{2}{*}{ Location } & \multirow{2}{*}{ Exposure Pathway } & \multirow{2}{*}{ Contaminent Class } & \multirow{2}{*}{ Contaminant } & \multirow{2}{*}{$\begin{array}{l}\text { Exposure Point } \\
\text { Concentration" }\end{array}$} & \multicolumn{2}{|c|}{ Chronic Daily Intake" } \\
\hline & & & & & & & $\begin{array}{l}\text { Carcinogenic } \\
\text { Effects }\end{array}$ & $\begin{array}{c}\text { Noncarcinogenic } \\
\text { Effects }\end{array}$ \\
\hline Adult Trespasser & Smear & Whole Building & Ingextion & Radionuclides & ${ }^{205} \mathrm{Ra}$ & $5.9 \mathrm{E}-03$ & $6.2 E+\infty$ & \\
\hline Adult Trespasser & Smear & Whole Building & Ingertion & Radionuclides & ${ }^{n} t_{R a}$ & $2.8 \mathrm{E}-02$ & $3.0 \mathrm{E}+01$ & \\
\hline Adult Trespasser & Smear & Whole Building & Ingestion & Radionuclides & 2nTh & $1.1 \mathrm{E}-02$ & $1.2 E+01$ & \\
\hline Adult Trespasser & Smear & Whole Building & Ingertion & Radionuclide: & ${ }^{200} \mathrm{Th}$ & 2.0E-02 & $2.1 E+01$ & \\
\hline Adult Trespasser & Smear & Whole Building & Ingertion & Radionuclide: & ${ }^{20} \mathrm{Th}$ & $1.1 \mathrm{E}-02$ & $1.2 \mathrm{E}+01$ & \\
\hline Adult Trespasser & Smear & Whole Building & Ingertion & Radionuclides & ${ }^{23} 323 \mathrm{U} U$ & $1.8 \mathrm{E}-02$ & $1.9 \mathrm{E}+01$ & \\
\hline Adult Treopasser & Smear & Whole Building & Ingeation & Radionuclides & ${ }^{23} \mathrm{U}$ & $1.8 \mathrm{E}-03$ & $1.9 E+00$ & \\
\hline Adult Trespasser & Smear & Whole Building & Ingestion & Radionuclides & ${ }^{20} \mathrm{Pu}$ & 9.5E-03 & $9.9 E+\infty$ & \\
\hline Adult Trespasser & Smear & Whole Building & Ingestion & Radionuclides & ${ }^{230} \mathrm{U}$ & $2.5 E-02$ & $2.6 E+01$ & \\
\hline Adult Trespasser & Smear & Whole Building & Ingestion & Radionuclides & ${ }_{290200} \mathrm{Pu}$ & $5.8 \mathrm{E}-02$ & $6.1 E+01$ & \\
\hline Adult Trespasser & Smear & Whole Building & Ingeution & Radionuclides & ${ }^{211} \mathrm{Am}$ & $7.4 \mathrm{E}-03$ & $7.7 \mathrm{E}+\infty 0$ & \\
\hline Adult Trespasser & Smear & Whole Building & Ingestion & Radionuclides & ${ }^{\infty} \mathrm{K}$ & $4.2 \mathrm{E}-01$ & $4.4 E+02$ & \\
\hline Adult Trespasser & Smear & Whole Building & Ingestion & Radionuclides & ${ }^{\infty} \mathrm{Co}$ & $1.0 \mathrm{E}-02$ & $1.1 \mathrm{E}+01$ & \\
\hline Adule Trespasser & Smear & Whole Building & Ingestion & Radionuclides & ${ }^{*} \mathrm{Sr}$ & $9.1 \mathrm{E}-01$ & $9.6 \mathrm{E}+02$ & \\
\hline Adult Trespasser & Smear & Whole Building & Inhalation & Radionuclides & ${ }^{\mathrm{MCs}}$ & $8.0 \mathrm{E}+01$ & $3.9 \mathrm{E}+00$ & \\
\hline Adult Trespasser & Smear & Whole Building & Inhalation & Radionuclides & ${ }^{n} x_{\mathrm{Ra}}$ & $5.9 \mathrm{E}-03$ & $2.9 \mathrm{E}-04$ & \\
\hline Adult Trespasser & Smear & Whole Building & Inhalation & Radionuclides & ${ }^{20} \mathrm{Ra}$ & $2.8 \mathrm{E}-02$ & $1.4 \mathrm{E}-03$ & \\
\hline Adult Trespasser & Smear & Whole Building & Inhalation & Radionuclides & ${ }^{n}$ Th & $1.1 \mathrm{E}-02$ & $5.5 \mathrm{E}-04$ & \\
\hline Adult Trespasser & Smear & Whole Building & Inhalation & Radionuclides & ${ }^{200} \mathrm{Th}$ & $2.0 \mathrm{E}-02$ & 9.9E-04 & \\
\hline Adult Trespaszer & Smear & Whole Building & Inhalation & Radionuclides & ${ }^{250} \mathrm{Th}$ & $1.1 \mathrm{E}-02$ & $5.6 \mathrm{E}-04$ & \\
\hline Adult Trespasser & Smear & Whole Building & Inhalation & Radionuclides & ${ }^{232323} \mathrm{U}$ & $1.8 \mathrm{E}-02$ & 8.7E-04 & \\
\hline
\end{tabular}

- Units are $\mathrm{pCi} / \mathrm{g}$

- Units are pCi-day 


\begin{tabular}{|c|c|c|c|c|c|c|c|c|}
\hline \multirow{2}{*}{ Receptor } & \multirow{2}{*}{ Media } & \multirow{2}{*}{ Location } & \multirow{2}{*}{ Exposure Pathway } & \multirow{2}{*}{ Contaminant Class } & \multirow{2}{*}{ Contaminant } & \multirow{2}{*}{$\begin{array}{l}\text { Exposure Point } \\
\text { Concentration" }\end{array}$} & \multicolumn{2}{|c|}{ Chronic Daily Intake" } \\
\hline & & & & & & & $\begin{array}{l}\text { Carcinogenic } \\
\text { Effocts }\end{array}$ & $\begin{array}{c}\text { Noncarcinogenic } \\
\text { Erfects }\end{array}$ \\
\hline Adult Trespasser & Smear & Whole Building & Inhalation & Radionuclides & ${ }^{23 s} U$ & $1.8 \mathrm{E}-03$ & 8.7E-05 & \\
\hline Adult Trespasser & Smear & Whole Building & Inhalation & Radionuclides & ${ }^{23} \mathrm{Pu}$ & 9.5E-03 & 4.6E-04 & \\
\hline Adult Trespasaer & Smear & Whole Building & Inhalation & Radionuclides & ${ }^{2 M} \mathrm{U}$ & $2.5 \mathrm{E}-02$ & $1.2 \mathrm{E}-03$ & \\
\hline Adult Trespasser & Smear & Whole Building & Inhalation & Radionuclides & ${ }^{2902200} \mathrm{Pu}$ & $5.8 \mathrm{E}-02$ & $2.8 \mathrm{E}-03$ & \\
\hline Adult Trespasser & Smear & Whole Building & Inhalation & Radionuclides & ${ }^{21} \mathrm{Am}$ & 7.4E-03 & $3.6 \mathrm{E}-04$ & \\
\hline Adult Trespasser & Smear & Whole Building & Inhalation & Radionuclides & ${ }^{\infty} \mathrm{K}$ & $4.2 \mathrm{E}-01$ & 2.1E-02 & \\
\hline Adult Trespasser & Smear & Whole Building & Inhalation & Radionuclides & ${ }^{\infty} \mathrm{Co}$ & $1.0 \mathrm{E}-02$ & $5.0 \mathrm{E}-04$ & \\
\hline Adult Trespasser & Smear & Whole Building & Inhalation & Radionuclides & ${ }^{\infty} \mathrm{Sr}$ & $9.1 \mathrm{E}-01$ & $4.5 \mathrm{E}-02$ & \\
\hline Adult Trespasser & Smear & Whole Building & External Exposure & Radionuclides & ${ }^{10} \mathrm{Cs}$ & $8.0 \mathrm{E}+01$ & $2.3 \mathrm{E}+03$ & \\
\hline Adult Trespasser & Smear & Whole Building & Extermal Exposure & Radionuclides & ${ }^{226} \mathrm{Ra}$ & $5.9 \mathrm{E}-03$ & $1.7 \mathrm{E}-01$ & \\
\hline Adult Trespasser & Smear & Whole Building & External Exposure & Radionuclides & ${ }^{2 n} \mathrm{Ra}$ & $2.8 \mathrm{E}-02$ & 8.1E-01 & \\
\hline Adult Trespasser & Smear & Whole Building & External Exposure & Radionuclides & ${ }^{2 n}$ Th & $1.1 \mathrm{E}-02$ & 3.3E-01 & \\
\hline Adult Trespasser & Smear & Whole Building & External Exposure & Radionuclides & ${ }^{230} \mathrm{Th}$ & $2.0 \mathrm{E}-0 \mathrm{Z}$ & $5.8 \mathrm{E}-0 \mathrm{I}$ & \\
\hline Adult Trespasser & Smear & Whole Building & External Exposure & Radionuclides & ${ }^{25} \mathrm{Th}$ & $1.1 \mathrm{E}-02$ & $3.3 \mathrm{E}-01$ & \\
\hline Adult Trespasser & Smear & Whole Building & External Exposure & Radionuclides & ${ }^{23325} \mathrm{U}$ & $1.8 \mathrm{E}-02$ & S.IE-01 & \\
\hline Adult Trespasser & Smear & Whole Building & External Exposure & Radionuclides & ${ }^{23} \mathrm{U}$ & $1.8 \mathrm{E}-03$ & $5.1 \mathrm{E}-02$ & \\
\hline Adult Trespasser & Smear & Whole Building & Extermal Exposure & Radionuclides & ${ }^{2 s s} \mathrm{Pu}$ & 9.5E-03 & $2.7 \mathrm{E}-01$ & \\
\hline Adult Trespasser & Smear & Whole Building & External Exposure & Radionuclide: & 240 & $2.5 \mathrm{E}-02$ & $7.2 \mathrm{E}-01$ & \\
\hline Adult Trespasser & Smear & Whole Building & External Exposure & Radionuclides & ${ }^{250250} \mathrm{Pu}$ & $5.8 \mathrm{E}-02$ & $1.7 E+\infty$ & \\
\hline Adult Trespasser & Smear & Whole Building & External Exposure & Radionuclides & ${ }^{211} \mathrm{Am}$ & $7.4 \mathrm{E}-03$ & 2.1E-01 & \\
\hline Adult Trespauser & Smear & Whole Building & Extemal Exposure & Radionuclides & ${ }^{\infty} \mathrm{K}$ & $4.2 \mathrm{E}-01$ & $1.2 \mathrm{E}+01$ & \\
\hline
\end{tabular}

- Units are pCi/g

- Units are pCi-day 


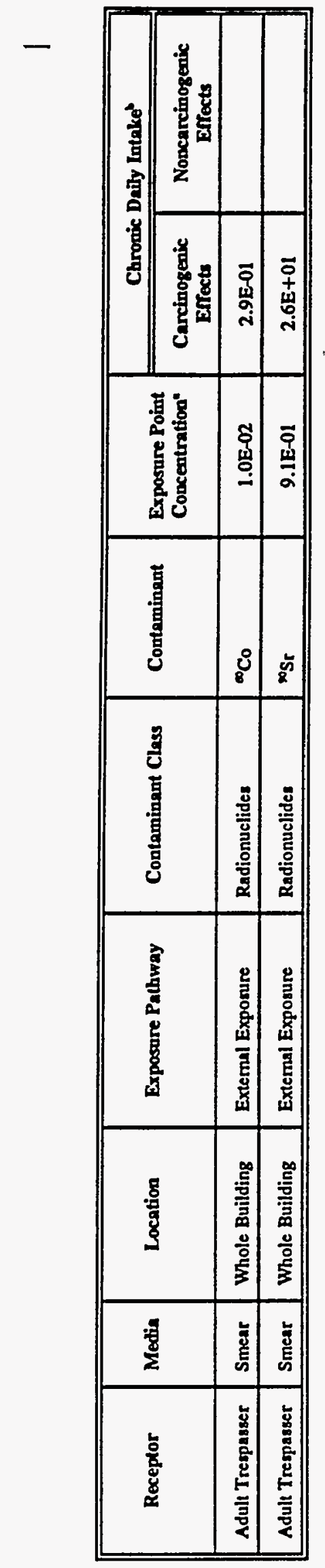

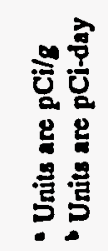


THIS PAGE INTENTIONALLY LEFT BLANK 


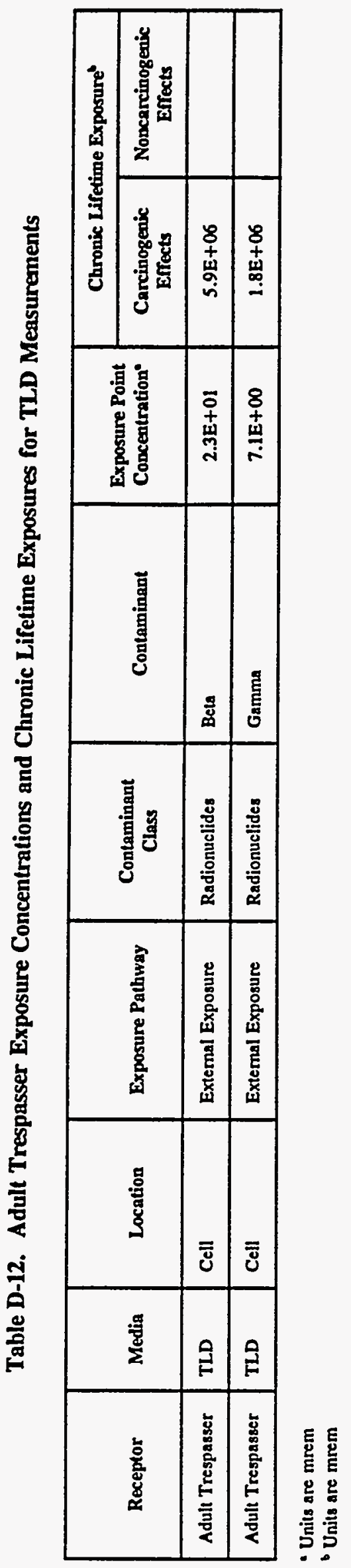


THIS PAGE INTENTIONALLY LEFT BLANK 
APPENDIX E

Risk Characterization Results 
Table E-1. Risk Characterization Results - Excess Cancer Risk Estimates - Maintenance Worker

\begin{tabular}{|c|c|c|c|c|c|c|c|}
\hline Contaminant & $\begin{array}{l}\text { Chronic } \\
\text { Daily } \\
\text { Intolke }\end{array}$ & $\begin{array}{l}\text { Slope } \\
\text { Factor }\end{array}$ & $\begin{array}{l}\text { Contominnat } \\
\text { Specific Risl }\end{array}$ & $\begin{array}{l}\text { Contamiant } \\
\text { Class Risk }\end{array}$ & $\begin{array}{c}\text { Total } \\
\text { Pathway } \\
\text { Risls }\end{array}$ & $\begin{array}{c}\text { Total } \\
\text { Contamiant } \\
\text { Risls }\end{array}$ & $\begin{array}{c}\text { Total } \\
\text { Area } \\
\text { Risk }\end{array}$ \\
\hline \multicolumn{8}{|c|}{ Exposure Pathway: Ingestion of Contaminants by Mointenonce Worker from Core Samples - Cell } \\
\hline Beryllium & 3.1E-06 & $4.3 \mathrm{E}+\infty$ & 1.3E-05 & & & & \\
\hline Total Inorganics & & & & 1.3E-05 & & & \\
\hline Aroclor-1254 & $5.9 \mathrm{E}-08$ & $7.7 \mathrm{E}+\infty 0$ & $4.6 \mathrm{E}-07$ & & & & \\
\hline Aroclor-1260 & $1.0 \mathrm{E}-07$ & 7.7E+ +0 & 7.7E-07 & & & & \\
\hline Total Organics & & & & $1.2 \mathrm{E}-06$ & & & \\
\hline${ }^{17} \mathrm{Cs}$ & $4.3 \mathrm{E}+05$ & 2.8E-11 & $1.2 \mathrm{E}-05$ & & & & \\
\hline${ }^{205} \mathrm{Ra}$ & $1.3 E+02$ & $1.2 \mathrm{E}-10$ & $1.5 \mathrm{E}-08$ & & & & \\
\hline $2 n_{T h}$ & $2.6 \mathrm{E}+02$ & $5.5 \mathrm{E}-11$ & $1.4 \mathrm{E}-08$ & & & & \\
\hline${ }^{250} \mathrm{Th}$ & $4.4 \mathrm{E}+02$ & $1.3 \mathrm{E}-11$ & 5.7E-09 & & & & \\
\hline${ }^{25} \mathrm{Th}$ & $2.7 E+02$ & $1.2 \mathrm{E}-11$ & 3.3E-09 & & & & \\
\hline $233 / 24 U$ & $4.1 E+02$ & $1.6 \mathrm{E}-11$ & $6.5 \mathrm{E}-09$ & & & & \\
\hline${ }^{254} \mathrm{Th}$ & $2.0 \mathrm{E}+03$ & $4.0 \mathrm{E}-12$ & $7.9 E-09$ & & & & \\
\hline${ }^{235} \mathrm{U}$ & $3.8 \mathrm{E}+01$ & $1.6 \mathrm{E}-11$ & $6.0 \mathrm{E}-10$ & & & & \\
\hline${ }^{238} \mathrm{Pu}$ & $1.4 \mathrm{E}+02$ & $2.2 \mathrm{E}-10$ & $3.1 \mathrm{E}-08$ & & & & \\
\hline${ }^{238} \mathrm{U}$ & $5.3 \mathrm{E}+02$ & $2.0 \mathrm{E}-11$ & 1.1E-08 & & & & \\
\hline${ }^{289200} \mathrm{Pu}$ & $1.3 \mathrm{E}+03$ & 2.3E-10 & $2.9 \mathrm{E}-07$ & & & & \\
\hline${ }^{40} \mathrm{~K}$ & $1.7 \mathrm{E}+03$ & $1.1 \mathrm{E}-11$ & $1.8 \mathrm{E}-08$ & & & & \\
\hline${ }^{60} \mathrm{Co}$ & 4.1E+01 & $1.5 \mathrm{E}-11$ & $6.1 \mathrm{E}-10$ & & & & \\
\hline${ }^{00} \mathrm{Sr}$ & $3.6 \mathrm{E}+03$ & $3.6 \mathrm{E}-11$ & $1.3 \mathrm{E}-07$ & & & & \\
\hline Tritium & $6.6 E+03$ & $5.4 \mathrm{E}-14$ & $3.6 \mathrm{E}-10$ & & & & - \\
\hline Total Radionuclides & & & & $1.3 \mathrm{E}-05$ & & & \\
\hline Total Pathway Risk & & & & & 2.7E-05 & & \\
\hline \multicolumn{8}{|c|}{ Exposure Pathway: Dermal Exposure of Contaminants by Maintenance Worker from Core Samples - Cel } \\
\hline Beryllium & $1.9 \mathrm{E}-07$ & $4.3 \mathrm{E}+02$ & 8.3E-05 & & & & \\
\hline Total Inorganics & & & & $8.3 \mathrm{E}-05$ & & & \\
\hline Aroclor-1254 & 3.7E-08 & $8.6 \mathrm{E}+\infty$ & $3.2 E-07$ & & & & \\
\hline Aroclor-1260 & $6.2 \mathrm{E}-08$ & $8.6 E+\infty 0$ & 5.3E-07 & & & & \\
\hline Total Organics & & & & $8.5 \mathrm{E}-07$ & & & \\
\hline Total Pathway Risk & & & & & $8.4 \mathrm{E}-05$ & & \\
\hline
\end{tabular}




\begin{tabular}{|c|c|c|c|c|c|c|c|}
\hline Contaminant & $\begin{array}{l}\text { Cluroxic } \\
\text { Daily } \\
\text { Intake }\end{array}$ & $\begin{array}{l}\text { Slope } \\
\text { Factor }\end{array}$ & $\begin{array}{l}\text { Contaminnat } \\
\text { Specific Risk }\end{array}$ & $\begin{array}{l}\text { Centamiont } \\
\text { Class Risk }\end{array}$ & $\begin{array}{c}\text { Total } \\
\text { Pathway } \\
\text { Risk }\end{array}$ & $\begin{array}{c}\text { Total } \\
\text { Contominnt } \\
\text { Risk }\end{array}$ & $\begin{array}{l}\text { Total } \\
\text { Aren } \\
\text { Rivk }\end{array}$ \\
\hline \multicolumn{8}{|c|}{ Exposure Pathway: Inhalation of Contaminants by Maintenance Worker from Core Samples - Cell } \\
\hline Arsenic & $4.2 \mathrm{E}-11$ & $5.0 \mathrm{E}+01$ & 2.1E-09 & - & & & \\
\hline Beryllium & $2.9 \mathrm{E}-10$ & $8.4 \mathrm{E}+\infty$ & 2.4E-09 & & & & \\
\hline Cadmium & $2.9 \mathrm{E}-09$ & $6.1 \mathrm{E}+\infty$ & $1.8 \mathrm{E}-08$ & & & & \\
\hline Chromium VI & $3.2 \mathrm{E}-09$ & $4.1 \mathrm{E}+01$ & $1.3 \mathrm{E}-07$ & & & & \\
\hline Total Inorganics & & & & $1.5 \mathrm{E}-07$ & & & \\
\hline${ }^{15} \mathrm{Cs}$ & $4.0 \mathrm{E}+01$ & $1.9 \mathrm{E}-11$ & $7.6 \mathrm{E}-10$ & & & & \\
\hline 20sa & $1.2 \mathrm{E}-02$ & 3.0E-09 & $4.8 \mathrm{E}-05$ & & & & \\
\hline${ }^{200} \mathrm{Th}$ & $2.5 \mathrm{E}-02$ & $7.8 \mathrm{E}-08$ & $1.9 \mathrm{E}-09$ & & & & \\
\hline${ }^{200} \mathrm{Th}$ & 4.1E-02 & $2.9 \mathrm{E}-08$ & $1.2 \mathrm{E}-09$ & & & & \\
\hline${ }^{20} \mathrm{Th}$ & $2.5 \mathrm{E}-02$ & 2.8E-08 & $7.1 \mathrm{E}-10$ & & & & \\
\hline${ }^{213} 23 x \mathrm{U}$ & $3.8 \mathrm{E}-02$ & $2.6 \mathrm{E}-08$ & $9.9 \mathrm{E}-10$ & & & & \\
\hline${ }^{22 x} \mathrm{Th}$ & $1.8 \mathrm{E}-01$ & $3.2 \mathrm{E}-11$ & $5.9 \mathrm{E}-12$ & & & & \\
\hline${ }^{215 U}$ & $3.5 \mathrm{E}-03$ & $2.5 E-08$ & $8.8 \mathrm{E}-11$ & & & & \\
\hline${ }^{258} \mathrm{Pu}$ & $1.3 \mathrm{E}-02$ & $3.9 \mathrm{E}-08$ & $5.1 \mathrm{E}-10$ & & & & \\
\hline 200 & $5.0 \mathrm{E}-02$ & $2.4 \mathrm{E}-08$ & $1.2 \mathrm{E}-09$ & & & & \\
\hline${ }^{298200} \mathrm{Pu}$ & $1.2 \mathrm{E}-01$ & $3.8 \mathrm{E}-08$ & $4.4 \mathrm{E}-09$ & & & & \\
\hline${ }^{40} \mathrm{~K}$ & $1.5 \mathrm{E}-01$ & $7.6 \mathrm{E}-12$ & $1.2 \mathrm{E}-12$ & & & & \\
\hline${ }^{60} \mathrm{Co}$ & $3.8 \mathrm{E}-03$ & $1.5 \mathrm{E}-10$ & $5.7 \mathrm{E}-13$ & & & & \\
\hline${ }^{90} \mathrm{Sr}$ & $3.4 \mathrm{E}-01$ & $6.2 \mathrm{E}-11$ & $2.1 \mathrm{E}-11$ & & & & \\
\hline Tritium & $6.2 \mathrm{E}-01$ & $7.8 E-14$ & 4.8E-14 & & & & \\
\hline Total Radionuciides & & & & $4.8 \mathrm{E}-05$ & & & \\
\hline Total Pathway Risk & & & & & 4.8E-05 & & \\
\hline \multicolumn{8}{|c|}{ Exposure Pathway: External Exposnre to Radionuclides by Maintenance Worker from Core Samples - Cell } \\
\hline${ }^{15} \mathrm{Cs}$ & $6.3 \mathrm{E}+03$ & $2.0 \mathrm{E}-06$ & $1.2 \mathrm{E}-02$ & & & & \\
\hline${ }^{2} \mathrm{Ra}$ & $1.8 \mathrm{E}+\infty$ & $6.0 \mathrm{E}-06$ & $1.1 E-05$ & & & & \\
\hline 20Th & $3.8 E+\infty 0$ & $5.6 \mathrm{E}-06$ & 2.1E-05 & & & & \\
\hline $200 \mathrm{Th}$ & $6.4 E+\infty$ & $5.4 \mathrm{E}-11$ & $3.5 \mathrm{E}-10$ & & & & \\
\hline${ }^{250} \mathrm{Th}$ & $4.0 \mathrm{E}+00$ & $2.6 \mathrm{E}-11$ & $1.0 \mathrm{E}-10$ & & & & \\
\hline${ }^{252534} \mathrm{U}$ & $5.9 \mathrm{E}+\infty 0$ & $3.0 \mathrm{E}-11$ & $1.8 \mathrm{E}-10$ & & & & \\
\hline${ }^{25} \mathrm{Th}$ & $2.9 \mathrm{E}+01$ & $3.5 \mathrm{E}-09$ & $1.0 \mathrm{E}-07$ & & & & \\
\hline${ }^{205} \mathrm{U}$ & $5.5 \mathrm{E}-01$ & $2.4 \mathrm{E}-07$ & $1.3 \mathrm{E}-07$ & & & & \\
\hline${ }^{238} \mathrm{Pu}$ & $2.1 E+\infty$ & $2.8 \mathrm{E}-11$ & $5.8 \mathrm{E}-11$ & & & & \\
\hline
\end{tabular}




\begin{tabular}{|c|c|c|c|c|c|c|c|}
\hline Contaminant & $\begin{array}{c}\text { Chrouic } \\
\text { Daily } \\
\text { Intalre }\end{array}$ & $\begin{array}{l}\text { Slope } \\
\text { Factor }\end{array}$ & $\begin{array}{l}\text { Contominnat } \\
\text { Specific Risk }\end{array}$ & $\begin{array}{l}\text { Contamiant } \\
\text { Class Risk }\end{array}$ & $\begin{array}{c}\text { Total } \\
\text { Pathway } \\
\text { Rislr }\end{array}$ & $\begin{array}{c}\text { Total } \\
\text { Contomint } \\
\text { Risl }\end{array}$ & $\begin{array}{l}\text { Total } \\
\text { Area } \\
\text { Risk }\end{array}$ \\
\hline${ }^{22 x} \mathrm{U}$ & $7.8 \mathrm{E}+\infty$ & $5.1 \mathrm{E}-08$ & 4.0E-07 & & & & \\
\hline${ }^{258200} \mathrm{Pu}$ & $1.8 \mathrm{E}+01$ & $1.7 \mathrm{E}-11$ & $3.1 \mathrm{E}-10$ & & & & \\
\hline${ }^{40} \mathrm{~K}$ & $2.4 \mathrm{E}+01$ & $5.4 \mathrm{E}-07$ & $1.3 \mathrm{E}-05$ & & & & \\
\hline${ }^{\infty} \mathrm{Co}$ & $5.9 \mathrm{E}-01$ & $8.6 \mathrm{E}-06$ & 5.1E-06 & & & & \\
\hline${ }^{90} \mathrm{Sr}$ & $5.3 E+01$ & $0.0 \mathrm{E}+00$ & $0.0 \mathrm{E}+00$ & & & & \\
\hline Tritium & $9.6 \mathrm{E}+01$ & $0.0 \mathrm{E}+\infty$ & $0.0 \mathrm{E}+\infty$ & & & - & \\
\hline Total Radionuclides & & & & $1.3 \mathrm{E}-02$ & & & \\
\hline Total Pathway Risk & & & & & $1.3 \mathrm{E}-02$ & & \\
\hline Total Inorganic Risk & & & & & & 9.6E-05 & \\
\hline Total Organic Risk & & & . & & & 2.1E-06 & \\
\hline Total Radionuclide Risk & & & & & & $1.3 \mathrm{E}-02$ & \\
\hline Total Area & & & & & & & $1.3 \mathrm{E}-02$ \\
\hline \multicolumn{8}{|c|}{ Exposure Pathway: Ingestion of Contaminants by Maintenance Worker from Core Somples - East Gallery } \\
\hline Beryllium & $7.0 \mathrm{E}-08$ & $4.3 \mathrm{E}+00$ & $3.0 \mathrm{E}-07$ & & & & \\
\hline Total Inorganics & & & & $3.0 \mathrm{E}-07$ & & & \\
\hline Aroclor-1254 & 3.0E-07 & 7.7E+00 & 2.3E-06 & & & & \\
\hline Aroclor-1260 & 4.5E-07 & $7.7 \mathrm{E}+\infty 0$ & $3.5 \mathrm{E}-06$ & & & & \\
\hline Bis(2-ethylhexyl)phthalate & $4.2 \mathrm{E}-08$ & $1.4 \mathrm{E}-02$ & $5.9 \mathrm{E}-10$ & & & & \\
\hline Total Organics & & & & $5.8 \mathrm{E}-06$ & & & \\
\hline${ }^{157} \mathrm{Cs}$ & $7.1 \mathrm{E}+03$ & $2.8 \mathrm{E}-11$ & $2.0 \mathrm{E}-07$ & & & & \\
\hline${ }^{20}{ }_{\mathrm{Ra}}$ & $1.1 \mathrm{E}+02$ & $1.2 \mathrm{E}-10$ & $1.4 \mathrm{E}-08$ & & & & \\
\hline $2{ }_{R a}$ & $1.2 \mathrm{E}+02$ & $1.0 \mathrm{E}-10$ & $1.2 \mathrm{E}-08$ & & & & \\
\hline $2 \times T h$ & $1.8 E+02$ & $5.5 \mathrm{E}-11$ & $9.6 \mathrm{E}-09$ & & & & \\
\hline${ }^{200} \mathrm{Th}$ & $3.8 \mathrm{E}+02$ & 1.3E-11 & $4.9 \mathrm{E}-09$ & & & & \\
\hline${ }^{200} \mathrm{Th}$ & $1.3 E+02$ & $1.2 \mathrm{E}-11$ & $1.6 \mathrm{E}-09$ & & & & \\
\hline${ }^{235254} U$ & $2.8 \mathrm{E}+02$ & $1.6 \mathrm{E}-11$ & $4.5 \mathrm{E}-09$ & & & & \\
\hline${ }^{258} \mathrm{U}$ & $2.4 E+02$ & $2.0 \mathrm{E}-11$ & $4.8 \mathrm{E}-09$ & & & & \\
\hline${ }^{211} \mathrm{Am}$ & $1.6 \mathrm{E}+02$ & $2.4 \mathrm{E}-10$ & $3.8 \mathrm{E}-08$ & & & & \\
\hline${ }^{\infty} \mathrm{K}$ & $1.7 E+03$ & $1.1 \mathrm{E}-11$ & $1.8 \mathrm{E}-08$ & & & & \\
\hline${ }^{\infty} \mathrm{Sr}$ & $2.5 \mathrm{E}+03$ & $3.6 \mathrm{E}-11$ & $9.1 \mathrm{E}-08$ & & & & \\
\hline Tritium & $8.0 \mathrm{E}+03$ & $5.4 \mathrm{E}-14$ & 4.3E-10 & & & & \\
\hline Total Radionuclides & & & & $4.0 \mathrm{E}-07$ & & & \\
\hline Total Pathway Risk & & & & & $6.5 \mathrm{E}-06$ & & \\
\hline
\end{tabular}




\begin{tabular}{|c|c|c|c|c|c|c|c|}
\hline Contaminant & $\begin{array}{l}\text { Chrodic } \\
\text { Daily } \\
\text { Intalce }\end{array}$ & $\begin{array}{l}\text { Slope } \\
\text { Factor }\end{array}$ & $\begin{array}{l}\text { Contaminnat } \\
\text { Specific Risk }\end{array}$ & $\begin{array}{l}\text { Contomient } \\
\text { Cless Risk }\end{array}$ & $\begin{array}{c}\text { Total } \\
\text { Pathway } \\
\text { Risk }\end{array}$ & $\begin{array}{c}\text { Total } \\
\text { Contamiant } \\
\text { Risk }\end{array}$ & $\begin{array}{l}\text { Total } \\
\text { Area } \\
\text { Riek }\end{array}$ \\
\hline \multicolumn{8}{|c|}{ Exposure Pathway: Dermal Exposure to Contaminants by Maintenance Worker from Core Somples - East Gallery } \\
\hline Beryllium & 4.4E-09 & $4.3 \mathrm{E}+02$ & $1.9 \mathrm{E}-06$ & & & & \\
\hline Total Inorganics & & & & $1.9 \mathrm{E}-06$ & & & \\
\hline Aroclor-1254 & $1.9 \mathrm{E}-07$ & $8.6 \mathrm{E}+00$ & $1.6 \mathrm{E}-06$ & & & & \\
\hline Aroclor-1260 & $2.8 \mathrm{E}-07$ & $8.6 \mathrm{E}+\infty 0$ & $2.4 \mathrm{E}-06$ & & & & \\
\hline Bis(2-ethylhexyl)phthalate & 2.6E-08 & 7.4E-02 & $1.9 \mathrm{E}-09$ & & & & \\
\hline Total Organics & & & & $4.0 \mathrm{E}-06$ & & & \\
\hline Total Pathway Risk & & & & & $5.9 \mathrm{E}-06$ & & \\
\hline \multicolumn{8}{|c|}{ Exposure Patbway: Inhalation of Contaminants by Maintenance Worker from Core Samples - East Gallery } \\
\hline Arsenic & $4.9 \mathrm{E}-11$ & $5.0 \mathrm{E}+01$ & $2.4 \mathrm{E}-09$ & & & & \\
\hline Beryllium & $6.5 \mathrm{E}-12$ & $8.4 \mathrm{E}+\infty 0$ & $5.5 \mathrm{E}-11$ & & & & \\
\hline Cadmium & 8.3E-11 & $6.1 \mathrm{E}+00$ & $5.1 \mathrm{E}-10$ & & & & \\
\hline Chromium VI & $1.4 \mathrm{E}-10$ & $4.1 \mathrm{E}+01$ & $5.6 \mathrm{E}-09$ & & & & \\
\hline Total Inorganics & & & & $3.6 \mathrm{E}-09$ & & & \\
\hline${ }^{157} \mathrm{Cs}$ & $6.6 \mathrm{E}-01$ & $1.9 \mathrm{E}-11$ & $1.3 \mathrm{E}-11$ & & & & \\
\hline${ }^{20} \mathrm{Ra}$ & $1.1 \mathrm{E}-02$ & $3.0 \mathrm{E}-09$ & 4.3E-05 & & & & \\
\hline $2=R_{a}$ & $1.1 \mathrm{E}-02$ & $6.9 E-10$ & $7.4 \mathrm{E}-12$ & & & & \\
\hline 200 h & $1.6 \mathrm{E}-02$ & $7.8 \mathrm{E}-08$ & $1.3 \mathrm{E}-09$ & & & & \\
\hline${ }^{200} \mathrm{Th}$ & $3.5 \mathrm{E}-02$ & $2.9 \mathrm{E}-08$ & $1.0 \mathrm{E}-09$ & & & & \\
\hline${ }^{20} \mathrm{Th}$ & $1.3 \mathrm{E}-02$ & $2.8 \mathrm{E}-08$ & 3.5E-10 & & & & \\
\hline${ }^{232324} U$ & $2.6 \mathrm{E}-02$ & $2.6 \mathrm{E}-08$ & $6.8 \mathrm{E}-10$ & & & & \\
\hline${ }^{2 \times} \mathrm{U}$ & $2.2 \mathrm{E}-02$ & $2.4 \mathrm{E}-08$ & $5.4 \mathrm{E}-10$ & & & & \\
\hline${ }^{24 !} \mathrm{Am}$ & $1.5 \mathrm{E}-02$ & $3.2 \mathrm{E}-08$ & 4.7E-10 & & & & \\
\hline${ }^{\infty} \mathrm{K}$ & $1.6 \mathrm{E}-01$ & $7.6 \mathrm{E}-12$ & $1.2 \mathrm{E}-12$ & & & & \\
\hline${ }^{\infty} \mathrm{Sr}$ & $2.4 \mathrm{E}-01$ & $6.2 \mathrm{E}-11$ & $1.5 \mathrm{E}-11$ & & & & \\
\hline Tritium & $7.5 \mathrm{E}-01$ & $7.8 \mathrm{E}-14$ & $5.8 \mathrm{E}-14$ & & & & \\
\hline Total Radionuclides & & & & $4.3 \mathrm{E}-05$ & & & \\
\hline Total Pathway Risk & & & & & 4.3E-05 & & \\
\hline \multicolumn{8}{|c|}{ Exposure Pathway: External Exposure to Radionuclides by Maintenance Worker from Core Semples - East Gallery } \\
\hline${ }^{157} \mathrm{Cs}$ & $1.0 \mathrm{E}+02$ & $2.0 \mathrm{E}-06$ & $2.1 \mathrm{E}-04$ & & & & \\
\hline${ }^{2 \times} \mathrm{Ra}$ & $1.6 \mathrm{E}+00$ & $6.0 \mathrm{E}-06$ & $9.9 \mathrm{E}-06$ & & & & \\
\hline $\operatorname{ma}$ & $1.7 \mathrm{E}+00$ & $2.9 \mathrm{E}-06$ & $4.9 \mathrm{E}-06$ & & & & \\
\hline 20Th & $2.6 \mathrm{E}+\infty$ & $5.6 \mathrm{E}-06$ & $1.4 \mathrm{E}-05$ & & & & \\
\hline
\end{tabular}




\begin{tabular}{|c|c|c|c|c|c|c|c|}
\hline Contaminant & $\begin{array}{c}\text { Chronic } \\
\text { Daily } \\
\text { Intake } \\
\end{array}$ & $\begin{array}{l}\text { Slope } \\
\text { Factor }\end{array}$ & $\begin{array}{l}\text { Contaminarat } \\
\text { Specific Risk }\end{array}$ & $\begin{array}{l}\text { Contamiant } \\
\text { Class Risk }\end{array}$ & $\begin{array}{c}\text { Total } \\
\text { Pathwray } \\
\text { Risk } \\
\end{array}$ & $\begin{array}{c}\text { Totol } \\
\text { Contamient } \\
\text { Risk } \\
\end{array}$ & $\begin{array}{l}\text { Totel } \\
\text { Area } \\
\text { Risk } \\
\end{array}$ \\
\hline${ }^{200} \mathrm{Th}$ & $5.5 E+\infty 0$ & $5.4 \mathrm{E}-11$ & $3.0 \mathrm{E}-10$ & & & & \\
\hline${ }^{20} \mathrm{Th}$ & $2.0 E+\infty 0$ & $2.6 \mathrm{E}-11$ & $5.1 E-11$ & & & & \\
\hline${ }^{23} 2525 \mathrm{U}$ & $4.1 E+\infty$ & $3.0 E-11$ & $1.2 \mathrm{E}-10$ & & & & \\
\hline 240 & $3.5 \mathrm{E}+\infty 0$ & $5.1 \mathrm{E}-08$ & $1.8 \mathrm{E}-07$ & & & & \\
\hline${ }^{201} \mathrm{Am}$ & $2.3 E+00$ & 4.9E-09 & $1.1 \mathrm{E}-08$ & & & & \\
\hline${ }^{\infty} \mathrm{K}$ & $2.5 \mathrm{E}+01$ & $5.4 \mathrm{E}-07$ & 1.3E-05 & & & & \\
\hline${ }^{90} \mathrm{Sr}$ & $3.7 E+01$ & $0.0 \mathrm{E}+\infty$ & $0.0 \mathrm{E}+\infty$ & & & & \\
\hline Tritium & $1.2 \mathrm{E}+02$ & $0.0 \mathrm{E}+\infty$ & $0.0 E+\infty$ & & & & \\
\hline Total Radionuclides & & & & 2.5E-04 & & & \\
\hline Total Pathway Risk & & & & & $2.5 \mathrm{E}-04$ & & \\
\hline Total Inorganic Risk & & & & & & $2.2 \mathrm{E}-06$ & \\
\hline Total Organic Risk & & & & & & $9.8 \mathrm{E}-06$ & \\
\hline Total Radionuclide Risk & & & & & & 2.9E-04 & \\
\hline Total Area & & & & & & & 3.1E-04 \\
\hline \multicolumn{8}{|c|}{ Exposure Pathway: Ingestion of Contaminants by Meintenance Worker from Sectiment in Cell } \\
\hline Aroclor-1254 & 7.2E-05 & 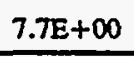 & 5.5E-04 & & & & \\
\hline Benzo(a)pyrene & $1.0 \mathrm{E}-06$ & $7.3 \mathrm{E}+\infty 0$ & 7.3E-06 & & & & \\
\hline Benzo(k)fluoranthene & 8.0E-06 & 7.3E-02 & $5.9 \mathrm{E}-07$ & & & & \\
\hline Bis(2-ethylhexyl)phthalate & 3.7E-07 & $1.4 \mathrm{E}-02$ & $5.1 \mathrm{E}-09$ & & & & \\
\hline Carbazole & 3.5E-07 & $2.0 \mathrm{E}-02$ & 7.0E-09 & & & & \\
\hline Chrysene & 3.3E-06 & $7.3 E-03$ & 2.4E-08 & & & & \\
\hline Dibenz(a,h)anthracene & $2.6 \mathrm{E}-07$ & $7.3 \mathrm{E}+\infty$ & $1.9 \mathrm{E}-06$ & & & & \\
\hline Indeno $(1,2,3-c d)$ pyrene & $1.7 \mathrm{E}-06$ & $7.3 \mathrm{E}-01$ & $1.2 \mathrm{E}-06$ & & & & \\
\hline Total Organics & & & & $5.6 \mathrm{E}-04$ & & & \\
\hline${ }^{15} \mathrm{Cs}$ & $9.6 \mathrm{E}+06$ & $2.8 \mathrm{E}-11$ & 2.7E-04 & & & & \\
\hline $20 \mathrm{Th}$ & $1.7 \mathrm{E}+02$ & $5.5 \mathrm{E}-11$ & $9.3 \mathrm{E}-09$ & & & & \\
\hline${ }^{20} \mathrm{Th}$ & $5.8 \mathrm{E}+03$ & $8.9 \mathrm{E}-11$ & $5.2 \mathrm{E}-07$ & & & & \\
\hline${ }^{200} \mathrm{Th}$ & $1.7 \mathrm{E}+02$ & $1.2 \mathrm{E}-11$ & $2.1 \mathrm{E}-09$ & & & & \\
\hline${ }^{235234} \mathrm{U}$ & $1.1 E+03$ & $1.6 \mathrm{E}-11$ & $1.7 \mathrm{E}-08$ & & & & \\
\hline${ }^{250} \mathrm{Pu}$ & $3.6 \mathrm{E}+02$ & $2.2 \mathrm{E}-10$ & $8.0 \mathrm{E}-08$ & & & & \\
\hline $25 \mathrm{U}$ & $7.0 \mathrm{E}+02$ & $2.0 \mathrm{E}-11$ & $1.4 \mathrm{E}-08$ & & & & \\
\hline${ }^{2091200} \mathrm{Pu}$ & $1.1 \mathrm{E}+04$ & 2.3E-10 & $2.5 \mathrm{E}-06$ & & & & \\
\hline${ }^{+0} \mathrm{~K}$ & $2.9 E+03$ & $1.1 \mathrm{E}-11$ & $3.2 \mathrm{E}-08$ & & & & \\
\hline
\end{tabular}




\begin{tabular}{|c|c|c|c|c|c|c|c|}
\hline Contaminant & $\begin{array}{l}\text { Chroxic } \\
\text { Daily } \\
\text { Intake }\end{array}$ & $\begin{array}{l}\text { Slope } \\
\text { Factor }\end{array}$ & $\begin{array}{l}\text { Contaminnat } \\
\text { Specific Risl }\end{array}$ & $\begin{array}{l}\text { Contamiant } \\
\text { Class Risls }\end{array}$ & $\begin{array}{c}\text { Total } \\
\text { Pathway } \\
\text { Risk }\end{array}$ & $\begin{array}{c}\text { Total } \\
\text { Contamiant } \\
\text { Risk }\end{array}$ & $\begin{array}{l}\text { Total } \\
\text { Area } \\
\text { Risk }\end{array}$ \\
\hline${ }^{60} \mathrm{Co}$ & $1.1 \mathrm{E}+03$ & $1.5 \mathrm{E}-11$ & $1.6 \mathrm{E}-08$ & & & & \\
\hline${ }^{20} \mathrm{Sr}$ & $3.0 \mathrm{E}+04$ & $3.6 \mathrm{E}-11$ & $1.1 \mathrm{E}-06$ & & & & \\
\hline Tritium & $3.1 \mathrm{E}+02$ & $5.4 \mathrm{E}-14$ & $1.7 \mathrm{E}-11$ & & & & \\
\hline Total Radionuclides & & & & 2.7E-04 & & & \\
\hline Total Pathway Risk & & & & & 8.3E-04 & & \\
\hline \multicolumn{8}{|c|}{ Exposure Pathway: Dermal Exposure to Contaminants by Mointenance Worker from Sediment in Cell } \\
\hline Aroclor-1254 & 4.5E-05 & $8.6 \mathrm{E}+\infty 0$ & $3.8 \mathrm{E}-04$ & & & & \\
\hline Benzo(a)pyrene & $6.2 \mathrm{E}-07$ & $2.4 E+01$ & $1.5 \mathrm{E}-05$ & & & & \\
\hline Benzo(k)fluoranthene & $5.0 \mathrm{E}-06$ & $2.4 \mathrm{E}-01$ & $1.2 \mathrm{E}-06$ & & & & \\
\hline Bis(2-ethylhexyl)phthalate & 2.3E-07 & $7.4 \mathrm{E}-02$ & $1.7 \mathrm{E}-08$ & & & & \\
\hline Carbazole & 2.2E-07 & $2.0 \mathrm{E}-02$ & $4.4 \mathrm{E}-09$ & & & & \\
\hline Chrysene & 2.1E-06 & $2.4 \mathrm{E}-02$ & $4.9 \mathrm{E}-08$ & & & & \\
\hline Dibenz(a,h)anthracene & $1.6 \mathrm{E}-07$ & $8.1 E+00$ & $1.3 \mathrm{E}-06$ & & & & \\
\hline Indeno( $1,2,3-\mathrm{cd})$ pyrene & $1.0 \mathrm{E}-06$ & $2.4 \mathrm{E}+00$ & 2.5E-06 & & & & \\
\hline Total Organics & & & & $4.0 \mathrm{E}-04$ & & & \\
\hline \multicolumn{8}{|l|}{ Total Pathway Risk } \\
\hline \multicolumn{8}{|c|}{ Exposure Pathway: Inhalation of Contaminants by Maintenance Worker from Setiment in Cell } \\
\hline Arsenic & $3.5 \mathrm{E}-10$ & $5.0 \mathrm{E}+01$ & $1.8 \mathrm{E}-08$ & & & & \\
\hline Cadmium & $9.8 \mathrm{E}-10$ & $6.1 E+\infty$ & $6.0 \mathrm{E}-09$ & & & & \\
\hline Chromium VI & $3.1 E-09$ & $4.1 \mathrm{E}+01$ & $1.3 \mathrm{E}-07$ & & & & \\
\hline Total Inorganics & & & & $1.5 \mathrm{E}-07$ & & & \\
\hline${ }^{15} \mathrm{Cs}$ & $9.0 \mathrm{E}+02$ & $1.9 \mathrm{E}-11$ & $1.7 \mathrm{E}-08$ & & & & \\
\hline${ }^{20}$ Th & $1.6 \mathrm{E}-02$ & $7.8 \mathrm{E}-08$ & $1.2 \mathrm{E}-09$ & & & & \\
\hline${ }^{29} \mathrm{Th}$ & $5.5 \mathrm{E}-01$ & 7.7E-08 & 4.2E-08 & & & & \\
\hline${ }^{200} \mathrm{Th}$ & $1.6 \mathrm{E}-02$ & $2.8 \mathrm{E}-08$ & $4.5 \mathrm{E}-10$ & & & & \\
\hline 232824 & $1.0 \mathrm{E}-01$ & $2.6 \mathrm{E}-08$ & $2.6 \mathrm{E}-09$ & & & & \\
\hline${ }^{258} \mathrm{Pu}$ & $3.4 \mathrm{E}-02$ & $3.9 E-08$ & $1.3 \mathrm{E}-09$ & & & & \\
\hline${ }^{2 x} \mathrm{U}$ & $6.5 \mathrm{E}-02$ & $2.4 E-08$ & $1.6 \mathrm{E}-09$ & & & & \\
\hline${ }^{29200 \mathrm{Pu}}$ & $1.0 \mathrm{E}+\infty 0$ & $3.8 \mathrm{E}-08$ & $3.9 \mathrm{E}-08$ & & & & \\
\hline${ }^{\infty} \mathrm{K}$ & $2.8 \mathrm{E}-01$ & $7.6 \mathrm{E}-12$ & 2.1E-12 & & & & \\
\hline${ }^{\infty} \mathrm{Co}$ & $1.0 \mathrm{E}-01$ & $1.5 \mathrm{E}-10$ & $1.5 \mathrm{E}-11$ & & & & \\
\hline${ }^{\infty} \mathrm{Sr}$ & $2.8 \mathrm{E}+00$ & $6.2 \mathrm{E}-11$ & $1.8 \mathrm{E}-10$ & & & & \\
\hline Tritium & $2.9 \mathrm{E}-02$ & $7.8 \mathrm{E}-14$ & $2.3 E-15$ & & & & \\
\hline
\end{tabular}




\begin{tabular}{|c|c|c|c|c|c|c|c|}
\hline Contaminant & $\begin{array}{c}\text { Chronic } \\
\text { Daily } \\
\text { Intake } \\
\end{array}$ & $\begin{array}{l}\text { Slope } \\
\text { Factor }\end{array}$ & $\begin{array}{l}\text { Contaminnat } \\
\text { Specific Risk }\end{array}$ & $\begin{array}{l}\text { Contamiant } \\
\text { Class Risk }\end{array}$ & $\begin{array}{c}\text { Total } \\
\text { Pathway } \\
\text { Risk }\end{array}$ & $\begin{array}{c}\text { Total } \\
\text { Contamiant } \\
\text { Risk }\end{array}$ & $\begin{array}{c}\text { Total } \\
\text { Area } \\
\text { Risk } \\
\end{array}$ \\
\hline Total Radionuclides & & & & $1.1 \mathrm{E}-07$ & & & \\
\hline Total Pathway Risk & & & & & $2.6 \mathrm{E}-07$ & & \\
\hline \multicolumn{8}{|c|}{ Exposure Pathway: Extermal Exposure to Radionuclides by Maintenance Worker from Sediment in Cell } \\
\hline${ }^{17} \mathrm{Cs}$ & $1.4 \mathrm{E}+05$ & $2.0 \mathrm{E}-06$ & $2.5 \mathrm{E}-01$ & & & & \\
\hline${ }^{20} \mathrm{Th}$ & $2.5 \mathrm{E}+00$ & $5.6 \mathrm{E}-06$ & $1.4 \mathrm{E}-05$ & & & & \\
\hline${ }^{209} \mathrm{Th}$ & $8.5 \mathrm{E}+01$ & $6.8 \mathrm{E}-07$ & $5.8 \mathrm{E}-05$ & & & & \\
\hline${ }^{20} \mathrm{Th}$ & $2.5 \mathrm{E}+\infty 0$ & $2.6 \mathrm{E}-11$ & $6.5 \mathrm{E}-11$ & & & & \\
\hline${ }^{233234} \mathrm{U}$ & $1.6 \mathrm{E}+01$ & $3.0 \mathrm{E}-11$ & $4.7 \mathrm{E}-10$ & & & & \\
\hline${ }^{2 s 8} \mathrm{Pu}$ & $5.3 \mathrm{E}+\infty 0$ & $2.8 \mathrm{E}-11$ & $1.5 \mathrm{E}-10$ & & & & \\
\hline${ }^{23 \times} \mathrm{U}$ & $1.0 \mathrm{E}+01$ & 5.1E-08 & $5.2 \mathrm{E}-07$ & & & & \\
\hline${ }^{239210} \mathrm{Pu}$ & $1.6 \mathrm{E}+02$ & 1.7E-11 & $2.7 \mathrm{E}-09$ & & & & \\
\hline${ }^{10} \mathrm{~K}$ & $4.3 \mathrm{E}+01$ & 5.4E-07 & $2.3 \mathrm{E}-05$ & & & & \\
\hline${ }^{\infty} \mathrm{Co}$ & $1.6 \mathrm{E}+01$ & $8.6 \mathrm{E}-06$ & $1.3 \mathrm{E}-04$ & & & & \\
\hline${ }^{8} \mathrm{Sr}$ & $4.5 E+02$ & $0.0 \mathrm{E}+00$ & $0.0 \mathrm{E}+\infty$ & & & & \\
\hline Tritium & $4.6 \mathrm{E}+\infty 0$ & $0.0 \mathrm{E}+00$ & $0.0 \mathrm{E}+00$ & & & & \\
\hline Total Radionuclides & & & & $2.5 \mathrm{E}-01$ & & & \\
\hline Total Pathway Risk & & & & & $2.5 \mathrm{E}-01$ & & \\
\hline Total Inorganic Risk & & & & & & $1.5 E-07$ & \\
\hline Total Organic Risk & & & & & & $9.6 \mathrm{E}-04$ & \\
\hline Total Radionuclide Risk & & & & & & $2.5 \mathrm{E}-01$ & \\
\hline Total Area & & & & & & & 2.5E-01 \\
\hline \multicolumn{8}{|c|}{ Exposure Pathway: Ingestion of Contaminants by Maintenance Worker from Core Samples - Whole Building } \\
\hline Beryllium & $2.2 \mathrm{E}-06$ & $4.3 \mathrm{E}+\infty$ & $9.3 E-06$ & & & & \\
\hline Total Inorganics & & & & $9.3 E-06$ & & & \\
\hline Aroclor-1254 & $2.5 E-07$ & $7.7 \varepsilon+\infty$ & $2.0 \mathrm{E}-06$ & & & & \\
\hline Aroclor- 1260 & $4.5 \mathrm{E}-07$ & $7.7 \mathrm{E}+00$ & $3.5 \mathrm{E}-06$ & & & & \\
\hline Bis(2-ethylhexyl)phthalate & $4.2 \mathrm{E}-08$ & $1.4 E-02$ & $5.9 \mathrm{E}-10$ & & & & \\
\hline Total Organice & & & & $5.4 \mathrm{E}-06$ & & & \\
\hline${ }^{157} \mathrm{Cs}$ & $4.3 E+05$ & $2.8 \mathrm{E}-11$ & $1.2 \mathrm{E}-0 \mathrm{~S}$ & & & & \\
\hline${ }^{206} \mathrm{Ra}$ & $1.3 \mathrm{E}+02$ & $1.2 \mathrm{E}-10$ & $1.5 \mathrm{E}-08$ & & & & \\
\hline${ }^{22} \mathrm{Ra}$ & $1.2 \mathrm{E}+02$ & $1.0 \mathrm{E}-10$ & $1.2 \mathrm{E}-08$ & & & & \\
\hline roTh & $2.4 \mathrm{E}+02$ & $5.5 \mathrm{E}-11$ & $1.3 \mathrm{E}-08$ & & & & \\
\hline${ }^{200} \mathrm{Th}$ & $4.4 \mathrm{E}+02$ & $1.3 \mathrm{E}-11$ & 5.7E-09 & & & & \\
\hline
\end{tabular}




\begin{tabular}{|c|c|c|c|c|c|c|c|}
\hline Contaminant & $\begin{array}{c}\text { Chronic } \\
\text { Daily } \\
\text { Intolke }\end{array}$ & $\begin{array}{l}\text { Slope } \\
\text { Factor }\end{array}$ & $\begin{array}{l}\text { Contaminnat } \\
\text { Specific Risk }\end{array}$ & $\begin{array}{l}\text { Contamiant } \\
\text { Class Risk }\end{array}$ & $\begin{array}{c}\text { Total } \\
\text { Pathway } \\
\text { Risk }\end{array}$ & $\begin{array}{l}\text { Total } \\
\text { Contamiant } \\
\text { Risk }\end{array}$ & $\begin{array}{l}\text { Total } \\
\text { Area } \\
\text { Rislk }\end{array}$ \\
\hline${ }^{200} \mathrm{Th}$ & $2.5 E+02$ & $1.2 \mathrm{E}-11$ & $3.0 \mathrm{E}-09$ & & & & \\
\hline 2332300 & $4.0 \mathrm{E}+02$ & $1.6 \mathrm{E}-11$ & $6.3 \mathrm{E}-09$ & & & & \\
\hline 25 Th & $2.0 \mathrm{E}+03$ & 4.0E-12 & $7.9 \mathrm{E}-09$ & & & & \\
\hline${ }^{25 U}$ & $3.8 \mathrm{E}+01$ & $1.6 \mathrm{E}-11$ & $6.0 \mathrm{E}-10$ & & & & \\
\hline${ }^{2 x} \mathrm{Pu}$ & $1.4 E+02$ & $2.2 \mathrm{E}-10$ & $3.1 \mathrm{E}-08$ & & & & \\
\hline${ }^{200} \mathrm{U}$ & $4.5 \mathrm{E}+02$ & $2.0 \mathrm{E}-11$ & $8.9 \mathrm{E}-09$ & & & & \\
\hline${ }^{292200} \mathrm{Pu}$ & $1.3 E+03$ & $2.3 E-10$ & $2.9 \mathrm{E}-07$ & & & & \\
\hline${ }^{2 H_{1}} \mathrm{Am}$ & $1.6 \mathrm{E}+02$ & $2.4 \mathrm{E}-10$ & $3.8 \mathrm{E}-08$ & & & & \\
\hline$\infty_{\mathrm{K}}$ & $1.7 \mathrm{E}+03$ & $1.1 \mathrm{E}-11$ & $1.8 \mathrm{E}-08$ & & & & \\
\hline${ }^{60} \mathrm{Co}$ & 4.1E+01 & $1.5 \mathrm{E}-11$ & $6.1 \mathrm{E}-10$ & & & & \\
\hline${ }^{\infty} \mathrm{Sr}$ & $3.6 \mathrm{E}+03$ & $3.6 \mathrm{E}-11$ & $1.3 \mathrm{E}-07$ & & & & \\
\hline Tritium & $8.0 \mathrm{E}+03$ & $5.4 \mathrm{E}-14$ & $4.3 \mathrm{E}-10$ & & & & \\
\hline Total Radionuclides & & & & $1.3 \mathrm{E}-05$ & & & \\
\hline Total Pathway Risk & & & & & $2.8 \mathrm{E}-05$ & & \\
\hline \multicolumn{8}{|c|}{ Exposure Pathway: Dermal Exposure of Contaminants by Maintenance Worker from Core Samples - Whole Building } \\
\hline Beryllium & $1.3 \mathrm{E}-07$ & $4.3 \mathrm{E}+02$ & $5.8 \mathrm{E}-05$ & & & & \\
\hline Total Inorganics & & & & $5.8 \mathrm{E}-05$ & & & \\
\hline Aroclor-1254 & $1.6 \mathrm{E}-07$ & $8.6 \mathrm{E}+\infty$ & $1.4 \mathrm{E}-0.6$ & & & & \\
\hline Aroclor-1260 & $2.8 \mathrm{E}-07$ & $8.6 \mathrm{E}+\infty$ & $2.4 \mathrm{E}-06$ & & & & \\
\hline Bis(2-ethylhexyl)phthalate & $2.6 \mathrm{E}-08$ & 7.4E-02 & $1.9 \mathrm{E}-09$ & & & & \\
\hline Total Organics & & & & $3.8 \mathrm{E}-06$ & & & \\
\hline Total Pathway Risk & & & & & $6.2 \mathrm{E}-05$ & & \\
\hline \multicolumn{8}{|c|}{ Exposure Pathway: Inhalation of Contaminants by Mainteasnce Worker firom Core Samples - Whole Building } \\
\hline Arsenic & 4.7E-11 & $5.0 \mathrm{E}+01$ & $2.3 \mathrm{E}-09$ & & & & \\
\hline Beryllium & $2.0 \mathrm{E}-10$ & $8.4 E+\infty 0$ & $1.7 \mathrm{E}-09$ & & & & \\
\hline Cadmium & 2.9E-09 & $6.1 \mathrm{E}+\infty 0$ & $1.8 \mathrm{E}-08$ & & & & \\
\hline Chromium VI & $3.2 \mathrm{E}-09$ & $4.1 E+01$ & $1.3 \mathrm{E}-07$ & & & & \\
\hline Total Inorganics & & & & $1.5 \mathrm{E}-07$ & & & \\
\hline${ }^{17} \mathrm{Cs}$ & $4.0 \mathrm{E}+01$ & $1.9 \mathrm{E}-11$ & $7.6 \mathrm{E}-10$ & & & & \\
\hline${ }^{2 \times} \mathrm{Ra}$ & $1.2 \mathrm{E}-02$ & $3.0 \mathrm{E}-09$ & $4.8 \mathrm{E}-05$ & & & & \\
\hline${ }^{2 x} \mathrm{Ra}$ & $1.1 \mathrm{E}-02$ & $6.9 \mathrm{E}-10$ & $7.4 \mathrm{E}-12$ & & & & \\
\hline $20 \mathrm{Th}$ & $2.2 \mathrm{E}-02$ & $7.8 \mathrm{E}-08$ & $1.7 \mathrm{E}-09$ & & & & \\
\hline${ }^{200} \mathrm{Th}$ & 4.1E-02 & $2.9 \mathrm{E}-08$ & $1.2 \mathrm{E}-09$ & & & & \\
\hline
\end{tabular}




\begin{tabular}{|c|c|c|c|c|c|c|c|}
\hline Contaminant & $\begin{array}{c}\text { Chronic } \\
\text { Daily } \\
\text { Intake }\end{array}$ & $\begin{array}{l}\text { Slope } \\
\text { Factor }\end{array}$ & $\begin{array}{l}\text { Contominnat } \\
\text { Specific Risl }\end{array}$ & $\begin{array}{l}\text { Contaminnt } \\
\text { Class Risk }\end{array}$ & $\begin{array}{c}\text { Total } \\
\text { Pathway } \\
\text { Risls }\end{array}$ & $\begin{array}{c}\text { Totsl } \\
\text { Contamiant } \\
\text { Risk }\end{array}$ & $\begin{array}{l}\text { Total } \\
\text { Area } \\
\text { Risl }\end{array}$ \\
\hline $200 \mathrm{Th}$ & $2.3 \mathrm{E}-02$ & $2.8 \mathrm{E}-08$ & $6.5 \mathrm{E}-10$ & & & & \\
\hline $25825 \mathrm{U}$ & $3.7 \mathrm{E}-02$ & $2.6 \mathrm{E}-08$ & $9.6 \mathrm{E}-10$ & & & & \\
\hline 2sth $\mathrm{Th}$ & 1.8E-01 & $3.2 \mathrm{E}-11$ & $5.9 \mathrm{E}-12$ & & & & \\
\hline 200 & $3.5 E-03$ & $2.5 \mathrm{E}-08$ & $8.8 \mathrm{E}-11$ & & & & \\
\hline${ }^{234} \mathrm{Pu}$ & $1.3 \mathrm{E}-02$ & $3.9 \mathrm{E}-08$ & 5.1E-10 & & & & \\
\hline${ }^{24} \mathrm{U}$ & $4.2 \mathrm{E}-02$ & $2.4 E-08$ & $1.0 \mathrm{E}-09$ & & & & \\
\hline${ }^{2508200} \mathrm{Pu}$ & $1.2 \mathrm{E}-01$ & $3.8 \mathrm{E}-08$ & $4.4 \mathrm{E}-09$ & & & & \\
\hline${ }^{2 \mu} \mathrm{Am}$ & $1.5 \mathrm{E}-02$ & $3.2 \mathrm{E}-08$ & $4.7 \mathrm{E}-10$ & . & & & \\
\hline${ }^{40} \mathrm{~K}$ & $1.6 \mathrm{E}-01$ & $7.6 \mathrm{E}-12$ & $1.2 \mathrm{E}-12$ & & & & \\
\hline${ }^{\infty} \mathrm{Co}$ & $3.8 \mathrm{E}-03$ & $1.5 \mathrm{E}-10$ & 5.TE-13 & & & & \\
\hline${ }^{\infty} \mathrm{Sr}$ & $3.4 \mathrm{E}-01$ & $6.2 \mathrm{E}-11$ & 2.1E-11 & & & & \\
\hline Tritium & 7.5E-01 & $7.8 \mathrm{E}-14$ & $5.8 \mathrm{E}-14$ & & & & \\
\hline Total Radionuclides & & & & 4.8E-05 & & & \\
\hline Total Pathway Risk & & & & & $4.8 \mathrm{E}-05$ & & \\
\hline \multicolumn{8}{|c|}{ Exposure Pathway: External Exposure to Radionnclides by Maintenance Worker from Core Samples - Whole Building } \\
\hline${ }^{17} \mathrm{Cs}$ & $6.3 E+03$ & $2.0 \mathrm{E}-06$ & $1.2 \mathrm{E}-02$ & & & & \\
\hline${ }^{2 \times} x_{\mathbf{R a}}$ & $1.8 \mathrm{E}+00$ & $6.0 \mathrm{E}-06$ & $1.1 \mathrm{E}-0 \mathrm{~S}$ & & & & \\
\hline$\dot{2}_{\mathrm{Ra}}$ & $1.7 \mathrm{E}+00$ & $2.9 \mathrm{E}-06$ & 4.9E-06 & & & & \\
\hline${ }^{20}$ Th & $3.5 E+00$ & $5.6 \mathrm{E}-06$ & $2.0 \mathrm{E}-05$ & & & & \\
\hline${ }^{200} \mathrm{Th}$ & $6.4 E+\infty$ & $5.4 \mathrm{E}-11$ & $3.4 \mathrm{E}-10$ & & & & \\
\hline${ }^{200} \mathrm{Th}$ & $3.7 E+\infty 0$ & $2.6 \mathrm{E}-11$ & $9.5 \mathrm{E}-11$ & & & & \\
\hline${ }^{233 / 24} \mathrm{U}$ & $5.8 \mathrm{E}+00$ & $3.0 \mathrm{E}-11$ & $1.7 \mathrm{E}-10$ & & & & \\
\hline${ }^{284} \mathrm{Th}$ & $2.9 E+01$ & $3.5 \mathrm{E}-09$ & $1.0 \mathrm{E}-07$ & & & & \\
\hline${ }^{20 S} \mathrm{U}$ & S.5E-01 & $2.4 \mathrm{E}-07$ & $1.3 \mathrm{E}-07$ & & & & \\
\hline${ }^{2 s 4} \mathrm{Pu}$ & $2.1 \mathrm{E}+\infty 0$ & $2.8 \mathrm{E}-11$ & $5.8 \mathrm{E}-11$ & & & & \\
\hline${ }^{250} \mathrm{U}$ & $6.5 E+\infty$ & 5.1E-08 & $3.3 \mathrm{E}-07$ & & & & \\
\hline${ }^{259200} \mathrm{Pu}$ & $1.8 \mathrm{E}+01$ & $1.7 E-11$ & $3.1 \mathrm{E}-10$ & & & & \\
\hline${ }^{241} \mathrm{Am}$ & $2.3 E+\infty$ & 4.9E-09 & $1.1 \mathrm{E}-08$ & & & & \\
\hline${ }^{\infty} \mathrm{K}$ & $2.5 E+01$ & $5.4 \mathrm{E}-07$ & $1.3 \mathrm{E}-05$ & & & & \\
\hline${ }^{\infty} \mathrm{Co}$ & $5.9 \mathrm{E}-01$ & 8.6E-06 & 5.1E-06 & & & & \\
\hline${ }^{90} \mathrm{Sr}$ & $5.3 E+01$ & $0.0 \mathrm{E}+\infty 0$ & $0.0 \mathrm{E}+\infty$ & & & & \\
\hline Tritium & $1.2 \mathrm{E}+02$ & $0.0 \mathrm{E}+\infty 0$ & $0.0 \mathrm{E}+\infty$ & & & & \\
\hline Total Radionuclides & & & & $1.3 E-02$ & & & \\
\hline
\end{tabular}




\begin{tabular}{|c|c|c|c|c|c|c|c|}
\hline Contaminant & $\begin{array}{l}\text { Chrotic } \\
\text { Daily } \\
\text { Intake }\end{array}$ & $\begin{array}{l}\text { Sope } \\
\text { Factor }\end{array}$ & $\begin{array}{l}\text { Contaminnat } \\
\text { Specific Rick }\end{array}$ & $\begin{array}{l}\text { Contominnt } \\
\text { Closs Risk }\end{array}$ & $\begin{array}{c}\text { Total } \\
\text { Patbway } \\
\text { Risk }\end{array}$ & $\begin{array}{c}\text { Total } \\
\text { Contamiont } \\
\text { Risk }\end{array}$ & $\begin{array}{c}\text { Total } \\
\text { Area } \\
\text { Risk }\end{array}$ \\
\hline Total Pathway Risk & & & & & $1.3 \mathrm{E}-02$ & & \\
\hline Total Inorganic Risk & & & & & & 6.7E-05 & \\
\hline Total Organic Risk & & & & & & $9.2 \mathrm{E}-06$ & \\
\hline Total Radionuclide Risk & & & & & & $1.3 \mathrm{E}-02$ & \\
\hline Total Area & & & & & & & $1.3 \mathrm{E}-02$ \\
\hline \multicolumn{8}{|c|}{ Exposure Pathway: Extemal Exposare to Radionuclides by Maintenance Worker from Direct Measurement Samples -.Cell } \\
\hline Beta-Gamms & $4.0 \mathrm{E}+05$ & $1.0 \mathrm{E}+00$ & 2.6E-01 & & & & \\
\hline Gamma & $3.5 \mathrm{E}+05$ & $1.0 \mathrm{E}+00$ & 2.2.E-01 & & & & \\
\hline Total Radionuclides & & & & $4.2 \mathrm{E}-01$ & & & \\
\hline Total Pathway Risk & & & & & 4.2E- 01 & & \\
\hline Total Radionuclide Risk & & & & & & 4.2E-01 & \\
\hline Total Area & & & & & & & 4.2E-01 \\
\hline \multicolumn{8}{|c|}{$\begin{array}{l}\text { Exposure Pathway: External Exposure to Radiounclides by Maintenance Worker from Direct Measurement Samples - East } \\
\text { Gallery }\end{array}$} \\
\hline Beta-Gamms & $5.6 \mathrm{E}+03$ & $1.0 \mathrm{E}+\infty$ & 4.1E-03 & & & & \\
\hline Gamma & $5.0 \mathrm{E}+03$ & $1.0 \mathrm{E}+\infty$ & 3.7E-03 & & & & \\
\hline Total Radionuclides & & & & $7.8 \mathrm{E}-03$ & & & \\
\hline Total Pathway Risk & & & & & $7.8 \mathrm{E}-03$ & & \\
\hline Total Radionuclide Risk & & & & & & $7.8 \mathrm{E}-03$ & \\
\hline Total Area & & & & & & & 7.8E-03 \\
\hline \multicolumn{8}{|c|}{$\begin{array}{l}\text { Exposure Pathway: External Exposure to Radionuclides by Maintenance Worker from Direct Measurement Samples - Whole } \\
\text { Building }\end{array}$} \\
\hline Beta-Gamma & $5.6 \mathrm{E}+05$ & $1.0 \mathrm{E}+00$ & $3.3 \mathrm{E}-01$ & & & & \\
\hline Gamma & $5.1 E+05$ & $1.0 \mathrm{E}+\infty 0$ & 3.1E-01 & & & & \\
\hline Total Radionuclides & & & & $5.4 \mathrm{E}-01$ & & & \\
\hline Total Pathway Risk & & & & & $5.4 \mathrm{E}-01$ & & \\
\hline Total Radionuclide Risk & & & & & & $5.4 \mathrm{E}-01$ & \\
\hline Total Area & & & & & & & $5.4 \mathrm{E}-01$ \\
\hline \multicolumn{8}{|c|}{ Exposure Pathway: Ingestion of Radiounclides by Maintenance Worker from Smear Samples - Cell } \\
\hline${ }^{13} \mathrm{Cs}$ & $4.5 \mathrm{E}+04$ & $2.8 \mathrm{E}-11$ & $1.3 \mathrm{E}-06$ & & & & \\
\hline${ }^{2 x} \mathrm{Ra}$ & $1.6 \mathrm{E}+00$ & $1.2 \mathrm{E}-10$ & $2.0 \mathrm{E}-10$ & & & & \\
\hline 20Th & $3.4 \mathrm{E}+00$ & $5.5 \mathrm{E}-11$ & $1.9 \mathrm{E}-10$ & & & & \\
\hline${ }^{200} \mathrm{Th}$ & $5.8 \mathrm{E}+\infty$ & $1.3 \mathrm{E}-11$ & $7.5 \mathrm{E}-11$ & & & & \\
\hline${ }^{20} \mathrm{Th}$ & $3.6 \mathrm{E}+\infty 0$ & $1.2 \mathrm{E}-11$ & 4.3E-11 & & & & \\
\hline
\end{tabular}




\begin{tabular}{|c|c|c|c|c|c|c|c|}
\hline Contaminant & $\begin{array}{l}\text { Chronic } \\
\text { Doily } \\
\text { Intake }\end{array}$ & $\begin{array}{l}\text { Sope } \\
\text { Fretor }\end{array}$ & $\begin{array}{l}\text { Contominnat } \\
\text { Specific Risl }\end{array}$ & $\begin{array}{l}\text { Contomiant } \\
\text { Class Risk }\end{array}$ & $\begin{array}{c}\text { Total } \\
\text { Pathway } \\
\text { Risk }\end{array}$ & $\begin{array}{l}\text { Total } \\
\text { Contamiant } \\
\text { Risk }\end{array}$ & $\begin{array}{l}\text { Total } \\
\text { Area } \\
\text { Risk }\end{array}$ \\
\hline $235 / 23 y$ & $5.3 E+\infty$ & $1.6 \mathrm{E}-11$ & $8.6 \mathrm{E}-11$ & & & & \\
\hline${ }^{225} U$ & 4.9E-01 & $1.6 \mathrm{E}-11$ & $7.9 \mathrm{E}-12$ & & & & \\
\hline${ }^{24 *} \mathrm{Pu}$ & $2.6 \mathrm{E}+00$ & $2.2 \mathrm{E}-10$ & $5.8 \mathrm{E}-10$ & & & & \\
\hline${ }^{228} \mathrm{U}$ & $7.0 \mathrm{E}+00$ & $2.0 \mathrm{E}-11$ & $1.4 \mathrm{E}-10$ & & & & \\
\hline${ }^{298520} \mathrm{Pu}$ & $1.6 \mathrm{E}+01$ & $2.3 E-10$ & $3.8 \mathrm{E}-09$ & & & & \\
\hline${ }^{10} \mathrm{~K}$ & $1.7 E+02$ & $1.1 \mathrm{E}-11$ & $1.9 \mathrm{E}-09$ & & & & \\
\hline${ }^{\infty 0} \mathrm{Co}$ & $4.2 \mathrm{E}+00$ & $1.5 \mathrm{E}-11$ & $6.3 \mathrm{E}-11$ & & & & \\
\hline${ }^{\infty} \mathrm{Sr}$ & $3.8 \mathrm{E}+02$ & $3.6 \mathrm{E}-11$ & $1.4 \mathrm{E}-08$ & & & & \\
\hline Total Radionuclides & & & & $1.3 \mathrm{E}-06$ & & - & \\
\hline Total Pathway Risk & & & & & $1.3 \mathrm{E}-06$ & & \\
\hline \multicolumn{8}{|c|}{ Exposure Pathway: Inhalation of Radionuclides by Maintenance Worker from Smear Samples - Cell } \\
\hline${ }^{15} \mathrm{Cs}$ & $4.2 \mathrm{E}+\infty$ & $1.9 \mathrm{E}-11$ & 7.9E-11 & & & & \\
\hline${ }^{20 x} \mathrm{Ra}$ & $1.5 \mathrm{E}-04$ & 3.0E-09 & $6.3 \mathrm{E}-07$ & & & & \\
\hline $2 \pi \mathrm{Th}$ & $3.2 \mathrm{E}-04$ & 7.8E-08 & 2.5E-11 & & & & \\
\hline${ }^{230} \mathrm{Th}$ & $5.4 \mathrm{E}-04$ & $2.9 \mathrm{E}-08$ & $1.6 \mathrm{E}-11$ & & & & \\
\hline${ }^{2 x}$ Th & 3.3E-04 & $2.8 \mathrm{E}-08$ & $9.3 \mathrm{E}-12$ & & & & \\
\hline${ }^{238234} U$ & $5.0 \mathrm{E}-04$ & $2.6 \mathrm{E}-08$ & $1.3 \mathrm{E}-11$ & & & & \\
\hline${ }^{235} \mathrm{U}$ & $4.6 \mathrm{E}-05$ & 2.5E-08 & $1.2 \mathrm{E}-12$ & & & & \\
\hline${ }^{200} \mathrm{Pu}$ & $2.5 \mathrm{E}-04$ & $3.9 \mathrm{E}-08$ & $9.6 \mathrm{E}-12$ & & & & \\
\hline${ }^{220} \mathrm{U}$ & $6.5 \mathrm{E}-04$ & $2.4 \mathrm{E}-08$ & $1.6 \mathrm{E}-11$ & & & & \\
\hline${ }^{299820} \mathrm{Pu}$ & $1.5 \mathrm{E}-03$ & $3.8 \mathrm{E}-08$ & $5.8 \mathrm{E}-11$ & & & & \\
\hline${ }^{\infty} \mathrm{K}$ & $1.6 \mathrm{E}-02$ & $7.6 \mathrm{E}-12$ & $1.2 \mathrm{E}-13$ & & & & \\
\hline${ }^{60} \mathrm{Co}$ & $3.9 \mathrm{E}-04$ & $1.5 \mathrm{E}-10$ & $5.9 \mathrm{E}-14$ & & & & \\
\hline${ }^{\infty} \mathrm{Sr}$ & $3.5 \mathrm{E}-02$ & $6.2 \mathrm{E}-11$ & $2.2 \mathrm{E}-12$ & & & & \\
\hline Total Radionuclides & & & & $6.3 \mathrm{E}-07$ & & & \\
\hline Total Pathway Risk & & & & & $6.3 \mathrm{E}-07$ & & \\
\hline \multicolumn{8}{|c|}{ Exposure Pathway: External Exposure to Radionoclides by Maintenence Worker from Smear Samples - Cell } \\
\hline${ }^{19} \mathrm{Cs}$ & $8.2 \mathrm{E}+02$ & $2.0 \mathrm{E}-06$ & $1.6 \mathrm{E}-03$ & & & & \\
\hline${ }^{20 \times} \mathrm{Ra}$ & $3.0 \mathrm{E}-02$ & $6.0 \mathrm{E}-06$ & $1.8 \mathrm{E}-07$ & & & & \\
\hline${ }^{2 n} \mathrm{Th}$ & $6.3 E-02$ & $5.6 \mathrm{E}-06$ & $3.5 \mathrm{E}-07$ & & & & \\
\hline${ }^{2 \times 0} \mathrm{Th}$ & $1.1 \mathrm{E}-01$ & $5.4 \mathrm{E}-11$ & 5.7E-12 & & & & \\
\hline${ }^{25} \mathrm{Th}$ & $6.5 \mathrm{E}-02$ & $2.6 \mathrm{E}-11$ & 1.7E-12 & & & & \\
\hline${ }^{202324} U$ & $9.8 \mathrm{E}-02$ & $3.0 \mathrm{E}-11$ & $2.9 \mathrm{E}-12$ & & & & \\
\hline
\end{tabular}




\begin{tabular}{|c|c|c|c|c|c|c|c|}
\hline Contaminant & $\begin{array}{l}\text { Chronic } \\
\text { Daily } \\
\text { Intole }\end{array}$ & $\begin{array}{l}\text { Sope } \\
\text { Factor }\end{array}$ & $\begin{array}{l}\text { Contominnat } \\
\text { Specific Risk }\end{array}$ & $\begin{array}{l}\text { Contomiont } \\
\text { Closs Risk }\end{array}$ & $\begin{array}{c}\text { Total } \\
\text { Pathway } \\
\text { Risl }\end{array}$ & $\begin{array}{c}\text { Total } \\
\text { Contaminnt } \\
\text { Rick }\end{array}$ & $\begin{array}{l}\text { Totol } \\
\text { Aren } \\
\text { Risk }\end{array}$ \\
\hline${ }^{23} \mathrm{U}$ & $9.0 \mathrm{E}-03$ & $2.4 \mathrm{E}-07$ & $2.2 \mathrm{E}-09$ & & & & \\
\hline${ }^{25 \times} \mathrm{Pu}$ & $4.8 \mathrm{E}-02$ & $2.8 \mathrm{E}-11$ & $1.3 \mathrm{E}-12$ & & & & \\
\hline${ }^{250} \mathrm{U}$ & $1.3 \mathrm{E}-01$ & $5.1 E-08$ & $6.5 \mathrm{E}-09$ & & & & \\
\hline $298200 \mathrm{Pu}$ & $3.0 \mathrm{E}-01$ & $1.7 \mathrm{E}-11$ & $5.1 \mathrm{E}-12$ & & & & \\
\hline$\omega_{\mathrm{K}}$ & $3.1 \mathrm{E}+00$ & $5.4 \mathrm{E}-07$ & $1.7 \mathrm{E}-06$ & & & & \\
\hline${ }^{\infty} \mathrm{Co}$ & 7.7E-02 & $8.6 \mathrm{E}-06$ & $6.6 \mathrm{E}-07$ & & & & \\
\hline${ }^{90} \mathrm{Sr}$ & $6.9 \mathrm{E}+\infty 0$ & $0.0 \mathrm{E}+\infty 0$ & $0.0 \mathrm{E}+\infty$ & & & & \\
\hline Total Radionuclides & & & & $1.6 \mathrm{E}-03$ & & & \\
\hline Total Pathway Risk & & & & & $1.6 \mathrm{E}-03$ & & \\
\hline Total Radionuclide Risk & & & & & & $1.6 \mathrm{E}-03$ & \\
\hline Total Area & & & & & & & $1.6 \mathrm{E}-03$ \\
\hline \multicolumn{8}{|c|}{ Exposure Pathway: Ingestion of Contaminants by Maintenance Worker from Smear Samples - East Gallery } \\
\hline${ }^{15} \mathrm{Cs}$ & $4.8 \mathrm{E}+03$ & $2.8 \mathrm{E}-11$ & $1.3 \mathrm{E}-07$ & & & & \\
\hline${ }^{2 \times R a}$ & $4.6 \mathrm{E}+00$ & $1.2 \mathrm{E}-10$ & 5.5E-10 & & & & \\
\hline${ }^{2 x} \mathrm{Ra}$ & $7.6 \mathrm{E}+01$ & $1.0 \mathrm{E}-10$ & $7.6 \mathrm{E}-09$ & & & & \\
\hline 20 Th & $7.1 E+\infty$ & $5.5 \mathrm{E}-11$ & $3.9 \mathrm{E}-10$ & & & & \\
\hline${ }^{200} \mathrm{Th}$ & $1.5 \mathrm{E}+01$ & $1.3 \mathrm{E}-11$ & $2.0 \mathrm{E}-10$ & & & & \\
\hline${ }^{25} \mathrm{Th}$ & $5.4 \mathrm{E}+\infty$ & $1.2 \mathrm{E}-11$ & $6.5 \mathrm{E}-11$ & & & & \\
\hline${ }^{23 \times 234} \mathrm{U}$ & $1.1 E+01$ & $1.6 \mathrm{E}-11$ & $1.8 \mathrm{E}-10$ & & & & \\
\hline${ }^{215 U}$ & $7.6 \mathrm{E}-01$ & $1.6 \mathrm{E}-11$ & $1.2 \mathrm{E}-11$ & & & & \\
\hline${ }^{258} \mathrm{Pu}$ & $4.9 \mathrm{E}+\infty 0$ & $2.2 \mathrm{E}-10$ & $1.1 \mathrm{E}-09$ & & & & \\
\hline${ }^{2000}$ & $8.8 \mathrm{E}+\infty$ & $2.0 \mathrm{E}-11$ & $1.8 \mathrm{E}-10$ & & & & \\
\hline${ }^{299500} \mathrm{Pu}$ & $4.9 \mathrm{E}+00$ & $2.3 E-10$ & $1.1 \mathrm{E}-09$ & & & & \\
\hline${ }^{2 n} \mathrm{Am}$ & $6.3 E+\infty 0$ & $2.4 \mathrm{E}-10$ & $1.5 \mathrm{E}-09$ & & & & \\
\hline${ }^{\infty} \mathrm{K}$ & $1.1 \mathrm{E}+03$ & $1.1 \mathrm{E}-11$ & $1.2 \mathrm{E}-08$ & & & & \\
\hline${ }^{20} \mathrm{Sr}$ & $1.7 \mathrm{E}+03$ & $3.6 \mathrm{E}-11$ & $6.1 \mathrm{E}-08$ & & & & \\
\hline Total Radionuclides & & & & $2.2 \mathrm{E}-07$ & & & \\
\hline Total Pathway Risk & & & & & $2.2 \mathrm{E}-07$ & & \\
\hline \multicolumn{8}{|c|}{ Exposure Pathway: Inhalation of Contaminants by Maintenence Worker from Smear Somples - East Gallery } \\
\hline${ }^{17} \mathrm{Cs}$ & $4.5 \mathrm{E}-01$ & $1.9 \mathrm{E}-11$ & 8.5E-12 & & & & \\
\hline${ }^{20} \mathrm{Ra}$ & 4.3E-04 & $3.0 \mathrm{E}-09$ & $1.8 \mathrm{E}-06$ & & & & \\
\hline $2 x \mathbf{R a}$ & $7.1 \mathrm{E}-03$ & $6.9 \mathrm{E}-10$ & $4.9 \mathrm{E}-12$ & & & & \\
\hline $200 \mathrm{Th}$ & $6.6 \mathrm{E}-04$ & $7.8 \mathrm{E}-08$ & $5.1 \mathrm{E}-11$ & & & & \\
\hline
\end{tabular}




\begin{tabular}{|c|c|c|c|c|c|c|c|}
\hline Coutaminant & $\begin{array}{l}\text { Chronic } \\
\text { Daily } \\
\text { Intoke }\end{array}$ & $\begin{array}{l}\text { Sope } \\
\text { Factor }\end{array}$ & $\begin{array}{l}\text { Contaminnat } \\
\text { Specific Risk }\end{array}$ & $\begin{array}{l}\text { Contominnt } \\
\text { Class Risk }\end{array}$ & $\begin{array}{c}\text { Total } \\
\text { Pathway } \\
\text { Risk }\end{array}$ & $\begin{array}{l}\text { Total } \\
\text { Contamiant } \\
\text { Risk }\end{array}$ & $\begin{array}{l}\text { Total } \\
\text { Area } \\
\text { Risk }\end{array}$ \\
\hline${ }^{200} \mathrm{Th}$ & $1.4 \mathrm{E}-03$ & 2.9E-08 & 4.1E-11 & & & & \\
\hline${ }^{250} \mathrm{Th}$ & 5.1E-04 & $2.8 \mathrm{E}-08$ & $1.4 \mathrm{E}-11$ & & & & \\
\hline${ }^{258253} U$ & $1.1 \mathrm{E}-03$ & $2.6 \mathrm{E}-08$ & $2.8 \mathrm{E}-11$ & & & & \\
\hline & 7.1E-05 & 2.5E-08 & $1.8 \mathrm{E}-12$ & & & & \\
\hline${ }^{24} \mathrm{Pu}$ & 4.6E-04 & $3.9 \mathrm{E}-08$ & $1.8 \mathrm{E}-11$ & & & & \\
\hline${ }^{248} \mathrm{U}$ & 8.3E-04 & $2.4 \mathrm{E}-08$ & $2.0 \mathrm{E}-11$ & & & & \\
\hline${ }^{259200} \mathrm{Pu}$ & 4.6E-04 & 3.8E-08 & $1.8 \mathrm{E}-11$ & & & & \\
\hline${ }^{241} \mathrm{Am}$ & $5.9 \mathrm{E}-04$ & $3.2 \mathrm{E}-08$ & $1.9 \mathrm{E}-11$ & & & & \\
\hline${ }^{\infty} \mathrm{K}$ & $1.1 \mathrm{E}-01$ & $7.6 \mathrm{E}-12$ & $8.0 \mathrm{E}-13$ & & & & \\
\hline${ }^{90} \mathrm{Sr}$ & $1.6 \mathrm{E}-01$ & $6.2 \mathrm{E}-11$ & $9.8 \mathrm{E}-12$ & & & & \\
\hline Total Radionuclides & & & & $1.8 \mathrm{E}-06$ & & & \\
\hline Total Pathway Risk & & & & & $1.8 \mathrm{E}-06$ & & \\
\hline \multicolumn{8}{|c|}{ Exposure Pathway: External Exposure to Radiomclides by Maintenance Worker from Smear Samples - East Gallery } \\
\hline${ }^{15} \mathrm{Cs}$ & $8.7 \mathrm{E}+01$ & $2.0 \mathrm{E}-06$ & $1.7 \mathrm{E}-04$ & & & & \\
\hline${ }^{2 \times} \mathrm{Ra}$ & $8.3 E-02$ & $6.0 \mathrm{E}-06$ & $5.0 \mathrm{E}-07$ & & & & \\
\hline${ }^{20+} \mathrm{Ra}$ & $1.4 E+\infty$ & $2.9 E-06$ & $4.0 \mathrm{E}-06$ & & & & \\
\hline $22 \mathrm{Th}$ & $1.3 \mathrm{E}-01$ & $5.6 \mathrm{E}-06$ & 7.2E-07 & & & & \\
\hline${ }^{200} \mathrm{Th}$ & $2.8 \mathrm{E}-01$ & $5.4 \mathrm{E}-11$ & $1.5 \mathrm{E}-11$ & & & & \\
\hline${ }^{20} \mathrm{Th}$ & 9.9E-02 & $2.6 \mathrm{E}-11$ & $2.6 \mathrm{E}-12$ & & & & \\
\hline${ }^{238234} U$ & 2.1E-01 & $3.0 \mathrm{E}-11$ & $6.2 \mathrm{E}-12$ & & & & \\
\hline${ }^{25 s} \mathrm{U}$ & $1.4 \mathrm{E}-02$ & $2.4 \mathrm{E}-07$ & $3.3 \mathrm{E}-09$ & & & & \\
\hline${ }^{280} \mathrm{Pu}$ & $9.0 \mathrm{E}-02$ & $2.8 \mathrm{E}-11$ & $2.5 \mathrm{E}-12$ & & & & \\
\hline${ }^{238} U$ & $1.6 \mathrm{E}-01$ & 5.1E-08 & 8.2E-09 & & & & \\
\hline${ }^{299220} \mathrm{Pu}$ & $9.0 \mathrm{E}-02$ & $1.7 \mathrm{E}-11$ & $1.5 \mathrm{E}-12$ & & & & \\
\hline${ }^{2 n 1} \mathrm{Am}$ & $1.2 \mathrm{E}-01$ & $4.9 \mathrm{E}-09$ & $5.6 \mathrm{E}-10$ & & & & \\
\hline${ }^{\infty} \mathrm{K}$ & $2.1 E+01$ & $5.4 \mathrm{E}-07$ & $1.1 E-05$ & & & & \\
\hline${ }^{\infty} \mathrm{Sr}$ & $3.1 E+01$ & $0.0 \mathrm{E}+\infty$ & $0.0 \mathrm{E}+\infty 0$ & & & & \\
\hline Total Radionuclides & & & & $1.9 \mathrm{E}-04$ & & & \\
\hline Total Pathway Risk & & & & & $1.9 \mathrm{E}-04$ & & \\
\hline Total Radionuclide Risk & & & & & & $1.9 \mathrm{E}-04$ & \\
\hline Total Area & & & & & & & $1.9 \mathrm{E}-04$ \\
\hline \multicolumn{8}{|c|}{ Expasure Pathway: Ingestion of Radionuclides by Maintenance Worker from Smear Samples - Whole Building } \\
\hline${ }^{17} \mathrm{Cs}$ & $2.5 E+04$ & $2.8 \mathrm{E}-11$ & $7.0 \mathrm{E}-07$ & & & & \\
\hline
\end{tabular}




\begin{tabular}{|c|c|c|c|c|c|c|c|}
\hline Contaminant & $\begin{array}{l}\text { Chronic } \\
\text { Daily } \\
\text { Intake }\end{array}$ & $\begin{array}{l}\text { Slope } \\
\text { Factor }\end{array}$ & $\begin{array}{l}\text { Contominnat } \\
\text { Specific Risl }\end{array}$ & $\begin{array}{l}\text { Contominnt } \\
\text { Class Risk }\end{array}$ & $\begin{array}{c}\text { Total } \\
\text { Pathway } \\
\text { Risk }\end{array}$ & $\begin{array}{l}\text { Totol } \\
\text { Contominnt } \\
\text { Risk }\end{array}$ & $\begin{array}{l}\text { Totel } \\
\text { Area } \\
\text { Rislk }\end{array}$ \\
\hline${ }^{2 \infty} \mathrm{Ra}$ & $1.9 E+\infty$ & $1.2 \mathrm{E}-10$ & $2.2 \mathrm{E}-10$ & & & & \\
\hline${ }^{2 x} \mathrm{Ra}$ & $8.8 \mathrm{E}+\infty 0$ & $1.0 \mathrm{E}-10$ & $8.8 \mathrm{E}-10$ & & & & \\
\hline $20 \mathrm{Th}$ & $3.5 \mathrm{E}+\infty$ & $5.5 \mathrm{E}-11$ & $1.9 \mathrm{E}-10$ & & & & \\
\hline${ }^{200} \mathrm{Th}$ & $6.3 E+\infty 0$ & $1.3 \mathrm{E}-11$ & $8.2 \mathrm{E}-11$ & & & & \\
\hline${ }^{200} \mathrm{Th}$ & $3.6 \mathrm{E}+\infty$ & $1.2 \mathrm{E}-11$ & 4.3E-11 & & & & \\
\hline${ }^{23 s} 2 \mathrm{sec} U$ & $5.5 \mathrm{E}+\infty$ & $1.6 \mathrm{E}-11$ & $8.9 \mathrm{E}-11$ & & & & \\
\hline${ }^{25 s} \mathrm{U}$ & $5.5 E-01$ & $1.6 \mathrm{E}-11$ & $8.9 \mathrm{E}-12$ & & & & \\
\hline${ }^{2 x} \mathrm{Pu}$ & $3.0 \mathrm{E}+\infty 0$ & $2.2 \mathrm{E}-10$ & $6.5 E-10$ & & & & \\
\hline${ }^{2 s} U$ & $7.8 \mathrm{E}+00$ & $2.0 \mathrm{E}-11$ & $1.6 \mathrm{E}-10$ & & & & \\
\hline${ }^{291200} \mathrm{Pu}$ & $1.8 \mathrm{E}+01$ & 2.3E-10 & $4.2 \mathrm{E}-09$ & & & & \\
\hline${ }^{211} \mathrm{Am}$ & $2.3 \mathrm{E}+\infty 0$ & $2.4 \mathrm{E}-10$ & $5.5 E-10$ & & & & \\
\hline${ }^{+\infty} \mathrm{K}$ & $1.3 \mathrm{E}+02$ & $1.1 \mathrm{E}-11$ & $1.5 \mathrm{E}-09$ & & & & \\
\hline${ }^{\infty} \mathrm{Co}$ & $3.2 \mathrm{E}+\infty$ & $1.5 \mathrm{E}-11$ & $4.8 \mathrm{E}-11$ & & & & \\
\hline${ }^{90} \mathrm{Sr}$ & $2.8 \mathrm{E}+02$ & $3.6 \mathrm{E}-11$ & $1.0 \mathrm{E}-08$ & & & & \\
\hline Total Radionuclides & & & & $7.2 \mathrm{E}-07$ & & & \\
\hline Total Pathway Risk & & & & & $7.2 \mathrm{E}-07$ & & \\
\hline \multicolumn{8}{|c|}{ Exposure Pathway: Inhalation of Radionuclides by Maintenance Worker from Smear Samples - Whole Building } \\
\hline${ }^{11} \mathrm{Cs}$ & $2.3 \mathrm{E}+00$ & $1.9 \mathrm{E}-11$ & $4.5 \mathrm{E}-11$ & & & & \\
\hline${ }^{205} R_{a}$ & $1.7 \mathrm{E}-04$ & $3.0 \mathrm{E}-09$ & 7.1E-07 & & & & \\
\hline${ }^{2 x} R_{2}$ & $8.2 E-04$ & $6.9 \mathrm{E}-10$ & $5.7 \mathrm{E}-13$ & & & & \\
\hline $28 \mathrm{Th}$ & $3.3 E-04$ & $7.8 \mathrm{E}-08$ & $2.6 \mathrm{E}-11$ & & & & \\
\hline${ }^{200} \mathrm{Th}$ & $5.9 \mathrm{E}-04$ & $2.9 \mathrm{E}-08$ & $1.7 \mathrm{E}-11$ & & & & \\
\hline${ }^{2 n} \mathrm{Th}$ & $3.3 E-04$ & $2.8 \mathrm{E}-08$ & 9.3E-12 & & & & \\
\hline${ }^{2232} 2 x U$ & $5.2 E-04$ & $2.6 \mathrm{E}-08$ & $1.3 \mathrm{E}-11$ & & & & \\
\hline${ }^{230} \mathrm{U}$ & $5.2 E-05$ & $2.5 E-08$ & $1.3 \mathrm{E}-12$ & & & & \\
\hline${ }^{250} \mathrm{Pu}$ & $2.8 \mathrm{E}-04$ & $3.9 \mathrm{E}-08$ & $1.1 \mathrm{E}-11$ & & & & \\
\hline${ }^{208} \mathrm{U}$ & 7.3E-04 & $2.4 \mathrm{E}-08$ & $1.8 \mathrm{E}-11$ & & & & \\
\hline${ }^{291200 \mathrm{Pu}}$ & $1.7 E-03$ & $3.8 \mathrm{E}-08$ & $6.4 \mathrm{E}-11$ & & & & \\
\hline${ }^{2 n 1} \mathrm{Am}$ & 2.2E-04 & $3.2 \mathrm{E}-08$ & $6.9 \mathrm{E}-12$ & & & & \\
\hline${ }^{+\infty} \mathrm{K}$ & $1.2 \mathrm{E}-02$ & $7.6 \mathrm{E}-12$ & $9.4 \mathrm{E}-14$ & & & & \\
\hline${ }^{\infty} \mathrm{Co}$ & $3.0 \mathrm{E}-04$ & $1.5 \mathrm{E}-10$ & 4.5E-14 & & & & \\
\hline${ }^{20} \mathrm{Sr}$ & 2.TE-02 & $6.2 \mathrm{E}-11$ & $1.6 \mathrm{E}-12$ & & & & \\
\hline Total Radionuclides & & & & 7.1E-07 & & & \\
\hline
\end{tabular}




\begin{tabular}{|c|c|c|c|c|c|c|c|}
\hline Contaminant & $\begin{array}{l}\text { Chromic } \\
\text { Daily } \\
\text { Intake }\end{array}$ & $\begin{array}{l}\text { Sope } \\
\text { Factor }\end{array}$ & $\begin{array}{l}\text { Contominnat } \\
\text { Specific Risk }\end{array}$ & $\begin{array}{l}\text { Contamiant } \\
\text { Class Risk }\end{array}$ & $\begin{array}{c}\text { Total } \\
\text { Pathway } \\
\text { Risk }\end{array}$ & $\begin{array}{l}\text { Total } \\
\text { Contamiant } \\
\text { Risk }\end{array}$ & $\begin{array}{l}\text { Total } \\
\text { Area } \\
\text { Risl }\end{array}$ \\
\hline Total Pathway Risk & & & & & 7.1E-07 & & \\
\hline \multicolumn{8}{|c|}{ Exposure Pathway: External Exposare to Radiomelides by Mointenance Worker from Smear Samples - Whale Building } \\
\hline${ }^{15} \mathrm{Cs}$ & $4.6 \mathrm{E}+02$ & $2.0 \mathrm{E}-06$ & 9.2E-04 & & & & \\
\hline${ }^{20} s_{\mathrm{Ra}}$ & $3.4 \mathrm{E}-02$ & $6.0 \mathrm{E}-06$ & $2.0 \mathrm{E}-07$ & & & & \\
\hline $2 \mathrm{Ra}$ & $1.6 \mathrm{E}-01$ & $2.9 \mathrm{E}-06$ & 4.7E-07 & & & & \\
\hline${ }^{20} \mathrm{Th}$ & $6.4 \mathrm{E}-02$ & $5.6 \mathrm{E}-06$ & $3.6 \mathrm{E}-07$ & & & & \\
\hline${ }^{200} \mathrm{Th}$ & $1.1 \mathrm{E}-01$ & $5.4 \mathrm{E}-11$ & $6.2 \mathrm{E}-12$ & & & & \\
\hline${ }^{25} \mathrm{Th}$ & $6.5 \mathrm{E}-02$ & $2.6 \mathrm{E}-11$ & $1.7 \mathrm{E}-12$ & & & & \\
\hline $25 \times 240$ & $1.0 \mathrm{E}-01$ & $3.0 \mathrm{E}-11$ & $3.0 \mathrm{E}-12$ & & & & \\
\hline${ }^{23} U$ & $1.0 \mathrm{E}-02$ & $2.4 \mathrm{E}-07$ & $2.4 E-09$ & & & & \\
\hline${ }^{24} \mathrm{Pu}$ & $5.4 \mathrm{E}-02$ & $2.8 \mathrm{E}-11$ & $1.5 \mathrm{E}-12$ & & & & \\
\hline${ }^{258} U$ & $1.4 \mathrm{E}-01$ & 5.1E-08 & $7.3 \mathrm{E}-09$ & & & & \\
\hline${ }^{298210} \mathrm{Pu}$ & $3.3 \mathrm{E}-01$ & $1.7 \mathrm{E}-11$ & $5.6 \mathrm{E}-12$ & & & & \\
\hline${ }^{241} \mathrm{Am}$ & $4.2 \mathrm{E}-02$ & $4.9 \mathrm{E}-09$ & 2.1E-10 & & & & \\
\hline${ }^{40} \mathrm{~K}$ & $2.4 \mathrm{E}+\infty 0$ & $5.4 \mathrm{E}-07$ & $1.3 \mathrm{E}-06$ & & & & \\
\hline${ }^{\infty} \mathrm{Co}$ & $5.8 \mathrm{E}-02$ & $8.6 \mathrm{E}-06$ & $5.0 \mathrm{E}-07$ & & & & \\
\hline${ }^{\infty} \mathrm{Sr}$ & $5.2 \mathrm{E}+\infty$ & $0.0 \mathrm{E}+\infty$ & $0.0 \mathrm{E}+00$ & & & & \\
\hline Total Radionuclides & & & & 9.2E-04 & & & \\
\hline Total Pathway Risk & & & & & $9.2 \mathrm{E}-04$ & & \\
\hline Total Radionuclide Risk & & & & & & $9.2 \mathrm{E}-04$ & \\
\hline Total Area & & & & & & & $9.2 \mathrm{E}-0.4$ \\
\hline \multicolumn{8}{|c|}{ Exposure Pathway: External Exposure to Radionochides by Maintenance Worker from TLD Measurements - Cell } \\
\hline Beta & $1.2 \mathrm{E}+06$ & $1.0 \mathrm{E}+\infty$ & 5.7E-01 & & & & \\
\hline Gamma & $3.6 \mathrm{E}+05$ & $1.0 \mathrm{E}+\infty 0$ & $2.3 \mathrm{E}-01$ & & & & \\
\hline Total Radionuclides & & & & $6.7 \mathrm{E}-01$ & & & \\
\hline Total Pathway Risk & & & & & $6.7 E-01$ & & \\
\hline Total Radionuclide Risk & & & & & & $6.7 \mathrm{E}-01$ & \\
\hline Totsl Area & & & & & & & $6.7 \mathrm{E}-01$ \\
\hline
\end{tabular}


THIS PAGE INTENTIONALLY LEFT BLANK 
Table E-2. Risk Characterization Results - Excess Cancer Risk Estimates - S\&M Worker

\begin{tabular}{|c|c|c|c|c|c|c|c|}
\hline Contaminant & $\begin{array}{c}\text { Chronic } \\
\text { Daily } \\
\text { Intolce }\end{array}$ & $\begin{array}{l}\text { Sope } \\
\text { Factor }\end{array}$ & $\begin{array}{l}\text { Contaminant } \\
\text { Specific } \\
\text { Risk }\end{array}$ & $\begin{array}{l}\text { Contamniant } \\
\text { Closs Risk }\end{array}$ & $\begin{array}{c}\text { Total } \\
\text { Pathway } \\
\text { Risk }\end{array}$ & $\begin{array}{c}\text { Total } \\
\text { Contaminent } \\
\text { Risk }\end{array}$ & $\begin{array}{l}\text { Total } \\
\text { Area } \\
\text { Risk }\end{array}$ \\
\hline \multicolumn{8}{|c|}{ Exposure Pathway: Ingestion of Contaminants by S\&M worker from Core Samples - Cell } \\
\hline Beryllium & 2.5E-08 & $4.3 \mathrm{E}+\infty$ & 1.1E-07 & & & & \\
\hline Total Inorganics & & & & $1.1 \mathrm{E}-07$ & & & \\
\hline Aroclor-1254 & $4.8 \mathrm{E}-10$ & $7.7 \mathrm{E}+\infty$ & $3.7 \mathrm{E}-09$ & & & & \\
\hline Aroclor- 1260 & $8.0 \mathrm{E}-10$ & $7.7 \mathrm{E}+\infty$ & $6.1 \mathrm{E}-09$ & & & & \\
\hline Total Organics & & & & $9.8 \mathrm{E}-09$ & & & \\
\hline${ }^{157} \mathrm{Cs}$ & $3.4 E+03$ & $2.8 \mathrm{E}-11$ & $9.6 \mathrm{E}-08$ & & & & \\
\hline${ }^{200} \mathrm{Ra}$ & $1.0 \mathrm{E}+00$ & $1.2 \mathrm{E}-10$ & $1.2 \mathrm{E}-10$ & & & & \\
\hline $2 \operatorname{Th}_{\mathrm{Th}}$ & $2.1 E+\infty$ & $5.5 \mathrm{E}-11$ & $1.2 \mathrm{E}-10$ & & & & \\
\hline${ }^{250} \mathrm{Th}$ & $3.5 \mathrm{E}+\infty 0$ & $1.3 \mathrm{E}-11$ & $4.6 \mathrm{E}-11$ & & & & \\
\hline $25 \mathrm{Th}$ & $2.2 E+\infty$ & $1.2 \mathrm{E}-11$ & $2.6 \mathrm{E}-11$ & & & & \\
\hline${ }^{23925} \mathrm{U}$ & $3.3 \mathrm{E}+00$ & $1.6 \mathrm{E}-11$ & $5.2 \mathrm{E}-11$ & & & & \\
\hline${ }^{23+} \mathrm{Th}$ & $1.6 \mathrm{E}+01$ & $4.0 \mathrm{E}-12$ & $6.3 \mathrm{E}-11$ & & & & \\
\hline${ }^{2 N S U}$ & $3.0 \mathrm{E}-01$ & $1.6 \mathrm{E}-11$ & $4.8 \mathrm{E}-12$ & & & & \\
\hline${ }^{220} \mathrm{Pu}$ & $1.1 E+\infty$ & $2.2 \mathrm{E}-10$ & $2.5 E-10$ & & & & \\
\hline 240 & $4.3 E+\infty 0$ & $2.0 \mathrm{E}-11$ & $8.5 E-11$ & & & & \\
\hline${ }^{2998200} \mathrm{Pu}$ & $1.0 \mathrm{E}+01$ & $2.3 E-10$ & 2.3E-09 & & & & \\
\hline${ }^{\infty} \mathrm{K}$ & $1.3 \mathrm{E}+01$ & 1.1E-11 & $1.5 \mathrm{E}-10$ & & & & \\
\hline${ }^{\infty 0} \mathrm{Co}$ & $3.3 \mathrm{E}-01$ & $1.5 E-11$ & $4.9 \mathrm{E}-12$ & & & & \\
\hline${ }^{\infty} \mathrm{Sr}$ & $2.9 \mathrm{E}+01$ & $3.6 \mathrm{E}-11$ & $1.0 \mathrm{E}-09$ & & & & \\
\hline Tritium & $5.3 E+01$ & $5.4 \mathrm{E}-14$ & $2.8 \mathrm{E}-12$ & & & & $\cdot$ \\
\hline Total Radionuclides & & & & $1.0 \mathrm{E}-07$ & & & \\
\hline Total Pathway Risk & & & & & $2.2 \mathrm{E}-07$ & & \\
\hline \multicolumn{8}{|c|}{ Exposure Pathway: Dermal Exposure of Contaminants by S\&M Worker from Core Samples - Cell } \\
\hline Beryllium & $1.5 E-09$ & $4.3 \mathrm{E}+02$ & $6.6 \mathrm{E}-07$ & & & & \\
\hline Total Inorganics & & & & $6.6 \mathrm{E}-07$ & & & \\
\hline Aroclor-1254 & $3.0 \mathrm{E}-10$ & $8.6 \mathrm{E}+\infty$ & 2.5E-09 & & & & \\
\hline Aroclor-1260 & $5.0 \mathrm{E}-10$ & $8.6 \mathrm{E}+00$ & 4.3E-09 & & & & \\
\hline Total Organics & & & & $6.8 \mathrm{E}-09$ & & & \\
\hline Total Pathway Risk & & & & & $6.7 \mathrm{E}-07$ & & \\
\hline
\end{tabular}




\begin{tabular}{|c|c|c|c|c|c|c|c|}
\hline Contaminant & $\begin{array}{l}\text { Chroaic } \\
\text { Daily } \\
\text { Intalce }\end{array}$ & $\begin{array}{l}\text { Sope } \\
\text { Factor }\end{array}$ & $\begin{array}{l}\text { Contaminant } \\
\text { Specific } \\
\text { Risk }\end{array}$ & $\begin{array}{c}\text { Contamniant } \\
\text { Class Risk }\end{array}$ & $\begin{array}{c}\text { Total } \\
\text { Pathway } \\
\text { Risk }\end{array}$ & $\begin{array}{c}\text { Total } \\
\text { Contaminant } \\
\text { Risk }\end{array}$ & $\begin{array}{l}\text { Totel } \\
\text { Area } \\
\text { Risk }\end{array}$ \\
\hline \multicolumn{8}{|c|}{ Exposure Pathway: Inhalation of Contaminants by S\&M Worker from Core Samples - Cell } \\
\hline Arsenic & 3.4E-13 & $5.0 \mathrm{E}+01$ & $1.7 \mathrm{E}-11$ & & & & \\
\hline Beryllium & $2.3 \mathrm{E}-12$ & $8.4 E+\infty 0$ & $1.9 \mathrm{E}-11$ & & & & \\
\hline Cadmium & $2.3 \mathrm{E}-11$ & $6.1 E+\infty$ & $1.4 \mathrm{E}-10$ & & & & \\
\hline Chromium VI & $2.6 \mathrm{E}-11$ & $4.1 \mathrm{E}+01$ & $1.0 \mathrm{E}-09$ & & & & \\
\hline Total Inorganics & & & & $1.2 \mathrm{E}-09$ & & & \\
\hline${ }^{157} \mathrm{Cs}$ & $3.2 \mathrm{E}-01$ & $1.9 \mathrm{E}-11$ & $6.1 \mathrm{E}-12$ & & & & \\
\hline${ }^{2} \mathrm{Ra}$ & $9.4 \mathrm{E}-05$ & $3.0 \mathrm{E}-09$ & $3.9 \mathrm{E}-07$ & & & & \\
\hline $\mathrm{nk}_{\mathrm{Th}}$ & $2.0 \mathrm{E}-04$ & $7.8 \mathrm{E}-08$ & $1.5 \mathrm{E}-11$ & & & & \\
\hline${ }^{200} \mathrm{Th}$ & 3.3E-04 & $2.9 \mathrm{E}-08$ & $9.5 \mathrm{E}-12$ & & & & \\
\hline${ }^{250} \mathrm{Th}$ & $2.0 \mathrm{E}-04$ & $2.8 \mathrm{E}-08$ & $5.7 \mathrm{E}-12$ & & & & \\
\hline${ }^{232524} \mathrm{U}$ & $3.0 \mathrm{E}-04$ & $2.6 \mathrm{E}-08$ & $7.9 \mathrm{E}-12$ & & & & \\
\hline $23 \mathrm{Th}$ & $1.5 \mathrm{E}-03$ & $3.2 \mathrm{E}-11$ & $4.7 \mathrm{E}-14$ & & & & \\
\hline${ }^{235} \mathrm{U}$ & $2.8 \mathrm{E}-05$ & $2.5 \mathrm{E}-08$ & $7.0 \mathrm{E}-13$ & & & & \\
\hline${ }^{23} \mathrm{Pu}$ & $1.1 \mathrm{E}-04$ & $3.9 \mathrm{E}-08$ & 4.1E-12 & & & & \\
\hline${ }^{230} \mathrm{U}$ & $4.0 \mathrm{E}-04$ & $2.4 \mathrm{E}-08$ & $9.5 E-12$ & & & & \\
\hline${ }^{2090200} \mathrm{Pu}$ & $9.3 \mathrm{E}-04$ & $3.8 \mathrm{E}-08$ & $3.5 \mathrm{E}-11$ & & & & \\
\hline${ }^{40} \mathrm{~K}$ & $1.2 \mathrm{E}-03$ & $7.6 \mathrm{E}-12$ & $9.4 \mathrm{E}-15$ & & & & \\
\hline${ }^{\infty} \mathrm{Co}$ & 3.0E-05 & $1.5 \mathrm{E}-10$ & 4.6E-15 & & & & \\
\hline${ }^{90} \mathrm{Sr}$ & $2.7 \mathrm{E}-03$ & $6.2 \mathrm{E}-11$ & $1.7 \mathrm{E}-13$ & & & & \\
\hline Tritium & $4.9 \mathrm{E}-03$ & $7.8 \mathrm{E}-14$ & $3.8 \mathrm{E}-16$ & & & & \\
\hline Total Radionuclides & & & & $3.9 \mathrm{E}-07$ & & & \\
\hline Total Pathway Risk & & & & & $3.9 \mathrm{E}-07$ & & \\
\hline \multicolumn{8}{|c|}{ Exposure Pathway: Extermal Exposure to Rationnclides by S\&M Worker firom Core Samples - Cell } \\
\hline${ }^{17} \mathrm{Cs}$ & $5.0 \mathrm{E}+01$ & $2.0 \mathrm{E}-06$ & $1.0 \mathrm{E}-04$ & & & & \\
\hline${ }^{2 \times R a}$ & $1.5 \mathrm{E}-02$ & $6.0 \mathrm{E}-06$ & 8.8E-08 & & & & \\
\hline $2 \mathrm{Th}$ & $3.1 E-02$ & $5.6 \mathrm{E}-06$ & $1.7 \mathrm{E}-07$ & & & & \\
\hline $200 \mathrm{Th}$ & 5.1E-02 & $5.4 \mathrm{E}-11$ & $2.8 \mathrm{E}-12$ & & & & \\
\hline${ }^{200} \mathrm{Th}$ & $3.2 \mathrm{E}-02$ & $2.6 \mathrm{E}-11$ & $8.3 \mathrm{E}-13$ & & & & \\
\hline${ }^{203234} U$ & 4.7E-02 & $3.0 \mathrm{E}-11$ & $1.4 \mathrm{E}-12$ & & & & \\
\hline${ }^{24 r} \mathrm{Th}$ & $2.3 \mathrm{E}-01$ & $3.5 \mathrm{E}-09$ & 8.1E-10 & & & & \\
\hline${ }^{23 s} \mathrm{U}$ & $4.4 \mathrm{E}-03$ & $2.4 \mathrm{E}-07$ & 1.1E-09 & & & & \\
\hline${ }^{25} \mathrm{Pu}$ & $1.6 \mathrm{E}-02$ & $2.8 \mathrm{E}-11$ & $4.6 \mathrm{E}-13$ & & & & \\
\hline
\end{tabular}




\begin{tabular}{|c|c|c|c|c|c|c|c|}
\hline Contaminent & $\begin{array}{c}\text { Chronic } \\
\text { Daily } \\
\text { Intake } \\
\end{array}$ & $\begin{array}{l}\text { Sope } \\
\text { Factor }\end{array}$ & $\begin{array}{c}\text { Contominent } \\
\text { Specific } \\
\text { Risk }\end{array}$ & $\begin{array}{l}\text { Contemnient } \\
\text { Cless Risk }\end{array}$ & $\begin{array}{c}\text { Total } \\
\text { Pathway } \\
\text { Risk } \\
\end{array}$ & $\begin{array}{c}\text { Totsl } \\
\text { Conteminant } \\
\text { Risk } \\
\end{array}$ & $\begin{array}{l}\text { Totel } \\
\text { Area } \\
\text { Risk } \\
\end{array}$ \\
\hline 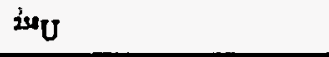 & $6.2 \mathrm{E}-02$ & $5.1 \mathrm{E}-08$ & $3.2 \mathrm{E}-09$ & & & & \\
\hline${ }^{29820 \mathrm{Pu}}$ & $1.5 \mathrm{E}-01$ & $1.7 \mathrm{E}-11$ & $2.5 \mathrm{E}-12$ & & & & \\
\hline${ }^{\infty} \mathrm{K}$ & $1.9 \mathrm{E}-01$ & $5.4 \mathrm{E}-07$ & $1.0 \mathrm{E}-07$ & & & & \\
\hline${ }^{\infty} \mathrm{Co}$ & 4.7E-03 & $8.6 \mathrm{E}-06$ & 4.1E-08 & & & & \\
\hline${ }^{90} \mathrm{Sr}$ & 4.2E-01 & $0.0 \mathrm{E}+00$ & $0.0 \mathrm{E}+\infty$ & & & & \\
\hline Tritium & 7.7E-01 & $0.0 \mathrm{E}+\infty$ & $0.0 \mathrm{E}+\infty$ & & & . & \\
\hline Total Radionuclides & & & & $1.0 \mathrm{E}-04$ & & & \\
\hline Total Pathway Risk & & & & & $1.0 \mathrm{E}-04$ & & \\
\hline Total Inorganic Risk & & & & & & $7.7 \mathrm{E}-07$ & \\
\hline Total Organic Risk & & & & & & $1.7 E-08$ & \\
\hline Total Radionuclide Risk & & & & & & $1.0 \mathrm{E}-04$ & \\
\hline Total Area & & & & & & & $1.0 \mathrm{E}-04$ \\
\hline \multicolumn{8}{|c|}{ Exposure Pathway: Ingestion of Contaminants by S\&M Worker from Core Samples - East Gallery } \\
\hline Beryllium & $5.6 \mathrm{E}-10$ & $4.3 E+\infty$ & $2.4 \mathrm{E}-09$ & & & & \\
\hline Total Inorganics & & & & $2.4 \mathrm{E}-09$ & & & \\
\hline Aroclor-1254 & $2.4 \mathrm{E}-09$ & $7.7 E+\infty$ & $1.8 \mathrm{E}-08$ & & & & \\
\hline Aroclor-1260 & $3.6 \mathrm{E}-09$ & $7.7 E+\infty$ & $2.8 \mathrm{E}-08$ & & & & \\
\hline Bis(2-ethylhexyl)phthalate & 3.4E-10 & $1.4 \mathrm{E}-02$ & 4.7E-12 & & & & \\
\hline Total Organics & & & & $4.6 \mathrm{E}-08$ & & & \\
\hline${ }^{15} \mathrm{Cs}$ & $5.7 E+01$ & $2.8 \mathrm{E}-11$ & $1.6 \mathrm{E}-09$ & & & & \\
\hline${ }^{20 \times} \mathrm{Ra}$ & $9.0 \mathrm{E}-01$ & $1.2 \mathrm{E}-10$ & $1.1 \mathrm{E}-10$ & & & & \\
\hline $2 \mathrm{r}_{\mathrm{R}}$ & $9.3 \mathrm{E}-01$ & $1.0 \mathrm{E}-10$ & 9.3E-11 & & & & \\
\hline${ }^{2 n} \mathrm{Th}$ & $1.4 \mathrm{E}+\infty 0$ & $5.5 \mathrm{E}-11$ & 7.7E-11 & & & & \\
\hline $250 \mathrm{Th}$ & $3.0 \mathrm{E}+\infty$ & 1.3E-11 & $3.9 \mathrm{E}-11$ & & & & \\
\hline${ }^{200} \mathrm{Th}$ & $1.1 E+\infty$ & $1.2 \mathrm{E}-11$ & $1.3 \mathrm{E}-11$ & & & & \\
\hline $242524 U$ & $2.3 E+\infty$ & $1.6 \mathrm{E}-11$ & 3.6E-11 & & & & \\
\hline${ }^{25} \mathrm{U}$ & $1.9 \mathrm{E}+\infty$ & $2.0 \mathrm{E}-11$ & $3.9 \mathrm{E}-11$ & & & & \\
\hline${ }^{20 t} \mathrm{Am}$ & $1.3 \mathrm{E}+\infty$ & $2.4 \mathrm{E}-10$ & $3.0 \mathrm{E}-10$ & & & & \\
\hline${ }^{\infty} \mathrm{K}$ & $1.3 \mathrm{E}+01$ & 1.1E-11 & $1.5 \mathrm{E}-10$ & & & & \\
\hline${ }^{90} \mathrm{Sr}$ & $2.0 \mathrm{E}+01$ & 3.6E-11 & $7.3 \mathrm{E}-10$ & & & & \\
\hline Tritium & $6.4 \mathrm{E}+01$ & $5.4 \mathrm{E}-14$ & 3.5E-12 & & & & \\
\hline Tolal Radionuclides & & & & $3.2 \mathrm{E}-09$ & & & \\
\hline Total Pathway Risk & & & & & $5.2 \mathrm{E}-08$ & & \\
\hline
\end{tabular}




\begin{tabular}{|c|c|c|c|c|c|c|c|}
\hline Contaminant & $\begin{array}{l}\text { Chroxic } \\
\text { Daily } \\
\text { Intolce }\end{array}$ & $\begin{array}{l}\text { Slope } \\
\text { Factor }\end{array}$ & $\begin{array}{c}\text { Contaminant } \\
\text { Specific } \\
\text { Risk }\end{array}$ & $\begin{array}{l}\text { Contamniant } \\
\text { Class Risk }\end{array}$ & $\begin{array}{c}\text { Total } \\
\text { Pathway } \\
\text { Risl }\end{array}$ & $\begin{array}{c}\text { Total } \\
\text { Contaminant } \\
\text { Risk }\end{array}$ & $\begin{array}{l}\text { Total } \\
\text { Aren } \\
\text { Risk }\end{array}$ \\
\hline \multicolumn{8}{|c|}{ Exposure Pathway: Dermal Exposure to Contaminents by S\&M Worker from Core Samples - East Gallery } \\
\hline Beryllium & $3.5 \mathrm{E}-11$ & $4.3 E+02$ & $1.5 \mathrm{E}-08$ & & & & \\
\hline Total Inorganics & & & & $1.5 \mathrm{E}-08$ & & & \\
\hline Aroclor-1254 & $1.5 \mathrm{E}-09$ & $8.6 \mathrm{E}+00$ & $1.3 E-08$ & & & & \\
\hline Aroclor-1260 & $2.3 \mathrm{E}-09$ & $8.6 \mathrm{E}+\infty 0$ & $1.9 \mathrm{E}-08$ & & & & \\
\hline Bis(2-ethylhexyl)phthalate & $2.1 \mathrm{E}-10$ & $7.4 \mathrm{E}-02$ & $1.5 \mathrm{E}-11$ & & & & \\
\hline Total Organics & & & & $3.2 \mathrm{E}-08$ & & & \\
\hline Total Pathway Risk & & & & & 4.7E-08 & & \\
\hline \multicolumn{8}{|c|}{ Exposure Pathway: Inhalation of Contaminants by S\&M Worker from Core Samples - East Gallery } \\
\hline Arsenic & $3.9 \mathrm{E}-13$ & $5.0 \mathrm{E}+01$ & $2.0 \mathrm{E}-11$ & & & & \\
\hline Beryilium & $5.2 \mathrm{E}-14$ & $8.4 \mathrm{E}+00$ & 4.4E-13 & & & & \\
\hline Cadmium & $6.7 \mathrm{E}-13$ & $6.1 E+\infty$ & 4.1E-12 & & & & \\
\hline Chromium VI & $1.1 \mathrm{E}-12$ & $4.1 \mathrm{E}+01$ & $4.4 \mathrm{E}-11$ & & & & \\
\hline Total Inorganics & & & & $6.8 \mathrm{E}-11$ & & & \\
\hline${ }^{17} \mathrm{Cs}$ & $5.3 \mathrm{E}-03$ & $1.9 \mathrm{E}-11$ & $1.0 \mathrm{E}-13$ & & & & \\
\hline${ }^{20} \mathrm{Rn}_{\mathrm{n}}$ & $8.4 \mathrm{E}-05$ & $3.0 \mathrm{E}-09$ & $3.5 \mathrm{E}-07$ & & & & \\
\hline${ }^{20} \mathrm{Ra}$ & 8.6E-05 & $6.9 \mathrm{E}-10$ & $6.0 \mathrm{E}-14$ & & & & \\
\hline 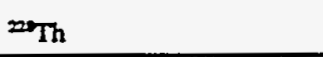 & $1.3 \mathrm{E}-04$ & $7.8 \mathrm{E}-08$ & $1.0 \mathrm{E}-11$ & & & & \\
\hline $200 \mathrm{Th}$ & $2.8 \mathrm{E}-04$ & $2.9 \mathrm{E}-08$ & $8.1 \mathrm{E}-12$ & & & & \\
\hline${ }^{20} \mathrm{Th}$ & $1.0 \mathrm{E}-04$ & $2.8 \mathrm{E}-08$ & $2.8 \mathrm{E}-12$ & & & & \\
\hline${ }^{2 \operatorname{sen} 2 \pi} U$ & $2.1 E-04$ & $2.6 \mathrm{E}-08$ & $5.5 \mathrm{E}-12$ & & & & \\
\hline${ }^{250} \mathrm{U}$ & $1.8 \mathrm{E}-04$ & $2.4 \mathrm{E}-08$ & $4.3 \mathrm{E}-12$ & & & & \\
\hline${ }^{211} \mathrm{Am}$ & $1.2 \mathrm{E}-04$ & $3.2 \mathrm{E}-08$ & 3.7E-12 & & & & \\
\hline${ }^{\infty} \mathrm{K}$ & $1.3 \mathrm{E}-03$ & $7.6 \mathrm{E}-12$ & $9.5 \mathrm{E}-15$ & & & & \\
\hline${ }^{\infty} \mathrm{Sr}$ & $1.9 \mathrm{E}-03$ & $6.2 \mathrm{E}-11$ & $1.2 \mathrm{E}-13$ & & & & \\
\hline Tritium & $6.0 \mathrm{E}-03$ & $7.8 \mathrm{E}-14$ & $4.7 \mathrm{E}-16$ & & & & \\
\hline Total Radionuclides & & & & $3.5 \mathrm{E}-07$ & & & \\
\hline Total Pathway Risk & & & & & $3.5 \mathrm{E}-07$ & & \\
\hline \multicolumn{8}{|c|}{ Exposure Pathway: External Exposure to Radionuclides by S\&M Worker from Core Samples - East Gallery } \\
\hline${ }^{157} \mathrm{Cs}$ & $8.3 \mathrm{E}-01$ & $2.0 \mathrm{E}-06$ & $1.7 \mathrm{E}-06$ & & & & \\
\hline${ }^{25 \mathrm{Ra}}$ & $1.3 \mathrm{E}-02$ & $6.0 \mathrm{E}-06$ & $7.9 \mathrm{E}-08$ & & & & \\
\hline${ }^{20} \mathrm{Rz}$ & $1.4 \mathrm{E}-02$ & $2.9 \mathrm{E}-06$ & $3.9 E-08$ & & & & \\
\hline neth & $2.0 \mathrm{E}-02$ & $5.6 \mathrm{E}-06$ & $1.1 \mathrm{E}-07$ & & & & \\
\hline
\end{tabular}




\begin{tabular}{|c|c|c|c|c|c|c|c|}
\hline Contaminant & $\begin{array}{l}\text { Chronic } \\
\text { Doily } \\
\text { Intake }\end{array}$ & $\begin{array}{l}\text { Sope } \\
\text { Factor }\end{array}$ & $\begin{array}{c}\text { Contominant } \\
\text { Specific } \\
\text { Risk }\end{array}$ & $\begin{array}{l}\text { Contamniant } \\
\text { Class Risk }\end{array}$ & $\begin{array}{c}\text { Total } \\
\text { Pathway } \\
\text { Risk }\end{array}$ & $\begin{array}{c}\text { Total } \\
\text { Contaminont } \\
\text { Risk }\end{array}$ & $\begin{array}{l}\text { Total } \\
\text { Area } \\
\text { Risk }\end{array}$ \\
\hline${ }^{200} \mathrm{Th}$ & $4.4 \mathrm{E}-02$ & $5.4 \mathrm{E}-11$ & 2.4E-12 & & & & \\
\hline${ }^{200} \mathrm{Th}$ & $1.6 \mathrm{E}-02$ & $2.6 \mathrm{E}-11$ & 4.1E-13 & & & & \\
\hline${ }^{231258} U$ & $3.3 \mathrm{E}-02$ & $3.0 \mathrm{E}-11$ & $9.9 \mathrm{E}-13$ & & & & \\
\hline 250 & $2.8 \mathrm{E}-02$ & 5.1E-08 & $1.4 \mathrm{E}-09$ & & & & \\
\hline${ }^{211} \mathrm{Am}$ & $1.8 \mathrm{E}-02$ & $4.9 \mathrm{E}-09$ & $8.9 \mathrm{E}-11$ & & & & \\
\hline${ }^{40} \mathrm{~K}$ & $2.0 \mathrm{E}-01$ & 5.4E- -07 & $1.1 \mathrm{E}-07$ & & & & \\
\hline${ }^{x} \mathrm{Sr}$ & $3.0 \mathrm{E}-01$ & $0.0 \mathrm{E}+00$ & $0.0 \mathrm{E}+\infty 0$ & & & & \\
\hline Tritium & $9.4 \mathrm{E}-01$ & $0.0 \mathrm{E}+\infty$ & $0.0 \mathrm{E}+\infty 0$ & $\therefore$ & & & \\
\hline Total Radionuclides & & & & $2.0 \mathrm{E}-06$ & & & \\
\hline Total Pathway Risk & & & & & 2.0E-06 & & \\
\hline Total Inorganic Risk & & & & & & 1.7E-08 & \\
\hline Total Organic Risk & & & & & & $7.8 \mathrm{E}-08$ & \\
\hline Total Radionuclide Risk & & & & & & 2.4E-06 & \\
\hline Total Area & & & & & & & 2.5E-06 \\
\hline \multicolumn{8}{|c|}{ Exposure Pathway: Ingestion of Contaminants by S\&M Worker from Sediment in Cell } \\
\hline Aroclor-1254 & 5.7E-07 & $7.7 \mathrm{E}+\infty$ & 4.4E-06 & & & & \\
\hline Benzo(a)pyrene & $8.0 \mathrm{E}-09$ & $7.3 E+\infty$ & $5.8 \mathrm{E}-08$ & & & & \\
\hline Benzo(k)fluoranthene & $6.4 \mathrm{E}-08$ & 7.3E-02 & 4.7E-09 & & & & \\
\hline Bis(2-ethylhexyl)phthatate & $2.9 \mathrm{E}-09$ & $1.4 \mathrm{E}-02$ & $4.1 \mathrm{E}-11$ & & & & \\
\hline Carbazole & $2.8 \mathrm{E}-09$ & $2.0 \mathrm{E}-02$ & $5.6 \mathrm{E}-11$ & & & & \\
\hline Chrysene & 2.7E-08 & 7.3E-03 & $1.9 \mathrm{E}-10$ & & & & \\
\hline Dibenz(a,h)anthracene & $2.1 E-09$ & $7.3 E+\infty$ & $1.5 \mathrm{E}-08$ & & & & \\
\hline Indeno(1,2,3-cd)pyrene & $1.3 \mathrm{E}-08$ & 7.3E-01 & $9.8 \mathrm{E}-09$ & & & & \\
\hline Total Organics & & & & $4.5 \mathrm{E}-06$ & & & \\
\hline${ }^{17} \mathrm{Cs}$ & $7.7 \mathrm{E}+04$ & $2.8 \mathrm{E}-11$ & $2.2 \mathrm{E}-06$ & & & & \\
\hline $200 \mathrm{Th}$ & $1.4 \mathrm{E}+00$ & $5.5 \mathrm{E}-11$ & $7.4 \mathrm{E}-11$ & & & & \\
\hline $\mathrm{Th}$ & $4.7 \mathrm{E}+01$ & $8.9 \mathrm{E}-11$ & $4.2 \mathrm{E}-09$ & & & & \\
\hline${ }^{20} \mathrm{Th}$ & $1.4 \mathrm{E}+00$ & $1.2 \mathrm{E}-11$ & $1.7 E-11$ & & & & \\
\hline${ }^{252} 252 \times$ & $8.6 \mathrm{E}+\infty 0$ & $1.6 \mathrm{E}-11$ & $1.4 \mathrm{E}-10$ & & & & \\
\hline 201Pu & $2.9 E+\infty 0$ & $2.2 \mathrm{E}-10$ & $6.4 \mathrm{E}-10$ & & & & \\
\hline${ }^{250} \mathrm{U}$ & $5.6 \mathrm{E}+\infty 0$ & 2.0E-11 & $1.1 \mathrm{E}-10$ & & & & \\
\hline${ }^{253200} \mathrm{Pu}$ & $8.9 E+01$ & 2.3E-10 & $2.0 \mathrm{E}-08$ & & & & \\
\hline${ }^{\infty} \mathrm{K}$ & $2.4 \mathrm{E}+01$ & $1.1 E-11$ & $2.6 \mathrm{E}-10$ & & & & \\
\hline
\end{tabular}




\begin{tabular}{|c|c|c|c|c|c|c|c|}
\hline Contaminant & $\begin{array}{l}\text { Chronic } \\
\text { Daily } \\
\text { Intake }\end{array}$ & $\begin{array}{l}\text { Sope } \\
\text { Factor }\end{array}$ & $\begin{array}{c}\text { Contaminant } \\
\text { Specific } \\
\text { Risk }\end{array}$ & $\begin{array}{c}\text { Contamniant } \\
\text { Class Risk }\end{array}$ & $\begin{array}{c}\text { Totol } \\
\text { Pathway } \\
\text { Risk }\end{array}$ & $\begin{array}{c}\text { Totol } \\
\text { Contaminant } \\
\text { Risk }\end{array}$ & $\begin{array}{l}\text { Total } \\
\text { Area } \\
\text { Risk }\end{array}$ \\
\hline${ }^{\infty} \mathrm{Co}$ & $8.6 \mathrm{E}+\infty$ & $1.5 \mathrm{E}-11$ & 1.3E-10 & & & & \\
\hline${ }^{80} \mathrm{Sr}$ & $2.4 \mathrm{E}+02$ & $3.6 \mathrm{E}-11$ & 8.8E- -09 & & & & \\
\hline Tritium & $2.5 \mathrm{E}+\infty$ & $5.4 \mathrm{E}-14$ & $1.4 \mathrm{E}-13$ & & & & \\
\hline Total Radionuclides & & & & 2.2E-06 & & & \\
\hline Total Pathway Risk & & & & & 6.7E-06 & & \\
\hline \multicolumn{8}{|c|}{ Exposure Pathway: Dermal Exposure to Contaminants by S\&M Worker from Sediment in Cell } \\
\hline Aroclor-1254 & $3.6 \mathrm{E}-07$ & $8.6 \mathrm{E}+00$ & 3.1E-06 & & & & \\
\hline Benzo(a)pyrene & $5.0 \mathrm{E}-09$ & $2.4 \mathrm{E}+01$ & $1.2 \mathrm{E}-07$ & & & & \\
\hline Benzo(k)fluoranthene & 4.0E-08 & $2.4 \mathrm{E}-01$ & $9.4 \mathrm{E}-09$ & & & & \\
\hline Bis(2-ethylhexyl)phthalate & $1.8 \mathrm{E}-09$ & $7.4 \mathrm{E}-02$ & 1.3E-10 & & & & \\
\hline Carbazole & $1.7 \mathrm{E}-09$ & $2.0 \mathrm{E}-02$ & $3.5 \mathrm{E}-11$ & & & & \\
\hline Chrysene & $1.7 \mathrm{E}-08$ & $2.4 \mathrm{E}-02$ & $3.9 \mathrm{E}-10$ & & & & \\
\hline Dibenz(a,h)anthracene & $1.3 \mathrm{E}-09$ & $8.1 \mathrm{E}+00$ & $1.1 \mathrm{E}-08$ & & & & \\
\hline Indeno(1,2,3-cd)pyrene & 8.4E-09 & $2.4 \mathrm{E}+00$ & $2.0 \mathrm{E}-08$ & & & & \\
\hline Total Organics & & & & $3.2 \mathrm{E}-06$ & & & \\
\hline Total Pathway Risk & & & & & $3.2 \mathrm{E}-06$ & & \\
\hline \multicolumn{8}{|c|}{ Exposure Pathway: Inhalation of Contaminants by S\&M Worker from Sediment in Cell } \\
\hline Arsenic & $2.8 \mathrm{E}-12$ & $5.0 \mathrm{E}+01$ & $1.4 \mathrm{E}-10$ & & & & \\
\hline Cadmium & $7.8 \mathrm{E}-12$ & $6.1 \mathrm{E}+00$ & $4.8 \mathrm{E}-11$ & & & & \\
\hline Chromium VI & 2.5E-11 & $4.1 \mathrm{E}+01$ & $1.0 \mathrm{E}-09$ & & & & \\
\hline Total Inorganics & & & & $1.2 \mathrm{E}-09$ & & & \\
\hline${ }^{157} \mathrm{Cs}$ & $7.2 \mathrm{E}+\infty$ & $1.9 \mathrm{E}-11$ & $1.4 \mathrm{E}-10$ & & & & \\
\hline $2 \mathrm{Th}$ & 1.3E-04 & $7.8 \mathrm{E}-08$ & $9.8 \mathrm{E}-12$ & & & & \\
\hline mTh & $4.4 \mathrm{E}-03$ & $7.7 \mathrm{E}-08$ & $3.4 \mathrm{E}-10$ & &. & & \\
\hline${ }^{200} \mathrm{Th}$ & $1.3 E-04$ & $2.8 \mathrm{E}-08$ & $3.6 \mathrm{E}-12$ & & & & \\
\hline${ }^{223} 25 x$ & $8.0 \mathrm{E}-04$ & $2.6 \mathrm{E}-08$ & 2.1E-11 & & & & \\
\hline${ }^{20} \mathrm{Pu}$ & $2.7 \mathrm{E}-04$ & $3.9 \mathrm{E}-08$ & $1.1 \mathrm{E}-11$ & & & & \\
\hline 240 & 5.2E-04 & $2.4 \mathrm{E}-08$ & $1.3 \mathrm{E}-11$ & & & & \\
\hline${ }^{299820} \mathrm{Pu}$ & 8.3E-03 & $3.8 \mathrm{E}-08$ & $3.1 \mathrm{E}-10$ & & & & \\
\hline${ }^{\infty} \mathrm{K}$ & $2.2 \mathrm{E}-03$ & $7.6 \mathrm{E}-12$ & $1.7 \mathrm{E}-14$ & & & & \\
\hline${ }^{50} \mathrm{Co}$ & $8.0 \mathrm{E}-04$ & $1.5 \mathrm{E}-10$ & $1.2 \mathrm{E}-13$ & & & & \\
\hline${ }^{00} \mathrm{Sr}$ & $2.3 E-02$ & $6.2 \mathrm{E}-11$ & $1.4 \mathrm{E}-12$ & & & & \\
\hline Tritium & $2.3 \mathrm{E}-04$ & $7.8 \mathrm{E}-14$ & $1.8 \mathrm{E}-17$ & & & & \\
\hline
\end{tabular}




\begin{tabular}{|c|c|c|c|c|c|c|c|}
\hline Contaminant & $\begin{array}{l}\text { Chronic } \\
\text { Doily } \\
\text { Intake } \\
\end{array}$ & $\begin{array}{l}\text { Slope } \\
\text { Factor }\end{array}$ & $\begin{array}{c}\text { Contaminant } \\
\text { Specific } \\
\text { Risk }\end{array}$ & $\begin{array}{l}\text { Contomniant } \\
\text { Class Rislk }\end{array}$ & $\begin{array}{c}\text { Total } \\
\text { Pathway } \\
\text { Risk }\end{array}$ & $\begin{array}{c}\text { Totsl } \\
\text { Contaminant } \\
\text { Risk }\end{array}$ & $\begin{array}{l}\text { Total } \\
\text { Area } \\
\text { Risk }\end{array}$ \\
\hline Total Radionuclides & & & & $8.5 E-10$ & & & \\
\hline Total Pathway Risk & & & & & $2.1 E-09$ & & \\
\hline \multicolumn{8}{|c|}{ Exposure Pathway: External Exposure to Radiomuclides by S\&M Worker from Sediment in Cell } \\
\hline${ }^{15} \mathrm{Cs}$ & $1.1 E+03$ & $2.0 \mathrm{E}-06$ & 2.3E-03 & & & & \\
\hline $20 \mathrm{Th}$ & $2.0 \mathrm{E}-02$ & $5.6 \mathrm{E}-06$ & 1.1E-07 & & & & \\
\hline${ }^{20} \mathrm{Th}$ & $6.8 \mathrm{E}-01$ & 6.8E-07 & 4.6E-07 & & & 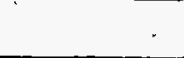 & \\
\hline${ }^{20} \mathrm{Th}$ & $2.0 \mathrm{E}-02$ & $2.6 \mathrm{E}-11$ & $5.2 \mathrm{E}-13$ & & & & \\
\hline${ }^{202825} \mathrm{U}$ & $1.3 \mathrm{E}-01$ & 3.0E-11 & 3.8E-12 & & & & \\
\hline${ }^{258} \mathrm{Pu}$ & $4.2 \mathrm{E}-02$ & $2.8 \mathrm{E}-11$ & $1.2 \mathrm{E}-12$ & & & & \\
\hline $25 \mathrm{U}$ & $8.2 \mathrm{E}-02$ & $5.1 \mathrm{E}-08$ & $4.2 E-09$ & & & & \\
\hline${ }^{208200} \mathrm{Pu}$ & $1.3 \mathrm{E}+\infty 0$ & $1.7 \mathrm{E}-11$ & $2.2 \mathrm{E}-11$ & & & & \\
\hline${ }^{40} \mathrm{~K}$ & 3.4E-01 & $5.4 \mathrm{E}-07$ & $1.9 \mathrm{E}-07$ & & & & \\
\hline${ }^{\infty} \mathrm{Co}$ & $1.3 \mathrm{E}-01$ & $8.6 \mathrm{E}-06$ & $1.1 \mathrm{E}-06$ & & & & \\
\hline${ }^{x} \mathrm{St}$ & $3.6 \mathrm{E}+00$ & $0.0 \mathrm{E}+\infty$ & $0.0 \mathrm{E}+\infty$ & & & & \\
\hline Tritium & $3.7 \mathrm{E}-02$ & $0.0 \mathrm{E}+\infty$ & $0.0 E+\infty$ & & & & \\
\hline Total Radionuclides & & & & $2.3 \mathrm{E}-03$ & & & \\
\hline Totul Pathway Risk & & & & & $2.3 \mathrm{E}-03$ & & \\
\hline Total Inorganic Risk & & & & & & $1.2 \mathrm{E}-09$ & \\
\hline Total Organic Risk & & & & & & 7.7E-06 & \\
\hline Total Radionuclide Risk & & & & & & $2.3 \mathrm{E}-03$ & \\
\hline Total Area & & & & & & & $2.3 \mathrm{E}-03$ \\
\hline \multicolumn{8}{|c|}{ Exposure Pathway: Ingestion of Contaminants by S\&M Worker from Core Samples - Whole Building } \\
\hline Beryllium & $1.7 \mathrm{E}-08$ & $4.3 \mathrm{E}+\infty$ & 7.4E-08 & & & & \\
\hline Total Inorganics & & & & 7.4E-08 & & & \\
\hline Aroclor-1254 & 2.0E-09 & $7.7 \mathrm{E}+\infty$ & $1.6 \mathrm{E}-08$ & & & & \\
\hline Aroclor-1260 & 3.6E- -09 & $7.7 \mathrm{E}+\infty$ & 2.8E-08 & & & & \\
\hline Bis(2-ethylhexyl)phthalate & 3.4E-10 & $1.4 \mathrm{E}-02$ & 4.7E-12 & & & & \\
\hline Total Organics & & & & 4.4E-08 & & & \\
\hline${ }^{17} \mathrm{Cs}$ & $3.4 \mathrm{E}+03$ & 2.8E-11 & $9.6 \mathrm{E}-08$ & & & & \\
\hline${ }^{2 \times} \mathrm{Ra}$ & $1.0 \mathrm{E}+00$ & $1.2 \mathrm{E}-10$ & $1.2 \mathrm{E}-10$ & & & & \\
\hline${ }^{2{ }^{2}} \mathrm{R}_{\mathrm{a}}$ & $9.3 \mathrm{E}-01$ & $1.0 \mathrm{E}-10$ & 9.3E-11 & & & & \\
\hline${ }^{20} \mathrm{Th}$ & $1.9 \mathrm{E}+00$ & $5.5 \mathrm{E}-11$ & $1.1 E-10$ & & & & \\
\hline${ }^{2 \times 0} \mathrm{Th}$ & $3.5 E+\infty 0$ & $1.3 \mathrm{E}-11$ & $4.5 \mathrm{E}-11$ & & & & \\
\hline
\end{tabular}




\begin{tabular}{|c|c|c|c|c|c|c|c|}
\hline Contaminant & $\begin{array}{l}\text { Chrouic } \\
\text { Doily } \\
\text { Intake }\end{array}$ & $\begin{array}{l}\text { Slope } \\
\text { Factor }\end{array}$ & $\begin{array}{l}\text { Contaminant } \\
\text { Specific } \\
\text { Risk }\end{array}$ & $\begin{array}{l}\text { Contamniant } \\
\text { Class Risk }\end{array}$ & $\begin{array}{c}\text { Total } \\
\text { Pathway } \\
\text { Risk }\end{array}$ & $\begin{array}{c}\text { Total } \\
\text { Contaminant } \\
\text { Risk }\end{array}$ & $\begin{array}{l}\text { Total } \\
\text { Area } \\
\text { Risk }\end{array}$ \\
\hline${ }^{200} \mathrm{Th}$ & $2.0 \mathrm{E}+\infty 0$ & $1.2 \mathrm{E}-11$ & 2.4E-11 & & & & \\
\hline${ }^{213254} U$ & $3.2 \mathrm{E}+\infty 0$ & $1.6 \mathrm{E}-11$ & 5.1E-11 & & & & \\
\hline${ }^{254} \mathrm{Th}$ & $1.6 \mathrm{E}+01$ & $4.0 \mathrm{E}-12$ & $6.3 \mathrm{E}-11$ & & & & \\
\hline${ }^{215 U}$ & $3.0 \mathrm{E}-01$ & $1.6 \mathrm{E}-11$ & $4.8 \mathrm{E}-12$ & & & & \\
\hline${ }^{24} \mathrm{Pu}$ & $1.1 \mathrm{E}+\infty$ & 2.2E-10 & $2.5 \mathrm{E}-10$ & & & & \\
\hline 2000 & $3.6 \mathrm{E}+\infty 0$ & 2.0E-11 & $7.1 \mathrm{E}-11$ & & & & \\
\hline${ }^{292810} \mathrm{Pu}$ & $1.0 \mathrm{E}+01$ & $2.3 \mathrm{E}-10$ & $2.3 \mathrm{E}-09$ & & & & \\
\hline${ }^{211} \mathrm{Am}$ & $1.3 \mathrm{E}+\infty 0$ & $2.4 \mathrm{E}-10$ & $3.0 \mathrm{E}-10$ & & & & \\
\hline${ }^{\infty} \mathrm{K}$ & $1.3 \mathrm{E}+01$ & $1.1 \mathrm{E}-11$ & $1.5 \mathrm{E}-10$ & & & & \\
\hline${ }^{\infty 0} \mathrm{Co}$ & $3.3 \mathrm{E}-01$ & $1.5 \mathrm{E}-11$ & $4.9 \mathrm{E}-12$ & & & & \\
\hline${ }^{\infty} \mathrm{Sr}$ & $2.9 E+01$ & $3.6 \mathrm{E}-11$ & $1.0 \mathrm{E}-09$ & & & & \\
\hline Tritium & $6.4 \mathrm{E}+01$ & $5.4 \mathrm{E}-14$ & $3.5 \mathrm{E}-12$ & & & & \\
\hline Total Radionuclides & & & & $1.0 \mathrm{E}-07$ & & & \\
\hline Total Pathway Risk & & & & & 2.2E-07 & & \\
\hline \multicolumn{8}{|c|}{ Exposure Pathway: Dermal Exposure of Contaminants by S\&M Worker from Core Samples - Whole Building } \\
\hline Beryllium & $1.1 \mathrm{E}-09$ & $4.3 \mathrm{E}+02$ & $4.6 \mathrm{E}-07$ & & & & \\
\hline Total Inorganics & & & & $4.6 \mathrm{E}-07$ & & & \\
\hline Aroclor-1254 & $1.3 \mathrm{E}-09$ & $8.6 \mathrm{E}+\infty$ & $1.1 \mathrm{E}-08$ & & & & \\
\hline Aroclor-1260 & $2.3 \mathrm{E}-09$ & $8.6 \mathrm{E}+\infty$ & $1.9 \mathrm{E}-08$ & & & & \\
\hline Bis(2-ethythexyl)phthalate & 2.1E-10 & $7.4 \mathrm{E}-02$ & $1.5 \mathrm{E}-11$ & & & & \\
\hline Toral Organics & & & & $3.0 \mathrm{E}-08$ & & & \\
\hline Total Pathway Risk & & & & & 4.9E-07 & & \\
\hline \multicolumn{8}{|c|}{ Exposure Pathway: Inhalation of Contaminants by S\&M Worker from Core Samples - Whole Building } \\
\hline Arsenic & 3.7E-13 & $5.0 \mathrm{E}+01$ & $1.9 \mathrm{E}-11$ & & & & \\
\hline Beryllium & $1.6 \mathrm{E}-12$ & $8.4 \mathrm{E}+\infty 0$ & $1.4 \mathrm{E}-11$ & & & & \\
\hline Cadmium & $2.3 \mathrm{E}-11$ & $6.1 \mathrm{E}+\infty 0$ & $1.4 \mathrm{E}-10$ & & & & \\
\hline Chromium VI & $2.6 \mathrm{E}-11$ & $4.1 \mathrm{E}+01$ & $1.0 \mathrm{E}-09$ & & & & \\
\hline Total Inorganics & & & & $1.2 \mathrm{E}-09$ & & & \\
\hline${ }^{17} \mathrm{Cs}$ & $3.2 \mathrm{E}-01$ & $1.9 \mathrm{E}-11$ & $6.1 \mathrm{E}-12$ & & & & \\
\hline${ }^{\infty} \mathrm{Ra}_{\mathrm{a}}$ & 9.4E-05 & $3.0 \mathrm{E}-09$ & $3.9 \mathrm{E}-07$ & & & & \\
\hline${ }^{2 x} \mathrm{Ra}$ & $8.6 \mathrm{E}-05$ & $6.9 \mathrm{E}-10$ & $6.0 \mathrm{E}-14$ & & & & \\
\hline $20 \mathrm{Th}$ & $1.8 \mathrm{E}-04$ & $7.8 \mathrm{E}-08$ & $1.4 \mathrm{E}-11$ & & & & \\
\hline${ }^{200} \mathrm{Th}$ & $3.2 \mathrm{E}-04$ & $2.9 \mathrm{E}-08$ & $9.4 \mathrm{E}-12$ & & & & \\
\hline
\end{tabular}




\begin{tabular}{|c|c|c|c|c|c|c|c|}
\hline Contaminant & $\begin{array}{l}\text { Chronic } \\
\text { Daily } \\
\text { Intake }\end{array}$ & $\begin{array}{l}\text { Stope } \\
\text { Factor }\end{array}$ & $\begin{array}{l}\text { Contaminant } \\
\text { Specific } \\
\text { Risk }\end{array}$ & $\begin{array}{l}\text { Contamniant } \\
\text { Class Risk }\end{array}$ & $\begin{array}{c}\text { Total } \\
\text { Pathway } \\
\text { Risk }\end{array}$ & $\begin{array}{c}\text { Total } \\
\text { Contaminant } \\
\text { Risk }\end{array}$ & $\begin{array}{l}\text { Total } \\
\text { Area } \\
\text { Risk }\end{array}$ \\
\hline${ }^{25} \mathrm{Th}$ & $1.9 \mathrm{E}-04$ & $2.8 \mathrm{E}-08$ & $5.2 \mathrm{E}-12$ & & & & \\
\hline${ }^{23 s 25 x} \mathrm{U}$ & $3.0 \mathrm{E}-04$ & $2.6 \mathrm{E}-08$ & $7.7 \mathrm{E}-12$ & & & & \\
\hline${ }^{254} \mathrm{Th}$ & $1.5 \mathrm{E}-03$ & $3.2 \mathrm{E}-11$ & $4.7 \mathrm{E}-14$ & & & & \\
\hline${ }^{23 S} \mathrm{U}$ & $2.8 \mathrm{E}-05$ & $2.5 \mathrm{E}-08$ & $7.0 \mathrm{E}-13$ & & & & \\
\hline${ }^{2 \mathrm{~N}} \mathrm{Pu}$ & $1.1 \mathrm{E}-04$ & $3.9 \mathrm{E}-08$ & 4.1E-12 & & & & \\
\hline $250 \mathrm{U}$ & 3.3E-04 & $2.4 \mathrm{E}-08$ & $8.0 \mathrm{E}-12$ & & & & \\
\hline${ }^{298200} \mathrm{Pu}$ & $9.3 \mathrm{E}-04$ & 3.8E-08 & $3.5 \mathrm{E}-11$ & & & & \\
\hline${ }^{2 \mu} \mathrm{Am}$ & $1.2 \mathrm{E}-04$ & $3.2 \mathrm{E}-08$ & $3.7 \mathrm{E}-12$ & . & & & \\
\hline${ }^{40} \mathrm{~K}$ & $1.3 \mathrm{E}-03$ & $7.6 \mathrm{E}-12$ & $9.5 E-15$ & & & & \\
\hline${ }^{\infty} \mathrm{Co}$ & $3.0 \mathrm{E}-05$ & $1.5 \mathrm{E}-10$ & 4.6E-15 & & & & \\
\hline${ }^{\infty} \mathrm{Sr}$ & $2.7 \mathrm{E}-03$ & $6.2 \mathrm{E}-11$ & $1.7 \mathrm{E}-13$ & & & & \\
\hline Tritium & $6.0 \mathrm{E}-03$ & $7.8 \mathrm{E}-14$ & 4.7E- 16 & & & & \\
\hline Total Radionuclides & & & & $3.9 \mathrm{E}-07$ & & & \\
\hline Total Pathway Risk & & & & & $3.9 \mathrm{E}-07$ & & \\
\hline \multicolumn{8}{|c|}{ Exposure Pathway: External Exposure to Radionoclides by S\&M Worker from Core Samples - Whole Building } \\
\hline${ }^{15} \mathrm{Cs}$ & $5.0 \mathrm{E}+01$ & 2.0E-06 & $1.0 \mathrm{E}-04$ & & & & \\
\hline $2 \mathrm{Ra}_{\mathrm{a}}$ & $1.5 \mathrm{E}-02$ & 6.0E-06 & $8.8 \mathrm{E}-08$ & & & & \\
\hline $2 x_{R_{a}}$ & $1.4 \mathrm{E}-02$ & $2.9 \mathrm{E}-06$ & $3.9 \mathrm{E}-08$ & & & & \\
\hline $2 \mathrm{Th}$ & $2.8 \mathrm{E}-02$ & $5.6 \mathrm{E}-06$ & $1.6 \mathrm{E}-07$ & & & & \\
\hline${ }^{200} \mathrm{Th}$ & $5.1 \mathrm{E}-02$ & 5.4E-11 & 2.7E-12 & & & & \\
\hline $25 \mathrm{Th}$ & $2.9 \mathrm{E}-02$ & $2.6 \mathrm{E}-11$ & 7.6E-13 & & & & \\
\hline 23258 & $4.6 \mathrm{E}-02$ & $3.0 E-11$ & $1.4 \mathrm{E}-12$ & & & & \\
\hline${ }^{24} \mathrm{Th}$ & 2.3E-01 & $3.5 \mathrm{E}-09$ & $8.1 E-10$ & & & & \\
\hline${ }^{25 S} \mathrm{U}$ & $4.4 \mathrm{E}-03$ & $2.4 \mathrm{E}-07$ & $1.1 \mathrm{E}-09$ & & & & \\
\hline${ }^{25 s} \mathrm{Pu}$ & $1.6 \mathrm{E}-02$ & $2.8 \mathrm{E}-11$ & 4.6E-13 & & & & \\
\hline${ }^{248} \mathrm{U}$ & $5.2 \mathrm{E}-02$ & $5.1 E-08$ & $2.7 \mathrm{E}-09$ & & & - & \\
\hline${ }^{252010} \mathrm{Pu}$ & $1.5 \mathrm{E}-01$ & $1.7 \mathrm{E}-11$ & $2.5 \mathrm{E}-12$ & & & & \\
\hline${ }^{201} \mathrm{Am}$ & $1.8 \mathrm{E}-02$ & $4.9 \mathrm{E}-09$ & $8.9 \mathrm{E}-11$ & & & & \\
\hline${ }^{\infty} \mathrm{K}$ & $2.0 \mathrm{E}-01$ & $5.4 \mathrm{E}-07$ & 1.1E-07 & & & & \\
\hline${ }^{\infty} \mathrm{Co}$ & 4.7E- -03 & 8.6E-06 & 4.1E-08 & & & & \\
\hline${ }^{x_{\mathrm{Sr}}}$ & $4.2 \mathrm{E}-01$ & $0.0 \mathrm{E}+\infty$ & $0.0 \mathrm{E}+\infty$ & & & & \\
\hline Tritium & 9.4E-01 & $0.0 \mathrm{E}+\infty$ & $0.0 \mathrm{E}+\infty$ & & & & \\
\hline Total Radionuclides & & & & $1.0 \mathrm{E}-04$ & & & \\
\hline
\end{tabular}




\begin{tabular}{|c|c|c|c|c|c|c|c|}
\hline Contaminant & $\begin{array}{l}\text { Chronic } \\
\text { Daily } \\
\text { Intalse }\end{array}$ & $\begin{array}{l}\text { Sope } \\
\text { Factor }\end{array}$ & $\begin{array}{c}\text { Contamingnt } \\
\text { Specific } \\
\text { Risk }\end{array}$ & $\begin{array}{l}\text { Contamniant } \\
\text { Class Risk }\end{array}$ & $\begin{array}{c}\text { Total } \\
\text { Pathway } \\
\text { Risk }\end{array}$ & $\begin{array}{c}\text { Total } \\
\text { Contaminant } \\
\text { Risk }\end{array}$ & $\begin{array}{l}\text { Total } \\
\text { Area } \\
\text { Risk }\end{array}$ \\
\hline Total Pathway Risk & & & & & $1.0 \mathrm{E}-04$ & & \\
\hline Total Inorganic Risk & & & & & & $5.4 E-07$ & \\
\hline Total Organic Risk & & & & & & $7.4 \mathrm{E}-08$ & \\
\hline Total Radionuclide Risk & & & & & & $1.0 \mathrm{E}-04$ & \\
\hline Total Area & & & & & & & $1.0 \mathrm{E}-04$ \\
\hline \multicolumn{8}{|c|}{ Exposure Pathway: External Exposure to Radionuchides by S\&M Worker from Direct Measurement Samples - Cell } \\
\hline Beta-Gamma & $3.2 E+03$ & $1.0 \mathrm{E}+\infty$ & 2.4E-03 & & & & \\
\hline Gamma & $2.8 \mathrm{E}+03$ & $1.0 \mathrm{E}+\infty$ & $2.0 \mathrm{E}-03$ & & & & \\
\hline Total Radionuclides & & & & $4.4 \mathrm{E}-03$ & & & \\
\hline Total Pathway Risk & & & & & 4.4E-03 & & \\
\hline Total Radionuclide Risk & & & & & & 4.4E-03 & \\
\hline Total Area & & & & & & & 4.4E-03 \\
\hline \multicolumn{8}{|c|}{ Exposure Pathway: External Exposure to Radionuclides by S\&M Worker from Direct Measurement Samples - East Gallery } \\
\hline Beta-Gamma & $4.5 \mathrm{E}+01$ & $1.0 \mathrm{E}+00$ & $3.3 \mathrm{E}-05$ & & & & \\
\hline Gamma & $4.0 \mathrm{E}+01$ & $1.0 \mathrm{E}+00$ & 2.9E-05 & & & & \\
\hline Total Radionuclides & & & & $6.2 \mathrm{E}-05$ & & & \\
\hline Total Pathway Risk & & & & & $6.2 E-05$ & & \\
\hline Total Radionuclide Risk & & & & & & $6.2 \mathrm{E}-05$ & \\
\hline Total Area & & & & & & & $6.2 \mathrm{E}-05$ \\
\hline \multicolumn{8}{|c|}{ Exposure Pathway: External Exposure to Radionuclides by S\&M Worker from Direct Measurement Samples - Whole Building } \\
\hline Beta-Gamma & $4.5 E+03$ & $1.0 \mathrm{E}+00$ & $3.3 \mathrm{E}-03$ & & & & \\
\hline Gamma & $4.1 E+03$ & $1.0 \mathrm{E}+00$ & $3.0 \mathrm{E}-03$ & & & & \\
\hline Total Radionuclides & & & & $6.2 \mathrm{E}-03$ & & & \\
\hline Total Pathway Risk & & & & & $6.2 \mathrm{E}-03$ & & \\
\hline Total Radionuclide Risk & & & & & & $6.2 \mathrm{E}-03$ & \\
\hline Total Area & & & & & & & $6.2 \mathrm{E}-03$ \\
\hline \multicolumn{8}{|c|}{ Exposure Pathway: Ingestion of Radionuclides by S\&M Worker from Smear Samples - Cell } \\
\hline${ }^{157} \mathrm{Cs}$ & $3.6 \mathrm{E}+02$ & $2.8 \mathrm{E}-11$ & $1.0 \mathrm{E}-08$ & & & & \\
\hline${ }^{2 \infty} \mathrm{Ra}$ & $1.3 \mathrm{E}-02$ & $1.2 \mathrm{E}-10$ & $1.6 \mathrm{E}-12$ & & & & \\
\hline${ }^{2 n} \mathrm{Th}$ & $2.8 \mathrm{E}-02$ & $5.5 \mathrm{E}-11$ & $1.5 \mathrm{E}-12$ & & & & \\
\hline${ }^{200} \mathrm{Th}$ & $4.6 \mathrm{E}-02$ & $1.3 \mathrm{E}-11$ & $6.0 \mathrm{E}-13$ & & & & \\
\hline${ }^{25} \mathrm{Th}$ & $2.9 \mathrm{E}-02$ & $1.2 \mathrm{E}-11$ & $3.4 \mathrm{E}-13$ & & & & \\
\hline${ }^{231223} \mathrm{U}$ & 4.3E-02 & $1.6 \mathrm{E}-11$ & $6.8 \mathrm{E}-13$ & & & & \\
\hline
\end{tabular}




\begin{tabular}{|c|c|c|c|c|c|c|c|}
\hline Contaminant & $\begin{array}{l}\text { Chronic } \\
\text { Daily } \\
\text { Intake }\end{array}$ & $\begin{array}{l}\text { Sope } \\
\text { Factor }\end{array}$ & $\begin{array}{c}\text { Conteminent } \\
\text { Specific } \\
\text { Risl. }\end{array}$ & $\begin{array}{l}\text { Contamniant } \\
\text { Class Risk }\end{array}$ & $\begin{array}{c}\text { Total } \\
\text { Pathway } \\
\text { Risk }\end{array}$ & $\begin{array}{c}\text { Total } \\
\text { Contaminant } \\
\text { Risk }\end{array}$ & $\begin{array}{l}\text { Total } \\
\text { Area } \\
\text { Risk }\end{array}$ \\
\hline${ }^{235} \mathrm{U}$ & 4.0E-03 & $1.6 \mathrm{E}-11$ & 6.3E-14 & & & & \\
\hline${ }^{230} \mathrm{Pu}$ & $2.1 \mathrm{E}-02$ & $2.2 \mathrm{E}-10$ & 4.6E-12 & & & & \\
\hline${ }^{240} \mathrm{U}$ & $5.6 \mathrm{E}-02$ & 2.0E-11 & $1.1 \mathrm{E}-12$ & & & & \\
\hline${ }^{258200 \mathrm{Pu}}$ & 1.3E-01 & $2.3 \mathrm{E}-10$ & $3.0 \mathrm{E}-11$ & & & & \\
\hline${ }^{10} \mathrm{~K}$ & $1.4 E+\infty 0$ & 1.1E-11 & $1.5 \mathrm{E}-11$ & & & & \\
\hline${ }^{\infty} \mathrm{Co}$ & $3.4 \mathrm{E}-02$ & 1.5E-11 & $5.1 \mathrm{E}-13$ & & & x & \\
\hline${ }^{90} \mathrm{Sr}$ & $3.0 \mathrm{E}+\infty$ & $3.6 \mathrm{E}-11$ & 1.1E-10 & & & & \\
\hline Total Radionuclides & & & & $1.0 \mathrm{E}-08$ & & & \\
\hline Total Pathway Risk & & & & & $1.0 \mathrm{E}-08$ & & \\
\hline \multicolumn{8}{|c|}{ Exposure Pathway: Inhalation of Radionoclides by S\&M Worker from Smear Samples - Cell } \\
\hline${ }^{15} \mathrm{Cs}$ & $3.3 \mathrm{E}-02$ & $1.9 \mathrm{E}-11$ & $6.3 \mathrm{E}-13$ & & & & \\
\hline${ }^{2 x} \mathrm{Ra}$ & $1.2 \mathrm{E}-06$ & $3.0 \mathrm{E}-09$ & $5.1 E-09$ & & & & \\
\hline $20 \mathrm{Th}$ & $2.6 \mathrm{E}-06$ & $7.8 \mathrm{E}-08$ & $2.0 \mathrm{E}-13$ & & & & \\
\hline${ }^{200} \mathrm{Th}$ & 4.3E-06 & $2.9 \mathrm{E}-08$ & $1.2 \mathrm{E}-13$ & & & & \\
\hline${ }^{250} \mathrm{Th}$ & $2.7 \mathrm{E}-06$ & $2.8 \mathrm{E}-08$ & $7.5 \mathrm{E}-14$ & & & & \\
\hline $233254 \mathrm{U}$ & $4.0 \mathrm{E}-06$ & $2.6 \mathrm{E}-08$ & $1.0 \mathrm{E}-13$ & & & & \\
\hline${ }^{2 N S U}$ & $3.7 \mathrm{E}-07$ & $2.5 \mathrm{E}-08$ & $9.2 \mathrm{E}-15$ & & & & \\
\hline${ }^{23 *} \mathrm{Pu}$ & $2.0 \mathrm{E}-06$ & $3.9 \mathrm{E}-08$ & $7.6 \mathrm{E}-14$ & & & & \\
\hline${ }^{2 \times} \mathrm{U}$ & $5.2 \mathrm{E}-06$ & $2.4 \mathrm{E}-08$ & $1.2 \mathrm{E}-13$ & & & & \\
\hline${ }^{250200 \mathrm{Pu}}$ & $1.2 \mathrm{E}-05$ & $3.8 E-08$ & 4.7E-13 & & & & \\
\hline${ }^{\infty} \mathrm{K}$ & $1.3 \mathrm{E}-04$ & $7.6 \mathrm{E}-12$ & $9.8 \mathrm{E}-16$ & & & & \\
\hline${ }^{\infty} \mathrm{Co}$ & $3.2 \mathrm{E}-06$ & $1.5 \mathrm{E}-10$ & 4.7E-16 & & & & \\
\hline${ }^{\infty} \mathrm{Sr}$ & $2.8 \mathrm{E}-04$ & $6.2 \mathrm{E}-11$ & $1.8 \mathrm{E}-14$ & & & & \\
\hline Total Radionuclides & & & & 5.1E-09 & & & \\
\hline Total Pathway Risk & & & & & 5.1E-09 & & \\
\hline \multicolumn{8}{|c|}{ Exposure Pathway: External Exposure to Radionuchides by S\&M Worker from Smear Samples - Cell } \\
\hline${ }^{17} \mathrm{Cs}$ & $6.5 E+\infty 0$ & $2.0 \mathrm{E}-06$ & $1.3 \mathrm{E}-05$ & & & & \\
\hline $206 \mathrm{Ra}$ & 2.4E-04 & $6.0 \mathrm{E}-06$ & $1.4 \mathrm{E}-09$ & & & & \\
\hline 20Th & $5.0 \mathrm{E}-04$ & $5.6 \mathrm{E}-06$ & $2.8 \mathrm{E}-09$ & & & & \\
\hline${ }^{200} \mathrm{Th}$ & 8.4E-04 & $5.4 \mathrm{E}-11$ & $4.5 \mathrm{E}-14$ & & & & \\
\hline $200 \mathrm{Th}$ & $5.2 \mathrm{E}-04$ & $2.6 \mathrm{E}-11$ & $1.4 \mathrm{E}-14$ & & & & \\
\hline $20252 x \mathrm{U}$ & $7.8 \mathrm{E}-04$ & $3.0 \mathrm{E}-11$ & $2.3 \mathrm{E}-14$ & & & & \\
\hline${ }^{2 s 3} \mathrm{U}$ & $7.2 \mathrm{E}-05$ & $2.4 \mathrm{E}-07$ & 1.7E-11 & & & & \\
\hline
\end{tabular}




\begin{tabular}{|c|c|c|c|c|c|c|c|}
\hline Contamingnt & $\begin{array}{l}\text { Chronic } \\
\text { Daily } \\
\text { Intake }\end{array}$ & $\begin{array}{l}\text { Sope } \\
\text { Factor }\end{array}$ & $\begin{array}{c}\text { Contominant } \\
\text { Specific } \\
\text { Risk }\end{array}$ & $\begin{array}{l}\text { Contamniant } \\
\text { Class Risk }\end{array}$ & $\begin{array}{c}\text { Total } \\
\text { Pathway } \\
\text { Risk }\end{array}$ & $\begin{array}{c}\text { Total } \\
\text { Contaminant } \\
\text { Risk }\end{array}$ & $\begin{array}{l}\text { Total } \\
\text { Area } \\
\text { Risk }\end{array}$ \\
\hline${ }^{2 s} \mathrm{Pu}$ & $3.8 \mathrm{E}-04$ & $2.8 \mathrm{E}-11$ & $1.1 \mathrm{E}-14$ & & & & \\
\hline${ }^{234} \mathrm{U}$ & $1.0 \mathrm{E}-03$ & 5.1E-08 & $5.2 \mathrm{E}-11$ & & & & \\
\hline${ }^{250200} \mathrm{Pu}$ & $2.4 \mathrm{E}-03$ & $1.7 \mathrm{E}-11$ & $4.1 E-14$ & & & & \\
\hline${ }^{40} \mathrm{~K}$ & $2.5 \mathrm{E}-02$ & $5.4 \mathrm{E}-07$ & $1.4 \mathrm{E}-08$ & & & & \\
\hline${ }^{\infty} \mathrm{Co}$ & $6.2 \mathrm{E}-04$ & $8.6 \mathrm{E}-06$ & $5.3 \mathrm{E}-09$ & & & & \\
\hline${ }^{\infty} \mathrm{Sr}$ & $5.5 \mathrm{E}-02$ & $0.0 \mathrm{E}+00$ & $0.0 \mathrm{E}+00$ & & & & \\
\hline Total Radionuclides & & & & $1.3 \mathrm{E}-05$ & & & \\
\hline Total Pathway Risk & & & & & $1.3 \mathrm{E}-05$ & & \\
\hline Total Radionuclide Risk & & & & & & $1.3 \mathrm{E}-05$ & \\
\hline Total Area & & & & & & & $1.3 \mathrm{E}-05$ \\
\hline \multicolumn{8}{|c|}{ Exposure Pathway: Ingestion of Radiomoclides by S\&M Worker from Smear Samples - East Gallery } \\
\hline${ }^{15} \mathrm{Cs}$ & $3.8 \mathrm{E}+01$ & $2.8 \mathrm{E}-11$ & $1.1 \mathrm{E}-09$ & & & & \\
\hline${ }^{2 \times} \mathrm{Ra}$ & $3.7 \mathrm{E}-02$ & $1.2 \mathrm{E}-10$ & $4.4 \mathrm{E}-12$ & & & & \\
\hline $2 \mathrm{Ra}$ & $6.1 \mathrm{E}-01$ & $1.0 \mathrm{E}-10$ & $6.1 \mathrm{E}-11$ & & & & \\
\hline 20 Th & $5.7 E-02$ & $5.5 \mathrm{E}-11$ & 3.1E-12 & & & & \\
\hline $30 \mathrm{Th}$ & $1.2 \mathrm{E}-01$ & $1.3 \mathrm{E}-11$ & $1.6 \mathrm{E}-12$ & & & & \\
\hline $200 \mathrm{Th}$ & $4.4 \mathrm{E}-02$ & $1.2 \mathrm{E}-11$ & $5.2 \mathrm{E}-13$ & & & & \\
\hline $2 \operatorname{sen} 2 x U$ & $9.1 \mathrm{E}-02$ & $1.6 \mathrm{E}-11$ & $1.5 \mathrm{E}-12$ & & & & \\
\hline${ }^{250} \mathrm{U}$ & $6.1 \mathrm{E}-03$ & $1.6 \mathrm{E}-11$ & $9.7 \mathrm{E}-14$ & & & & \\
\hline${ }^{2 x 8} \mathrm{Pu}$ & $4.0 \mathrm{E}-02$ & 2.2E-10 & 8.7E-12 & & & & \\
\hline${ }^{258} \mathrm{U}$ & 7.1E-02 & $2.0 \mathrm{E}-11$ & $1.4 \mathrm{E}-12$ & & & & \\
\hline $209200 \mathrm{Pu}$ & $4.0 \mathrm{E}-02$ & $2.3 \mathrm{E}-10$ & $9.1 \mathrm{E}-12$ & & & & \\
\hline${ }^{201} \mathrm{Am}$ & $5.1 \mathrm{E}-02$ & $2.4 \mathrm{E}-10$ & $1.2 \mathrm{E}-11$ & & & & \\
\hline${ }^{\infty} \mathrm{K}$ & $9.0 \mathrm{E}+\infty$ & $1.1 \mathrm{E}-11$ & $9.9 \mathrm{E}-11$ & & & & \\
\hline${ }^{\infty} \mathrm{Sr}$ & $1.4 \mathrm{E}+01$ & $3.6 \mathrm{E}-11$ & $4.9 \mathrm{E}-10$ & & & & \\
\hline Totsl Radionuclides & & & & $1.8 \mathrm{E}-09$ & & & \\
\hline Total Pathway Risk & & & & & $1.8 \mathrm{E}-09$ & & \\
\hline \multicolumn{8}{|c|}{ Exposure Pathway: Inhalation of Radionuclides by S\&M Worker from Smear Samples - East Gallery } \\
\hline${ }^{137} \mathrm{Cs}$ & $3.6 \mathrm{E}-03$ & $1.9 \mathrm{E}-11$ & $6.8 \mathrm{E}-14$ & & & & \\
\hline${ }^{205} \mathbf{R a}$ & $3.4 \mathrm{E}-06$ & $3.0 \mathrm{E}-09$ & $1.4 \mathrm{E}-08$ & & & & \\
\hline$=\mathbf{R a}$ & $5.6 \mathrm{E}-05$ & $6.9 \mathrm{E}-10$ & $3.9 \mathrm{E}-14$ & & & & \\
\hline 20 Th & $5.3 \mathrm{E}-06$ & $7.8 \mathrm{E}-08$ & 4.1E-13 & & & & \\
\hline${ }^{200} \mathrm{Th}$ & $1.1 E-05$ & $2.9 \mathrm{E}-08$ & $3.3 \mathrm{E}-13$ & & & & \\
\hline
\end{tabular}




\begin{tabular}{|c|c|c|c|c|c|c|c|}
\hline Contaminant & $\begin{array}{c}\text { Chronic } \\
\text { Daily } \\
\text { Intake }\end{array}$ & $\begin{array}{l}\text { Slope } \\
\text { Factor }\end{array}$ & $\begin{array}{c}\text { Contaminant } \\
\text { Specific } \\
\text { Risk }\end{array}$ & $\begin{array}{l}\text { Contamniant } \\
\text { Class Risk }\end{array}$ & $\begin{array}{c}\text { Total } \\
\text { Pathway } \\
\text { Risk }\end{array}$ & $\begin{array}{c}\text { Total } \\
\text { Contaminant } \\
\text { Risk }\end{array}$ & $\begin{array}{l}\text { Total } \\
\text { Area } \\
\text { Risk }\end{array}$ \\
\hline $200 \mathrm{Th}$ & 4.1E-06 & $2.8 \mathrm{E}-08$ & $1.1 E-13$ & & & & \\
\hline 235250 & $8.5 \mathrm{E}-06$ & $2.6 \mathrm{E}-08$ & $2.2 E-13$ & & & & \\
\hline${ }^{25 S U}$ & 5.7E-07 & 2.5E-08 & $1.4 \mathrm{E}-14$ & & & & \\
\hline${ }^{250} \mathrm{Pu}$ & $3.7 E-06$ & $3.9 E-08$ & $1.4 \mathrm{E}-13$ & & & & \\
\hline${ }^{2 x} \mathrm{U}$ & $6.6 \mathrm{E}-06$ & 2.4E-08 & $1.6 \mathrm{E}-13$ & & & & \\
\hline 2910upp & 3.7E-06 & $3.8 \mathrm{E}-08$ & $1.4 \mathrm{E}-13$ & & & - & \\
\hline${ }^{211} \mathrm{Am}$ & 4.7E-06 & 3.2E-08 & $1.5 \mathrm{E}-13$ & & & & \\
\hline${ }^{*} \mathrm{~K}$ & 8.4E-04 & $7.6 \mathrm{E}-12$ & $6.4 \mathrm{E}-15$ & & & & \\
\hline${ }^{\infty 0} \mathrm{Sr}$ & $1.3 \mathrm{E}-03$ & $6.2 \mathrm{E}-11$ & $7.9 \mathrm{E}-14$ & & & & \\
\hline Toul Radionuclides & & & & $1.4 \mathrm{E}-08$ & & & \\
\hline Toul Pathway Risk & & & & & $1.4 \mathrm{E}-08$ & & \\
\hline \multicolumn{8}{|c|}{ Exposure Pathway: External Exposure to Radionaclides by S\&M Worker from Smear Samples - East Gallery } \\
\hline${ }^{17} \mathrm{Cs}$ & 7.0E-01 & $2.0 \mathrm{E}-06$ & $1.4 \mathrm{E}-06$ & & & & \\
\hline${ }^{20 \times} \mathbf{R a}$ & $6.7 \mathrm{E}-04$ & $6.0 \mathrm{E}-06$ & 4.0E-09 & & & & \\
\hline${ }^{2 x} \mathrm{Ra}$ & $1.1 \mathrm{E}-02$ & $2.9 \mathrm{E}-06$ & 3.2E-08 & & & & \\
\hline${ }^{20} \mathrm{Th}$ & $1.0 \mathrm{E}-03$ & $5.6 \mathrm{E}-06$ & $5.8 \mathrm{E}-09$ & & & & \\
\hline${ }^{200} \mathrm{Th}$ & $2.2 \mathrm{E}-03$ & $5.4 \mathrm{E}-11$ & $1.2 \mathrm{E}-13$ & & & & \\
\hline${ }^{20} \mathrm{Th}$ & $7.9 \mathrm{E}-04$ & $2.6 \mathrm{E}-11$ & $2.1 \mathrm{E}-14$ & & & & \\
\hline${ }^{21223} U$ & $1.7 \mathrm{E}-03$ & $3.0 \mathrm{E}-11$ & $5.0 \mathrm{E}-14$ & & & & \\
\hline${ }^{215} \mathrm{U}$ & 1.1E-04 & $2.4 \mathrm{E}-07$ & $2.7 \mathrm{E}-11$ & & & & \\
\hline${ }^{2 s \mathrm{Pu}} \mathrm{Pu}$ & $7.2 \mathrm{E}-04$ & $2.8 \mathrm{E}-11$ & $2.0 \mathrm{E}-14$ & & & & \\
\hline${ }^{200} \mathrm{U}$ & $1.3 E-03$ & 5.1E-08 & $6.6 \mathrm{E}-11$ & & & & \\
\hline $291200 \mathrm{Pu}$ & $7.2 \mathrm{E}-04$ & 1.7E-11 & $1.2 \mathrm{E}-14$ & & & & \\
\hline${ }^{211} \mathrm{Am}$ & $9.2 \mathrm{E}-04$ & $4.9 \mathrm{E}-09$ & $4.5 \mathrm{E}-12$ & & & & \\
\hline${ }^{+\infty} \mathrm{K}$ & $1.6 \mathrm{E}-01$ & $5.4 \mathrm{E}-07$ & $8.9 \mathrm{E}-08$ & & & & \\
\hline${ }^{\infty} \mathrm{Sr}$ & $2.5 \mathrm{E}-01$ & $0.0 E+\infty$ & $0.0 \mathrm{E}+\infty 0$ & & & & \\
\hline Total Radionuclides & & & & $1.5 \mathrm{E}-06$ & & & \\
\hline Total Pathway Risk & & & & & $1.5 \mathrm{E}-06$ & & \\
\hline Total Radionuclide Risk & & & & & & $1.5 \mathrm{E}-06$ & \\
\hline Total Ares & & & & & & & $1.5 \mathrm{E}-06$ \\
\hline \multicolumn{8}{|c|}{ Exposure Pathway: Ingestion of Radionuclides by S\&M Worker from Smear Samples - Whole Building } \\
\hline${ }^{19} \mathrm{Cs}$ & $2.0 \mathrm{E}+02$ & $2.8 \mathrm{E}-11$ & $5.6 \mathrm{E}-09$ & & & & \\
\hline${ }^{2 s} s_{a}$ & $1.5 E-02$ & $1.2 \mathrm{E}-10$ & $1.8 \mathrm{E}-12$ & & & & \\
\hline
\end{tabular}




\begin{tabular}{|c|c|c|c|c|c|c|c|}
\hline Contaminant & $\begin{array}{c}\text { Chronic } \\
\text { Daily } \\
\text { Intake }\end{array}$ & $\begin{array}{l}\text { Sope } \\
\text { Factor }\end{array}$ & $\begin{array}{c}\text { Contaminant } \\
\text { Specific } \\
\text { Risl }\end{array}$ & $\begin{array}{l}\text { Contamniant } \\
\text { Class Risk }\end{array}$ & $\begin{array}{c}\text { Total } \\
\text { Pathway } \\
\text { Rislr }\end{array}$ & $\begin{array}{c}\text { Total } \\
\text { Contaminant } \\
\text { Risk }\end{array}$ & $\begin{array}{l}\text { Total } \\
\text { Area } \\
\text { Risk }\end{array}$ \\
\hline${ }^{2} R_{a}$ & $7.1 E-02$ & $1.0 \mathrm{E}-10$ & $7.1 \mathrm{E}-12$ & & & & \\
\hline $20 \mathrm{Th}$ & $2.8 \mathrm{E}-02$ & 5.5E-11 & $1.6 \mathrm{E}-12$ & & & & \\
\hline${ }^{20} \mathrm{Th}$ & $5.0 \mathrm{E}-02$ & 1.3E-11 & $6.5 \mathrm{E}-13$ & & & & \\
\hline${ }^{200} \mathrm{Th}$ & $2.9 \mathrm{E}-02$ & $1.2 \mathrm{E}-11$ & $3.4 \mathrm{E}-13$ & & & & \\
\hline $251234 U$ & $4.4 \mathrm{E}-02$ & $1.6 \mathrm{E}-11$ & $7.1 \mathrm{E}-13$ & & & & \\
\hline${ }^{215} \mathrm{U}$ & 4.4E-03 & $1.6 \mathrm{E}-11$ & $7.1 \mathrm{E}-14$ & & & & \\
\hline${ }^{2 s 8} \mathrm{Pu}$ & $2.4 \mathrm{E}-02$ & 2.2E-10 & $5.2 \mathrm{E}-12$ & & & & \\
\hline${ }^{234} \mathrm{U}$ & $6.3 \mathrm{E}-02$ & $2.0 \mathrm{E}-11$ & $1.3 \mathrm{E}-12$ & & & & \\
\hline${ }^{259200} \mathrm{Pu}$ & $1.4 \mathrm{E}-01$ & 2.3E-10 & 3.3E-11 & & & & \\
\hline${ }^{211} \mathrm{Am}$ & $1.8 \mathrm{E}-02$ & $2.4 \mathrm{E}-10$ & $4.4 \mathrm{E}-12$ & & & & \\
\hline${ }^{+\infty} \mathrm{K}$ & $1.1 \mathrm{E}+\infty$ & $1.1 \mathrm{E}-11$ & $1.2 \mathrm{E}-11$ & & & & \\
\hline${ }^{80} \mathrm{Co}$ & $2.6 \mathrm{E}-02$ & $1.5 \mathrm{E}-11$ & $3.8 \mathrm{E}-13$ & & & & \\
\hline${ }^{\infty} \mathrm{Sr}$ & $2.3 E+00$ & $3.6 \mathrm{E}-11$ & $8.2 \mathrm{E}-11$ & & & & \\
\hline Tocal Radionuclides & & & & $5.8 \mathrm{E}-09$ & & & \\
\hline Total Pathway Risk & & & & & $5.8 \mathrm{E}-09$ & & \\
\hline \multicolumn{8}{|c|}{ Exposure Pathway: Inhalation of Radionculides by S\&M Worker from Smear Samples - Whole Building } \\
\hline${ }^{17} \mathrm{Cs}$ & $1.9 \mathrm{E}-02$ & $1.9 E-11$ & $3.6 \mathrm{E}-13$ & & & & \\
\hline${ }^{2} \mathrm{Ra}$ & $1.4 \mathrm{E}-06$ & $3.0 \mathrm{E}-09$ & $5.7 \mathrm{E}-09$ & & & & \\
\hline $2 x_{R}$ & $6.6 \mathrm{E}-06$ & $6.9 \mathrm{E}-10$ & $4.5 \mathrm{E}-15$ & & & & \\
\hline 20 Th & $2.6 \mathrm{E}-06$ & $7.8 \mathrm{E}-08$ & $2.1 \mathrm{E}-13$ & & & & \\
\hline${ }^{200} \mathrm{Th}$ & 4.7E-06 & $2.9 \mathrm{E}-08$ & $1.4 \mathrm{E}-13$ & & & & \\
\hline${ }^{20} \mathrm{Th}$ & $2.7 \mathrm{E}-06$ & $2.8 \mathrm{E}-08$ & $7.5 \mathrm{E}-14$ & & & & \\
\hline${ }^{232 \pi} \mathrm{U}$ & $4.1 \mathrm{E}-06$ & $2.6 \mathrm{E}-08$ & $1.1 \mathrm{E}-13$ & & & & \\
\hline${ }^{2 w U}$ & $4.1 E-07$ & $2.5 \mathrm{E}-08$ & $1.0 \mathrm{E}-14$ & & & & \\
\hline${ }^{2 s} \mathrm{Pu}$ & $2.2 \mathrm{E}-06$ & $3.9 \mathrm{E}-08$ & $8.6 E-14$ & & & & \\
\hline 2500 & $5.9 \mathrm{E}-06$ & $2.4 \mathrm{E}-08$ & $1.4 \mathrm{E}-13$ & & & & \\
\hline${ }^{2095200 \mathrm{Pu}}$ & $1.4 \mathrm{E}-05$ & $3.8 \mathrm{E}-08$ & $5.1 \mathrm{E}-13$ & & & & \\
\hline${ }^{2 n} \mathrm{Am}$ & $1.7 \mathrm{E}-06$ & $3.2 \mathrm{E}-08$ & $5.5 E-14$ & & & & \\
\hline${ }^{\infty} \mathrm{K}$ & $9.8 \mathrm{E}-05$ & $7.6 \mathrm{E}-12$ & $7.5 \mathrm{E}-16$ & & & & \\
\hline${ }^{50} \mathrm{Co}$ & $2.4 \mathrm{E}-06$ & $1.5 \mathrm{E}-10$ & $3.6 \mathrm{E}-16$ & & & & \\
\hline${ }^{90} \mathrm{Sr}$ & $2.1 \mathrm{E}-04$ & $6.2 \mathrm{E}-11$ & $1.3 \mathrm{E}-14$ & & & & \\
\hline Total Radionuclides & & & & $5.7 \mathrm{E}-09$ & & & \\
\hline Total Pathway Risk & & & & & $5.7 \mathrm{E}-09$ & & \\
\hline
\end{tabular}




\begin{tabular}{|c|c|c|c|c|c|c|c|}
\hline Contaminant & $\begin{array}{c}\text { Chronic } \\
\text { Daily } \\
\text { Intake }\end{array}$ & $\begin{array}{l}\text { Sope } \\
\text { Factor }\end{array}$ & $\begin{array}{c}\text { Contominant } \\
\text { Specific } \\
\text { Risk }\end{array}$ & $\begin{array}{l}\text { Contomniant } \\
\text { Class Risk }\end{array}$ & $\begin{array}{c}\text { Total } \\
\text { Pathway } \\
\text { Risk }\end{array}$ & $\begin{array}{c}\text { Total } \\
\text { Contaminant } \\
\text { Risk }\end{array}$ & $\begin{array}{l}\text { Total } \\
\text { Area } \\
\text { Risk }\end{array}$ \\
\hline \multicolumn{8}{|c|}{ Exposure Pathway: External Exposure to Rationuclides by S\&M Worker from Smear Samples - Whole Building } \\
\hline${ }^{17} \mathrm{Cs}$ & $3.7 E+\infty 0$ & 2.0E-06 & 7.3E-06 & & & & \\
\hline${ }^{20} \mathrm{Ra}$ & 2.7E-04 & $6.0 \mathrm{E}-06$ & $1.6 \mathrm{E}-09$ & & & & \\
\hline${ }^{20} \mathrm{Ra}$ & $1.3 \mathrm{E}-03$ & 2.9E-06 & 3.7E-09 & & & & \\
\hline${ }^{220} \mathrm{Th}$ & $5.2 \mathrm{E}-04$ & $5.6 \mathrm{E}-06$ & $2.9 \mathrm{E}-09$ & & & & \\
\hline${ }^{200} \mathrm{Th}$ & $9.2 \mathrm{E}-04$ & $5.4 \mathrm{E}-11$ & $5.0 \mathrm{E}-14$ & & & & \\
\hline $250 \mathrm{Th}$ & $5.2 \mathrm{E}-04$ & $2.6 \mathrm{E}-11$ & $1.4 \mathrm{E}-14$ & & & & \\
\hline${ }^{25 s 25} \mathrm{U}$ & 8.1E-04 & $3.0 \mathrm{E}-11$ & 2.4E-14 & & & & \\
\hline${ }^{25} U$ & 8.1E-05 & $2.4 E-07$ & $1.9 \mathrm{E}-11$ & & & & \\
\hline $250 \mathrm{Pu}$ & 4.3E-04 & $2.8 \mathrm{E}-11$ & $1.2 \mathrm{E}-14$ & & & & \\
\hline${ }^{220} \mathrm{U}$ & $1.1 \mathrm{E}-03$ & S.1E-08 & $5.8 \mathrm{E}-11$ & & & & \\
\hline${ }^{299200} \mathrm{Pu}$ & $2.6 \mathrm{E}-03$ & $1.7 \mathrm{E}-11$ & $4.5 \mathrm{E}-14$ & & & & \\
\hline${ }^{211} \mathrm{Am}$ & $3.4 \mathrm{E}-04$ & $4.9 \mathrm{E}-09$ & $1.7 \mathrm{E}-12$ & & & & \\
\hline${ }^{40} \mathrm{~K}$ & $1.9 \mathrm{E}-02$ & $5.4 \mathrm{E}-07$ & $1.0 \mathrm{E}-08$ & & & & \\
\hline${ }^{\infty} \mathrm{Co}_{0}$ & 4.7E-04 & $8.6 \mathrm{E}-06$ & $4.0 \mathrm{E}-09$ & & & & \\
\hline${ }^{\infty} \mathrm{Sr}$ & $4.2 \mathrm{E}-02$ & $0.0 \mathrm{E}+\infty$ & $0.0 \mathrm{E}+\infty$ & & & & \\
\hline Total Radionuclides & & & & $7.4 \mathrm{E}-06$ & & & \\
\hline Total Pathway Risk & & & & & $7.4 \mathrm{E}-06$ & & \\
\hline Total Radionuclide Risk & & & & & & $7.4 \mathrm{E}-06$ & \\
\hline Total Are: & & & & & & & $7.4 \mathrm{E}-06$ \\
\hline \multicolumn{8}{|c|}{ Exposure Pathway: External Exposure to Radionuclides by S\&M Worker from TLD Measurements - Cell } \\
\hline Ben & $9.4 \mathrm{E}+03$ & $1.0 \mathrm{E}+\infty 0$ & $6.8 \mathrm{E}-03$ & & & & \\
\hline Gamma & $2.8 \mathrm{E}+03$ & $1.0 \mathrm{E}+\infty$ & 2.1E-03 & & & & \\
\hline Total Radionuclides & & & & $8.9 \mathrm{E}-03$ & & & \\
\hline Total Pathway Risk & & & & & 8.9E-03 & & \\
\hline Total Radionuclide Risk & & & & & & $8.9 \mathrm{E}-03$ & \\
\hline Total Area & & & & & & & $8.9 E-03$ \\
\hline
\end{tabular}


Table E-3. Hazard Results - Noncarcinogenic Effects (Maintenance Worker)

\begin{tabular}{|c|c|c|c|c|c|c|c|}
\hline \multirow{2}{*}{ Chemcial } & \multirow{2}{*}{$\begin{array}{c}\text { Arerage } \\
\text { Daily Intole } \\
\text { (mg/kg-day) }\end{array}$} & \multirow{2}{*}{$\begin{array}{l}\text { Reference Dose } \\
\text { (mg/kg-day) }\end{array}$} & \multicolumn{5}{|c|}{ Hazard Quotient/ Hazard Index } \\
\hline & & & $\begin{array}{c}\text { Contaminant } \\
\text { Specific }\end{array}$ & $\begin{array}{c}\text { Contaminant } \\
\text { Class }\end{array}$ & Pathway & $\begin{array}{l}\text { Contominant } \\
\text { Class Total }\end{array}$ & $\begin{array}{l}\text { Total } \\
\text { Area }\end{array}$ \\
\hline \multicolumn{8}{|c|}{ Exposure Palhway: Ingestion of Chemicals by Maintenance Worker from Core Samples - Cell } \\
\hline Arsenic & 1.3E-06 & $3.0 \mathrm{E}-04$ & 4.2E-03 & & & & \\
\hline Barium & $3.5 \mathrm{E}-04$ & $7.0 \mathrm{E}-02$ & $5.0 \mathrm{E}-03$ & & & $\cdot$ & \\
\hline Beryllium & 8.6E-06 & $5.0 \mathrm{E}-03$ & 1.7E-03 & & & & \\
\hline Cadmium & 8.8E-05 & $1.0 \mathrm{E}-03$ & $8.8 E-02$ & & & & \\
\hline Chromium VI & $9.6 \mathrm{E}-05$ & $5.0 \mathrm{E}-03$ & $1.9 \mathrm{E}-02$ & & & & \\
\hline Manganere & 4.7E-03 & $1.4 \mathrm{E}-01$ & $3.3 \mathrm{E}-02$ & & & & \\
\hline Mereury & $1.7 \mathrm{E}-07$ & $3.0 \mathrm{E}-04$ & $5.5 \mathrm{E}-04$ & & & & \\
\hline Nickel & $6.8 \mathrm{E}-05$ & 2.0E-02 & $3.4 \mathrm{E}-03$ & & & & \\
\hline Vanadium & $1.2 \mathrm{E}-04$ & $7.0 \mathrm{E}-03$ & $1.7 \mathrm{E}-02$ & & & & \\
\hline Zine & $1.7 \mathrm{E}-02$ & $3.0 \mathrm{E}-01$ & 5.7E-02 & & & & \\
\hline Total Inorganics & & & & 2.3E-01 & & & \\
\hline Pathway Hazard Index & & & & & 2.3E-01 & & \\
\hline \multicolumn{8}{|c|}{ Exposure Pathway: Dermal Exposure of Chemicals to Maintenance Worker from Core Samples - Cell } \\
\hline Arsenic & $7.9 \mathrm{E}-08$ & $1.2 \mathrm{E}-04$ & $6.5 \mathrm{E}-04$ & & & & \\
\hline Barium & $2.2 \mathrm{E}-0.5$ & $4.9 \mathrm{E}-03$ & $4.5 E-03$ & & & & \\
\hline Beryllium & $5.4 \mathrm{E}-07$ & $5.0 \mathrm{E}-05$ & $1.1 \mathrm{E}-02$ & & & & \\
\hline Cadmium & $5.5 E-06$ & $1.0 \mathrm{E}-05$ & $5.5 \mathrm{E}-01$ & & & & \\
\hline Chromium VI & $6.0 \mathrm{E}-06$ & $1.0 \mathrm{E}-04$ & $6.0 \mathrm{E}-02$ & & & & \\
\hline Manganese & $2.9 \mathrm{E}-04$ & $5.6 \mathrm{E}-03$ & $5.2 \mathrm{E}-02$ & & & $\cdot$ & \\
\hline Mercury & $1.0 \mathrm{E}-08$ & $3.0 \mathrm{E}-08$ & $3.5 \mathrm{E}-01$ & . & & & \\
\hline Nickel & $4.2 E-06$ & $5.4 \mathrm{E}-03$ & $7.8 \mathrm{E}-04$ & & & & \\
\hline Vanadium & $7.3 E-06$ & $7.0 \mathrm{E}-05$ & $1.0 \mathrm{E}-01$ & & & & \\
\hline Zine & $1.1 \mathrm{E}-03$ & $6.0 \mathrm{E}-02$ & $1.8 \mathrm{E}-02$ & & & & \\
\hline Total Inorganics & & & & $1.1 E+\infty$ & & & \\
\hline Pathway Hazard Index & & & & & $1.1 \mathrm{E}+00$ & & \\
\hline \multicolumn{8}{|c|}{ Exposure Pathway: Inhalation of Chemicals by Maintenance Worker from Core Samples - Cell } \\
\hline Barium & 3.3E-08 & $1.4 \mathrm{E}-04$ & 2.3E-04 & & & & \\
\hline Manganese & $4.4 \mathrm{E}-07$ & $1.4 \mathrm{E}-05$ & $3.0 \mathrm{E}-02$ & & & & \\
\hline Mercury & $1.6 \mathrm{E}-11$ & $8.6 \mathrm{E}-05$ & $1.8 \mathrm{E}-07$ & & & & \\
\hline
\end{tabular}




\begin{tabular}{|c|c|c|c|c|c|c|c|}
\hline \multirow{2}{*}{ Chemcial } & \multirow{2}{*}{$\begin{array}{c}\text { Average } \\
\text { Daily Intolse } \\
\text { (mg/kg-day) }\end{array}$} & \multirow{2}{*}{$\begin{array}{l}\text { Reference Dose } \\
\text { (mg/kg-day) }\end{array}$} & \multicolumn{5}{|c|}{ Hazand Quotient/ Hazand Index } \\
\hline & & & $\begin{array}{l}\text { Contaminint } \\
\text { Specific }\end{array}$ & $\begin{array}{c}\text { Contaminant } \\
\text { Class }\end{array}$ & Pathway & $\begin{array}{l}\text { Contaminant } \\
\text { Class Total }\end{array}$ & $\begin{array}{l}\text { Total } \\
\text { Area }\end{array}$ \\
\hline Total Inorganics & & & & 3.1E-02 & & & \\
\hline Pathway Hazard Index & & & & & 3.1E-02 & & \\
\hline Total Inorganic Risk & & & & & & $1.4 \mathrm{E}+00$ & \\
\hline Total Area & & & & & & & $1.4 \mathrm{E}+00$ \\
\hline \multicolumn{8}{|c|}{ Expasure Pathway: Ingestion of Chemicals by Maintenance Worker from Core Samples - East Gallery } \\
\hline Arsenic & $1.5 \mathrm{E}-06$ & 3.0E-04 & $4.9 \mathrm{E}-03$ & & & & \\
\hline Barium & $1.0 \mathrm{E}-05$ & $7.0 \mathrm{E}-02$ & 1.5E-04 & . & & & \\
\hline Beryllium & 2.0E-07 & 5.0E-03 & $3.9 \mathrm{E}-05$ & & & & \\
\hline Cadmium & $2.5 \mathrm{E}-05$ & $1.0 \mathrm{E}-03$ & $2.5 E-03$ & & & & \\
\hline Chromium VI & 4.1E-06 & $5.0 \mathrm{E}-03$ & $8.1 E-04$ & & & & \\
\hline Manganese & $1.3 \mathrm{E}-04$ & $1.4 \mathrm{E}-01$ & 9.3E-04 & & & & \\
\hline Nickei & $2.9 \mathrm{E}-06$ & $2.0 \mathrm{E}-02$ & 1.5E-04 & & & & \\
\hline Vansdium & 4.5E-06 & $7.0 \mathrm{E}-03$ & $6.4 \mathrm{E}-04$ & & & & \\
\hline Zinc & $4.8 \mathrm{E}-04$ & $3.0 \mathrm{E}-01$ & $1.6 \mathrm{E}-03$ & & & & \\
\hline Total Inorganics & & & & $1.2 \mathrm{E}-02$ & & & \\
\hline Bis(2-ethylhexyl)phthalate & $1.2 \mathrm{E}-07$ & $2.0 \mathrm{E}-02$ & $5.9 \mathrm{E}-06$ & & & & \\
\hline Dimethyl phthalate & 2.7E-08 & $1.0 \mathrm{E}+01$ & $2.7 \mathrm{E}-09$ & & & & \\
\hline Total Organics & & & & $5.9 E-06$ & & & \\
\hline Pathway Hazsard Index & & & & & $1.2 \mathrm{E}-02$ & & \\
\hline \multicolumn{8}{|c|}{ Exposure Pathway: Dermal Exposure of Chemicals to Maintenance Worker from Core Samples - East Gallery } \\
\hline Arsenic & $9.2 \mathrm{E}-08$ & $1.2 \mathrm{E}-04$ & $7.4 \mathrm{E}-04$ & & & & \\
\hline Barium & $6.5 \mathrm{E}-07$ & $4.9 \mathrm{E}-03$ & $1.3 \mathrm{E}-04$ & & & & \\
\hline Beryilium & $1.2 \mathrm{E}-08$ & $5.0 \mathrm{E}-05$ & $2.4 \mathrm{E}-04$ & & & & \\
\hline Cadmium & $1.6 \mathrm{E}-07$ & $1.0 \mathrm{E}-05$ & $1.6 \mathrm{E}-02$ & & & & \\
\hline Chromium VI & $2.5 \mathrm{E}-07$ & $1.0 \mathrm{E}-04$ & $2.5 \mathrm{E}-03$ & & & & \\
\hline Manganese & $8.1 \mathrm{E}-06$ & $5.6 \mathrm{E}-03$ & $1.4 E-03$ & & & & \\
\hline Nickel & $1.8 \mathrm{E}-07$ & $5.4 \mathrm{E}-03$ & $3.4 \mathrm{E}-05$ & & & & \\
\hline Vanadium & $2.8 \mathrm{E}-07$ & $7.0 \mathrm{E}-0.5$ & 4.0E-03 & & & & \\
\hline Zine & $3.0 \mathrm{E}-05$ & $6.0 \mathrm{E}-02$ & $5.0 \mathrm{E}-04$ & & & & \\
\hline Total Inorganics & & & & $2.5 \mathrm{E}-02$ & & & \\
\hline Bis(2-ethylhexyl)phthalate & $7.3 \mathrm{E}-08$ & $3.8 \mathrm{E}-03$ & $1.9 \mathrm{E}-05$ & & & & \\
\hline Dimethyl phthalate & $1.7 \mathrm{E}-08$ & $1.0 \mathrm{E}+01$ & $1.7 \mathrm{E}-05$ & & & & \\
\hline
\end{tabular}




\begin{tabular}{|c|c|c|c|c|c|c|c|}
\hline \multirow{2}{*}{ Chemcial } & \multirow{2}{*}{$\begin{array}{c}\text { Average } \\
\text { Doily Intake } \\
\text { (mg/kg-day) }\end{array}$} & \multirow{2}{*}{$\begin{array}{l}\text { Referexce Dose } \\
\text { (mg/kg-day) }\end{array}$} & \multicolumn{5}{|c|}{ Hazand Quotient/ Hazand Index } \\
\hline & & & $\begin{array}{l}\text { Contaminent } \\
\text { Specific }\end{array}$ & $\begin{array}{c}\text { Contominant } \\
\text { Closs }\end{array}$ & Pathway & $\begin{array}{l}\text { Contaminant } \\
\text { Class Total }\end{array}$ & $\begin{array}{l}\text { Total } \\
\text { Area }\end{array}$ \\
\hline Total Organics & & & & $1.9 \mathrm{E}-05$ & & & \\
\hline Pathway Hazard Index & & & & & 2.5E-02 & & \\
\hline \multicolumn{8}{|c|}{ Exposure Pathway: Inhalation of Chemicals by Maintenance Worker from Core Samples - East Gallery } \\
\hline Barium & $9.8 \mathrm{E}-10$ & $1.4 \mathrm{E}-04$ & $6.8 \mathrm{E}-06$ & & & & \\
\hline Manganere & $1.2 \mathrm{E}-08$ & $1.4 \mathrm{E}-05$ & $8.5 \mathrm{E}-04$ & & * & & \\
\hline Total Inorganics & & & & 8.5E-04 & & & \\
\hline Pathway Hazard Index. & & & & & 8.5E-04 & & \\
\hline Total Inorganic Risk & & & & & & $3.8 \mathrm{E}-02$ & \\
\hline Total Organic Risk & & & & & & $2.5 E-05$ & \\
\hline Total Area & & & & & & & 3.8E-02 \\
\hline \multicolumn{8}{|c|}{ Exposure Pathway: Ingestion of Chemicals by Maintenance Worker from Sediment in Cell } \\
\hline Antimony & $1.8 \mathrm{E}-05$ & 4.0E-04 & $4.4 \mathrm{E}-02$ & & & $\dot{0}$ & \\
\hline Arsenic & $1.1 \mathrm{E}-05$ & $3.0 \mathrm{E}-04$ & $3.5 \mathrm{E}-02$ & & & & \\
\hline Barium & $5.9 \mathrm{E}-05$ & $7.0 \mathrm{E}-02$ & 8.4E-04 & & & & \\
\hline Cadmium & 2.9E-05 & $1.0 \mathrm{E}-03$ & $2.9 E-02$ & & & & \\
\hline Chromium VI & 9.2E-05 & $5.0 \mathrm{E}-03$ & $1.8 \mathrm{E}-02$ & & & & \\
\hline Cyanide & $2.8 \mathrm{E}-07$ & $2.0 \mathrm{E}-02$ & $1.4 \mathrm{E}-05$ & & & & \\
\hline Manganese & 6.4E-04 & $1.4 \mathrm{E}-01$ & $4.6 \mathrm{E}-03$ & & & & \\
\hline Mercury & 9.1E-06 & 3.0E-04 & $3.0 \mathrm{E}-02$ & & & & \\
\hline Nickel & $8.3 E-05$ & $2.0 \mathrm{E}-02$ & $4.1 E-03$ & & & & \\
\hline Selenium & 4.3E-07 & $5.0 \mathrm{E}-03$ & $8.5 E-05$ & & & & \\
\hline Zinc & $3.6 \mathrm{E}-03$ & $3.0 \mathrm{E}-01$ & $1.2 \mathrm{E}-02$ & & & $\cdot$ & \\
\hline Total Inorganics & & & & $1.8 \mathrm{E}-01$ & & & \\
\hline Acenaphthene & 4.7E-07 & $6.0 \mathrm{E}-02$ & $7.8 \mathrm{E}-06$ & & & & \\
\hline Acetone & 4.4E- -08 & $1.0 \mathrm{E}-01$ & 4.4E-07 & & & & \\
\hline Anthracene & $9.3 E-07$ & $3.0 \mathrm{E}-01$ & $3.1 E-06$ & & & & \\
\hline Bis(2-ethyihexyl)phthalate & $1.0 \mathrm{E}-06$ & $2.0 \mathrm{E}-02$ & 5.1E-05 & & & & \\
\hline Fluoranthene & $1.3 \mathrm{E}-05$ & $4.0 \mathrm{E}-02$ & 3.3E-04 & & & & \\
\hline Fluorene & 4.2E-07 & $4.0 \mathrm{E}-02$ & $1.0 \mathrm{E}-05$ & & & & \\
\hline Pyrene & 1.1E-05 & $3.0 \mathrm{E}-02$ & $3.6 \mathrm{E}-04$ & & & & \\
\hline Total Organics & & & & $7.6 \mathrm{E}-04$ & & & \\
\hline Pathway Hazard Index & & & & & $1.8 \mathrm{E}-01$ & & \\
\hline
\end{tabular}




\begin{tabular}{|c|c|c|c|c|c|c|c|}
\hline \multirow{2}{*}{ Chemcial } & \multirow{2}{*}{$\begin{array}{c}\text { Average } \\
\text { Daity Intalce } \\
\text { (mg/kg-day) }\end{array}$} & \multirow{2}{*}{$\begin{array}{c}\text { Reference Dose } \\
\text { (mg/kg-day) }\end{array}$} & \multicolumn{5}{|c|}{ Hazard Quotient/ Hazard Index } \\
\hline & & & $\begin{array}{l}\text { Contominunt } \\
\text { Specific }\end{array}$ & $\begin{array}{c}\text { Contominont } \\
\text { Class }\end{array}$ & Pathway & $\begin{array}{l}\text { Contaminant } \\
\text { Class Total }\end{array}$ & $\begin{array}{l}\text { Totol } \\
\text { Area }\end{array}$ \\
\hline \multicolumn{8}{|c|}{ Exposure Pathway: Dermal Exposure of Chemicals to Maintenance Worker from Sediment in Cell } \\
\hline Antimony & $1.1 E-06$ & $8.0 \mathrm{E}-06$ & $1.4 \mathrm{E}-01$ & & & & \\
\hline Arsenic & $6.6 \mathrm{E}-07$ & $1.2 \mathrm{E}-04$ & $5.3 \mathrm{E}-03$ & & & & \\
\hline Barium & 3.7E-06 & 4.9E-03 & 7.5E-04 & & & & \\
\hline Cadmium & $1.8 \mathrm{E}-06$ & $1.0 \mathrm{E}-05$ & $1.8 \mathrm{E}-01$ & & & & \\
\hline Chromium VI & $5.8 \mathrm{E}-06$ & $1.0 \mathrm{E}-04$ & $5.8 \mathrm{E}-02$ & & & & \\
\hline Cyanide & $1.8 \mathrm{E}-08$ & 8.0E-03 & 2.2E-06 & & & & \\
\hline Manganese & $4.0 \mathrm{E}-05$ & $5.6 \mathrm{E}-03$ & $7.1 \mathrm{E}-03$ & & & & \\
\hline Mereury & 5.7E-07 & $3.0 \mathrm{E}-08$ & $1.9 \mathrm{E}+0 \mathrm{I}$ & & & & \\
\hline Nickel & $5.2 \mathrm{E}-06$ & $5.4 \mathrm{E}-03$ & $9.6 \mathrm{E}-04$ & & & & \\
\hline Selenium & 2.7E-08 & $2.2 \mathrm{E}-03$ & $1.2 \mathrm{E}-05$ & & & & \\
\hline Zine & 2.3E-04 & $6.0 \mathrm{E}-02$ & $3.8 \mathrm{E}-03$ & & & & \\
\hline Total Inorganics & & & & $1.9 \mathrm{E}+01$ & & & \\
\hline Acenaphthene & $2.9 \mathrm{E}-07$ & $6.0 \mathrm{E}-02$ & $4.9 \mathrm{E}-06$ & & & & \\
\hline Acetone & 2.7E-08 & 8.3E-02 & 3.3E-07 & & & & \\
\hline Anthracene & $5.8 \mathrm{E}-07$ & $2.3 E-01$ & $2.5 \mathrm{E}-06$ & & & & \\
\hline Bis(2-ethylhexyl)phthalate & $6.4 \mathrm{E}-07$ & $3.8 \mathrm{E}-03$ & 1.7E-04 & & & & \\
\hline Fluoranthene & $8.2 \mathrm{E}-06$ & $1.2 \mathrm{E}-02$ & $6.6 \mathrm{E}-0.4$ & & & & \\
\hline Fluorene & $2.6 \mathrm{E}-07$ & 4.0E-02 & $6.5 E-06$ & & & & \\
\hline Pyrene & 6.7E-06 & $9.3 \mathrm{E}-03$ & $7.2 \mathrm{E}-04$ & & & & \\
\hline Total Organics & & & & $1.6 \mathrm{E}-03$ & & & \\
\hline Pathway Hazard Index & & & & & $1.9 E+01$ & & \\
\hline \multicolumn{8}{|c|}{ Exposure Pathway: Inhalation of Chemicals by Maintenance Worker from Sediment in Cell } \\
\hline Barium & $5.5 \mathrm{E}-09$ & $1.4 \mathrm{E}-04$ & $3.8 \mathrm{E}-05$ & & & & \\
\hline Manganese & $6.0 \mathrm{E}-08$ & $1.4 \mathrm{E}-05$ & $4.2 \mathrm{E}-03$ & & & & \\
\hline Mercury & 8.5E-10 & $8.6 \mathrm{E}-05$ & $1.0 \mathrm{E}-0.5$ & & & & \\
\hline Total Inorganics & & & & $4.2 \mathrm{E}-03$ & & & \\
\hline Pathway Hazard Index & & & & & 4.2E-03 & & \\
\hline Total Inorganic Risk & & & & & & $2.0 \mathrm{E}+01$ & \\
\hline Total Organic Risk & & & & & & 2.3E-03 & \\
\hline Total Area & & & & & & & $2.0 \mathrm{E}+01$ \\
\hline
\end{tabular}




\begin{tabular}{|c|c|c|c|c|c|c|c|}
\hline \multirow{2}{*}{ Chemcial } & \multirow{2}{*}{$\begin{array}{c}\text { Average } \\
\text { Daily Intolke } \\
\text { (mg//g-day) }\end{array}$} & \multirow{2}{*}{$\begin{array}{l}\text { Reference Dose } \\
\text { (mg/kg-day) }\end{array}$} & \multicolumn{5}{|c|}{ Hazard Quotient/ Hazand Index } \\
\hline & & & $\begin{array}{l}\text { Contaminont } \\
\text { Specific }\end{array}$ & $\begin{array}{l}\text { Contominant } \\
\text { Class }\end{array}$ & Patbway & $\begin{array}{l}\text { Contaminant } \\
\text { Class Total }\end{array}$ & $\begin{array}{l}\text { Total } \\
\text { Area }\end{array}$ \\
\hline \multicolumn{8}{|c|}{ Exposure Pathway: Ingestion of Chemicals by Mointenance Worker from Core Somples - Whole Building } \\
\hline Arsenic & $1.4 \mathrm{E}-06$ & 3.0E-04 & 4.7E-03 & & & & \\
\hline Barium & 3.5E-04 & $7.0 \mathrm{E}-02$ & $5.0 \mathrm{E}-03$ & & & & \\
\hline Beryllium & 6.0E-06 & $5.0 \mathrm{E}-03$ & $1.2 E-03$ & & & & \\
\hline Cadmium & 8.8E-05 & $1.0 \mathrm{E}-03$ & 8.8E-02 & & & & \\
\hline Chromium VI & $9.6 \mathrm{E}-05$ & 5.0E-03 & $1.9 \mathrm{E}-02$ & & & & \\
\hline Manganese & 4.7E-03 & $1.4 \mathrm{E}-01$ & $3.3 E-02$ & & & & \\
\hline Mercury & $1.6 \mathrm{E}-07$ & 3.0E-04 & 5.2E-04 & & & & \\
\hline Nicke! & $6.8 \mathrm{E}-05$ & $2.0 \mathrm{E}-02$ & $3.4 \mathrm{E}-03$ & & & & \\
\hline Vanadium & $1.2 \mathrm{E}-04$ & $7.0 \mathrm{E}-03$ & 1.7E-02 & & & & \\
\hline Zinc & $1.7 \mathrm{E}-02$ & $3.0 \mathrm{E}-01$ & 5.7E-02 & & & $\cdot$ & \\
\hline Total Inorganics & & & & 2.3E-01 & & & \\
\hline Bis(2-ethylhexyl)phthalate & $1.2 \mathrm{E}-07$ & $2.0 \mathrm{E}-02$ & 5.9E-06 & & & & \\
\hline Dimethyl phthalate & $2.7 \mathrm{E}-08$ & $1.0 \mathrm{E}+01$ & 2.7E-09 & & & & \\
\hline Total Organics & & & & 5.9E-06 & & & \\
\hline Pathway Hazard Index & & & & & 2.3E-01 & & \\
\hline \multicolumn{8}{|c|}{ Exposure Pathway: Dermal Exposure of Chemicals to Maintenance Worker from Core Samples - Whole Building } \\
\hline Arsenic & 8.7E-08 & $1.2 \mathrm{E}-04$ & 7.1E-04 & & & & \\
\hline Barium & 2.2E-05 & $4.9 E-03$ & $4.5 E-03$ & & & & \\
\hline Beryllium & $3.8 \mathrm{E}-07$ & $5.0 \mathrm{E}-05$ & 7.5E-03 & & & & \\
\hline Cadmium & $5.5 \mathrm{E}-06$ & $1.0 \mathrm{E}-05$ & $5.5 \mathrm{E}-01$ & & & & \\
\hline Chromium VI & $6.0 \mathrm{E}-06$ & $1.0 \mathrm{E}-04$ & $6.0 \mathrm{E}-02$ & & & $\cdot$ & \\
\hline Manganese & $2.9 \mathrm{E}-04$ & $5.6 \mathrm{E}-03$ & $5.2 \mathrm{E}-02$ & & & & \\
\hline Mercury & 9.7E-09 & $3.0 \mathrm{E}-08$ & $3.2 \mathrm{E}-01$ & & & & \\
\hline Nickel & $4.2 \mathrm{E}-06$ & $5.4 \mathrm{E}-03$ & $7.8 \mathrm{E}-04$ & & & & \\
\hline Vanadium & $7.3 \mathrm{E}-06$ & $7.0 \mathrm{E}-05$ & $1.0 \mathrm{E}-01$ & & & & \\
\hline Zinc & $1.1 \mathrm{E}-03$ & $6.0 \mathrm{E}-02$ & $1.8 \mathrm{E}-02$ & & & & \\
\hline Total Inorganics & & & & $1.1 \mathrm{E}+00$ & & & \\
\hline Bis(2-ethylhexyl)phthalate & $7.3 \mathrm{E}-08$ & $3.8 \mathrm{E}-03$ & $1.9 E-05$ & & & & \\
\hline Dimethyl phthalate & $1.7 \mathrm{E}-08$ & $1.0 E+01$ & $1.7 \mathrm{E}-09$ & & & & \\
\hline Total Organics & & & & $1.9 \mathrm{E}-05$ & & & \\
\hline Pathway Hazard Index & & & & & $1.1 E+00$ & & \\
\hline
\end{tabular}




\begin{tabular}{|c|c|c|c|c|c|c|c|}
\hline \multirow{2}{*}{ Chemcial } & \multirow{2}{*}{$\begin{array}{c}\text { Average } \\
\text { Daily Intake } \\
\text { (mp/lo-day) }\end{array}$} & \multirow{2}{*}{$\begin{array}{l}\text { Reference Dose } \\
\text { (mg/kg-day) }\end{array}$} & \multicolumn{5}{|c|}{ Hazard Quotient/ Hazand Index } \\
\hline & & & $\begin{array}{l}\text { Contaminunt } \\
\text { Specific }\end{array}$ & $\begin{array}{c}\text { Contaminant } \\
\text { Class }\end{array}$ & Pathway & $\begin{array}{l}\text { Contominant } \\
\text { Class Total }\end{array}$ & $\begin{array}{l}\text { Total } \\
\text { Area }\end{array}$ \\
\hline \multicolumn{8}{|c|}{ Exposure Pathway: Inhalation of Chemicals by Maintenance Worker from Core Samples - Whole Building } \\
\hline Barium & $3.3 \mathrm{E}-08$ & $1.4 \mathrm{E}-04$ & 2.3E-04 & & & & \\
\hline Manganese & 4.4E-07 & $1.4 \mathrm{E}-05$ & $3.0 \mathrm{E}-02$ & & & & \\
\hline Mercury & $1.5 \mathrm{E}-11$ & 8.6E-05 & 1.7E-07 & & & & \\
\hline Total Inorganics & & & & $3.1 \mathrm{E}-02$ & & & \\
\hline Pathway Hazard Index & & & & & 3.1E-02 & & \\
\hline Total Inorganic Risk & & & & & & $1.4 \mathrm{E}+\infty$ & \\
\hline Total Organic Risk & & & & & & 2.5E-05 & \\
\hline Total Area & & & & & & & $1.4 \mathrm{E}+00$ \\
\hline
\end{tabular}


Table E-4. Hrourd Results - Noncarcinogenic Effects (S\&M Worker)

\begin{tabular}{|c|c|c|c|c|c|c|c|}
\hline \multirow{2}{*}{ Chemcial } & \multirow{2}{*}{$\begin{array}{c}\text { Average Daily } \\
\text { Intake } \\
\text { (mg/kg-day) }\end{array}$} & \multirow{2}{*}{$\begin{array}{c}\text { Reference } \\
\text { Dase } \\
\text { (mg/kg-day) }\end{array}$} & \multicolumn{5}{|c|}{ Hazand Quotient/ Hazard Index } \\
\hline & & & $\begin{array}{c}\text { Contominant } \\
\text { Specific }\end{array}$ & $\begin{array}{c}\text { Contominant } \\
\text { Class }\end{array}$ & Pathwray & $\begin{array}{c}\text { Contuminont } \\
\text { Clavs Total }\end{array}$ & $\begin{array}{l}\text { Total } \\
\text { Area }\end{array}$ \\
\hline \multicolumn{8}{|c|}{ Exposure Pathway: Ingestion of Chemicals by S\&M Worker from Core Samples - Cell } \\
\hline Arsenic & $1.0 \mathrm{E}-08$ & $3.0 \mathrm{E}-04$ & 3.4E-05 & & & & \\
\hline Barium & 2.8E-06 & $7.0 \mathrm{E}-02$ & $4.0 \mathrm{E}-05$ & & & & \\
\hline Beryllium & $6.9 \mathrm{E}-08$ & 5.0E-03 & 1.4E-05 & & & & \\
\hline Cadmium & 7.0E-07 & $1.0 \mathrm{E}-03$ & 7.0E-04 & & & & \\
\hline Chromium VI & 7.7E-07 & 5.0E-03 & $1.5 \mathrm{E}-04$ & & & & \\
\hline Manganese & 3.7E-05 & $1.4 \mathrm{E}-01$ & 2.7E-04 & & & & \\
\hline Mercury & $1.3 E-09$ & $3.0 \mathrm{E}-04$ & 4.4E-06 & & & & \\
\hline Nickel & $5.4 \mathrm{E}-07$ & $2.0 \mathrm{E}-02$ & 2.7E-05 & & & & \\
\hline Vanadium & $9.4 \mathrm{E}-07$ & $7.0 \mathrm{E}-03$ & $1.3 \mathrm{E}-04$ & & & & \\
\hline Zinc & $1.4 \mathrm{E}-04$ & $3.0 \mathrm{E}-01$ & $4.6 \mathrm{E}-04$ & & & & \\
\hline Total Inorganics & & & & $1.8 \mathrm{E}-03$ & & & \\
\hline Pathway Hazard Index & & & & & $1.8 \mathrm{E}-03$ & & \\
\hline \multicolumn{8}{|c|}{ Exposure Pathway: Dermal Exposure of Chemicals to S\&M Worker from Core Semples - Cell } \\
\hline Arsenic & $6.3 \mathrm{E}-10$ & $1.2 \mathrm{E}-04$ & $5.2 \mathrm{E}-06$ & & & & \\
\hline Barium & $1.8 \mathrm{E}-07$ & $4.9 \mathrm{E}-03$ & $3.6 \mathrm{E}-05$ & & & & \\
\hline Beryllium & 4.3E-09 & $5.0 \mathrm{E}-05$ & 8.6E-05 & & & & \\
\hline Cadmium & 4.4E-08 & $1.0 \mathrm{E}-05$ & 4.4E-03 & & & & \\
\hline Chromium VI & $4.8 \mathrm{E}-08$ & $1.0 \mathrm{E}-04$ & 4.8E-04 & & & & \\
\hline Manganese & 2.3E-06 & $5.6 \mathrm{E}-03$ & 4.2E-04 & & & - & \\
\hline Mercury & 8.3E-11 & $3.0 \mathrm{E}-08$ & 2.8E-03 & & & & \\
\hline Nickel & $3.4 \mathrm{E}-08$ & $5.4 \mathrm{E}-03$ & $6.2 \mathrm{E}-06$ & & & & \\
\hline Vanadium & $5.9 \mathrm{E}-08$ & $7.0 \mathrm{E}-05$ & 8.4E-04 & & & & \\
\hline Zinc & $8.5 E-06$ & $6.0 \mathrm{E}-02$ & $1.4 \mathrm{E}-04$ & & & & \\
\hline Total Inorganics & & & & $9.2 E-03$ & & & \\
\hline Pathway Hazard Index & & & & & $9.2 \mathrm{E}-03$ & & \\
\hline \multicolumn{8}{|c|}{ Exposure Pathway: Ishalation of Chemicals by S\&M Worker from Core Samples - Cell } \\
\hline Barium & $2.6 E-10$ & $1.4 \mathrm{E}-04$ & $1.8 \mathrm{E}-06$ & & & & \\
\hline Manganese & $3.5 \mathrm{E}-09$ & $1.4 \mathrm{E}-05$ & - 2.4E-04 & & & & \\
\hline Mercury & $1.2 \mathrm{E}-13$ & 8.6E-05 & 1.4E-09 & & & & \\
\hline
\end{tabular}




\begin{tabular}{|c|c|c|c|c|c|c|c|}
\hline \multirow{2}{*}{ Chemcial } & \multirow{2}{*}{$\begin{array}{l}\text { Average Doily } \\
\text { Intalce } \\
\text { (mg/kg-day) }\end{array}$} & \multirow{2}{*}{$\begin{array}{c}\text { Reference } \\
\text { Dose } \\
\text { (mg/kg-day) }\end{array}$} & \multicolumn{5}{|c|}{ Hazard Quotient/ Hazard Index } \\
\hline & & & $\begin{array}{c}\text { Contominast } \\
\text { Specific }\end{array}$ & $\begin{array}{c}\text { Contaminant } \\
\text { Class } \\
\end{array}$ & Pathway & $\begin{array}{c}\text { Contaminant } \\
\text { Class Total }\end{array}$ & $\begin{array}{l}\text { Total } \\
\text { Area }\end{array}$ \\
\hline Total Inorganics & & & & 2.5E-04 & & & \\
\hline Pathway Hazard Index & & & & & 2.5E-04 & & \\
\hline Total Inorganic Risk & & & & & & $1.1 \mathrm{E}-02$ & \\
\hline Total Area & & & & & & & 1.1E-02 \\
\hline \multicolumn{8}{|c|}{ Exposure Pathway: Ingestion of Chemicals by S\&M Worker from Core Samples - East Gallery } \\
\hline Arsenic & $1.2 \mathrm{E}-08$ & 3.0E-04 & 3.9E-05 & & & & \\
\hline Barium & $8.4 \mathrm{E}-08$ & 7.0E-02 & $1.2 \mathrm{E}-06$ & . & & & \\
\hline Beryllium & $1.6 \mathrm{E}-09$ & $5.0 \mathrm{E}-03$ & $3.1 \mathrm{E}-07$ & & & & \\
\hline Cadmium & $2.0 \mathrm{E}-08$ & $1.0 \mathrm{E}-03$ & $2.0 \mathrm{E}-05$ & & & & \\
\hline Chromium VI & $3.2 \mathrm{E}-08$ & $5.0 \mathrm{E}-03$ & $6.5 \mathrm{E}-06$ & & & & \\
\hline Manganese & $1.0 \mathrm{E}-06$ & $1.4 \mathrm{E}-01$ & $7.4 \mathrm{E}-06$ & & & & \\
\hline Nickel & 2.3E-08 & $2.0 \mathrm{E}-02$ & $1.2 \mathrm{E}-06$ & & & & \\
\hline Vanadium & $3.6 \mathrm{E}-08$ & $7.0 \mathrm{E}-03$ & $5.1 \mathrm{E}-06$ & & & & \\
\hline Zinc & $3.8 \mathrm{E}-06$ & $3.0 \mathrm{E}-01$ & $1.3 \mathrm{E}-05$ & & & & \\
\hline Total Inorganics & & & & $9.4 \mathrm{E}-05$ & & & \\
\hline Bis(2-ethylhexyi)phthalate & \multicolumn{2}{|c|}{$9.4 \mathrm{E}-10$} & 2.0E-02 & 4.7E-08 & & & \\
\hline Dimethyl phthalate & $2.2 \mathrm{E}-10$ & $1.0 \mathrm{E}+01$ & 2.2E-11 & & & & \\
\hline Total Organics & & & & 4.7E-08 & & & \\
\hline Pathway Hazad Index & & & & & $9.4 \mathrm{E}-05$ & & \\
\hline \multicolumn{8}{|c|}{ Exposure Pathway: Dermal Exposure of Chemicals to S\&M Worker from Core Samples - East Gallery } \\
\hline Arsenic & $7.3 \mathrm{E}-10$ & $1.2 \mathrm{E}-0.4$ & $6.0 \mathrm{E}-06$ & & & & \\
\hline Barium & $5.2 \mathrm{E}-09$ & $4.9 \mathrm{E}-03$ & $1.1 \mathrm{E}-06$ & & & & \\
\hline Beryilium & $9.8 \mathrm{E}-11$ & $5.0 \mathrm{E}-05$ & $2.0 \mathrm{E}-06$ & & & & \\
\hline Cadmium & $1.2 \mathrm{E}-09$ & $1.0 \mathrm{E}-05$ & $1.2 \mathrm{E}-04$ & & & & \\
\hline Chromium VI & $2.0 \mathrm{E}-09$ & $1.0 \mathrm{E}-04$ & $2.0 \mathrm{E}-05$ & & & & \\
\hline Manganese & $6.5 \mathrm{E}-08$ & $5.6 \mathrm{E}-03$ & $1.2 \mathrm{E}-05$ & & & & \\
\hline Nickel & $1.5 E-09$ & $5.4 \mathrm{E}-03$ & 2.7E-07 & & & & \\
\hline Vanadium & $2.2 \mathrm{E}-09$ & $7.0 \mathrm{E}-05$ & $3.2 \mathrm{E}-05$ & & & & \\
\hline Zine & 2.4E-07 & $6.0 \mathrm{E}-02$ & $4.0 \mathrm{E}-06$ & & & & \\
\hline Total Inorganics & & & & $2.0 \mathrm{E}-04$ & & & \\
\hline Bis(2-ethylhexyl)phthalate & \multicolumn{2}{|c|}{$5.9 \mathrm{E}-10$} & $3.8 \mathrm{E}-03$ & $1.5 \mathrm{E}-07$ & & & \\
\hline Dimethyl phthalate & $1.3 \mathrm{E}-10$ & $1.0 \mathrm{E}+01$ & 1.3E-11 & & & & \\
\hline
\end{tabular}




\begin{tabular}{|c|c|c|c|c|c|c|c|}
\hline \multirow{2}{*}{ Chemcial } & \multirow{2}{*}{$\begin{array}{c}\text { Average Daily } \\
\text { Intake } \\
\text { (mg/kg-day) }\end{array}$} & \multirow{2}{*}{$\begin{array}{c}\text { Reference } \\
\text { Dose } \\
\text { (mg/kg-day) }\end{array}$} & \multicolumn{5}{|c|}{ Hazand Quotieat/ Hazard Index } \\
\hline & & & $\begin{array}{l}\text { Contaminant } \\
\text { Specific }\end{array}$ & $\begin{array}{l}\text { Contaminant } \\
\text { Class }\end{array}$ & Pathway & $\begin{array}{l}\text { Contaminant } \\
\text { Class Total }\end{array}$ & $\begin{array}{l}\text { Total } \\
\text { Area }\end{array}$ \\
\hline Total Organics & & & & $1.5 \mathrm{E}-07$ & & & \\
\hline Pathway Hazard Index & & & & & 2.0E-04 & & \\
\hline \multicolumn{8}{|c|}{ Exposure Pathway: Inhalation of Chemicals by S\&M Worker from Core Samples - East Gallery } \\
\hline Barium & $7.8 \mathrm{E}-12$ & $1.4 \mathrm{E}-04$ & $5.5 E-08$ & & & & \\
\hline Manganese & 9.7E-11 & $1.4 \mathrm{E}-05$ & $6.8 \mathrm{E}-06$ & & & & \\
\hline Total Inorganics & & & & $6.8 \mathrm{E}-06$ & & & \\
\hline Pathway Hazard Index & & & & & $6.8 \mathrm{E}-06$ & & \\
\hline Total Inorganic Risk & & & & & & $3.0 \mathrm{E}-04$ & \\
\hline Total Organic Risk & & & & & & $2.0 \mathrm{E}-07$ & \\
\hline Total Area & & & & & & & $3.0 \mathrm{E}-04$ \\
\hline \multicolumn{8}{|c|}{ Exposure Pathway: Ingestion of Chemicals by S\&M Worker from Sediment in Cell } \\
\hline Antimony & $1.4 \mathrm{E}-07$ & 4.0E-04 & $3.5 \mathrm{E}-04$ & & & & \\
\hline Arsenic & 8.4E-08 & $3.0 \mathrm{E}-04$ & $2.8 \mathrm{E}-04$ & & & & \\
\hline Barium & 4.7E-07 & $7.0 \mathrm{E}-02$ & $6.7 \mathrm{E}-06$ & & & & \\
\hline Cadmium & $2.4 \mathrm{E}-07$ & $1.0 \mathrm{E}-03$ & $2.4 \mathrm{E}-04$ & & & & \\
\hline Chromium VI & 7.4E-07 & $5.0 \mathrm{E}-03$ & $1.5 \mathrm{E}-04$ & & & & \\
\hline Cyanide & $2.3 \mathrm{E}-09$ & $2.0 \mathrm{E}-02$ & $1.1 \mathrm{E}-07$ & & & & \\
\hline Manganese & $5.1 E-06$ & $1.4 \mathrm{E}-01$ & $3.7 \mathrm{E}-05$ & & & & \\
\hline Mercury & $7.3 \mathrm{E}-08$ & 3.0E-04 & $2.4 \mathrm{E}-04$ & & & & \\
\hline Nickel & $6.6 \mathrm{E}-07$ & $2.0 \mathrm{E}-02$ & 3.3E-05 & & & & \\
\hline Selenium & $3.4 \mathrm{E}-09$ & $5.0 \mathrm{E}-03$ & $6.8 \mathrm{E}-07$ & & & & \\
\hline Zine & $2.9 \mathrm{E}-05$ & $3.0 \mathrm{E}-01$ & $9.7 \mathrm{E}-05$ & & & & \\
\hline Total Inorganics & & & & $1.4 \mathrm{E}-03$ & & & \\
\hline Acensphthene & $3.8 \mathrm{E}-09$ & $6.0 \mathrm{E}-02$ & $6.3 E-08$ & & & & \\
\hline Acetone & $3.5 \mathrm{E}-10$ & $1.0 \mathrm{E}-01$ & $3.5 E-09$ & & & & \\
\hline Anthracene & $7.4 \mathrm{E}-09$ & $3.0 \mathrm{E}-01$ & $2.5 \mathrm{E}-08$ & & & & \\
\hline Bis(2-ethylhexyl)phthalate & \multicolumn{2}{|c|}{$8.2 E-09$} & $2.0 \mathrm{E}-02$ & $4.1 \mathrm{E}-07$ & & & \\
\hline Fluoranthene & $1.1 \mathrm{E}-07$ & $4.0 \mathrm{E}-02$ & $2.6 \mathrm{E}-06$ & & & & \\
\hline Fluorene & $3.3 \mathrm{E}-09$ & $4.0 \mathrm{E}-02$ & $8.3 E-08$ & & & & \\
\hline Pyrene & $8.6 E-08$ & $3.0 \mathrm{E}-02$ & $2.9 \mathrm{E}-06$ & & & & \\
\hline Total Organics & & & & $6.1 E-06$ & & & \\
\hline Pathway Hazard Index & & & & & $1.4 \mathrm{E}-03$ & & \\
\hline
\end{tabular}




\begin{tabular}{|c|c|c|c|c|c|c|c|}
\hline \multirow{2}{*}{ Chemcial } & \multirow{2}{*}{$\begin{array}{c}\text { Average Daily } \\
\text { Intalke } \\
\text { (mg/kg-day) }\end{array}$} & \multirow{2}{*}{$\begin{array}{c}\text { Reference } \\
\text { Dose } \\
\text { (mg/lo-day) }\end{array}$} & \multicolumn{5}{|c|}{ Hazard Quotient/ Hazard Index } \\
\hline & & & $\begin{array}{c}\text { Contominart } \\
\text { Specific }\end{array}$ & $\begin{array}{c}\text { Contaminant } \\
\text { Class }\end{array}$ & Pathway & $\begin{array}{c}\text { Contaninent } \\
\text { Class Total }\end{array}$ & $\begin{array}{l}\text { Total } \\
\text { Area }\end{array}$ \\
\hline \multicolumn{8}{|c|}{ Exposure Pathway: Dermal Exposure of Chemicals to S\&M Worker from Sediment in Cell } \\
\hline Antimony & 8.8E-09 & 8.0E-06 & $1.1 \mathrm{E}-03$ & & & & \\
\hline Arsenic & 5.3E-09 & $1.2 \mathrm{E}-04$ & 4.3E-05 & & & & \\
\hline Barium & $2.9 \mathrm{E}-08$ & 4.9E-03 & $6.0 \mathrm{E}-06$ & & & & \\
\hline Cadmium & $1.5 \mathrm{E}-08$ & $1.0 \mathrm{E}-05$ & $1.5 \mathrm{E}-03$ & & & & \\
\hline Chromium VI & 4.6E-08 & $1.0 \mathrm{E}-04$ & 4.6E-04 & & & & \\
\hline Cyanide & $1.4 \mathrm{E}-10$ & 8.0E-03 & $1.8 \mathrm{E}-08$ & & & & \\
\hline Manganese & 3.2E-07 & $5.6 \mathrm{E}-03$ & 5.7E-05 & & & & \\
\hline Mercury & $4.6 \mathrm{E}-09$ & $3.0 \mathrm{E}-08$ & $1.5 \mathrm{E}-01$ & & & & \\
\hline Nickel & 4.1E-08 & $5.4 \mathrm{E}-03$ & $7.6 \mathrm{E}-06$ & & & & \\
\hline Selenium & 2.1E-10 & $2.2 \mathrm{E}-03$ & $9.7 \mathrm{E}-08$ & & & & \\
\hline Zine & $1.8 \mathrm{E}-06$ & $6.0 \mathrm{E}-02$ & 3.0E-05 & & & & \\
\hline Total Inorganics & & & & $1.6 \mathrm{E}-01$ & & & \\
\hline Acenaphthene & $2.3 \mathrm{E}-09$ & $6.0 \mathrm{E}-02$ & $3.9 E-08$ & & & & \\
\hline Acetone & 2.2E-10 & $8.3 \mathrm{E}-02$ & $2.6 \mathrm{E}-09$ & & & & \\
\hline Anthracene & 4.6E-09 & 2.3E-01 & $2.0 \mathrm{E}-08$ & & & & \\
\hline Bis(2-ethylhexyl)phthalate & \multicolumn{2}{|c|}{ 5.1E- 09} & $3.8 \mathrm{E}-03$ & $1.3 \mathrm{E}-06$ & & & \\
\hline Fluoranthene & $6.6 \mathrm{E}-08$ & $1.2 \mathrm{E}-02$ & $5.3 \mathrm{E}-06$ & & & & \\
\hline Fluorene & 2.1E-09 & $4.0 \mathrm{E}-02$ & $5.2 \mathrm{E}-08$ & & & & \\
\hline Pyrene & $5.4 \mathrm{E}-08$ & $9.3 \mathrm{E}-03$ & $5.8 \mathrm{E}-06$ & & & & \\
\hline Total Organics & & & & $1.3 E-05$ & & & \\
\hline Pathway Hazard Index & & & & & $1.6 \mathrm{E}-01$ & & \\
\hline \multicolumn{8}{|c|}{ Exposure Pathway: Inhalation of Chemicals by S\&M Worker from Sediment in Cell } \\
\hline Barium & $4.4 E-11$ & $1.4 \mathrm{E}-04$ & 3.1E-07 & & & & \\
\hline Manganese & 4.8E-10 & $1.4 \mathrm{E}-05$ & 3.4E-05 & & & & \\
\hline Mercury & $6.8 \mathrm{E}-12$ & $8.6 \mathrm{E}-05$ & $8.0 \mathrm{E}-08$ & & & & \\
\hline Total Inorganics & & & & 3.4E-05 & & & \\
\hline Pathway Hazard Index & & & & & 3.4E-05 & & \\
\hline Total Inorganic Risk & & & & & & $1.6 \mathrm{E}-01$ & \\
\hline Total Organic Risk & & & & & & $1.9 \mathrm{E}-05$ & \\
\hline Total Area & & & & & & & $1.6 \mathrm{E}-01$ \\
\hline
\end{tabular}




\begin{tabular}{|c|c|c|c|c|c|c|c|}
\hline \multirow{2}{*}{ Chemcial } & \multirow{2}{*}{$\begin{array}{l}\text { Arerage Daily } \\
\text { Intake } \\
\text { (mg/kg-day) }\end{array}$} & \multirow{2}{*}{$\begin{array}{c}\text { Reference } \\
\text { Dose } \\
\text { (mg/k8-day) }\end{array}$} & \multicolumn{5}{|c|}{ Hazard Quotient/ Hazand Index } \\
\hline & & & $\begin{array}{c}\text { Contaminant } \\
\text { Specific }\end{array}$ & $\begin{array}{c}\text { Contaminant } \\
\text { Class }\end{array}$ & Pathway & $\begin{array}{c}\text { Contaminant } \\
\text { Class Total }\end{array}$ & $\begin{array}{l}\text { Total } \\
\text { Area }\end{array}$ \\
\hline \multicolumn{8}{|c|}{ Exposure Pathway: Ingestion of Chemicals by S\&M Worker from Core Samples - Whole Building } \\
\hline Arsenic & $1.1 E-08$ & $3.0 \mathrm{E}-04$ & 3.7E-05 & & & & \\
\hline Barium & $2.8 \mathrm{E}-06$ & $7.0 \mathrm{E}-02$ & $4.0 \mathrm{E}-05$ & & & & \\
\hline Beryllium & $4.8 \mathrm{E}-08$ & $5.0 \mathrm{E}-03$ & $9.7 \mathrm{E}-06$ & & & & \\
\hline Cadmium & $7.0 \mathrm{E}-07$ & $1.0 \mathrm{E}-03$ & $7.0 \mathrm{E}-04$ & & & & \\
\hline Chromium VI & 7.7E-07 & $5.0 \mathrm{E}-03$ & $1.5 \mathrm{E}-04$ & & & & \\
\hline Manganese & 3.7E-05 & $1.4 \mathrm{E}-01$ & 2.7E-04 & & & & \\
\hline Mereury & $1.2 \mathrm{E}-09$ & $3.0 \mathrm{E}-04$ & 4.1E-06 & & & & \\
\hline Nickel & $5.4 \mathrm{E}-07$ & $2.0 \mathrm{E}-02$ & $2.7 \mathrm{E}-05$ & & & & \\
\hline Vanadium & $9.4 \mathrm{E}-07$ & $7.0 \mathrm{E}-03$ & $1.3 \mathrm{E}-04$ & & & & \\
\hline Zinc & $1.4 \mathrm{E}-04$ & $3.0 \mathrm{E}-01$ & $4.6 \mathrm{E}-04$ & & & & \\
\hline Total Inorganics & & & & $1.8 \mathrm{E}-03$ & & & \\
\hline Bis(2-ethylhexyl)phthalate & $9.4 \mathrm{E}-10$ & $2.0 \mathrm{E}-02$ & 4.7E-08 & & & & \\
\hline Dimethyl phthaiate & $2.2 \mathrm{E}-10$ & $1.0 \mathrm{E}+01$ & 2.2E-11 & & & & \\
\hline Total Organics & & & & 4.7E-08 & & & \\
\hline Pathway Hazard Index & & & & & $1.8 \mathrm{E}-03$ & & \\
\hline \multicolumn{8}{|c|}{ Exposure Pathway: Dermal Exposure of Chemicals to S\&M Worker from Core Samples - Whole Buiking } \\
\hline Arsenic & 7.0E-10 & $1.2 \mathrm{E}-04$ & $5.7 \mathrm{E}-06$ & & & & \\
\hline Barium & $1.8 \mathrm{E}-07$ & $4.9 \mathrm{E}-03$ & $3.6 \mathrm{E}-05$ & & & & \\
\hline Beryllium & $3.0 \mathrm{E}-09$ & $5.0 \mathrm{E}-05$ & $6.0 \mathrm{E}-05$ & & & & \\
\hline Cadmium & 4.4E-08 & $1.0 \mathrm{E}-05$ & 4.4E-03 & & & & \\
\hline Chromium VI & $4.8 E-08$ & $1.0 \mathrm{E}-04$ & $4.8 \mathrm{E}-04$ & & & $\cdot$ & \\
\hline Manganese & $2.3 \mathrm{E}-06$ & $5.6 \mathrm{E}-03$ & 4.2E-04 & & & & \\
\hline Mercury & $7.8 \mathrm{E}-11$ & $3.0 \mathrm{E}-08$ & $2.6 \mathrm{E}-03$ & & & & \\
\hline Nickel & $3.4 \mathrm{E}-08$ & $5.4 \mathrm{E}-03$ & $6.2 \mathrm{E}-06$ & & & & \\
\hline Vanadium & $5.9 \mathrm{E}-08$ & 7.0E-05 & $8.4 \mathrm{E}-04$ & & & & \\
\hline Zinc & 8.5E-06 & $6.0 \mathrm{E}-02$ & $1.4 \mathrm{E}-04$ & & & & \\
\hline Total Inorganics & & & & $9.0 \mathrm{E}-03$ & & & \\
\hline Bis(2-ethylhexyl)phthalate & $5.9 \mathrm{E}-10$ & $3.8 \mathrm{E}-03$ & $1.5 \mathrm{E}-07$ & & & & \\
\hline Dimethyl phthalate & $1.3 \mathrm{E}-10$ & $1.0 \mathrm{E}+01$ & 1.3E-11 & & & & \\
\hline Total Organics & & & & $1.5 \mathrm{E}-07$ & & & \\
\hline Pathway Hazard Idnex & & & & & $9.0 \mathrm{E}-03$ & & \\
\hline
\end{tabular}




\begin{tabular}{|c|c|c|c|c|c|c|c|}
\hline \multirow{2}{*}{ Chemcial } & \multirow{2}{*}{$\begin{array}{c}\text { Average Daily } \\
\text { Intake } \\
\text { (mg//g-day) }\end{array}$} & \multirow{2}{*}{$\begin{array}{c}\text { Reference } \\
\text { Dase } \\
\text { (mp/kg-day) }\end{array}$} & \multicolumn{5}{|c|}{ Hazard Quotient/ Hazand Index } \\
\hline & & & $\begin{array}{l}\text { Contaminant } \\
\text { Specific }\end{array}$ & $\begin{array}{c}\text { Contaminant } \\
\text { Class }\end{array}$ & Patbway & $\begin{array}{c}\text { Contaminant } \\
\text { Class Total }\end{array}$ & $\begin{array}{l}\text { Total } \\
\text { Area }\end{array}$ \\
\hline \multicolumn{8}{|c|}{ Exposure Pathway: Inhalation of Cbemicals by S\&M Worker from Core Somples - Whole Building } \\
\hline Barium & $2.6 \mathrm{E}-10$ & $1.4 \mathrm{E}-04$ & $1.8 \mathrm{E}-06$ & & & & \\
\hline Manganese & 3.5E-09 & $1.4 \mathrm{E}-05$ & 2.4E-04 & & & & \\
\hline Mercury & $1.2 \mathrm{E}-13$ & $8.6 \mathrm{E}-05$ & $1.4 \mathrm{E}-09$ & & & & \\
\hline Total Inorganics & & & & $2.5 \mathrm{E}-04$ & & & \\
\hline Pathway Hazard Index & & & & & $2.5 E=-4$ & & \\
\hline Total Inorganic Risk & & & & & & 1.1E-02 & \\
\hline Total Organic Risk & & & & & & 2.0E-07 & \\
\hline Total Area & & & & & & & $1.1 \mathrm{E}-02$ \\
\hline
\end{tabular}


Table E-5. Risk Characterization Results - Excess Cancer Risk Estimates - Adult Trespasser

\begin{tabular}{|c|c|c|c|c|c|c|c|}
\hline Contaminant & $\begin{array}{l}\text { Chronic } \\
\text { Daily } \\
\text { Intake }\end{array}$ & $\begin{array}{l}\text { Slope } \\
\text { Factor }\end{array}$ & $\begin{array}{l}\text { Contaminant } \\
\text { Specific } \\
\text { Risk }\end{array}$ & $\begin{array}{c}\text { Contaminant } \\
\text { Class Risk }\end{array}$ & $\begin{array}{c}\begin{array}{c}\text { Total } \\
\text { Pathway }\end{array} \\
\text { Risk }\end{array}$ & $\begin{array}{c}\text { Total } \\
\text { Contaminant } \\
\text { Risk }\end{array}$ & $\begin{array}{l}\text { Total } \\
\text { Area } \\
\text { Risk }\end{array}$ \\
\hline \multicolumn{8}{|c|}{ Exposure Pathway: Ingestion of Contominants by Adult Trespesser from Core Samples - Cell } \\
\hline Beryllium & $1.0 \mathrm{E}-05$ & $4.3 E+\infty$ & 4.4E-05 & & & & \\
\hline Total Inorganics & & & & $4.4 \mathrm{E}-05$ & & & \\
\hline Aroclor-1254 & 2.0E-07 & $7.7 E+\infty$ & $1.5 \mathrm{E}-06$ & & & & \\
\hline Aroclor-1260 & $3.3 \mathrm{E}-07$ & $7.7 \mathrm{E}+00$ & $2.6 \mathrm{E}-06$ & & & & \\
\hline Total Organics & & & & 4.1E-06 & & & \\
\hline${ }^{17} \mathrm{Cs}$ & $1.4 \mathrm{E}+06$ & $2.8 \mathrm{E}-11$ & $4.0 \mathrm{E}-05$ & & & & \\
\hline${ }^{208} \mathrm{Re}$ & $4.2 \mathrm{E}+02$ & $1.2 \mathrm{E}-10$ & $5.1 \mathrm{E}-08$ & & & & \\
\hline${ }^{2 x} \mathrm{Th}$ & $8.8 \mathrm{E}+02$ & $5.5 \mathrm{E}-11$ & $4.9 \mathrm{E}-08$ & & & & \\
\hline${ }^{200} \mathrm{Th}$ & $1.5 \mathrm{E}+03$ & 1.3E-11 & $1.9 \mathrm{E}-08$ & & & & \\
\hline${ }^{20} \mathrm{Th}$ & $9.1 \mathrm{E}+02$ & $1.2 \mathrm{E}-11$ & $1.1 \mathrm{E}-08$ & & & & \\
\hline${ }^{233234} U$ & $1.4 \mathrm{E}+03$ & $1.6 \mathrm{E}-11$ & $2.2 \mathrm{E}-08$ & & & & \\
\hline${ }^{224} \mathrm{Th}$ & $6.6 \mathrm{E}+03$ & 4.0E-12 & 2.7E-08 & & & & \\
\hline${ }^{22 s} U$ & $1.3 E+02$ & $1.6 \mathrm{E}-11$ & $2.0 \mathrm{E}-09$ & & & & \\
\hline${ }^{23 \mathrm{Pu}}$ & 4.7E+02 & $2.2 \mathrm{E}-10$ & $1.0 \mathrm{E}-07$ & & & & \\
\hline${ }^{280} \mathrm{U}$ & $1.8 E+03$ & $2.0 \mathrm{E}-11$ & $3.6 \mathrm{E}-08$ & & & & \\
\hline${ }^{259820} \mathrm{Pu}$ & $4.2 E+03$ & 2.3E-10 & 9.7E-07 & & & & \\
\hline${ }^{\infty} \mathrm{K}$ & $5.5 E+03$ & $1.1 \mathrm{E}-11$ & $6.1 \mathrm{E}-08$ & & & & \\
\hline${ }^{\infty} \mathrm{Co}$ & $1.4 \mathrm{E}+02$ & $1.5 \mathrm{E}-11$ & 2.0E-09 & & & & \\
\hline${ }^{\infty} \mathrm{Sr}$ & $1.2 E+04$ & $3.6 \mathrm{E}-11$ & 4.4E-07 & & & & \\
\hline Tritium & $2.2 \mathrm{E}+04$ & $5.4 \mathrm{E}-14$ & $1.2 \mathrm{E}-09$ & & & & \\
\hline Total Radionuclides & & & & $4.2 \mathrm{E}-05$ & & & \\
\hline Total Pathway Risk & & & & & $9.0 \mathrm{E}-05$ & & \\
\hline \multicolumn{8}{|c|}{ Exposure Pathway: Dermal Exposure to Contominants by Adult Trespasser from Core Samples- Cell } \\
\hline Beryllium & 5.5E-07 & $4.3 \mathrm{E}+02$ & 2.4E-04 & & & & \\
\hline Total Inorganics & & & & $2.4 \mathrm{E}-04$ & & & \\
\hline Aroclor-1254 & $1.1 \mathrm{E}-07$ & $8.6 \mathrm{E}+\infty$ & 9.1E-07 & & & & \\
\hline Aroclor-1260 & $1.8 \mathrm{E}-07$ & $8.6 \mathrm{E}+\infty$ & $1.5 \mathrm{E}-06$ & & & & \\
\hline Total Organics & & & & $2.4 \mathrm{E}-06$ & & & \\
\hline Total Pathway Risk & & & & & 2.4E-04 & & \\
\hline
\end{tabular}




\begin{tabular}{|c|c|c|c|c|c|c|c|}
\hline Contaminant & $\begin{array}{l}\text { Chronic } \\
\text { Doily } \\
\text { Intoke }\end{array}$ & $\begin{array}{l}\text { Stope } \\
\text { Factor }\end{array}$ & $\begin{array}{l}\text { Contominnnt } \\
\text { Specific } \\
\text { Risl }\end{array}$ & $\begin{array}{l}\text { Contaminant } \\
\text { Class Rislc }\end{array}$ & $\begin{array}{c}\text { Total } \\
\text { Pathway } \\
\text { Risl }\end{array}$ & $\begin{array}{c}\text { Total } \\
\text { Contaminant } \\
\text { Risl }\end{array}$ & $\begin{array}{l}\text { Total } \\
\text { Area } \\
\text { Risk }\end{array}$ \\
\hline \multicolumn{8}{|c|}{ Exposure Pathway: Inhalation of Contaminants by Adult Trespasser from Core Samples - Cell } \\
\hline Arsenic & 7.1E-11 & $5.0 \mathrm{E}+01$ & $3.6 \mathrm{E}-09$ & & & & \\
\hline Beryllium & $4.8 \mathrm{E}-10$ & $8.4 \mathrm{E}+\infty$ & 4.1E-09 & & & & \\
\hline Cadmium & $4.9 \mathrm{E}-09$ & $6.1 \mathrm{E}+00$ & $3.0 \mathrm{E}-08$ & & & & \\
\hline Chromium VI & $5.4 \mathrm{E}-09$ & $4.1 \mathrm{E}+01$ & $2.2 \mathrm{E}-07$ & & & & \\
\hline Total Inorganics & & & & $2.6 \mathrm{E}-07$ & & & \\
\hline${ }^{15} \mathrm{Cs}$ & 6.7E+01 & $1.9 \mathrm{E}-11$ & $1.3 \mathrm{E}-09$ & & & & \\
\hline${ }^{2 \times \mathbf{R a}}$ & $2.0 \mathrm{E}-02$ & $3.0 \mathrm{E}-09$ & 8.1E-05 & & & & \\
\hline 20Th & $4.1 \mathrm{E}-02$ & 7.8E-08 & $3.2 \mathrm{E}-09$ & & & & \\
\hline${ }^{200} \mathrm{Th}$ & $6.9 \mathrm{E}-02$ & $2.9 \mathrm{E}-08$ & $2.0 \mathrm{E}-09$ & & & & \\
\hline $2 \times \mathrm{Th}$ & $4.3 \mathrm{E}-02$ & 2.8E-08 & $1.2 \mathrm{E}-09$ & & & & \\
\hline${ }^{232} 23 x \mathrm{U}$ & $6.4 E-02$ & 2.6E-08 & $1.7 \mathrm{E}-09$ & & & & \\
\hline${ }^{25} \mathrm{Th}$ & 3.1E-01 & $3.2 \mathrm{E}-11$ & $9.9 \mathrm{E}-12$ & & & & \\
\hline${ }^{235} \mathrm{U}$ & $5.9 \mathrm{E}-03$ & $2.5 E-08$ & $1.5 \mathrm{E}-10$ & & & & \\
\hline${ }^{280} \mathrm{Pu}$ & $2.2 \mathrm{E}-02$ & $3.9 E-08$ & $8.6 \mathrm{E}-10$ & & & & \\
\hline${ }^{2 x 8} \mathrm{U}$ & 8.3E-02 & $2.4 \mathrm{E}-08$ & $2.0 \mathrm{E}-09$ & & & & \\
\hline${ }^{209200 \mathrm{Pu}}$ & $2.0 E-01$ & $3.8 \mathrm{E}-08$ & $7.5 \mathrm{E}-09$ & & & & \\
\hline${ }^{\infty} \mathrm{K}$ & $2.6 \mathrm{E}-01$ & $7.6 \mathrm{E}-12$ & $2.0 \mathrm{E}-12$ & & & & \\
\hline${ }^{\circ} \mathrm{Co}$ & $6.4 \mathrm{E}-03$ & 1.5E-10 & $9.6 \mathrm{E}-13$ & & & & \\
\hline${ }^{90} \mathrm{Sr}$ & $5.7 \mathrm{E}-01$ & $6.2 \mathrm{E}-11$ & $3.5 \mathrm{E}-11$ & & & & \\
\hline Tritium & $1.0 \mathrm{E}+\infty$ & $7.8 \mathrm{E}-14$ & 8.1E-14 & & & & \\
\hline Total Radionuclides & & & & $8.1 E-05$ & & & - \\
\hline Total Pathway Risk & & & & & $8.1 E-05$ & & \\
\hline \multicolumn{8}{|c|}{ Exposure Pathway: Extemal Exposure to Radionuclides by Adult Trespasser from Core Samples - Cell } \\
\hline${ }^{157} \mathrm{Cs}$ & $3.2 \mathrm{E}+04$ & $2.0 \mathrm{E}-06$ & $6.1 \mathrm{E}-02$ & & & & \\
\hline${ }^{20} \mathrm{Ra}$ & $9.2 E+\infty$ & $6.0 \mathrm{E}-05$ & $5.5 \mathrm{E}-05$ & & & & \\
\hline $20 \mathrm{Th}$ & $1.9 \mathrm{E}+01$ & $5.6 \mathrm{E}-06$ & 1.1E-04 & & & & \\
\hline${ }^{230} \mathrm{Th}$ & $3.2 \mathrm{E}+01$ & $5.4 \mathrm{E}-11$ & $1.7 \mathrm{E}-09$ & & & & \\
\hline $25 \mathrm{Th}$ & $2.0 \mathrm{E}+01$ & $2.6 \mathrm{E}-11$ & $5.2 \mathrm{E}-10$ & & & & \\
\hline${ }^{232} 23 \mathrm{U}$ & $3.0 \mathrm{E}+01$ & $3.0 \mathrm{E}-11$ & $9.0 \mathrm{E}-10$ & & & & \\
\hline${ }^{25+} \mathrm{Th}$ & $1.5 E+02$ & $3.5 \mathrm{E}-09$ & $5.1 E-07$ & & & & \\
\hline${ }^{223} \mathrm{U}$ & $2.8 E+\infty 0$ & $2.4 \mathrm{E}-07$ & $6.6 \mathrm{E}-07$ & & & & \\
\hline${ }^{250} \mathrm{Pu}$ & $1.0 \mathrm{E}+01$ & $2.8 \mathrm{E}-11$ & $2.9 \mathrm{E}-10$ & & & & \\
\hline
\end{tabular}




\begin{tabular}{|c|c|c|c|c|c|c|c|}
\hline Contaminant & $\begin{array}{l}\text { Chronic } \\
\text { Daily } \\
\text { Intoke }\end{array}$ & $\begin{array}{l}\text { Sope } \\
\text { Factor }\end{array}$ & $\begin{array}{l}\text { Contaminant } \\
\text { Specific } \\
\text { Risl }\end{array}$ & $\begin{array}{c}\text { Contaminant } \\
\text { Class Risk }\end{array}$ & $\begin{array}{c}\text { Total } \\
\text { Pathway } \\
\text { Risk }\end{array}$ & $\begin{array}{c}\text { Total } \\
\text { Contaminant } \\
\text { Risk }\end{array}$ & $\begin{array}{l}\text { Total } \\
\text { Area } \\
\text { Risle }\end{array}$ \\
\hline${ }^{258} \mathrm{U}$ & $3.9 \mathrm{E}+01$ & 5.1E-08 & $2.0 \mathrm{E}-06$ & & & & \\
\hline${ }^{250200 \mathrm{Pus}}$ & $9.2 \mathrm{E}+01$ & $1.7 \mathrm{E}-11$ & $1.6 \mathrm{E}-09$ & & & & \\
\hline${ }^{40} \mathrm{~K}$ & $1.2 \mathrm{E}+02$ & 5.4E-07 & $6.6 \mathrm{E}-05$ & & & & \\
\hline${ }^{\infty} \mathrm{Co}$ & $3.0 \mathrm{E}+\infty$ & $8.6 \mathrm{E}-\infty 6$ & $2.6 \mathrm{E}-05$ & & & & \\
\hline${ }^{90} \mathrm{Sr}$ & $2.7 \mathrm{E}+02$ & $0.0 \mathrm{E}+00$ & $0.0 \mathrm{E}+00$ & & & & \\
\hline Tritium & $4.9 \mathrm{E}+02$ & $0.0 \mathrm{E}+00$ & $0.0 \mathrm{E}+\infty$ & & & & \\
\hline Total Radionuclides & & & & $6.2 \mathrm{E}-02$ & & & \\
\hline Total Pathway Risk & & & & & $6.2 \mathrm{E}-02$ & & \\
\hline Total Inorganic Risk & & & & & & $2.8 \mathrm{E}-04$ & \\
\hline Total Organic Risk & & & & & & 6.5E-06 & \\
\hline Total Radionuclide Risk & " & & & & & $6.2 \mathrm{E}-02$ & \\
\hline Total Area & & & & & & & $6.2 \mathrm{E}-02$ \\
\hline \multicolumn{8}{|l|}{ Total Pathway Risk } \\
\hline \multicolumn{8}{|c|}{ Exposure Pathway: Ingestion of Contaminants by Adult Trespasser from Core Samples - East Gallery } \\
\hline Beryllium & $2.3 \mathrm{E}-07$ & $4.3 \mathrm{E}+\infty 0$ & $1.0 \mathrm{E}-06$ & & & & \\
\hline Total Inorganics & & & & $1.0 \mathrm{E}-06$ & & & \\
\hline Aroclor-1254 & $1.0 \mathrm{E}-06$ & $7.7 \mathrm{E}+\infty 0$ & 7.7E-06 & & & & \\
\hline Aroclor- 1260 & $1.5 \mathrm{E}-06$ & $7.7 \mathrm{E}+\infty$ & $1.2 \mathrm{E}-05$ & & & & \\
\hline Bis(2-ethylhexyl)phthalate & $1.4 \mathrm{E}-07$ & 1.4E-02 & $2.0 \mathrm{E}-09$ & & & & \\
\hline Total Organics & & & & $1.9 \mathrm{E}-05$ & & & \\
\hline${ }^{19} \mathrm{Cs}$ & $2.4 \mathrm{E}+04$ & $2.8 \mathrm{E}-11$ & $6.7 \mathrm{E}-07$ & & & & \\
\hline${ }^{20} \mathrm{Ra}$ & $3.8 \mathrm{E}+02$ & $1.2 \mathrm{E}-10$ & $4.5 \mathrm{E}-08$ & & & & $\dot{-}$ \\
\hline ins: & $3.9 \mathrm{E}+02$ & $1.0 \mathrm{E}-10$ & $3.9 \mathrm{E}-08$ & & & & \\
\hline${ }^{20} \mathrm{Th}$ & $5.9 E+02$ & $5.5 \mathrm{E}-11$ & $3.2 \mathrm{E}-08$ & & & & \\
\hline${ }^{200} \mathrm{Th}$ & $1.3 \mathrm{E}+03$ & $1.3 \mathrm{E}-11$ & $1.6 \mathrm{E}-08$ & & & & \\
\hline${ }^{200} \mathrm{Th}$ & $4.5 E+02$ & $1.2 \mathrm{E}-11$ & $5.4 \mathrm{E}-09$ & & & & \\
\hline $25 / 25 \mathrm{U}$ & $9.5 \mathrm{E}+02$ & $1.6 \mathrm{E}-11$ & $1.5 \mathrm{E}-08$ & & & & \\
\hline${ }^{280} \mathrm{U}$ & $8.1 E+02$ & $2.0 \mathrm{E}-11$ & $1.6 \mathrm{E}-08$ & & & & \\
\hline${ }^{2 ! 1} \mathrm{Am}$ & $5.3 \mathrm{E}+02$ & $2.4 \mathrm{E}-10$ & $1.3 \mathrm{E}-07$ & & & & \\
\hline${ }^{\infty} \mathrm{K}$ & $5.6 \mathrm{E}+03$ & $1.1 E-11$ & $6.2 \mathrm{E}-08$ & & & & \\
\hline${ }^{\circ} \mathrm{Sr}$ & $8.5 E+03$ & $3.6 \mathrm{E}-11$ & $3.1 \mathrm{E}-07$ & & & & \\
\hline Tritium & $2.7 E+04$ & $5.4 \mathrm{E}-14$ & $1.5 \mathrm{E}-09$ & & & & \\
\hline Total Radionuclides & & & & $1.3 \mathrm{E}-06$ & & & \\
\hline
\end{tabular}




\begin{tabular}{|c|c|c|c|c|c|c|c|}
\hline Contaminant & $\begin{array}{l}\text { Chronic } \\
\text { Daily } \\
\text { Intake }\end{array}$ & $\begin{array}{l}\text { Slope } \\
\text { Factor }\end{array}$ & $\begin{array}{l}\text { Contaminant } \\
\text { Specific } \\
\text { Risk }\end{array}$ & $\begin{array}{l}\text { Contaminant } \\
\text { Class Risk }\end{array}$ & $\begin{array}{c}\text { Total } \\
\text { Pathway } \\
\text { Risk }\end{array}$ & $\begin{array}{c}\text { Total } \\
\text { Contaminant } \\
\text { Risk }\end{array}$ & $\begin{array}{l}\text { Total } \\
\text { Area } \\
\text { Risk }\end{array}$ \\
\hline Total Pathway Risk & & & & & 2.1E-05 & & \\
\hline \multicolumn{8}{|c|}{ Exposure Pathway: Dermal Exposure to Contamingnts by Adult Trespasser from Core Samples - East Gallery } \\
\hline Beryllium & $1.2 E-08$ & $4.3 \mathrm{E}+02$ & 5.4E-06 & & & & \\
\hline Total Inorganics & & & & $5.4 \mathrm{E}-06$ & & & \\
\hline Aroclor-1254 & 5.3E-07 & $8.6 \mathrm{E}+00$ & 4.5E-06 & & & & \\
\hline Aroclor-1260 & 8.1E-07 & $8.6 \mathrm{E}+\infty 0$ & $6.9 \mathrm{E}-06$ & & & & \\
\hline Bis(2-ethylhexyl)phthalate & 7.5E-08 & $7.4 \mathrm{E}-02$ & $5.5 \mathrm{E}-09$ & & & & \\
\hline Total Organics & & & & $1.1 \mathrm{E}-05$ & & & \\
\hline Total Pathway Risk & & & & & $1.6 \mathrm{E}-05$ & & \\
\hline \multicolumn{8}{|c|}{ Exposure Pathway: Inhalation of Contaminants by Adult Trespasser from Core Samples - East Gallery } \\
\hline Arsenic & $8.2 \mathrm{E}-11$ & $5.0 \mathrm{E}+01$ & 4.1E-09 & & & & \\
\hline Beryilium & $1.1 \mathrm{E}-11$ & $8.4 \mathrm{E}+\infty 0$ & $9.2 \mathrm{E}-11$ & & & & \\
\hline Cadmium & $1.4 \mathrm{E}-10$ & $6.1 \mathrm{E}+\infty$ & $8.5 \mathrm{E}-10$ & & & & \\
\hline Chromium VI & $2.3 \mathrm{E}-10$ & $4.1 \mathrm{E}+01$ & 9.3E-09 & & & & \\
\hline Total Inorganics & & & & $1.4 \mathrm{E}-08$ & & & \\
\hline${ }^{157} \mathrm{Cs}$ & $1.1 \mathrm{E}+\infty$ & $1.9 \mathrm{E}-11$ & 2.1E-11 & & & & \\
\hline${ }^{208 \mathrm{Ra}}$ & $1.8 \mathrm{E}-02$ & $3.0 \mathrm{E}-09$ & 7.3E-05 & & & & \\
\hline 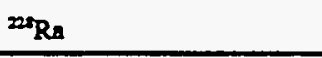 & $1.8 E-02$ & $6.9 E-10$ & $1.3 \mathrm{E}-11$ & & & & \\
\hline ExTh & 2.7E-02 & $7.8 \mathrm{E}-08$ & $2.1 \mathrm{E}-09$ & & & & \\
\hline${ }^{20} \mathrm{Th}$ & $5.9 \mathrm{E}-02$ & $2.9 \mathrm{E}-08$ & $1.7 \mathrm{E}-09$ & & & & \\
\hline${ }^{20} \mathrm{Th}$ & $2.1 E-02$ & $2.8 \mathrm{E}-08$ & $5.9 \mathrm{E}-10$ & & & & \\
\hline${ }^{252524} U$ & $4.4 \mathrm{E}-02$ & $2.6 \mathrm{E}-08$ & $1.1 \mathrm{E}-09$ & & & & \\
\hline${ }^{200} \mathrm{U}$ & $3.8 \mathrm{E}-02$ & $2.4 \mathrm{E}-08$ & $9.1 \mathrm{E}-10$ & & & & \\
\hline${ }^{241} \mathrm{Am}$ & $2.5 E-02$ & $3.2 \mathrm{E}-08$ & $7.8 \mathrm{E}-10$ & & & & \\
\hline${ }^{\infty} \mathrm{K}$ & $2.6 \mathrm{E}-01$ & $7.6 \mathrm{E}-12$ & $2.0 \mathrm{E}-12$ & & & & \\
\hline${ }^{\infty} \mathrm{Sr}$ & $4.0 \mathrm{E}-01$ & $6.2 \mathrm{E}-11$ & $2.5 \mathrm{E}-11$ & & & & \\
\hline Tritium & $1.3 \mathrm{E}+\infty$ & $7.8 \mathrm{E}-14$ & $9.8 \mathrm{E}-14$ & & & & \\
\hline Total Rsdionuclides & & & & 7.3E-05 & & & \\
\hline Total Pathway Risk & & & & & 7.3E-05 & & \\
\hline \multicolumn{8}{|c|}{ Exposure Pathway: External Exposure to Radionuclides by Adult Trespasser from Core Samples - East Gallery } \\
\hline${ }^{157} \mathrm{Cs}$ & $5.2 \mathrm{E}+02$ & $2.0 \mathrm{E}-06$ & $1.0 \mathrm{E}-03$ & & & & \\
\hline${ }^{20} \mathrm{Ra}$ & $8.3 \mathrm{E}+00$ & $6.0 \mathrm{E}-06$ & $5.0 \mathrm{E}-05$ & & & & \\
\hline $2 \pi \mathrm{Ra}$ & $8.5 \mathrm{E}+00$ & $2.9 \mathrm{E}-06$ & $2.5 E-05$ & & & & \\
\hline
\end{tabular}




\begin{tabular}{|c|c|c|c|c|c|c|c|}
\hline Contaminant & $\begin{array}{l}\text { Chronic } \\
\text { Daily } \\
\text { Intake }\end{array}$ & $\begin{array}{l}\text { Sope } \\
\text { Factor }\end{array}$ & $\begin{array}{l}\text { Contaminont } \\
\text { Specific } \\
\text { Risk }\end{array}$ & $\begin{array}{c}\text { Contaminant } \\
\text { Class Risl }\end{array}$ & $\begin{array}{c}\text { Total } \\
\text { Pathway } \\
\text { Risk }\end{array}$ & $\begin{array}{c}\text { Total } \\
\text { Contaminant } \\
\text { Risk }\end{array}$ & $\begin{array}{l}\text { Total } \\
\text { Area } \\
\text { Risk }\end{array}$ \\
\hline $\operatorname{monh}$ & $1.3 \mathrm{E}+01$ & $5.6 \mathrm{E}-06$ & 7.2E-05 & & & & \\
\hline${ }^{200} \mathrm{Th}$ & $2.8 \mathrm{E}+01$ & $5.4 \mathrm{E}-11$ & $1.5 \mathrm{E}-09$ & & & & \\
\hline $2 m \mathrm{Th}$ & $9.9 \mathrm{E}+\infty$ & $2.6 \mathrm{E}-11$ & $2.6 \mathrm{E}-10$ & & & & \\
\hline${ }^{29 S 23} U$ & $2.1 \mathrm{E}+01$ & $3.0 \mathrm{E}-11$ & $6.2 \mathrm{E}-10$ & & & & \\
\hline 250 & $1.8 \mathrm{E}+01$ & S.1E-08 & $9.0 \mathrm{E}-07$ & & & & \\
\hline${ }^{211} \mathrm{Am}$ & $1.2 \mathrm{E}+01$ & $4.9 \mathrm{E}-09$ & $5.6 \mathrm{E}-08$ & & & & \\
\hline${ }^{10} \mathrm{~K}$ & $1.2 E+02$ & 5.4E-07 & $6.7 \mathrm{E}-05$ & & & & \\
\hline${ }^{\infty} \mathrm{Sr}$ & $1.9 E+02$ & $0.0 \mathrm{E}+\infty 0$ & $0.0 \mathrm{E}+00$ & & & & \\
\hline Tritium & $5.9 \mathrm{E}+02$ & $0.0 \mathrm{E}+\infty 0$ & $0.0 \mathrm{E}+00$ & & & & \\
\hline Total Radionuclides & & & & $1.3 \mathrm{E}-03$ & & & \\
\hline Toral Pathway Risk & & & & & $1.3 \mathrm{E}-03$ & & \\
\hline Total Inorganic Risk & & & & & & $6.4 \mathrm{E}-06$ & \\
\hline Total Organic Risk & & & & & & 3.1E-05 & \\
\hline Total Radionuclide Risk & & & & & & $1.3 \mathrm{E}-03$ & \\
\hline Total Area & & & & & & & $1.4 E-03$ \\
\hline \multicolumn{8}{|c|}{ Exposure Pathway: Ingestion of Contaminants by Adult Trespasser from Sediment in Cell } \\
\hline Aroclor-1254 & $2.4 \mathrm{E}-04$ & 7.7E+ $+\infty$ & $1.9 \mathrm{E}-03$ & & & & \\
\hline Benzo(a)pyrene & $3.3 \mathrm{E}-06$ & $7.3 \mathrm{E}+\infty 0$ & 2.4E-05 & & & & \\
\hline Berzo(k)fluoranthene & 2.7E-05 & $7.3 \mathrm{E}-02$ & $2.0 \mathrm{E}-06$ & & & & \\
\hline Bis(2-ethylhexyl)phthalate & $1.2 \mathrm{E}-06$ & $1.4 \mathrm{E}-02$ & $1.7 \mathrm{E}-08$ & & & & \\
\hline Carbazole & $1.2 \mathrm{E}-06$ & $2.0 \mathrm{E}-02$ & $2.3 \mathrm{E}-08$ & & & & \\
\hline Chrysene & $1.1 \mathrm{E}-05$ & $7.3 \mathrm{E}-03$ & $8.1 E-08$ & & & & - \\
\hline Dibenz $(a, h)$ anthracene & $8.8 \mathrm{E}-07$ & $7.3 \mathrm{E}+\infty 0$ & $6.4 \mathrm{E}-06$ & & & & \\
\hline Indeno(1,2,3-cd)pyrene & $5.6 \mathrm{E}-06$ & 7.3E-01 & 4.1E-06 & & & & \\
\hline Total Organics & & & & $1.9 \mathrm{E}-03$ & & & \\
\hline${ }^{15} \mathrm{Cs}$ & $3.2 E+07$ & $2.8 \mathrm{E}-11$ & $9.1 E-04$ & & & & \\
\hline${ }^{20} \mathrm{Th}$ & $5.7 \mathrm{E}+02$ & 5.5E-11 & $3.1 \mathrm{E}-08$ & & & & \\
\hline${ }^{2} \mathrm{Th}$ & $2.0 \mathrm{E}+04$ & $8.9 \mathrm{E}-11$ & $1.7 \mathrm{E}-06$ & & & & \\
\hline${ }^{20} \mathrm{Th}$ & $5.8 \mathrm{E}+02$ & $1.2 \mathrm{E}-11$ & $6.9 \mathrm{E}-09$ & & & & \\
\hline${ }^{231234} \mathrm{U}$ & $3.6 E+03$ & $1.6 \mathrm{E}-11$ & $5.8 \mathrm{E}-08$ & & & & \\
\hline${ }^{234} \mathrm{Pu}$ & $1.2 E+03$ & 2.2E-10 & $2.7 \mathrm{E}-07$ & & & & \\
\hline${ }^{238} \mathrm{U}$ & $2.4 \mathrm{E}+03$ & $2.0 \mathrm{E}-11$ & 4.7E-08 & & & & \\
\hline${ }^{258220} \mathrm{Pu}$ & $3.7 E+04$ & 2.3E-10 & $8.5 \mathrm{E}-06$ & & & & \\
\hline
\end{tabular}




\begin{tabular}{|c|c|c|c|c|c|c|c|}
\hline Contaminant & $\begin{array}{c}\text { Chronic } \\
\text { Daily } \\
\text { Intake }\end{array}$ & $\begin{array}{l}\text { Sope } \\
\text { Factor }\end{array}$ & $\begin{array}{l}\text { Contaminant } \\
\text { Specific } \\
\text { Risk }\end{array}$ & $\begin{array}{c}\text { Contaminant } \\
\text { Class Risk }\end{array}$ & $\begin{array}{c}\text { Total } \\
\text { Pathway } \\
\text { Risk }\end{array}$ & $\begin{array}{c}\text { Total } \\
\text { Contaminant } \\
\text { Risk }\end{array}$ & $\begin{array}{l}\text { Total } \\
\text { Area } \\
\text { Risk }\end{array}$ \\
\hline${ }^{10} \mathrm{~K}$ & $9.9 \mathrm{E}+03$ & $1.1 E-11$ & $1.1 E-07$ & & & & \\
\hline${ }^{60} \mathrm{Co}$ & $3.6 E+03$ & $1.5 \mathrm{E}-11$ & $5.4 \mathrm{E}-08$ & & & & \\
\hline${ }^{\infty 0} \mathrm{Sr}$ & $1.0 \mathrm{E}+05$ & $3.6 \mathrm{E}-11$ & 3.7E-06 & & & & \\
\hline Tritium & $1.1 E+03$ & $5.4 \mathrm{E}-14$ & 5.7E-11 & & & & \\
\hline Total Radionuclides & & & & $9.2 \mathrm{E}-04$ & & & \\
\hline Total Pathway Risk & & & & & $2.8 \mathrm{E}-03$ & & \\
\hline \multicolumn{8}{|c|}{ Exposure Pathway: Dermal Exposure to Contaminents by Adult Trespasser from Sediment in Cell } \\
\hline Aroclor-1254 & $1.3 \mathrm{E}-04$ & $8.6 \mathrm{E}+00$ & $1.1 \mathrm{E}-03$ & & & & \\
\hline Benzo(a)pyrene & $1.8 \mathrm{E}-06$ & $2.4 \mathrm{E}+01$ & $4.2 \mathrm{E}-05$ & & & & \\
\hline Benzo(z)fluoranthene & $1.4 \mathrm{E}-05$ & $2.4 E-01$ & $3.4 \mathrm{E}-06$ & & & & \\
\hline Bis(2-ethylhexyl)phthalate & $6.5 \mathrm{E}-07$ & 7.4E-02 & 4.8E-08 & & & & \\
\hline Carbazole & $6.2 E-07$ & $2.0 \mathrm{E}-02$ & $1.2 \mathrm{E}-08$ & & & & \\
\hline Chrysene & $5.9 \mathrm{E}-06$ & $2.4 \mathrm{E}-02$ & $1.4 \mathrm{E}-07$ & & & & \\
\hline Dibenz $(a, h)$ anthracene & 4.7E-07 & $8.1 E+\infty$ & $3.8 \mathrm{E}-06$ & & & & \\
\hline Indeno(1,2,3-cd)pyrene & $3.0 \mathrm{E}-06$ & $2.4 \mathrm{E}+\infty 0$ & $7.0 \mathrm{E}-06$ & & & & \\
\hline Total Organics & & & & $1.1 \mathrm{E}-03$ & & & \\
\hline Total Pathway Risk & & & & & $1.1 \mathrm{E}-03$ & & \\
\hline \multicolumn{8}{|c|}{ Exposure Pathway: Inhalation of Contaminants by Adult Trespasser from Sediment In Cell } \\
\hline Arsenic & $5.9 \mathrm{E}-10$ & $5.0 \mathrm{E}+01$ & 2.9E-08 & & & & \\
\hline Cadmium & $1.6 \mathrm{E}-09$ & $6.1 \mathrm{E}+\infty$ & $1.0 \mathrm{E}-08$ & & & & \\
\hline Chromium VI & $5.2 \mathrm{E}-09$ & $4.1 E+01$ & $2.1 \mathrm{E}-07$ & & & & \\
\hline Total Inorganics & & & & $2.5 \mathrm{E}-07$ & & & $\cdot$ \\
\hline${ }^{15} \mathrm{Cs}$ & $1.5 \mathrm{E}+03$ & $1.9 \mathrm{E}-11$ & $2.9 \mathrm{E}-08$ & & & & \\
\hline$=\mathrm{Th}$ & $2.6 \mathrm{E}-02$ & $7.8 \mathrm{E}-08$ & $2.1 E-09$ & . & & & \\
\hline $20 \mathrm{Th}$ & $9.2 E-01$ & 7.7E-08 & $7.1 \mathrm{E}-08$ & & & & \\
\hline $200 \mathrm{Th}$ & $2.7 E-02$ & $2.8 E-08$ & $7.5 \mathrm{E}-10$ & & & & \\
\hline${ }^{2525230} U$ & $1.7 \mathrm{E}-01$ & $2.6 \mathrm{E}-08$ & 4.4E-09 & & & & \\
\hline${ }^{20} \mathrm{Pu}$ & $5.7 \mathrm{E}-02$ & $3.9 \mathrm{E}-08$ & $2.2 \mathrm{E}-09$ & & & & \\
\hline${ }^{240} \mathrm{U}$ & 1.1E-01 & 2.4E-08 & $2.6 \mathrm{E}-09$ & & & & \\
\hline${ }^{251200 \mathrm{Pu}}$ & $1.7 E+\infty$ & $3.8 \mathrm{E}-08$ & $6.6 \mathrm{E}-08$ & & & & \\
\hline${ }^{\circ} \mathrm{K}$ & $4.6 \mathrm{E}-01$ & $7.6 \mathrm{E}-12$ & $3.5 \mathrm{E}-12$ & & & & \\
\hline${ }^{60} \mathrm{Co}$ & 1.7E- 01 & $1.5 \mathrm{E}-10$ & $2.5 \mathrm{E}-11$ & & & & \\
\hline${ }^{\infty} \mathrm{Sr}$ & $4.8 E+\infty 0$ & $6.2 \mathrm{E}-11$ & $3.0 \mathrm{E}-10$ & & & & \\
\hline
\end{tabular}




\begin{tabular}{|c|c|c|c|c|c|c|c|}
\hline Contaminant & $\begin{array}{l}\text { Chronic } \\
\text { Daily } \\
\text { Intake }\end{array}$ & $\begin{array}{l}\text { Slope } \\
\text { Factor }\end{array}$ & $\begin{array}{l}\text { Contominnnt } \\
\text { Specific } \\
\text { Risk }\end{array}$ & $\begin{array}{l}\text { Contaminant } \\
\text { Class Risk }\end{array}$ & $\begin{array}{c}\text { Total } \\
\text { Pathway } \\
\text { Risk }\end{array}$ & $\begin{array}{c}\text { Total } \\
\text { Contaminant } \\
\text { Risl. }\end{array}$ & $\begin{array}{l}\text { Total } \\
\text { Area } \\
\text { Risk }\end{array}$ \\
\hline Tritium & $4.9 \mathrm{E}-02$ & $7.8 \mathrm{E}-14$ & $3.8 \mathrm{E}-15$ & & & & \\
\hline Total Radionuclides & & & & $1.8 \mathrm{E}-07$ & & & \\
\hline Total Pathway Risk & & & & & 4.3E-07 & & \\
\hline \multicolumn{8}{|c|}{ Exposure Pathway: Extemal Exposure to Radionnclides by Adult Trespasser from Sediment in Cell } \\
\hline${ }^{15} \mathrm{Cs}$ & $7.1 E+05$ & 2.0E-06 & $7.6 \mathrm{E}-01$ & & & & \\
\hline 25eTh & $1.2 \mathrm{E}+01$ & $5.6 \mathrm{E}-06$ & 7.0E-05 & & & & \\
\hline $20 \mathrm{Th}$ & $4.3 E+02$ & $6.8 \mathrm{E}-07$ & $2.9 \mathrm{E}-04$ & & & & \\
\hline $20 \mathrm{Th}$ & $1.3 \mathrm{E}+01$ & $2.6 \mathrm{E}-11$ & 3.3E-10 & & & & \\
\hline $235250 \mathrm{U}$ & $7.9 \mathrm{E}+01$ & $3.0 \mathrm{E}-11$ & $2.4 \mathrm{E}-09$ & & & & \\
\hline${ }^{228} \mathrm{Pu}$ & $2.7 \mathrm{E}+01$ & 2.8E-11 & 7.5E-10 & & & & \\
\hline${ }^{250} \mathrm{U}$ & $5.2 E+01$ & 5.1E-08 & $2.6 \mathrm{E}-06$ & & & & \\
\hline${ }^{239210} \mathrm{Pu}$ & $8.1 \mathrm{E}+02$ & $1.7 \mathrm{E}-11$ & $1.4 \mathrm{E}-08$ & & & & \\
\hline${ }^{10} \mathrm{~K}$ & $2.2 E+02$ & 5.4E-07 & $1.2 \mathrm{E}-04$ & & & & \\
\hline${ }^{\infty} \mathrm{Co}$ & $7.9 \mathrm{E}+01$ & $8.6 \mathrm{E}-06$ & $6.8 \mathrm{E}-04$ & & & & \\
\hline${ }^{\infty} \mathrm{Sr}$ & $2.2 E+03$ & $0.0 \mathrm{E}+00$ & $0.0 \mathrm{E}+\infty 0$ & & & & \\
\hline Tritum & $2.3 E+01$ & $0.0 \mathrm{E}+\infty 0$ & $0.0 \mathrm{E}+00$ & & & & \\
\hline Total Radionuclides & & & & $7.6 \mathrm{E}-01$ & & & \\
\hline Total Pathway Risk & & & & & $7.6 \mathrm{E}-01$ & & \\
\hline Toul Inorganic Risk & & & & & & $2.5 \mathrm{E}-07$ & \\
\hline Total Organic Risk & & & & & & $3.0 \mathrm{E}-03$ & \\
\hline Total Radionuclide Risk & & & & & & $7.6 \mathrm{E}-01$ & \\
\hline Toul Ares & & & & & & & $7.6 \mathrm{E}-01$ \\
\hline \multicolumn{8}{|c|}{ Exposure Pathway: Ingestion of Contominants by Adult Trespasser from Core Samples - Whole Building } \\
\hline Beryllium & $7.2 \mathrm{E}-06$ & $4.3 \mathrm{E}+00$ & 3.1E-05 & & & & \\
\hline Total Inorganics & & & & $3.1 E-05$ & & & \\
\hline Aroclor-1254 & 8.5E-07 & $7.7 \mathrm{E}+\infty 0$ & $6.6 \mathrm{E}-06$ & & & & \\
\hline Aroclor-1260 & $1.5 \mathrm{E}-06$ & $7.7 E+\infty$ & $1.2 \mathrm{E}-05$ & & & & \\
\hline Bis(2-ethylhexyl)phthatate & $1.4 \mathrm{E}-07$ & $1.4 E-02$ & $2.0 \mathrm{E}-09$ & & & & \\
\hline Total Organics & & & & $1.8 \mathrm{E}-05$ & & & \\
\hline${ }^{17} \mathrm{Cs}$ & $1.4 \mathrm{E}+06$ & $2.8 \mathrm{E}-11$ & $4.0 \mathrm{E}-05$ & & & & \\
\hline${ }^{20} \mathrm{Ra}$ & $4.2 \mathrm{E}+02$ & $1.2 \mathrm{E}-10$ & $5.1 E-08$ & & & & \\
\hline${ }^{20} \mathrm{Rs}$ & $3.9 E+02$ & $1.0 \mathrm{E}-10$ & $3.9 \mathrm{E}-08$ & & & & \\
\hline $2 n T h$ & $8.1 E+02$ & $5.5 \mathrm{E}-11$ & 4.4E-08 & & & & \\
\hline
\end{tabular}




\begin{tabular}{|c|c|c|c|c|c|c|c|}
\hline Contaminant & $\begin{array}{c}\text { Chranic } \\
\text { Daily } \\
\text { Intolke }\end{array}$ & $\begin{array}{l}\text { Sope } \\
\text { Factor }\end{array}$ & $\begin{array}{l}\text { Contominant } \\
\text { Specific } \\
\text { Risk }\end{array}$ & $\begin{array}{l}\text { Contaminant } \\
\text { Class Risk }\end{array}$ & $\begin{array}{c}\text { Total } \\
\text { Pathway } \\
\text { Risk } \\
\end{array}$ & $\begin{array}{c}\text { Total } \\
\text { Contaminant } \\
\text { Risk }\end{array}$ & $\begin{array}{l}\text { Total } \\
\text { Area } \\
\text { Risk }\end{array}$ \\
\hline${ }^{250} \mathrm{Th}$ & $1.5 \mathrm{E}+03$ & $1.3 \mathrm{E}-11$ & $1.9 \mathrm{E}-08$ & & & & \\
\hline${ }^{20} \mathrm{Th}$ & $8.4 \mathrm{E}+02$ & $1.2 \mathrm{E}-11$ & $1.0 \mathrm{E}-08$ & & & & \\
\hline${ }^{239} 24 \mathrm{U}$ & $1.3 E+03$ & $1.6 \mathrm{E}-11$ & $2.1 \mathrm{E}-08$ & & & & \\
\hline 25hTh & $6.6 \mathrm{E}+03$ & $4.0 \mathrm{E}-12$ & 2.7E-08 & & & & \\
\hline${ }^{235} \mathrm{U}$ & $1.3 \mathrm{E}+02$ & $1.6 \mathrm{E}-11$ & $2.0 \mathrm{E}-09$ & & & & \\
\hline${ }^{258} \mathrm{Pu}$ & $4.7 \mathrm{E}+02$ & $2.2 \mathrm{E}-10$ & $1.0 \mathrm{E}-07$ & & & & \\
\hline${ }^{200} \mathrm{U}$ & $1.5 \mathrm{E}+03$ & 2.0E-11 & $3.0 \mathrm{E}-08$ & & & & \\
\hline${ }^{258200} \mathrm{Pu}$ & $4.2 \mathrm{E}+03$ & $2.3 \mathrm{E}-10$ & 9.7E-07 & & & & \\
\hline${ }^{2 n 1} \mathrm{Am}$ & $5.3 \mathrm{E}+02$ & $2.4 \mathrm{E}-10$ & $1.3 \mathrm{E}-07$ & & & & \\
\hline${ }^{40} \mathrm{~K}$ & $5.6 \mathrm{E}+03$ & $1.1 \mathrm{E}-11$ & $6.2 \mathrm{E}-08$ & & & & \\
\hline${ }^{60} \mathrm{Co}$ & $1.4 \mathrm{E}+02$ & $1.5 \mathrm{E}-11$ & $2.0 \mathrm{E}-09$ & & & & \\
\hline${ }^{20} \mathrm{Sr}$ & $1.2 E+04$ & $3.6 \mathrm{E}-11$ & 4.4E-07 & & & & \\
\hline Tritium & 2.7E+04 & $5.4 \mathrm{E}-14$ & $1.5 \mathrm{E}-09$ & & & & \\
\hline Toral Radionuclides & & & & $4.2 \mathrm{E}-05$ & & & \\
\hline Totsl Pathway Risk & & & & & 9.1E-05 & & \\
\hline \multicolumn{8}{|c|}{ Exposure Pathway: Dermal Exposure to Contaminents by Adult Trespasser from Core Samples - Whole Building } \\
\hline Beryllium & $3.8 \mathrm{E}-07$ & $4.3 \mathrm{E}+02$ & $1.7 \mathrm{E}-04$ & & & & \\
\hline Total Inorganics & & & & $1.7 E-04$ & & & \\
\hline Aroclor-1254 & 4.5E-07 & $8.6 \mathrm{E}+00$ & $3.9 \mathrm{E}-06$ & & & & \\
\hline Aroclor-1260 & 8.1E-07 & $8.6 \mathrm{E}+\infty 0$ & $6.9 E-06$ & & & & \\
\hline Bis(2-ethylhexyl)phthalate & 7.5E-08 & $7.4 \mathrm{E}-02$ & $5.5 \mathrm{E}-09$ & & & & \\
\hline Total Organics & & & & $1.1 \mathrm{E}-05$ & & & $\cdot$ \\
\hline Total Pathway Risk & & & & & $1.8 \mathrm{E}-04$ & & \\
\hline \multicolumn{8}{|c|}{ Exposure Pathway: Inhalation of Contaminants by Adult Trespasser from Core Samples - Whole Building } \\
\hline Arsenic & $7.8 \mathrm{E}-11$ & $5.0 \mathrm{E}+01$ & $3.9 \mathrm{E}-09$ & & & & \\
\hline Beryllium & $3.4 \mathrm{E}-10$ & $8.4 \mathrm{E}+\infty 0$ & $2.8 \mathrm{E}-09$ & & & & \\
\hline Cadmium & 4.9E-09 & $6.1 \mathrm{E}+00$ & $3.0 \mathrm{E}-08$ & & & & \\
\hline Chromium VI & $5.4 \mathrm{E}-09$ & $4.1 E+01$ & 2.2E-07 & & & & \\
\hline Total Inorganics & & & & $2.6 \mathrm{E}-07$ & & & \\
\hline${ }^{17} \mathrm{Cs}$ & $6.7 \mathrm{E}+01$ & $1.9 \mathrm{E}-11$ & $1.3 \mathrm{E}-09$ & & & & \\
\hline${ }^{2} \mathrm{Ra}$ & $2.0 \mathrm{E}-02$ & $3.0 \mathrm{E}-09$ & 8.1E-05 & & & & \\
\hline$=\mathrm{Ra}_{\mathrm{R}}$ & $1.8 \mathrm{E}-02$ & $6.9 \mathrm{E}-10$ & $1.3 \mathrm{E}-11$ & & & & \\
\hline 2sih & $3.8 \mathrm{E}-02$ & $7.8 \mathrm{E}-08$ & $2.9 \mathrm{E}-09$ & & & & \\
\hline
\end{tabular}




\begin{tabular}{|c|c|c|c|c|c|c|c|}
\hline Contaminant & $\begin{array}{l}\text { Chronic } \\
\text { Daily } \\
\text { Intoke }\end{array}$ & $\begin{array}{l}\text { Sope } \\
\text { Fector }\end{array}$ & $\begin{array}{l}\text { Contaminant } \\
\text { Spacific } \\
\text { Risk }\end{array}$ & $\begin{array}{l}\text { Contaminant } \\
\text { Class Risk }\end{array}$ & $\begin{array}{c}\begin{array}{c}\text { Total } \\
\text { Pathway }\end{array} \\
\text { Risk } \\
\end{array}$ & $\begin{array}{c}\text { Total } \\
\text { Contaminant } \\
\text { Risk }\end{array}$ & $\begin{array}{l}\text { Total } \\
\text { Area } \\
\text { Risk }\end{array}$ \\
\hline${ }^{200} \mathrm{Th}$ & $6.8 \mathrm{E}-02$ & $2.9 \mathrm{E}-08$ & $2.0 \mathrm{E}-09$ & & & & \\
\hline${ }^{20} \mathrm{Th}$ & $3.9 \mathrm{E}-02$ & $2.8 \mathrm{E}-08$ & $1.1 \mathrm{E}-09$ & & & & \\
\hline${ }^{23} 3234 \mathrm{U}$ & $6.2 \mathrm{E}-02$ & $2.6 \mathrm{E}-08$ & $1.6 \mathrm{E}-09$ & & & & \\
\hline${ }^{234} \mathrm{Th}$ & $3.1 \mathrm{E}-01$ & $3.2 \mathrm{E}-11$ & $9.9 \mathrm{E}-12$ & & & & \\
\hline${ }^{25 s} \mathrm{U}$ & $5.9 \mathrm{E}-03$ & 2.5E-08 & $1.5 \mathrm{E}-10$ & & & & \\
\hline${ }^{200} \mathrm{Pu}$ & 2.2E-02 & 3.9E-08 & $8.6 \mathrm{E}-10$ & & & & \\
\hline${ }^{230} \mathrm{U}$ & $7.0 \mathrm{E}-02$ & 2.4E-08 & $1.7 \mathrm{E}-09$ & & & & \\
\hline${ }^{23020} \mathrm{Pu}$ & $2.0 \mathrm{E}-01$ & $3.8 \mathrm{E}-08$ & $7.5 \mathrm{E}-09$ & & & & \\
\hline${ }^{24} \mathrm{Am}$ & $2.5 \mathrm{E}-02$ & 3.2E-08 & $7.8 \mathrm{E}-10$ & & & & \\
\hline${ }^{\infty} \mathrm{K}$ & $2.6 \mathrm{E}-01$ & $7.6 \mathrm{E}-12$ & $2.0 \mathrm{E}-12$ & & & & \\
\hline${ }^{60} \mathrm{Co}$ & $6.4 E-03$ & $1.5 E-10$ & $9.6 E-13$ & & & & \\
\hline${ }^{\infty} \mathrm{Sr}$ & $5.7 \mathrm{E}-01$ & $6.2 \mathrm{E}-11$ & $3.5 E-11$ & & & & \\
\hline Tritium & $1.3 \mathrm{E}+\infty$ & $7.8 \mathrm{E}-14$ & $9.8 E-14$ & & & & \\
\hline Total Radionuclides & & & & 8.1E-05 & & & \\
\hline Total Pathway Risk & & & & & 8.1E-05 & & \\
\hline \multicolumn{8}{|c|}{ Exposare Pathway: External Exposure to Radionuclides by Adult Trespasser from Core Samples - Whole Building } \\
\hline${ }^{177} \mathrm{Cs}$ & $3.2 \mathrm{E}+04$ & $2.0 \mathrm{E}-06$ & $6.1 \mathrm{E}-02$ & & & & \\
\hline${ }^{208} \mathrm{Rs}$ & $9.2 \mathrm{E}+\infty$ & $6.0 \mathrm{E}-06$ & $5.5 E-05$ & & & & \\
\hline $2 \pi \mathrm{Ra}$ & $8.5 E+\infty$ & $2.9 \mathrm{E}-06$ & $2.5 \mathrm{E}-05$ & & & & \\
\hline${ }^{20}$ Th & $1.8 \mathrm{E}+01$ & $5.6 \mathrm{E}-06$ & $9.9 E-05$ & & & & \\
\hline${ }^{200} \mathrm{Th}$ & $3.2 E+01$ & $5.4 \mathrm{E}-11$ & $1.7 \mathrm{E}-09$ & & & & \\
\hline${ }^{200} \mathrm{Th}$ & $1.8 \mathrm{E}+01$ & $2.6 \mathrm{E}-11$ & $4.8 \mathrm{E}-10$ & & & & - \\
\hline $233234 \mathrm{U}$ & $2.9 \mathrm{E}+01$ & $3.0 \mathrm{E}-11$ & 8.7E-10 & & & & \\
\hline 2stTh & $1.5 \mathrm{E}+02$ & $3.5 \mathrm{E}-09$ & 5.1E-07 & & & & \\
\hline${ }^{23 s} \mathrm{U}$ & $2.8 \mathrm{E}+00$ & $2.4 E-07$ & $6.6 \mathrm{E}-07$ & & & & \\
\hline${ }^{258} \mathrm{Pu}$ & $1.0 \mathrm{E}+01$ & $2.8 \mathrm{E}-11$ & $2.9 \mathrm{E}-10$ & & & & \\
\hline${ }^{210} \mathrm{U}$ & $3.3 E+01$ & $5.1 \mathrm{E}-08$ & $1.7 \mathrm{E}-06$ & & & & \\
\hline${ }^{2591200} \mathrm{Pu}$ & $9.2 \mathrm{E}+01$ & $1.7 \mathrm{E}-11$ & $1.6 \mathrm{E}-09$ & & & & \\
\hline${ }^{2 n} \mathrm{Am}$ & $1.2 \mathrm{E}+01$ & $4.9 \mathrm{E}-09$ & $5.6 \mathrm{E}-08$ & & & & \\
\hline${ }^{\infty} \mathrm{K}$ & $1.2 \mathrm{E}+02$ & $5.4 \mathrm{E}-07$ & $6.7 \mathrm{E}-05$ & & & & \\
\hline${ }^{60} \mathrm{Co}$ & $3.0 \mathrm{E}+\infty 0$ & 8.6E-06 & $2.6 \mathrm{E}-05$ & & & & \\
\hline${ }^{90} \mathrm{Sr}$ & $2.7 \mathrm{E}+02$ & $0.0 \mathrm{E}+\infty$ & $0.0 \mathrm{E}+\infty$ & & & & \\
\hline Tritium & $5.9 \mathrm{E}+02$ & $0.0 \mathrm{E}+00$ & $0.0 \mathrm{E}+00$ & & & & \\
\hline
\end{tabular}




\begin{tabular}{|c|c|c|c|c|c|c|c|}
\hline Contaminant & $\begin{array}{c}\text { Chronic } \\
\text { Daily } \\
\text { Intake }\end{array}$ & $\begin{array}{l}\text { Sope } \\
\text { Factor }\end{array}$ & $\begin{array}{c}\text { Contominant } \\
\text { Specific } \\
\text { Risk }\end{array}$ & $\begin{array}{l}\text { Contaminant } \\
\text { Class Risk }\end{array}$ & $\begin{array}{c}\text { Total } \\
\text { Pathway } \\
\text { Risk }\end{array}$ & $\begin{array}{c}\text { Total } \\
\text { Contaminant } \\
\text { Risk }\end{array}$ & $\begin{array}{l}\text { Totol } \\
\text { Area } \\
\text { Risk }\end{array}$ \\
\hline Total Radionuclides & & & & $6.2 \mathrm{E}-02$ & & & \\
\hline Total Pathway Risk & & & & & $6.2 \mathrm{E}-02$ & & \\
\hline Total Inorganic Risk & & & & & & 2.0E-04 & \\
\hline Total Organic Risk & & & & & & $2.9 E-05$ & \\
\hline Total Radionuclide Risk & & & & & & $6.2 \mathrm{E}-02$ & \\
\hline Total Area & & & & & & & $6.2 \mathrm{E}-02$ \\
\hline \multicolumn{8}{|c|}{ Exposure Pathway: External Exposure to Radionnchides by Adult Trespasser from Direct Measurement Samples - Cell } \\
\hline Beta-Gamma & $2.0 \mathrm{E}+06$ & $1.0 \mathrm{E}+\infty$ & 7.7E-01 & & & & \\
\hline Gamma & $1.8 E+06$ & $1.0 \mathrm{E}+\infty$ & $7.2 \mathrm{E}-01$ & & & & \\
\hline Total Radionuclides & & & & $9.4 \mathrm{E}-01$ & & & \\
\hline Total Pathway Risk & & & & & $9.4 \mathrm{E}-01$ & & \\
\hline Total Radionuclide Risk & & & & & & $9.4 \mathrm{E}-01$ & \\
\hline Total Area & & & & & & & $9.4 \mathrm{E}-01$ \\
\hline \multicolumn{8}{|c|}{ Exposure Pathway: Extermal Exposure to Radionoclides by Adult Trespasser from Direct Measurement Samples - East Gallery } \\
\hline Beta-Gamma & $2.8 \mathrm{E}+04$ & $1.0 \mathrm{E}+00$ & $2.0 \mathrm{E}-02$ & & & & \\
\hline Gamma & $2.5 E+04$ & $1.0 \mathrm{E}+00$ & $1.8 \mathrm{E}-02$ & & & & \\
\hline Total Radionuclides & & & & $3.8 \mathrm{E}-02$ & & & \\
\hline Total Pathway Risk & & & & & $3.8 \mathrm{E}-02$ & & \\
\hline Total Radionuclide Risk & & & & & & $3.8 \mathrm{E}-02$ & \\
\hline Total Area & & & & & & & $3.8 \mathrm{E}-02$ \\
\hline \multicolumn{8}{|c|}{$\begin{array}{l}\text { Exposure Pathway: External Exposure to Radionnclides by Adult Trespasser from Direct Measurement Samples - Whole } \\
\text { Building }\end{array}$} \\
\hline Beta-Gamma & $2.8 \mathrm{E}+06$ & $1.0 \mathrm{E}+00$ & 8.7E-01 & & & & \\
\hline Gamma & $2.6 \mathrm{E}+06$ & $1.0 \mathrm{E}+00$ & 8.5E-01 & & & & \\
\hline Total Radionuclides & & & & $9.8 \mathrm{E}-01$ & & & \\
\hline Total Pathway Risk & & & & & $9.8 \mathrm{E}-01$ & & \\
\hline Total Radionuclide Risk & & & & & & $9.8 \mathrm{E}-01$ & \\
\hline Total Ares & & & & & & & $9.8 \mathrm{E}-01$ \\
\hline \multicolumn{8}{|c|}{ Exposure Pathway: Ingestion of Radionuclides by Adult Trespasser from Sinear Samples - Cell } \\
\hline${ }^{15} \mathrm{Cs}$ & $1.5 \mathrm{E}+05$ & $2.8 \mathrm{E}-11$ & 4.2E-06 & & & & \\
\hline${ }^{2 x} \mathrm{Ra}$ & $5.5 E+00$ & $1.2 \mathrm{E}-10$ & $6.6 \mathrm{E}-10$ & & & & \\
\hline $20 \mathrm{Th}$ & $1.2 \mathrm{E}+01$ & $5.5 E-11$ & $6.4 \mathrm{E}-10$ & & & & \\
\hline${ }^{200} \mathrm{Th}$ & $1.9 \mathrm{E}+01$ & 1.3E-11 & $2.5 \mathrm{E}-10$ & & & & \\
\hline
\end{tabular}




\begin{tabular}{|c|c|c|c|c|c|c|c|}
\hline Cantaminant & $\begin{array}{c}\text { Chronic } \\
\text { Daily } \\
\text { Intake }\end{array}$ & $\begin{array}{l}\text { Sope } \\
\text { Foctor }\end{array}$ & $\begin{array}{c}\text { Contaminant } \\
\text { Specific } \\
\text { Risl }\end{array}$ & $\begin{array}{c}\text { Contaminant } \\
\text { Class Risl }\end{array}$ & $\begin{array}{c}\begin{array}{c}\text { Total } \\
\text { Pathway }\end{array} \\
\text { Risk } \\
\end{array}$ & $\begin{array}{c}\text { Total } \\
\text { Contaminant } \\
\text { Risk }\end{array}$ & $\begin{array}{l}\text { Total } \\
\text { Area } \\
\text { Risk }\end{array}$ \\
\hline${ }^{200} \mathrm{Th}$ & $1.2 \mathrm{E}+01$ & $1.2 \mathrm{E}-11$ & $1.4 \mathrm{E}-10$ & & & & \\
\hline${ }^{232524} U$ & $1.8 \mathrm{E}+01$ & $1.6 \mathrm{E}-11$ & $2.9 \mathrm{E}-10$ & & & & \\
\hline${ }^{25} \mathrm{U}$ & $1.7 \mathrm{E}+00$ & $1.6 \mathrm{E}-11$ & 2.7E-11 & & & & \\
\hline${ }^{22 x \mathrm{Pu}}$ & $8.8 E+00$ & $2.2 \mathrm{E}-10$ & $1.9 \mathrm{E}-09$ & & & & \\
\hline${ }^{28 x} \mathrm{U}$ & $2.3 \mathrm{E}+01$ & $2.0 \mathrm{E}-11$ & 4.7E-10 & & & & \\
\hline${ }^{299200} \mathrm{Pu}$ & $5.5 \mathrm{E}+01$ & 2.3E-10 & 1.3E-08 & & & & \\
\hline${ }^{\infty} \mathrm{K}$ & $5.8 \mathrm{E}+02$ & $1.1 \mathrm{E}-11$ & $6.4 \mathrm{E}-09$ & & & & \\
\hline${ }^{60} \mathrm{Co}$ & $1.4 \mathrm{E}+01$ & $1.5 \mathrm{E}-11$ & $2.1 \mathrm{E}-10$ & & & & \\
\hline${ }^{90} \mathrm{Sr}$ & $1.3 \mathrm{E}+03$ & $3.6 \mathrm{E}-11$ & $4.6 \mathrm{E}-08$ & & & & \\
\hline Total Radionuclides & & & & $4.3 \mathrm{E}-06$ & & & \\
\hline Total Pathway Risk & & & & & 4.3E-06 & & \\
\hline \multicolumn{8}{|c|}{ Exposure Pathway: Inhalation of Radionuclides by Adult Trespasser from Smear Samples Cell } \\
\hline${ }^{15} \mathrm{Cs}$ & $7.0 \mathrm{E}+\infty 0$ & $1.9 \mathrm{E}-11$ & $1.3 \mathrm{E}-10$ & & & & \\
\hline${ }^{20} \mathrm{Ra}$ & $2.6 \mathrm{E}-04$ & $3.0 \mathrm{E}-09$ & $1.1 E-06$ & & & & \\
\hline 220Th & $5.4 \mathrm{E}-04$ & $7.8 \mathrm{E}-08$ & $4.2 \mathrm{E}-11$ & & & & \\
\hline${ }^{200} \mathrm{Th}$ & $9.0 \mathrm{E}-04$ & $2.9 \mathrm{E}-08$ & $2.6 E-11$ & & & & \\
\hline $25 \mathrm{Th}$ & $5.6 \mathrm{E}-04$ & $2.8 \mathrm{E}-08$ & $1.6 \mathrm{E}-11$ & & & & \\
\hline${ }^{293253} \mathrm{U}$ & $8.4 \mathrm{E}-04$ & $2.6 \mathrm{E}-08$ & $2.2 \mathrm{E}-11$ & & & & \\
\hline${ }^{230} \mathrm{U}$ & 7.7E-05 & $2.5 E-08$ & $1.9 \mathrm{E}-12$ & & & & \\
\hline${ }^{23} \mathrm{Pu}$ & 4.1E-04 & $3.9 \mathrm{E}-08$ & $1.6 \mathrm{E}-11$ & & & & \\
\hline${ }^{250} U$ & $1.1 \mathrm{E}-03$ & $2.4 \mathrm{E}-08$ & $2.6 E-11$ & & & & \\
\hline${ }^{298210} \mathrm{Pu}$ & $2.6 E-03$ & $3.8 \mathrm{E}-08$ & $9.8 \mathrm{E}-11$ & & & & . \\
\hline${ }^{40} \mathrm{~K}$ & $2.7 \mathrm{E}-02$ & $7.6 \mathrm{E}-12$ & $2.0 \mathrm{E}-13$ & & & & \\
\hline${ }^{\infty} \mathrm{Co}$ & $6.6 \mathrm{E}-04$ & $1.5 \mathrm{E}-10$ & $9.9 \mathrm{E}-14$ & & & & \\
\hline${ }^{00} \mathrm{Sr}$ & $5.9 \mathrm{E}-02$ & $6.2 \mathrm{E}-11$ & $3.7 \mathrm{E}-12$ & & & & \\
\hline Total Radionuclides & & & & $1.1 \mathrm{E}-06$ & & & \\
\hline Total Pathway Risk & & & & & $1.1 \mathrm{E}-06$ & & \\
\hline \multicolumn{8}{|c|}{ Exposure Pathway: External Exposure to Radionuclides by Adult Trespasser from Smear Samples - Cell } \\
\hline${ }^{17} \mathrm{Cs}$ & $4.1 E+03$ & $2.0 \mathrm{E}-06$ & 8.2E-03 & & & & \\
\hline${ }^{2 x} \mathrm{Ra}$ & $1.5 \mathrm{E}-01$ & $6.0 \mathrm{E}-06$ & $9.1 \mathrm{E}-07$ & & & & \\
\hline $20 \mathrm{Th}$ & $3.2 E-01$ & $5.6 \mathrm{E}-06$ & $1.8 \mathrm{E}-06$ & & & & \\
\hline${ }^{200} \mathrm{Th}$ & $5.3 \mathrm{E}-01$ & $5.4 \mathrm{E}-11$ & 2.9E-11 & & & & \\
\hline${ }^{20} \mathrm{Th}$ & $3.3 \mathrm{E}-01$ & $2.6 \mathrm{E}-11$ & $8.5 \mathrm{E}-12$ & & & & \\
\hline
\end{tabular}




\begin{tabular}{|c|c|c|c|c|c|c|c|}
\hline Contaminant & $\begin{array}{l}\text { Chronic } \\
\text { Daily } \\
\text { Intalce }\end{array}$ & $\begin{array}{l}\text { Sope } \\
\text { Factor }\end{array}$ & $\begin{array}{c}\text { Contaminant } \\
\text { Specific } \\
\text { Risk }\end{array}$ & $\begin{array}{l}\text { Contaminant } \\
\text { Class Risk }\end{array}$ & $\begin{array}{c}\text { Total } \\
\text { Pathway } \\
\text { Risk }\end{array}$ & $\begin{array}{l}\text { Total } \\
\text { Contaminant } \\
\text { Risk }\end{array}$ & $\begin{array}{l}\text { Total } \\
\text { Aren } \\
\text { Risk }\end{array}$ \\
\hline $201234 U$ & 4.9E- 01 & $3.0 \mathrm{E}-11$ & $1.5 \mathrm{E}-11$ & & & & \\
\hline${ }^{25 S} \mathrm{U}$ & $4.5 \mathrm{E}-02$ & $2.4 \mathrm{E}-07$ & $1.1 \mathrm{E}-08$ & & & & \\
\hline${ }^{250} \mathrm{Pu}$ & 2.4E-01 & $2.8 \mathrm{E}-11$ & $6.8 \mathrm{E}-12$ & & & & \\
\hline${ }^{230} \mathrm{U}$ & $6.4 \mathrm{E}-01$ & $5.1 \mathrm{E}-08$ & 3.3E-08 & & & & \\
\hline${ }^{209200 \mathrm{Pu}}$ & $1.5 \mathrm{E}+\infty$ & $1.7 \mathrm{E}-11$ & $2.6 \mathrm{E}-11$ & & & & \\
\hline${ }^{\infty} \mathrm{K}$ & $1.6 \mathrm{E}+01$ & $5.4 \mathrm{E}-07$ & $8.5 E-06$ & & & & \\
\hline${ }^{\infty} \mathrm{Co}$ & $3.9 \mathrm{E}-01$ & $8.6 \mathrm{E}-06$ & $3.3 \mathrm{E}-06$ & & & & \\
\hline${ }^{\infty} S_{r}$ & $3.5 \mathrm{E}+01$ & $0.0 E+\infty$ & $0.0 \mathrm{E}+00$ & & & & \\
\hline Total Radionuclides & & & & $8.2 E-03$ & & & \\
\hline Total Pathway Risk & & & & & $8.2 \mathrm{E}-03$ & & \\
\hline Total Radionuclide Risk & & & & & & $8.2 \mathrm{E}-03$ & \\
\hline Total Area & & & & & & & 8.2E-03 \\
\hline \multicolumn{8}{|c|}{ Exposure Pathway: Ingestion of Radionuclides by Adult Trespasser from Smear Samples - East Gallery } \\
\hline${ }^{17} \mathrm{Cs}$ & $1.6 \mathrm{E}+04$ & $2.8 \mathrm{E}-11$ & $4.5 \mathrm{E}-07$ & & & & \\
\hline${ }^{20} \mathrm{Ra}$ & $1.5 \mathrm{E}+01$ & $1.2 \mathrm{E}-10$ & $1.8 \mathrm{E}-09$ & & & & \\
\hline${ }^{2 *} \mathrm{Ra}$ & $2.5 E+02$ & $1.0 \mathrm{E}-10$ & $2.5 \mathrm{E}-08$ & & & & \\
\hline 200 Th & $2.4 E+01$ & $5.5 \mathrm{E}-11$ & $1.3 \mathrm{E}-09$ & & & & \\
\hline${ }^{200} \mathrm{Th}$ & $5.1 \mathrm{E}+01$ & $1.3 \mathrm{E}-11$ & $6.6 \mathrm{E}-10$ & & & & \\
\hline $200 \mathrm{Th}$ & $1.8 \mathrm{E}+01$ & $1.2 \mathrm{E}-11$ & $2.2 \mathrm{E}-10$ & & & & \\
\hline${ }^{2023250} \mathrm{U}$ & $3.8 \mathrm{E}+01$ & $1.6 \mathrm{E}-11$ & $6.1 \mathrm{E}-10$ & & & & \\
\hline${ }^{25} \mathrm{U}$ & $2.6 \mathrm{E}+\infty$ & $1.6 \mathrm{E}-11$ & $4.1 \mathrm{E}-11$ & & & & \\
\hline${ }^{210} \mathrm{Pu}$ & $1.7 E+01$ & $2.2 \mathrm{E}-10$ & $3.6 \mathrm{E}-09$ & & & & $\dot{-}$ \\
\hline 200 & $3.0 \mathrm{E}+01$ & $2.0 \mathrm{E}-11$ & $5.9 \mathrm{E}-10$ & & & & \\
\hline${ }^{20250} \mathrm{Pu}$ & $1.7 \mathrm{E}+01$ & 2.3E-10 & $3.8 \mathrm{E}-09$ & & & & \\
\hline${ }^{21} \mathrm{Am}$ & $2.1 E+01$ & 2.4E-10 & $5.1 \mathrm{E}-09$ & & & & \\
\hline$\infty_{\mathrm{K}}$ & $3.8 \mathrm{E}+03$ & $1.1 \mathrm{E}-11$ & $4.2 \mathrm{E}-08$ & & & & \\
\hline${ }^{\circ} \mathrm{Sr}$ & $5.7 \mathrm{E}+03$ & $3.6 \mathrm{E}-11$ & 2.1E-07 & & & & \\
\hline Total Radionuciides & & & & $7.4 \mathrm{E}-07$ & & & \\
\hline Total Pathway Risk & & & & & $7.4 \mathrm{E}-07$ & & \\
\hline \multicolumn{8}{|c|}{ Exposure Pathway: Inhalation of Radionuclides by Adult Trespasser from Smear Samples - East Gallery } \\
\hline${ }^{15} \mathrm{Cs}$ & $7.5 \mathrm{E}-01$ & $1.9 \mathrm{E}-11$ & $1.4 \mathrm{E}-11$ & & & & \\
\hline 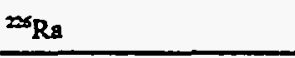 & $7.2 \mathrm{E}-04$ & $3.0 \mathrm{E}-09$ & $3.0 \mathrm{E}-06$ & & & & \\
\hline$=2 \mathrm{Ra}$ & $1.2 \mathrm{E}-02$ & $6.9 \mathrm{E}-10$ & $8.2 \mathrm{E}-12$ & & & & \\
\hline
\end{tabular}




\begin{tabular}{|c|c|c|c|c|c|c|c|}
\hline Contaminant & $\begin{array}{l}\text { Chronic } \\
\text { Daily } \\
\text { Intake }\end{array}$ & $\begin{array}{l}\text { Slope } \\
\text { Factor }\end{array}$ & $\begin{array}{l}\text { Contaminant } \\
\text { Specific } \\
\text { Risk }\end{array}$ & $\begin{array}{l}\text { Contaminant } \\
\text { Class Risk }\end{array}$ & $\begin{array}{c}\text { Total } \\
\text { Pathway } \\
\text { Risk }\end{array}$ & $\begin{array}{c}\text { Totol } \\
\text { Contaminant } \\
\text { Risk }\end{array}$ & $\begin{array}{l}\text { Total } \\
\text { Area } \\
\text { Risk }\end{array}$ \\
\hline${ }^{20} \mathrm{Th}$ & $1.1 \mathrm{E}-03$ & $7.8 \mathrm{E}-08$ & $8.6 E-11$ & & & & \\
\hline${ }^{200} \mathrm{Th}$ & $2.4 \mathrm{E}-03$ & $2.9 \mathrm{E}-08$ & $6.9 E-11$ & & & & \\
\hline${ }^{200} \mathrm{Th}$ & $8.5 \mathrm{E}-04$ & $2.8 \mathrm{E}-08$ & $2.4 E-11$ & & & & \\
\hline $235234 \mathrm{U}$ & $1.8 \mathrm{E}-03$ & $2.6 \mathrm{E}-08$ & $4.6 E-11$ & & & & \\
\hline${ }^{235} \mathrm{U}$ & $1.2 \mathrm{E}-04$ & 2.5E-08 & $3.0 \mathrm{E}-12$ & & & & \\
\hline${ }^{2 s 0} \mathrm{Pu}$ & 7.7E-04 & $3.9 \mathrm{E}-08$ & 3.0E-11 & & & & \\
\hline${ }^{210} U$ & $1.4 \mathrm{E}-03$ & 2.4E-08 & $3.3 \mathrm{E}-11$ & & & & \\
\hline${ }^{2591200} \mathrm{Pu}$ & 7.7E-04 & $3.8 \mathrm{E}-08$ & $2.9 \mathrm{E}-11$ & & & & \\
\hline${ }^{211} \mathrm{Am}$ & $9.9 \mathrm{E}-04$ & $3.2 \mathrm{E}-08$ & $3.2 \mathrm{E}-11$ & & & & \\
\hline${ }^{+\infty} \mathrm{K}$ & $1.8 \mathrm{E}-01$ & $7.6 \mathrm{E}-12$ & $1.3 \mathrm{E}-12$ & & & & \\
\hline${ }^{90} \mathrm{Sr}$ & 2.7E-01 & $6.2 \mathrm{E}-11$ & 1.7E-11 & & & & \\
\hline Total Radionuclides & & & & $3.0 \mathrm{E}-06$ & & & \\
\hline Total Pathway Risk & & & & & $3.0 \mathrm{E}-06$ & & \\
\hline \multicolumn{8}{|c|}{ Exposure Pathway: External Exposure to Radionuclides by Adult Trespasser from Smear Samples - East Gallery } \\
\hline${ }^{197} \mathrm{Cs}$ & $4.4 \mathrm{E}+02$ & 2.0E-06 & $8.8 \mathrm{E}-04$ & & & & \\
\hline${ }^{208} \mathbf{R a}$ & $4.2 \mathrm{E}-01$ & $6.0 \mathrm{E}-06$ & $2.5 \mathrm{E}-06$ & & & & \\
\hline $220 \mathrm{Ra}$ & $7.0 \mathrm{E}+\infty$ & 2.9E-06 & $2.0 \mathrm{E}-05$ & & & & \\
\hline $2 \mathrm{Th}$ & $6.5 \mathrm{E}-01$ & $5.6 \mathrm{E}-06$ & $3.6 \mathrm{E}-06$ & & & & \\
\hline${ }^{200} \mathrm{Th}$ & $1.4 E+\infty 0$ & $5.4 \mathrm{E}-11$ & $7.5 \mathrm{E}-11$ & & & & \\
\hline $200 \mathrm{Th}$ & $5.0 \mathrm{E}-01$ & $2.6 \mathrm{E}-11$ & $1.3 \mathrm{E}-11$ & & & & \\
\hline $231254 \mathrm{U}$ & $1.0 \mathrm{E}+\infty 0$ & 3.0E-11 & $3.1 \mathrm{E}-11$ & & & & \\
\hline${ }^{215 U}$ & $7.0 \mathrm{E}-02$ & $2.4 \mathrm{E}-07$ & 1.7E-08 & & & & . \\
\hline${ }^{250} \mathrm{Pu}$ & $4.5 \mathrm{E}-01$ & $2.8 \mathrm{E}-11$ & $1.3 \mathrm{E}-11$ & & & & \\
\hline${ }^{24} \mathrm{U}$ & 8.1E-01 & 5.1E-08 & $4.2 \mathrm{E}-08$ & & & & \\
\hline${ }^{298 n} \mathrm{Pu}$ & $4.5 \mathrm{E}-01$ & 1.7E-11 & 7.7E-12 & & & & \\
\hline${ }^{211} \mathrm{Am}$ & $5.8 \mathrm{E}-01$ & $4.9 \mathrm{E}-09$ & $2.8 \mathrm{E}-09$ & & & & \\
\hline${ }^{\infty} \mathrm{K}$ & $1.0 \mathrm{E} \div 02$ & $5.4 E-07$ & $5.6 \mathrm{E}-05$ & & & & \\
\hline${ }^{\infty} \mathrm{Sr}$ & $1.6 \mathrm{E}+02$ & $0.0 \mathrm{E}+00$ & $0.0 \mathrm{E}+\infty 0$ & & & & \\
\hline Total Redionuclides & & & & $9.6 \mathrm{E}-04$ & & & \\
\hline Total Pathway Risk & & & & & $9.6 \mathrm{E}-04$ & & \\
\hline Total Radionuclide Risk & & & & & & $9.7 \mathrm{E}-04$ & \\
\hline Toul Area & & & & & & & 9.7E-04 \\
\hline
\end{tabular}




\begin{tabular}{|c|c|c|c|c|c|c|c|}
\hline Contaminant & $\begin{array}{l}\text { Chronic } \\
\text { Daily } \\
\text { Intake }\end{array}$ & $\begin{array}{l}\text { Sope } \\
\text { Factor }\end{array}$ & $\begin{array}{l}\text { Contaminant } \\
\text { Specific } \\
\text { Risl }\end{array}$ & $\begin{array}{l}\text { Contaminnnt } \\
\text { Class Risk }\end{array}$ & $\begin{array}{c}\text { Total } \\
\text { Pathway } \\
\text { Risk }\end{array}$ & $\begin{array}{c}\text { Total } \\
\text { Contaminant } \\
\text { Risk }\end{array}$ & $\begin{array}{l}\text { Total } \\
\text { Area } \\
\text { Risk }\end{array}$ \\
\hline \multicolumn{8}{|c|}{ Exposure Pathway: Ingestion of Radionnclides by Adult Trespasser from Smear Samples - Whole Building } \\
\hline${ }^{13} \mathrm{Cs}$ & $8.4 \mathrm{E}+04$ & $2.8 \mathrm{E}-11$ & $2.4 \mathrm{E}-06$ & & & & \\
\hline${ }^{20} \mathrm{Ra}$ & $6.2 \mathrm{E}+\infty$ & $1.2 \mathrm{E}-10$ & 7.5E-10 & & & & \\
\hline${ }^{20} \mathbf{R a}$ & $3.0 \mathrm{E}+01$ & $1.0 \mathrm{E}-10$ & $3.0 \mathrm{E}-09$ & & & & \\
\hline $20 \mathrm{Th}$ & $1.2 \mathrm{E}+01$ & $5.5 \mathrm{E}-11$ & $6.5 \mathrm{E}-10$ & & & & \\
\hline${ }^{200} \mathrm{Th}$ & $2.1 \mathrm{E}+01$ & $1.3 \mathrm{E}-11$ & 2.7E-10 & & & & \\
\hline${ }^{200} \mathrm{Th}$ & $1.2 \mathrm{E}+01$ & $1.2 \mathrm{E}-11$ & $1.4 \mathrm{E}-10$ & & & & \\
\hline${ }^{234234} \mathrm{U}$ & $1.9 \mathrm{E}+01$ & $1.6 \mathrm{E}-11$ & $3.0 \mathrm{E}-10$ & & & & \\
\hline 250 & $1.9 \mathrm{E}+\infty 0$ & $1.6 \mathrm{E}-11$ & $3.0 \mathrm{E}-11$ & & & & \\
\hline${ }^{23} \mathrm{Pu}$ & $9.9 \mathrm{E}+00$ & $2.2 \mathrm{E}-10$ & 2.2E-09 & & & & \\
\hline 200 & $2.6 \mathrm{E}+01$ & $2.0 \mathrm{E}-11$ & 5.3E-10 & & & & \\
\hline${ }^{299200} \mathrm{Pu}$ & $6.1 \mathrm{E}+01$ & $2.3 \mathrm{E}-10$ & $1.4 \mathrm{E}-08$ & & & & \\
\hline${ }^{211} \mathrm{Am}$ & $7.7 \mathrm{E}+\infty$ & $2.4 \mathrm{E}-10$ & $1.9 \mathrm{E}-09$ & & & & \\
\hline${ }^{10} \mathrm{~K}$ & $4.4 \mathrm{E}+02$ & $1.1 \mathrm{E}-11$ & $4.9 \mathrm{E}-09$ & & & & \\
\hline${ }^{\infty} \mathrm{Co}$ & $1.1 E+01$ & $1.5 \mathrm{E}-11$ & $1.6 \mathrm{E}-10$ & & & & \\
\hline${ }^{\infty} \mathrm{Sr}$ & $9.6 \mathrm{E}+02$ & $3.6 \mathrm{E}-11$ & $3.4 \mathrm{E}-08$ & & & & \\
\hline Total Radionuclides & & & & $2.4 \mathrm{E}-06$ & & & \\
\hline Total Pathway Risk & & & & & $2.4 \mathrm{E}-06$ & & \\
\hline \multicolumn{8}{|c|}{ Exposure Patbway: Inhalation of Radionuclides by Adult Trespasser from Smear Samples - Whole Building } \\
\hline${ }^{17} \mathrm{Cs}$ & $3.9 \mathrm{E}+\infty$ & $1.9 \mathrm{E}-11$ & $7.5 \mathrm{E}-11$ & & & & \\
\hline${ }^{2 x} \mathrm{Ra}$ & $2.9 \mathrm{E}-04$ & $3.0 \mathrm{E}-09$ & $1.2 \mathrm{E}-06$ & & & & \\
\hline $2 \pi \mathrm{Ra}$ & $1.4 \mathrm{E}-03$ & $6.9 \mathrm{E}-10$ & $9.5 \mathrm{E}-13$ & & & & . \\
\hline $20 \mathrm{Th}$ & $5.5 E-04$ & $7.8 \mathrm{E}-08$ & 4.3E-11 & & & & \\
\hline $20 \mathrm{Th}$ & $9.9 \mathrm{E}-04$ & $2.9 \mathrm{E}-0.8$ & $2.9 E-11$ & & & & \\
\hline $200 \mathrm{Th}$ & $5.6 \mathrm{E}-04$ & $2.8 \mathrm{E}-08$ & $1.6 \mathrm{E}-11$ & & & & \\
\hline${ }^{231254} \mathrm{U}$ & $8.7 \mathrm{E}-04$ & $2.6 \mathrm{E}-08$ & $2.3 \mathrm{E}-11$ & & & & \\
\hline WU & 8.7E-05 & $2.5 \mathrm{E}-08$ & 2.2E-12 & & & & \\
\hline${ }^{24} \mathrm{Pu}$ & $4.6 \mathrm{E}-04$ & $3.9 \mathrm{E}-08$ & $1.8 \mathrm{E}-11$ & & & & \\
\hline $200 \mathrm{U}$ & $1.2 \mathrm{E}-03$ & $2.4 \mathrm{E}-08$ & 3.0E-11 & & & & \\
\hline${ }^{259200} \mathrm{Pu}$ & $2.8 \mathrm{E}-03$ & $3.8 \mathrm{E}-08$ & $1.1 \mathrm{E}-10$ & & & & \\
\hline${ }^{2 n} \mathrm{Am}$ & $3.6 \mathrm{E}-04$ & 3.2E- -08 & $1.2 \mathrm{E}-11$ & & & & \\
\hline${ }^{\infty} \mathrm{K}$ & $2.1 \mathrm{E}-02$ & $7.6 \mathrm{E}-12$ & $1.6 \mathrm{E}-13$ & & & & \\
\hline${ }^{50} \mathrm{Co}$ & $5.0 \mathrm{E}-04$ & $1.5 \mathrm{E}-10$ & $7.5 \mathrm{E}-14$ & & & & \\
\hline
\end{tabular}




\begin{tabular}{|c|c|c|c|c|c|c|c|}
\hline Contominant & $\begin{array}{c}\text { Chronic } \\
\text { Daily } \\
\text { Intake }\end{array}$ & $\begin{array}{l}\text { Slope } \\
\text { Factor }\end{array}$ & $\begin{array}{l}\text { Contaminant } \\
\text { Specific } \\
\text { Risk }\end{array}$ & $\begin{array}{l}\text { Contaminant } \\
\text { Class Risk }\end{array}$ & $\begin{array}{c}\text { Totol } \\
\text { Pathway } \\
\text { Risk }\end{array}$ & $\begin{array}{c}\text { Total } \\
\text { Contaminant } \\
\text { Risk }\end{array}$ & $\begin{array}{l}\text { Total } \\
\text { Area } \\
\text { Risk }\end{array}$ \\
\hline${ }^{\infty} \mathrm{Sr}$ & 4.5E-02 & $6.2 \mathrm{E}-11$ & $2.8 \mathrm{E}-12$ & & & & \\
\hline Total Radionuclides & & & & $1.2 \mathrm{E}-06$ & & & \\
\hline Total Pathway Risk & & & & & $1.2 \mathrm{E}-06$ & & \\
\hline \multicolumn{8}{|c|}{ Exposure Pathway: External Exposure to Radionuclides by Adult Trespasser from Smear Samples - Whole Buiding } \\
\hline${ }^{197} \mathrm{Cs}$ & $2.3 \mathrm{E}+03$ & $2.0 \mathrm{E}-06$ & $4.6 \mathrm{E}-03$ & & & & \\
\hline${ }^{206} \mathrm{Ra}$ & $1.7 \mathrm{E}-01$ & $6.0 \mathrm{E}-06$ & $1.0 \mathrm{E}-06$ & & & & \\
\hline 2ra & 8.1E-01 & 2.9E-06 & $2.4 \mathrm{E}-06$ & & & & \\
\hline $22 \mathrm{Th}$ & $3.3 \mathrm{E}-01$ & $5.6 \mathrm{E}-06$ & $1.8 \mathrm{E}-06$ & & & & \\
\hline${ }^{250} \mathrm{Th}$ & $5.8 \mathrm{E}-01$ & 5.4E-11 & $3.1 E-11$ & & & & \\
\hline${ }^{200} \mathrm{Th}$ & $3.3 \mathrm{E}-01$ & $2.6 \mathrm{E}-11$ & $8.5 \mathrm{E}-12$ & & & & \\
\hline${ }^{23 \times 234} \mathrm{U}$ & $5.1 E-01$ & $3.0 \mathrm{E}-11$ & $1.5 \mathrm{E}-11$ & & & & \\
\hline${ }^{219} \mathrm{U}$ & $5.1 \mathrm{E}-02$ & $2.4 \mathrm{E}-07$ & $1.2 \mathrm{E}-08$ & & & & \\
\hline${ }^{2 s 0} \mathrm{Pu}$ & 2.7E-01 & $2.8 \mathrm{E}-11$ & $7.6 \mathrm{E}-12$ & & & & \\
\hline${ }^{22010} \mathrm{U}$ & $7.2 \mathrm{E}-01$ & $5.1 \mathrm{E}-08$ & 3.7E-08 & & & & \\
\hline${ }^{299200} \mathrm{Pu}$ & $1.7 \mathrm{E}+\infty 0$ & $1.7 \mathrm{E}-11$ & $2.8 \mathrm{E}-11$ & & & & \\
\hline${ }^{2 n} \mathrm{Am}$ & $2.1 \mathrm{E}-01$ & $4.9 E-09$ & $1.0 \mathrm{E}-09$ & & & & \\
\hline${ }^{10} \mathrm{~K}$ & $1.2 \mathrm{E}+01$ & $5.4 \mathrm{E}-07$ & $6.6 \mathrm{E}-06$ & & & & \\
\hline${ }^{60} \mathrm{Co}$ & $2.9 \mathrm{E}-01$ & $8.6 \mathrm{E}-06$ & $2.5 E-06$ & & & & \\
\hline${ }^{\infty} \mathrm{Sr}$ & $2.6 \mathrm{E}+01$ & $0.0 \mathrm{E}+\infty 0$ & $0.0 \mathrm{E}+00$ & & & & \\
\hline Total Radionuclides & & & & $4.6 \mathrm{E}-03$ & & & \\
\hline Total Pathway Risk & & & & & $4.6 \mathrm{E}-03$ & & \\
\hline Total Radionuclide Risk & & & & & & $4.6 \mathrm{E}-03$ & - \\
\hline Total Area & & & & & & & $4.6 E-03$ \\
\hline \multicolumn{8}{|c|}{ Exposure Pathway: External Exposure to Radionoclides by Adult Trespasser from TLD Measurements - Cell } \\
\hline Beta & $5.9 \mathrm{E}+06$ & $1.0 \mathrm{E}+\infty 0$ & $9.9 \mathrm{E}-01$ & & & & \\
\hline Gamms & $1.8 \mathrm{E}+06$ & $1.0 \mathrm{E}+\infty$ & $7.3 \mathrm{E}-01$ & & & & \\
\hline Total Radionuclides & & & & $1.0 \mathrm{E}+00$ & & & \\
\hline Total Pathway Risk & & & & & $1.0 \mathrm{E}+00$ & & \\
\hline Total Radionuclide Risk & & & & & & $1.0 \mathrm{E}+\infty$ & \\
\hline Total Ares & & & & & & & $1.0 \mathrm{E}+\infty 0$ \\
\hline
\end{tabular}


THIS PAGE INTENTIONALLY LEFT BLANK 
Table E-6. Hazard Results - Noncarcinogenic Effects (Adult Trespasser)

\begin{tabular}{|c|c|c|c|c|c|c|c|}
\hline \multirow{2}{*}{ Chemcial } & \multirow{2}{*}{$\begin{array}{c}\text { Average } \\
\text { Daily Intalke } \\
\text { (mg/kg-day) }\end{array}$} & \multirow{2}{*}{$\begin{array}{c}\text { Reference } \\
\text { Dose } \\
\text { (mg/kg-day) }\end{array}$} & \multicolumn{5}{|c|}{ Hazard Quotient/ Hazard Index } \\
\hline & & & $\begin{array}{c}\text { Contaminant } \\
\text { Specific }\end{array}$ & $\begin{array}{c}\text { Contominant } \\
\text { Class }\end{array}$ & Pathway & $\begin{array}{c}\text { Contominant } \\
\text { Class Total }\end{array}$ & $\begin{array}{l}\text { Total } \\
\text { Area } \\
\end{array}$ \\
\hline \multicolumn{8}{|c|}{ Exposure Pathway: Ingestion of Chemicals by Adult Trespasser from Core Samples - Cell } \\
\hline Arsenic & $3.6 \mathrm{E}-06$ & 3.0E-04 & $1.2 \mathrm{E}-02$ & & & & \\
\hline Barium & $9.9 \mathrm{E}-04$ & $7.0 \mathrm{E}-02$ & $1.4 \mathrm{E}-02$ & & & & \\
\hline Beryllium & 2.4E-05 & $5.0 \mathrm{E}-03$ & $4.8 \mathrm{E}-03$ & & & & \\
\hline Cadmium & 2.5E-04 & $1.0 \mathrm{E}-03$ & 2.5E-01 & & & & \\
\hline Chromium VI & 2.7E-04 & $5.0 \mathrm{E}-03$ & $5.4 \mathrm{E}-02$ & & & & \\
\hline Manganese & $1.3 \mathrm{E}-02$ & $1.4 \mathrm{E}-01$ & $9.3 \mathrm{E}-02$ & & & & \\
\hline Mercury & 4.7E-07 & $3.0 \mathrm{E}-04$ & $1.6 \mathrm{E}-03$ & & & & \\
\hline Nickel & $1.9 \mathrm{E}-04$ & $2.0 \mathrm{E}-02$ & $9.5 \mathrm{E}-03$ & & & & \\
\hline Vanadium & 3.3E-04 & 7.0E-03 & 4.7E-02 & & & & \\
\hline Zinc & $4.8 \mathrm{E}-02$ & $3.0 \mathrm{E}-01$ & $1.6 \mathrm{E}-01$ & & & & \\
\hline Total Inorganics & & & & $6.4 \mathrm{E}-01$ & & & \\
\hline Pathway Hazard Index & & & & & 6.4E-01 & & \\
\hline \multicolumn{8}{|c|}{ Exposure Pathway: Dermol Exposure of Chemicals to Adult Trespasser from Core Samples - Cell } \\
\hline Arsenic & $1.9 \mathrm{E}-07$ & $1.2 \mathrm{E}-04$ & $1.5 \mathrm{E}-03$ & & & & \\
\hline Barium & $5.2 \mathrm{E}-05$ & $4.9 \mathrm{E}-03$ & $1.1 \mathrm{E}-02$ & & & & \\
\hline Beryllium & $1.3 \mathrm{E}-06$ & $5.0 \mathrm{E}-05$ & $2.6 \mathrm{E}-02$ & & & & \\
\hline Cadmium & $1.3 \mathrm{E}-05$ & $1.0 \mathrm{E}-05$ & $1.3 \mathrm{E}+00$ & & & & \\
\hline Chromium VI & $1.4 \mathrm{E}-05$ & $1.0 \mathrm{E}-04$ & $1.4 \mathrm{E}-01$ & & & & \\
\hline Manganese & $6.9 \mathrm{E}-04$ & $5.6 \mathrm{E}-03$ & $1.2 \mathrm{E}-01$ & & & - & \\
\hline Mercury & $2.5 E-08$ & $3.0 \mathrm{E}-08$ & 8.2E-01 & & & & \\
\hline Nickel & $1.0 \mathrm{E}-05$ & $5.4 \mathrm{E}-03$ & $1.9 \mathrm{E}-03$ & & & & \\
\hline Vanadium & 1.7E-05 & $7.0 \mathrm{E}-05$ & 2.5E-01 & & & & \\
\hline Zinc & $2.5 E-03$ & $6.0 \mathrm{E}-02$ & $4.2 \mathrm{E}-02$ & & & & \\
\hline Total Inorganics & & & & $2.7 \mathrm{E}+00$ & & & \\
\hline Pathway Hazard Index & & & & & $2.7 \mathrm{E}+00$ & & \\
\hline \multicolumn{8}{|c|}{ Exposure Pathway: Inhalation of Chemicals by Adult Trespasser from Core Samples - Cell } \\
\hline Barium & $4.6 \mathrm{E}-08$ & $1.4 \mathrm{E}-04$ & $3.2 \mathrm{E}-04$ & & & & \\
\hline Manganese & $6.1 \mathrm{E}-07$ & $1.4 \mathrm{E}-05$ & $4.3 \mathrm{E}-02$ & & & & \\
\hline Mercury & $2.2 \mathrm{E}-11$ & $8.6 \mathrm{E}-05$ & 2.5E-07 & & & & \\
\hline
\end{tabular}




\begin{tabular}{|c|c|c|c|c|c|c|c|}
\hline \multirow{2}{*}{ Chemcial } & \multirow{2}{*}{$\begin{array}{c}\text { Average } \\
\text { Daily Intake } \\
\text { (mg/k8-day) }\end{array}$} & \multirow{2}{*}{$\begin{array}{c}\text { Reference } \\
\text { Dose } \\
\text { (mg/kg-day) }\end{array}$} & \multicolumn{5}{|c|}{ Hazard Quotient/ Hazard Index } \\
\hline & & & $\begin{array}{l}\text { Contrminant } \\
\text { Specific }\end{array}$ & $\begin{array}{c}\text { Contaminant } \\
\text { Class }\end{array}$ & Pathway & $\begin{array}{l}\text { Contominant } \\
\text { Class Total }\end{array}$ & $\begin{array}{l}\text { Total } \\
\text { Area }\end{array}$ \\
\hline Total Inorganics & & & & 4.3E-02 & & & \\
\hline Pathway Hazard Index & & & & & 4.3E-02 & & \\
\hline Total Inorganic Risk & & & & & & $3.4 \mathrm{E}+00$ & \\
\hline Total Area & & & & & & & $3.4 \mathrm{E}+\infty$ \\
\hline \multicolumn{8}{|c|}{ Exposure Pathway: Ingestion of Chemicals by Adult Trespasser from Core Samples - East Gallery } \\
\hline Arsenic & 4.1E-06 & 3.0E-04 & $1.4 E-02$ & & & & \\
\hline Barium & $2.9 \mathrm{E}-05$ & $7.0 \mathrm{E}-02$ & $4.2 \mathrm{E}-04$ & . & & & \\
\hline Beryllium & 5.5E-07 & 5.0E-03 & $1.1 \mathrm{E}-04$ & & & & \\
\hline Cadmium & 7.0E-06 & $1.0 \mathrm{E}-03$ & $7.0 \mathrm{E}-03$ & & & & \\
\hline Chromium VI & 1.1E-05 & $5.0 \mathrm{E}-03$ & $2.3 \mathrm{E}-03$ & & & & \\
\hline Manganese & $3.6 \mathrm{E}-04$ & $1.4 \mathrm{E}-01$ & $2.6 \mathrm{E}-03$ & & & & \\
\hline Nickel & 8.2E-06 & $2.0 \mathrm{E}-02$ & $4.1 \mathrm{E}-04$ & & & & \\
\hline Vansdium & $1.2 \mathrm{E}-05$ & $7.0 \mathrm{E}-03$ & $1.8 \mathrm{E}-03$ & & & & \\
\hline Zinc & $1.3 E-03$ & 3.0E-01 & $4.5 \mathrm{E}-03$ & & & & \\
\hline Total Inorganics & & & & $3.3 \mathrm{E}-02$ & & & \\
\hline Bis(2-ethylhexyl)phthalate & $3.3 \mathrm{E}-07$ & $2.0 \mathrm{E}-02$ & $1.6 \mathrm{E}-05$ & & & & \\
\hline Dimethyl phthalate & 7.5E-08 & $1.0 \mathrm{E}+01$ & 7.5E-09 & & & & \\
\hline Total Organics & & & & $1.6 \mathrm{E}-05$ & & & \\
\hline Pathway Hazard Index & & & & & $3.3 \mathrm{E}-02$ & & \\
\hline \multicolumn{8}{|c|}{ Exposure Pathway: Dermal Exposure of Chemicals to Adult Trespasser from Core Samples - East Gallery } \\
\hline Arsenic & $2.2 \mathrm{E}-07$ & $1.2 \mathrm{E}-04$ & $1.8 \mathrm{E}-03$ & & & & \\
\hline Barium & $1.6 \mathrm{E}-06$ & $4.9 \mathrm{E}-03$ & $3.2 \mathrm{E}-04$ & & & & \\
\hline Beryllium & $2.9 \mathrm{E}-08$ & $5.0 E-05$ & $5.8 \mathrm{E}-04$ & & & & \\
\hline Cadmium & $3.7 \mathrm{E}-07$ & $1.0 \mathrm{E}-05$ & $3.7 E-02$ & & & & \\
\hline Chromium VI & $6.0 \mathrm{E}-07$ & $1.0 \mathrm{E}-04$ & $6.0 \mathrm{E}-03$ & & & & \\
\hline Manganese & $1.9 \mathrm{E}-05$ & $5.6 \mathrm{E}-03$ & $3.4 \mathrm{E}-03$ & & & & \\
\hline Nickel & 4.4E-07 & $5.4 E-03$ & 8.1E-05 & & & & \\
\hline Vanadium & $6.6 \mathrm{E}-07$ & 7.0E-05 & $9.4 \mathrm{E}-03$ & & & & \\
\hline Zine & 7.1E-05 & $6.0 \mathrm{E}-02$ & $1.2 \mathrm{E}-03$ & & & & \\
\hline Total Inorganics & & & & $6.0 \mathrm{E}-02$ & & & \\
\hline Bis(2-ethylhexyl)phthalate & $1.7 \mathrm{E}-07$ & $3.8 \mathrm{E}-03$ & $4.6 \mathrm{E}-05$ & & & & \\
\hline Dimethyl phthalate & $4.0 \mathrm{E}-08$ & $1.0 \mathrm{E}+01$ & 4.0E-09 & & & & \\
\hline
\end{tabular}




\begin{tabular}{|c|c|c|c|c|c|c|c|}
\hline \multirow{2}{*}{ Chemcial } & \multirow{2}{*}{$\begin{array}{c}\text { Average } \\
\text { Daily Intoke } \\
\text { (mg/kg-day) }\end{array}$} & \multirow{2}{*}{$\begin{array}{l}\text { Reference } \\
\text { Dose } \\
\text { (mg/kg-day) }\end{array}$} & \multicolumn{5}{|c|}{ Hazand Quotient/ Hazand Index } \\
\hline & & & $\begin{array}{l}\text { Contominant } \\
\text { Specific }\end{array}$ & $\begin{array}{c}\text { Contaminant } \\
\text { Class }\end{array}$ & Pathway & $\begin{array}{c}\text { Contaminant } \\
\text { Class Total }\end{array}$ & $\begin{array}{l}\text { Total } \\
\text { Area }\end{array}$ \\
\hline Total Organics & & & & 4.6E-05 & & & \\
\hline Pathway Hazard Index & & & & & $6.0 \mathrm{E}-02$ & & \\
\hline \multicolumn{8}{|c|}{ Exposure Pathway: Inhalation of Chemicals by Adult Trespasser from Core Samples - East Gallery } \\
\hline Barium & $1.4 \mathrm{E}-09$ & 1.4E-04 & 9.6E-06 & & & & \\
\hline Manganese & $1.7 \mathrm{E}-08$ & $1.4 \mathrm{E}-05$ & $1.2 \mathrm{E}-03$ & & & & \\
\hline Total Inorganics & & & & $1.2 \mathrm{E}-03$ & & & \\
\hline Pathway Hazard Index & & & & . & $1.2 \mathrm{E}-03$ & & \\
\hline Total Inorganic Risk & & & & & & $9.4 \mathrm{E}-02$ & \\
\hline Total Organic Risk & & & & & & $6.2 \mathrm{E}-05$ & \\
\hline Total Area & & & & & & & 9.4E-02 \\
\hline \multicolumn{8}{|c|}{ Exposure Pathway: Ingestion of Chemicals by Adult Trespasser from Sediment in Cen } \\
\hline Antimony & $5.0 \mathrm{E}-05$ & $4.0 \mathrm{E}-04$ & $1.2 \mathrm{E}-01$ & & & & \\
\hline Arsenic & $2.9 \mathrm{E}-05$ & 3.0E-04 & $9.8 \mathrm{E}-02$ & & & & \\
\hline Barium & $1.6 \mathrm{E}-04$ & $7.0 \mathrm{E}-02$ & 2.3E-03 & & & & \\
\hline Cadmium & 8.2E-05 & $1.0 \mathrm{E}-03$ & $8.2 \mathrm{E}-02$ & & & & \\
\hline Chromium VI & $2.6 \mathrm{E}-04$ & $5.0 \mathrm{E}-03$ & $5.2 \mathrm{E}-02$ & & & & \\
\hline Cyanide & $7.9 \mathrm{E}-07$ & $2.0 \mathrm{E}-02$ & 4.0E-05 & & & & \\
\hline Manganese & $1.8 \mathrm{E}-03$ & $1.4 \mathrm{E}-01$ & $1.3 \mathrm{E}-02$ & & & & \\
\hline Mercury & $2.6 \mathrm{E}-05$ & $3.0 \mathrm{E}-04$ & $8.5 E-02$ & & & & \\
\hline Nickel & 2.3E-04 & $2.0 \mathrm{E}-02$ & $1.2 \mathrm{E}-02$ & & & & \\
\hline Selenium & $1.2 \mathrm{E}-05$ & $5.0 \mathrm{E}-03$ & $2.4 \mathrm{E}-04$ & & & & \\
\hline Zine & $1.0 \mathrm{E}-02$ & $3.0 \mathrm{E}-01$ & $3.4 \mathrm{E}-02$ & & & & \\
\hline Total Inorganics & & & & $5.0 \mathrm{E}-01$ & & & \\
\hline Acenaphthene & $1.3 \mathrm{E}-06$ & $6.0 \mathrm{E}-02$ & $2.2 \mathrm{E}-05$ & & & & \\
\hline Acetone & $1.2 \mathrm{E}-07$ & $1.0 \mathrm{E}-01$ & $1.2 \mathrm{E}-06$ & & & & \\
\hline Anthracene & $2.6 \mathrm{E}-06$ & $3.0 \mathrm{E}-01$ & 8.7E-06 & & & & \\
\hline Bis(2-ethylhexyl)phthalate & $2.9 \mathrm{E}-06$ & $2.0 \mathrm{E}-02$ & $1.4 \mathrm{E}-04$ & & & & \\
\hline Fluoranthene & 3.7E-05 & $4.0 \mathrm{E}-02$ & $9.2 \mathrm{E}-04$ & & & & \\
\hline Fluorene & $1.2 \mathrm{E}-06$ & 4.0E-02 & 2.9E-05 & & & & \\
\hline Pyrene & $3.0 \mathrm{E}-05$ & $3.0 \mathrm{E}-02$ & $1.0 \mathrm{E}-03$ & & & & \\
\hline Total Organics & & & & $2.1 E-03$ & & & \\
\hline Pathway Hazard Index & & & & & $5.0 \mathrm{E}-01$ & & \\
\hline
\end{tabular}




\begin{tabular}{|c|c|c|c|c|c|c|c|}
\hline \multirow{2}{*}{ Chemcial } & \multirow{2}{*}{$\begin{array}{c}\text { Average } \\
\text { Daily Intake } \\
\text { (mg//kg-day) }\end{array}$} & \multirow{2}{*}{$\begin{array}{c}\text { Reference } \\
\text { Dose } \\
\text { (mg/kg-day) }\end{array}$} & \multicolumn{5}{|c|}{ Hazard Quotient/ Hazard Index } \\
\hline & & & $\begin{array}{l}\text { Contaminant: } \\
\text { Specific }\end{array}$ & $\begin{array}{c}\text { Contaminant } \\
\text { Class }\end{array}$ & Pathway & $\begin{array}{c}\text { Contaminant } \\
\text { Class Total }\end{array}$ & $\begin{array}{l}\text { Total } \\
\text { Area }\end{array}$ \\
\hline \multicolumn{8}{|c|}{ Exposure Pathway: Dermal Exposure of Chemicals to Adult Trespasser froin Sediment in Cell } \\
\hline Antimony & $2.6 \mathrm{E}-06$ & $8.0 \mathrm{E}-06$ & $3.3 \mathrm{E}-01$ & & & & \\
\hline Arsenic & $1.6 \mathrm{E}-\infty 6$ & $1.2 \mathrm{E}-04$ & $1.3 \mathrm{E}-02$ & & & & \\
\hline Barium & 8.7E-06 & $4.9 \mathrm{E}-03$ & $1.8 \mathrm{E}-03$ & & & & \\
\hline Cadmium & $4.4 \mathrm{E}-06$ & $1.0 \mathrm{E}-05$ & $4.4 \mathrm{E}-01$ & & & & \\
\hline Chromium VI & $1.4 \mathrm{E}-05$ & $1.0 \mathrm{E}-04$ & $1.4 \mathrm{E}-01$ & & & & \\
\hline Cyanide & $4.2 \mathrm{E}-08$ & $8.0 \mathrm{E}-03$ & $5.3 \mathrm{E}-06$ & & & & \\
\hline Manganese & $9.5 \mathrm{E}-05$ & $5.6 \mathrm{E}-03$ & $1.7 \mathrm{E}-02$ & & & & \\
\hline Mercury & $1.4 \mathrm{E}-06$ & $3.0 \mathrm{E}-08$ & $4.5 \mathrm{E}+01$ & & & & \\
\hline Nickel • & $1.2 \mathrm{E}-05$ & $5.4 \mathrm{E}-03$ & 2.3E-03 & & & & \\
\hline Selenium & $6.3 E-08$ & $2.2 \mathrm{E}-03$ & $2.9 \mathrm{E}-05$ & & & & \\
\hline Zinc & $5.4 \mathrm{E}-04$ & $6.0 \mathrm{E}-02$ & $9.0 \mathrm{E}-03$ & & & & \\
\hline Total Inorganics & & & & $4.6 \mathrm{E}+01$ & & & \\
\hline Acenaphthene & $7.0 \mathrm{E}-07$ & $6.0 \mathrm{E}-02$ & $1.2 \mathrm{E}-05$ & & & & \\
\hline Acetone & $6.5 \mathrm{E}-08$ & $8.3 E-02$ & $7.9 \mathrm{E}-07$ & & & & \\
\hline Anthracene & $1.4 \mathrm{E}-06$ & $2.3 \mathrm{E}-01$ & $6.1 E-06$ & & & & \\
\hline Bis(2-ethylhexyl)phthalate & $1.5 \mathrm{E}-06$ & $3.8 \mathrm{E}-03$ & $4.0 \mathrm{E}-04$ & & & & \\
\hline Fluoranthene & $2.0 \mathrm{E}-05$ & $1.2 \mathrm{E}-02$ & $1.6 \mathrm{E}-03$ & & & & \\
\hline Fluorene & $6.2 \mathrm{E}-07$ & $4.0 \mathrm{E}-02$ & $1.5 \mathrm{E}-05$ & & & & \\
\hline Pyrene & $1.6 \mathrm{E}-05$ & $9.3 E-03$ & $1.7 \mathrm{E}-03$ & & & & \\
\hline Total Organics & & & & $3.7 \mathrm{E}-03$ & & & \\
\hline Pathway Hazard Idnex & & & & & $4.6 \mathrm{E}+01$ & & \\
\hline \multicolumn{8}{|c|}{ Exposure Pathway: Inhalation of Chemicals by Adult Trespasser from Sediment in Cell } \\
\hline Barium & 7.7E-09 & $1.4 \mathrm{E}-04$ & $5.4 \mathrm{E}-05$ & & & & \\
\hline Manganese & $8.4 \mathrm{E}-08$ & $1.4 \mathrm{E}-05$ & $5.9 \mathrm{E}-03$ & & & & \\
\hline Mercury & $1.2 \mathrm{E}-09$ & $8.6 \mathrm{E}-05$ & $1.4 \mathrm{E}-05$ & & & & \\
\hline Total Inorganics & & & & $5.9 \mathrm{E}-03$ & & & \\
\hline Pathway Hazard Index & & & & & $5.9 \mathrm{E}-03$ & & \\
\hline Total Inorganic Risk & & & & & & 4.TE+01 & \\
\hline Total Organic Risk & & & & & & $5.9 \mathrm{E}-03$ & \\
\hline Total Area & & & & & & & $4.7 \mathrm{E}+01$ \\
\hline
\end{tabular}




\begin{tabular}{|c|c|c|c|c|c|c|c|}
\hline \multirow{2}{*}{ Chemcial } & \multirow{2}{*}{$\begin{array}{c}\text { Avernge } \\
\text { Doily Intake } \\
\text { (mg//g-day) }\end{array}$} & \multirow{2}{*}{$\begin{array}{c}\text { Reference } \\
\text { Dose } \\
\text { (mp/kg-day) }\end{array}$} & \multicolumn{5}{|c|}{ Hazand Quotient/ Hazand Index } \\
\hline & & & $\begin{array}{l}\text { Contaminant } \\
\text { Specific }\end{array}$ & $\begin{array}{c}\text { Contominant } \\
\text { Closs }\end{array}$ & Pathway & $\begin{array}{l}\text { Contaminant } \\
\text { Class Total }\end{array}$ & $\begin{array}{l}\text { Total } \\
\text { Area }\end{array}$ \\
\hline \multicolumn{8}{|c|}{ Exposure Pathway: Ingestion of Chemicals by Adult Trespesser from Core Samples - Whole Building } \\
\hline Arsenic & $3.9 \mathrm{E}-06$ & 3.0E-04 & $1.3 \mathrm{E}-02$ & & & & \\
\hline Barium & $9.9 \mathrm{E}-04$ & $7.0 \mathrm{E}-02$ & $1.4 \mathrm{E}-02$ & & & & \\
\hline Beryllium & $1.7 \mathrm{E}-05$ & $5.0 \mathrm{E}-03$ & $3.4 \mathrm{E}-03$ & & & & \\
\hline Cadmium & $2.5 \mathrm{E}-04$ & $1.0 \mathrm{E}-03$ & $2.5 \mathrm{E}-01$ & & & & \\
\hline Chromium VI & 2.7E-04 & $5.0 \mathrm{E}-03$ & $5.4 E-02$ & & & & \\
\hline Manganere & $1.3 \mathrm{E}-02$ & $1.4 \mathrm{E}-01^{\circ}$ & 9.3E-02 & & & & \\
\hline Mercury & 4.4E-07 & 3.0E-04 & $1.5 \mathrm{E}-03$ & & & & \\
\hline Nickel & $1.9 \mathrm{E}-04$ & $2.0 \mathrm{E}-02$ & $9.5 \mathrm{E}-03$ & & & & \\
\hline Vanadium & $3.3 \mathrm{E}-04$ & $7.0 \mathrm{E}-03$ & 4.7E-02 & & & & \\
\hline Zinc & $4.8 \mathrm{E}-02$ & $3.0 \mathrm{E}-01$ & $1.6 \mathrm{E}-01$ & & & & \\
\hline Total Inorganics & & & & $6.4 \mathrm{E}-01$ & & & \\
\hline Bis(2-ethylhexyl)phthalate & 3.3E-07 & $2.0 \mathrm{E}-02$ & $1.6 E-05$ & & & & \\
\hline Dimethyl phthalate & $7.5 \mathrm{E}-08$ & $1.0 \mathrm{E}+01$ & $7.5 \mathrm{E}-09$ & & & & \\
\hline Total Organics & & & & $1.6 \mathrm{E}-05$ & & & \\
\hline Pathway Hazard Index & & & & & $6.4 \mathrm{E}-01$ & & \\
\hline \multicolumn{8}{|c|}{ Exposure Pathway: Dermal Exposure of Chemicals to Adult Trespasser from Core Samples - Whole Building } \\
\hline Arsenic & 2.1E-07 & $1.2 \mathrm{E}-04$ & 1.7E-03 & & & & \\
\hline Barium & $5.2 \mathrm{E}-05$ & $4.9 \mathrm{E}-03$ & $1.1 \mathrm{E}-02$ & & & & \\
\hline Beryllium & $9.0 \mathrm{E}-07$ & $5.0 \mathrm{E}-05$ & $1.8 \mathrm{E}-02$ & & & & \\
\hline Cadmium & $1.3 \mathrm{E}-05$ & $1.0 \mathrm{E}-05$ & $1.3 \mathrm{E}+\infty 0$ & & & & \\
\hline Chromium VI & $1.4 \mathrm{E}-05$ & $1.0 \mathrm{E}-04$ & $1.4 \mathrm{E}-01$ & & & & \\
\hline Manganese & $6.9 \mathrm{E}-04$ & $5.6 \mathrm{E}-03$ & $1.2 \mathrm{E}-01$ & & & & \\
\hline Mercury & $2.3 \mathrm{E}-08$ & $3.0 \mathrm{E}-08$ & 7.7E-01 & & & & \\
\hline Nickel & $1.0 \mathrm{E}-05$ & $5.4 \mathrm{E}-03$ & $1.9 \mathrm{E}-03$ & & & & \\
\hline Vanadium & $1.7 \mathrm{E}-05$ & $7.0 \mathrm{E}-05$ & $2.5 \mathrm{E}-01$ & & & & \\
\hline Zinc & $2.5 \mathrm{E}-03$ & $6.0 \mathrm{E}-02$ & $4.2 \mathrm{E}-02$ & & & & \\
\hline Total Inorganics & & & & $2.7 E+00$ & & & \\
\hline Bis(2-ethylhexyl)phthalate & $1.7 \mathrm{E}-07$ & $3.8 \mathrm{E}-03$ & $4.6 \mathrm{E}-05$ & & & & \\
\hline Dimethyl phthalate & $4.0 \mathrm{E}-08$ & $1.0 \mathrm{E}+01$ & $4.0 \mathrm{E}-09$ & & & & \\
\hline Total Organics & & & & $4.6 \mathrm{E}-05$ & & & \\
\hline Pathway Hazard Index & & & & & $2.7 \mathrm{E}+\infty$ & & \\
\hline
\end{tabular}




\begin{tabular}{|c|c|c|c|c|c|c|c|}
\hline \multirow{2}{*}{ Chemcial } & \multirow{2}{*}{$\begin{array}{c}\text { Average } \\
\text { Daily Intake } \\
\text { (mg/kg-day) }\end{array}$} & \multirow{2}{*}{$\begin{array}{c}\text { Reference } \\
\text { Dose } \\
\text { (mg/kg-day) }\end{array}$} & \multicolumn{5}{|c|}{ Hazard Quotient/ Hazard Index } \\
\hline & & & $\begin{array}{l}\text { Contaminant } \\
\text { Specific }\end{array}$ & $\begin{array}{c}\text { Contaminant } \\
\text { Closs }\end{array}$ & Pathway & $\begin{array}{c}\text { Contaminnnt } \\
\text { Class Total }\end{array}$ & $\begin{array}{l}\text { Total } \\
\text { Area }\end{array}$ \\
\hline \multicolumn{8}{|c|}{ Exposure Pathway: Inhalation of Chemicals by Adult Trespasser from Core Samples - Whole Building } \\
\hline Barium & 4.6E-08 & $1.4 \mathrm{E}-04$ & 3.2E-04 & & & & \\
\hline Manganese & 6.1E-07 & 1.4E-05 & 4.3E-02 & & & & \\
\hline Mercury & 2.0E-11 & 8.6E-05 & $2.4 \mathrm{E}-07$ & & & & \\
\hline Total Inorganics & & & & 4.3E-02 & & & \\
\hline Pathway Hazard Index & & & & & 4.3E- 02 & & \\
\hline Total Inorganic Risk & & & & . & & $3.3 E+00$ & \\
\hline Total Organic Risk & & & & & & $6.2 \mathrm{E}-05$ & \\
\hline Total Area & & & & & & & $3.3 \mathrm{E}+\infty$ \\
\hline
\end{tabular}


1. T. Burwinkle

2. K. Constant

3. F. Cretella

4. K. Golden

5. J. Hooyman

6. M. Jugan

7. L. Kaiser

8. S. Kerr

9. W. Mcginn

10-16. G. Mandry

17. P. Moor

18. S. Robers

19. I. Smith

20. Central Research Library

21-25. ER Document Management Center

26-28. Laboratory Records Department

29. ORNL Patent Section 